\title{
RECTANGULAR DIAGRAMS OF SURFACES: DISTINGUISHING LEGENDRIAN KNOTS
}

\author{
IVAN DYNNIKOV AND MAXIM PRASOLOV
}

\begin{abstract}
In an earlier paper we introduced rectangular diagrams of surfaces and showed that any isotopy class of a surface in the three-sphere can be presented by a rectangular diagram. Here we study transformations of those diagrams and introduce basic moves that allow the transition between diagrams representing isotopic surfaces. We also introduce more general combinatorial objects called mirror diagrams and various moves for them that can be used to transform presentations of isotopic surfaces to each other. The moves are divided into two (non-exclusive) types so that, vaguely speaking, type I moves commute with type II ones. This commutation is the matter of the main technical result of the paper. We use it as well as a relation of the moves to Giroux's convex surfaces to propose a new method for distinguishing Legendrian knots. We apply this method to show that two Legendrian knots having topological type $6_{2}$ are not equivalent. More applications of the method will be the subject of subsequent papers.
\end{abstract}

\section{Contents}

1. Introduction

1.1. Prerequisites

1.2. Some general conventions and notation

1.3. Organization of the paper

2. A test for equivalence of Legendrian links

2.1. Dividing configurations

2.2. An invariant of Legendrian links

2.3. Distinguishing Legendrian links

2.4. An example: the knot $6_{2}$

3. Basic moves of rectangular diagrams of surfaces

3.1. Notation. Vertex types

3.2. Wrinkle moves and stabilization moves

3.3. Twisting a 'rectangular' surface around the boundary

3.4. Exchange moves

3.5. Weak equivalence of dividing configurations via neutral basic moves $\quad 30$

4. Spatial ribbon graphs $\quad 32$

4.1. The basic concept 33

4.2. Enhanced and patched spatial ribbon graphs 34

4.3. Morphisms of spatial ribbon graphs $\quad 35$

4.4. Handle moves of spatial ribbon graphs

5. Mirror diagrams $\quad 37$

5.1. Representing ribbon graphs by mirror diagrams 37

5.2. Simple boundary circuits and framed rectangular diagrams of links $\quad 41$

5.3. Rectangular diagrams of a surface as mirror diagrams

5.4. Canonic dividing configurations

6. Elementary moves of mirror diagrams 43

This work was performed at the Steklov International Mathematical Center and supported by the Ministry of Science and Higher Education of the Russian Federation (agreement no. 075-15-2019-1614). The work of M. Prasolov has also been partially supported by the Young Russian Mathematics award. 
6.1. Conventions

6.2. Elementary moves of mirror diagrams (without enhancement) 44

6.3. Elementary moves of enhanced mirror diagrams

6.4. Stable equivalence via elementary moves 48

7. More transformations. Neat decompositions 51

7.1. Preliminary remarks and conventions 51

7.2. Neutral moves

7.3. Split moves and merge moves 5

7.4. Handle decomposition of $\overparen{M}$ and partial homeomorphisms $h_{M}^{M^{\prime}}$

8. Mirror diagrams and the standard contact structure $\quad 62$

8.1. Legendrian graphs $\quad 62$

8.2. Divided Legendrian graphs

8.3. Type II moves from the point of view of $\xi_{+}$-divided Legendrian ribbon graphs 68

8.4. Subdiagrams of mirror diagrams $\quad 70$

8.5. Invariance of $\mathscr{I} \quad 73$

9. Commutation theorems $\quad 75$

9.1. Formulation and the strategy of the proof $\quad 75$

\begin{tabular}{lll|}
9.2 . Splitting routes and splitting paths & 78 \\
\hline
\end{tabular}

9.3. Generalized split and generalized merge moves 82

9.4. Commutation of moves 86

9.5. Similarity of splitting routes 94

9.6. First commutation property of generalized type II merge moves 105

9.7. Second commutation property of generalized type II merge moves, non-special case 110

9.8. Generalized wrinkle moves and flexibility $\quad 119$

9.9. Second commutation property of generalized type II merge moves, special case 121

9.10. Generalized type II bypass removals. First commutation property 125

9.11. Second commutation property of generalized type II bypass removals 130

9.12. Proof of the commutation theorems

Appendix A: Finding realizations

Appendix B: Flypes $\quad 141$

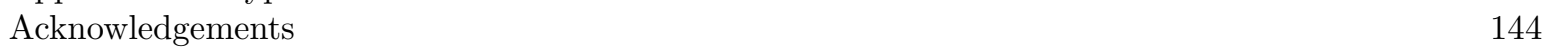

\begin{tabular}{lr} 
References & 144 \\
\hline
\end{tabular}

\section{INTRODUCTION}

For a pre-introduction the reader is referred to the work 12 .

This paper is focused on combinatorial properties of certain objects that generalize rectangular diagrams of links and provide for a nice way to represent surfaces and ribbon graphs in the three-sphere. We call these objects rectangular diagrams of surfaces, and mirror diagrams, respectively. The combinatorial formalism we develop here is strongly related to two contact structures of $\mathbb{S}^{3}$, the standard one, $\xi_{+}$, and its mirror image, $\xi_{-}$. This is by no means unexpected since rectangular diagrams of links are already known to provide for a convenient framework to study Legendrian links in $\mathbb{S}^{3}$.

Various diagrams of 'rectangular' kind that we consider represent topological objects that are in a nice position with respect to both contact structures $\xi_{+}$and $\xi_{-}$, simultaneously. Namely, links represented in the 'rectangular' way are Legendrian with respect to both contact structures, and surfaces represented in the 'rectangular' way are convex in Giroux's sense, also with respect to both contact structures. So, each single 'rectangular' object represents two topological objects that are of interest from the contact topology point of view. The key circumstance about these two objects is their mutual independence to an extent allowed by the topological settings.

In the case of links, this independence, which is discovered in [11, means that, for any $\xi_{+}$-Legendrian link type $\mathscr{L}_{+}$and $\xi_{-}$-Legendrian link type $\mathscr{L}_{-}$which belong to the same topological link type, there 
is a rectangular diagram representing both of them. Moreover, any stabilization and destabilization of one of these Legendrian link types can be realized, without altering the other, by elementary moves of rectangular diagrams.

The independence of any such $\mathscr{L}_{+}$and $\mathscr{L}_{-}$has remarkable consequences, one of which is a proof of the Jones conjecture [11. Another consequence is an algorithm for computing the maximal possible Thurston-Bennequin number of Legendrian links having a given topological link type.

In the present paper we formulate and prove a similar independence property for Giroux's convex surfaces. A simplified version of this property says that if $F_{+}$and $F_{-}$are isotopic closed surfaces in $\mathbb{S}^{3}$ such that $F_{+}$is convex (in Giroux's sense) with respect to $\xi_{+}$, and $F_{-}$is convex with respect to $\xi_{-}$, then there is a rectangular diagram of a surface $\Pi$ such that the associated surface $\widehat{\Pi}$ is isotopic to $F_{+}$through $\xi_{+}$-convex surfaces and to $F_{-}$through $\xi_{-}$-convex surfaces. The importance of this fact comes from the existence of an algorithm that, given certain combinatorial information about $F_{+}$and $F_{-}$, produces finitely many candidates for such $\Pi$ among which at least one has the required properties.

The information supplied to the algorithm includes the combinatorial structure of the dividing sets $\delta_{+}$ and $\delta_{-}$of $F_{+}$and $F_{-}$, respectively, and their mutual position. By dividing sets one means certain onedimensional submanifolds in Giroux's convex surfaces defined with a reference to the respective contact structures. The isotopy class of a dividing set is a very strong invariant of a convex surface as discovered by E. Giroux [25. The mutual position of $\delta_{+}$and $\delta_{-}$is understood as follows. The surfaces $F_{+}$and $F_{-}$ are identified using the given isotopy between them, so $\delta_{+}$and $\delta_{-}$are viewed as submanifolds in a single surface. After putting them in general position the union $\delta_{+} \cup \delta_{-}$is a finite one-dimensional CW-subcomplex of the surface.

The algorithm mentioned above takes a combinatorial description of $\delta_{+} \cup \delta_{-}$as the input and produces finitely many rectangular diagrams of a surface such that at least one of them has the desired properties, that is, represents a surface isotopic to $F_{+}$through $\xi_{+}$-convex surfaces and to $F_{-}$through $\xi_{-}$-convex ones. This can be used, for an arbitrary given one-dimensional submanifold $\delta$ of a surface $F \in \mathbb{S}^{3}$, to decide whether or not the isotopy class of $\delta$ can be realized by a dividing set of a convex surface isotopic to $F$.

This approach extends, with some limitations, to surfaces with Legendrian boundary, providing for a powerful tool for distinguishing Legendrian knots.

The comparing of Legendrian knots having the same classical invariants (which are topological type, Thurston-Bennequin number, and rotation number) is a difficult problem in general. The first success in this direction was made in Yu. Chekanov's work [5, where he distinguished two Legendrian knots having topological type $5_{2}$ by means of new algebraic invariants. The latter were extracted from a differential graded algebra associated with a Lagrangian projection of the knot. Similar construction appeared about the same time in Ya. Eliashberg's work [17.

Further algebraic invariants having proved useful to distinguish Legendrian knots are constructed by D. Fuchs [24, L. Ng [37, 36], P.Pushkar' and Yu. Chekanov [40, and by P. Ozsváth, Z. Szabó, and D. Thurston [38.

However, algebraic invariants do not provide for a systematic way to compare Legendrian knots. There are still many examples of pairs of Legendrian knot types for which the existing evidence suggests that they are distinct but the known computable invariants fail to confirm that. The reader is referred to the Legendrian knot atlas by W. Chongchitmate and L. Ng [6] for an overview of the state of the art.

A number of classification results on Legendrian knots, which are due to Y. Eliashberg and M. Fraser [18, 19, J.Etnyre and K. Honda [20, J.Etnyre, D. LaFountain, and B. Tosun[21], J.Etnyre, L. Ng, and V. Vértesi [22, J. Etnyre and V. Vértesi [23, are obtained by means of a different approach based on the study of Giroux's convex surfaces and characteristic foliations.

Even if Legendrian types are classified for a given topological type of a knot, it may be still difficult to recognize them if the knot is not Legendrian simple. This is where the technique of the present paper may also be useful, since it allows one, under certain circumstances, to find convex surfaces with desired structure of the dividing set or to prove that no such surface exists. Combined with the ideas of the paper [30] by K. Honda, W. Kazez, and G. Matić, where Haken hierarchies built from convex surfaces are studied, this approach has a potential to yield a complete algorithm for comparing Legendrian knots. 
The biggest difficulty with making this work comes from the fact that the topological orientationpreserving symmetry group of a knot may be infinite and is unknown in general1. If this group is known to be trivial for a given knot type, then comparing Legendrian knots of this topological type becomes fairly easy as explained in [15, 16. In particular, Conjecture 1 of 12 is confirmed there, as well as a number of conjectures of [6] about concrete knots with trivial orientation-preserving symmetry group. This includes the topological types $9_{42}-9_{45}, 10_{128}$, and $10_{160}$.

In order to illustrate our method here, we picked the simplest unresolved case from [6], which deals with two conjecturably inequivalent Legendrian knots having topological type $6_{2}$. We show that the knots are indeed not Legendrian equivalent. Up to this writing, we have also confirmed, in a similar fashion, the conjectures of [6] about Legendrian knots having topological type $7_{6}$ and maximizing the Thurston-Bennequin number [13]. This is the example we tried next after $6_{2}$. It looks quite feasible to resolve all open question in [ 6] in the near future by means of the method described in this paper.

The techniques developed in this paper was originally motivated by an attempt to extend the monotonic simplification approach of 7 to general links. This approach utilized the ideas of the preceding works by J. Birman and W. Menasco [3, 4, and P. Cromwell [8. W. Menasco's work 34, though not explicitly used here, has given us a hint for understanding the deep connection between the monotonic simplification approach and contact topology (the graphs $G_{++} \cup G_{--}$and $G_{+-} \cup G_{-+}$in that work are close analogues of the Giroux graphs, which are behind the scenes of our method as outlined in Section 8).

1.1. Prerequisites. We will use the following definitions and notation from paper [12]:

- coordinate system $(\theta, \varphi, \tau)$ on $\mathbb{S}^{3}$ coming from the join presentation $\mathbb{S}^{3}=\mathbb{S}^{1} * \mathbb{S}^{1}$,

- torus projection,

- rectangular diagram of a link,

- link $\widehat{R}$ associated with a rectangular diagram of a link $R$,

- connected component of a rectangular diagram of a link,

- oriented rectangular diagram of a link,

- cusp-free curve,

- framed link,

- framed rectangular diagram of a link,

- rectangular diagram of a graph,

- admissible framing of a link of the form $\widehat{R}$,

- graph $\widehat{G}$ associated with a rectangular diagram of a graph $G$,

- a rectangle $r \subset \mathbb{T}^{2}$,

- tile $\widehat{r}$ associated with a rectangle $r \in \mathbb{T}^{2}$;

- rectangular diagram of a surface,

- surface $\widehat{\Pi}$ associated with a rectangular diagram of a surface $\Pi$,

- boundary of a rectangular diagram of a surface,

- the contact structures $\xi_{ \pm}$on $\mathbb{S}^{3}$

- Thurston-Bennequin numbers $\operatorname{tb}_{ \pm}(L)$,

- relative Thurston-Bennequin numbers $\operatorname{tb}_{ \pm}(L ; F)$,

- surface with corners,

- contact line element field,

- Giroux's convex surface,

- 0 -arc and -1-arc,

- ((very) nice) characteristic foliation,

- the Giroux graph of a convex surface with a very nice characteristic foliation,

- an extended Giroux graph of a convex surface with a very nice characteristic foliation,

- equivalence of Giroux's convex surfaces with corners,

- exchange moves of rectangular diagrams of links,

- type I (type II) (de)stabilization of a rectangular diagram of a link.

\footnotetext{
${ }^{1}$ Since submitting the original version of this paper we have found a way to overcome this difficulty. In a future paper we will show that equivalence of Legendrian knots is decidable.
} 
1.2. Some general conventions and notation. We work in the piecewise smooth category. Surfaces that we consider are assumed to be $C^{1}$-smooth and are allowed to have corners at the boundary according to [12, Definition 5]. Isotopies of various objects in $\mathbb{S}^{3}$ are understood as those that can be extended to ambient piecewise- $C^{1}$ isotopies.

Unless otherwise specified, an isotopy of a surface is assumed to be performed within the class of surfaces with corners so that the tangent plane to the surface depends continuously on $(x, t)$, where $x$ is a point of the surface, and $t$ is the isotopy parameter. This matters when we consider an isotopy of a surface with corners relative to its boundary - the tangent plane to the surface at a singularity of the boundary must remain fixed during the isotopy. More general isotopies, which are not required to keep the surface in the class of surfaces with corners, are referred to as $C^{0}$-isotopies. 'An isotopy in the class of Giroux's convex surfaces' is understood as stated in [12, Definition 25].

If $X$ is an arc or a surface we use the notation int $(X)$ for $X \backslash \partial X$.

If $\Pi$ is a rectangular diagram of a surface (see [12, Definition 1], by a vertex of $\Pi$ we call a vertex of any rectangle $r \in \Pi$, and by an occupied level of $\Pi$ we mean a meridian $\{\theta\} \times \mathbb{S}^{1}$ or a longitude $\mathbb{S}^{1} \times\{\varphi\}$ of $\mathbb{T}^{2}=\mathbb{S}^{1} \times \mathbb{S}^{1}$ that contains a vertex of $\Pi$ (see Subsection 3.1 for details).

The intersection points of the associated surface $\widehat{\Pi}$ (see [12, Definition 9]) with the circles $\mathbb{S}_{\tau=1}^{1}$ and $\mathbb{S}_{\tau=0}^{1}$ are called the vertices of $\widehat{\Pi}$, and the sides of the tiles in $\widehat{\Pi}$ the edges of $\widehat{\Pi}$.

Throughout the paper $\mathbb{S}^{1}$ stands for an oriented circle. If $p, q \in \mathbb{S}^{1}$ are two distinct points, we denote by $[p ; q]$ the $\operatorname{arc} \alpha \subset \mathbb{S}^{1}$ such that, if we view it as a 1-chain, then $\partial \alpha=q-p$. Accordingly, $[p ; q),(p ; q]$, and $(p ; q)$ denote $[p ; q] \backslash\{q\},[p ; q] \backslash\{p\}$, and $[p ; q] \backslash\{p, q\}$, respectively.

We use a similar notation for intervals of meridians and longitudes of $\mathbb{T}^{2}$ (which are also oriented). For instance, if $p$ and $q$ are two distinct points of a meridian $m$, then $(p ; q)$ and $(q ; p)$ refer to disjoint intervals of $m$.

In order to avoid confusion, we denote by $(x, y)$ a point with coordinates $x$ and $y$, and by $(x ; y),[x ; y]$, etc., the intervals between $x$ and $y$.

1.3. Organization of the paper. In Section 2 we describe the main application of the machinery developed in this paper. As an illustration of the method, we demonstrate how two Legendrian knots whose inequivalence has been previously unknown can be distinguished. Some technical details of the proof are placed in the Appendix A at the end of the paper.

In Section 3 we introduce certain transformations, called basic moves, of rectangular diagrams of surfaces, and discuss their properties. The discussion of one of the basic moves, called a flype, is postponed till Appendix B since it is not involved in the proof of the main result.

Section 4 consists mostly of definitions of various objects related to spatial ribbon graphs. One of the ideas behind our approach is that dealing with ribbon graphs, which are essentially one-dimensional objects, is much easier than dealing with surfaces, whereas ribbon graphs may carry enough information about the surfaces that we want to study.

Spatial ribbon graphs are conveniently represented by yet another kind of rectangular diagrams, which we call mirror diagrams. These are introduced in Section 5 . The moves that generate important equivalence relations between mirror diagrams are defined in Section [6. In Section 7 further moves are introduced, and various relations between them are established.

In section 8 we discuss the connection between contact topology and mirror diagrams in a more detail. Though this connection is the actual origin of the ideas behind the key technical result of this paper, which is the relative commutation theorem, its formalization appears to us more difficult than presenting the proof in purely combinatorial terms. The latter is done in Section 9 where the formulation of the commutation theorems is also given.

\section{A TEST FOR EQUIVALENCE OF LEGENDRIAN LINKS}

\subsection{Dividing configurations.}

Definition 2.1. Let $F$ be a compact surface (with or without boundary, not necessarily orientable and connected). By an abstract dividing set on $F$ we mean an oriented one-dimensional submanifold $\delta \subset F$ such that $\delta \cap \partial F=\partial \delta$ and the following holds: if $d \subset F$ is a closed embedded disc such that $d \cap \delta \subset \partial d$, 
then $\partial d$ admits an orientation that agrees with the orientation of $\delta$ on every arc in $\partial d \cap \delta$. Additionally, each non-orientable connected component of $F$ is required to have a non-empty intersection with $\delta$.

Another characterization of an abstract dividing set $\delta \subset F$ is as follows. Let $F^{\prime}$ be the compact surface obtained from $F$ by cutting along $\delta$ (so that $\delta$ is doubled), and $\pi: F^{\prime} \rightarrow F$ be the natural projection. Then $\delta$ is an abstract dividing set if and only if $F^{\prime}$ admits an orientation such that the induced orientation of $\partial F^{\prime}$ coincides on $\pi^{-1}(\delta)$ with the one inherited from $\delta$.

Definition 2.2. An ordered pair $D=\left(\delta^{+}, \delta^{-}\right)$of abstract dividing sets on $F$ is called a dividing configuration on $F$. A dividing configuration $\left(\delta^{+}, \delta^{-}\right)$is called admissible if the following holds:

(1) $\delta^{+}$and $\delta^{-}$are transverse to one another;

(2) each connected component of $\delta^{+}$non-trivially intersects $\delta^{-}$, and vice versa;

(3) the intersection $\delta^{+} \cap \delta^{-}$is disjoint from $\partial F$;

(4) each connected component of $F \backslash\left(\delta^{+} \cup \delta^{-}\right)$is contractible and has either empty or contractible intersection with $\partial F$.

Dividing configurations (or abstract dividing sets) related by a self-homeomorphism of $F$ homotopic to the identity are regarded as equivalent.

Two dividing configurations $\left(\delta_{1}^{+}, \delta_{1}^{-}\right),\left(\delta_{2}^{+}, \delta_{2}^{-}\right)$on $F$ are weakly equivalent if $\delta_{1}^{+}$is equivalent to $\delta_{2}^{+}$ and $\delta_{1}^{-}$is equivalent to $\delta_{2}^{-}$, that is, if there are two self-homeomorphisms of $F$ homotopic to the identity such that one of them takes $\delta_{1}^{+}$to $\delta_{2}^{+}$, and the other $\delta_{1}^{-}$to $\delta_{2}^{-}$.

Lemma 2.1. Let $D=\left(\delta^{+}, \delta^{-}\right)$be a dividing configuration on a compact surface $F$. An admissible dividing configuration on $F$ weakly equivalent to $D$ exists if and only if the following two conditions hold:

(1) any connected component of $F$ has a non-empty intersection with both $\delta^{+}$and $\delta^{-}$;

(2) any connected component of $\partial F$ has a non-empty intersection with $\delta^{+} \cup \delta^{-}$.

We omit the easy proof.

The homeomorphism class of a compact surface $F$ endowed with an admissible dividing configuration $D=\left(\delta^{+}, \delta^{-}\right)$is a simple combinatorial object that can be encoded as follows. Number all the intersection points of $\delta^{+}$with $\delta^{-}$. For $\gamma$ a connected component of $\delta^{+}$or $\delta^{-}$, denote by $s(\gamma)$ the sequence obtained by listing the numbers of all the points from $\delta^{+} \cap \delta^{-}$contained in $\gamma$ in the order they follow on $\gamma$. If $\gamma$ is a closed curve, we start from any of the points, go around $\gamma$ once, and finish by repeating the starting number. Let $\gamma_{i}^{+}, i=1, \ldots, k$, be the connected components of $\delta^{+}$, and $\gamma_{i}^{-}, i=1, \ldots, l$, the connected components of $\delta^{-}$.

One can see that the homeomorphism class of $(F, D)$ can be recovered from the following data:

$$
\left(\left\{s\left(\gamma_{1}^{+}\right), \ldots, s\left(\gamma_{k}^{+}\right)\right\},\left\{s\left(\gamma_{1}^{-}\right), \ldots, s\left(\gamma_{l}^{-}\right)\right\}\right),
$$

which will be referred to as a dividing code of $(F, D)$. There is an arbitrariness in the definition of a dividing code, and there are certain restrictions on the data that can occur as a dividing code of a surface endowed with a dividing configuration. We need not discuss these issues here.

Note, however, that the set of all possible dividing codes of an admissible dividing configuration is determined by any single one. Two dividing codes are said to be isomorphic if they are associated with the same homeomorphism class of surfaces endowed with an admissible dividing configuration.

Definition 2.3. Let $\Pi$ be a rectangular diagram of a surface (see [12, Section 2] for the definition). A dividing configuration $D=\left(\delta^{+}, \delta^{-}\right)$on the associated surface $\widehat{\Pi}$ will be said to be canonic if the intersection of $\delta^{+}$(respectively, $\delta^{-}$) with every tile $\widehat{r}, r \in \Pi$, is an arc connecting the midpoints of two opposite sides of $\widehat{r}$, and the functions $\theta, \varphi$ (respectively, $-\theta, \varphi$ ) are locally increasing on $\delta^{+}$(respectively, $\delta^{-}$); see Figure 2.1.

This definition is motivated by the fact that any surface of the form $\widehat{\Pi}$ is convex in Giroux's sense with respect to both contact structures $\xi_{+}$and $\xi_{-}$, and submanifolds $\delta^{ \pm}$forming a canonic dividing configuration are suitable for the respective dividing sets, see [12, Subsection 4.4]. 

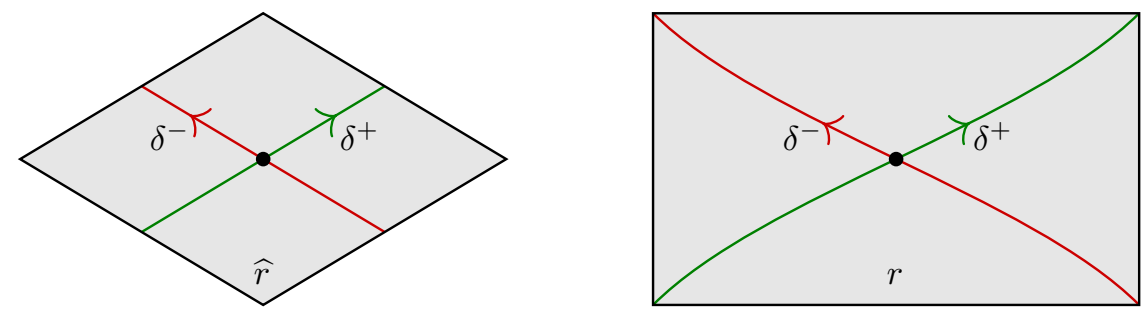

FiguRE 2.1. A canonic dividing configuration in a single tile and its torus projection

Note that if $\left(\delta^{+}, \delta^{-}\right)$is a canonic dividing configuration of $\widehat{\Pi}$, then every tile of $\widehat{\Pi}$ contains exactly one intersection point of $\delta^{+}$with $\delta^{-}$. Thus, there is a natural bijection $\delta^{+} \cap \delta^{-} \leftrightarrow \Pi$.

A canonic dividing configuration of $\widehat{\Pi}$ is an object that is dual to the tiling of $\widehat{\Pi}$ in a natural sense. Namely, if $\widehat{\Pi}$ is a closed surface, then its canonic dividing configuration makes up the 1-skeleton of a cell decomposition of $\widehat{\Pi}$ dual to the tiling.

Proposition 2.1. For any rectangular diagram of a surface $\Pi$ :

(i) there exists a canonic dividing configuration of $\widehat{\Pi}$;

(ii) a canonic dividing configuration of $\widehat{\Pi}$ is admissible;

(iii) any two canonic dividing configurations of $\widehat{\Pi}$ are equivalent.

The proof is easy and left to the reader. We only remark that part (i) of this proposition just says that the oriented submanifolds $\delta^{ \pm}$in Definition 2.3 satisfy the orientation compatibility condition of Definition 2.1.

Definition 2.4. Let $D=\left(\delta^{+}, \delta^{-}\right)$be a dividing configuration on a compact surface $F$. By a realization of $D$ we call a pair $(\Pi, \phi)$ in which $\Pi$ is a rectangular diagram of a surface, and $\phi$ is an embedding $\phi$ : $F \rightarrow \mathbb{S}^{3}$ such that $\phi(F)=\widehat{\Pi}$ and $\phi$ takes $D$ to a canonic dividing configuration on $\widehat{\Pi}$.

If $F \subset \mathbb{S}^{3}$ is a compact surface embedded in $\mathbb{S}^{3}$, and $D$ is a dividing configuration on $F$, then by a proper realization of $D$ we call a realization $(\Pi, \phi)$ such that $\phi$ can be extended to an orientationpreserving self-homeomorphism of $\mathbb{S}^{3}$. Such an extension will be assumed to be chosen for each proper realization and denoted by the same letter as the embedding $F \rightarrow \mathbb{S}^{3}$ being extended.

All the useful information about a realization $(\Pi, \phi)$ of $D=\left(\delta^{+}, \delta^{-}\right)$is actually contained in the diagram $\Pi$ equipped with the induced correspondence between the rectangles of $\Pi$ and the points from $\delta^{+} \cap$ $\delta^{-}$, so we will often omit mentioning the corresponding embedding $\phi$ and refer to the diagram $\Pi$ as a realization of $D$.

Suppose we are given a dividing code $\left(\left\{s_{1}^{+}, \ldots, s_{k}^{+}\right\},\left\{s_{1}^{-}, \ldots, s_{l}^{-}\right\}\right)$of an admissible dividing configuration, and want to find its realizations. If properness of the realization is not required, then this task amounts to finding rectangular diagrams $\Pi$ that admit a numeration of rectangles such that every number assigned to a rectangle $r \in \Pi$ appears in the sequence $s_{i}^{+}$for some $i$ (and also in the sequence $s_{j}^{-}$for some $j$ ) and the following holds:

(1) the top right vertex of the $i$ th rectangle of $\Pi$ coincides with the bottom left vertex of the $j$ th one if and only if $i$ is followed by $j$ in one of the sequences $s_{m}^{+}, m=1, \ldots, k$;

(2) the top left vertex of the $i$ th rectangle of $\Pi$ coincides with the bottom right vertex of the $j$ th one if and only if $i$ is followed by $j$ in one of the sequences $s_{m}^{-}, m=1, \ldots, l$.

Example 2.1. Shown in Figure 2.2 on the left is a Seifert surface for the Figure Eight knot endowed with a dividing configuration $D=\left(\delta^{+}, \delta^{-}\right)$, with $\delta^{+}$shown in green and $\delta^{-}$in red (see Convention 2.1 below for further clarification). This configuration can be encoded as follows:

$$
\{(1,2,3,4,5),(6),(7)\},\{(1,7,5,6,1),(2),(3),(4)\} .
$$


The central picture in Figure 2.2 provides an example of a proper realization of $D$ (more precisely, it is a rectangular diagram of a surface that gives rise to a proper realization of $D$ ). The intersection points of $\delta^{+}$and $\delta^{-}$and the rectangles of the realization are numbered accordingly.

The right picture in Figure 2.2 provides an example of a realization of $D$ that is not proper. Indeed, one can see that the boundary of the corresponding surface is unknotted.
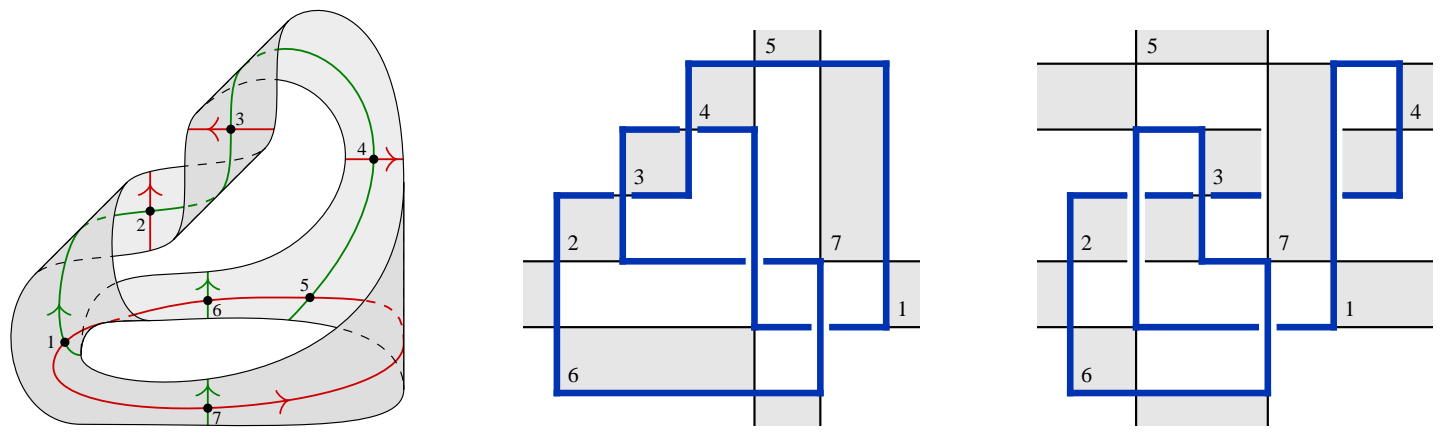

Figure 2.2. A surface with a dividing configuration (left), a proper realization of this configuration (center), and a realization that is not proper (right)

Convention 2.1. The style in which the pictures in Figure 2.2 are drawn will be accepted throughout the paper. Namely, if a picture is supposed to show a dividing configuration $\left(\delta^{+}, \delta^{-}\right)$, then $\delta^{+}$will be shown in green and $\delta^{-}$in red.

A canonic dividing configuration $\left(\delta^{+}, \delta^{-}\right)$of a surface presented by a rectangular diagram is never displayed at the diagram because it has a standard form in each rectangle (as shown in Figure 2.1) and carries no additional information. The rectangles are typically numbered, and the points of $\delta^{+} \cap \delta^{-}$are numbered accordingly.

In our illustrations, rectangular diagrams of a surface will often be accompanied by a rectangular diagram of their boundary link (as the two diagrams in Figure 2.2). The latter will be drawn in the most natural way for human perception, which usually corresponds neither to the boundary framing (see [12, Definitions 12-14 and Figure 12]) nor to the boundary circuits (see Definition [5.2 below). We draw the horizontal edges passing under all the rectangles, and the vertical edges passing over all the rectangles with exceptions made for the rectangles having a side that is contained in an edge. We find this way of drawing our diagrams the most suggestive.

Definition 2.5. Two rectangular diagrams of a surface (of a link, of a graph) are said to be combinatorially equivalent (or of the same combinatorial type) if one can be taken to the other by a selfhomeomorphism of $\mathbb{T}^{2}$ that has the form $(\theta, \varphi) \mapsto(f(\theta), g(\varphi))$, where $f$ and $g$ are orientation-preserving self-homeomorphisms of $\mathbb{S}^{1}$.

Example 2.1 demonstrates that canonic dividing configurations of combinatorially non-equivalent diagrams may have the same dividing code. The following statement is obvious.

Proposition 2.2. If $D$ is a canonic dividing configuration of a rectangular diagram $\Pi$ of a surface, then the isomorphism class of a dividing code of $D$ is uniquely determined by the combinatorial type of $\Pi$.

For any $n$, there are only finitely many combinatorial types of rectangular diagrams of surfaces having exactly $n$ rectangles.

\subsection{An invariant of Legendrian links.}

Convention 2.2. By a a link in this paper, we mean a piecewise smooth link in $\mathbb{S}^{3}$ whose components are ordered. Thus, by saying that a $\operatorname{link} L=\cup_{i} L_{i}$ is taken to $L^{\prime}=\cup_{i} L_{i}^{\prime}$ by a homeomorphism $\phi$, where $L_{i}$ and $L_{i}^{\prime}$ are connected components, $i=1,2, \ldots$, we mean that $\phi$ takes $L_{i}$ to $L_{i}^{\prime}$ for each applicable $i$. If 
the links $L$ and $L^{\prime}$ are oriented (which will typically be the case), then by writing $\phi(L)=L^{\prime}$ we assume that $\phi$ preserves the orientation.

The same refers to rectangular diagrams of links: connected components are silently assumed to be ordered, and the components of the associated link are assumed to be ordered respectively.

Definition 2.6. Let $L$ be a fixed oriented link, and let $R$ be an oriented rectangular diagram of a link such that $L$ and $\widehat{R}$ are isotopic. Let also $F \subset \mathbb{S}^{3}$ be a compact surface such that $F \cap L$ is a sublink of $\partial F$. We say that $F$ is +-compatible (respectively, --compatible) with $R$ if for some (and then any) orientation-preserving homeomorphism $\phi: \mathbb{S}^{3} \rightarrow \mathbb{S}^{3}$ taking $L$ to $\widehat{R}$ we have tb+ $(K, \phi(F)) \leqslant 0$ (respectively, tb- $(K, \phi(F)) \leqslant 0)$ for any connected component $K$ of $\phi(L \cap \partial F)$.

This definition is motivated by Theorem 1 of $\left[12\right.$, which states, in the present terms, that the $C^{0}-$ isotopy class of a surface $F$ can be represented by a rectangular diagram so that a link $L$ related to $F$ as above is simultaneously represented by a given rectangular diagram of a link $R$, if and only if $F$ is both +-compatible and --compatible with $R$.

Definition 2.7. Let $L \subset \mathbb{S}^{3}$ be a fixed oriented link, and let $F \subset \mathbb{S}^{3}$ be a fixed compact surface such that $F \cap L$ is a sublink of $\partial F$. Let also $R$ be an oriented rectangular diagram of a link. We say that an abstract dividing set $\delta \subset F$ is properly +-realizable (respectively, properly --realizable) at $R$ if there exists an abstract dividing set $\delta^{\prime} \subset F$ and a proper realization $(\Pi, \phi)$ of $\left(\delta, \delta^{\prime}\right)$ (respectively, of $\left(\delta^{\prime}, \delta\right)$ ) such that $\phi(L)=\widehat{R}$. In this case we also say that $(\Pi, \phi)$ is a proper + -realization of $\delta$ (respectively, a proper--realization of $\delta$ ) at $R$.

The set of equivalence classes of all properly +-realizable (respectively, properly --realizable) abstract dividing sets at $R$ will be denoted by $\mathscr{I}_{F, L,+}(R)$ (respectively, $\mathscr{I}_{F, L,-}(R)$ ).

Theorem 2.1. Let $L \subset \mathbb{S}^{3}$ be a fixed oriented link, and let $F \subset \mathbb{S}^{3}$ be a fixed compact surface such that $F \cap L$ is a sublink of $\partial F$. Let also $R, R^{\prime}$ be oriented rectangular diagrams of a link such that the links $\widehat{R}$ and $\widehat{R}^{\prime}$ are equivalent to L, and $F$ is --compatible with both of them. If $\widehat{R}$ and $\widehat{R}^{\prime}$ are equivalent as Legendrian links, then $\mathscr{I}_{F, L,+}(R)=\mathscr{I}_{F, L,+}\left(R^{\prime}\right)$.

If one replaces the standard contact structure $\xi_{+}$by its mirror image $\xi_{-}$in the definition of a Legendrian link, then the above statement holds for $\mathscr{I}_{F, L,-}$ in place of $\mathscr{I}_{F, L,+}$ and +-compatibility in place of -compatibility.

This statement is a consequence of the representability result of [12], properties of dividing sets of Giroux's convex surfaces [25], and the fact that, for a rectangular diagram of a surface $\Pi$, the associated surface $\widehat{\Pi}$ is convex in Giroux's sense with respect to both contact structures $\xi_{ \pm}$, and a pair of dividing sets of $\widehat{\Pi}$ with respect to $\xi_{+}$and $\xi_{-}$forms a canonic dividing configuration on $\widehat{\Pi}$.

A proof can also been given in a more combinatorial manner, which will be done in Section 8 .

2.3. Distinguishing Legendrian links. In view of Theorem 2.1, to distinguish Legendrian links presented by oriented rectangular diagrams $R_{1}$ and $R_{2}$, say, with $\widehat{R}_{1}$ and $\widehat{R}_{2}$ isotopic to a fixed oriented link $L$, it suffices to show that $\delta \in \mathscr{I}_{F, L,+}\left(R_{1}\right)$ and $\delta \notin \mathscr{I}_{F, L,+}\left(R_{2}\right)$ hold for some surface $F \subset \mathbb{S}^{3}$ such that $F \cap L$ is a sublink of $\partial F$ and $F$ is --compatible with $R_{1}, R_{2}$, and an abstract dividing set $\delta$ on $F$. Clearly, the claim $\delta \in \mathscr{I}_{F, L,+}(R)$, if true, can be confirmed by producing a realization explicitly. The main achievement of this paper is a method for proving, under certain circumstances, claims of the opposite kind, $\delta \notin \mathscr{I}_{F, L,+}(R)$. The method is based on the following statement.

Theorem 2.2. Let $L \subset \mathbb{S}^{3}$ be a fixed oriented link, $F \subset \mathbb{S}^{3}$ a fixed compact surface such that $F \cap L$ is a sublink of $\partial F$, and let $R$ be an oriented rectangular diagram of a link. Let also $\delta_{0}$ and $\delta_{1}$ be two abstract dividing sets on $F$. Assume that there exist a proper + -realization $\left(\Pi_{0}, \phi_{0}\right)$ of $\delta_{0}$ and a proper --realization $\left(\Pi_{1}, \phi_{1}\right)$ of $\delta_{1}$ at $R$. Assume also that there exists a $C^{0}$-isotopy $\phi_{t}, t \in[0 ; 1]$, from $\phi_{0}$ to $\phi_{1}$ fixed at $L$.

Then there exists an admissible dividing configuration $\left(\delta^{+}, \delta^{-}\right)$weakly equivalent to $\left(\delta_{0}, \delta_{1}\right)$, and for any such dividing configuration there exist a proper realization $(\Pi, \xi)$ of $\left(\delta^{+}, \delta^{-}\right)$and a rectangular diagram of a link $R^{\prime}$ such that $\xi(L)=\widehat{R}^{\prime}$ and $R^{\prime}$ is obtained from $R$ by a finite sequence of exchange moves. 
Proof. We use here many intermediate results that are distributed over the Sections 3 9, We also use terminology and notation introduced in those sections. So, logically this proof belongs to the end of Section 9. However, we place it here in belief that the reader will profit from seeing the general idea and the structure of the proof before falling into details (some of which may be pretty boring).

By the assumption of the theorem $\Pi_{0}$ and $\Pi_{1}$ are rectangular diagrams of a surface such that $\phi_{i}(F)=$ $\widehat{\Pi}_{i}, i=0,1$. The embedding $\phi_{0}$ realizes the dividing configuration $\left(\delta_{0}, \delta_{0}^{\prime}\right)$ with some $\delta_{0}^{\prime}$, and $\phi_{1}$ realizes $\left(\delta_{1}^{\prime}, \delta_{1}\right)$ with some $\delta_{1}^{\prime}$. We give the proof in eight steps, of which the most tricky is Step 6.

Step 1. Prove the existence of an admissible dividing configuration $\left(\delta^{+}, \delta^{-}\right)$weakly equivalent to $\left(\delta_{0}, \delta_{1}\right)$ : Let $\gamma$ be a connected component of $\partial F$, and let $Q_{0}$ and $Q_{1}$ be the components of $\partial \Pi_{0}$ and $\partial \Pi_{1}$, respectively, corresponding to $\gamma$, that is, such that $\widehat{Q}_{0}=\phi_{0}(\gamma)$ and $\widehat{Q}_{1}=\phi_{1}(\gamma)$. Then the number of points in $\gamma \cap \delta_{0}$ (respectively, in $\gamma \cap \delta_{1}$ ) is equal to $-2 \mathrm{tb}_{+}\left(Q_{0} ; \Pi_{0}\right)$ (respectively, to $-2 \mathrm{tb}_{-}\left(Q_{1} ; \Pi_{1}\right)$ ) 2 . We also have

$$
\mathrm{tb}_{+}\left(Q_{0} ; \Pi_{0}\right)+\mathrm{tb}_{-}\left(Q_{1} ; \Pi_{1}\right)=\mathrm{tb}_{+}\left(Q_{0}\right)-\mathrm{lk}\left(\gamma, \gamma^{F}\right)+\mathrm{tb}_{-}\left(Q_{1}\right)+\mathrm{lk}\left(\gamma, \gamma^{F}\right)=\mathrm{tb}_{+}\left(Q_{0}\right)+\mathrm{tb}_{-}\left(Q_{1}\right) .
$$

The latter sum must be negative, since, by [11, Theorem 7], there exists a rectangular diagram of a knot $Q$ such that $\left(\mathrm{tb}_{+}(Q), \mathrm{tb}_{-}(Q)\right)=\left(\mathrm{tb}_{+}\left(Q_{0}\right), \mathrm{tb}_{-}\left(Q_{1}\right)\right)$. The number of vertices in $Q$ equals $-2\left(\mathrm{tb}_{+}(Q)+\right.$ $\left.\mathrm{tb}_{-}(Q)\right)$, hence $\mathrm{tb}_{+}(Q)+\mathrm{tb}_{-}(Q)<0$.

Thus, for any connected component $\gamma$ of $\partial F$, at least one of the sets $\gamma \cap \delta_{0}$ and $\gamma \cap \delta_{1}$ is not empty. The intersection of any connected component of $F$ with both $\delta_{0}$ and $\delta_{1}$ is also not empty, since, by the assumption of the theorem, the abstract dividing sets $\delta_{0}$ and $\delta_{1}$ have realizations (+- or --). By Lemma 2.1 there exists an admissible dividing configuration $\left(\delta^{+}, \delta^{-}\right)$weakly equivalent to $\left(\delta_{0}, \delta_{1}\right)$.

Step 2. Reduce to the case when $L \subset \partial F$ :

Let $\gamma$ be a connected component of $L \backslash \partial F$, and let $Q$ be the connected component of $R$ corresponding to $\gamma$. Let also

$$
\left(\theta_{1}, \varphi_{1}\right),\left(\theta_{1}, \varphi_{2}\right),\left(\theta_{2}, \varphi_{2}\right), \ldots,\left(\theta_{m}, \varphi_{m}\right),\left(\theta_{m}, \varphi_{1}\right)
$$

be the vertices of $Q$ listed in the order they follow on $Q$. For $\varepsilon>0$ denote by $\Pi^{\varepsilon}$ the following collection of rectangles:

$$
\Pi^{\varepsilon}=\left\{\left[\theta_{i} ; \theta_{i}+\varepsilon\right] \times\left[\varphi_{i}+\varepsilon ; \varphi_{i+1}\right],\left[\theta_{i}+\varepsilon ; \theta_{i+1}\right] \times\left[\varphi_{i+1} ; \varphi_{i+1}+\varepsilon\right]\right\}_{i=1, \ldots, m},
$$

where indices are regarded modulo $m$. If $\varepsilon$ is small enough, then $\Pi^{\varepsilon}$ is a rectangular diagram of a surface, and the associated surface $\widehat{\Pi}^{\varepsilon}$ is an annulus disjoint from $\widehat{\Pi}_{0}$ and $\widehat{\Pi}_{1}$. Fix such an $\varepsilon$ from now on.

We can find an annulus $A \subset \mathbb{S}^{3}$ disjoint from $F$ such that $\gamma \subset \partial A$ and the linking number of the connected components of $\partial A$ is the same as that of the connected components of $\partial \widehat{\Pi}^{\varepsilon}$. (All such annuli are pairwise $C^{0}$-isotopic relative to $F \cup L$, so the choice does not matter.)

We can extend $\phi_{0}$ and $\phi_{1}$ to $A$ so that $\phi_{0}(A)=\phi_{1}(A)=\widehat{\Pi}^{\varepsilon}$. Then replace $F, \Pi_{0}$, and $\Pi_{1}$ by $F \cup A$, $\Pi_{0} \cup \Pi^{\varepsilon}$, and $\Pi_{1} \cup \Pi^{\varepsilon}$, respectively. This reduces the number of connected components of $L$ not contained in $\partial F$, whereas all assumptions of the theorem still hold after extending $\delta_{0}$ and $\delta_{1}$ to $A$ accordingly.

Thus, we may assume from now on that $L \subset \partial F$.

Step 3. Reduce to the case when the isotopy $\phi$ complies with the conventions introduced in Subsection 1.2 and for any point $p \in \widehat{R}$, the tangent plane to $\phi_{t}(F)$ at $p$ remains fixed during the isotopy:

There is no problem to make $\phi C^{1}$-smooth outside $\partial F$ and also at all points $x \in \partial F$ such that $\phi_{t}(x)$ is fixed and is not a singularity of $\partial\left(\phi_{t}(F)\right)$. This smoothness is assumed in the sequel.

Suppose that there is a connected component $Q$ of $R$ such that the tangent plane to $\phi_{t}(F)$ at $p \in$ $\widehat{Q} \backslash\left(\mathbb{S}_{\tau=0}^{1} \cup \mathbb{S}_{\tau=1}^{1}\right)$ varies when $t$ runs from 0 to 1 . Suppose also that $\mathrm{b}_{+}\left(Q ; \Pi_{0}\right)<0$ (observe that $\left.\operatorname{tb}_{+}\left(Q ; \Pi_{0}\right)=\operatorname{tb}_{+}\left(Q ; \Pi_{1}\right)\right)$. It follows from Proposition 3.1 that we can adjust $\Pi_{0}$ and $\phi$ so that:

(1) the assumptions of the theorem still hold;

(2) $\widehat{\Pi}_{0}$ is altered only slightly (in terms of the $C^{0}$-topology);

(3) for any connected component $Q^{\prime} \neq Q$ of $R$, any point $p \in \widehat{Q}^{\prime} \backslash\left(\mathbb{S}_{\tau=0}^{1} \cup \mathbb{S}_{\tau=1}^{1}\right)$, and $t \in[0,1]$, the tangent plane to $\phi_{t}(F)$ at $p$ is unaltered;

\footnotetext{
${ }^{2}$ To simplify notation, we define $\operatorname{tb}_{ \pm}(Q ; \Pi)$ as $\operatorname{tb}_{ \pm}(\widehat{Q} ; \widehat{\Pi})$.
} 
(4) the tangent plane to $\phi_{t}(F)$ at any point $p \in \widehat{Q}$ is defined and does not depend on $t$ after the adjustment.

If $\mathrm{tb}_{-}\left(Q ; \Pi_{0}\right)<0$ we can similarly adjust $\Pi_{1}$ instead of $\Pi_{0}$.

At least one of the inequalities $\operatorname{tb}_{+}\left(Q ; \Pi_{0}\right)<0$ and $\mathrm{tb}_{-}\left(Q ; \Pi_{0}\right)<0$ must hold, since $2\left(-\mathrm{tb}_{+}\left(Q ; \Pi_{0}\right)-\right.$ $\left.\operatorname{tb}_{-}\left(Q ; \Pi_{0}\right)\right)$ is the number of vertices in $Q$. Therefore, after doing finitely many such adjustments we come to the situation in which the tangent plane to $\phi_{t}(F)$ at any point $p \in \widehat{R}$ does not depend on $t$, and $\phi$ is an isotopy complying with the conventions introduced in Subsection 1.2. We assume this from now on.

Step 4. Make holes (switch to mirror diagrams):

Let $M=M\left(\Pi_{0}\right)$ and $M^{\prime}=M\left(\Pi_{1}\right)$ be the enhanced mirror diagrams associated with $\Pi_{0}$ and $\Pi_{1}$, respectively (see Section 5), and let $\eta$ be the morphism of the respective spatial ribbon graphs $\widehat{M} \rightarrow \widehat{M^{\prime}}$ induced by the isotopy $\phi$ (see Subsection 4.3 for the definition of a morphism), that is, the one defined by $\left(\widehat{\Pi}_{0}, \widehat{\Pi}_{1}, \phi_{1} \circ \phi_{0}^{-1}\right) \in \eta$.

The diagrams $M$ and $M^{\prime}$ have a common simple collection $C$ of boundary circuits that represents the framed rectangular diagram of a link $(R, f)$, where $f$ is the framing opposite to $\left.f^{\Pi_{0}}\right|_{R}=\left.f^{\Pi_{1}}\right|_{R}$.

Step 5. Find a sequence of moves representing the isotopy:

By Theorem 6.1 there exists a sequence of elementary moves preserving all boundary circuits in $C$

$$
M=M_{0} \stackrel{\eta_{1}}{\longmapsto} M_{1} \stackrel{\eta_{2}}{\longmapsto} M_{2} \stackrel{\eta_{3}}{\longmapsto} \ldots \stackrel{\eta_{N}}{\longmapsto} M_{N}=M^{\prime}
$$

such that $\eta=\eta_{N} \circ \ldots \circ \eta_{2} \circ \eta_{1}$.

Step 6. Rearrange the moves using the relative commutation theorem:

By Proposition 5.3 the enhanced mirror diagrams $M$ and $M^{\prime}$ have simple essential boundary. Since $C \subset$ $\partial_{\mathrm{e}} M \cap \partial_{\mathrm{e}} M^{\prime}$, it follows from Proposition 9.2 that $M$ and $M^{\prime}$ are flexible relative to $C$.

Now by Theorem 9.2 the sequence (2.1) can be modified so that, after the modification, it will consist of elementary moves together with some number of jump moves, and remain $C$-delicate (see Definition 9.1), and for some $k \in[0, N]$ the $C$-delicate subsequences $M_{0} \stackrel{\eta_{1}}{\longmapsto} M_{1} \stackrel{\eta_{2}}{\longmapsto} M_{2} \stackrel{\eta_{3}}{\longmapsto} \ldots \stackrel{\eta_{k}}{\longmapsto} M_{k}$ and $M_{k} \stackrel{\eta_{k+1}}{\longmapsto}$ $M_{k+1} \stackrel{\eta_{k+2}}{\longmapsto} M_{k+2} \stackrel{\eta_{k+3}}{\longmapsto} \ldots \stackrel{\eta_{N}}{\longmapsto} M_{N}$ will be of type I and type II, respectively. We fix these sequence and number $k$ from now on and denote the morphisms $\eta_{k} \circ \ldots \circ \eta_{2} \circ \eta_{1}: \widehat{M} \rightarrow \widehat{M}_{k}$ and $\eta_{N} \circ \ldots \eta_{k+2} \circ \eta_{k+1}$ : $\widehat{M}_{k} \rightarrow M^{\prime}$ by $\eta_{\mathrm{I}}$ and $\eta_{\mathrm{II}}$, respectively.

Step 7. Patch the holes:

Let $S$ be a surface carried by $\widehat{M}_{k}$ such that some isotopy inducing the morphism $\eta_{\mathrm{I}}$ brings $\widehat{\Pi}_{0}$ to $S$, and let $\psi$ be a homeomorphism $\widehat{\Pi}_{0} \rightarrow S$ such that $\left(\widehat{\Pi}_{0}, S, \psi\right) \in \eta_{\mathrm{I}}$. Clearly we also have $\left(\widehat{\Pi}_{1}, S, \psi \circ \phi_{0} \circ \phi_{1}^{-1}\right) \in$ $\eta_{\mathrm{II}}$.

By Lemma 5.1 the surface $S$ can be chosen in the form $\widehat{\Pi}$ for some rectangular diagram of a surface $\Pi$. We assume for the rest of the proof that such a choice has been made. Since the sequence (2.1) is $C$ delicate we may also assume that $\psi$ takes $\widehat{R}$ to a union of essential boundary circuits of $\widehat{M}_{k}$ that has the form $\widehat{R}^{\prime}$, where $R^{\prime}$ is a rectangular diagram of a link. Moreover, it follows that such $R^{\prime}$ (viewed combinatorially) is obtained from $R$ by a sequence of exchange moves.

Let $\left(\delta_{+}, \delta_{-}\right)$be a canonic dividing configuration of $\widehat{\Pi}$. Since the enhanced mirror diagrams $M$ and $M^{\prime}$ are associated with rectangular diagrams of surfaces, all inessential boundary circuits of $M$ and $M^{\prime}$ are simultaneously +-negligible and --negligible (see Definition 8.12). It now follows from Lemma 8.4 that the abstract dividing set $\left(\psi \circ \phi_{0}\right)\left(\delta_{0}\right)$ is equivalent to $\delta_{+}$, and, by symmetry, $\left(\psi \circ \phi_{0}\right)\left(\delta_{1}\right)$ is equivalent to $\delta_{-}$. In other words, $\left(\Pi, \psi \circ \phi_{0}\right)$ is a realization of the dividing configuration $\left(\left(\psi \circ \varphi_{0}\right)^{-1}\left(\delta_{+}\right),\left(\psi \circ \varphi_{0}\right)^{-1}\left(\delta_{-}\right)\right)$, which is weakly equivalent to $\left(\delta_{0}, \delta_{1}\right)$. Since $S=\widehat{\Pi}$ has been chosen isotopic to $\widehat{\Pi}_{0}$, with the isotopy realizing $\eta_{\mathrm{I}}$, this realization is proper. We also have $\psi\left(\phi_{0}(L)\right)=\widehat{R}^{\prime}$.

Step 8. Produce realizations for all admissible dividing configurations weakly equivalent to $\left(\delta_{0}, \delta_{1}\right)$ from a single one:

By this point, we have shown that for some dividing configuration on $F$ weakly equivalent to $\left(\delta_{0}, \delta_{1}\right)$, 
there exists a proper realization $(\Pi, \xi)$ such that $\xi(L)$ has the form $\widehat{R}^{\prime}$, where $R^{\prime}$ is obtained from $R$ by a sequence of exchange moves. It follows from Proposition 3.2 and Lemma 3.6 that the same holds for any admissible dividing configuration on $F$ weakly equivalent to $\left(\delta_{0}, \delta_{1}\right)$, which concludes the proof of the theorem.

Theorem 2.2 immediately implies the following.

Corollary 2.1. Let $L$ be a fixed oriented link, and let $F \subset \mathbb{S}^{3}$ be a fixed compact surface such that $F \cap L$ is a sublink of $\partial F$. Let also $R$ be an oriented rectangular diagram of a link such that $\widehat{R}$ is isotopic to L. Finally, let $A$ be a family of abstract dividing sets on $F$ such that, for any orientation-preserving self-homeomorphism $\phi$ of $\mathbb{S}^{3}$ taking $L$ to $\widehat{R}$, there exist $\delta^{\prime} \in A$ and a proper --realization $\left(\Pi, \phi^{\prime}\right)$ of $\delta^{\prime}$ at $R$ with $\phi^{\prime} C^{0}$-isotopic to $\phi$ relative to $L$.

Then, for an abstract dividing set $\delta \subset F$, we have $\delta \in \mathscr{I}_{F, L,+}(R)$ if and only if, for some $\delta^{\prime} \in A$, the following two conditions hold:

(1) there exists an admissible dividing configuration $\left(\delta^{+}, \delta^{-}\right)$weakly equivalent to $\left(\delta, \delta^{\prime}\right)$;

(2) whenever $\left(\delta^{+}, \delta^{-}\right)$is such a configuration, there exist a rectangular diagram of a link $R^{\prime}$ obtained from $R$ by a finite sequence of exchange moves, and a proper realization $\left(\Pi^{\prime}, \phi\right)$ of $\left(\delta^{+}, \delta^{-}\right)$such that $\phi(L)=\widehat{R}^{\prime}$.

Definition 2.8. A family $A$ of abstract dividing sets satisfying the assumptions of Corollary 2.1 will be called --representative for $R$. One similarly defines a +-representative family, by applying the symme$\operatorname{try} \xi_{+} \leftrightarrow \xi_{-}$.

Corollary 2.1 is most useful when one can find a finite --representative family $A$ for $R$. In this case, it is a finite procedure to check whether or not $\delta \in \mathscr{I}_{F, L,+}(R)$. Indeed, for each $\delta^{\prime} \in A$ it is easy to find an admissible dividing configuration $\left(\delta^{+}, \delta^{-}\right)$weakly equivalent to $\left(\delta, \delta^{\prime}\right)$, and, due to Proposition 2.2, each dividing configuration admits, up to combinatorial equivalence, only finitely many realizations, which can be searched.

For each found realization, one should, of course, check whether it is proper or not. This amounts to comparing certain Haken three-manifolds with boundary pattern, which is doable in general [33] but sometimes is simply not needed because no realization exists.

2.4. An example: the knot $6{ }_{2}$. We illustrate how the method described above works by proving the following statement.

Proposition 2.3. The following two Legendrian knots, which have topological type $6_{2}$, presented by front projections are not equivalent.
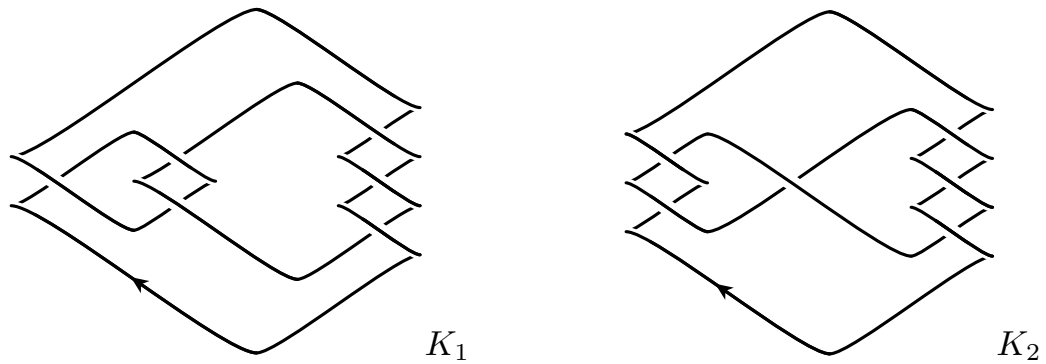

Proof. Shown in Figure 2.3 are oriented rectangular diagrams $R_{1}$ and $R_{2}$ such that $\widehat{R}_{i}$ is equivalent to $K_{i}$ as a $\xi_{+}$-Legendrian knot, $i=1,2$. The knots $\widehat{R}_{1}$ and $\widehat{R}_{2}$ are equivalent as $\xi_{-}$-Legendrian knots. Indeed, there is a sequence of moves including exchange moves and type II stabilizations and destabilizations transforming $R_{1}$ to $R_{2}$. It is sketched in Figure 2.4.

One easily finds that $\operatorname{tb}_{+}\left(K_{1}\right)=\mathrm{tb}_{+}\left(K_{2}\right)=-7$, so, if $F$ is any Seifert surface for $\widehat{R}_{i}, i=1,2$, then we have $\operatorname{tb}_{+}\left(\widehat{R}_{i} ; F\right)=-7<0$. Therefore, for any oriented knot $K$ having topological type $6_{2}$, and any Seifert surface $F$ for $K$, we have by Theorem 2.1 $\mathscr{I}_{F, K,-}\left(R_{1}\right)=\mathscr{I}_{F, K,-}\left(R_{2}\right)$. 

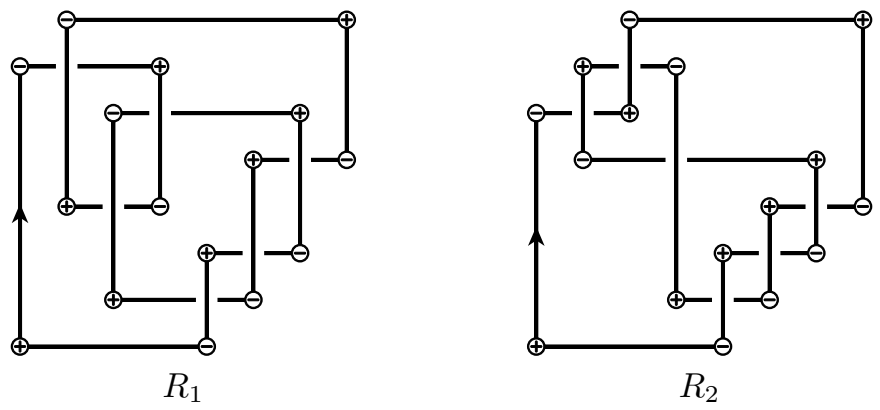

Figure 2.3. Rectangular presentations of $K_{1}$ and $K_{2}$

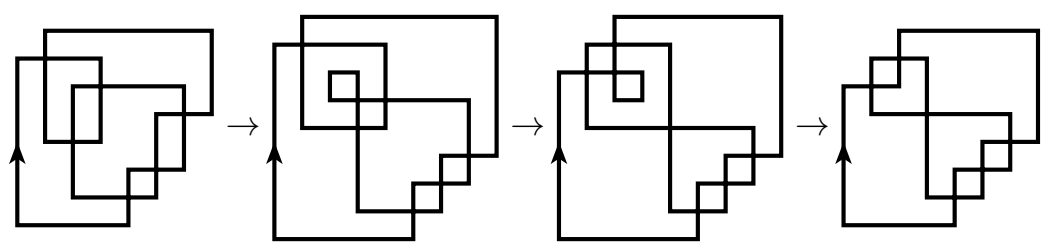

FIgURE 2.4. Transforming $R_{1}$ to $R_{2}$ by exchange moves and type II (de)stabilizations

We are going to show that $\mathscr{I}_{F, L,+}\left(R_{1}\right) \neq \mathscr{I}_{F, L,+}\left(R_{2}\right)$ for a specific choice of $F$ and $L=\partial F$. This specific choice is presented in Figure 2.5] in the rectangular form. What is shown in Figure 2.5] is a rectangular diagram of a surface, which we denote by $\Pi^{*}$, together with the rectangular diagram of the boundary link, and some additional data.

It is a direct check that:

(1) the boundary of $\Pi^{*}$ coincides - combinatorially — with $R_{1}$ (provided that the orientation of $\partial \Pi^{*}$ is chosen as in Figure 2.5);

(2) $\widehat{\Pi}$ is a genus two orientable surface with a single boundary component (there are 18 rectangles in $\Pi^{*}$, which correspond to the tiles of $\widehat{\Pi}^{*}, 44$ vertices of $\Pi^{*}$, which correspond to the sides of the tiles, and 23 occupied levels, which correspond to the vertices of the tiles, thus the Euler characteristic is $18-44+23=-3$ );

(3) the loops on $\widehat{\Pi}^{*}$ whose torus projections are indicated in Figure 2.5 by curved lines with various arrowheads generate the fundamental group of $\widehat{\Pi}^{*}$ and cut $\widehat{\Pi}^{*}$ into an octagon disc with a hole.

Moreover, one can verify that, after the cutting, a canonic dividing configuration $\left(\delta_{+}, \delta_{-}\right)$of $\widehat{\Pi}^{*}$ looks as shown in Figure 2.6 on the left (with Convention 2.1] in force). An intersection of $\delta_{+}$and $\delta_{-}$is marked by a black dot if the surface is shown near this point in the same orientation in Figure 2.6 as in the torus projection in Figure 2.5. and by a white dot otherwise.

Now we are going to apply Corollary 2.1 in which we put $L=\widehat{R}_{1}, R=R_{2}$, and $F=\widehat{\Pi}^{*}$. By Theorem 2.1. since $\widehat{R}_{1}$ and $\widehat{R}_{2}$ are equivalent as $\xi_{-}$-Legendrian knots and tb $\left(R_{2}\right)=-7<0$, there exists a --realization of $\delta_{-}$at $R_{2}$. However, the one-element set $\left\{\delta_{-}\right\}$is not known to be (and actually is not) --representative for $R_{2}$, so Corollary 2.1 cannot be applied immediately.

To construct a --representative family we must take into account the symmetry group of the knot $6_{2}$, that is, the mapping class group of the pair $\left(\mathbb{S}^{3}, \widehat{R}_{1}\right)$. This group is known to be isomorphic to the dihedral group $D_{2} \cong \mathbb{Z}_{2} \oplus \mathbb{Z}_{2}$, see [41, 31, but this includes two elements inverting the orientation of $\widehat{R}_{1}$, which do not bother us. The subgroup of orientation-preserving elements is just $\mathbb{Z}_{2}$. The only nontrivial element of this subgroup can be represented by an orientation-preserving self-homeomorphism $\sigma$ of $\mathbb{S}^{3}$ that preserves the surface $\widehat{\Pi}^{*}$ and sends each generator $x_{i}, i=1,2,3,4$, indicated in Figure 2.5 to its inverse. 


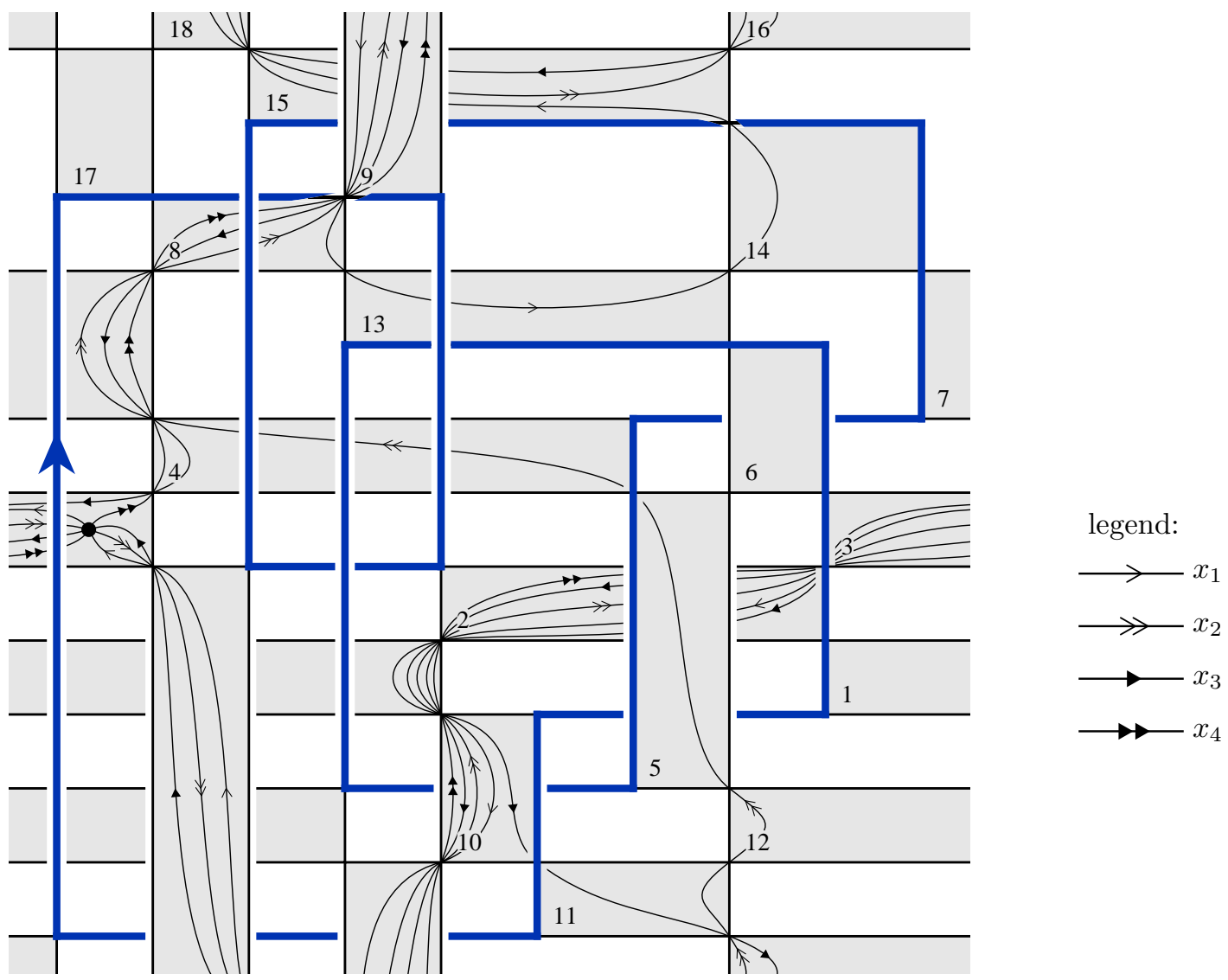

FiguRE 2.5. Rectangular diagram $\Pi^{*}$ of a Seifert surface for $\widehat{R}_{1}$

In order to make the reader able to verify this, we note that the knot $6_{2}$ is fibered 43 , 35] and has genus two 42. So, the surface $\widehat{\Pi}^{*}$ can be taken for a fiber. The loops $x_{i}, i=1,2,3,4$, freely generate $\pi_{1}\left(\widehat{\Pi}^{*}\right)$. The complement of $\widehat{R}_{1}$ can be identified with the mapping torus of a self-homeomorphism of $\widehat{\Pi}^{*} \backslash \partial \widehat{\Pi}^{*}$ inducing the following automorphism of $\pi_{1}\left(\widehat{\Pi}^{*}\right)$ :

$$
x_{1} \mapsto x_{1} x_{2} x_{1}^{2}, \quad x_{2} \mapsto x_{1}^{-2} x_{3} x_{1}^{-1}, \quad x_{3} \mapsto x_{1} x_{4} x_{1}^{2}, \quad x_{4} \mapsto x_{1}^{-1} .
$$

Thus, the fundamental group of $\mathbb{S}^{3} \backslash \widehat{R}_{1}$ has the following presentation:

$$
\left\langle x_{1}, x_{2}, x_{3}, x_{4}, t ; t x_{1} t^{-1}=x_{1} x_{2} x_{1}^{2}, t x_{2} t^{-1}=x_{1}^{-2} x_{3} x_{1}^{-1}, t x_{3} t^{-1}=x_{1} x_{4} x_{1}^{2}, t x_{4} t^{-1}=x_{1}^{-1}\right\rangle .
$$

All the generators of this presentation are shown in Figure 2.7. One can check the above relations directly by switching to the Wirtinger presentation.

Now one can easily see that the formulas

$$
x_{1} \mapsto x_{1}^{-1}, \quad x_{2} \mapsto x_{2}^{-1}, \quad x_{3} \mapsto x_{3}^{-1}, \quad x_{4} \mapsto x_{4}^{-1}, \quad t \mapsto x_{1} t
$$

define an automorphism of $\pi_{1}\left(\mathbb{S}^{3} \backslash \widehat{R}_{1}\right.$ ), which corresponds to a self-homeomorphism $\sigma$ of $\mathbb{S}^{3} \backslash \widehat{R}_{1}$ (extendable to the whole of $\mathbb{S}^{3}$ ). We claim that such a homeomorphism represents the sought-for element of the symmetry group. Indeed, it follows from (2.2) that the orientations of $\widehat{\Pi}^{*}, \widehat{R}_{1}$, and $\mathbb{S}^{3}$ are preserved, the automorphism has order two and represents a non-trivial element in the group of outer automorphisms of $\pi_{1}\left(\mathbb{S}^{3} \backslash \widehat{R}_{1}\right)$. 

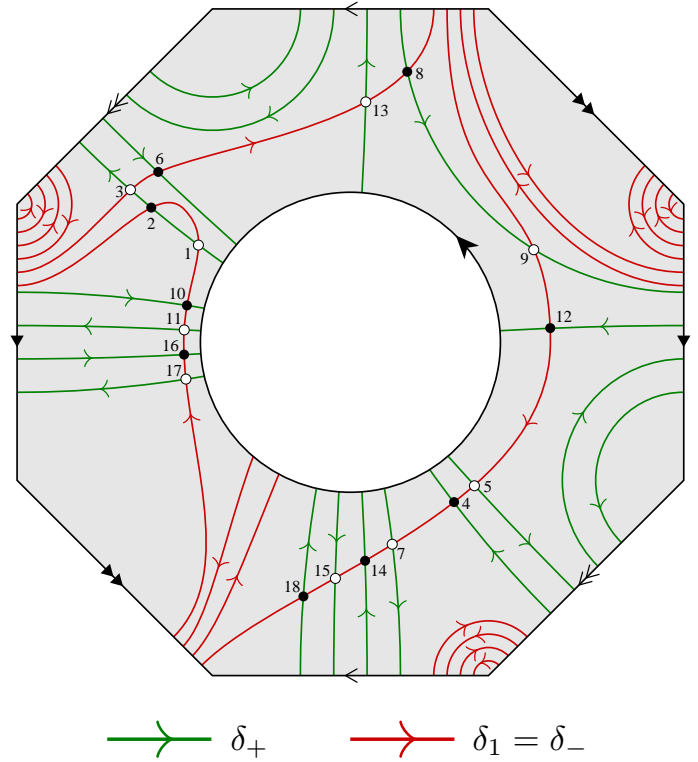

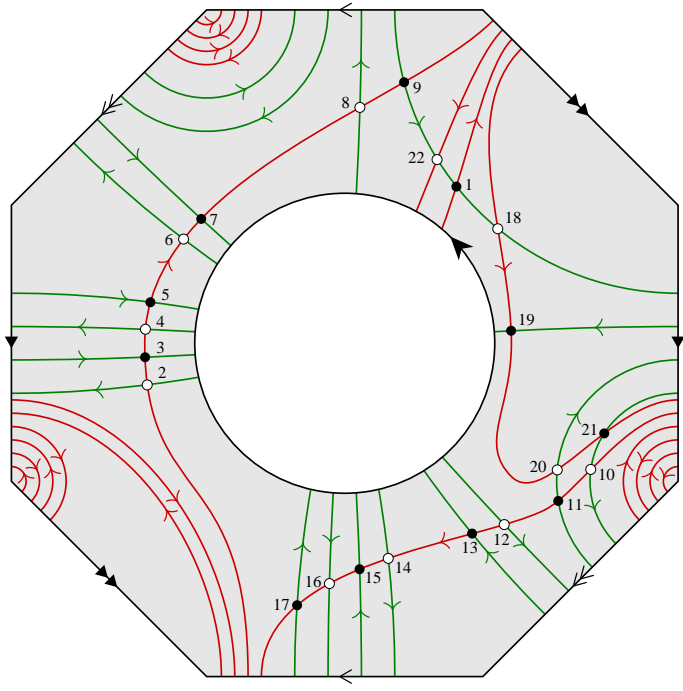

$\longrightarrow \delta_{+} \quad \longrightarrow \delta_{2}=\sigma\left(\delta_{-}\right)$

Figure 2.6. Dividing configurations $\left(\delta_{+}, \delta_{-}\right)$and $\left(\delta_{+}, \sigma\left(\delta_{-}\right)\right)$

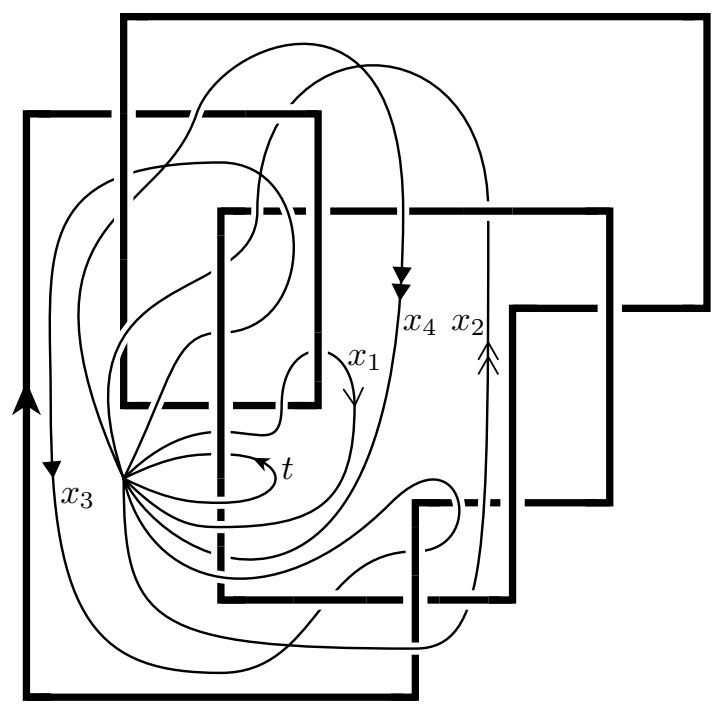

Figure 2.7. Generators $x_{1}, x_{2}, x_{3}, x_{4}, t$ of $\pi_{1}\left(\mathbb{S}^{3} \backslash \widehat{R}_{1}\right)$

The homeomorphism $\sigma$ can be chosen to be an involution. Moreover, in terms of the punctured octagon obtained from $\widehat{\Pi}^{*}$ by cutting along $x_{i}, i=1,2,3,4$, the map $\left.\sigma\right|_{\widehat{\Pi}^{*}}$ can be turned into a rotation by $\pi$ around the center.

We now claim that the family $A=\left\{\delta_{-}, \sigma\left(\delta_{-}\right)\right\}$is --representative for $R_{2}$. Indeed, let $\left(\Pi_{1}, \phi_{1}\right)$ be a proper --realization of $\delta_{-}$at $R_{2}$. Then $\left(\Pi_{1}, \phi_{1} \circ \sigma\right)$ is a proper --realization of $\sigma\left(\delta_{-}\right)$. By construction, any orientation-preserving self-homeomorphism of $\mathbb{S}^{3}$ that takes $L=\widehat{R}_{1}$ to $\widehat{R}_{2}$ is isotopic relative to $L$ either to $\phi_{1}$ or to $\phi_{1} \circ \sigma$, hence $A$ is --representative. 
The right picture in Figure 2.6 shows the dividing configuration $\left(\delta_{+}, \sigma\left(\delta_{-}\right)\right)$, which, for our particular choice of $\delta_{ \pm}$shown in Figure 2.6, is admissible.

By Corollary 2.1, we have $\delta_{+} \in \mathscr{I}_{\widehat{\Pi}^{*}, \widehat{R}_{1},+}\left(R_{2}\right)$ if and only if there exists a proper realization $\left(\Pi^{\prime}, \phi\right)$ of one of the dividing configurations $\left(\delta_{+}, \delta_{-}\right)$or $\left(\delta_{+}, \sigma\left(\delta_{-}\right)\right)$, such that $\partial \Pi^{\prime}$ is obtained from $R_{2}$ by exchange moves. The diagram $R_{2}$ does not admit any exchange move (we call such diagrams rigid), so, we may additionally demand that $\partial \Pi^{\prime}=R_{2}$. An exhaustive search, which is finite (and very small in this case), of all (combinatorial types of) rectangular diagrams of surfaces $\Pi^{\prime}$ that give rise to a realization of $\left(\delta_{+}, \delta_{-}\right)$or $\left(\delta_{+}, \sigma\left(\delta_{-}\right)\right)$shows that none of them has $\partial \Pi^{\prime}=R_{2}$. Therefore, $\delta_{+} \notin \mathscr{I}_{\widehat{\Pi}^{*}, \widehat{R}_{1},+}$, and hence the Legendrian knots $\widehat{R}_{1}$ and $\widehat{R}_{2}$ are not equivalent.

Moreover, the exhaustive search shows that the configuration $\left(\delta_{+}, \delta_{-}\right)$has, up to combinatorial equivalence, only one realization, the one we started with. The configuration $\left(\delta_{+}, \sigma\left(\delta_{-}\right)\right)$has only two realizations, which are shown in Figure 2.8. (These realizations are proper as the boundary knots of the
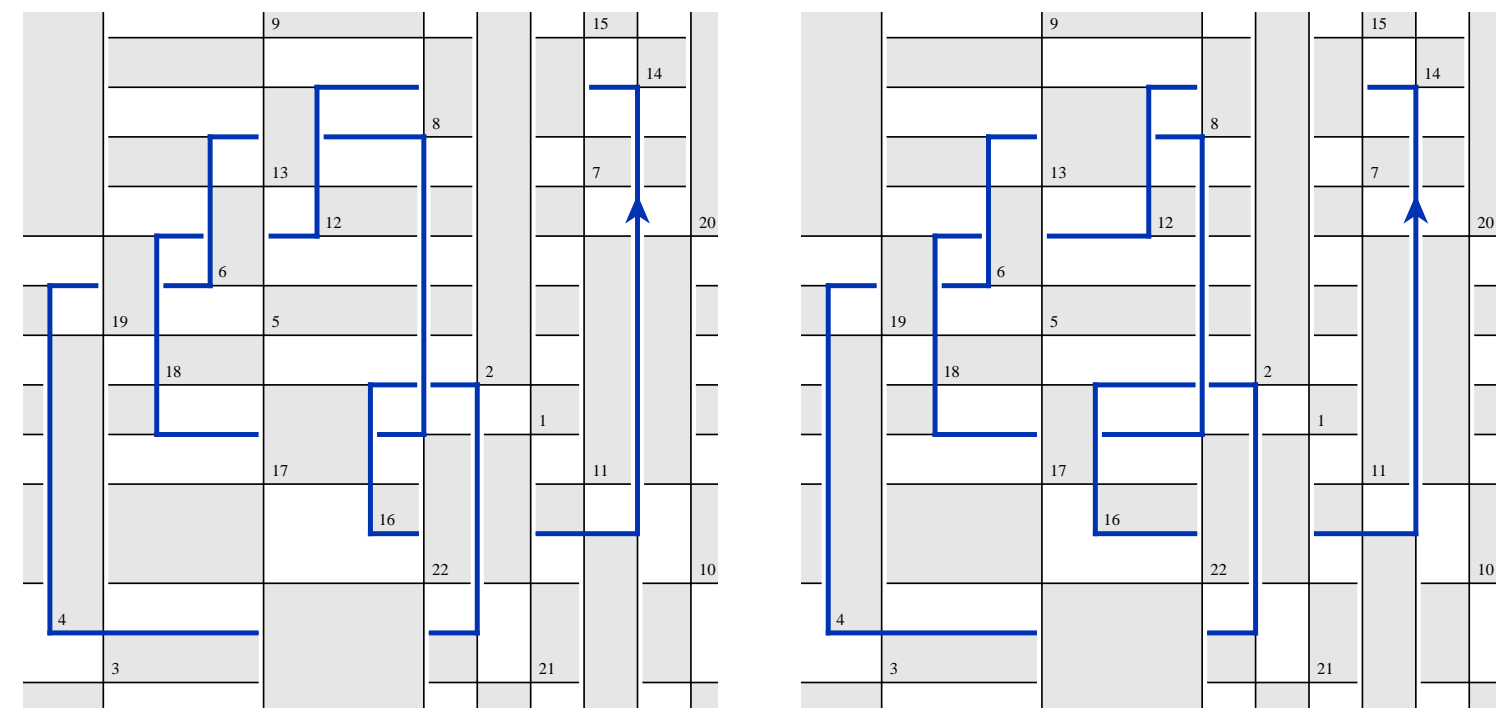

FigurE 2.8. The only two realizations of $\left(\delta_{+}, \sigma\left(\delta_{-}\right)\right)$

obtained surfaces have topological type $6_{2}$, and there is only one $C^{0}$-isotopy class of genus two Seifert surfaces for this knot.)

A proof that there are no more combinatorial types of realizations of $\left(\delta_{+}, \delta_{-}\right)$and $\left(\delta_{+}, \sigma\left(\delta_{-}\right)\right)$is given in the Appendix A. We also sketch here an 'ideological' argument that allows one, in this particular case, to see that there is no realization $\left(\Pi^{\prime}, \phi\right)$ of $\left(\delta_{+}, \delta_{-}\right)$or $\left(\delta_{+}, \sigma\left(\delta_{-}\right)\right)$with $\partial \Pi^{\prime}=R_{2}$.

For brevity, we will say that a dividing configuration $D$ is compatible with a rectangular diagram of a knot $R$ if there is a proper realization $\left(\Pi^{\prime}, \phi\right)$ of $D$ such that $\partial \Pi^{\prime}$ can be obtained from $R$ by exchange moves. We also say that two rectangular diagrams of a knot $R^{\prime}$ and $R^{\prime \prime}$, say, are bi-Legendrian equivalent if the knots $\widehat{R}^{\prime}$ and $\widehat{R}^{\prime \prime}$ are Legendrian equivalent with respect to both contact structures $\xi_{+}$and $\xi_{-}$.

For an oriented rectangular diagram of a knot $R$, we denote by $\rho(R)$ the diagram obtained from $R$ by reflecting in the origin, that is, by the map $(\theta, \varphi) \mapsto(-\theta,-\varphi)$, and reversing the orientation. We learn from Figure 2.8 that $\left(\delta_{+}, \sigma\left(\delta_{-}\right)\right)$is compatible with $\rho\left(R_{1}\right)$. The diagrams $R_{1}$ and $\rho\left(R_{1}\right)$ are known to be bi-Legendrian equivalent [6] (in the notation of [6], if $R$ represents $L$, then $\rho(R)$ represents $-\mu(L)$ ). Two transitions between $R_{1}$ and $\rho\left(R_{1}\right)$ via elementary moves, one without type II (de)stabilizations and the other without type I (de)stabilizations are sketched in Figure 2.9], where each arrow marked 'I' or 'II' stands for an operation that can be decomposed into a stabilization of the respective type, a few exchange moves, and a destabilization of the same type as the preceding stabilization. (These operations 


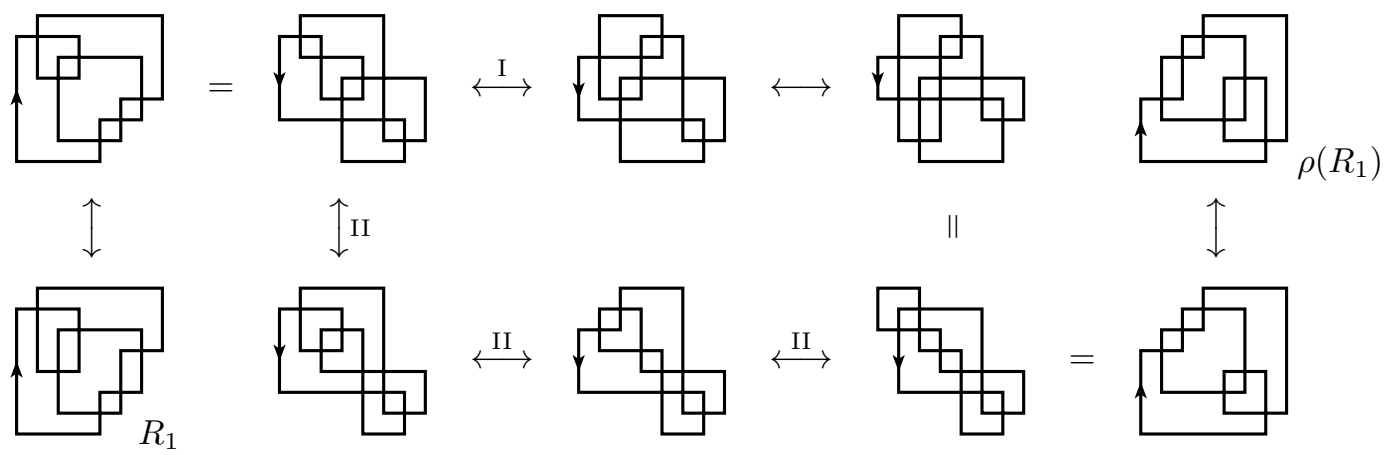

FiguRE 2.9. Bi-Legendrian equivalence of $R_{1}$ and $\rho\left(R_{1}\right)$

are particular cases of flypes introduced in 9.) The unmarked arrows stand for (a composition of) exchange moves. The equality signs mean combinatorial equivalence.

One can deduce from the fact that $\left(\delta_{+}, \delta_{-}\right)$is compatible with $R_{1}$ and $\left(\delta_{+}, \sigma\left(\delta_{-}\right)\right)$is compatible with $\rho\left(R_{1}\right)$ that whenever $R$ is an oriented rectangular diagram of a knot bi-Legendrian equivalent to $R_{1}$, the following two conditions are equivalent:

(1) $\left(\delta_{+}, \delta_{-}\right)$is compatible with $R$;

(2) $\left(\delta_{+}, \sigma\left(\delta_{-}\right)\right)$is compatible with $\rho(R)$.

Now observe that $\delta_{+}$and $\delta_{-}$intersect in a 'non-optimal' way: they have a bigon, that is, a disc enclosed by two arcs one of which is a subset of $\delta_{+}$and the other of $\delta_{-}$(the endpoints of the arcs are numbered 1 and 2 in the left picture in Figure 2.6), and this bigon cannot be reduced, since the reduction would produce a non-admissible dividing configuration. This means by Lemma 3.9 that the configuration $\left(\delta_{+}, \delta_{-}\right)$is incompatible with any rigid diagram of a non-trivial knot. In particular, it is incompatible with $R_{2}$ and $\rho\left(R_{2}\right)$. This implies that neither $\left(\delta_{+}, \delta_{-}\right)$nor $\left(\delta_{+}, \sigma\left(\delta_{-}\right)\right)$is compatible with $R_{2}$.

\section{BASIC MOVES OF RECTANGULAR DIAGRAMS OF SURFACES}

In this section and in Appendix B we introduce moves of rectangular diagrams of surfaces that preserve the isotopy class of the represented surface, and allow transition between diagrams representing isotopic surfaces.

We will refer to all the transformations of rectangular diagrams of surfaces introduced below in this section and in Appendix B as basic moves. These include: (half-)wrinkle creation and reduction moves, (de)stabilization moves, exchange moves, and flypes. Some of them are assigned a type (I or II), and the others are neutral (have no type).

Theorem 3.1. Let $\Pi$ and $\Pi^{\prime}$ be rectangular diagrams of a surface. The surfaces $\widehat{\Pi}$ and $\widehat{\Pi}^{\prime}$ are isotopic if and only if $\Pi^{\prime}$ can be obtained from $\Pi$ by a sequence of basic moves.

The surfaces $\widehat{\Pi}$ and $\widehat{\Pi}^{\prime}$ are equivalent as Giroux's convex surfaces with respect to $\xi_{+}$(respectively, $\left.\xi_{-}\right)$ if and only if $\Pi^{\prime}$ can be obtained from $\Pi$ by a sequence of type I (respectively, type II) and neutral basic moves.

Although the hard part of this theorem, namely, the sufficiency of the basic moves for transition between isotopic (convex) surfaces, sounds as the most fundamental result among the statements formulated in this section, it is not needed to establish our main result, which is Theorem 2.2. For this reason, and in order not to overload the paper, we omit the proof of Theorem 3.1 here. The proof of the first part of the theorem appears in [14. The proof of the second part will be published elsewhere.

We also skip some details of the proof that Giroux's convexity can be maintained in the transition from $\widehat{\Pi}$ to $\widehat{\Pi}^{\prime}$ as stated in Theorem [3.1. since we don't use the relation to Giroux's convex surfaces in this strong form. What we do use is the invariance of the isotopy classes of certain dividing sets, which is a consequence of the above mentioned relation, but can be established without reference to Giroux's 
convexity. We also do use in the proof of Theorem 2.2 some results of this section of which the most crucial ones are Propositions 3.1 and 3.2 ,

Now we proceed with the definition of basic moves.

3.1. Notation. Vertex types. We recall from 12 that we use the coordinate system $\theta, \varphi, \tau$ on $\mathbb{S}^{3}$ coming from the join presentation of $\mathbb{S}^{3}$. For $v=\left(\theta_{0}, \varphi_{0}\right) \in \mathbb{T}^{2}$ we denote by $\widehat{v}$ the arc written in these coordinates as $\left\{\left(\theta_{0}, \varphi_{0}, \tau\right): \tau \in[0 ; 1]\right\}$. For a rectangle $r=\left[\theta_{1} ; \theta_{2}\right] \times\left[\varphi_{1} ; \varphi_{2}\right]$ we denote by $\widehat{r}$ the tile associated with $r$ (see [12, Subsection 2.3]).

For $\theta, \varphi \in \mathbb{S}^{1}$ we also use the notation $m_{\theta}$ for the meridian $\{\theta\} \times \mathbb{S}^{1} \subset \mathbb{T}^{2}$, and $\ell_{\varphi}$ for the longitude $\mathbb{S}^{1} \times$ $\{\varphi\}$. By $\widehat{m}_{\theta}$ and $\widehat{\ell}_{\varphi}$ we denote the endpoints of the $\operatorname{arc} \widehat{(\theta, \varphi)}$, lying on $\mathbb{S}_{\tau=1}^{1}$ and $\mathbb{S}_{\tau=0}^{1}$, respectively.

Let $\Pi$ be a rectangular diagram of a surface, and let $\left(\delta_{+}, \delta_{-}\right)$be a canonic dividing configuration of $\widehat{\Pi}$. If $r$ is a rectangle of $\Pi$, then the tile $\widehat{r}$ contains a unique intersection point of $\delta_{+}$and $\delta_{-}$, which we denote by $\dot{r}$. If $v$ is a vertex of $\Pi$, then there is a unique arc of $\left(\delta_{+} \cup \delta_{-}\right) \backslash\left(\delta_{+} \cap \delta_{-}\right)$intersecting $\widehat{v}$, which we denote by $\stackrel{v}{v}$. Finally, the closure of every connected component of $\widehat{\Pi} \backslash\left(\delta_{+} \cup \delta_{-}\right)$is a disc containing a unique vertex of the tiling of $\widehat{\Pi}$, and if this vertex is $\widehat{a}$, where $a$ is an occupied level of $\Pi$, then the corresponding disc will be denoted by $\stackrel{\circ}{a}$.

Summarizing, if $\Pi$ is a rectangular diagram of a surface we have the following one-to-one correspondences between objects related to the diagram $\Pi$, to the associated surface $\widehat{\Pi}$, and to a canonic dividing configuration $\left(\delta_{+}, \delta_{-}\right)$of $\widehat{\Pi}$ (see Figure 3.1):

\begin{tabular}{l|l|l}
$x$ & $\widehat{x}$ & $\stackrel{\circ}{x}$ \\
\hline a rectangle of $\Pi$ & a tile of $\widehat{\Pi}$ & a point of $\delta_{+} \cap \delta_{-}$ \\
\hline a vertex of $\Pi$ & an edge of $\widehat{\Pi}$ & $\begin{array}{l}\text { a connected component of } \\
\left(\delta_{+} \cup \delta_{-}\right) \backslash\left(\delta_{+} \cap \delta_{-}\right)\end{array}$ \\
\hline an occupied level of $\Pi$ & a vertex of $\widehat{\Pi}$ & $\begin{array}{l}\text { the closure of a connected } \\
\text { component of } \widehat{\Pi} \backslash\left(\delta_{+} \cup \delta_{-}\right)\end{array}$
\end{tabular}
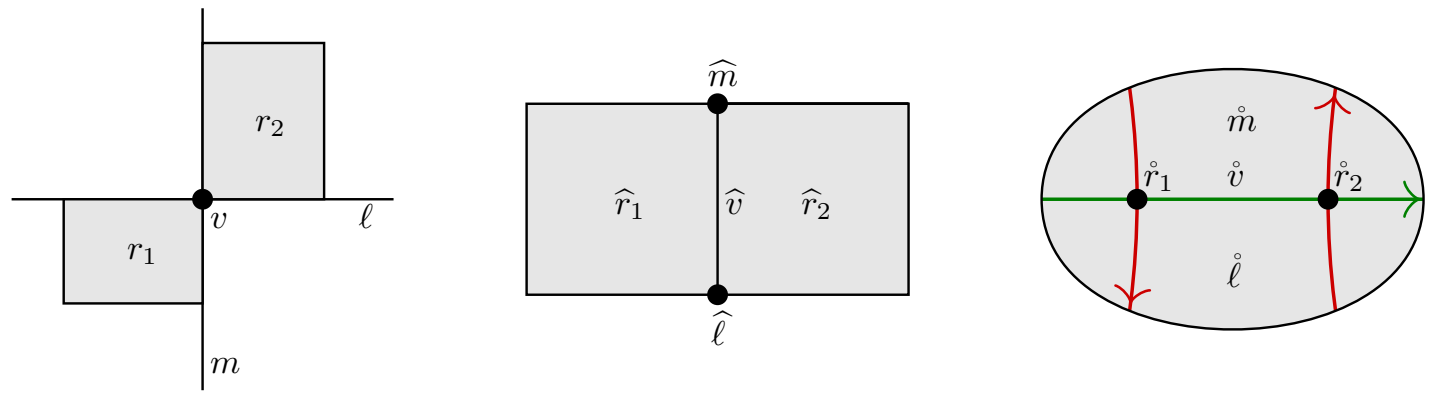

Figure 3.1. Correspondence between objects related to $\Pi$, to $\widehat{\Pi}$, and to $\left(\delta_{+}, \delta_{-}\right)$

Definition 3.1. If $r=\left[\theta_{1} ; \theta_{2}\right] \times\left[\varphi_{1} ; \varphi_{2}\right] \subset \mathbb{T}^{2}$ is a rectangle, then the points $\left(\theta_{1}, \varphi_{1}\right),\left(\theta_{2}, \varphi_{2}\right)$ will be called the \-vertices of $r$ and the points $\left(\theta_{1}, \varphi_{2}\right),\left(\theta_{2}, \varphi_{1}\right)$ the $/$-vertices of $r$. A vertex $v$ is called $a \backslash$ vertex (respectively, a /-vertex) of a rectangular diagram $\Pi$ if it is a \-vertex (respectively, a /-vertex) of some rectangle $r \in \Pi$. It follows from the definition of a rectangular diagram of a surface that no point can be simultaneously a \-vertex and a /-vertex of $\Pi$.

Equivalently, $v$ is a $\backslash$-vertex (respectively, $/$-vertex) of $\Pi$ if $\stackrel{\circ}{v} \subset \delta_{+}$(respectively, $\stackrel{\circ}{v} \subset \delta_{-}$), where $\left(\delta_{+}, \delta_{-}\right)$is a canonic dividing configuration of $\widehat{\Pi}$.

3.2. Wrinkle moves and stabilization moves. The moves defined in this subsection are similar to each other in nature. However, wrinkle and half-wrinkle moves are neutral, and (de)stabilization moves have type I or type II. 
Definition 3.2. Let $\Pi$ be a rectangular diagram of a surface, and let $v_{1}=\left(\theta_{0}, \varphi_{1}\right)$ and $v_{2}=\left(\theta_{0}, \varphi_{2}\right)$ be a \-vertex and a /-vertex of $\Pi$, respectively, lying on the same meridian $m_{\theta_{0}}$. Choose an $\varepsilon>0$ so that no meridian in $\left[\theta_{0}-2 \varepsilon ; \theta_{0}+2 \varepsilon\right] \times \mathbb{S}^{1} \subset \mathbb{T}^{2}$ other than $m_{\theta_{0}}$ is an occupied level of $\Pi$. Also choose an orientation-preserving self-homeomorphism $\psi$ of the interval $\left[\theta_{0}-2 \varepsilon ; \theta_{0}+2 \varepsilon\right]$.

Let $\Pi^{\prime}$ be the rectangular diagram of a surface obtained from $\Pi$ by making the following modifications:

(1) every rectangle of the form $\left[\theta_{0} ; \theta_{1}\right] \times\left[\varphi^{\prime} ; \varphi^{\prime \prime}\right]$ (respectively, $\left.\left[\theta_{1} ; \theta_{0}\right] \times\left[\varphi^{\prime} ; \varphi^{\prime \prime}\right]\right)$ with $\left[\varphi^{\prime} ; \varphi^{\prime \prime}\right] \subset\left[\varphi_{1} ; \varphi_{2}\right]$ is replaced by $\left[\psi\left(\theta_{0}+\varepsilon\right) ; \theta_{1}\right] \times\left[\varphi^{\prime} ; \varphi^{\prime \prime}\right]$ (respectively, by $\left.\left[\theta_{1} ; \psi\left(\theta_{0}+\varepsilon\right)\right] \times\left[\varphi^{\prime} ; \varphi^{\prime \prime}\right]\right)$;

(2) every rectangle of the form $\left[\theta_{0} ; \theta_{1}\right] \times\left[\varphi^{\prime} ; \varphi^{\prime \prime}\right]$ (respectively, $\left.\left[\theta_{1} ; \theta_{0}\right] \times\left[\varphi^{\prime} ; \varphi^{\prime \prime}\right]\right)$ with $\left[\varphi^{\prime} ; \varphi^{\prime \prime}\right] \subset\left[\varphi_{2} ; \varphi_{1}\right]$ is replaced by $\left[\psi\left(\theta_{0}-\varepsilon\right) ; \theta_{1}\right] \times\left[\varphi^{\prime} ; \varphi^{\prime \prime}\right]$ (respectively, by $\left.\left[\theta_{1} ; \psi\left(\theta_{0}-\varepsilon\right)\right] \times\left[\varphi^{\prime} ; \varphi^{\prime \prime}\right]\right)$;

(3) two new rectangles are added, $\left[\psi\left(\theta_{0}-\varepsilon\right) ; \psi\left(\theta_{0}\right)\right] \times\left[\varphi_{1} ; \varphi_{2}\right]$ and $\left[\psi\left(\theta_{0}\right) ; \psi\left(\theta_{0}+\varepsilon\right)\right] \times\left[\varphi_{2} ; \varphi_{1}\right]$.

Then we say that the passage from $\Pi$ to $\Pi^{\prime}$ is a vertical wrinkle creation move. The inverse operation is referred to as a vertical wrinkle reduction move.

Horizontal wrinkle creation and reduction moves are defined similarly with the roles of $\theta$ and $\varphi$ exchanged.

A vertical wrinkle move is illustrated in Figure 3.2. The left pair of pictures shows how the rectangular diagram changes, the top right pair of pictures shows how the corresponding tiling of $\widehat{\Pi}$ changes, and the
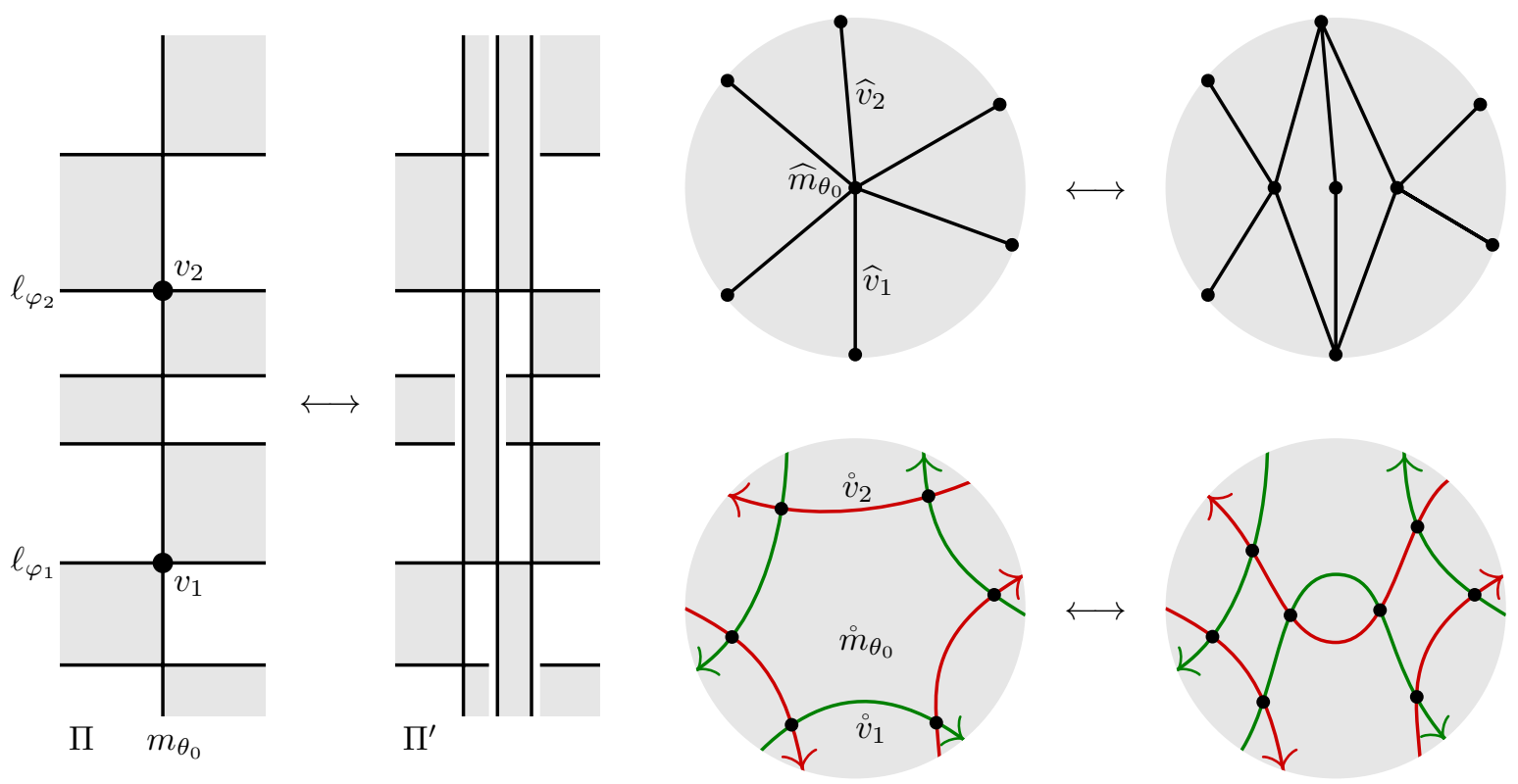

FiguRE 3.2. A vertical wrinkle move

bottom right pair of pictures demonstrates the change in the canonic dividing configuration of $\widehat{\Pi}$.

The combinatorial type of the obtained diagram $\Pi^{\prime}$ in Definition 3.2 does not depend on the homeomorphism $\psi$, and, in many situations, the reader may safely forget about $\psi$ by assuming $\psi=$ id. However, the flexibility arising from the arbitrariness in the choice of $\psi$ will sometimes be useful. Namely, by choosing $\psi$ so that $\psi\left(\theta_{0}-\varepsilon\right)=\theta_{0}$ or $\psi\left(\theta_{0}+\varepsilon\right)=\theta_{0}$ (we keep using the notation from Definition 3.2) we will reduce the number of rectangles of the diagram $\Pi$ that are modified by the move. In particular, if $m_{\theta_{0}}$ contains vertices of $\partial \Pi$, then an appropriate choice of $\psi$ will allow us to keep the boundary of the diagram fixed, that is, to have $\partial \Pi^{\prime}=\partial \Pi$.

Similar homeomorphisms, also denoted by $\psi$, appear in Definitions 3.4 3.6. and 3.8 for a like reason.

Lemma 3.1. Let $\Pi \mapsto \Pi^{\prime}$ be a wrinkle creation move, and let $D, D^{\prime}$ be canonic dividing configurations of $\widehat{\Pi}$ and $\widehat{\Pi}^{\prime}$, respectively. Then there exists an isotopy bringing $(\widehat{\Pi}, D)$ to $\left(\widehat{\Pi}^{\prime}, D^{\prime \prime}\right)$, where $D^{\prime \prime}$ is a dividing 
configuration on $\widehat{\Pi}^{\prime}$ weakly equivalent to $D^{\prime}$. If, additionally, we have $\partial \Pi=\partial \Pi^{\prime}$, then the isotopy can be chosen to be fixed on $\partial \widehat{\Pi}$.

Moreover, $\widehat{\Pi}$ and $\widehat{\Pi}^{\prime}$ are isotopic in the class of Giroux's convex surfaces with respect to either of the contact structures $\xi_{+}$and $\xi_{-}$, and if $\partial \Pi=\partial \Pi^{\prime}$, then the isotopy can be chosen to be fixed on $\partial \widehat{\Pi}$.

Proof. We use the notation from Definition 3.2 Let $U$ be a small open neighborhood of the domain $\left[\theta_{0}-\right.$ $\left.\varepsilon ; \theta_{0}+\varepsilon\right] * \mathbb{S}^{1} \subset \mathbb{S}^{3}$. If $U$ is chosen small enough, then it is homeomorphic to a three-ball and intersects each of the surfaces $\widehat{\Pi}$ and $\widehat{\Pi}^{\prime}$ in a two-disc. Indeed, the intersection $\widehat{\Pi} \cap U$ is a small neighborhood of the star graph in $\widehat{\Pi}$ formed by the edges of the tiling that emanate from $\widehat{m}_{\theta_{0}}$. The intersection $\widehat{\Pi}^{\prime} \cap U$ is a small neighborhood, in $\widehat{\Pi}^{\prime}$, of the union of the edges of the new tiling that emanate from $\widehat{m}_{\theta_{0}+\varepsilon}$ and $\widehat{m}_{\theta_{0}-\varepsilon}$ with the two new tiles.

One can also see that the surfaces $\widehat{\Pi}$ and $\widehat{\Pi}^{\prime}$ are close to one another outside $U$. Moreover, we have $\partial \widehat{\Pi} \backslash U=\partial \widehat{\Pi}^{\prime} \backslash U$, and both intersections $\partial \widehat{\Pi} \cap U, \partial \widehat{\Pi}^{\prime} \cap U$ are either empty or consist of a single arc. This implies the existence of the required isotopy from $\widehat{\Pi}$ to $\widehat{\Pi}^{\prime}$.

In order to see that an isotopy from $\widehat{\Pi}$ to $\widehat{\Pi}^{\prime}$ can be performed in the class of Giroux's convex surfaces we note that the only 'large' modification of the surface $\widehat{\Pi}$ occurs near the disc $m_{\theta_{0}} * \mathbb{S}^{1}$. The modification consists in cutting $\widehat{\Pi}$ along the arc $\widehat{v}_{1} \cup \widehat{v}_{2}$, then shifting the banks of the cut off one another by a $C^{1}$-small deformation, and finally gluing a disc consisting of two new tiles in the obtained hole.

Due to the symmetry between $\xi_{+}$and $\xi_{-}$it suffices to discuss the convexity issue with respect to $\xi_{+}$. We observe that the only portion of the dividing set $\delta_{+}$that is involved in the 'large' modification is a subarc of the arc $\dot{v}_{1}$, and we have just seen that the portion of the surface affected by this modification is a disc. The technique of [27] (see also [32]) allows to construct an isotopy for this modification that avoids non-convex surfaces.

Now we direct our attention to the modification of a canonic dividing configuration that occurs as a result of a wrinkle creation move.

Definition 3.3. Let $D=\left(\delta_{+}, \delta_{-}\right)$and $D^{\prime}=\left(\delta_{+}^{\prime}, \delta_{-}^{\prime}\right)$ be two dividing configurations on a surface $F$, such that they are isotopic outside of an open two-disc $d \subset F$ and satisfy the following conditions:

(1) each intersection $\delta_{+} \cap d, \delta_{-} \cap d, \delta_{+}^{\prime} \cap d, \delta_{-}^{\prime} \cap d$ is an arc;

(2) the $\operatorname{arcs} \delta_{+} \cap d$ and $\delta_{-} \cap d$ are disjoint;

(3) the $\operatorname{arcs} \delta_{+}^{\prime} \cap d$ and $\delta_{-}^{\prime} \cap d$ intersect transversely in two points.

Then we say that $D^{\prime}$ is obtained from $D$ by a bigon creation, and $D$ from $D^{\prime}$ by a bigon reduction. The disc in $d$ enclosed by two arcs one of which is contained in $\delta_{+}^{\prime}$ and the other in $\delta_{-}^{\prime}$ is called a bigon of $\left(\delta_{+}^{\prime}, \delta_{-}^{\prime}\right)$.

One can see from Figure 3.2 that the passage from $D^{\prime \prime}$ to $D^{\prime}$ in Lemma 3.1 is a composition of a bigon creation and an isotopy in $\widehat{\Pi}^{\prime}$. The following Lemma shows that any bigon creation can be 'realized' by a wrinkle creation move.

Lemma 3.2. Let $\Pi$ be a rectangular diagram of a surface, and let $\left(\delta_{+}, \delta_{-}\right)$be a canonic dividing configuration of $\widehat{\Pi}$. Let also $\left(\delta_{+}^{\prime}, \delta_{-}^{\prime}\right)$ be a dividing configuration on $\widehat{\Pi}$ obtained from $\left(\delta_{+}, \delta_{-}\right)$by a bigon creation. Then there exists a proper realization $\left(\Pi^{\prime}, \phi\right)$ of $\left(\delta_{+}^{\prime}, \delta_{-}^{\prime}\right)$ such that $\Pi \mapsto \Pi^{\prime}$ is a wrinkle creation move.

Proof. Let $d$ be as in Definition 3.3 . Then the $\operatorname{arcs} \delta_{+} \cap d$ and $\delta_{-} \cap d$ are subarcs of $\stackrel{\circ}{1}_{1}$ and $\stackrel{v}{2}_{2}$, respectively, for some \-vertex $v_{1}$ and $/$-vertex $v_{2}$ of $\Pi$. These two vertices lie on the same occupied level of $\Pi$, since there is clearly a connected component $\Omega$ of $\widehat{\Pi} \backslash\left(\delta_{+} \cup \delta_{-}\right)$such that both $\stackrel{\circ}{v}_{1}$ and $\stackrel{\circ}{2}_{2}$ contribute to the boundary of $\Omega$. Without loss of generality we may assume that they lie on the same meridian $m_{\theta_{0}}$. Then we can apply a wrinkle creation move exactly as described in Definition 3.2. Let $\Pi^{\prime}$ be the obtained diagram, and let $\phi$ be a homeomorphism from $\widehat{\Pi}$ to $\widehat{\Pi}^{\prime}$ that arises from the isotopy discussed in Lemma 3.1 Then $\phi$ takes $\left(\delta_{+}^{\prime}, \delta_{-}^{\prime}\right)$ to a dividing configuration on $\widehat{\Pi}^{\prime}$ equivalent to a canonic one. The claim follows.

Definition 3.4. Let $\Pi, v_{1}, v_{2}$, and $\psi$ be as in Definition 3.2 and suppose additionally that we have $v_{1}, v_{2} \in \partial \Pi$. Let $\Pi^{\prime}$ be obtained from $\Pi$ as described in Definition 3.2 with the following one distinction: 
- if $\Pi$ has no rectangle of the form $\left[\theta_{0} ; \theta_{1}\right] \times\left[\varphi^{\prime} ; \varphi^{\prime \prime}\right]$ or $\left[\theta_{1} ; \theta_{0}\right] \times\left[\varphi^{\prime} ; \varphi^{\prime \prime}\right]$ with $\left[\varphi^{\prime} ; \varphi^{\prime \prime}\right] \subset\left[\varphi_{1} ; \varphi_{2}\right]$, we do not add the rectangle $\left[\psi\left(\theta_{0}\right) ; \psi\left(\theta_{0}+\varepsilon\right)\right] \times\left[\varphi_{2} ; \varphi_{1}\right] ;$

- if $\Pi$ has no rectangle of the form $\left[\theta_{0} ; \theta_{1}\right] \times\left[\varphi^{\prime} ; \varphi^{\prime \prime}\right]$ or $\left[\theta_{1} ; \theta_{0}\right] \times\left[\varphi^{\prime} ; \varphi^{\prime \prime}\right]$ with $\left[\varphi^{\prime} ; \varphi^{\prime \prime}\right] \subset\left[\varphi_{2} ; \varphi_{1}\right]$, we do not add the rectangle $\left[\psi\left(\theta_{0}-\varepsilon\right) ; \psi\left(\theta_{0}\right)\right] \times\left[\varphi_{1} ; \varphi_{2}\right]$.

One of these two cases must occur.

Then we say that the passage from $\Pi$ to $\Pi^{\prime}$ is a vertical half-wrinkle creation move, and the inverse operation is a vertical half-wrinkle reduction move.

Horizontal half-wrinkle moves are defined similarly with the roles of $\theta$ and $\varphi$ exchanged.

A half-wrinkle move is illustrated in Figure 3.3. One can see that in the case $v_{1}, v_{2} \in \partial \Pi$ the respective
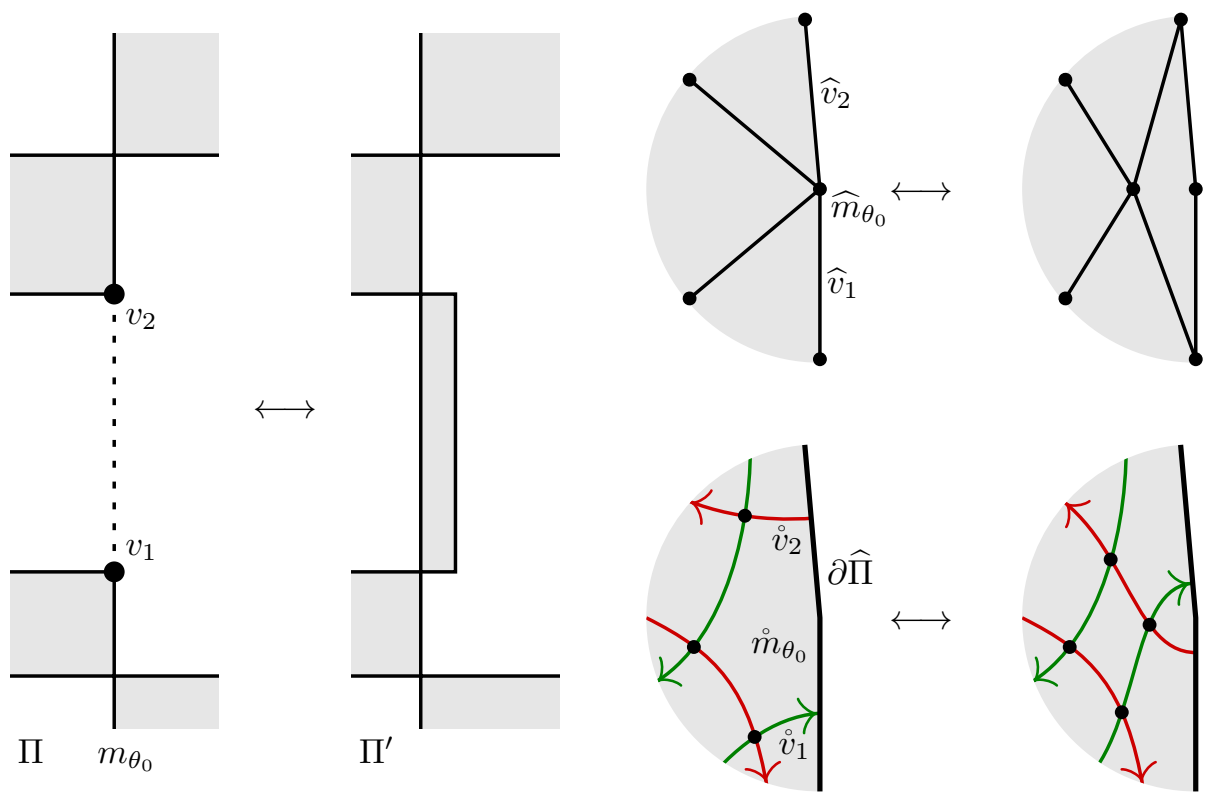

FiguRE 3.3. A vertical half-wrinkle move

wrinkle creation move can be decomposed into two half-wrinkle creation moves, hence the name.

There is a full analogue of Lemma 3.1 for half-wrinkle moves.

Lemma 3.3. Let $\Pi \mapsto \Pi^{\prime}$ be a half-wrinkle creation move, and let $D, D^{\prime}$ be canonic dividing configurations of $\widehat{\Pi}$ and $\widehat{\Pi}^{\prime}$, respectively. Then there exists a $C^{0}$-isotopy that brings $(\widehat{\Pi}, D)$ to $\left(\widehat{\Pi}^{\prime}, D^{\prime \prime}\right)$, where $D^{\prime \prime}$ is a dividing configuration on $\widehat{\Pi}^{\prime}$ weakly equivalent to $D^{\prime}$. If, additionally, we have $\partial \Pi=\partial \Pi^{\prime}$, then the isotopy can be chosen to be fixed on $\partial \widehat{\Pi}$.

Moreover, $\widehat{\Pi}$ and $\widehat{\Pi}^{\prime}$ are isotopic in the class of Giroux's convex surfaces with respect to either of the contact structures $\xi_{+}$and $\xi_{-}$, and if $\partial \Pi=\partial \Pi^{\prime}$, then the isotopy can be chosen to keep the boundary of the surface arbitrarily $C^{0}$-close to $\partial \widehat{\Pi}$.

We omit the proof, which is similar to that of Lemma 3.1

Remark 3.1. A small complication of the formulation of Lemma 3.3, if compared to Lemma 3.1, is due to the fact that the boundaries $\partial \widehat{\Pi}$ and $\partial \widehat{\Pi}^{\prime}$ have singularities, some of which may need to be smoothed in order to perform an isotopy from $\widehat{\Pi}$ to $\widehat{\Pi}^{\prime}$ through Giroux's convex surfaces. This is because the boundary framings induced by $\Pi$ and $\Pi^{\prime}$ are now different, which was not the case in Lemma 3.1 .

Definition 3.5. Let $\left(\delta_{+}, \delta_{-}\right)$and $\left(\delta_{+}^{\prime}, \delta_{-}^{\prime}\right)$ be two dividing configurations on a surface $F$. Assume that there is a closed disc $b \subset F$ whose boundary consists of three arcs $\alpha, \beta, \gamma$, such that $\alpha \subset \delta_{+}^{\prime}, \beta \subset \delta_{-}^{\prime}$, and $\gamma \subset \partial F$. Such a disc will be called a half-bigon of $\left(\delta_{+}^{\prime}, \delta_{-}^{\prime}\right)$. 
Assume also that there is an open neighborhood $d$ of $b$ in $F$ homeomorphic to a half-disc such that the following holds:

(1) each intersection $\delta_{+} \cap d, \delta_{-} \cap d, \delta_{+}^{\prime} \cap d, \delta_{-}^{\prime} \cap d$ is a half-closed arc;

(2) the $\operatorname{arcs} \delta_{+} \cap d$ and $\delta_{-} \cap d$ are disjoint;

(3) the arcs $\delta_{+}^{\prime} \cap d$ and $\delta_{-}^{\prime} \cap d$ intersect transversely in a single point.

Then we say that the transition $\left(\delta_{+}, \delta_{-}\right) \mapsto\left(\delta_{+}^{\prime}, \delta_{-}^{\prime}\right)$ is a half-bigon creation and the inverse one is a half-bigon reduction.

Lemma 3.4. Let $\Pi$ be a rectangular diagram of a surface, and let $\left(\delta_{+}, \delta_{-}\right)$be a canonic dividing configuration of $\widehat{\Pi}$. Let also $\left(\delta_{+}^{\prime}, \delta_{-}^{\prime}\right)$ be a dividing configuration obtained from $\left(\delta_{+}, \delta_{-}\right)$by a half-bigon creation. Then there exists a proper realization $\left(\Pi^{\prime}, \phi\right)$ of $\left(\delta_{+}^{\prime}, \delta_{-}^{\prime}\right)$ such that $\Pi \mapsto \Pi^{\prime}$ is a half-wrinkle creation move.

The proof, which is similar to that of Lemma 3.2, is omitted.

Definition 3.6. Let $\Pi, v_{1}, v_{2}$, and $\psi$ be as in Definition 3.2 except that $v_{2} \in m_{\theta_{0}}$ is not a vertex of $\Pi$ and, moreover, $v_{2}$ does not belong to the boundary of any rectangle in $\Pi$. Let $\Pi^{\prime}$ be obtained from $\Pi$ by exactly the same modification as the one described in Definition 3.2 . Then the passage from $\Pi$ to $\Pi^{\prime}$ is called a type I stabilization move and the inverse one a type I destabilization move.

Similarly, if $v_{2}$ is as in Definition 3.2. and $v_{1} \in m_{\theta_{0}}$ does not belong to the boundary of any rectangle, then the passage from $\Pi$ to $\Pi^{\prime}$ is called a type II stabilization move and the inverse one a type II destabilization move.

If the roles of $\theta$ and $\varphi$ coordinates are exchanged in this definition the obtained moves are still called type I or type II (de)stabilization moves, respectively.

An example of a (de)stabilization move is shown in Figure 3.4
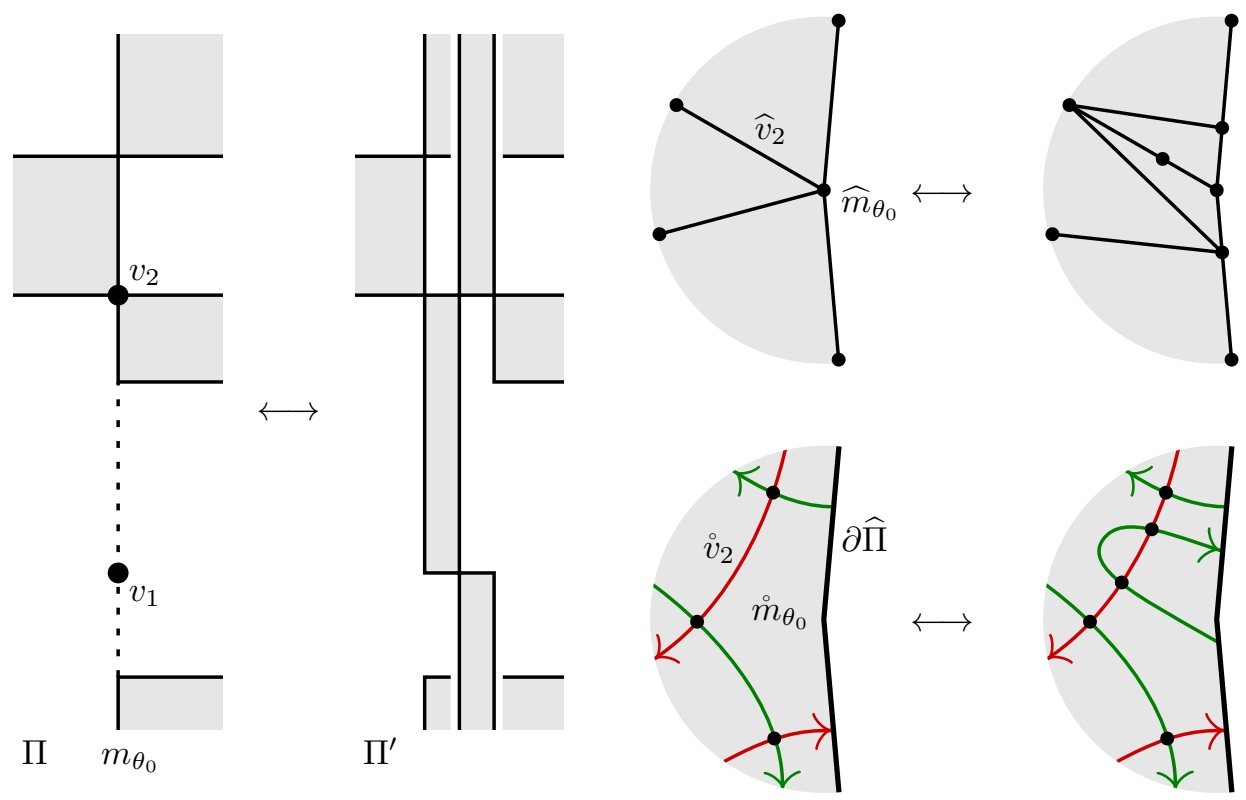

FiguRE 3.4. A type II stabilization/destabilization moves

Lemma 3.5. Let $\Pi \mapsto \Pi^{\prime}$ be a stabilization move, and let $\left(\delta_{+}, \delta_{-}\right),\left(\delta_{+}^{\prime}, \delta_{-}^{\prime}\right)$ be canonic dividing configurations of $\widehat{\Pi}$ and $\widehat{\Pi}^{\prime}$, respectively. Then there is an isotopy that brings $\left(\widehat{\Pi}, \delta_{+}\right)$to $\left(\widehat{\Pi}^{\prime}, \delta_{+}^{\prime}\right)$ if the stabilization is of type $I$, and $\left(\widehat{\Pi}, \delta_{-}\right)$to $\left(\widehat{\Pi}^{\prime}, \delta_{-}^{\prime}\right)$ if the stabilization is of type II. Moreover, the isotopy can be chosen 
to keep the surface in the class of Giroux's convex surfaces with respect to $\xi_{+}$if the stabilization is of type I, and with respect to $\xi_{-}$if the stabilization is of type II.

The proof is again similar to that of Lemma 3.1 and is omitted. Note that now the boundary of the surface is necessarily modified. This modification preserves the equivalence class of $\widehat{\partial \Pi}$ as a Legendrian link with respect to $\xi_{+}$if the stabilization is of type I, and with respect to $\xi_{-}$if the stabilization is of type II.

Note also that if $\Pi \mapsto \Pi^{\prime}$ is a type I (respectively, type II) stabilization of a rectangular diagram of a surface, then $\partial \Pi \mapsto \partial \Pi^{\prime}$ is a type I (respectively, type II) stabilization of a rectangular diagram of a link (in the generalized sense of [7]). Therefore, a stabilization does always change the equivalence class of $\widehat{\Pi}$ as a Giroux's convex surface with respect to one of the contact structures $\xi_{-}$or $\xi_{+}$, depending on whether it is of type I or type II. This can be seen from the fact that the boundary link $\partial \widehat{\Pi}$ undergoes a Legendrian stabilization with respect to one of the contact structures, as well as from the fact that a new arc is added to the respective dividing set.

Remark 3.2. There are two more, naturally defined, moves of rectangular diagrams of surfaces that induce a stabilization of the boundary. They are shown in Figure 3.5. The first one is an addition of a rectangle
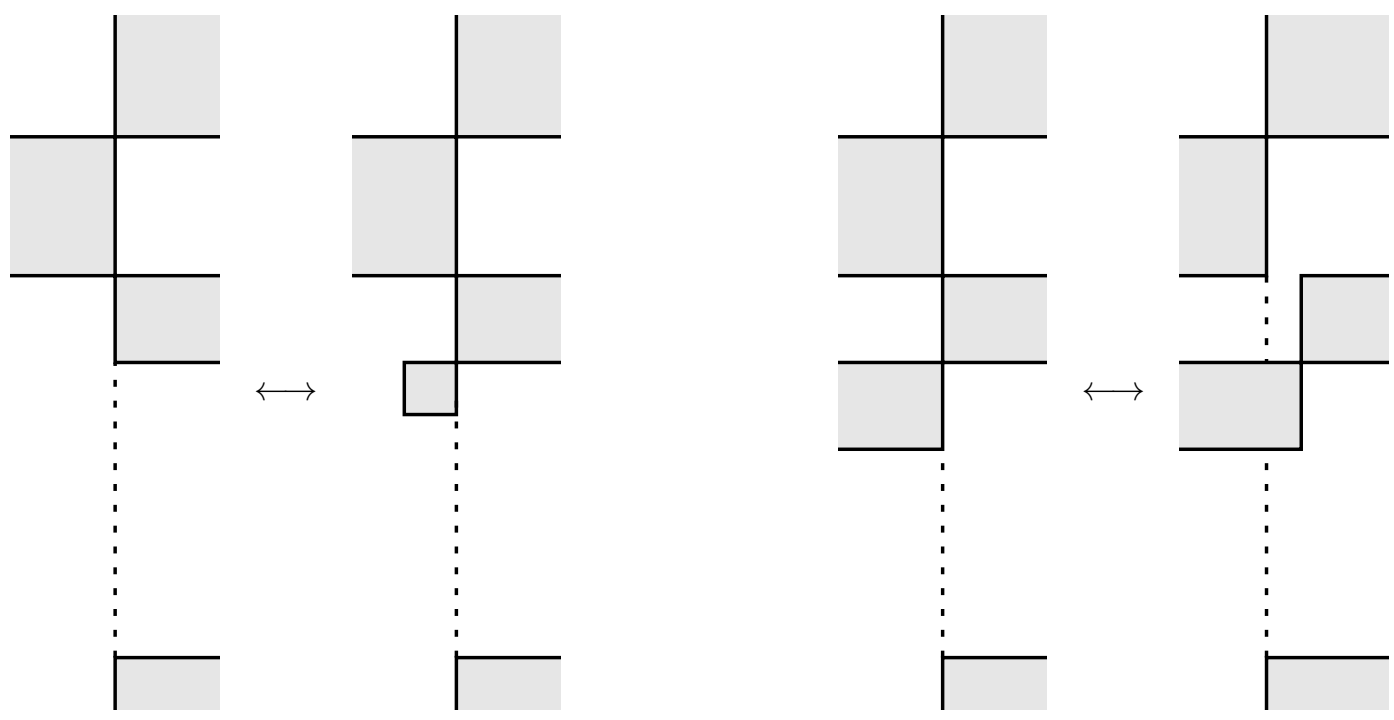

FiguRE 3.5. Two more ways to stabilize the boundary

sharing exactly one vertex with the diagram. The second move consists in a 'splitting' of an occupied level containing an edge of the boundary into two occupied levels close to the original one. Though these moves look pretty simple we don't include them into the list of basic moves. We leave it as an exercise to the reader to show explicitly that these moves can be decomposed into basic moves.

3.3. Twisting a 'rectangular' surface around the boundary. In [12, Subsection 2.5] we defined a framing of a cusp-free link $L$ in $\mathbb{S}^{3}$ as a way, viewed up to smooth isotopy, to attach a union of annuli (which should be a surface with corners) to $L$. Recall that a framing in our sense contains more information than just the self-linking numbers of the components. We also introduced in 12 the concept of a framing of a rectangular diagram of a link as an ordering of each pair of vertices that forms an edge of the diagram. When $f$ is a framing we use the signs $<_{f}$ and $>_{f}$ to denote the corresponding relation (note that this is not an ordering, sometimes not even a partial ordering, on the set all vertices of the diagram). 
Let $(R, f)$ be a framed rectangular diagram of a link, and let $v$ be a vertex of $R$. Let also $v_{1}$ and $v_{2}$ be vertices of $R$ such that $\left\{v, v_{1}\right\}$ is a vertical edge of $R$, and $\left\{v, v_{2}\right\}$ is a horizontal edge. We call $v a /-v e r t e x$ (respectively, a \-vertex) of $(R, f)$ if $v_{1}<_{f} v<_{f} v_{2}$ or $v_{1}>_{f} v>_{f} v_{2}$ (respectively, $v_{1}<_{f} v>_{f} v_{2}$ or $\left.v_{1}>_{f} v<_{f} v_{2}\right)$.

If $R$ is connected, then for any framing $f$ of $R$ there is a unique framing $f^{\prime} \neq f$ such that $(R, f)$ and $\left(R, f^{\prime}\right)$ have the same set of $/$-vertices. This framing is defined by $u<_{f^{\prime}} v \Leftrightarrow u>_{f} v$. We say that such $f^{\prime}$ is opposite to $f$.

Every rectangular diagram of a surface $\Pi$ defines a framing on the rectangular diagram of a link $\partial \Pi$ through the rule given in [12, Proposition 3]. We call this framing the boundary framing of $\partial \Pi$ induced by $\Pi$ and denote by $f^{\Pi}$. One can see that $v$ is a /-vertex (respectively, a \-vertex) of $\left(\partial \Pi, f^{\Pi}\right)$ if and only if $v$ is a $/$-vertex (respectively, a \-vertex) of $\Pi$.

When $\Pi$ is a rectangular diagram of a surface and $R$ is a rectangular diagram of a link such that $R \subset$ $\partial \Pi$, we denote by $\mathrm{tb}_{+}(R ; \Pi)$ (respectively, by tb- $\left.(R ; \Pi)\right)$ the Thurston-Bennequin number $\mathrm{tb}(\widehat{R} ; \widehat{\Pi})$ (respectively, tb- $(\widehat{R} ; \widehat{\Pi})$ (see [12, Definitions 16 and 17$])$. These numbers have a very simple combinatorial meaning: $-\mathrm{tb}_{+}(R ; \Pi)$ (respectively, $\left.-\mathrm{tb}_{-}(R ; \Pi)\right)$ is one half of the number of \-vertices (respectively, $/$-vertices) of $\Pi$ in $R$.

Proposition 3.1. Let $\Pi$ be a rectangular diagram of a surface, and let $Q$ be a connected component of the rectangular diagram of a link $R=\partial \Pi$. Let also $F \subset \mathbb{S}^{3}$ be a compact surface with the following properties:

(1) $\partial F=\partial \widehat{\Pi}$;

(2) there is a tubular open neighborhood $U$ of $\widehat{Q}$ such that $\bar{U}$ intersects each of $F$ and $\widehat{\Pi}$ in an annulus, and we have $F \backslash U=\widehat{\Pi} \backslash U$;

(3) at every point of $\widehat{Q}$ the surface $F$ is tangent either to $\xi_{+}$or $\xi_{-}$.

Then there exists a rectangular diagram of a surface $\Pi^{\prime}$ with the following properties:

(1) $\partial \Pi^{\prime}=\partial \Pi$;

(2) there is a $C^{0}$-isotopy $\phi$ from $\widehat{\Pi}$ to $\widehat{\Pi}^{\prime}$ fixed on $\partial \widehat{\Pi}$ and composed of two isotopies: the first, $\phi^{\prime}$, brings $\widehat{\Pi}$ to $F$, and the second, $\phi^{\prime \prime}$, brings $F$ to $\widehat{\Pi}^{\prime}$, such that the following holds:

(a) $\phi^{\prime}$ is fixed outside $U$;

(b) $\phi^{\prime \prime}$ is $C^{1}$-smooth, it preserves the tangent plane to the surface at every point of $\partial F$, and for all $t \in[0 ; 1], \phi_{t}^{\prime \prime}$ is $C^{1}$-close to the identity outside $U$;

(c) if $\mathrm{tb}_{+}(Q ; \Pi)<0$ (respectively, $\left.\mathrm{tb}_{-}(Q ; \Pi)<0\right)$ then $\phi$ brings $\delta_{+}$(respectively, $\left.\delta_{-}\right)$to an abstract dividing set on $\widehat{\Pi}^{\prime}$ equivalent to $\delta_{+}^{\prime}$ (respectively, to $\left.\delta_{-}^{\prime}\right)$, where $\left(\delta_{+}, \delta_{-}\right)$and $\left(\delta_{+}^{\prime}, \delta_{-}^{\prime}\right)$ are canonic dividing configurations of $\widehat{\Pi}$ and $\widehat{\Pi}^{\prime}$, respectively.

Proof. It follows from the assumptions of the proposition that there exists a $C^{0}$-isotopy $\psi:[0 ; 1] \times \widehat{\Pi} \rightarrow \mathbb{S}^{3}$ from $\widehat{\Pi}$ to $F$ such that $\left.\psi_{t}\right|_{\widehat{\Pi} \backslash U}=$ id for all $t \in[0 ; 1]$. Clearly, such an isotopy can be chosen to be $C^{1}-$ smooth outside $[0 ; 1] \times\left(\widehat{Q} \cap\left(\mathbb{S}_{\tau=0}^{1} \cup \mathbb{S}_{\tau=1}^{1}\right)\right)$.

Let $v_{1}, v_{2}, \ldots, v_{n}$ be all the vertices of $Q$ numbered in the order they follow on $Q$ (choose any orientation of $Q$ if no one is given), starting from an arbitrarily chosen vertex. Their indices are regarded modulo $n$, that is, $v_{i+n}=v_{i}$.

For every vertex $v$ of $Q$ and each point $p \in \operatorname{int}(\widehat{v})$, let $\lambda(p)$ be the signed angle by which the tangent plane $T_{p} \psi_{t}(\widehat{\Pi})$ rotates around the arc $\widehat{v}$ when $t$ runs from 0 to 1 . The function $\lambda$ can be extended continuously to the whole curve $\widehat{Q}$. Since the surfaces $\widehat{\Pi}$ and $F$ have the same tangent planes at any point $p \in \widehat{Q} \cap\left(\mathbb{S}_{\tau=0}^{1} \cup \mathbb{S}_{\tau=1}^{1}\right)$, the value of $\lambda(p)$ at any such point is an integer multiple of $\pi$. Denote by $k_{i}$ the integer such that $\lambda$ (the end point of $\left.\widehat{v}_{i}\right)=k_{i} \pi$.

Denote by $f$ the framing of $Q$ corresponding to the admissible framing of $\widehat{Q}$ induced by $F$. For this framing, a vertex $v \in Q$ has type ' $/$ ' if $F$ is tangent to $\xi_{+}$along $\widehat{v}$, and type ' $\backslash$ ' if $F$ is tangent to $\xi_{-}$ along $\widehat{v}$. 
The relative twist of $\xi_{-}$with respect to $\xi_{+}$along $\widehat{v}, v \in \mathbb{T}^{2}$, is equal to $\pi$. Therefore,

$$
k_{i}-k_{i-1}=\left\{\begin{aligned}
1 & \text { if } v_{i} \text { is a } / \text {-vertex of }\left(Q, f^{\Pi}\right) \text { and a } \backslash \text {-vertex of }(Q, f), \\
-1 & \text { if } v_{i} \text { is a } / \text {-vertex of }(Q, f) \text { and a } \backslash \text {-vertex of }\left(Q, f^{\Pi}\right), \\
0 & \text { if the types of } v \text { as a vertex of }(Q, f) \text { and }\left(Q, f^{\Pi}\right) \text { coincide. }
\end{aligned}\right.
$$

Now we proceed by induction in the sum $\sum_{i=1}^{n}\left|k_{i}\right|$, which will be referred to as the framing distance between $\widehat{\Pi}$ and $F$. The induction base is trivial. Indeed, if the framing distance between $\widehat{\Pi}$ and $F$ is zero, then the isotopy $\psi$ above can be chosen so that the tangent plane to the surface be fixed at any point of $\widehat{Q}$ during the isotopy. We simply take $\Pi^{\prime}=\Pi$.

Suppose that $\sum_{i=1}^{n}\left|k_{i}\right|>0$. To make the induction step, we show how to find a rectangular diagram of a surface $\Pi_{1}$ such that the surface $\widehat{\Pi}_{1}$ is $C^{1}$-close to $\widehat{\Pi}$ outside $U$, intersects $\bar{U}$ in an annulus, and has a smaller framing distance from $F$ than $\widehat{\Pi}$ has. There are several cases to consider.

Case 1: there exist $l \leqslant m$ such that $k_{l}=k_{l+1}=\ldots=k_{m}>0$ and $k_{l-1}=k_{m+1}=k_{l}-1$.

By construction, $v_{l}$ is a $/$-vertex, and $v_{m+1}$ is a \-vertex of $\Pi$. Therefore, there exists $j \in[l ; m]$ such that $v_{j}$ is a $/$-vertex, and $v_{j+1}$ is a \-vertex of $\Pi$. By construction, the pair $\left(v_{j}, v_{j+1}\right)$ is an edge of $Q$.

We can, therefore, apply a half-wrinkle creation move $\Pi \mapsto \Pi_{1}$ so that the diagram is modified in a small neighborhood of the occupied level of $\Pi$ containing $v_{j}$ and $v_{j+1}$. Moreover, we can ensure that the boundary of the diagram is not modified, $\partial \Pi_{1}=\partial \Pi$. Then $v_{j}$ will become a \-vertex, and $v_{j+1}$ will become a $/$-vertex of $\Pi_{1}$. One can see from this that the passage from $\Pi$ to $\Pi_{1}$ reduces the framing distance to $F$ by 1 .

The rectangle added to the diagram $\Pi$ to obtain $\Pi_{1}$ can be chosen arbitrarily narrow, stretched in the direction of the respective edge of $Q$. This ensures that the corresponding tile is contained in $U$, and that $\widehat{\Pi}$ and $\widehat{\Pi}_{1}$ are $C^{1}$-close to each other outside $U$. A $C^{0}$-isotopy from $\widehat{\Pi}_{\text {to }} \widehat{\Pi}_{1}$ arising in this way, which perturbs the surfaces only slightly (in the $C^{1}$ sense) outside $U$, brings a canonic dividing configuration of $\widehat{\Pi}$ to a dividing configuration weakly equivalent to a canonic dividing configuration of $\widehat{\Pi}_{1}$ (see Lemma 3.3).

Case 2: there exist $l \leqslant m$ such that $k_{l}=k_{l+1}=\ldots=k_{m}<0$ and $k_{l-1}=k_{m+1}=k_{l}+1$.

This case is symmetric to the previous one and is left to the reader.

Case 3: all $k_{i}$ are equal, and we have $\mathrm{tb}_{+}(Q ; \Pi), \mathrm{tb}_{-}(Q ; \Pi)<0$.

If all $k_{i}$ are positive, we find $j$ such that $v_{j}$ is a $/$-vertex, and $v_{j+1}$ is a \-vertex of $\Pi$, and then proceed as in Case 1 . If all $k_{i}$ 's are negative, we find $j$ such that $v_{j}$ is a $\backslash$-vertex, and $v_{j+1}$ is a $/$-vertex of $\Pi$, and then proceed symmetrically to Case 1 .

Case 4: all $k_{i}$ are equal, and we have $\mathrm{tb}_{+}(Q ; \Pi)=0$.

In this case, $Q$ consists only of $/$-vertices of $\Pi$.

For an $\varepsilon>0$ such that $\varepsilon$ is smaller than the distance between any two parallel occupied levels of $\Pi$, we define rectangular diagrams of a surface $\Pi_{\varepsilon}$ and $\Pi_{-\varepsilon}$ as follows.

To obtain $\Pi_{\varepsilon}$, first, shift all occupied levels, vertical and horizontal, containing vertices of $Q$ by $-\varepsilon$. Then, for any $u=\left(\theta_{1}, \varphi_{1}\right)$ and $v=\left(\theta_{2}, \varphi_{2}\right)$ such that $\{u, v\}$ is an edge of $Q$ and $u<_{f} \Pi v$, add the rectangle $\left[\theta_{1}-\varepsilon, \theta_{1}\right] \times\left[\varphi_{2}, \varphi_{1}-\varepsilon\right]$ to the diagram if $\{u, v\}$ is a vertical edge of $Q$, and the rectangle $\left[\theta_{2}, \theta_{1}-\right.$ $\varepsilon] \times\left[\varphi_{1}-\varepsilon, \varphi_{1}\right]$ if $\{u, v\}$ is a horizontal edge of $Q$. Figure 3.6 shows how the diagram is changed near a vertex $v$ of $Q$.

Similarly, to obtain $\Pi_{-\varepsilon}$, first, shift all occupied levels, vertical and horizontal, containing vertices of $Q$ by $\varepsilon$. Then for any $u=\left(\theta_{1}, \varphi_{1}\right)$ and $v=\left(\theta_{2}, \varphi_{2}\right)$ such that $\{u, v\}$ is an edge of $Q$ and $u<_{f} \Pi v$, add the rectangle $\left[\theta_{1}, \theta_{1}+\varepsilon\right] \times\left[\varphi_{2}+\varepsilon, \varphi_{1}\right]$ to the diagram if $\{u, v\}$ is a vertical edge of $Q$, and the rectangle $\left[\theta_{2}+\varepsilon, \theta_{1}\right] \times\left[\varphi_{1}, \varphi_{1}+\varepsilon\right]$ if $\{u, v\}$ is a horizontal edge of $Q$.

One can see that if $\varepsilon$ is small, then each of the surfaces $\widehat{\Pi}_{\varepsilon}$ and $\widehat{\Pi}_{-\varepsilon}$ is $C^{1}$-close to a surface obtained from $\widehat{\Pi}$ by attaching a narrow collar along $\widehat{Q}$, and this collar has the form of a union of tiles which correspond to the narrow rectangles added to the diagram. For one of the passages $\Pi \mapsto \Pi_{\varepsilon}$ and $\Pi \mapsto \Pi_{-\varepsilon}$ all $k_{i}$ 's are incremented by 1 , and for the other, all $k_{i}$ 's are dropped by 1 . So, the framing distances from 

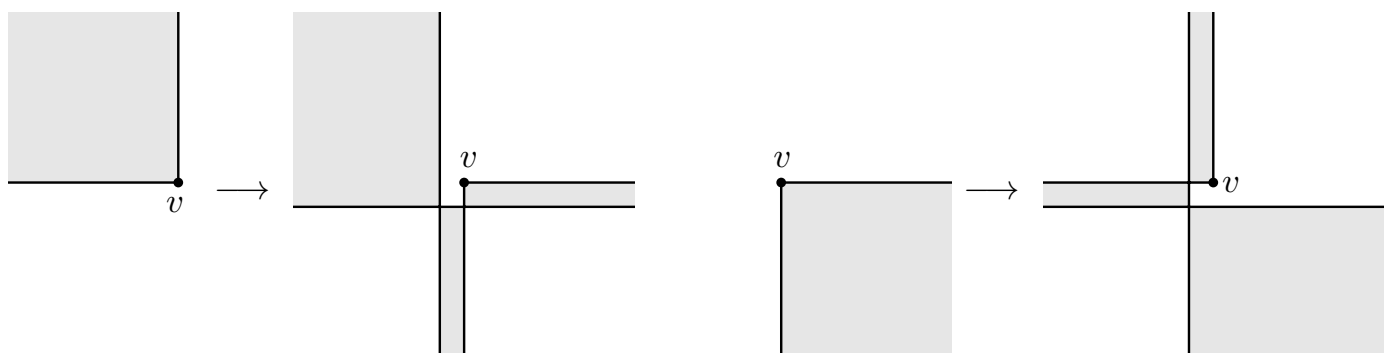

Figure 3.6. Twisting $\widehat{\Pi}$ around $\widehat{Q} \subset \partial \widehat{\Pi}$ in the case $\operatorname{tb}_{+}(Q ; \Pi)=0$

the corresponding surfaces to $F$ are changed by $n$ and $-n$, respectively. We choose for $\Pi_{1}$ the one of the two diagrams $\Pi_{\varepsilon}$ and $\Pi_{-\varepsilon}$ from which the framing distance to $F$ is smaller. This choice depends on the orientation of $Q$ and the framing $Q^{\Pi}$.

Denote by $\left(\delta_{+}^{0}, \delta_{-}^{0}\right)$ a canonic dividing configuration of the collar added to $\widehat{\Pi}$ to obtain $\widehat{\Pi}_{1}$. One can see that $\delta_{+}^{0}$ is a separating simple closed curve, whereas $\delta_{-}^{0}$ consists of non-separating arcs that prolong some connected components of $\delta_{-}$. Therefore, if $\left(\delta_{1+}, \delta_{1-}\right)$ is a canonic dividing configuration of $\widehat{\Pi}_{1}$, then a $C^{0}$-small deformation that takes $\widehat{\Pi}$ to $\widehat{\Pi}_{1}$ also takes $\delta_{-}$to an abstract dividing set equivalent to $\delta_{1-}$.

Case 5: all $k_{i}$ are equal, and we have tb- $(Q ; \Pi)=0$.

This case is symmetric to Case 4 .

The induction step follows.

Remark 3.3. In addition to the assertion of Proposition 3.1, one can show by means of the general theory of Giroux's convex surfaces that there exists an isotopy from $\widehat{\Pi}$ to $\widehat{\Pi}^{\prime}$ in the class of Giroux's convex surfaces with respect to $\xi_{+}$if $\operatorname{tb}_{+}(Q ; \Pi)<0$, and with respect to $\xi_{-}$if $\mathrm{tb}_{-}(Q ; \Pi)<0$. If both conditions hold, this fact also follows from Lemmas 3.1 and 3.3 and the reasoning in the proof of Proposition 3.1. If only one of the conditions holds, one can construct a decomposition of the collar addition illustrated in Figure 3.6 into basic moves excluding type I (respectively, type II) moves if $\operatorname{tb}_{+}(Q ; \Pi)=0$ (respectively, if tb_ $(Q ; \Pi)=0)$, and then apply the respective lemmas from this section.

3.4. Exchange moves. Lemmas 3.2 and 3.4 say, vaguely speaking, that any (half-)bigon creation in a canonic dividing configuration can be realized by applying a (half-)wrinkle move to the corresponding diagram. Here we consider the inverse operations, (half-)bigon reductions, which require more care.

If a canonic dividing configuration has a bigon or a half-bigon, this does not necessarily imply that the (half-)bigon can be immediately reduced by a (half-)wrinkle move. One of the obstructions comes from the fact that the two new rectangles created as a result of a wrinkle creation (or one rectangle in the case of a half-wrinkle creation) are thin in the sense of the following definition.

Definition 3.7. A rectangle $r=\left[\theta_{1} ; \theta_{2}\right] \times\left[\varphi_{1} ; \varphi_{2}\right]$ of a rectangular diagram of a surface $\Pi$ is called vertically thin (respectively, horizontally thin) if there are no vertical occupied levels of $\Pi$ in the domain $\theta \in\left(\theta_{1} ; \theta_{2}\right)$ (respectively, in the domain $\varphi \in\left(\varphi_{1} ; \varphi_{2}\right)$ ).

If a canonic dividing configuration of $\widehat{\Pi}$ has a bigon, but the rectangles corresponding to the bigon's corners are not thin, a wrinkle reduction reducing this bigon is not applicable to $\Pi$. Whether or not a rectangle is thin cannot, in general, be read off the combinatorial structure of the canonic dividing configuration. However, some rectangles can be made thin by exchange moves, which are defined below, without modification of that structure. Exchange moves are, by definition, neutral.

Definition 3.8. Let $\Pi$ be a rectangular diagram of a surface, and let $\theta_{1}, \theta_{2}, \theta_{3}, \varphi_{1}, \varphi_{2} \in \mathbb{S}^{1}$ be such that:

(1) we have $\theta_{2} \in\left(\theta_{1} ; \theta_{3}\right)$;

(2) the rectangles $r_{1}=\left[\theta_{1} ; \theta_{2}\right] \times\left[\varphi_{1} ; \varphi_{2}\right]$ and $r_{2}=\left[\theta_{2} ; \theta_{3}\right] \times\left[\varphi_{2} ; \varphi_{1}\right]$ contain no vertices of $\Pi$;

(3) the vertices of $r_{1}$ and $r_{2}$ are disjoint from the rectangles of $\Pi$. 
Let $f: \mathbb{S}^{1} \rightarrow \mathbb{S}^{1}$ be a map that is identical on $\left[\theta_{3} ; \theta_{1}\right]$, and exchanges the intervals $\left(\theta_{1} ; \theta_{2}\right)$ and $\left(\theta_{2} ; \theta_{3}\right)$ :

$$
f(\theta)=\left\{\begin{array}{l}
\theta-\theta_{2}+\theta_{3}, \text { if } \theta \in\left(\theta_{1}, \theta_{2}\right], \\
\theta-\theta_{2}+\theta_{1}, \text { if } \theta \in\left(\theta_{2}, \theta_{3}\right) .
\end{array}\right.
$$

Choose a self-homeomorphism $\psi$ of $\mathbb{S}^{1}$ identical on $\left[\theta_{3} ; \theta_{1}\right]$, and let

$$
\Pi^{\prime}=\left\{\left[\psi\left(f\left(\theta^{\prime}\right)\right) ; \psi\left(f\left(\theta^{\prime \prime}\right)\right)\right] \times\left[\varphi^{\prime} ; \varphi^{\prime \prime}\right]:\left[\theta^{\prime} ; \theta^{\prime \prime}\right] \times\left[\varphi^{\prime} ; \varphi^{\prime \prime}\right] \in \Pi\right\} .
$$

Then we say that the passage from $\Pi$ to $\Pi^{\prime}$, or the other way, is a vertical exchange move. An example is shown in Figure 3.7

A horizontal exchange move is defined similarly with the roles of $\theta$ and $\varphi$ exchanged.
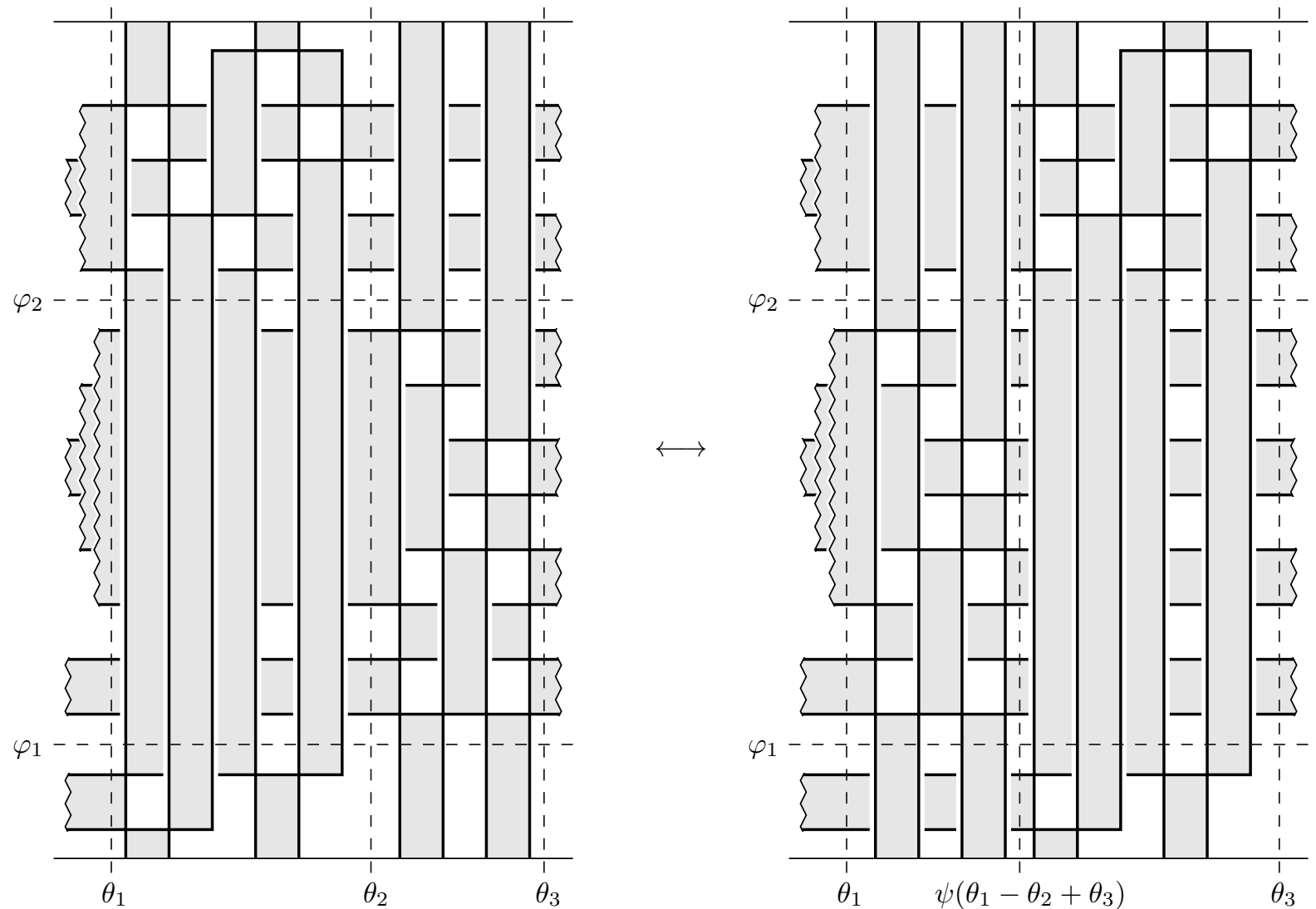

FiguRE 3.7. An exchange move

The following lemma is obvious.

Lemma 3.6. If $\Pi \mapsto \Pi^{\prime}$ is an exchange move of rectangular diagrams of a surface, then the rectangular diagrams of a link $\partial \Pi$ and $\partial \Pi^{\prime}$ are connected by a finite sequence of exchange moves.

Lemma 3.7. Let $\Pi \mapsto \Pi^{\prime}$ be an exchange move, and let $D, D^{\prime}$ be canonic dividing configurations of $\widehat{\Pi}$ and $\widehat{\Pi}^{\prime}$, respectively. Then there exists an isotopy bringing $(\widehat{\Pi}, D)$ to $\left(\widehat{\Pi}^{\prime}, D^{\prime}\right)$. Moreover, $\widehat{\Pi}$ and $\widehat{\Pi}^{\prime}$ are isotopic in the class of Giroux's convex surfaces with respect to either of the contact structures $\xi_{+}$and $\xi_{-}$.

Proof. Due to symmetry it suffices to show the existence of an isotopy from $(\widehat{\Pi}, D)$ to $\left(\widehat{\Pi}^{\prime}, D^{\prime}\right)$ in the class of Giroux's convex surfaces with respect to $\xi_{+}$. We may assume without loss of generality that $0 \notin\left[\theta_{1} ; \theta_{3}\right]$, and treat the $\theta$-coordinate of any point from $\left[\theta_{1} ; \theta_{3}\right] * \mathbb{S}^{1}$ as a real number in the interval $(0,2 \pi)$. 
We use the notation from Definition 3.8 . Denote also by $\widetilde{\mathscr{G}}$ the 1 -skeleton of the tiling of $\widehat{\Pi}$, that is,

$$
\widetilde{\mathscr{G}}=\bigcup_{v \text { is a vertex of } \Pi} \widehat{v} .
$$

Similarly, let $\widetilde{\mathscr{G}^{\prime}}$ be the 1-skeleton of the tiling of $\widehat{\Pi}^{\prime}$.

Let $m_{\theta^{1}}, \ldots, m_{\theta^{k}}$ be the occupied meridians of $\Pi$ in the interval $\theta \in\left[\theta_{1} ; \theta_{3}\right]$, listed in the increasing order, $\theta_{1}<\theta^{1}<\theta^{2}<\ldots<\theta^{k}<\theta_{3}$. Denote $\psi\left(f\left(\theta^{i}\right)\right)$ by $\theta^{\prime i}$. The points $p_{i}=\widehat{m}_{\theta^{i}}$ and $p_{i}^{\prime}=\widehat{m}_{\theta^{\prime} i}$ are vertices of $\widetilde{\mathscr{G}}$ and $\widetilde{\mathscr{G}^{\prime}}$, respectively, $i=1, \ldots, k$. All the other vertices of $\widetilde{\mathscr{G}}$ are the same as those of $\widetilde{\mathscr{G}^{\prime}}$.

Let $\phi:[0 ; 1] \times \widetilde{\mathscr{G}} \rightarrow \mathbb{S}^{3}$ be a homotopy such that:

(1) for all $t \in[0,1]$ the image $\phi_{t}(\widetilde{\mathscr{G}})$ has the form $\widehat{X}, X \subset \mathbb{T}^{2}$;

(2) $\phi_{0}=\left.\mathrm{id}\right|_{\widetilde{G}}$

(3) $\phi_{t}\left(p_{i}\right)=\widehat{m}_{(1-t) \theta^{i}+t \theta^{\prime i}}, i=1, \ldots, k, t \in[0 ; 1]$;

(4) $\phi_{t}(p)=p, t \in[0 ; 1]$, if $p \in \widetilde{\mathscr{G}} \cap\left(\mathbb{S}_{\tau=0}^{1} \cup \mathbb{S}_{\tau=1}^{1}\right)$ is not in $\left\{p_{1}, \ldots, p_{k}\right\}$.

Such a homotopy is clearly unique.

The homotopy $\phi$ is not an isotopy if $\theta^{1} \in\left(\theta_{1} ; \theta_{2}\right)$ and $\theta^{k} \in\left(\theta_{2} ; \theta_{3}\right)$, but it is not very far from being an isotopy. For any $t \in[0 ; 1]$ the map $\phi_{t}$ is an immersion and there are only finitely many moments $t$ when it is not an embedding. Namely, this occurs when $\phi_{t}\left(p_{i}\right)=\phi_{t}\left(p_{j}\right)$ for some $i, j, 1 \leqslant i<j \leqslant k$. In this case, we necessarily have $\theta^{i} \in\left(\theta_{1} ; \theta_{2}\right)$ and $\theta^{j} \in\left(\theta_{2} ; \theta_{3}\right)$.

For all $t \in[0 ; 1]$ the images of all edges of $\widetilde{\mathscr{G}}$ incident to the vertex $p_{i}$ under the map $\phi_{t}$ are contained in the domain $\mathbb{S}_{\tau=1}^{1} *\left(\varphi_{2} ; \varphi_{1}\right)$ if $\theta^{i} \in\left[\theta_{1} ; \theta_{2}\right]$, and in the domain $\mathbb{S}_{\tau=1}^{1} *\left(\varphi_{1} ; \varphi_{2}\right)$ if $\theta^{i} \in\left[\theta_{2} ; \theta_{3}\right]$. We can disturb $\phi_{t}$ slightly so that for all $t \in(0 ; 1)$ the image $\phi_{t}(e)$ of any edge $e$ of $\widetilde{\mathscr{G}}$ remains a Legendrian arc (with respect to $\xi_{+}$), and the point $\phi_{t}\left(p_{i}\right)$ is contained in the domain $\varphi \in\left(\varphi_{2} ; \varphi_{1}\right), \tau<1$ if $\theta^{i} \in\left(\theta_{1} ; \theta_{2}\right)$, and in the domain $\varphi \in\left(\varphi_{1} ; \varphi_{2}\right), \tau<1$ if $\theta^{i} \in\left(\theta_{2} ; \theta_{3}\right)$.

Then for each $t \in[0 ; 1]$ the map $\phi_{t}$ becomes an embedding, and $\phi$ becomes an isotopy from $\widehat{\mathscr{G}}$ to $\widehat{\mathscr{G}^{\prime}}$ through Legendrian graphs.

The isotopy $\phi$ can be extended to the hole surface $\widehat{\Pi}$ so that $\phi_{1}(\widehat{\Pi})=\widehat{\Pi}^{\prime}$. Moreover, for any $t_{0} \in[0 ; 1]$ the extension can be chosen so that the surface $\phi_{t}(\widehat{\Pi})$ is convex at the moment $t=t_{0}$. This follows from the fact that convexity is a generic property. It also follows that the surface $\phi_{t}(\widehat{\Pi})$ will remain convex for $t$ close enough to $t_{0}$.

Since the interval $[0 ; 1]$ is compact we can find finitely many points $0=t_{0}<t_{1}<\ldots<t_{n}=1$ and extensions $\phi^{1}, \ldots, \phi^{n}$ of the isotopy $\phi$ to $\widehat{\Pi}$ such that the surface $\phi_{t}^{i}(\widehat{\Pi})$ is convex for $t \in\left[t_{i-1} ; t_{i}\right]$. Denote by $F_{i-1}$ the surface $\phi_{t_{i-1}}^{i}(\widehat{\Pi})$, and by $F_{i}^{\prime}$ the surface $\phi_{t_{i}}^{i}(\widehat{\Pi})$. So far we have isotopies from $\widehat{\Pi}=F_{0}$ to $F_{1}^{\prime}$, from $F_{1}$ to $F_{2}^{\prime}, \ldots$, from $F_{n-1}$ to $F_{n}^{\prime}=\widehat{\Pi}^{\prime}$ through convex surfaces. We also know that, for each $i=1, \ldots, n-1$, the convex surfaces $F_{i}^{\prime}$ and $F_{i}$ are isotopic relative to $\widetilde{\mathscr{G}}{ }_{i}=\phi_{t_{i}}(\widetilde{\mathscr{G}})$. By construction, the connected components of $F_{i}^{\prime} \backslash \widetilde{\mathscr{G}}_{i}$ and $F_{i} \backslash \widetilde{\mathscr{G}}_{i}$ are open discs intersecting any dividing set of the respective surface in a single arc. By using [12, Lemma 6] one shows that there is an isotopy from $F_{i}^{\prime}$ to $F_{i}$ through convex surfaces.

The constructed isotopy brings every tile of $\widehat{\Pi}$ to a tile of $\widehat{\Pi}^{\prime}$, hence it brings $D$ to a dividing configuration on $\widehat{\Pi}^{\prime}$ equivalent to $D^{\prime}$. The claim follows.

Lemma 3.8. Let $\Pi$ be a rectangular diagram of a surface, and let $\left(\delta_{+}, \delta_{-}\right)$be a canonic dividing configuration of $\widehat{\Pi}$. Let also $\left(\delta_{+}^{\prime}, \delta_{-}^{\prime}\right)$ be an admissible dividing configuration obtained from $\left(\delta_{+}, \delta_{-}\right)$by a (half-)bigon reduction. Then there exists a proper realization $\left(\Pi^{\prime}, \phi\right)$ of $\left(\delta_{+}^{\prime}, \delta_{-}^{\prime}\right)$ such that $\Pi^{\prime}$ can be obtained from $\Pi$ by a sequence consisting of two or less exchange moves and one (half-)wrinkle reduction.

Proof. Consider the case of a bigon reduction. The bigon of $\left(\delta_{+}, \delta_{-}\right)$that is being reduced must be bounded by two arcs of the form $\stackrel{\circ}{v}_{1}$ and $\stackrel{\circ}{v}_{2}$, where $v_{1}$ is a /-vertex and $v_{2}$ is a \-vertex of $\Pi$. The common endpoints of $\stackrel{\circ}{1}_{1}$ and $\stackrel{\circ}{2}_{2}$ must have the form $\stackrel{\circ}{1}_{1}, \stackrel{\circ}{2}_{2}$, where $r_{1}$ and $r_{2}$ are two rectangles of $\Pi$ that share the vertices $v_{1}, v_{2}$. 
Without loss of generality we may assume that $v_{1}$ and $v_{2}$ lie at the same vertical occupied level of $\Pi$, which is $\theta=\widetilde{\theta}_{2}$, and the rectangles $r_{1}$ and $r_{2}$ have form $\left[\widetilde{\theta}_{1} ; \widetilde{\theta}_{2}\right] \times\left[\widetilde{\varphi}_{1} ; \widetilde{\varphi}_{2}\right]$ and $\left[\widetilde{\theta}_{2} ; \widetilde{\theta}_{3}\right] \times\left[\widetilde{\varphi}_{2} ; \widetilde{\varphi}_{1}\right]$, respectively. (Note that the situation $\widetilde{\theta}_{1}=\widetilde{\theta}_{3}$ is impossible as otherwise $\widehat{r}_{1} \cup \widehat{r}_{2}$ would be a sphere containing exactly two intersection points of $\delta_{+}$and $\delta_{-}$, in which case the reduction of the bigon would produce an inadmissible dividing configuration.)

Pick an $\varepsilon>0$ smaller than one half of the distance between any two parallel occupied levels of $\Pi$. Then $\Pi$ satisfies the conditions of Definition 3.8 after putting $\theta_{1}=\widetilde{\theta}_{1}+\varepsilon, \theta_{2}=\widetilde{\theta}_{2}-\varepsilon, \theta_{3}=\widetilde{\theta}_{3}+\varepsilon$, $\varphi_{1}=\widetilde{\varphi}_{1}-\varepsilon, \varphi_{2}=\widetilde{\varphi}_{2}+\varepsilon$ (consult Figure 3.8), and we can apply the respective exchange move (we put $\psi=\mathrm{id})$.
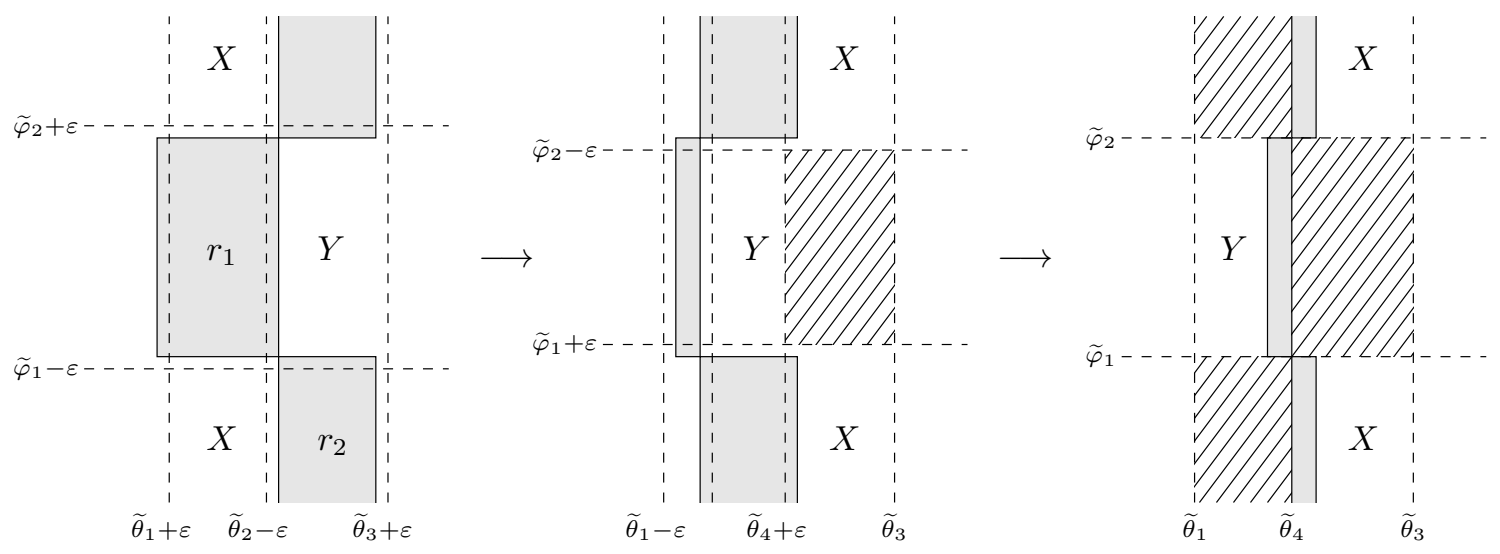

FiguRE 3.8. Exchange moves in a bigon reduction procedure. The blocks of vertices $X$ and $Y$ move horizontally. The hatched areas are free of vertices of the diagram

As a result of the move the rectangles $r_{1}$ and $r_{2}$ are transformed into $\left[\widetilde{\theta}_{1} ; \widetilde{\theta}_{1}+2 \varepsilon\right] \times\left[\varphi_{1} ; \varphi_{2}\right]$ and $\left[\widetilde{\theta}_{1}+\right.$ $\left.2 \varepsilon ; \widetilde{\theta}_{4}+2 \varepsilon\right] \times\left[\varphi_{2} ; \varphi_{1}\right]$, respectively, where $\widetilde{\theta}_{4}=\widetilde{\theta}_{1}-\widetilde{\theta}_{2}+\widetilde{\theta}_{3}$.

The obtained diagram satisfies the conditions of Definition 3.8 for $\theta_{1}=\widetilde{\theta}_{1}-\varepsilon, \theta_{2}=\widetilde{\theta}_{1}+3 \varepsilon, \theta_{3}=\widetilde{\theta}_{4}+\varepsilon$, $\varphi_{1}=\widetilde{\varphi}_{1}+\varepsilon, \varphi_{2}=\widetilde{\varphi}_{2}-\varepsilon$. After applying the respective exchange move the two rectangles that $r_{1}$ and $r_{2}$ are transformed to are thin.

As a result of the two exchange moves the combinatorial structure of the canonic configuration of $\Pi$ is unaltered. Now the bigon can be reduced by a wrinkle reduction move, provided that the move is applicable. This is the case if and only if at most one of the meridians $m_{\tilde{\theta}_{1}}$ and $m_{\tilde{\theta}_{3}}$ contains vertices of $\partial \Pi$, which is equivalent to saying that the bigon reduction yields an admissible dividing configuration. Indeed, the admissibility of the configuration obtained by the bigon reduction can be violated only at the region that is obtained by merging the discs ${\stackrel{\circ}{\tilde{\theta}_{1}}}_{\widetilde{\theta}_{1}}$ and ${\stackrel{\circ}{m_{\tilde{\theta}}}}_{\widetilde{H}}$, and this happens if and only if both discs have a non-empty intersection with $\partial \widehat{\Pi}$.

In the case of a half-bigon reduction the proof is similar and left to the reader.

As one can see from the above proof, the hypothesis that a bigon reduction yields an admissible dividing configuration is essential in Lemma 3.8. The following example shows that this hypothesis does not always hold.

Example 3.1. The dividing configuration in the left picture of Figure 2.6 contains a bigon (with corners marked 1 and 2) that cannot be reduced. The respective rectangles (see Figure 2.5) are horizontally thin but a wrinkle reduction is not applicable since both rectangles contain boundary vertices of the diagram.

Lemma 3.9. Let $D=\left(\delta_{+}, \delta_{-}\right)$be an admissible dividing configuration on a surface $F$, and let $b$ be a bigon of $\delta_{+}$and $\delta_{-}$such that the reduction of b produces a non-admissible dividing configuration. Let also $F_{0}$ be the connected component of $F$ containing $b$. Then at least one of the following holds:

(1) for any realization $(\Pi, \phi)$ of $D$, the rectangular diagram of a link $\partial \Pi$ admits an exchange move; 
(2) for any realization $(\Pi, \phi)$ of $D$, the boundary $\partial \Pi$ represents an unknot;

(3) $F_{0}$ contains exactly two intersection points of $\delta_{+}$and $\delta_{-}$, and $F_{0}$ is homeomorphic to the sphere $\mathbb{S}^{2}$.

Proof. Let $(\Pi, \phi)$ be a realization of $D$, and let $r_{1}, r_{2}$ be the rectangles corresponding to the points in $\delta_{+} \cap \delta_{-} \cap \partial b$. Suppose that $\widehat{r}_{1} \cup \widehat{r}_{2}$ is not homeomorphic to $\mathbb{S}^{2}$ (otherwise Case (3) occurs). Then $r_{1}$ and $r_{2}$ share exactly two vertices. Let $x$ be the occupied level containing these vertices. The rectangles $r_{1}$ and $r_{2}$ can be made thin by exchange moves on $\Pi$. This may already result in an exchange move on $\partial \Pi$, in which case we are done. Suppose otherwise.

Since the reduction of $b$ produces a non-admissible dividing configuration, both occupied levels parallel to $x$ that contain unshared vertices of $r_{1}$ and $r_{2}$ contain edges of $\partial \Pi$. Let $x_{1}, x_{2}$ be these occupied levels, and let $\left\{v_{1}, v_{2}\right\}=\partial \Pi \cap x_{1},\left\{v_{3}, v_{4}\right\}=\partial \Pi \cap x_{2}$. Denote by $y_{1}, y_{2}, y_{3}, y_{4}$ the occupied levels of $\Pi$ perpendicular to $x$ and passing through $v_{1}, v_{2}, v_{3}, v_{4}$, respectively.

If $y_{1}, y_{2}, y_{3}, y_{4}$ are pairwise distinct, then the edges $\left\{v_{1}, v_{2}\right\},\left\{v_{3}, v_{4}\right\}$ can be exchanged. Suppose that some of $y_{1}, y_{2}, y_{3}, y_{4}$ coincide.

An edge $\left\{v^{\prime}, v^{\prime \prime}\right\}$ of a rectangular diagram of a link $R$ will be said to be short if there is an annulus $A \subset$ $\mathbb{T}^{2}$ of the form $\left[\theta_{1} ; \theta_{2}\right] \times \mathbb{S}^{1}$ or $\mathbb{S}^{1} \times\left[\varphi_{1} ; \varphi_{2}\right]$ such that $v^{\prime}$ and $v^{\prime \prime}$ lie on different components of $\partial A$, and there are no vertices of $R$ in $\operatorname{int}(A)$. Such an edge can always be exchanged with one of the neighboring parallel edges unless the other two edges contained in $\partial A$ are also short.

Without loss of generality we may assume $y_{1}=y_{3}$. Then $\left\{v_{1}, v_{3}\right\}$ is a short edge of $\partial \Pi$, which means that either $\partial \Pi$ admits an exchange move or all edges of the connected component of $\partial \Pi$ containing $v_{1}$ are short. In the latter case one can see that $\partial \Pi$ represents an unknot.

\subsection{Weak equivalence of dividing configurations via neutral basic moves.}

Proposition 3.2. Let $F \subset \mathbb{S}^{3}$ be a compact surface, and let $D=\left(\delta_{+}, \delta_{-}\right)$be a dividing configuration on $F$. Let also $(\Pi, \phi)$ be a proper realization of $D$. Then for any admissible dividing configuration $D^{\prime}=\left(\delta_{+}^{\prime}, \delta_{-}^{\prime}\right)$ on $F$ weakly equivalent to $D$, there exists a proper realization $\left(\Pi^{\prime}, \phi^{\prime}\right)$ of $D^{\prime}$ such that $\Pi^{\prime}$ can be obtained from $\Pi$ by finitely many wrinkle, half-wrinkle, and exchange moves.

Proof. Due to Lemmas 3.2, 3.4. and 3.8 it suffices to show that $D^{\prime}$ can be obtained from $D$ by a sequence of isotopies and (half-)bigon reductions and creations so that every step of the process results in an admissible dividing configuration.

We may assume without loss of generality that the surface $F$ is connected. Otherwise the procedure below should be repeated for each connected component of $F$.

Suppose that $F$ is not homeomorphic to the sphere $\mathbb{S}^{2}$.

The admissibility of a dividing configuration is preserved under isotopies and (half-)bigon creations, so our strategy is to modify $D$ and $D^{\prime}$ by isotopies and (half-)bigon creations so that they eventually coincide.

We start by applying an isotopy to either $D$ or $D^{\prime}$ so as to have $\delta_{+}=\delta_{+}^{\prime}$. We assume this equality to hold from now on. By a small perturbation of $\delta_{+}$we can make it transverse to $\delta_{-}$and $\delta_{-}^{\prime}$.

As follows from the results of [26] if $\delta$ is a realizable abstract dividing set on $F \neq \mathbb{S}^{2}$, then any closed connected component of $\delta$ is homotopically non-trivial in $F$. However, a connected component of $\delta$ can be an arc that cuts off a half-disc from $F$. We call such an arc trivial. We will now reduce the general case to the case when $\delta_{-}$has no trivial arcs.

To this end, for each arc of $\delta_{-}^{\prime}$, we pull its endpoints along $\partial F$ toward the respective points of $\partial \delta_{-}$ so as to have $\partial \delta_{-}=\partial \delta_{-}^{\prime}$ and to make $\delta_{-}$and $\delta_{-}^{\prime}$ isotopic relative to $\partial \delta_{-}$. The idea is illustrated in Figure 3.9 This can be done so that $D^{\prime}$ undergoes only isotopies and half-bigon creations.

Now there is a union $\mathscr{U} \subset F$ of finitely many open discs such that

(1) the closure $\overline{\mathscr{U}}$ of $\mathscr{U}$ is disjoint from $\delta_{+}, \delta_{-}$, and $\delta_{-}^{\prime}$;

(2) $D$ and $D^{\prime}$ are still weakly equivalent on $F \backslash \mathscr{U}$;

(3) no arc in $\delta_{-}$is trivial in $F \backslash \mathscr{U}$.

The discs can be chosen in a small vicinity of the common endpoints of $\delta_{-}$and $\delta_{-}^{\prime}$.

In what follows we will apply isotopies to $\delta_{ \pm}$and $\delta_{ \pm}^{\prime}$ so that during the isotopies the curves will remain disjoint from $\overline{\mathscr{U}}$. 

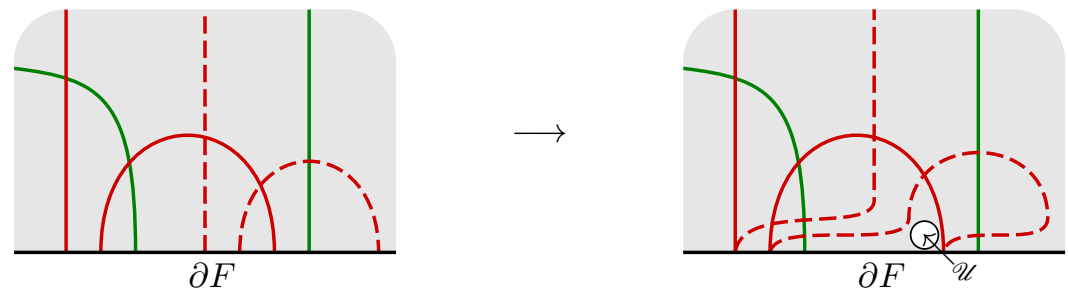

legend:

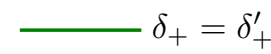

FiguRE 3.9. Pulling the endpoints of $\delta_{-}^{\prime}$ toward the respective points of $\partial \delta_{-}$

By a small perturbation of $\delta_{-}^{\prime}$ we make it transverse to $\delta_{-}$, and make $\partial \delta_{-}^{\prime}$ disjoint from $\partial \delta_{-}$. Let $b$ be a (half-)bigon of $\left(\delta_{-}, \delta_{-}^{\prime}\right)$ disjoint from $\mathscr{U}$. By applying, if necessary, an isotopy to $\delta_{+}=\delta_{+}^{\prime}$ that results in creation of (half-)bigons of $D$ and/or $D^{\prime}$ we can achieve that the intersection $\delta_{+} \cap b$ consists of arcs having one endpoint at $\delta_{-}$and the other at $\delta_{-}^{\prime}$; see Figure 3.10. Then we can reduce the (half-)bigon $b$
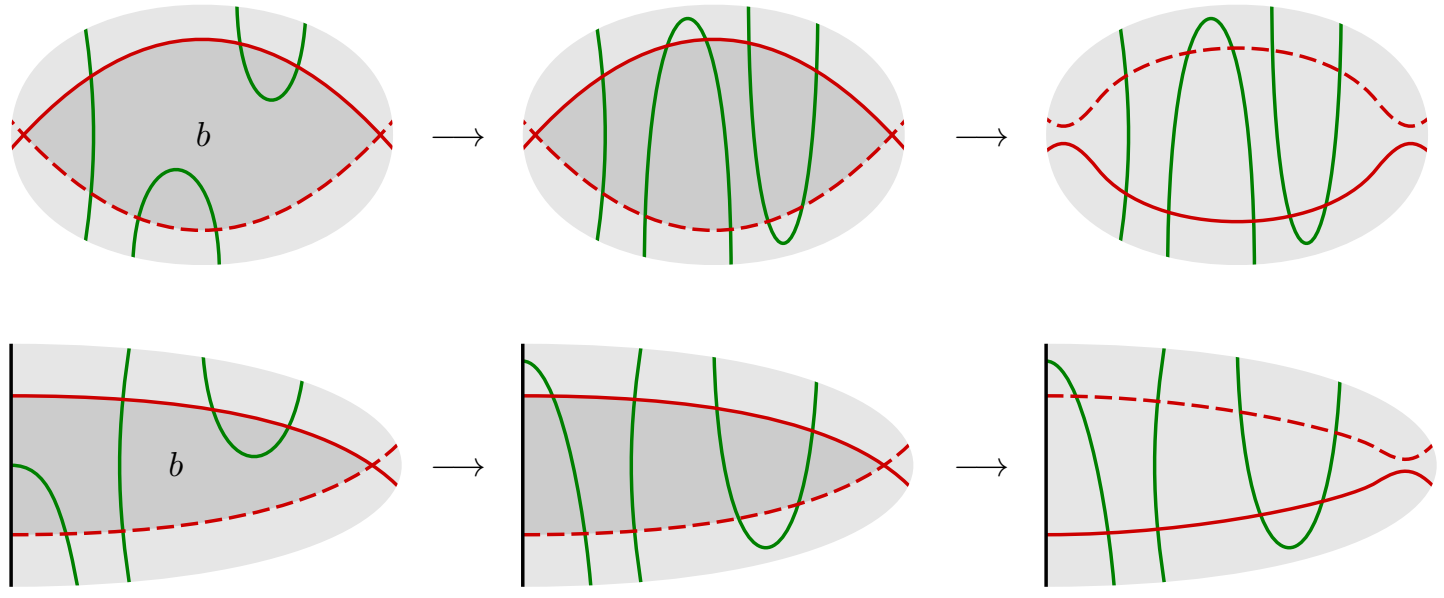

Figure 3.10. Reducing (half-)bigons of $\left(\delta_{-}, \delta_{-}^{\prime}\right)$. See Figure 3.9 for the legend

so that the equivalence classes of $D$ and $D^{\prime}$ remain unchanged.

Proceeding as above we reduce all bigons and half-bigons of $\left(\delta_{-}, \delta_{-}^{\prime}\right)$ contained in $F \backslash \mathscr{U}$. Since there is an isotopy from $\delta_{-}$to $\delta_{-}^{\prime}$ in $F \backslash \mathscr{U}$ that keeps $\partial \delta_{-}$in $\partial F$, after completing the (half-)bigon reductions the abstract dividing sets $\delta_{-}$and $\delta_{-}^{\prime}$ are almost disjoint in the following sense.

Two abstract dividing sets $\delta_{1}, \delta_{2} \subset F$ are called almost disjoint if, for any connected components $\gamma_{1}$, $\gamma_{2}$ of $\delta_{1}, \delta_{2}$, respectively, one of the following holds:

(1) $\gamma_{1}$ and $\gamma_{2}$ are disjoint;

(2) $\gamma_{1}$ and $\gamma_{2}$ are one-sided closed curves isotopic to one another and having a single intersection point.

Let $\gamma$ be a two-sided closed connected component of $\delta_{-}$. Since $\delta_{-}$and $\delta_{-}^{\prime}$ are almost disjoint, and $\gamma$ is homotopically non-trivial in $F \backslash \mathscr{U}$, there exists an annulus $A \subset F \backslash \overline{\mathscr{U}}$ such that its intersection with $\delta_{-} \cup \delta_{-}^{\prime}$ consists of all the connected components of $\delta_{-}$and $\delta_{-}^{\prime}$ that are isotopic either to $\gamma$ or $-\gamma$ (where $-\gamma$ stands for $\gamma$ with reversed orientation) and all these components are homotopically non-trivial in $A$.

By applying, if necessary, an isotopy to $\delta_{+}$that results in creation of bigons of $D$ and/or $D^{\prime}$ we can make the intersection $\delta_{+} \cap A$ consist of non-separating arcs in $A$ each of which intersects each of the connected components of $\delta_{-}$and $\delta_{-}^{\prime}$ exactly once; see Figure 3.11. Then we apply an isotopy to $\delta_{-} \cap A$ that will bring it to $\delta_{-}^{\prime} \cap A$ keeping inside $A$, without altering the equivalence class of $D$. After that we will have $\delta_{-} \cap A=\delta_{-}^{\prime} \cap A$. 

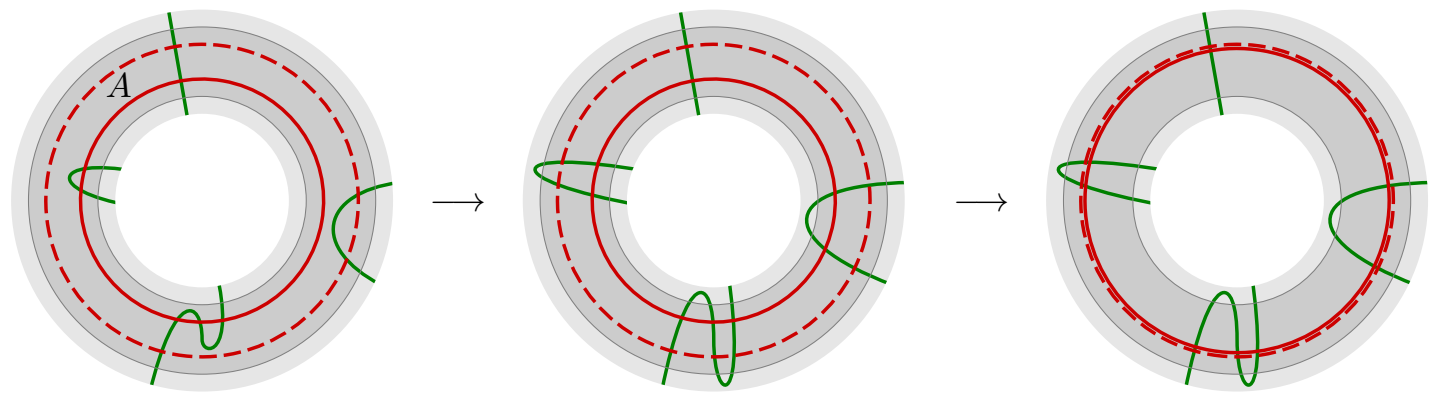

Figure 3.11. Finally making $\delta_{-}$and $\delta_{-}^{\prime}$ coinciding. See Figure 3.9 for the legend

A similar procedure works for connected components of $\delta_{-}$that are arcs. Instead of an annulus we consider a 'half-annulus', and also allow half-bigon creations for $D$ and $D^{\prime}$.

Finally, let $\gamma$ be a one-sided closed component of $\delta_{-}$. There is a connected component $\gamma^{\prime}$ of $\delta_{-}^{\prime}$ isotopic to $\gamma$, and we have $\left|\gamma \cap \gamma^{\prime}\right|=1$. These two curves enclose a disc with two boundary points identified, and a small neighborhood of this disc is homeomorphic to a Möbius strip; see Figure 3.12. The intersection

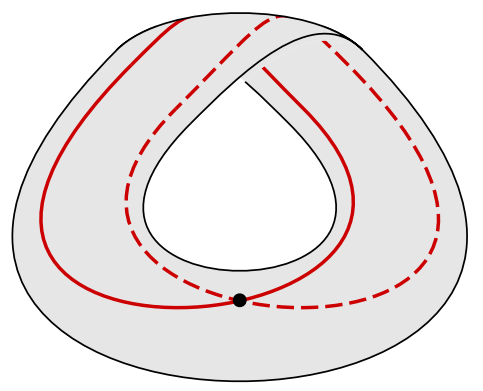

FIgURE 3.12. An unavoidable intersection of two isotopic one-sided simple closed curves

of $\delta_{+}$with this disc is treated in the same way as in the case of an ordinary bigon (see Figure 3.10), and after that $\gamma$ can be isotoped to $\gamma^{\prime}$ without distorting the equivalence class of $D$.

We repeat this procedure for any connected component of $\delta_{-}$that does not yet coincide with the respective component of $\delta_{-}^{\prime}$, and keep doing so until we get $\delta_{-}=\delta_{-}^{\prime}$. The case $F \neq \mathbb{S}^{2}$ is done.

Now suppose that $F$ is homeomorphic to $\mathbb{S}^{2}$. Since $D$ is a realizable configuration, it follows from the results of [26] that each of $\delta_{+}$and $\delta_{-}$is a single circle. A dividing configuration $\left(\delta_{1}, \delta_{2}\right)$ on $\mathbb{S}^{2}$ with $\delta_{1} \cong \delta_{2} \cong \mathbb{S}^{1}$ is admissible if and only if $\delta_{1}$ and $\delta_{2}$ intersect transversely and $\delta_{1} \cap \delta_{2} \neq \varnothing$.

So, for $F \cong \mathbb{S}^{2}$ we go another way: we keep reducing bigons of $D$ until we have $\left|\delta_{+} \cap \delta_{-}\right|=2$. This produces only admissible configurations during the process. Do the same with $D^{\prime}$. The configurations $D$ and $D^{\prime}$ become equivalent.

\section{SPATial RibBOn GRAPHS}

The aim of this section is mainly to fix the terminology that we operate in the sequel. In simple words, a spatial ribbon graph is the 1-skeleton of a cell decomposition of a compact surface embedded in $\mathbb{S}^{3}$, endowed with an additional structure that allows to recover the surface up to certain equivalence (which amounts to an isotopy if the surface is connected). The boundary circuits of a spatial ribbon graph encode the boundary components of the represented surface and the attachment maps of the two-cells.

The role of an enhancement of a spatial ribbon graph is to distinguish between these two types of boundary circuits. However, for all important applications known by far, it suffices to work with the 
'default' choice of enhancement, by which all boundary circuits that can be attachment maps of a twocell (such boundary circuits are called patchable) are declared as such. So, at the expense of losing some generality, the reader may safely ignore any mentioning of enhancements.

Morphisms of spatial ribbon graphs are designed to represent homeomorphisms of the surfaces represented by the graphs, viewed up to isotopy.

\subsection{The basic concept.}

Definition 4.1. By a spatial graph we mean a finite one-dimensional (or zero-dimensional) CW-complex $\Gamma$ embedded in $\mathbb{R}^{3}$ so that the restriction of the embedding to each 1-cell is piecewise smooth.

We put no restrictions on the presence of multiple edges or loops in spatial graphs and don't demand spatial graphs to be connected. We do not distinguish between two spatial graphs if they coincide as subsets of $\mathbb{R}^{3}$, which means that any edge of a spatial graph can be subdivided by new vertices, and this is not regarded as a change of the graph.

Points of $\Gamma$ that have no neighborhood homeomorphic to an open interval are called true vertices of $\Gamma$.

Definition 4.2. By a simple circuit in a spatial graph $\Gamma$ we mean any simple closed curve in $\Gamma$.

By an almost simple circuit in $\Gamma$ we mean a closed curve in $\Gamma$ that does not pass more than twice through any point of $\Gamma$ which is not a true vertex. More precisely, an almost simple circuit is an equivalence class of continuous maps $f: \mathbb{S}^{1} \rightarrow \Gamma$ such that $\left|f^{-1}(p)\right| \leqslant 2$ whenever $p \in \Gamma$ is not a true vertex of $\Gamma$, where two maps $f$ and $g$ are regarded equivalent if $f \circ h_{1}=g \circ h_{2}$ for some degree- \pm 1 continuous maps $h_{1}, h_{2}: \mathbb{S}^{1} \rightarrow \mathbb{S}^{1}$.

Note that an almost simple circuit can be presented by a constant map, that is, a map whose image is a single point (which should be a true vertex).

Definition 4.3. Let $\Gamma$ be a spatial graph, and let $F$ be a compact surface embedded in $\mathbb{S}^{3}$ such that $\Gamma \subset F$. Let also $\gamma$ be an almost simple circuit in $\Gamma$, and $\widetilde{\gamma}$ a connected component of $\partial F$. We say that $\gamma$ is $a$ projection of $\widetilde{\gamma}$ to $\Gamma$ in $F$ if there is a map $f: \mathbb{S}^{1} \times[0 ; 1] \rightarrow \mathbb{S}^{3}$ such that:

(1) $F \cup f\left(\mathbb{S}^{1} \times[0 ; 1]\right)$ is a compact surface;

(2) the restriction $\left.f\right|_{\mathbb{S}^{1} \times[0 ; 1)}$ is an embedding;

(3) $\left.f\right|_{\mathbb{S}^{1} \times\{1\}}$ represents $\gamma$;

(4) there exists a continuous map $g: \mathbb{S}^{1} \rightarrow[0 ; 1]$ such that the map $t \mapsto f(t, g(t)), t \in \mathbb{S}^{1}$, is a parametrization of $\widetilde{\gamma}$.

Definition 4.4. A spatial ribbon graph is a 4 -tuple $\rho=\left(\Gamma_{\rho}, \partial \rho, V_{\rho}, S_{\rho}\right)$ in which $\Gamma_{\rho}$ is a spatial graph, $\partial \rho$ is a finite family of (not necessarily distinct) almost simple circuits in $\Gamma_{\rho}, V_{\rho}$ is a finite subset of $\Gamma_{\rho}$ containing all true vertices of $\Gamma_{\rho}$ and intersecting any simple closed curve in $\Gamma_{\rho}$ at least twice, and $S_{\rho}$ is a non-empty class of compact surfaces each endowed with a bijection between $\partial \rho$ and the set of boundary components of $\partial F$, such that:

(1) for any surface $F \in S_{\rho}$ the following holds:

(a) $F$ contains $\Gamma_{\rho}$;

(b) the inclusion $\Gamma_{\rho} \hookrightarrow F$ is a homotopy equivalence;

(c) for any connected component $\gamma$ of $\partial F$ the corresponding circuit in $\partial \rho$ is a projection of $\gamma$ to $\Gamma_{\rho}$ in $F$;

(2) for any two surfaces $F, F^{\prime} \in S_{\rho}$ the following holds:

(a) if $p \in V_{\rho}$, then the tangent planes $T_{p} F, T_{p} F^{\prime}$ to $F$ and $F^{\prime}$ at $p$ coincide;

(b) there is an isotopy from $F$ to $F^{\prime}$ within the class $S_{\rho}$;

(3) $S_{\rho}$ is maximal, that is, for any surface $F^{\prime}$ endowed with a bijection between connected components of $\partial F^{\prime}$ and the elements of $\partial \rho$ such that $F^{\prime}$ can be obtained from some $F \in S_{\rho}$ by an isotopy that keeps the tangent plane to the surface at any point $p \in V_{\rho}$ fixed, we have $F^{\prime} \in S_{\rho}$.

The elements of $\partial \rho$ are called boundary circuits of $\rho$, and the set $\partial \rho$ the boundary of $\rho$. The elements of $V_{\rho}$ are called vertices of $\rho$, and the closure of any connected component of $\Gamma_{\rho} \backslash V_{\rho}$ is called an edge of $\rho$. 
An explanation is in order.

If the graph $\Gamma_{\rho}$ in this definition has a connected component $\gamma$ homeomorphic to a circle, then $\partial \rho$ may contain two copies of $\gamma$, which are regarded as different elements of $\partial \rho$. Unless we need to deal with such graphs a simpler definition can be used instead: a spatial ribbon graph is a triple $\rho=\left(\Gamma_{\rho}, V_{\rho}, S_{\rho}\right)$ in which $\Gamma_{\rho}$ is a spatial graph, $V_{\rho}$ is a finite subset of $\Gamma_{\rho}$ containing all true vertices of $\Gamma_{\rho}$ and intersecting any simple closed curve in $\Gamma_{\rho}$ at least twice, and $S_{\rho}$ is a non-empty isotopy class of compact surfaces in $\mathbb{S}^{3}$ having $\Gamma_{\rho}$ as a deformation retract and fixed tangent planes at all points in $V_{\rho}$. Boundary circuits of $\rho$ are then defined as the projections of boundary components of any surface $F \in S_{\rho}$.

Indeed, let $\Gamma$ be a spatial graph, and let $F$ be a compact surface containing $\Gamma$ and retracting to $\Gamma$. Let also $V$ be a finite subset of $\Gamma$ containing all true vertices and intersecting each simple closed curve in $\Gamma$ at least twice. One can see that every connected component of $\partial F$ has a unique projection to $\Gamma$ in $F$, so, by demanding $S_{\rho} \ni F$, a spatial ribbon graph $\rho$ (in the sense of Definition 4.4) with $\Gamma_{\rho}=\Gamma$, $V_{\rho}=V$, and $\partial \rho$ being the family of projections of the connected components of $\partial F$ to $\Gamma$ in $F$ is defined uniquely. If $\Gamma$ has no circular connected components, then no two boundary components of $\partial F$ have the same projection, so, there is a unique bijection between $\partial \rho$ and the set of connected components of $\partial F$ satisfying condition (1c) from Definition 4.4 .

However, the simpler definition may cause ambiguity in certain context if a connected component of $\Gamma$ is homeomorphic to $\mathbb{S}^{1}$ and the corresponding connected component of $F$ is an annulus. In this case, two boundary components of $\partial F$ have the same projections to $\Gamma$, and thus there is no one-to-one correspondence between boundary components of $F$ and boundary circuits of the spatial ribbon graph defined by $\Gamma, F$, and $V$.

Definition 4.5. Let $\gamma$ be a simple boundary circuit of a spatial ribbon graph $\rho$. Then, clearly, there is a surface with corners $F \in S_{\rho}$ such that the connected component of $\partial F$ corresponding to $\gamma$ coincides with $\gamma$.

In this situation, the framing of $\gamma$ obtained by restricting the boundary framing induced by $F$ to $\gamma$ (see [12, Definitions 12 and 13]) is said to be induced by $\rho$. Clearly, it does not depend on a particular choice of $F$.

The induced framing is similarly defined for any link whose components are pairwise disjoint simple boundary circuits of $\rho$.

\subsection{Enhanced and patched spatial ribbon graphs.}

Definition 4.6. Let $F$ be a compact surface in $\mathbb{S}^{3}$. A boundary component $\gamma \subset \partial F$ is called patchable if there is an embedded closed disc $d \subset \mathbb{S}^{3}$ such that $\partial d \cap F=\gamma$.

A boundary circuit of a spatial ribbon graph $\rho$ is called patchable if the corresponding boundary component of some (and then any) surface $F \in S_{\rho}$ is patchable.

Let $\gamma_{1}, \ldots, \gamma_{k}$ be some patchable connected components of $\partial F$. We say that a compact embedded surface $F^{\prime} \subset \mathbb{S}^{3}$ is obtained from $F$ by patching the holes $\gamma_{1}, \ldots, \gamma_{k}$ if $F^{\prime}$ contains $F$ and $F^{\prime} \backslash F$ is a union of open discs $d_{1}, \ldots, d_{k}$ such that $\partial \bar{d}_{i}=\gamma_{i}, i=1, \ldots, k$. The boundary components $\gamma_{i}$ will then be said to be patched in $F^{\prime}$.

Definition 4.7. Let $\gamma$ be a patchable boundary circuit of a spatial ribbon graph $\rho$, and let $p: \mathbb{D}^{2} \rightarrow \mathbb{S}^{3}$ be a continuous map from a closed two-disc $\mathbb{D}^{2}$ to the three-sphere such that:

(1) the restriction of $p$ to $\operatorname{int}\left(\mathbb{D}^{2}\right)$ is an embedding;

(2) the image of $\operatorname{int}\left(\mathbb{D}^{2}\right)$ under $p$ is disjoint from $\Gamma_{\rho}$;

(3) the restriction of $p$ to $\partial \mathbb{D}^{2}$ represents $\gamma$;

(4) there is a surface $F \in S_{\rho}$ such that $F \cup p\left(\mathbb{D}^{2}\right)$ is a surface obtained from $F$ by patching the hole corresponding to $\gamma$.

Then we say that the pair $\left(\mathbb{D}^{2}, p\right)$ is a patching disc for $\gamma$ in $\rho$. Two patching discs $\left(\mathbb{D}_{1}^{2}, p_{1}\right)$ and $\left(\mathbb{D}_{2}^{2}, p_{2}\right)$ are equivalent if $p_{1}\left(\mathbb{D}_{1}^{2}\right)=p_{2}\left(\mathbb{D}_{2}^{2}\right)$.

Since the equivalence class of a patching disc $\left(\mathbb{D}^{2}, p\right)$ is defined uniquely by the image $p\left(\mathbb{D}^{2}\right)$, we will sometimes refer to the latter as a patching disc. Note, however, that $p\left(\mathbb{D}^{2}\right)$ is generally not a disc and even not necessarily a surface (for instance, it can be a disc with two boundary points identified). 
Definition 4.8. By an enhanced spatial ribbon graph we call a 5-tuple $\rho=\left(\Gamma_{\rho}, \partial \rho, V_{\rho}, S_{\rho}, H_{\rho}\right)$ in which $\rho_{0}=\left(\Gamma_{\rho}, \partial \rho, V_{\rho}, S_{\rho}\right)$ is a spatial ribbon graph and $H_{\rho}$ is a subset of the set of patchable boundary circuits of $\rho_{0}$.

A boundary circuit $\gamma \in \partial \rho$ is said to be an inessential boundary circuit (or a hole) of $\rho$ if $\gamma \in H_{\rho}$, and otherwise an essential boundary circuit of $\rho$. For any $F \in S_{\rho}$, connected components of $\partial F$ corresponding to essential (respectively, inessential) boundary circuits of $\rho$ also are called essential (respectively, inessential). Inessential boundary components of $\partial F$ are also called holes.

By a patching of an enhanced spatial ribbon graph $\rho$ we mean an isotopy class of pairs of compact surfaces $\left(F_{0}, F\right)$ such that $F_{0} \in S_{\rho}$ and $F$ is obtained by patching all the holes of $F_{0}$. An enhanced spatial ribbon graph endowed with a patching is called a patched spatial ribbon graph.

In other words, an enhanced spatial ribbon graph is a spatial ribbon graph with a declaration which of the patchable boundary circuits are subject to patching, but without specifying a concrete way of patching. If the underlying graph $\Gamma_{\rho}$ is connected then the complement to a regular neighborhood of $\Gamma_{\rho}$ is an irreducible manifold, which implies that up to isotopy there is only one way to patch each patchable boundary circuit. So, in this case, enhancement is just equivalent to patching.

Definition 4.9. The set of all essential boundary circuits of an enhanced ribbon graph $\rho$ is called the essential boundary of $\rho$ and denoted by $\partial_{\mathrm{e}} \rho$. For any $F \in S_{\rho}$ the union of essential connected components of $\partial F$ is denoted by $\partial_{\mathrm{e}} F$.

Definition 4.10. Let $(\rho, P)$ be a patched spatial ribbon graph. A surface $F$ is said to be carried by $(\rho, P)$ if $\left(F_{0}, F\right) \in P$ for some $F_{0} \in S_{\rho}$. If, additionally, $\partial F=\cup_{K \in \partial_{\mathrm{e}} \rho} K$ holds we say that $F$ is properly carried by $(\rho, P)$.

A surface is said to be carried (respectively, properly carried) by an enhanced spatial ribbon graph $\rho$ if it is carried (respectively, properly carried) by $(\rho, P)$ for some patching $P$ of $\rho$.

One can see that a surface properly carried by $(\rho, P)$ exists if and only if $\partial_{\mathrm{e}} \rho$ consists of pairwise disjoint simple circuits.

Definition 4.11. Let $\rho$ and $\rho^{\prime}$ be two enhanced spatial ribbon graphs, and let $L$ be a framed link whose components are common essential boundary circuits of $\rho$ and $\rho^{\prime}$ endowed with the induced framing, which is the same for $\rho$ and $\rho^{\prime}$.

The enhanced spatial ribbon graphs $\rho$ and $\rho^{\prime}$ are said to be stably equivalent relative to $L$ if $V_{\rho} \cap L=$ $V_{\rho^{\prime}} \cap L$ and one can choose their patchings $P$ and $P^{\prime}$, respectively, so that for some (and then any) pairs of surfaces $\left(F_{0}, F\right) \in P$ and $\left(F_{0}^{\prime}, F^{\prime}\right) \in P^{\prime}$ the following holds:

(1) $L \subset \partial F, L \subset \partial F^{\prime}$ (as a framed link);

(2) $F$ can be brought to $F^{\prime}$ by a isotopy fixed on $L$ that preserves the tangent plane to $F$ at any point $p \in V_{\rho} \cap L$ and brings essential (respectively, inessential) boundary components of $F$ to essential (respectively, inessential) boundary components of $F^{\prime}$.

Note that, if enhanced spatial ribbon graphs $\rho$ and $\rho^{\prime}$ are stably equivalent relative to $L$, then for any patching $P$ of $\rho$ one can find a patching $P^{\prime}$ of $\rho^{\prime}$ such that the patched spatial ribbon graphs $(\rho, P)$ and $\left(\rho^{\prime}, P^{\prime}\right)$ are stably equivalent. This implies, in particular, that stable equivalence is transitive, which justifies the term 'equivalence'.

4.3. Morphisms of spatial ribbon graphs. For an enhanced spatial ribbon graph $\rho$ we denote by $\widetilde{S}_{\rho}$ the class of all surfaces $F$ that can be obtained from a surface $F_{0} \in S_{\rho}$ by a partial patching, that is, by patching any family of holes (including the extreme cases of no holes and all holes). Each surface $F \in \widetilde{S}_{\rho}$ is assumed to be endowed with a bijection inherited from some $F_{0} \subset F, F_{0} \in S_{\rho}$, between connected components of $\partial F$ and the boundary circuits of $\rho$ that are not patched in $F$.

In particular, $\widetilde{S}_{\rho}$ contains all surfaces from $S_{\rho}$ and all surfaces carried by $\rho$.

Definition 4.12. Let $\rho_{1}$ and $\rho_{2}$ be enhanced spatial ribbon graphs. By a morphism from $\rho_{1}$ to $\rho_{2}$ we call a maximal non-empty class $\eta$ of triples $\left(F_{1}, F_{2}, h\right)$ in which $F_{1} \in \widetilde{S}_{\rho_{1}}, F_{2} \in \widetilde{S}_{\rho_{2}}$, and $h: F_{1} \rightarrow F_{2}$ is an embedding, such that 
(1) for any $\left(F_{1}, F_{2}, h\right) \in \eta$ there exist surfaces $F_{1}^{\prime}$ and $F_{2}^{\prime}$ carried by $\rho_{1}$ and $\rho_{2}$, respectively, and a homeomorphism $h^{\prime}: F_{1}^{\prime} \rightarrow F_{2}^{\prime}$ such that $F_{1}^{\prime} \supset F_{1}, F_{2}^{\prime} \supset F_{2},\left.h^{\prime}\right|_{F_{1}}=h$, and $\left(F_{1}^{\prime}, F_{2}^{\prime}, h^{\prime}\right) \in \eta$;

(2) for any two triples $\left(F_{1}, F_{2}, h\right),\left(F_{1}, F_{2}, h^{\prime}\right) \in \eta$ such that $F_{i}$ is carried by $\rho_{i}, i=1,2$, the embeddings $h$ and $h^{\prime}$ are isotopic;

(3) if $\left(F_{1}, F_{2}, h\right) \in \eta$ and there are isotopies from $F_{1}$ and $F_{2}$ to surfaces $F_{1}^{\prime}$ and $F_{2}^{\prime}$ within the classes $\widetilde{S}_{\rho_{1}}$ and $\widetilde{S}_{\rho_{2}}$, respectively, inducing homeomorphisms $h_{1}: F_{1} \rightarrow F_{1}^{\prime}$ and $h_{2}: F_{2} \rightarrow F_{2}^{\prime}$, then $\left(F_{1}^{\prime}, F_{2}^{\prime}, h_{2} \circ h \circ h_{1}^{-1}\right) \in \eta$.

Informally speaking, a morphism from $\rho$ to $\rho^{\prime}$ is a way, viewed up to isotopy, to identify surfaces carried by $\rho$ with those carried by $\rho^{\prime}$. In order to fix such an identification it suffices to choose a single homeomorphism from a surface $F$ carried by $\rho$ to a surface $F^{\prime}$ carried by $\rho^{\prime}$. Since we view it up to isotopy it suffices to know the restriction of this homeomorphism to any subsurface $F_{0} \subset F, F_{0} \in \widetilde{S}_{\rho}$. So the idea is to include into $\eta$ all maps that give rise, after patching the holes, to the same, up to isotopy, identification between surfaces carried by $\rho$ with surfaces carried by $\rho^{\prime}$.

The composition $\eta_{2} \circ \eta_{1}$ of two composable morphisms $\eta_{1}: \rho_{1} \rightarrow \rho_{2}$ and $\eta_{2}: \rho_{2} \rightarrow \rho_{3}$ is defined in the obvious way: if $\left(F_{1}, F_{2}, h_{1}\right) \in \eta_{1}$ and $\left(F_{2}, F_{3}, h_{2}\right) \in \eta_{2}$, then $\left(F_{1}, F_{3}, h_{2} \circ h_{1}\right) \in \eta_{2} \circ \eta_{1}$. Clearly, there is an identity morphism for every enhanced spatial ribbon graph, and every morphism has an inverse.

\subsection{Handle moves of spatial ribbon graphs.}

Definition 4.13. Let $\rho$ and $\rho^{\prime}$ be enhanced spatial ribbon graphs, and let $d$ be a patching disc for $\rho^{\prime}$ such that the following holds:

(1) $\Gamma_{\rho^{\prime}} \backslash \Gamma_{\rho}$ is an open simple arc;

(2) for some $F \in S_{\rho}$ and $F^{\prime} \in S_{\rho^{\prime}}$ we have $F^{\prime} \cup d=F$ and $\partial_{\mathrm{e}} F=\partial_{\mathrm{e}} F^{\prime}$;

(3) $V_{\rho^{\prime}} \cap \Gamma_{\rho}=V_{\rho}$.

Then we say that the transition from $\rho$ to $\rho^{\prime}$ is a handle addition, and the inverse operation is a handle removal.

The patching disc $d$ will be said to be associated with the handle addition $\rho \mapsto \rho^{\prime}$. We also associate with this move a morphism $\eta$ from $\rho$ to $\rho^{\prime}$ by requesting that $\left(F, F,\left.\mathrm{id}\right|_{F}\right) \in \eta$. To state that $\eta$ is the associated morphism we write $\rho \stackrel{\eta}{\mapsto} \rho^{\prime}$.

This definition means, in particular, that for every surface $F^{\prime} \in S_{\rho^{\prime}}$ there is a surface $F \in S_{\rho}$ (which is not the same as in the definition above) such that $F^{\prime}$ is obtained from $F$ by attaching a 1-handle to $\partial F$; see Figure 4.1. This is done so that all but exactly one of the boundary components of $F$ are preserved,

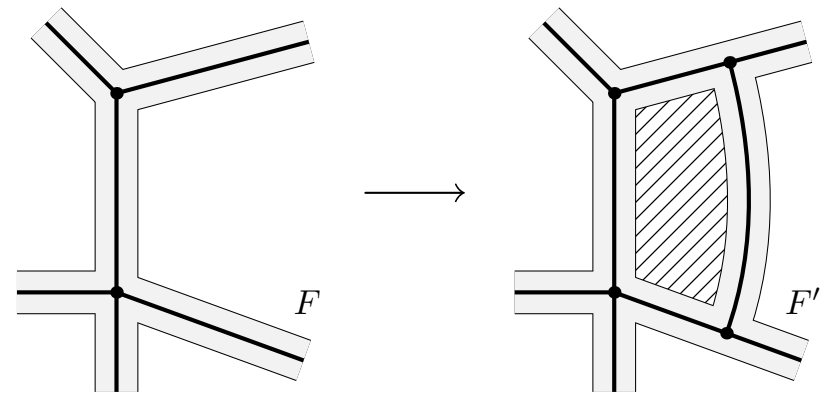

FiguRE 4.1. Handle addition

and the exceptional one $\gamma$, say, is replaced by two components $\gamma_{1}^{\prime}, \gamma_{2}^{\prime}$, say, of $\partial F^{\prime}$. If $\gamma$ is an essential component of $\partial F$, then exactly one of $\gamma_{1}^{\prime}$ and $\gamma_{2}^{\prime}$ is an essential component of $\partial F^{\prime}$. Otherwise both of them are inessential. Any connected component of $\partial F$ that is also contained in $\partial F^{\prime}$ is essential for $F^{\prime}$ if and only if it is essential for $F$.

Lemma 4.1. Let $\rho$ and $\rho^{\prime}$ be enhanced spatial ribbon graphs such that $\Gamma_{\rho^{\prime}}=\Gamma_{\rho}$, $\partial \rho^{\prime}=\partial \rho, H_{\rho^{\prime}}=H_{\rho}$, $V_{\rho^{\prime}} \supset V_{\rho}, F_{\rho^{\prime}} \subset F_{\rho}$. Then $\rho$ and $\rho^{\prime}$ are related by a finite sequence of handle additions and removals preserving any boundary circuit $c \in \partial \rho$ that satisfies $c \cap V_{\rho}=c \cap V_{\rho^{\prime}}$. 
Proof. Clearly it suffices to prove the statement in the case when $V_{\rho^{\prime}} \backslash V_{\rho}$ is a single point $p$. This is assumed below.

Let $\alpha$ be the edge of $\rho$ that contains $p$. Pick a surface $F \in S_{\rho^{\prime}}$. There is another simple arc $\beta$, say, in $F$ such that $\beta \cap \Gamma_{\rho}=\partial \beta=\partial \alpha$ and the $\operatorname{arcs} \alpha, \beta$ co-bound a disc $d \subset F$.

Let $\rho \mapsto \rho_{1} \mapsto \rho_{2} \mapsto \rho_{3}$ be a sequence of two handle additions and one handle removal, following in the alternating order, associated with $d$ : we add $\beta$, then remove $\alpha$, then put $\alpha$ back. The move $\rho_{2} \mapsto \rho_{3}$, which puts $\alpha$ back, can be chosen so that $\operatorname{int}(\alpha) \cap V_{\rho_{3}}=\{p\}$. Then $\rho_{3} \mapsto \rho^{\prime}$ will be a handle removal, which completes the job.

Proposition 4.1. Let $\rho$ and $\rho^{\prime}$ be two enhanced spatial ribbon graphs, and let $L$ be a framed link whose components are common essential boundary circuits of $\rho$ and $\rho^{\prime}$ endowed with the induced framing, which is the same for $\rho$ and $\rho^{\prime}$. Let also $\eta$ be a morphism from $\rho$ to $\rho^{\prime}$. Then the following two conditions are equivalent:

(1) $\rho$ and $\rho^{\prime}$ are stably equivalent relative to L, and some isotopy realizing this stable equivalence induces the morphism $\eta$;

(2) there exists a sequence of handle additions and removals

$$
\rho=\rho_{0} \stackrel{\eta_{1}}{\longmapsto} \rho_{1} \ldots \stackrel{\eta_{N}}{\longmapsto} \rho_{N}=\rho^{\prime}
$$

each of which preserves the framed boundary circuits contained in $L$, and we have $\eta=\eta_{N} \circ \ldots \circ \eta_{1}$.

Proof. The implication $(2) \Rightarrow(1)$ is easy and is left to the reader. We will prove the converse one.

Assume that Condition (1) holds, and suppose, additionally, that there is a surface $F$ carried by both $\rho$ and $\rho^{\prime}$ such that $\partial F \supset L$ and $\left(F, F\right.$, id $\left.\left.\right|_{F}\right) \in \eta$. Suppose also that the intersection $\Gamma_{\rho} \cap \Gamma_{\rho^{\prime}} \backslash L$ is finite.

By Lemma 4.1 we may arbitrarily subdivide edges of a spatial ribbon graph by means of handle additions and removals, without touching the boundary circuits disjoint from the interior of these edges. Therefore, we may assume without loss of generality that $\Gamma_{\rho} \cap \Gamma_{\rho^{\prime}} \backslash L$ is a subset of $V_{\rho} \cap V_{\rho^{\prime}}$.

There exists a graph $\Gamma \subset F$ containing $\Gamma_{\rho} \cup \Gamma_{\rho^{\prime}}$ and having no one-valent vertices and no true vertices outside $V_{\rho} \cup V_{\rho^{\prime}}$. It can be turned into an enhanced spatial ribbon graph $\rho^{\prime \prime}$ with $\Gamma_{\rho^{\prime \prime}}=\Gamma, V_{\rho^{\prime \prime}}=V_{\rho} \cup V_{\rho^{\prime}}$, so that $F$ be carried by $\rho^{\prime \prime}$. One can see that $\rho^{\prime \prime}$ can then be obtained from each of $\rho^{\prime}$ and $\rho^{\prime \prime}$ by a sequence of handle additions, and all the associated morphisms of spatial ribbon graphs will contain the triple $\left(F, F\right.$, id $\left.\left.\right|_{F}\right)$, hence Condition (2) holds in this case.

Now we drop the assumption on the finiteness of $\Gamma_{\rho} \cap \Gamma_{\rho^{\prime}} \backslash L$. One can find an enhanced spatial ribbon graph $\rho^{\prime \prime}$ carrying $F$ such that $V_{\rho^{\prime \prime}} \cap L=V_{\rho} \cap L$, and both $\Gamma_{\rho} \cap \Gamma_{\rho^{\prime \prime}} \backslash L$ and $\Gamma_{\rho^{\prime}} \cap \Gamma_{\rho^{\prime \prime}} \backslash L$ are finite. By the above argument there are sequences of handle additions and removals producing $\rho^{\prime \prime}$ from $\rho$, and $\rho^{\prime}$ from $\rho^{\prime \prime}$, and all the associated morphisms contain the triple $\left(F, F,\left.\mathrm{id}\right|_{F}\right)$. Hence Condition (2) holds in this case, too.

Now let $\rho$ and $\rho^{\prime}$ be arbitrary enhanced spatial ribbon graphs and let $\eta$ be a morphism from $\rho$ to $\rho^{\prime}$. Assume that Condition (1) holds, that is, there are surfaces $F$ and $F^{\prime}$ carried by $\rho$ and $\rho^{\prime}$, respectively, and an isotopy from $F$ to $F^{\prime}$ fixed on $L$ and inducing the morphism $\eta$.

One can find a sequence of surfaces $F=F_{1}, F_{2}, \ldots, F_{n}=F^{\prime}$ such that, for every $i=1, \ldots, n-1$, there is an isotopy from $F_{i}$ to $F_{i+1}$ fixed outside of an open ball that intersects $F_{i}$ in an open disc (or a half-disc) $d_{i}$ satisfying $d_{i} \cap L=\varnothing$ and $\overline{F_{i} \backslash \overline{d_{i}}} \supset V_{\rho} \cap L$. Such surfaces and isotopies can be chosen so that the isotopy from $F$ to $F^{\prime}$ obtained by concatenating the latter is arbitrarily close to the original one, and hence also induces $\eta$. For every $i=1, \ldots, n-1$, choose an enhanced spatial ribbon graph $\rho_{i}$ with $V_{\rho_{i}} \cap L=V_{\rho} \cap L$ so that $\Gamma_{\rho_{i}}$ is contained in $F_{i} \backslash d_{i}$, and $F_{i}$ is carried by $\rho_{i}$. Set also $\rho_{0}=\rho$ and $\rho_{n}=\rho^{\prime}$.

By construction, each surface $F_{i}, i=1, \ldots, n$, contains $\Gamma_{\rho_{i}}$ and $\Gamma_{\rho_{i-1}}$. Therefore, for each $i=1, \ldots, n$, there exists a sequence of handle additions and removals that produces $\rho_{i}$ from $\rho_{i-1}$. Choose morphisms $\eta_{i}$ from $\rho_{i-1}$ to $\rho_{i}$ so that $\left(F_{i}, F_{i}\right.$, id $\left.\left.\right|_{F_{i}}\right) \in \eta_{i}, i=1, \ldots, n$. Clearly we will have $\eta=\eta_{n} \circ \ldots \circ \eta_{1}$. This implies Condition (2) in the general case.

\section{Mirror Diagrams}

5.1. Representing ribbon graphs by mirror diagrams. Here comes our main instrument. 
Definition 5.1. By a mirror diagram we call a 4-tuple $M=\left(\Theta_{M}, \Phi_{M}, E_{M}, T_{M}\right)$ in which $\left(\Theta_{M}, \Phi_{M}, E_{M}\right)$ is a rectangular diagram of a graph (see [12, Definition 22]) and $T_{M}$ is a map from $E_{M}$ to the set of two symbols, ' \' and ' $/$ '. An element $v \in E_{M}$ such that $T_{M}(v)=$ ' '' (respectively, $T_{M}(v)=$ ' $/$ ') is referred to as $a \backslash$-mirror (respectively, a /-mirror). We also call $T_{M}(v)$ the type of the mirror $v$. The meridians $m_{\theta}, \theta \in \Theta_{M}$, and the longitudes $\ell_{\varphi}, \varphi \in \Phi_{M}$, are called vertical and horizontal occupied levels of $M$, respectively. The set $\left\{m_{\theta}: \theta \in \Theta_{M}\right\} \cup\left\{\ell_{\varphi}: \varphi \in \Phi_{M}\right\}$ of all occupied levels of $M$ is denoted by $L_{M}$.

To represent a mirror diagram $M$ graphically we draw all occupied levels of $M$ in a square, which is thought of as a cut torus, and mark each mirror by a dash slanted at $\pm \pi / 4$ according to the type of the mirror. When there is no mirror at the intersection of two occupied levels we draw the vertical occupied level passing over the horizontal one; see Figure 5.1.

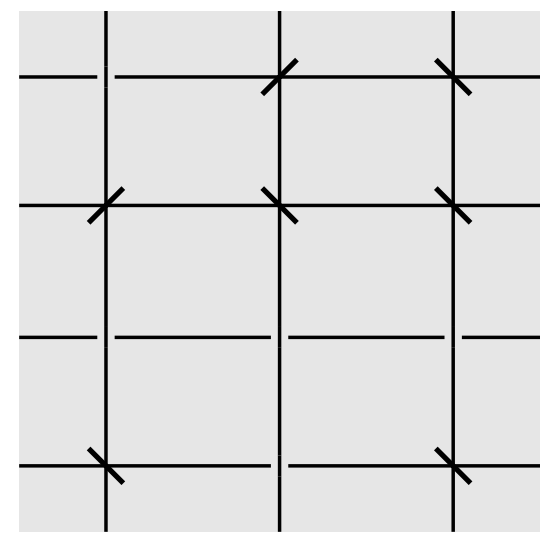

FiguRE 5.1. A mirror diagram

Definition 5.2. A boundary circuit of a mirror diagram $M$ is a closed path of a ray of light traveling along the occupied levels of $M$ and getting reflected off the mirrors of $M$; see Figure 5.2 In particular, if some occupied level of $M$ contains no mirrors, then this level alone forms a boundary circuit. The set

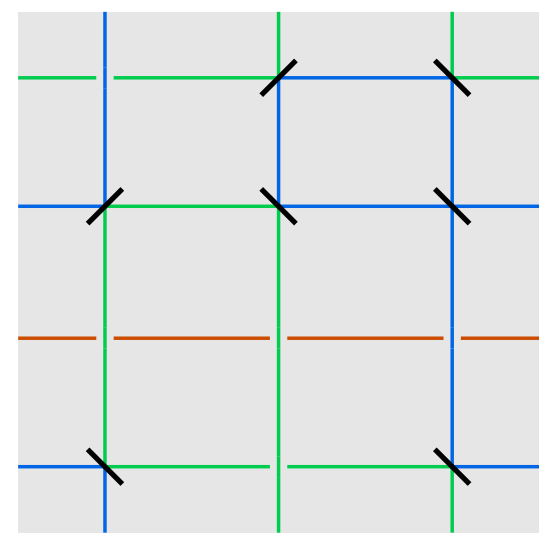

FiguRE 5.2. Boundary circuits of a mirror diagram. Each circuit is shown in different color

of all boundary circuits of $M$ will be denoted by $\partial M$ and called the boundary of $M$.

Mirror diagrams represent spatial ribbon graphs in $\mathbb{S}^{3}$ as we now describe.

Let $M$ be a mirror diagram. Denote by $G_{M}$ the rectangular diagram of a graph $\left(\Theta_{M}, \Phi_{M}, E_{M}\right)$. All edges of the graph $\widehat{G}_{M}$ are tangent to both contact structures $\xi_{+}$and $\xi_{-}$(see [12, Section 3]), hence there exists a compact surface $F$ such that (recall that we don't demand the surfaces to be $C^{2}$-smooth): 
(1) $\widehat{G}_{M}$ is contained in the interior of $F$;

(2) $F$ is tangent to $\xi_{+}$along $\widehat{\mu}$ if $\mu$ is a /-mirror of $M$;

(3) $F$ is tangent to $\xi_{-}$along $\widehat{\mu}$ if $\mu$ is a $\backslash$-mirror of $M$.

Pick such a surface $F$ and fix it from now on. For a subset $X \subset \mathbb{S}^{3}$ and a real $\varepsilon>0$ we denote by $U_{\varepsilon}(X)$ the open $\varepsilon$-neighborhood of $X$, and by $\bar{X}$ the closure of $X$.

For a small enough $\varepsilon>0$ the closure of the intersection $F \cap U_{\varepsilon}\left(\widehat{G}_{M} \cap\left(\mathbb{S}_{\tau=0}^{1} \cup \mathbb{S}_{\tau=1}^{1}\right)\right)$ is a union of pairwise disjoint discs, each containing a single point in $\widehat{G}_{M} \cap\left(\mathbb{S}_{\tau=0}^{1} \cup \mathbb{S}_{\tau=1}^{1}\right)$, and being such that the torus projection of its boundary is close to the corresponding occupied level of $M$. We fix such an $\varepsilon$ and, for each $x \in L_{M}$, denote by $\widehat{x}$ the disc corresponding to $x$.

Now take $\delta>0$ much smaller than $\varepsilon$ so that the closure of $F \cap U_{\delta}\left(\widehat{G}_{M}\right) \backslash U_{\varepsilon}\left(\mathbb{S}_{\tau=0}^{1} \cup \mathbb{S}_{\tau=1}^{1}\right)$ is a union of pairwise disjoint discs, each intersecting a single arc of the form $\widehat{\mu}, \mu \in E_{M}$. We denote the disc intersecting $\widehat{\mu}$, where $\mu \in E_{M}$, by $\widehat{\mu}$. It has the form of a narrow strip joining the discs $\widehat{m}$ and $\widehat{\ell}$, where $m$ and $\ell$ are the meridian and the longitude through $\mu$, and twisted around $\widehat{\mu}$ by $\pi / 2$ in the direction that depends on the type of the mirror $\mu$ in $M$; see Figure 5.3 .
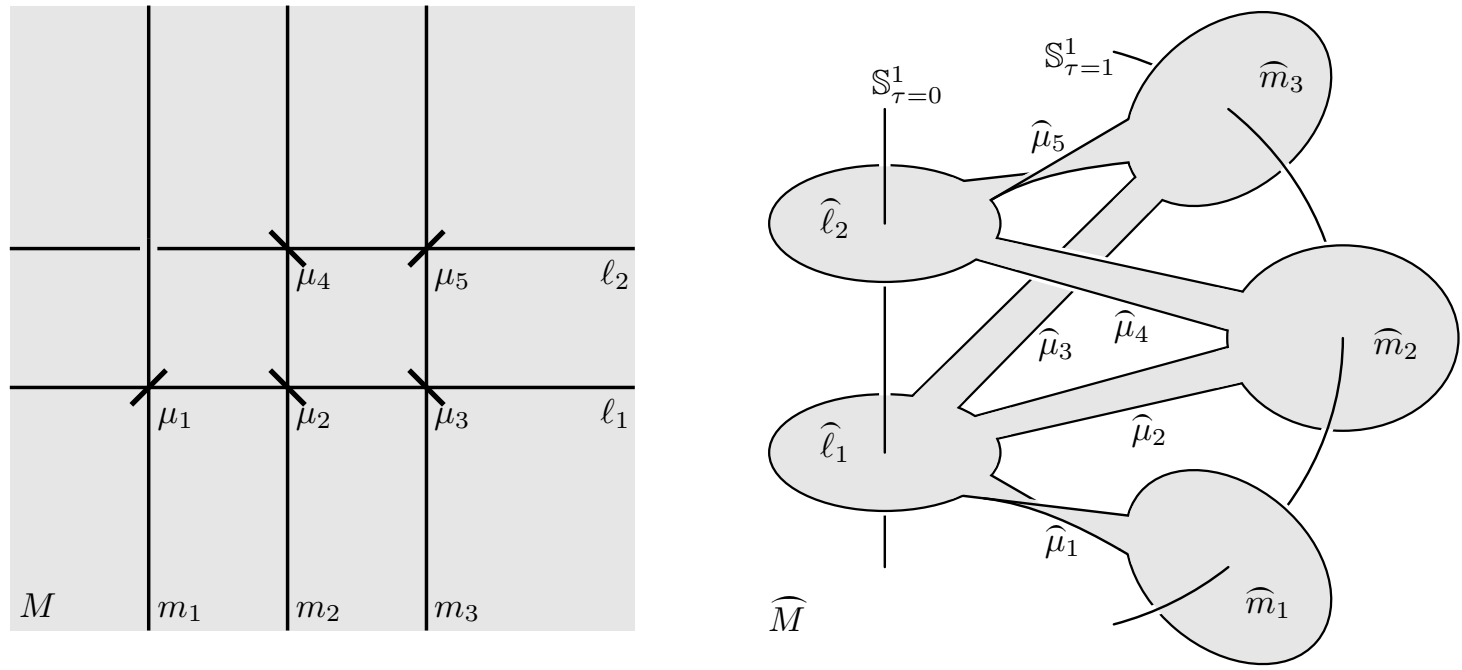

Figure 5.3. A mirror diagram $M$ and the corresponding surface $\overparen{M}$

The union $\left(\cup_{x \in L_{M}} \widehat{x}\right) \cup\left(\cup_{\mu \in E_{M}} \widehat{\mu}\right)$ is a surface with corners, which we denote by $\widehat{M}$. It is easy to see that $\overparen{M}$ retracts to $\widehat{G}_{M}$, and the torus projection of each connected component of $\partial \widehat{M}$ approximates a unique boundary circuit of $M$ (the smaller $\varepsilon$ and $\delta$, the better is the accuracy). This establishes a natural one-to-one correspondence between the boundary components of $\overparen{M}$ and the boundary circuits of $M$.

More formally, this correspondence is defined as follows. Let $x$ be an occupied level of $M$, and let $\mu_{1}, \ldots, \mu_{k}$ be all mirrors of $M$ lying on $x$ numbered according to their cyclic order in $x$. There is a natural one-to-one correspondence between connected components of $x \backslash\left\{\mu_{1}, \ldots, \mu_{k}\right\}$ and the connected components of $\widehat{x} \cap \partial \widehat{M}$. Namely, if $k \geqslant 2$ and $\alpha=\left(\mu_{i}, \mu_{i+1(\bmod k)}\right)$, then the component of $\widehat{x} \cap \partial \widehat{M}$ corresponding to $\alpha$, which we denote by $\widehat{\alpha}$, is defined by requesting that the torus projection of $\partial \hat{\alpha}$ projects into a subinterval of $\left(\mu_{i}, \mu_{i+1(\bmod k)}\right)$ along the $\varphi$-direction if $x$ is a longitude, and into a subinterval of $\left(\mu_{i}, \mu_{i+1(\bmod k)}\right)$ along the $\theta$-direction if $x$ is a meridian. If $k=0$ or $k=1$, then both sets $x \backslash\left\{\mu_{1}, \ldots, \mu_{k}\right\}$ and $\widehat{x} \cap \partial \widehat{M}$ are connected, so the bijection in question is the only possible one. If $c$ is the boundary circuit of $M$ that contains $\alpha$, then the respective connected component of $\partial \widehat{M}$, which we denote by $\widehat{c}$, is the one that contains $\hat{\alpha}$. One can see that this rule being applied to all occupied levels $x$ establishes a one-to-one correspondence between $\partial M$ and the set of all connected components of $\partial \widehat{M}$. 
Definition 5.3. The spatial ribbon graph $\widehat{M}$ associated with a mirror diagram $M$ is defined by putting

$$
\Gamma_{\widehat{M}}=\widehat{G}_{M}, \quad V_{\widehat{M}}=\widehat{G}_{M} \cap\left(\mathbb{S}_{\tau=0}^{1} \cup \mathbb{S}_{\tau=1}^{1}\right),
$$

and letting $S_{\widehat{M}}$ be such that $\overparen{M} \in S_{\widehat{M}}$. This means, in particular, that $\partial \widehat{M}$ consists of the projections of the connected components of $\partial \widehat{M}$ to $\widehat{G}_{M}$ in $\widehat{M}$. According to the discussion above there is a natural bijection between $\partial \widehat{M}$ and $\partial M$.

A mirror diagram $M$ is called connected if so is the graph $\widehat{G}_{M}$. A mirror diagram $M_{0}$ is referred to as a connected component of a mirror diagram $M$ if $\widehat{M}_{0}$ is a connected component of $\widehat{M}$.

Note that a spatial ribbon graph associated with a mirror diagram $M$ is a well defined object whereas the definition of the surface $\overparen{M}$ contains a large arbitrariness. However, we will often refer to surfaces of the form $\overparen{M}$ in our explanations, silently assuming that some reasonable choice has been made for all such surfaces that are being currently considered, and this choice is natural in the present context.

Note also that if $M$ is a mirror diagram, then any surface $F \in S_{\widehat{M}}$ is orthogonal to $\mathbb{S}_{\tau=0}^{1} \cup \mathbb{S}_{\tau=1}^{1}$ at any point in $\Gamma_{\widehat{M}} \cap\left(\mathbb{S}_{\tau=0}^{1} \cup \mathbb{S}_{\tau=1}^{1}\right)$.

Definition 5.4. Let $\rho$ be a(n enhanced) spatial ribbon graph, and let $F_{0}, F_{1}$ be two surfaces. We say that $F_{0}$ and $F_{1}$ are isotopic relative to $\rho$ if there is an isotopy from $F_{0}$ to $F_{1}$ fixed on $\Gamma_{\rho}$ through surfaces each of which contains a subsurface from $S_{\rho}$. In particular, $F_{0}$ and $F_{1}$ are meant to contain subsurfaces $F_{0}^{\prime}, F_{1}^{\prime} \in S_{\rho}$, respectively.

Practically, this definition means that $F_{0}$ (equivalently, $F_{1}$ ) contains a subsurface from $S_{\rho}$, and there is an isotopy from $F_{0}$ to $F_{1}$ that is fixed on $\Gamma_{\rho}$ and preserves the tangent plane to the surface at any vertex of $\rho$.

Lemma 5.1. Let $M$ be a mirror diagram, and let $F$ be a compact surface containing a subsurface $F^{\prime} \in$ $S_{\widehat{M}}$. Suppose that each connected component of $\partial F$ is either contained in $\Gamma_{\widehat{M}}$ or disjoint from $\Gamma_{\widehat{M}}$. Then there exists a rectangular diagram of a surface $\Pi$ such that $\widehat{\Pi}$ is isotopic to $F$ relative to $\widehat{M}$.

Proof. Since $F \supset F^{\prime} \in S_{\widehat{M}}$, the surface $F$ is isotopic relative to $\widehat{M}$ to a surface which is tangent to $\xi_{+}$ along $\widehat{\mu}$ if $\mu$ is a $/$-mirror of $M$, and to $\xi_{-}$if $\mu$ is a $\backslash$-mirror. So, in what follows we assume that this tangency condition holds for $F$ from the beginning, and use only isotopies preserving it.

Denote by $L$ the link $\partial F \backslash \Gamma_{\widehat{M}}$. By analogy with [12, Lemma 2] one can show that, for any $k \in \mathbb{Z}$, there exists a rectangular diagram of a link $R$ such that $\widehat{R}$ is isotopic to $L$ relative to $\Gamma_{\widehat{M}}$, and each connected

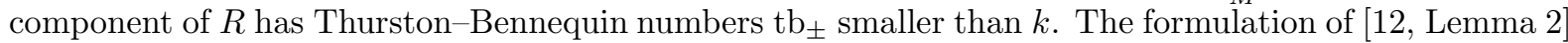
is different from this claim only in involving a rectangular diagram of a link in place of a more general rectangular diagram of a graph, which is $G_{M}$, but this does not affect the proof.

By choosing $k$ small enough and taking $R$ as above one can find a surface $F^{\prime}$ such that

(1) $\partial F^{\prime} \backslash \Gamma_{\widehat{M}}=\widehat{R}$

(2) for any connected component $K$ of $\partial F^{\prime}$ we have $\mathrm{tb}_{+}\left(K ; F^{\prime}\right)$, tb- $\left(K ; F^{\prime}\right) \leqslant 0$;

(3) there is an isotopy from $F$ to $F^{\prime}$ fixed on $\Gamma_{\widehat{M}}$ and preserving the tangency with $\xi_{+}$or $\xi_{-}$along $\Gamma_{\widehat{M}}$.

By [12, Proposition 5] there exists an isotopy fixed on $\Gamma_{\widehat{M}} \cup \widehat{R}$ and preserving the tangency with $\xi_{+}$ or $\xi_{-}$along $\Gamma_{\widehat{M}}$, from $F^{\prime}$ to a surface of the form $\widehat{\Pi}$, where $\Pi$ is a rectangular diagram of a surface.

Definition 5.5. A boundary circuit of a mirror diagram $M$ is called patchable if the corresponding boundary circuit of $\widehat{M}$ is patchable.

Definition 5.6. An enhanced mirror diagram is a 5-tuple $M=\left(\Theta_{M}, \Phi_{M}, E_{M}, T_{M}, H_{M}\right)$ in which $M_{0}=$ $\left(\Theta_{M}, \Phi_{M}, E_{M}, T_{M}\right)$ is a mirror diagram, and $H_{M}$ is a subset of the set of patchable boundary circuits of $M_{0}$. The non-patchable boundary circuits of $M_{0}$ as well as the patchable ones that are not elements of $H_{M}$ are called essential boundary circuits of $M$. The elements of $H_{M}$ are called inessential boundary circuits of $M$ or holes. The set $(\partial M) \backslash H_{M}$ is called the essential boundary of $M$ and denoted by $\partial_{\mathrm{e}} M$. 
If $M$ is an enhanced mirror diagram we denote by $\widehat{M}$ the enhanced spatial ribbon graph having $\widehat{M}_{0}$ as the underlying spatial ribbon graph, such that the inessential boundary circuits of $\widehat{M}$ are exactly those that correspond to the elements of $H_{M}$.

Every mirror diagram $M$ will be also regarded as an enhanced one with $H_{M}$ being the set of all patchable boundary circuits of $M$.

\subsection{Simple boundary circuits and framed rectangular diagrams of links.}

Definition 5.7. A boundary circuit of a mirror diagram is said to be simple if it hits:

(1) at least one mirror,

(2) each mirror at most once, and

(3) at most two mirrors at each occupied level.

A collection of simple boundary circuits whose union satisfies (2) and (3) above is also termed simple.

Recall that a framing $f$ of a rectangular diagram of a link is an ordering $>_{f}$ of each pair of vertices forming an edge [12, Definition 14] (see also Subsection 3.3 above).

A simple collection of boundary circuits of a mirror diagram is an object of exactly the same kind as the one used to represent a framed rectangular diagram of a link (see Definition 14 in 12 and the discussion afterwards). Thus, with every simple collection $C=\left\{c_{1}, \ldots, c_{k}\right\}$ of boundary circuits of a mirror diagram we associate a framed rectangular diagram of a link $(R(C), f(C))$ by letting $R(C)$ be the set of all mirrors hit by the circuits $c_{i}, i=1, \ldots, k$, (with the types of the mirrors forgotten), and letting $f(C)$ be a framing of $R(C)$ such that for any edge $\left\{v_{1}, v_{2}\right\}$ of $R(C)$ we have $v_{2}>_{f(C)} v_{1}$ if and only if $\left[v_{1} ; v_{2}\right] \subset \bigcup_{i=1}^{k} c_{i}$.

For a framing $f$ of a rectangular diagram of a link $R$, we denote by $\widehat{f}$ the respective admissible framing of the link $\widehat{R}$.

For a boundary circuit $c$ of a mirror diagram $M$ we denote by $\widehat{c}$ the respective boundary circuit of the spatial ribbon graph $\widehat{M}$. The following statement, proof of which is left to the reader, is an easy consequence of the definitions.

Proposition 5.1. A boundary circuit c of a mirror diagram $M$ is simple if and only if so is the boundary circuit $\widehat{c}$ of $\widehat{M}$.

A collection $C=\left\{c_{1}, \ldots, c_{k}\right\}$ of boundary circuits of a mirror diagram $M$ is simple if and only if the boundary circuits $\widehat{c}_{1}, \ldots, \widehat{c}_{k}$ of $\widehat{M}$ are all simple and pairwise disjoint. In this case we have $\widehat{R(C)}=$ $\widehat{c}_{1} \cup \ldots \cup \widehat{c}_{k}$. Moreover, the framing induced by $\widehat{M}$ on $\widehat{R(C)}$ is opposite to $\widehat{f(C)}$.

Recall that links presented by rectangular diagrams are Legendrian with respect to both contact structures $\xi_{+}$and $\xi_{-}$. The following statement is an easy observation, proof of which is also omitted.

Proposition 5.2. Let $c$ be a simple boundary circuit of a mirror diagram $M$, and let $F \in S_{\widehat{M}}$ be a surface such that $\widehat{c} \subset \partial F$. Let also $k$ and $l$ be the numbers of times the circuit $c$ hits /-mirrors and $\backslash$-mirrors, respectively. Then the Thurston-Bennequin numbers of $\widehat{c}$ relative to $F$ are as follows:

$$
\mathrm{tb}_{+}(\widehat{c} ; F)=-l / 2, \quad \mathrm{tb}_{-}(\widehat{c} ; F)=-k / 2 .
$$

This motivates the following definition.

Definition 5.8. Let $c$ be a boundary circuit of a mirror diagram $M$ such that $c$ hits $/$-mirrors and $\backslash$-mirrors $k$ times and $l$ times, respectively. By the Thurston-Bennequin numbers of $c$, denoted $\mathrm{tb}_{+}(c)$ and $\mathrm{tb}_{-}(c)$, we mean the numbers $-l / 2$ and $-k / 2$, respectively.

It is elementary to see that $\mathrm{tb}_{+}(c)$ and $\mathrm{tb}_{-}(c)$ are always integers even if $c$ is not a simple boundary circuit. 


\subsection{Rectangular diagrams of a surface as mirror diagrams.}

Definition 5.9. For every rectangular diagram of a surface $\Pi$, the associated enhanced mirror diagram $M(\Pi)$ is defined as follows:

- $L_{M(\Pi)}$ is the set of all occupied levels of $\Pi$;

- $E_{M(\Pi)}$ is the set of vertices of $\Pi$, each of which has the same type (' $/$ ' or ' $\backslash$ ') in $M(\Pi)$ as in $\Pi$;

- $H_{M(\Pi)}=\{\partial r: r \in \Pi\}$.

Definition 5.10. Let $M$ be an enhanced mirror diagram. We say that a rectangular diagram of a surface $\Pi$ is carried (respectively, properly carried) by $M$ if the surface $\widehat{\Pi}$ is carried (respectively, properly carried) by $\widehat{M}$.

Propositions 5.3 and 5.4 below follow immediately from these definitions.

Proposition 5.3. Any rectangular diagram of a surface $\Pi$ is properly carried by $M(\Pi)$.

The map $\Pi \mapsto M(\Pi)$ is a one-to-one correspondence between rectangular diagrams of a surface and enhanced mirror diagrams $M$ satisfying the following two conditions:

(1) each inessential boundary circuit of $M$ has the form of the boundary of a rectangle $r \subset \mathbb{T}^{2}$ such that the interior of $r$ is disjoint from $E_{M}$;

(2) the essential boundary $\partial_{\mathrm{e}} M$ of $M$ is simple.

Proposition 5.4. Let $M$ be a mirror diagram, and let $\Pi$ be a rectangular diagram of a surface properly carried by $M$. Then the rectangular diagram of the link $R\left(\partial_{\mathrm{e}} M\right)$ coincides with $\partial \Pi$, whereas the framing $f\left(\partial_{\mathrm{e}} M\right)$ is opposite to the boundary framing of $\partial \Pi$ induced by $\Pi$.

Proposition 5.5. Let $M$ be an enhanced mirror diagram. A rectangular diagram of a surface properly carried by $M$ exists if and only if the essential boundary of $M$ is simple.

Proof. The 'only if' direction is obvious.

To prove the 'if' direction note that the simplicity of $\partial_{\mathrm{e}} M$ implies that there exists a surface $F$ properly carried by $\widehat{M}$. Proposition 5 of [12] (in which we put $X_{1}=E_{M}, X_{2}=\varnothing$ ) implies that there exists a surface of the form $\widehat{\Pi}$ isotopic to $F$ relative to $\Gamma_{\widehat{M}}$, where $\Pi$ is a rectangular diagram of a surface. Such a diagram $\Pi$ is properly carried by $M$.

\subsection{Canonic dividing configurations.}

Definition 5.11. Let $M$ be a mirror diagram. A dividing configuration $\left(\delta_{+}, \delta_{-}\right)$on a surface $F \in S_{\widehat{M}}$ is said to be a canonic dividing configuration of $F$ if the following holds:

(1) $\delta_{+} \cup \delta_{-}$is a union of pairwise disjoint arcs each intersecting $\Gamma_{\widehat{M}}$ exactly once;

(2) $\delta_{+}$(respectively, $\delta_{-}$) intersects each arc of the form $\widehat{\mu}$, where $\mu$ is a $\backslash$-mirror (respectively, a $\zeta$-mirror) of $M$, exactly once, at the midpoint $(\tau=1 / 2)$, and the intersection is transverse;

(3) the coorientation of each arc $\widehat{\mu} \subset \Gamma_{\widehat{M}}$ defined by $\delta_{+} \cup \delta_{-}$at the midpoint agrees with the one induced on $\widehat{\mu}$ by the gradient of $\left.\varphi\right|_{F}$ near the circle $\mathbb{S}_{\tau=1}^{1}$.

One can see that a canonic dividing configuration on any surface $F \in S_{\rho}$ is defined uniquely up to equivalence. On can also see that it is never admissible.

Example 5.1. A canonic dividing configuration on the surface $\overparen{M}$ can be obtained as follows. Take for $\delta_{+}$ (respectively, for $\delta_{-}$) the intersection of all strips of the form $\hat{\mu}$, where $\mu$ is a \-mirror (respectively, a $/$-mirror), with the torus $\mathbb{T}_{\tau=1 / 2}^{2}$, and orient it so that the coordinate $\varphi$ increases on each connected component of $\delta_{+} \cup \delta_{-}$in the positive direction.

We conclude the section by the following statement, which is not formally needed to establish our main results, but reveals the differential-geometric meaning of canonic dividing configurations.

Proposition 5.6. Let $M$ be an enhanced mirror diagram with simple essential boundary, and let $F$ be a surface properly carried by $\widehat{M}$ such that $F$ is convex in Giroux's sense with respect to both contact 
structures $\xi_{+}$and $\xi_{-}$(for instance, a surface of the form $\widehat{\Pi}$ with $\Pi$ a rectangular diagram of a surface properly carried by $M)$. Let $\delta_{+}$and $\delta_{-}$be dividing sets of $F$ corresponding to $\xi_{+}$and $\xi_{-}$, respectively.

Then for any sufficiently small closed neighborhood $F_{0}$ of $\Gamma_{\widehat{M}}$ in $F$ the pair $\left(\delta_{+} \cap F_{0}, \delta_{-} \cap F_{0}\right)$ is a dividing configuration in $F_{0}$ equivalent to a canonic one.

Proof. Consider the contact structure $\xi_{+}$. The dividing set $\delta_{+}$can be represented as the set of zeros of the restriction of the two-valued function $\alpha(l)$ to the surface $F$, where $l$ is a contact line element field on $\mathbb{S}^{3}$ transverse to $F$, and $\alpha$ is a standard contact form (that is, a 1-form such that $\xi_{+}=\operatorname{ker} \alpha$ ).

Let $\beta$ be a regular fiber of the characteristic foliation $\mathscr{F}$ of the surface $F$ approaching singularities at the endpoints. In a small neighborhood of $\beta$ the field $l$ has the form $\{v,-v\}$, where $v$ is a contact vector field. Fix such $v$ from now on and orient $\beta$ so that $\alpha(w)>0$ whenever $u$ is a positive tangent vector to $\beta$, and $w$ is a tangent vector to $F$ such that $(u, w, v)$ is a positively oriented basis of the three-space.

One can show that if we traverse $\beta$ in the positive direction, then the sign of $\alpha(v)$ can change only from ' + ' to ' - ' but not the other way. So, it may change at most once. If $\beta$ is a 0 -arc (see 12, Subsection 4.3] for the definition), then the signs of $\alpha(v)$ at the endpoints of $\beta$ coincide, and if $\beta$ is a -1 -arc, then the signs are opposite. Therefore, any 0 -arc is disjoint from $\delta_{+}$, whereas any -1 -arc intersects $\delta_{+}$exactly once.

Note also that $\delta_{+}$must be disjoint from the singularities of $\mathscr{F}$, and no regular fiber of $\mathscr{F}$ can be a 1 -arc as the surface $F$ is assumed to be convex.

If $\mu$ is a $/$-mirror of $M$, then $\widehat{\mu}$ is an arc with singularities of $\mathscr{F}$ at the endpoints. The total rotation of the contact plane $\xi_{+}(p)$ with respect to the tangent plane $T_{p} F$ when $p$ traverses $\widehat{\mu}$ is zero. Therefore, $\widehat{\mu}$ consists of singularities of $\mathscr{F}$ and 0 -arcs, and thus is disjoint from $\delta_{+}$.

If $\mu$ is a $\backslash$-mirror, then the total rotation of the contact plane $\xi_{+}(p)$ with respect to the tangent plane $T_{p} F$ when $p$ traverses $\widehat{\mu}$ is equal to $-\pi$. Therefore, $\widehat{\mu}$ consists of singularities of $\mathscr{F}$, some number (may be zero) of 0 -arcs, and a single -1 -arc, and hence intersects $\delta_{+}$exactly once.

By symmetry an edge $\widehat{\mu}$ intersects $\delta_{-}$once if $\mu$ is a $/$-mirror and do not intersect it if $\mu$ is a $\backslash$-mirror. The claim follows.

\section{Elementary MOVES OF MiRRor DiAGRAMS}

6.1. Conventions. Denote by $r_{-}, r_{\mid}, r_{/}, r \backslash$ the reflections of the torus $\mathbb{T}^{2}$ about the lines $\varphi=0, \theta=0$, $\varphi=\theta$, and $\varphi=-\theta$, respectively:

$$
r_{-}(\theta, \varphi)=(\theta,-\varphi), \quad r_{\mid}(\theta, \varphi)=(-\theta, \varphi), \quad r_{/}(\theta, \varphi)=(\varphi, \theta), \quad r \backslash(\theta, \varphi)=(-\varphi,-\theta) .
$$

For a mirror diagram $M$ we define

$$
\begin{array}{ll}
r_{-}(M)=\left(\Theta_{M},-\Phi_{M}, r_{-}\left(E_{M}\right), \nu \circ T_{M} \circ r_{-}\right), & r_{\mid}(M)=\left(-\Theta_{M}, \Phi_{M}, r_{\mid}\left(E_{M}\right), \nu \circ T_{M} \circ r_{\mid}\right), \\
r /(M)=\left(\Phi_{M}, \Theta_{M}, r_{/}\left(E_{M}\right), T_{M} \circ r_{/}\right), & r \backslash(M)=\left(-\Phi_{M},-\Theta_{M}, r \backslash\left(E_{M}\right), T_{M} \circ r \backslash\right),
\end{array}
$$

where $-X$ with $X \subset \mathbb{S}^{1}$, stands for $\{-x: x \in X\}$, and $\nu$ is the map exchanging the symbols ' $\backslash$ ' and ' $/$ '.

Convention 6.1. Whenever we give a name to a transformation $M \mapsto M^{\prime}$ of mirror diagrams, the same name is meant to be given to the transformations $r_{-}(M) \mapsto r_{-}\left(M^{\prime}\right), r_{\mid}(M) \mapsto r_{\mid}\left(M^{\prime}\right), r \backslash(M) \mapsto r \backslash\left(M^{\prime}\right)$, and $r /(M) \mapsto r /\left(M^{\prime}\right)$.

Whenever a transformation $M \mapsto M^{\prime}$ is said to be of type $\mathrm{I}$ it is meant that the transformations $r(M) \mapsto r \backslash\left(M^{\prime}\right), r_{/}(M) \mapsto r_{/}\left(M^{\prime}\right)$ are of type I, and that the transformations $r_{-}(M) \mapsto r_{-}\left(M^{\prime}\right)$, $r_{\mid}(M) \mapsto r_{\mid}\left(M^{\prime}\right)$ are of type II.

Due to this symmetry, any statement about the moves has an equivalent 'dual' one obtained by exchanging types I and II of moves and types ' $>$ ' and ' $/$ ' of mirrors. We may, without a special notice, refer to a previously proven statement when the dual one should be used instead.

Convention 6.2. To represent graphically a transformation of mirror diagrams we often draw a portion of each diagram where the change occurs. Indicated occupied levels may have arbitrarily many mirrors outside the shown region unless their endpoints are marked by perpendicular dashes: $\longmapsto$. In the latter case, the occupied level is supposed to contain mirrors only in the shown portion of the diagram. 
A dotted line …... in the pictures of transformations indicate an arbitrary family of parallel occupied levels, which is supposed to be unchanged by the transformation. A gray box marked with a letter stands for an arbitrary family of mirrors at the intersection of the indicated occupied levels unless otherwise specified. The family of mirrors inside such boxes may be shifted as a whole by the considered moves.

Convention 6.3. Sometimes, in order to illustrate a transformation $M \mapsto M^{\prime}$ of mirror diagrams we draw the parts of the surfaces $\overparen{M}$ and $\widehat{M}^{\prime}$ in which the surfaces differ, without pretending to show realistically their positions in the three-space. However, if two discs of the form $\widehat{x}, \widehat{x}^{\prime}$, where $x$ and $x^{\prime}$ are parallel occupied levels, are close to one another according to the context, we may draw them overlapping, one over the other:

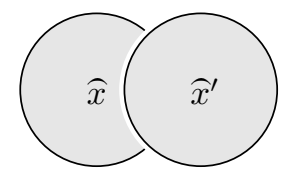

Every strip of the form $\widehat{\mu}$, where $\mu$ is a mirror of a mirror diagram $M$, is marked in our pictures by a small arc with endpoints at $\partial \widehat{M}$, intersecting $\widehat{\mu}$ exactly once. The arc is made red or green depending on the type of the mirror. If $\mu$ is of type ' $\backslash$ ' this arc is green, and otherwise red:
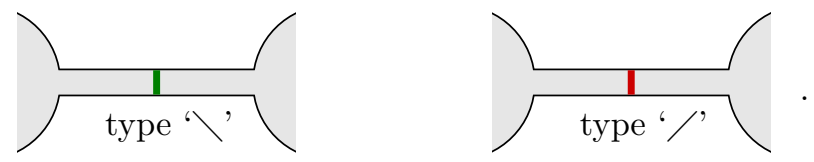

These arcs represent a canonic dividing configuration $\left(\delta_{+}, \delta_{-}\right)$of $\overparen{M}$ with $\delta_{+}$shown in green and $\delta_{-}$in red, which accords with Convention 2.1. We do not show the orientation of these arcs in the pictures.

Convention 6.4. Quite commonly, objects like rectangular diagrams are viewed up to combinatorial equivalence, without distinction between two diagrams that can be obtained from one another by a selfhomeomorphism of $\mathbb{T}^{2}$ of the form $(\theta, \varphi) \mapsto(f(\theta), g(\varphi))$, where $f$ and $g$ are degree 1 self-homeomorphisms of $\mathbb{S}^{1}$. This is not the case here. When we deal with mirror diagrams the exact position of some occupied levels does matter. More precisely, when a diagram transforms, it is important to observe which mirrors stay fixed and which are altered. For instance, from combinatorial point of view a transformation may look as an exchange of two occupied levels. But geometrically one of the levels may stay fixed, and it will matter which one of the two does so and which one moves.

6.2. Elementary moves of mirror diagrams (without enhancement). The transformations of mirror diagrams introduced in this section, namely, extension/elimination, elementary bypass addition/removal, and slide moves, are called elementary moves. First we introduce them for ordinary mirror diagrams and then extend the definitions to enhanced ones.

Definition 6.1. Let $M$ and $M^{\prime}$ be mirror diagrams such that $M^{\prime}$ is obtained from $M$ by adding a new occupied level and a $/$-mirror at the intersection of the new occupied level with an existing occupied level of $M$. Then we say that the passage $M \mapsto M^{\prime}$ is a type I extension move, and the inverse operation a type I elimination move; see Figure 6.1 (a).

Definition 6.2. Let $M$ and $M^{\prime}$ be mirror diagrams such that for some $\theta_{1} \neq \theta_{2}, \varphi_{1} \neq \varphi_{2}$ the following holds:

(1) $\left(\theta_{1}, \varphi_{2}\right)$ and $\left(\theta_{2}, \varphi_{1}\right)$ are $/$-mirrors of both $M$ and $M^{\prime}$;

(2) $\left(\theta_{1}, \varphi_{1}\right)$ is a $\backslash$-mirror of both $M$ and $M^{\prime}$;

(3) $\left(\theta_{2}, \varphi_{2}\right)$ is a $\backslash$-mirror of $M$ but not of $M^{\prime}$;

(4) there are no more mirrors of $M$ or $M^{\prime}$ in $r=\left[\theta_{1} ; \theta_{2}\right] \times\left[\varphi_{1} ; \varphi_{2}\right]$;

(5) $M$ and $M^{\prime}$ have the same set of mirrors outside $r$ and the same set of occupied levels.

Then we say that $M^{\prime}$ is obtained from $M$ by a type I elementary bypass removal, and $M$ is obtained from $M^{\prime}$ by a type I elementary bypass addition; see Figure 6.1 (b). 
Definition 6.3. Let $M$ and $M^{\prime}$ be mirror diagrams such that for some $\theta_{1} \neq \theta_{2}, \varphi_{1} \neq \varphi_{2}$ the following holds:

(1) $\left(\theta_{1}, \varphi_{1}\right),\left(\theta_{1}, \varphi_{2}\right)$ and $\left(\theta_{2}, \varphi_{1}\right)$ are $/$-mirrors of $M$;

(2) $\left(\theta_{1}, \varphi_{2}\right),\left(\theta_{2}, \varphi_{1}\right)$ and $\left(\theta_{2}, \varphi_{2}\right)$ are $/$-mirrors of $M^{\prime}$;

(3) there are no more mirrors of $M$ or $M^{\prime}$ in $r=\left[\theta_{1} ; \theta_{2}\right] \times\left[\varphi_{1} ; \varphi_{2}\right]$;

(4) $M$ and $M^{\prime}$ have the same set of mirrors outside $r$ and the same sets of occupied levels.

Then we say that $M^{\prime}$ is obtained from $M$ by a type I slide move; see Figure 6.1 (c).

(a)

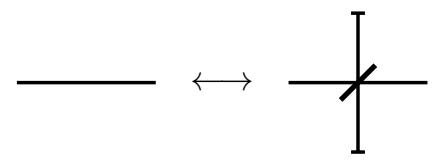

(b)

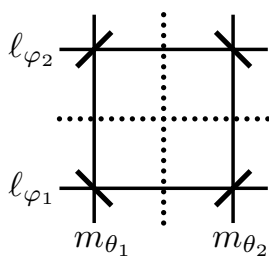

(c)

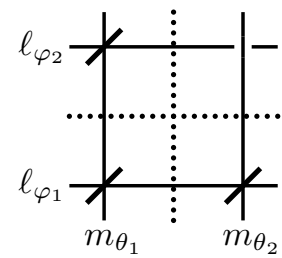

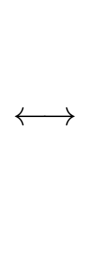
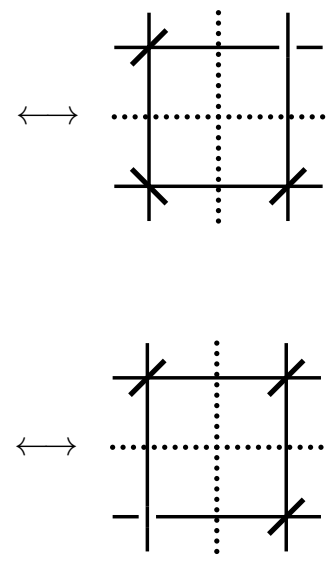
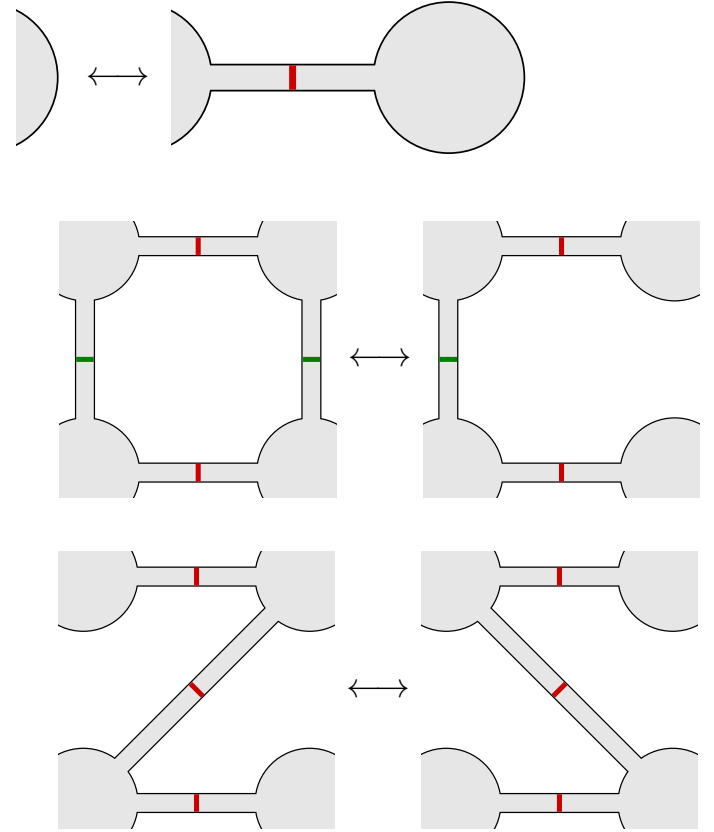

FiguRE 6.1. Type I elementary moves of mirror diagrams: (a) extension/elimination; (b) elementary bypass removal/addition; (c) slide

Observe that, due to Convention 6.1, the inverse operation to a slide move is also a slide move, and of the same type.

Remark 6.1. As one can learn from Figure 6.2 any slide move can be decomposed into a sequence of moves including extension, elimination, elementary bypass addition, and elementary bypass removal moves. So, the set of transformations that are decomposable into elementary moves does not depend on whether or not the latter include slide moves. However, we will need to work with transformations that are decomposable into elementary moves of one type, either I or II, and then the inclusion of slides into elementary moves will matter. One can see that the decomposition in Figure 6.2 involves moves of both types. This cannot be avoided in general.

6.3. Elementary moves of enhanced mirror diagrams. The occupied levels at which mirrors are explicitly mentioned in Definitions 6.1, 6.2, and 6.3 and shown in Figure 6.1 are referred to as involved in the respective move.

Definition 6.4. Let $M$ and $M^{\prime}$ be enhanced mirror diagrams, and let $M_{0}, M_{0}^{\prime}$ be the underlying mirror diagrams (with enhancement forgotten). Assume that $M_{0} \mapsto M_{0}^{\prime}$ is an elementary move. Then the move $M \mapsto M^{\prime}$ bears the same name (extension, elimination, elementary bypass addition, elementary bypass removal, or slide) and the same type (I or II) as $M_{0} \mapsto M_{0}^{\prime}$ does, provided that: 

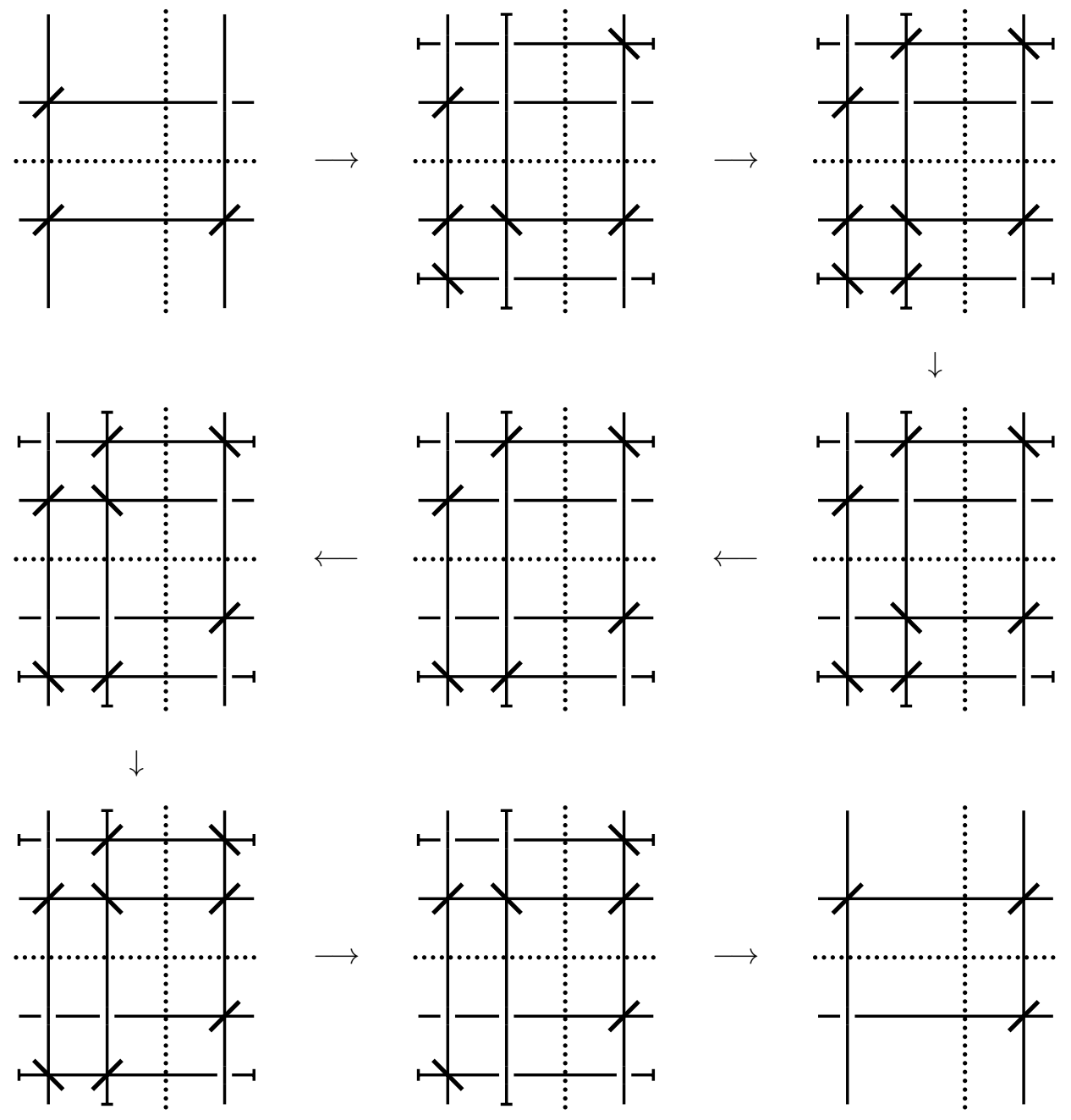

Figure 6.2. Decomposition of a slide move into elementary moves of other kinds

(1) for any boundary circuit $c$ of $M$ that shares a non-trivial subinterval of an occupied level not involved in the move with a boundary circuit $c^{\prime}$ of $M^{\prime}$, either both $c$ and $c^{\prime}$ are essential or both are inessential (for $M$ and $M^{\prime}$, respectively);

(2) the number of essential boundary circuits of $M$ is equal to that of $M^{\prime}$.

We now explain in more detail what this definition means for each kind of elementary moves. First, note that for any elementary move $M \mapsto M^{\prime}$ any common boundary circuit of $M$ and $M^{\prime}$ is either essential for both of them or inessential for both of them.

Let $M \mapsto M^{\prime}$ be an extension or elimination move. Then there are unique $c \in \partial M$ and $c^{\prime} \in \partial M^{\prime}$ such that $c \notin \partial M^{\prime}$ and $c^{\prime} \notin \partial M$. The essentialness or inessentialness of any boundary circuit in $\partial M \backslash\{c\}=$ $\partial M^{\prime} \backslash\left\{c^{\prime}\right\}$ is preserved. The boundary circuit $c$ is essential for $M$ if and only if so is $c^{\prime}$ for $M^{\prime}$.

Now let $M \mapsto M^{\prime}$ be an elementary bypass removal. We use the notation from Definition 6.2, $M$ has two boundary circuits that are not in $\partial M^{\prime}$, one of which is $c_{1}=\partial r$. Denote the other by $c_{2}$. They are replaced in $M^{\prime}$ by a single boundary circuit $c^{\prime}$ that can be viewed as the connected sum of $c_{1}$ and $c_{2}$. The boundary circuit $c_{1}$ of $M$ must be inessential. The boundary circuit $c_{2}$ is essential if and only if so is $c^{\prime}$. The essentialness of any boundary circuit in $\partial M \backslash\left\{c_{1}, c_{2}\right\}=\partial M^{\prime} \backslash\left\{c^{\prime}\right\}$ is preserved by the move. 
Finally let $M \mapsto M^{\prime}$ be a slide move. We use the notation from Definition 6.3. There are two, not necessarily distinct, boundary circuits $c_{1}, c_{2}$ of $M$ that are replaced by another two, $c_{1}^{\prime}, c_{2}^{\prime}$, say, so that $c_{i}^{\prime}$ coincides with $c_{i}$ outside $r$; see Figure 6.3. We have $c_{1}=c_{2}$ if and only if $c_{1}^{\prime}=c_{2}^{\prime}$. The circuit $c_{i}$
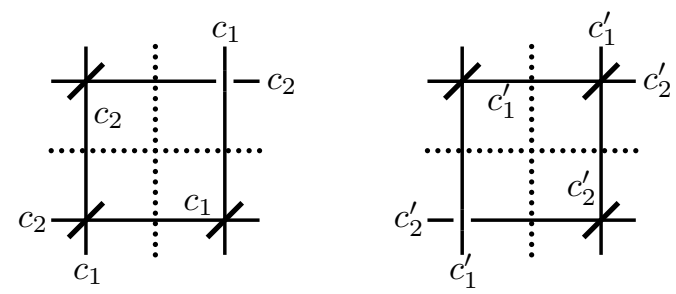

FiguRE 6.3. The correspondence between modified boundary circuits before and after a slide move

is essential for $M$ if and only if so is $c_{i}^{\prime}$ for $M^{\prime}, i=1,2$. The essentialness of any boundary circuit from $\partial M \backslash\left\{c_{1}, c_{2}\right\}=\partial M^{\prime} \backslash\left\{c_{1}^{\prime}, c_{2}^{\prime}\right\}$ is preserved by the move.

Remark 6.2. Let $M_{0}$ be the underlying mirror diagram of an enhanced mirror diagram $M$, and let $M_{0} \mapsto$ $M_{0}^{\prime}$ be an elementary move. If this move is an extension, elimination, or a slide move, then there exists a unique enhanced mirror diagram $M^{\prime}$ with the underlying mirror diagram $M_{0}^{\prime}$ such that $M \mapsto M^{\prime}$ is also an extension, elimination, or slide move, respectively.

If $M_{0} \mapsto M_{0}^{\prime}$ is an elementary bypass removal, then the respective $M^{\prime}$ may not exist. Indeed, a boundary circuit of $M$ having form of the boundary of a rectangle, which disappears in $M^{\prime}$, must be inessential for $M^{\prime}$ to exist. If it is essential, the move does not fit into the definition of the enhanced version of the move. If $M^{\prime}$ exists, then it is unique in this case.

If $M_{0} \mapsto M_{0}^{\prime}$ is an elementary bypass addition, then the respective $M^{\prime}$ always exists but it may not be unique. This arises from the fact that $M_{0}$ and $M_{0}^{\prime}$ may fit into the settings of Definition 6.2. with Convention 6.1 in force, in two different ways.

To see this let $r_{1}, r_{2} \subset \mathbb{T}^{2}$ two rectangles sharing a vertex and being disjoint otherwise. Suppose that both $\partial r_{1}$ and $\partial r_{2}$ are boundary circuits of $M_{0}^{\prime}$ and there are no mirrors of $M_{0}^{\prime}$ inside the rectangles $r_{1}, r_{2}$. Suppose also that the mirror added by the move $M_{0} \mapsto M_{0}^{\prime}$ is the common vertex of $r_{1}$ and $r_{2}$. Denote by $c$ the boundary circuit of $M$ that is replaced by $\partial r_{1}, \partial r_{2}$ as a result of the move. If $c$ is essential for $M$, then exactly one of $\partial r_{1}, \partial r_{2}$ must be essential for $M^{\prime}$, but it may be either of them.

A similar situation occurs when two rectangles whose boundaries are boundary circuits of $M_{0}^{\prime}$ share two or four vertices.

It can also happen that a transformation $M \mapsto M^{\prime}$ fits into the definition of a slide move in two different ways, but in this case only one boundary circuit is modified, so the enhancement of $M$ prescribes that of $M^{\prime}$.

Remark 6.3. Conditions (1) and (2) of Definition 6.4 are always satisfied when all patchable boundary circuits of $M$ and $M^{\prime}$ are inessential, which is the 'default' option.

Definition 6.5. For every elementary move $M \mapsto M^{\prime}$ of enhanced mirror diagrams we define the associated morphism of the respective enhanced spatial ribbon graphs as follows.

If $M \mapsto M^{\prime}$ is an extension move, the associated morphism $\eta$ is defined by $\left(\overparen{M}^{\prime}, \overparen{M^{\prime}}\right.$, id $\left.\left.\right|_{M^{\prime}}\right) \in \eta$.

If $M \mapsto M^{\prime}$ is an elementary bypass addition, then any surface carried by $\widehat{M}^{\prime}$ is also carried by $\widehat{M}$. Take any such surface $F$. The associated morphism $\eta$ is defined by the condition $\left(F, F,\left.\operatorname{id}\right|_{F}\right) \in \eta$.

For elimination moves and elementary bypass removals the associated morphisms are defined as the inverses to the morphisms associated with the inverses of the respective moves.

Now let $M \mapsto M^{\prime}$ be a slide move. We use the notation from Definition 6.3. Denote also the mirrors at $\left(\theta_{1}, \varphi_{1}\right),\left(\theta_{2}, \varphi_{1}\right),\left(\theta_{1}, \varphi_{2}\right),\left(\theta_{2}, \varphi_{2}\right)$ by $\mu_{1}, \mu_{2}, \mu_{3}, \mu_{4}$, respectively, and the discs

$$
\widehat{m}_{\theta_{1}} \cup \widehat{m}_{\theta_{2}} \cup \widehat{\ell}_{\varphi_{1}} \cup \widehat{\ell}_{\varphi_{2}} \cup \widehat{\mu}_{1} \cup \widehat{\mu}_{2} \cup \widehat{\mu}_{3} \subset \overparen{M} \quad \text { and } \quad \widehat{m}_{\theta_{1}} \cup \widehat{m}_{\theta_{2}} \cup \widehat{\ell}_{\varphi_{1}} \cup \widehat{\ell}_{\varphi_{2}} \cup \widehat{\mu}_{2} \cup \widehat{\mu}_{3} \cup \widehat{\mu}_{4} \subset \widehat{M}^{\prime}
$$


by $d$ and $d^{\prime}$, respectively. Let $h$ be a homeomorphism from $\overparen{M}$ to $\widehat{M}^{\prime}$ such that $\left.h\right|_{M \backslash d}=$ id. If $d$ is not a connected component of $\overparen{M}$, then this determines $h$ up to isotopy. If $d$ is a connected component of $\overparen{M}$ we demand additionally that $\left.h\right|_{d}: d \rightarrow d^{\prime}$ is orientation-preserving, where the orientations of $d$ and $d^{\prime}$ are chosen so that they agree on $d \cap d^{\prime}$.

The associated morphism $\eta$ is now defined by the condition $\left(\overparen{M}, \widehat{M}^{\prime}, h\right) \in \eta$.

To state that $\eta$ is the morphism associated with the move $M \mapsto M^{\prime}$ we write $M \stackrel{\eta}{\mapsto} M^{\prime}$.

6.4. Stable equivalence via elementary moves. We now express relative stable equivalence of spatial ribbon graphs presented by mirror diagrams, in combinatorial terms.

Theorem 6.1. Let $M$ and $M^{\prime}$ be enhanced mirror diagrams, and let $C=\left\{c_{1}, \ldots, c_{k}\right\}$ be a simple family of their common essential boundary circuits. Let also $\eta$ be a morphism from $\widehat{M}$ to $\widehat{M}^{\prime}$. The following two conditions are equivalent.

(1) The enhanced ribbon graphs $\widehat{M}$ and $\widehat{M}^{\prime}$ are stably equivalent relative to $L=\bigcup_{i=1}^{k} \widehat{c}_{i}$, and some isotopy realizing this stable equivalence induces $\eta$.

(2) There exists a sequence of elementary moves

$$
M=M_{0} \stackrel{\eta_{1}}{\longmapsto} M_{1} \stackrel{\eta_{2}}{\longmapsto} \ldots \stackrel{\eta_{n}}{\longmapsto} M_{n}=M^{\prime}
$$

preserving all the boundary circuits in $C$, such that $\eta=\eta_{n} \circ \ldots \circ \eta_{1}$.

Proof. The part $(2) \Rightarrow(1)$ is quite obvious and left to the reader. The inverse implication is a consequence of Lemmas 6.1 and 6.2 below.

Lemma 6.1. Let $M$ and $M^{\prime}$ be enhanced mirror diagrams, and let $C=\left\{c_{1}, \ldots, c_{k}\right\}$ be a simple family of their common essential boundary circuits. If the enhanced ribbon graphs $\widehat{M}$ and $\widehat{M^{\prime}}$ are stably equivalent relative to the framed link $L=\bigcup_{i=1}^{k} \widehat{c}_{i}$, and $\eta$ is a morphism from $\widehat{M}$ to $\widehat{M}^{\prime}$ induced by an isotopy realizing the stable equivalence, then there exists a sequence of enhanced mirror diagrams

$$
M=M_{0}, M_{1}, \ldots, M_{n}=M^{\prime}
$$

such that each transition $\widehat{M}_{i-1} \stackrel{\eta_{i}}{\longmapsto} \widehat{M}_{i}, i=1, \ldots, n$, is a handle addition or a handle removal preserving all the boundary circuits in $L$, and we have $\eta=\eta_{n} \circ \ldots \circ \eta_{1}$.

Proof. We construct the sought-for sequence of handle additions and removals in four steps.

Step 1. Reduce to the case when $E_{M} \cap E_{M^{\prime}}$ consists only of the mirrors that are hit by the boundary circuits $c_{i}, i=1, \ldots, k$ :

Suppose that both $M$ and $M^{\prime}$ have a mirror at $\left(\theta_{0}, \varphi_{0}\right)$ (not necessarily of the same type) that is not hit by any $c_{i}$. Suppose also that it is a $/$-mirror of $M$ (the case of a \-mirror is similar). Choose $\varepsilon>0$ so small that $\varnothing=\left(\theta_{0} ; \theta_{0}+\varepsilon\right] \cap \Theta_{M}=\left(\theta_{0} ; \theta_{0}+\varepsilon\right] \cap \Theta_{M^{\prime}}=\left[\varphi_{0}-\varepsilon ; \varphi_{0}\right) \cap \Phi_{M}=\left[\varphi_{0}-\varepsilon ; \varphi_{0}\right) \cap \Phi_{M^{\prime}}$.

Let $M_{1}$ be a mirror diagram obtained from $M$ by the following sequence of elementary moves (see Figure 6.4):

- two extension moves that add new occupied levels, $m_{\theta_{0}+\varepsilon}$ and $\ell_{\varphi_{0}-\varepsilon}$, and two $\backslash$-mirrors at $\left(\theta_{0}+\right.$ $\left.\varepsilon, \varphi_{0}\right)$ and $\left(\theta_{0}, \varphi_{0}-\varepsilon\right)$;

- an elementary bypass addition that inserts a $/$-mirror at $\left(\theta_{0}+\varepsilon, \varphi_{0}-\varepsilon\right)$.

Denote by $\eta_{1}$ the morphism from $\widehat{M}$ to $\widehat{M}_{1}$ that is the composition of the morphisms associated with these moves.

Let $M_{2}$ be the diagram obtained from $M_{1}$ by an elementary bypass removal that deletes the mirror at $\left(\theta_{0}, \varphi_{0}\right)$, and let $\eta_{2}$ be the associated morphism from $\widehat{M}_{1}$ to $\widehat{M}_{2}$. One can see that $\widehat{M} \mapsto \widehat{M}_{1}$ is a handle addition, and $\widehat{M}_{1} \mapsto \widehat{M}_{2}$ is a handle removal.

The enhanced spatial ribbon graphs $\widehat{M}_{2}$ and $\widehat{M}^{\prime}$ are still stably equivalent relative to $L$, and there is an isotopy realizing this stable equivalence that induces $\eta \circ \eta_{1}^{-1} \circ \eta_{2}^{-1}$. The set $E_{M_{2}}$ shares fewer elements with $E_{M^{\prime}}$ than $E_{M}$ does. By repeating this step finitely many times we get an enhanced mirror 


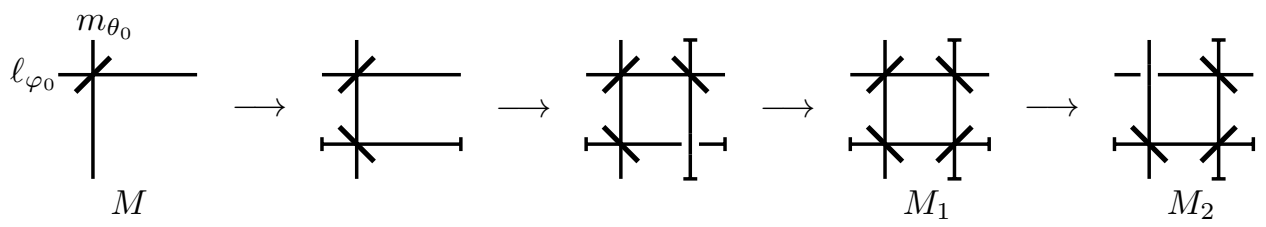

FiguRE 6.4. Removing an unwanted mirror by elementary moves

diagram $M^{\prime \prime}$ that is obtained from $M$ by a sequence of elementary moves preserving $L$, and is such that all mirrors in $E_{M^{\prime \prime}} \cap E_{M^{\prime}}$ are hit by some $c_{i}, i \in\{1, \ldots, k\}$. We redenote $M^{\prime \prime}$ by $M$ and proceed to the next step.

Step 2. Find a transition from $\widehat{M}$ to $\widehat{M}^{\prime}$ via spatial ribbon graphs, and put them into a single surface $F$ :

Denote by $M \cup M^{\prime}$ the mirror diagram defined by the following conditions: $L_{M \cup M^{\prime}}=L_{M} \cup L_{M^{\prime}}$, $E_{M \cup M^{\prime}}=E_{M} \cup E_{M^{\prime}},\left.T_{M \cup M^{\prime}}\right|_{E_{M}}=T_{M},\left.T_{M \cup M^{\prime}}\right|_{E_{M^{\prime}}}=T_{M^{\prime}}$. It exists since $T_{M}$ and $T_{M^{\prime}}$ agree on $E_{M} \cap$ $E_{M^{\prime}}$.

By Proposition 4.1 there exists a sequence of handle additions and removals

$$
\widehat{M}=\rho_{0} \stackrel{\eta_{1}}{\longmapsto} \rho_{1} \stackrel{\eta_{2}}{\longmapsto} \ldots \stackrel{\eta_{N}}{\longmapsto} \rho_{N}=\widehat{M}^{\prime}
$$

preserving the boundary circuits $\widehat{c}_{i}, i=1, \ldots, k$, and such that $\eta=\eta_{N} \circ \ldots \circ \eta_{2} \circ \eta_{1}$. Such a sequence will be called a guiding sequence for the transition $M \mapsto M^{\prime}$.

A generic choice of a guiding sequence ensures that the edges of the graphs $\Gamma_{\rho_{i}}$ that are added or removed in the course of transforming $\Gamma_{\rho_{0}}$ into $\Gamma_{\rho_{N}}$ do not have unnecessary intersections with each other, and all the graphs $\Gamma_{\rho_{i}}, i=0, \ldots, N$, are contained in a single compact surface $F$ such that, for each $i$, there is a surface $F_{i} \in S_{\rho_{i}}$ contained in $F$ and containing an open neighborhood of $\Gamma_{\rho_{i}}$ in $F$. Let $\Gamma$ be the union $\bigcup_{i=1}^{k} \Gamma_{\rho_{k}}$. We may also assume that $F$ is tangent to $\xi_{+}$along $\widehat{\mu}$ if $\mu$ is a $/$-mirror of $M \cup M^{\prime}$ and to $\xi_{-}$if $\mu$ is a $\backslash$-mirror of $M \cup M^{\prime}$.

Step 3. Make the surface $F$ 'rectangular':

By Lemma 5.1 there is an isotopy relative to $\widehat{M \cup M^{\prime}}$ from $F$ to a surface of the form $\widehat{\Pi}$, where $\Pi$ is a rectangular diagram of a surface. This isotopy takes $\rho_{i}, i=0, \ldots, N$, to spatial ribbon graphs that still form a guiding sequence for the transition $M \mapsto M^{\prime}$. So we may assume from the beginning that $F=\widehat{\Pi}$. Step 4. Make $\Gamma$ a subcomplex of the tiling of $\Pi$ :

Fix an open neighborhood $U \subset \widehat{\Pi}$ of $\Gamma_{\widehat{M \cup M^{\prime}}}$ such that $U$ retracts to $\Gamma_{\widehat{M \cup M^{\prime}}}$, and $U \cap \Gamma_{\rho_{i}}$ retracts to $\Gamma_{\widehat{M \cup M^{\prime}}}$ for any $i=1, \ldots, N-1$. By using wrinkle creation moves we can find a rectangular diagram of a surface $\Pi_{1}$ and a homeomorphism $h: \widehat{\Pi} \rightarrow \widehat{\Pi}_{1}$ such that

(1) $\Pi_{1}$ is obtained from $\Pi$ by finitely many wrinkle creation moves;

(2) there is an isotopy relative to $\widehat{M \cup M^{\prime}}$ from id $\left.\right|_{\widehat{\Pi}}$ to $h$;

(3) the preimage of the tiling of $\widehat{\Pi}_{1}$ under $h$ is arbitrarily fine outside $U$, i.e. for any rectangle $r \in \Pi_{1}$ the diameter of $h^{-1}(\widehat{r}) \backslash U$ is smaller than any $\delta>0$ chosen in advance.

This means that such $\Pi_{1}$ and $h$ can be chosen so that $h(\Gamma)$ is contained in the 1-skeleton of the tiling of $\widehat{\Pi}_{1}$.

We replace $\Pi$ by $\Pi_{1}$, and $\rho_{i}$ by $h\left(\rho_{i}\right), i=0,1, \ldots, N$. The new $\rho_{i}$ 's still form a guiding sequence for the transition $M \mapsto M^{\prime}$ and each $\rho_{i}$ now has the form $\widehat{M}_{i}$, where $M_{i}$ is an enhanced mirror diagram. This completes the proof.

Lemma 6.2. Let $M$ and $M^{\prime}$ be enhanced mirror diagrams such that $\widehat{M} \stackrel{\eta}{\mapsto} \widehat{M}^{\prime}$ is a handle addition. Then there is a sequence

$$
M=M_{0} \stackrel{\eta_{1}}{\longmapsto} M_{1} \stackrel{\eta_{2}}{\longmapsto} \ldots \stackrel{\eta_{n}}{\longmapsto} M_{n}=M^{\prime}
$$

of elementary moves preserving all common boundary circuits of $M$ and $M^{\prime}$ such that $\eta=\eta_{n} \circ \ldots \circ \eta_{2} \circ \eta_{1}$. 
Proof. By Lemma 5.1 a surface $F \in \widetilde{S}_{\widehat{M}}$ containing a patching disc associated with the move $M \mapsto M^{\prime}$ can be found in the form $\widehat{\Pi}$, where $\Pi$ is a rectangular diagram of a surface. We fix the surface $F$ from now on. In the construction below, for all $i$ we will have $F \in \widetilde{S}_{\widehat{M}_{i}}$ and $\left(F, F,\left.\operatorname{id}\right|_{F}\right) \in \eta_{i}$, so the equality $\eta=\eta_{n} \circ \ldots \circ \eta_{2} \circ \eta_{1}$ will hold trivially.

The number of tiles of $F=\widehat{\Pi}$ that form a patching disc associated with the handle addition $\widehat{M} \mapsto \widehat{M}^{\prime}$ will be called the complexity of this handle addition. The proof of the Lemma is by induction in this complexity.

Suppose that the move $M \mapsto M^{\prime}$ has complexity 1 . This means that there is a patching disc of the form $\widehat{r}, r \in \Pi$. Clearly, $M^{\prime}$ can be obtained from $M$ by zero, one, two, or three extension moves followed by an elementary bypass addition. This gives the induction base.

Suppose that the move $M \mapsto M^{\prime}$ has complexity $k>1$. Let $d \subset F$ be a patching disc associated with the move $M \mapsto M^{\prime}$ consisting of $k$ tiles of $F$. The disc $d$ can be cut into two nontrivial parts by a simple $\operatorname{arc} \alpha \subset \bigcup_{i=1}^{k} \partial \widehat{r}_{i}$ such that at least one of the endpoints of $\alpha$ lies in $\Gamma_{\widehat{M}}$. Denote by $M_{1}$ and $M_{1}^{\prime}$ the mirror diagrams defined by the following conditions:

(1) $\Gamma_{\widehat{M}_{1}}=\Gamma_{\widehat{M}} \cup \alpha, \Gamma_{\widehat{M}_{1}^{\prime}}=\Gamma_{\widehat{M}^{\prime}} \cup \alpha$;

(2) some surfaces $F_{1} \in S_{\widehat{M}_{1}}$ and $F_{1}^{\prime} \in S_{\widehat{M}_{1}^{\prime}}$ are tangent to $d$ along $d \cap \Gamma_{\widehat{M}_{1}}$ and $d \cap \Gamma_{\widehat{M}_{1}^{\prime}}$, respectively. There are the following two cases to consider.

Case 1. Both endpoints of $\alpha$ lie in $\Gamma_{\widehat{M}}$. Then for appropriate enhancements of $M_{1}$ and $M_{1}^{\prime}$ both transitions $M \mapsto M_{1}$ and $M_{1} \mapsto M_{1}^{\prime}$ are handle additions of complexity smaller than $k$, and the transition $M_{1}^{\prime} \mapsto M^{\prime}$ is a handle removal of complexity smaller than $k$; see Figure 6.5
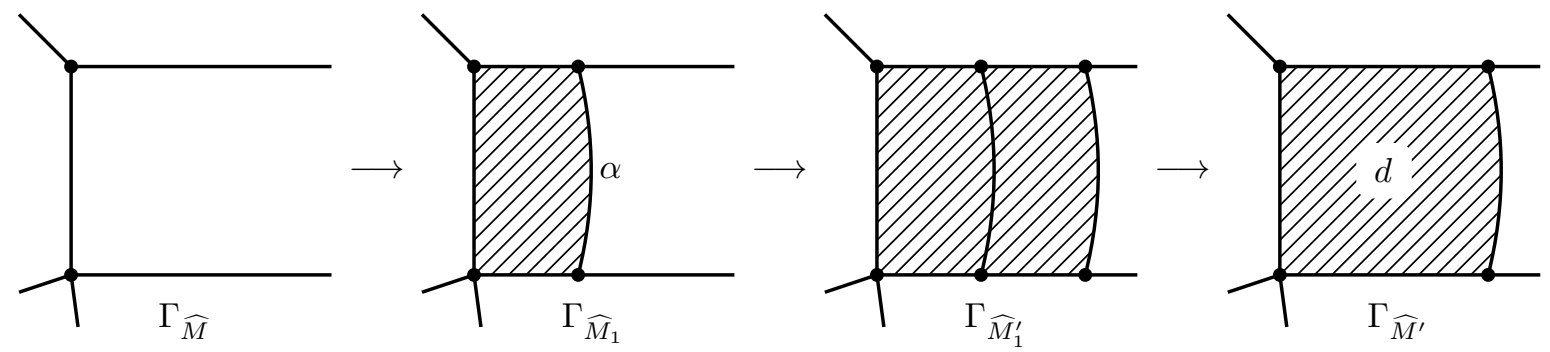

Figure 6.5. Induction step in Lemma 6.2, Case 1

Case 2. Only one endpoint of $\alpha$ lies in $\Gamma_{\widehat{M}}$. Then for appropriate enhancements of $M_{1}$ and $M_{1}^{\prime}$ the transition $M \mapsto M_{1}$ decomposes into a few extension moves. The transition $M_{1} \mapsto M_{1}^{\prime}$ decomposes into two handle additions of complexity smaller than $k$. Finally, the transition $M_{1}^{\prime} \mapsto M^{\prime}$ is again a handle removal of complexity smaller than $k$; see Figure 6.6 .
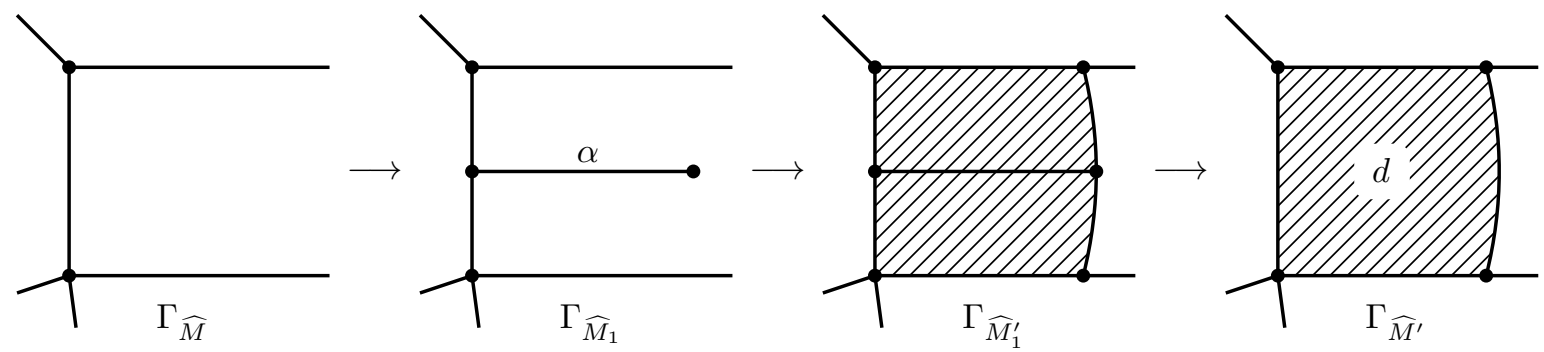

Figure 6.6. Induction step in Lemma 6.2, Case 2

Both cases give the induction step. 


\section{More transformations. Neat decompositions}

7.1. Preliminary remarks and conventions. This is a purely technical section in which we introduce further families of moves of mirror diagrams that are needed to establish our main results, and show how to decompose the new moves into elementary ones.

For brevity we give definitions in terms of ordinary, that is, not enhanced, mirror diagrams, and extend them to enhanced mirror diagrams by using the following convention.

Convention 7.1. Definition 6.4 extends to all the moves defined in this section, with involved occupied levels understood in a broader sense. Namely, on occupied level is involved if it contains the mirrors explicitly mentioned in the definition of the move and/or mirrors whose position is changed by the move.

Additionally, if the definition of a move $M \mapsto M^{\prime}$ prescribes $M$ or $M^{\prime}$ to have a circuit $c$ that hits exactly four mirrors, each on one side (this occurs in the cases of bridge, wrinkle, and double split moves), then $c$ is demanded to be inessential in the extension of the definition to enhanced mirror diagrams.

Definition 7.1. Let $M \mapsto M^{\prime}$ be an elementary move or one of the moves defined in this section, and let $c$ and $c^{\prime}$ be essential boundary circuits of $M$ and $M^{\prime}$, respectively. We say that the move $M \mapsto M^{\prime}$ transforms $c$ to $c^{\prime}$ if $c$ and $c^{\prime}$ either share a nondegenerate interval of a common occupied level of $M$ and $M^{\prime}$ which is not involved in the move, or both $c$ and $c^{\prime}$ are contained entirely in the occupied levels involved in the move.

We also use this terminology for subsets of the essential boundaries of $M$ and $M^{\prime}: C \subset \partial_{\mathrm{e}} M$ is transformed to $C^{\prime} \subset \partial_{\mathrm{e}} M$ if each $c \in C$ is transformed to some $c^{\prime} \in C^{\prime}$, and for each $c^{\prime} \in C^{\prime}$ there is a $c \in C$ transformed to $c^{\prime}$.

For each kind of moves $M \mapsto M^{\prime}$ that we define in this section, it is elementary to show that the relation ' $c$ is transformed to $c$ ' is a one-to-one correspondence between essential boundaries $\partial_{\mathrm{e}} M$ and $\partial_{\mathrm{e}} M$ '.

Every move $M \mapsto M^{\prime}$ that we define below is accompanied by an associated morphism of the respective enhanced spatial ribbon graphs $\widehat{M} \rightarrow \widehat{M^{\prime}}$. By writing $M \stackrel{\eta}{\mapsto} M^{\prime}$ we always mean that $\eta$ is the associated morphism.

Definition 7.2. Let a transformation $M \stackrel{\eta}{\mapsto} M^{\prime}$ of enhanced mirror diagrams be decomposed into a sequence of other transformations:

$$
M=M_{0} \stackrel{\eta_{1}}{\longmapsto} M_{1} \stackrel{\eta_{2}}{\longmapsto} \ldots \stackrel{\eta_{k}}{\longmapsto} M_{k}=M^{\prime},
$$

each endowed with a morphism of the respective enhanced spatial ribbon graphs, and let $C$ be a collection of boundary circuits of $M$ preserved by the transformation $M \stackrel{\eta}{\mapsto} M^{\prime}$. We say that decomposition (7.1) is $C$-neat if the following holds:

(1) each boundary circuit in $C$ is preserved by all the transformations $M_{i-1} \mapsto M_{i}, i=1, \ldots, k$;

(2) for any $0 \leqslant i_{1}<i_{2} \leqslant k$, whenever an essential boundary circuit $c$ of $M_{i_{1}}$ and the respective boundary circuit $c^{\prime}$ of $M_{i_{2}}$ have negative Thurston-Bennequin number tb + (respectively, tb_), that is, hit at least one \-mirror (respectively, /-mirror), so have the corresponding boundary circuits of all $M_{j}$ 's with $j \in\left[i_{1} ; i_{2}\right]$;

(3) $\eta=\eta_{k} \circ \ldots \circ \eta_{2} \circ \eta_{1}$

If $C$ includes all boundary circuits of $M$ preserved by the transformation $M \stackrel{\eta}{\mapsto} M^{\prime}$, a $C$-neat decomposition (7.1) is called neat for short.

We will often use the following obvious properties of $C$-neat decompositions.

Lemma 7.1. Let $M_{0} \stackrel{\eta_{1}}{\longmapsto} M_{1} \stackrel{\eta_{2}}{\longmapsto} \ldots \stackrel{\eta_{k}}{\longmapsto} M_{k}$ be a decomposition of a transformation $M_{0} \stackrel{\eta}{\longmapsto} M_{k}$ of enhanced mirror diagrams preserving a collection $C$ of boundary circuits, and let $i, j$ be such that $0 \leqslant i<$ $j \leqslant k$.

The following statements are equivalent:

(1) the decomposition $M_{0} \stackrel{\eta_{1}}{\longmapsto} M_{1} \stackrel{\eta_{2}}{\longmapsto} \ldots \stackrel{\eta_{k}}{\longmapsto} M_{k}$ of $M_{0} \stackrel{\eta}{\longmapsto} M_{k}$ is C-neat;

(2) the decomposition $M_{k} \stackrel{\eta_{k}^{-1}}{\longmapsto} \ldots \stackrel{\eta_{2}^{-1}}{\longmapsto} M_{1} \stackrel{\eta_{1}^{-1}}{\longmapsto} M_{0}$ of $M_{k} \stackrel{\eta^{-1}}{\longmapsto} M_{0}$ is $C$-neat; 
(3) the decompositions $M_{0} \stackrel{\eta_{1}}{\longmapsto} M_{1} \stackrel{\eta_{2}}{\longmapsto} \ldots \stackrel{\eta_{i}}{\longmapsto} M_{i} \stackrel{\eta_{j} \circ \eta_{j-1} \circ \ldots \circ \eta_{i+1}}{\longmapsto} M_{j} \stackrel{\eta_{j+1}}{\longmapsto} \ldots \stackrel{\eta_{k}}{\longmapsto} M_{k}$ and $M_{i} \stackrel{\eta_{i+1}}{\longmapsto}$ $M_{i+1} \stackrel{\eta_{i+2}}{\longmapsto} \ldots \stackrel{\eta_{j}}{\longmapsto} M_{j}$ of $M_{0} \stackrel{\eta}{\longmapsto} M_{k}$ and $M_{i} \stackrel{\eta_{j} \circ \eta_{j-1} \circ \ldots \circ \eta_{i+1}}{\longmapsto} M_{j}$, respectively, are $C$-neat.

Definition 7.3. A transformation $M \stackrel{\eta}{\mapsto} M^{\prime}$ of enhanced mirror diagrams is said to be safe-to-bringforward if, for any essential boundary circuit $c \in \partial M$ and the corresponding boundary circuit $c^{\prime} \in \partial M^{\prime}$, we have $\mathrm{tb}_{+}(c)<0 \Rightarrow \mathrm{tb}_{+}\left(c^{\prime}\right)<0$ and $\mathrm{tb}_{-}(c)<0 \Rightarrow \mathrm{tb}_{-}\left(c^{\prime}\right)<0$.

If these conditions hold only for tb+ (respectively, for tb-), then the transformation $M \stackrel{\eta}{\mapsto} M^{\prime}$ is said to be +-safe-to-bring-forward (respectively, --safe-to-bring-forward).

The inverse of a safe-to-bring-forward (respectively, +-safe-to-bring-forward or --safe-to-bring-forward) transformation is said to be safe-to-postpone (respectively, +-safe-to-postpone or --safe-to-postpone).

If a transformation is safe-to-bring-forward and safe-to-postpone, it is said to be safe. Similarly, for \pm -versions.

The reason for these names is the following statement, which is also obvious.

Lemma 7.2. Let $M_{0} \stackrel{\eta_{1}}{\longmapsto} M_{1} \stackrel{\eta_{2}}{\longmapsto} M_{2}$ be a decomposition of a transformation $M_{0} \stackrel{\eta}{\longmapsto} M_{2}, \eta=\eta_{2} \circ \eta_{1}$, and let $C \subset \partial M_{0}$, be a collection of boundary circuits preserved by all three transformations $M_{0} \stackrel{\eta}{\mapsto} M_{2}$, $M_{0} \stackrel{\eta_{1}}{\longmapsto} M_{1}$, and $M_{1} \stackrel{\eta_{2}}{\longmapsto} M_{2}$. Then the decomposition $M_{0} \stackrel{\eta_{1}}{\longmapsto} M_{1} \stackrel{\eta_{2}}{\longmapsto} M_{2}$ is $C$-neat once at least one of the following holds:

(1) $M_{0} \stackrel{\eta_{1}}{\longmapsto} M_{1}$ is safe-to-bring-forward;

(2) $M_{1} \stackrel{\eta_{2}}{\longmapsto} M_{2}$ is safe-to-postpone;

(3) $M_{0} \stackrel{\eta_{1}}{\longmapsto} M_{1}$ is +-safe-to-bring-forward and $M_{1} \stackrel{\eta_{2}}{\longmapsto} M_{2}$ is --safe-to-postpone;

(4) $M_{0} \stackrel{\eta_{1}}{\longmapsto} M_{1}$ is --safe-to-bring-forward and $M_{1} \stackrel{\eta_{2}}{\longmapsto} M_{2}$ is +-safe-to-postpone.

In what follows, all decompositions that are claimed to be neat can be obtained from the trivial one (just a single transformation) by recursively applying one of the following two operations or their inverses:

(1) replacing some move in the decomposition by a sequence of two moves for which one of the cases (1)-(4) of Lemma 7.2 occurs;

(2) append a safe-to-postpone move at the end of the sequence or a safe-to-bring-forward at the beginning.

Due to Lemmas 7.1 and 7.2 this guarantees that all the obtained decompositions obey Condition (2) of Definition 7.2

Observe that all type I elementary moves are +-safe, and all type II elementary moves are --safe. Slide moves of both types are just safe. Type I extension moves and elementary bypass removals are --safe-tobring-forward, whereas type I elimination moves and elementary bypass additions are --safe-to-postpone. Symmetrically for type II moves.

Condition (3) in Definition 7.2 is very important, but in the situations we need to consider, either it holds trivially or follows from an easy check. For instance, if the modified part of the surface is a disc some part of whose boundary is fixed, then we need not worry about Condition (3), since it holds true automatically.

For the reasons mentioned above, when we prove below that some decomposition is neat, we concentrate on showing that the result of the obtained transformation is what we claim it to be, and don't always provide separate comments why Conditions (2) and (3) of Definition 7.2 are satisfied.

7.2. Neutral moves. It is important for us to distinguish between type I and type II elementary moves. However, there are certain transformations of mirror diagrams that can be decomposed into a few elementary moves of either type (and thus are safe). We call such transformations neutral moves. They include additions and removals of a bridge, jump moves, wrinkle creations and reductions, and double split and merge moves, which are defined below.

Definition 7.4. Let $M$ and $M^{\prime}$ be mirror diagrams such that for some $\theta_{1} \neq \theta_{2}, \varphi_{1} \neq \varphi_{2}$ the following holds:

(1) $\mu_{1}=\left(\theta_{1}, \varphi_{1}\right)$ is a $\backslash$-mirror of both $M$ and $M^{\prime}$; 
(2) $\mu_{2}=\left(\theta_{2}, \varphi_{1}\right)$ is a $/$-mirror of both $M$ and $M^{\prime}$;

(3) $\ell_{\varphi_{2}}$ is not an occupied level of $M$;

(4) $\mu_{3}=\left(\theta_{1}, \varphi_{2}\right)$ is a $/$-mirror of $M^{\prime}$;

(5) $\mu_{4}=\left(\theta_{2}, \varphi_{2}\right)$ is a $\backslash$-mirror of $M^{\prime}$;

(6) there are no other mirrors of $M$ or $M^{\prime}$ in $r=\left[\theta_{1} ; \theta_{2}\right] \times\left[\varphi_{1} ; \varphi_{2}\right]$;

(7) $M$ and $M^{\prime}$ have the same set of mirrors outside $r$, and we have $\Theta_{M}=\Theta_{M^{\prime}}, \Phi_{M}=\Phi_{M^{\prime}} \backslash\left\{\varphi_{2}\right\}$.

Then we say that $M^{\prime}$ is obtained from $M$ by adding a bridge, and $M$ is obtained from $M^{\prime}$ by removing a bridge; see Figure 7.1 (a).

The morphism $\eta$ associated with the bridge addition $M \mapsto M^{\prime}$ is defined by $\left(\overparen{M}, \overparen{M},\left.\mathrm{id}\right|_{\overparen{M}}\right) \in \eta$.

Definition 7.5. Let $M$ and $M^{\prime}$ be mirror diagrams such that for some $\theta_{1} \neq \theta_{2}$ and $\varphi_{0}$ the following holds:

(1) $\mu_{1}=\left(\theta_{1}, \varphi_{0}\right)$ is a $\backslash$-mirror of $M$ and a $/$-mirror of $M^{\prime}$;

(2) $\mu_{2}=\left(\theta_{2}, \varphi_{0}\right)$ is a $\backslash$-mirror of $M^{\prime}$ and a $/$-mirror of $M$;

(3) there are no more mirrors of $M$ or $M^{\prime}$ at $\ell_{\varphi_{0}}$;

(4) $M$ and $M^{\prime}$ have the same set of mirrors outside $\ell_{\varphi_{0}}$, and the same sets of occupied levels.

Then we say that $M$ and $M^{\prime}$ are obtained from one another by a twist move; see Figure 7.1 (b).

The morphism $\eta$ associated with the twist move $M \mapsto M^{\prime}$ is defined by the condition $\left(\overparen{M}, \widehat{M}^{\prime}, h\right) \in \eta$, where $h$ is a homeomorphism $\overparen{M} \rightarrow \widehat{M}^{\prime}$ identical outside of $\widehat{\mu}_{1} \cup \widehat{\mu}_{2} \cup \widehat{\ell}_{\varphi_{0}}$.

(a)

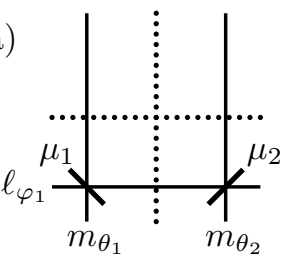

(b)

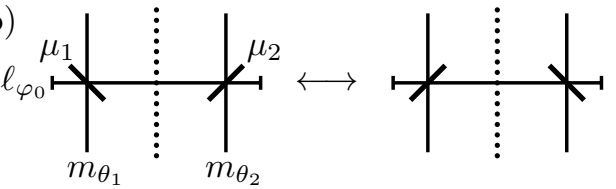

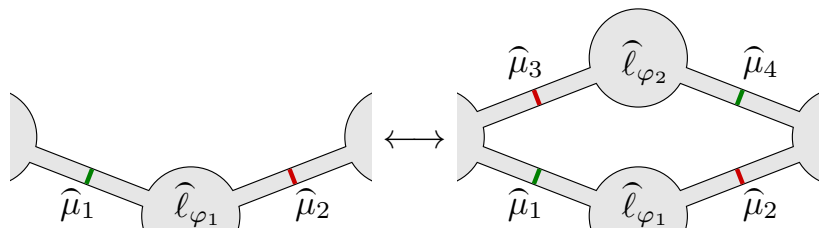

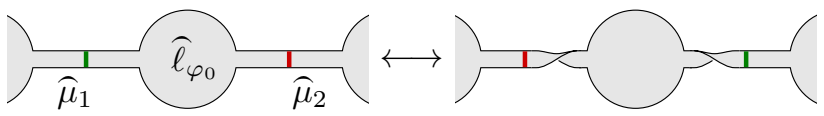

Figure 7.1. (a) Bridge moves and (b) twist moves

Definition 7.6. Let $M$ be a mirror diagrams such that $\ell_{\varphi_{1}}$ is an occupied level of $M$ and $\ell_{\varphi_{2}}$ is not an occupied level of $M$. Assume that for some $\theta_{1} \neq \theta_{2}$ all mirrors at $\ell_{\varphi_{1}}$ occur in the interval $\left(\theta_{1} ; \theta_{2}\right) \times\left\{\varphi_{1}\right\}$, and there are no mirrors of $M$ in $\left(\theta_{1} ; \theta_{2}\right) \times\left(\varphi_{1} ; \varphi_{2}\right)$. Let $M^{\prime}$ be obtained from $M$ by replacing the occupied level $\ell_{\varphi_{1}}$ with $\ell_{\varphi_{2}}$, and each mirror of the form $\left(\theta, \varphi_{1}\right)$ with a mirror at $\left(\theta, \varphi_{2}\right)$ of the same type. Then we say that $M \mapsto M^{\prime}$ is a jump move; see Figure 7.2 .

The morphism $\eta$ associated with the jump move $M \mapsto M^{\prime}$ is defined by the condition $\left(\overparen{M}, \widehat{M}^{\prime}, h\right) \in \eta$, where $h$ is a homeomorphism $\overparen{M} \rightarrow \overparen{M}^{\prime}$ such that $\left.h\right|_{\widehat{y}}$ is an orientation-preserving self-homeomorphism of $\hat{y}$ whenever $y$ is an occupied level of $M$ distinct from $\ell_{\varphi_{1}}$, or a mirror located outside $\ell_{\varphi_{1}}$, and $h$ takes $\widehat{\ell}_{\varphi_{1}}$ to $\widehat{\ell}_{\varphi_{2}}$ preserving the coorientation defined by $d \varphi$.

Definition 7.7. Let a mirror diagram $M$ have a $\backslash$-mirror $\mu_{1}$ at $\left(\theta_{1}, \varphi_{0}\right)$ and a $/$-mirror $\mu_{2}$ at $\left(\theta_{2}, \varphi_{0}\right)$. Let $\varphi_{0}, \varphi_{1}, \varphi_{2}, \varphi_{3} \in \mathbb{S}^{1}$ be such that $\varphi_{0} \in\left[\varphi_{1} ; \varphi_{3}\right], \varphi_{2} \in\left(\varphi_{1} ; \varphi_{3}\right)$, and there are no horizontal occupied levels $\ell_{\varphi}$ of $M$ with $\varphi \in\left[\varphi_{1} ; \varphi_{3}\right], \varphi \neq \varphi_{0}$. Let a mirror diagram $M^{\prime}$ be obtained from $M$ by:

(1) replacing the occupied level $\ell_{\varphi_{0}}$ by three occupied levels $\ell_{\varphi_{i}}, i=1,2,3$;

(2) replacing each mirror at $\left(\theta, \varphi_{0}\right)$, with $\theta \in\left(\theta_{1} ; \theta_{2}\right)$, by a mirror at $\left(\theta, \varphi_{3}\right)$ of the same type; 


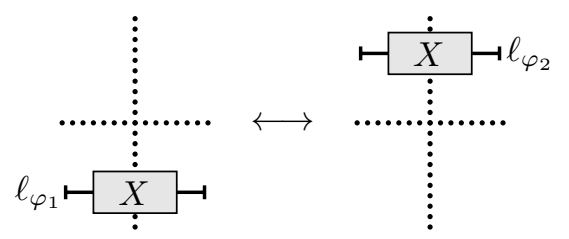

Figure 7.2. A jump move
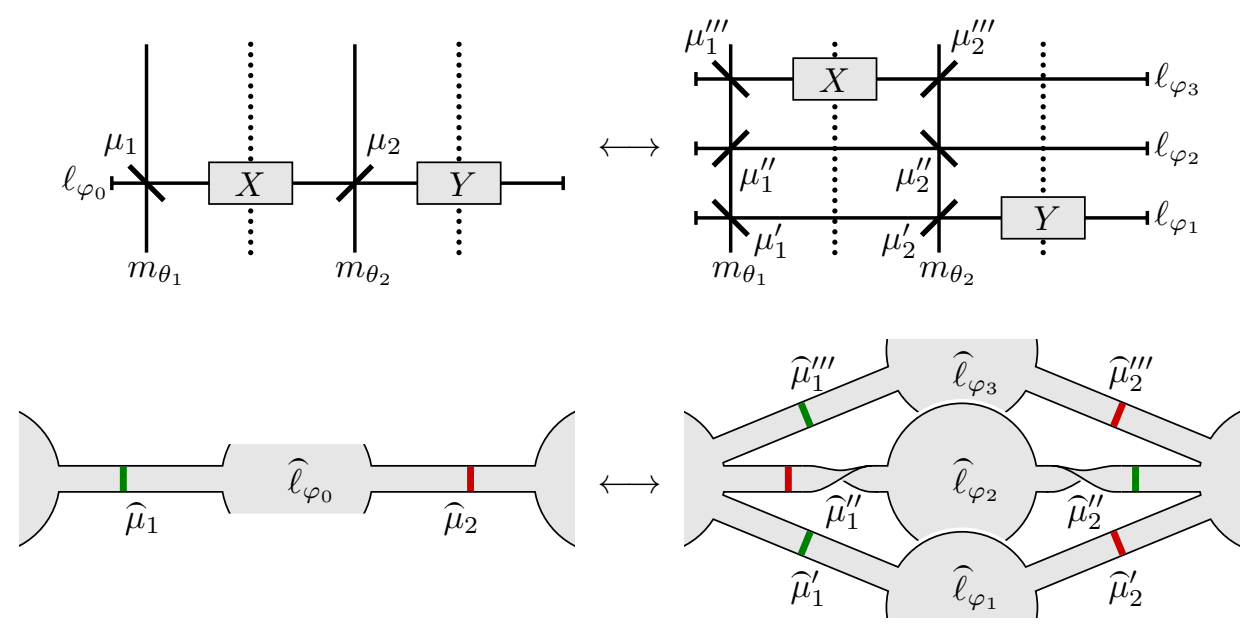

FIGURE 7.3. Wrinkle creation and reduction moves

(3) replacing each mirror at $\left(\theta, \varphi_{0}\right)$, with $\theta \in\left(\theta_{2} ; \theta_{1}\right)$, by a mirror at $\left(\theta, \varphi_{1}\right)$ of the same type;

(4) replacing the mirrors $\mu_{1}$ and $\mu_{2}$ by $\backslash$-mirrors $\mu_{1}^{\prime}=\left(\theta_{1}, \varphi_{1}\right), \mu_{2}^{\prime \prime}=\left(\theta_{2}, \varphi_{2}\right), \mu_{1}^{\prime \prime \prime}=\left(\theta_{1}, \varphi_{3}\right)$ and $/$ mirrors $\mu_{2}^{\prime}=\left(\theta_{2}, \varphi_{1}\right), \mu_{1}^{\prime \prime}=\left(\theta_{1}, \varphi_{2}\right), \mu_{2}^{\prime \prime \prime}=\left(\theta_{2}, \varphi_{3}\right)$.

Then we say that $M \mapsto M^{\prime}$ is a wrinkle creation move, and the inverse passage is a wrinkle reduction move; see Figure 7.3. The mirrors $\mu_{1}$ and $\mu_{2}$ of $M$ are referred to as the ramification mirrors of the move $M \mapsto M^{\prime}$.

The morphism $\eta$ associated with the wrinkle move $M \mapsto M^{\prime}$ is defined as follows. Let $F$ be a surface obtained from $\widehat{M}^{\prime}$ by patching the two new holes, that is, the holes corresponding to the two boundary circuits of $M^{\prime}$ that hit the mirrors at $\ell_{\varphi_{2}}$.

We demand $(\overparen{M}, F, h) \in \eta$, where $h$ is a homeomorphism $\overparen{M} \rightarrow F$ such that $\left.h\right|_{\widehat{y}}$ is an orientationpreserving self-homeomorphism of $\widehat{y}$ whenever $y$ is an occupied level of $M$ different from $\ell_{\varphi_{0}}$, or a mirror located outside $\ell_{\varphi_{0}}$.

Definition 7.8. Let $M$ and $M^{\prime}$ be as in Definition 7.7 and let $M^{\prime \prime}$ be obtained from $M^{\prime}$ by removing the occupied level $\ell_{\varphi_{2}}$ together with the mirrors $\mu_{1}^{\prime \prime}, \mu_{2}^{\prime \prime}$. Then the transition $M \mapsto M^{\prime \prime}$ is called a double split move, and the inverse one a double merge move; see Figure 7.4. One can see that $M^{\prime} \mapsto M^{\prime \prime}$ is a bridge removal. The mirrors $\mu_{1}$ and $\mu_{2}$ of $M$ are called the splitting mirrors of the move $M \mapsto M^{\prime \prime}$, and the intervals $\left\{\theta_{1}\right\} \times\left(\varphi_{1} ; \varphi_{3}\right),\left\{\theta_{2}\right\} \times\left(\varphi_{1} ; \varphi_{3}\right)$ the $\backslash$-splitting gap and the $/$-splitting gap, respectively.

The morphism associated with the move $M \mapsto M^{\prime \prime}$ is defined as the composition of the morphisms associated with the wrinkle creation move $M \mapsto M^{\prime}$ and the bridge removal $M^{\prime} \mapsto M^{\prime \prime}$.

Recall that the symmetries $r \backslash, r_{/}, r_{-}$, and $r_{\mid}$should be applied to all the definitions of our moves, see Convention 6.1. Recall also that these definitions are supposed to be extended to the case of enhanced mirror diagrams, see Convention 7.1 . 

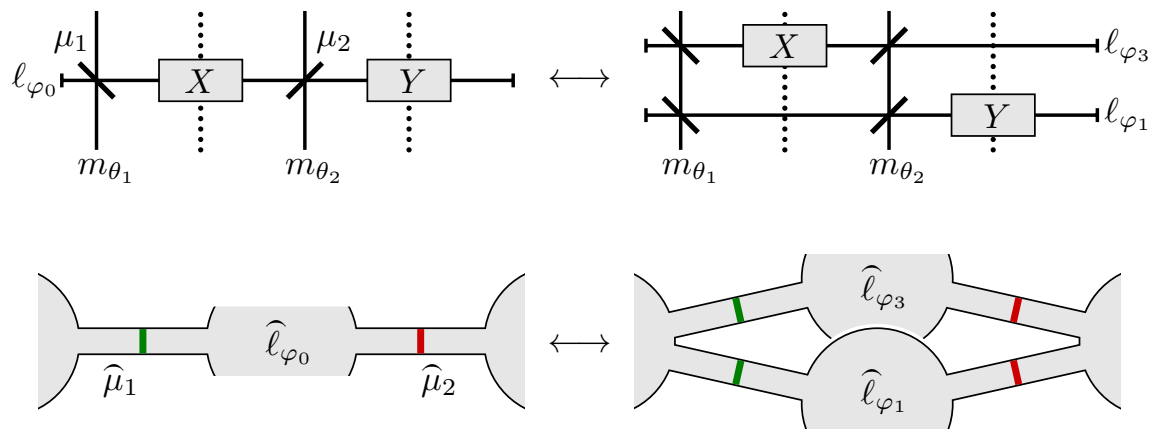

Figure 7.4. Double split and double merge moves

Lemma 7.3. Every bridge, twist, jump, wrinkle creation, wrinkle reduction, double split, or double merge move of enhanced mirror diagrams admits a neat decomposition into type $T$ elementary moves with $T$ being any of I and II.

Proof. Due to the symmetry of the definitions of the moves it suffices to produce a neat decomposition into type I moves. It is elementary to see in each case below that the decomposition is neat (see the discussion after Definition (7.2).

Bridge moves. Bridge addition consists in adding a new occupied level and two mirrors at it. This can be done in two steps: (1) add a new occupied level and the one of the two mirrors that has type $/ ;(2)$ add the second mirror, which is of type \. The first operation is a type I extension move, and the second a type I elementary bypass addition.

Twist moves. A twist move can be neatly decomposed into several bridge moves. Indeed, let $\ell_{\varphi}$ be the occupied level at which one can perform a twist move. Let $\varepsilon>0$ be so small that there are no other occupied levels $\ell_{\varphi^{\prime}}$ of the diagram with $\varphi^{\prime} \in[\varphi-\varepsilon ; \varphi+\varepsilon]$. Add a bridge at $\ell_{\varphi+\varepsilon}$ and remove the bridge at $\ell_{\varphi}$. The result is almost what we want but the new pair of mirrors occurs at a level different from $\ell_{\varphi}$. To fix this add a bridge at $\ell_{\varphi-\varepsilon}$, remove the one at $\ell_{\varphi+\varepsilon}$, add one at $\ell_{\varphi}$, and finally, remove the one at $\ell_{\varphi-\varepsilon}$.

Since the statement has already been settled for bridge moves it also holds for twist moves.

Jump moves. We use the notation from Definition 7.6, and proceed by induction in $n$, where $n$ is the number of mirrors at the level $\ell_{\varphi_{1}}$ excluding the 'rightmost' one if it is a $/$-mirror.

If $n=0$, then either there are no mirrors at $\ell_{\varphi_{1}}$, or there is a single $/$-mirror. In the latter case, the jump move is a composition of a type I extension move (which adds the new mirror and the occupied level $\ell_{\varphi_{2}}$ to the diagram) and an elimination move (which removes the old mirror and the occupied level $\left.\ell_{\varphi_{1}}\right)$.

If there are no mirrors at $\ell_{\varphi_{1}}$ choose a free meridian $m_{\theta_{0}}$, add $m_{\theta_{0}}$ and a $/$-mirror at $\ell_{\varphi_{1}} \cap m_{\theta_{0}}$ by an extension move, then proceed as before to replace $\ell_{\varphi_{1}}$ by $\ell_{\varphi_{2}}$, and finally, eliminate the mirror at $\ell_{\varphi_{2}} \cap m_{\theta_{0}}$ and the occupied level $m_{\theta_{0}}$.

For the induction step there are the following three cases to consider.

Case 1: the two 'rightmost' mirrors at $\ell_{\varphi_{1}}$ are of the /-type. We proceed as shown in Figure 7.5.

Case 2: the 'rightmost' mirror at $\ell_{\varphi_{1}}$ is of the /-type, and the preceding one of the $\backslash$-type. We proceed as shown in Figure 7.6 .

Case 3: the 'rightmost' mirror at $\ell_{\varphi_{1}}$ is of the \-type. Then we add, by a type I extension move, a $/$-mirror on the right of it at $\ell_{\varphi_{1}}$ together with a new vertical occupied level, then proceed as in Case 2 , and finally apply an elimination move to erase the 'auxiliary' occupied level and the mirror on it.

All this is done by elementary type I moves and bridge moves, which have already been shown to be neatly decomposable into type I elementary moves. 

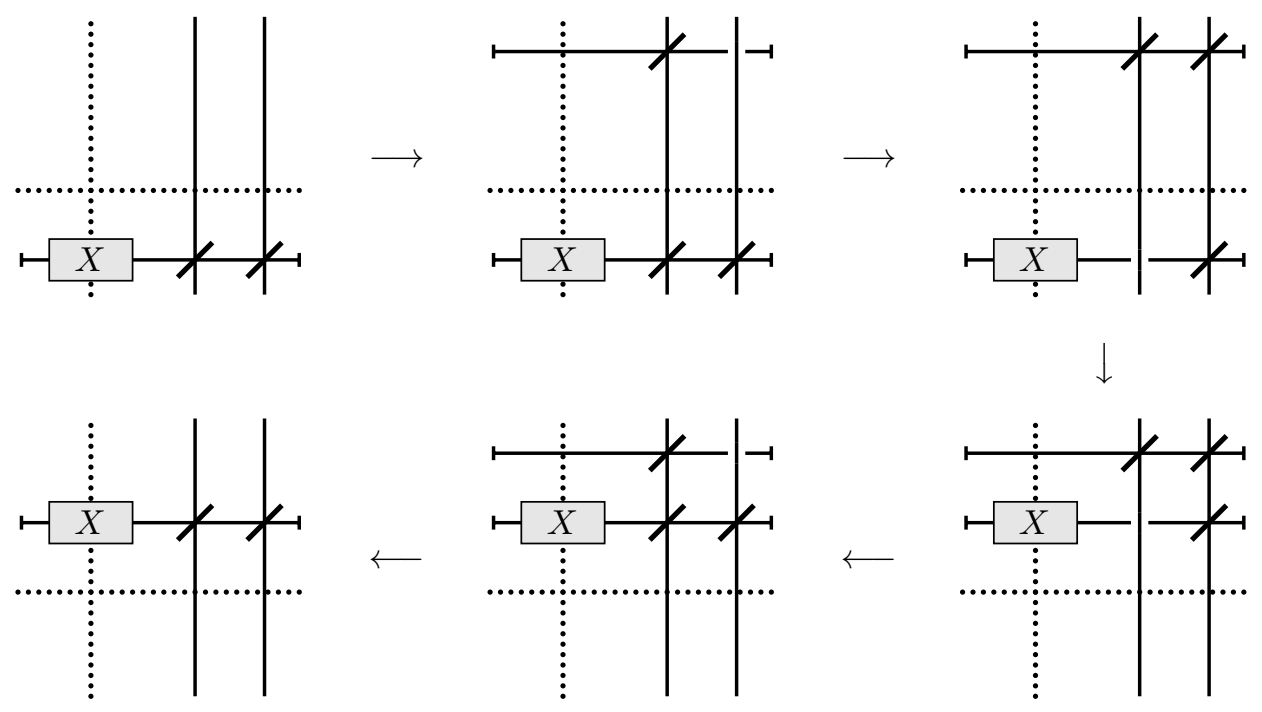

FigURE 7.5. Induction step for the decomposition of a jump move, Case 1
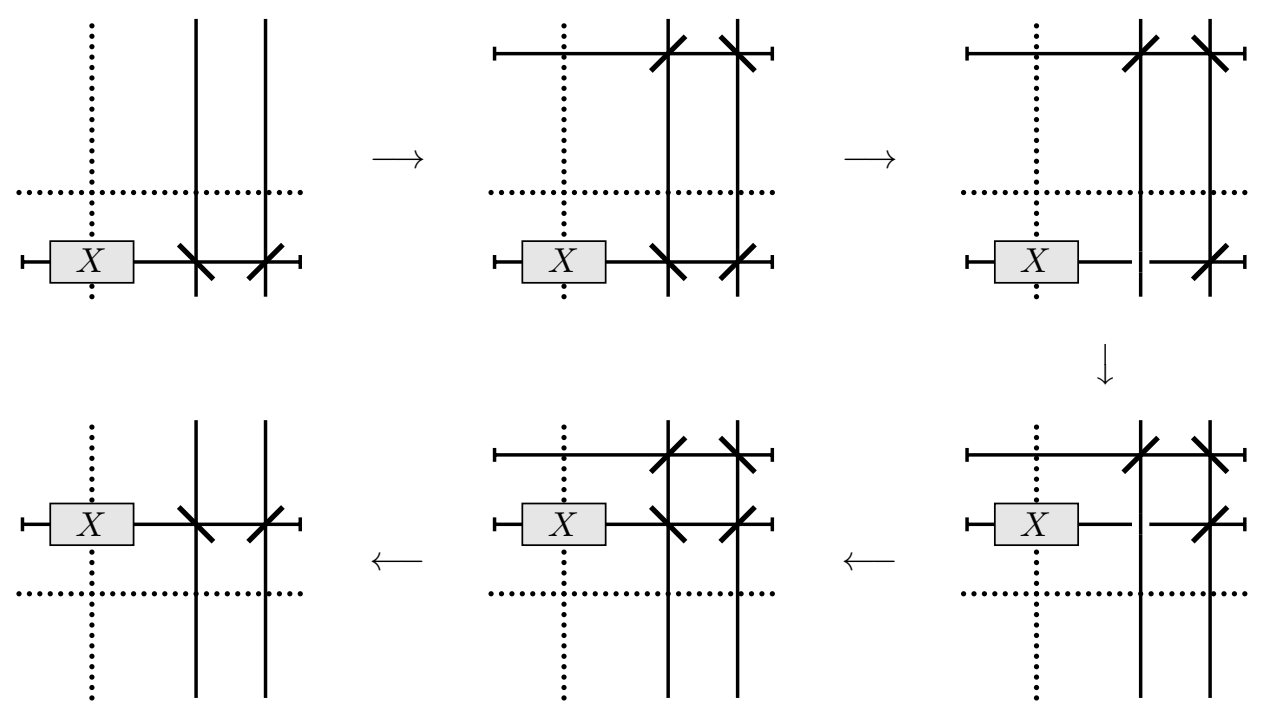

FigURE 7.6. Induction step for the decomposition of a jump move, Case 2

Wrinkle moves. We use the notation from Definition 7.7. We assume that the mirrors in the gray box $Y$ stay fixed, that is, $\varphi_{0}=\varphi_{1}$. The case when the mirrors in $X$ stay fixed is symmetric to this one. If none of the cases occur we first apply a jump move (which is neatly decomposed into type I elementary moves as shown above) to take the block $Y$ to the desired position and then proceed as described below.

The proof is by induction in the number $n$ of mirrors of $M$ located in the block $X$, that is, in $\left(\theta_{1} ; \theta_{2}\right) \times\left\{\varphi_{1}\right\}$. If $n=0$ the wrinkle creation move decomposes neatly into two bridge moves, which have already been shown to be neatly decomposable into type I elementary moves.

The induction step is similar to that in the case of a jump move. It is sketched in Figure 7.7, where $Z$ stands for a mirror of either type. 

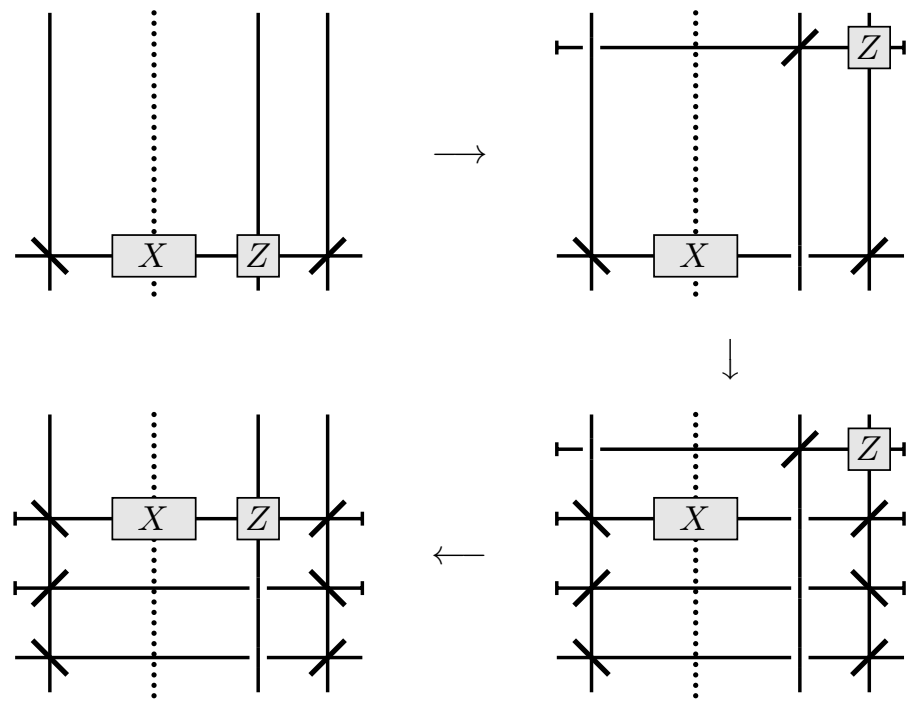

FIGURE 7.7. Induction step for the decomposition of a wrinkle move

Double split and double merge moves. These are, by definition, neatly decomposed into a wrinkle move and a bridge move, which have been discussed above.

7.3. Split moves and merge moves. The moves defined below are similar in nature to double split moves, but unlike the latter they are not neutral.

Definition 7.9. Let $\theta_{1}, \theta_{2}, \varphi_{0}, \varphi_{1}, \varphi_{2} \in \mathbb{S}^{1}$ be such that $\theta_{1} \neq \theta_{2}, \varphi_{1} \neq \varphi_{2}, \varphi_{0} \in\left[\varphi_{1} ; \varphi_{2}\right]$, and let $M$ be a mirror diagram with a $/$-mirror $\mu$ at $\left(\theta_{2}, \varphi_{0}\right)$, such that $M$ has no occupied level $\ell_{\varphi}$ with $\varphi \in\left[\varphi_{1} ; \varphi_{2}\right]$, $\varphi \neq \varphi_{0}$, and no mirror at $\left(\theta_{1}, \varphi_{0}\right)$. Let $M^{\prime}$ be a mirror diagram obtained from $M$ by the following alterations:

(1) replacing the occupied level $\ell_{\varphi_{0}}$ by two occupied levels $\ell_{\varphi_{1}}$ and $\ell_{\varphi_{2}}$;

(2) replacing $\mu$ by two $/$-mirrors $\mu^{\prime}$ and $\mu^{\prime \prime}$ at $\left(\theta_{2}, \varphi_{1}\right)$ and $\left(\theta_{2}, \varphi_{2}\right)$, respectively;

(3) replacing each mirror at $\left(\theta, \varphi_{0}\right)$ with $\theta \in\left(\theta_{1} ; \theta_{2}\right)$ by a mirror at $\left(\theta, \varphi_{2}\right)$ of the same type;

(4) replacing each mirror at $\left(\theta, \varphi_{0}\right)$ with $\theta \in\left(\theta_{2} ; \theta_{1}\right)$ by a mirror at $\left(\theta, \varphi_{1}\right)$ of the same type.

Then we say that the passage $M \mapsto M^{\prime}$ is a type I split move, and the inverse one is a type I merge move; see Figure 7.8. The level $\ell_{\varphi_{0}}$ is said to be split in the transition from $M$ to $M^{\prime}$, and the levels $\ell_{\varphi_{1}}$ and $\ell_{\varphi_{2}}$

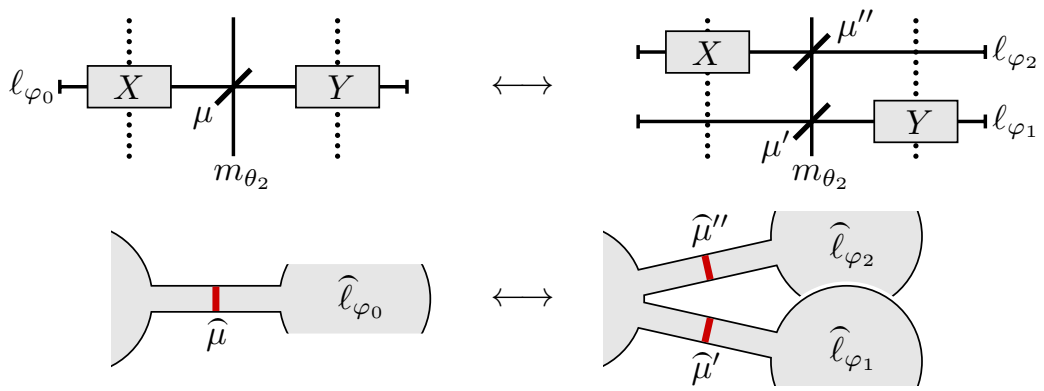

FiguRE 7.8. Type I split/merge moves

are said to be merged in the inverse transition. 
The mirror $\mu$ is referred to as the splitting mirror, the point $\left(\theta_{1}, \varphi_{0}\right)$ as the snip point, and the interval $\left\{\theta_{2}\right\} \times\left(\varphi_{1} ; \varphi_{2}\right)$ as the splitting gap of the split move $M \mapsto M^{\prime}$.

The morphism $\eta$ associated with the merge move $M^{\prime} \mapsto M$ is defined by $\left(\widehat{M}^{\prime}, \overparen{M}, h\right) \in \eta$, where $h$ is an embedding $\overparen{M^{\prime}} \rightarrow \overparen{M}$ such that $\left.h\right|_{\widehat{y}}$ is an orientation-preserving self-homeomorphism of $\widehat{y}$ whenever $y$ is an occupied level of $M^{\prime}$ distinct from $\ell_{\varphi_{1}}, \ell_{\varphi_{2}}$, or a mirror located outside of these two levels, and $h\left(\widehat{\ell}_{\varphi_{1}} \cup\right.$ $\left.\widehat{\ell}_{\varphi_{2}}\right) \subset \widehat{\ell}_{\varphi_{0}}$.

Lemma 7.4. A type I (respectively, type II) split move of mirror diagrams admits a neat decomposition into type I (respectively, type II) elementary moves.

Proof. The proof is by induction, which is similar to that in the cases of a jump move and of a wrinkle move in Lemma 7.3 . We leave it to the reader.

Lemma 7.5. Any type II slide move and type II elementary bypass addition or removal admits a neat decomposition into a sequence of moves from the following list:

- type II split moves,

- type II merge moves,

- neutral moves.

Proof. A decomposition of a slide move is shown in Figure 7.9. We need one split move, then a jump move, and finally a merge move.
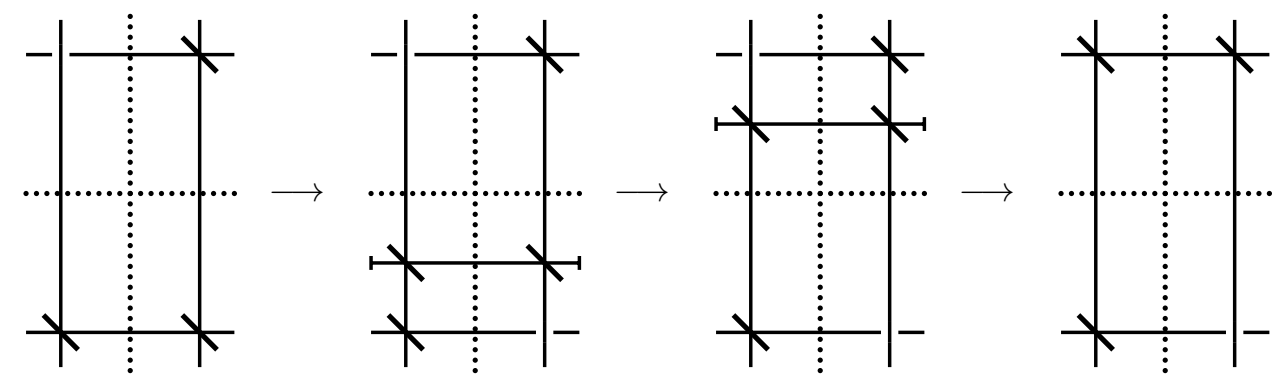

FIGURE 7.9. A neat decomposition of a type II slide move into type II split/merge moves and a jump move

A decomposition of a type II elementary bypass addition is shown in Figure 7.10 . We need a bridge addition and then a type II merge move. For a type II elementary bypass removal the sequence is inverse, and we have a type II split move followed by a bridge removal.

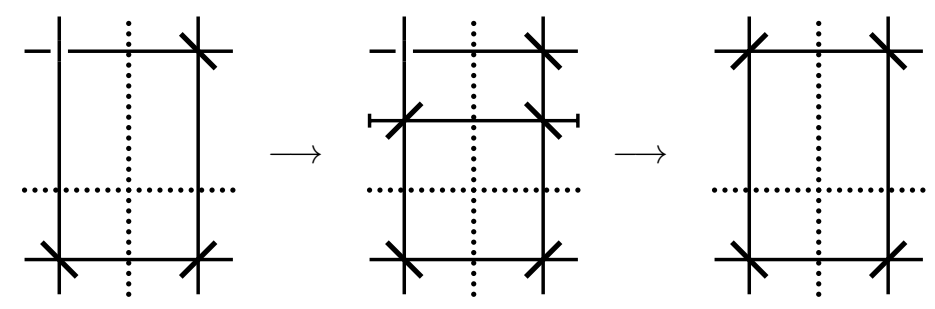

FIGURE 7.10. A neat decomposition of a type II elementary bypass addition into a bridge move and a type II merge move 
Lemma 7.6. Let $M \mapsto M^{\prime}$ be a type II extension move of enhanced mirror diagrams, and let $c$ be the boundary circuit of $M$ modified by this move. Assume that $\mathrm{tb}_{+}(c)<0$. Then the move $M \mapsto M^{\prime}$ admits a neat decomposition into type II split moves and neutral moves.

Proof. The proof is by induction in the smallest number of hops it takes to go along $c$ from the position where a new \-mirror, which we denote by $\mu$, is inserted to an existing \-mirror, which we denote by $\mu_{0}$.

If $\mu_{0}$ is 'immediately visible' from $\mu$, that is, if $\mu$ is inserted in a straight line segment contained in $c$ one of whose endpoints is $\mu_{0}$. The extension move can be decomposed neatly into a type II split move and a jump move; see Figure [7.11] This gives the induction base.

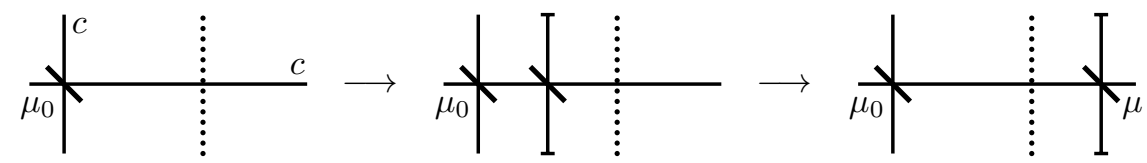

Figure 7.11. Decomposition of an extension move into a split move and a jump move

If $\mu$ is in $k$ hops from $\mu_{0}, k>1$, insert a $\backslash$-mirror $\mu^{\prime}$ in a straight-line portion of $c$ adjacent to the one containing $\mu$, so that the new mirror is in $k-1$ hops from $\mu_{0}$, and then apply two bridge moves as shown in Figure 7.12 to 'transfer' it to the desired position. This gives the induction step.

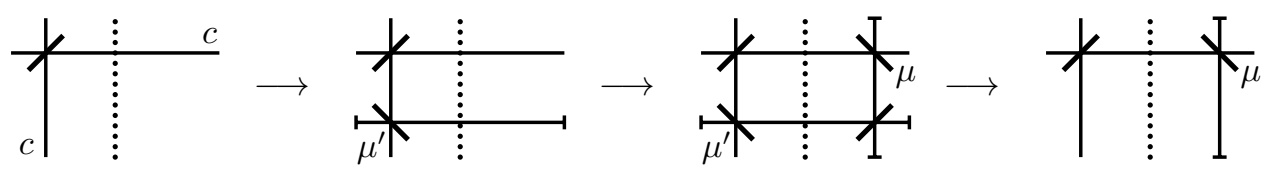

FiguRE 7.12. Adding a \-mirror and 'moving' it along the boundary circuit $c$ by bridge moves

Lemma 7.7. Any type II split move of enhanced mirror diagrams admits a neat decomposition into a sequence of moves one of which is a type II elementary bypass removal, and the others are type I elementary moves.

Proof. Figure 7.13 demonstrates a decomposition into a sequence of the following moves: a type I extension move, a wrinkle creation, a type II bypass removal, a bridge removal, a type I elimination move. The wrinkle and bridge moves should be then decomposed neatly into type I moves, which is possible by Lemma 7.3 .

7.4. Handle decomposition of $\overparen{M}$ and partial homeomorphisms $h_{M}^{M^{\prime}}$. Surfaces of the form $\overparen{M}$, where $M$ is a mirror diagram, naturally come with a handle decomposition. The 0-handles of the decomposition are discs $\widehat{x}, x \in L_{M}$, and the 1-handles are strips $\widehat{\mu}, \mu \in E_{M}$. Additionally, every

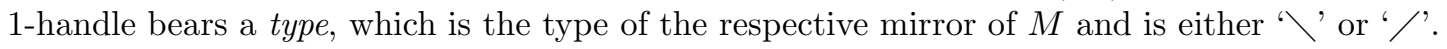

For certain previously defined moves $M \mapsto M^{\prime}$ of mirror diagrams, we introduce here a partial homeomorphism $h_{M}^{M^{\prime}}$ from $\overparen{M}$ to $\widehat{M}^{\prime}$ preserving the handle decomposition. By this we mean a homeomorphism from a compact subsurface $F \subset \widehat{M}$ to a compact subsurface $F^{\prime} \subset \widehat{M}^{\prime}$ such that $F$ and $F^{\prime}$ inherit a handle decomposition structure from $M$ and $M^{\prime}$, respectively, and $h_{M}^{M^{\prime}}$ takes 0-handles of $F$ to 0-handles of $F^{\prime}$ and 1-handles of $F$ to 1-handles of $F^{\prime}$. The partial homeomorphism $h_{M}^{M^{\prime}}$ will also respect the type of each 1-handle and the coorientations of 0-handles defined by the normal vector fields to $\xi_{+}$and $\xi_{-}$.

The partial homeomorphism $h_{M}^{M^{\prime}}$ will be also extendable to the whole of $\mathbb{S}^{3}$ and, after extension, take some surface carried by $\widehat{M}$ to a surface carried by $\widehat{M^{\prime}}$ so as to induce the morphism $\widehat{M} \rightarrow \widehat{M}^{\prime}$ associated with the move $M \mapsto M^{\prime}$. In each case, the surfaces $F$ and $F^{\prime}$ are obtained from $\overparen{M}$ and $\widehat{M}^{\prime}$ by 'as little 

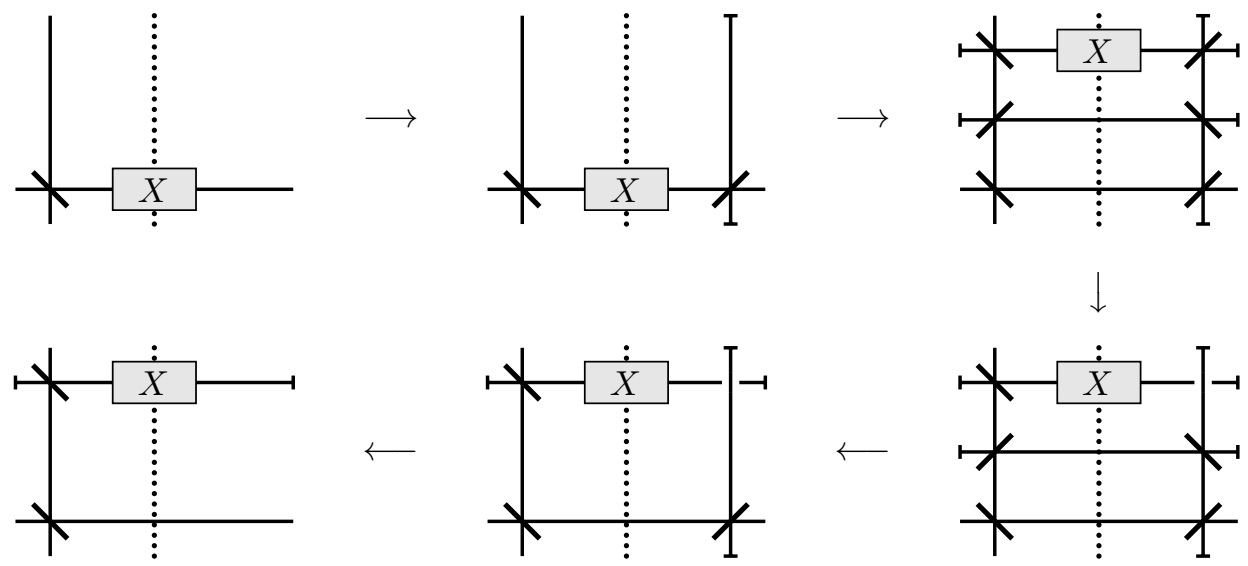

Figure 7.13. A neat decomposition of a type II split move into a type II elementary bypass removal, type I moves, and neutral moves

modifications as possible' for allowing $h_{M}^{M^{\prime}}$ to exist. The modifications will include the trivial one (no modification at all), removing some handles, and cutting along a normal arc (see below).

If $h_{M}^{M^{\prime}}$ is already defined we set $h_{M^{\prime}}^{M}=\left(h_{M}^{M^{\prime}}\right)^{-1}$ for the inverse move.

The moves for which we introduce the partial homeomorphism $h_{M}^{M^{\prime}}$ include the following ones: extension/elimination, elementary bypass addition/removal, jump, wrinkle creation/reduction, and split/merge moves.

Note that $h_{M}^{M^{\prime}}$ will depend not only on the diagrams $M$ and $M^{\prime}$ but also on how the move $M \mapsto M^{\prime}$ is interpreted. For instance, sometimes a transformation of mirror diagrams can be viewed as an extension move and as a split move, and the respective partial homeomorphisms $h_{M}^{M^{\prime}}$ will be quite different.

Let $M$ be a(n enhanced) mirror diagram, and let $\mu$ be a mirror of $M$. By the lateral boundary of the strip $\widehat{\mu}$ we call the closure of $\partial \widehat{\mu} \backslash \bigcup_{x \in L_{M}} \widehat{x}$.

Definition 7.10. Let $M$ be a(n enhanced) mirror diagram. A simple arc $\beta$ in the surface $\overparen{M}$ is normal if the following holds:

(1) $\beta$ is transverse to $\partial \widehat{x}$ for every occupied level $x$ of $M$;

(2) the intersection of $\beta$ with every strip $\hat{\mu}, \mu \in E_{M}$, consists of arcs each of which has endpoints at the boundaries of two different discs of the form $\widehat{x}, x \in L_{M}$, and is disjoint from the lateral boundary of $\widehat{\mu}$;

(3) $\partial \beta \subset \bigcup_{x \in L_{M}} \partial \widehat{x}$.

If $\beta \subset \overparen{M}$ is a normal arc, then by cutting $\overparen{M}$ along $\beta$ we mean removing from $\overparen{M}$ a small open neighborhood $U$ of $\beta$ such that for any $y \in L_{M}$ or $y \in E_{M}$ the subset $\widehat{y} \backslash U$ is a deformation retract of $\widehat{y} \backslash \beta$.

It follows from this definition that the surface obtained by cutting $\overparen{M}$ along a normal arc inherits the handle decomposition structure.

Definition 7.11. Let $M$ and $M^{\prime}$ be enhanced mirror diagrams, and let $M \mapsto M^{\prime}$ be one of the moves for which we define below the partial homeomorphism $h_{M}^{M^{\prime}}$. Let also $y$ be an occupied level or a mirror of $M$, and $y^{\prime}$ an occupied level or a mirror, respectively, of $M^{\prime}$. We say that $y$ is a predecessor of $y^{\prime}$, and $y^{\prime}$ is a successor of $y$ for the move $M \mapsto M^{\prime}$ if $h_{M}^{M^{\prime}}$ takes some portion of $\widehat{y}$ to some portion of $\widehat{y}^{\prime}$. We use the notation $y \mapsto y^{\prime}$ to express this fact.

Now we consider the moves $M \mapsto M^{\prime}$ for which we define the partial homeomorphism $h_{M}^{M^{\prime}}$, case by case. 
Case 1: $M \mapsto M^{\prime}$ is an extension move.

The domain $F$ and the image $F^{\prime}$ of $h_{M}^{M^{\prime}}$ is the whole of $\overparen{M}$, and $h_{M}^{M^{\prime}}$ is just the identity map. The new occupied level and the new mirror of $M^{\prime}$ have no predecessors. For the inverse move these occupied level and mirror of $M^{\prime}$ have no successors.

All the other occupied levels and mirrors of $M$ and $M^{\prime}$ are the same, so each of them is a predecessor and a successor of itself for both moves $M \mapsto M^{\prime}$ and $M^{\prime} \mapsto M$.

Case 2: $M \mapsto M^{\prime}$ is an elementary bypass addition.

Similarly to the previous case we have $F=F^{\prime}=\overparen{M}, h_{M}^{M^{\prime}}=\left.\mathrm{id}\right|_{F}$. The mirror of $M^{\prime}$ that is not present in $M$ has no predecessor for the move $M \mapsto M^{\prime}$, and no successor for the inverse move. All the mirrors and occupied levels of $M$ are successors and predecessors of themselves for both moves.

Case 3: $M \mapsto M^{\prime}$ is a jump move.

The partial homeomorphism $h_{M}^{M^{\prime}}$ is a homeomorphism $\overparen{M} \rightarrow \widehat{M}^{\prime}$ that takes each handle $\widehat{y}$, where $y \in E_{M}$ or $y \in L_{M}$, to itself if $y$ is preserved by the move, and to $\widehat{y}^{\prime}$ if Definition [7.6 says that $y$ is replaced by $y^{\prime}$ in $M^{\prime}$. In the latter case, if $y$ and $y^{\prime}$ are occupied levels, the homeomorphism $h_{M}^{M^{\prime}}$ restricted to $\widehat{y}$ is demanded to preserve the orientation that comes from the coorientation of $\mathbb{S}_{\tau=0}^{1} \cup \mathbb{S}_{\tau=1}^{1}$.

Case 4: $M \mapsto M^{\prime}$ is a wrinkle creation.

We use the notation from Definition 7.7. From the combinatorial point of view, we have the following predecessor/successor pairs:

- $\mu_{1} \mapsto \mu_{1}^{\prime}, \mu_{1} \mapsto \mu_{1}^{\prime \prime \prime}, \mu_{2} \mapsto \mu_{2}^{\prime}, \mu_{2} \mapsto \mu_{2}^{\prime \prime \prime}$,

- $\ell_{\varphi_{0}} \mapsto \ell_{\varphi_{1}}, \ell_{\varphi_{0}} \mapsto \ell_{\varphi_{3}}$,

- $\left(\theta, \varphi_{0}\right) \mapsto\left(\theta, \varphi_{3}\right)$ if $\theta \in\left(\theta_{1} ; \theta_{2}\right)$ and $\left(\theta, \varphi_{0}\right) \in E_{M}$,

- $\left(\theta, \varphi_{0}\right) \mapsto\left(\theta, \varphi_{1}\right)$ if $\theta \in\left(\theta_{2} ; \theta_{1}\right)$ and $\left(\theta, \varphi_{0}\right) \in E_{M}$,

- $y \mapsto y$ if $y \in E_{M} \backslash \ell_{\varphi_{0}}$ or $y \in L_{M} \backslash\left\{\ell_{\varphi_{0}}\right\}$.

The mirrors $\mu_{1}^{\prime \prime}, \mu_{2}^{\prime \prime}$ and the occupied level $\ell_{\varphi_{2}}$ of $M^{\prime}$ have no predecessors. Let $M^{\prime \prime}$ be the mirror diagram obtained by removing these three elements from $M^{\prime}$ (this is a bridge removal). We put $F^{\prime}=\overparen{M}^{\prime \prime}$, and this is the image of $h_{M}^{M^{\prime}}$; see Figure 7.14
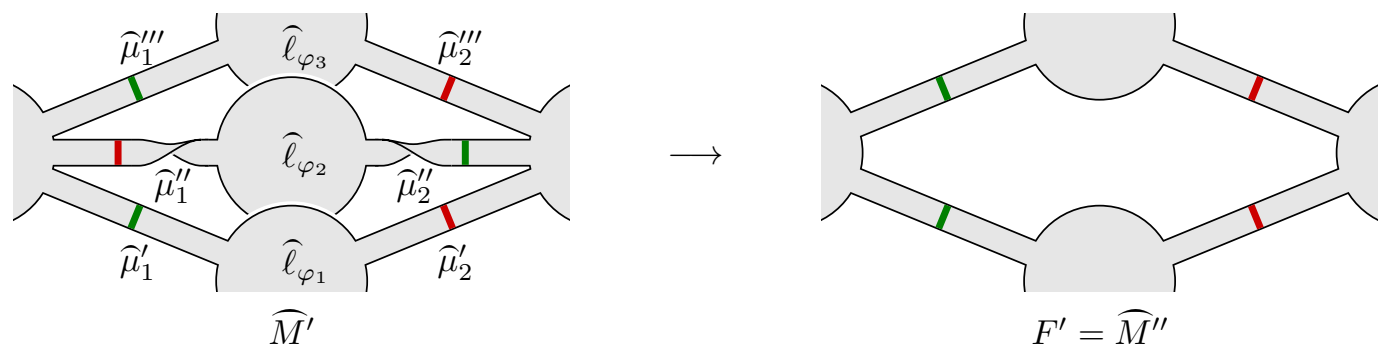

Figure 7.14. The image of $h_{M}^{M^{\prime}}$ in the case of a wrinkle creation

Let $\beta$ be a normal arc in $\widehat{M}$ that cuts the strips $\widehat{\mu}_{1}, \widehat{\mu}_{2}$ and the disc $\widehat{\ell}_{\varphi_{0}}$ into halves, and is disjoint from the interiors of all other handles in $\widehat{M}$. We let the domain of $h_{M}^{M^{\prime}}$ be a surface $F$ obtained from $\overparen{M}$ by cutting it along $\beta$; see Figure 7.15
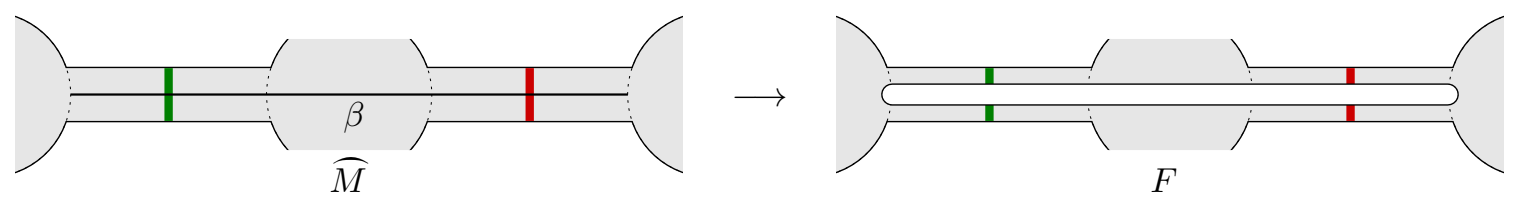

Figure 7.15. The domain of $h_{M}^{M^{\prime}}$ in the case of a wrinkle creation 
The surfaces $F$ and $F^{\prime}$ have the same handle decomposition structure, and the homeomorphism $h_{M}^{M^{\prime}}$ : $F \rightarrow F^{\prime}$ is defined naturally.

Case 5: $M \mapsto M^{\prime}$ is a double split move.

$F, F^{\prime}$ and $h_{M}^{M^{\prime}}$ are exactly as in the previous case. The difference is that now $h_{M}^{M^{\prime}}$ is an onto map. The splitting mirrors and the occupied level of $M$ containing them each have two successors in $M^{\prime}$, all the other elements of $M$ have a unique successor. All mirrors and occupied levels of $M^{\prime}$ have a unique predecessor in $M$.

Case 6: $M \mapsto M^{\prime}$ is a split move.

We use the notation from Definition 7.9. We have the following predecessor/successor pairs for the split move

- $M \mapsto M^{\prime}: \mu \mapsto \mu^{\prime}, \mu \mapsto \mu^{\prime \prime}$,

- $\ell_{\varphi_{0}} \mapsto \ell_{\varphi_{1}}, \ell_{\varphi_{0}} \mapsto \ell_{\varphi_{2}}$

- $\left(\theta, \varphi_{0}\right) \mapsto\left(\theta, \varphi_{2}\right)$ if $\theta \in\left(\theta_{1} ; \theta_{2}\right)$ and $\left(\theta, \varphi_{0}\right) \in E_{M}$,

- $\left(\theta, \varphi_{0}\right) \mapsto\left(\theta, \varphi_{1}\right)$ if $\theta \in\left(\theta_{2} ; \theta_{1}\right)$ and $\left(\theta, \varphi_{0}\right) \in E_{M}$

- $y \mapsto y$ if $y \in E_{M} \backslash \ell_{\varphi_{0}}$ or $y \in L_{M} \backslash\left\{\ell_{\varphi_{0}}\right\}$.

The image $F^{\prime}$ of $h_{M}^{M^{\prime}}$ is the whole of $\widehat{M}^{\prime}$. The domain of $h_{M}^{M^{\prime}}$ is obtained from $\overparen{M}$ by cutting along an $\operatorname{arc} \beta$ that cuts the strip $\widehat{\mu}$ and the disc $\hat{\ell}_{\varphi_{0}}$ into halves; see Figure 7.16.
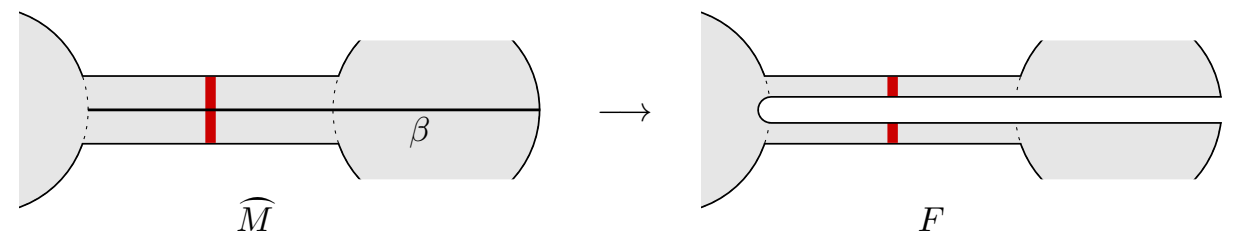

Figure 7.16. The domain of $h_{M}^{M^{\prime}}$ in the case of a split move

\section{Mirror diagrams AND the STANDARd CONTACT StruCture}

In contrast to the previous section, this one is mostly ideological. We start by discussing the connection between the formalism of mirror diagrams, Legendrian graphs, and Giroux's convex surfaces. When the nature of this connection is understood the results of this paper including the commutation theorems in Section 9 should appear less surprising. However, we present their proofs mostly in combinatorial terms, keeping the reference to contact topology, which is the actual source of the ideas, to a minimum. In particular, among numerous definitions and statements given in this section, we formally use in the sequel only Definitions 8.12 and 8.13 , and Lemmas 8.2 and 8.3 We also omit the 'enhanced' versions, which can be easily guessed, of Definitions 8.3 and 8.5 8.11, as well as of Proposition 8.1, Theorem 8.3, and Theorem 8.4. Without enhancements, 'essential' and 'inessential' referring to a boundary circuit or a boundary component mean 'non-patchable' and 'patchable', respectively.

We conclude this section by proving Theorem 2.1] in a combinatorial manner.

8.1. Legendrian graphs. Recall from [12, Section 3] that a Legendrian graph is a spatial graph $\Gamma$ whose edges are everywhere tangent to the standard contact structure $\xi_{+}$and such that any simple arc in $\Gamma$ is cusp-free.

Definition 8.1. A spatial ribbon graph $\rho$ is said to be a Legendrian ribbon graph (with respect to the standard contact structure $\xi_{+}$) if $\Gamma_{\rho}$ is a Legendrian graph, all edges of $\rho$ are smooth arcs, and there is a surface $F \in S_{\rho}$ tangent to $\xi_{+}$at all points of $\Gamma_{\rho}$.

Remark 8.1. The surface $F$ in this definition need not, and typically cannot, be $C^{2}$-smooth. 
It is easy to see that a Legendrian ribbon graph $\rho$ is uniquely determined by the spatial graph $\Gamma_{\rho}$ and the set of vertices $V_{\rho}$, and, moreover, $\Gamma_{\rho}$ may be an arbitrary Legendrian graph. So, there is no essential difference between the concept of a Legendrian graph and that of a Legendrian ribbon graph. However, the context of spatial ribbon graphs suggests to introduce a weaker equivalence relation than the one that looks more natural in the context of Legendrian graphs (see [12, Definition 21]).

Definition 8.2. Two Legendrian ribbon graphs $\rho_{0}$ and $\rho_{1}$ are said to be Legendrian equivalent (with respect to $\left.\xi_{+}\right)$if there is an isotopy $F_{t}, t \in[0,1]$, from a surface $F_{0} \in S_{\rho_{0}}$ to a surface $F_{1} \in S_{\rho_{1}}$ such that, for any $t \in[0,1]$, the surface $F_{t}$ is tangent to $\xi_{+}$along a Legendrian graph $\Gamma_{t}$ which is a deformation retract of $F_{t}$.

In terms of the respective Legendrian graphs this equivalence relation coincides with the one introduced in [1] and studied also in [39, where Legendrian graphs are considered modulo isotopy in the class of Legendrian graphs and edge contraction/blow-up operations.

Generalized rectangular diagrams of [39] are, in the present terms, essentially the same thing as rectangular diagrams of a graph. (In contrast to [39, we allow Legendrian graphs to have isolated vertices. This difference plays no important role.) If $G$ is such a diagram then $\widehat{G}$ is a Legendrian graph. The associated Legendrian ribbon graph is then $\widehat{M}$, where $M$ is the mirror diagram obtained from $G$ by assigning the ' $/$ ' type to every vertex of $G$.

With this correspondence, the vertex addition moves in [39] translate exactly into type I extension moves of mirror diagrams, the end shifts of type L in [39] translate into moves that are decomposed easily into type I extension and slide moves, and the commutations in [39] become a particular case of jump moves if the mirror diagrams are viewed up to combinatorial equivalence.

On the other hand, the type I slide moves translate back to transformations that are easily decomposed into elementary moves of type L from [39]. Thus, from Theorems 3.2 and 2.2 of [39] and Lemma 7.3 above we have the following.

Theorem 8.1. Any Legendrian ribbon graph is Legendrian equivalent to a Legendrian ribbon graph of the form $\widehat{M}$, where $M$ is a mirror diagram having only /-mirrors.

Let $M_{1}$ and $M_{2}$ be mirror diagrams having only /-mirrors. The Legendrian ribbon graphs $\widehat{M}_{1}$ and $\widehat{M}_{2}$ are Legendrian equivalent if and only if $M_{1}$ and $M_{2}$ can be obtained from each other by type I elementary moves (which can only be extension, elimination, and slide moves).

Note that no analogue of stable equivalence makes sense for Legendrian ribbon graphs. This is because there cannot exist any 'non-trivial' patchable boundary circuits of such a graph. More precisely, a boundary circuit of a Legendrian ribbon graph $\rho$ is patchable if and only if it belongs to a contractible connected component of $\Gamma_{\rho}$ (that is, a tree). This can be deduced from the well known result of Bennequin [2] on the non-existence of overtwisted discs (i.e. discs tangent to $\xi_{+}$along the whole boundary), and this implies, in particular, that handle addition is impossible if we restrict ourselves to the class of Legendrian ribbon graphs.

Note also that if a mirror diagram has only $/$-mirrors, then the only elementary moves applicable to it are extensions, eliminations, and slides (which keep the diagram free of $\backslash$-mirrors).

As the following statement shows, the equivalence relation for Legendrian ribbon graphs introduced in Definition 8.2 is quite natural in the context of Giroux's convex surfaces. By $\mathscr{G}(F)$ we denote the Giroux graph of $F$ (see [12, Definition 27]).

Theorem 8.2. (i) Let $F$ and $F^{\prime}$ be convex surfaces with Legendrian boundaries and very nice characteristic foliations. Assume that they are isotopic in the class of convex surfaces. Then the Legendrian ribbon graphs associated with Legendrian graphs $\mathscr{G}(F)$ and $\mathscr{G}\left(F^{\prime}\right)$ are Legendrian equivalent.

(ii) Let $F$ be a convex surface with Legendrian boundary and very nice characteristic foliation, and let $\rho$ be a Legendrian ribbon graph equivalent to the one associated with $\mathscr{G}(F)$. Then there exists a convex surface $F^{\prime}$ isotopic to $F$ through convex surfaces such that

(1) $\partial F^{\prime}$ is Legendrian;

(2) the characteristic foliation of $F^{\prime}$ is very nice; 
(3) $\mathscr{G}\left(F^{\prime}\right)$ coincides with $\Gamma_{\rho}$.

The proof will be published elsewhere. We don't use this result in the sequel.

8.2. Divided Legendrian graphs. Here we discuss what general mirror diagrams are if viewed up to type I elementary moves. To start, let us forget for a while about contact structures.

Definition 8.3. By a divided spatial ribbon graph we mean a spatial ribbon graph $\rho$ in which each surface $F \in S_{\rho}$ is endowed with an isotopy class $\Delta(F)$ of abstract dividing sets so that the following holds:

(1) for any $F \in S_{\rho}$ there exists a representative $\delta \in \Delta(F)$ consisting of arcs each of which intersects $\Gamma_{\rho}$ exactly once and transversely (in the topological sense), at a point which is not a true vertex of $\Gamma_{\rho}$;

(2) for any $F, F^{\prime} \in S_{\rho}$ any isotopy in the class $S_{\rho}$ that brings $F$ to $F^{\prime}$ (respecting the correspondence between connected components of $\partial F$ and $\partial F^{\prime}$ with the elements of $\left.\partial \rho\right)$ also brings $\Delta(F)$ to $\Delta\left(F^{\prime}\right)$.

Two divided spatial ribbon graphs $\rho$ and $\rho^{\prime}$ are said to be equivalent if a surface $F \in S_{\rho}$ can be brought to a surface $F^{\prime} \in S_{\rho^{\prime}}$ by an isotopy that also brings $\Delta(F)$ to $\Delta\left(F^{\prime}\right)$.

Definition 8.4. Let $F$ be a surface contained in another surface $F^{\prime}$, and let $\delta$ and $\delta^{\prime}$ be abstract dividing sets on $F$ and $F^{\prime}$, respectively. We say that $\delta^{\prime}$ extends $\delta$ if $\delta=\delta^{\prime} \cap F$ and any connected component of $\delta^{\prime}$ has a non-empty intersection with $F$.

If surfaces $F$ and $F^{\prime}$ are endowed with isotopy classes of abstract dividing sets $\Delta(F)$ and $\Delta\left(F^{\prime}\right)$, respectively, we write $F \subset_{\mathrm{d}} F^{\prime}$ to state that $F \subset F^{\prime}$ and any $\delta \in \Delta(F)$ can be extended to some $\delta^{\prime} \in$ $\Delta\left(F^{\prime}\right)$.

Definition 8.5. Let $\rho$ be a divided ribbon graph. An inessential boundary circuit $\gamma$ of $\rho$ is called negligible if, for some (and then any) surface $F \in S_{\rho}$ and some (and then any) representative $\delta \in \Delta(F)$, the connected component $\widetilde{\gamma}$ of $\partial F$ corresponding to $\gamma$ meets $\delta$ exactly twice. In this case, the component $\widetilde{\gamma}$ of $\partial F$ is also called negligible.

The reason why we single out this type of hole is the following one. If a surface $F^{\prime}$ is obtained from a surface $F \in S_{\rho}$ by patching a negligible hole, where $\rho$ is a divided ribbon graph, then there is a unique way to endow $F^{\prime}$ with an isotopy class of abstract dividing sets so as to have $F \subset_{\mathrm{d}} F^{\prime}$.

For a divided spatial ribbon graph $\rho$, we denote by $\bar{S}_{\rho}$ the class of all surfaces $F$, each endowed with an isotopy class $\Delta(F)$ of abstract dividing sets, such that $F$ is obtained from some $F_{0} \in S_{\rho}$ by patching all negligible holes, and we have $F_{0} \subset_{\mathrm{d}} F$.

Definition 8.6. Two divided spatial ribbon graphs $\rho$ and $\rho^{\prime}$ are said to be stably equivalent if a surface $F \in \bar{S}_{\rho}$ can be brought to $F \in \bar{S}_{\rho^{\prime}}$ by an isotopy that also takes $\Delta(F)$ to $\Delta\left(F^{\prime}\right)$.

Definition 8.7. Let $\rho$ be a divided ribbon graph, and let $F_{0} \in S_{\rho}, \delta_{0} \in \Delta(F)$. Two connected components $\beta_{1}$ and $\beta_{2}$, say, of $\delta_{0}$ are said to be coherent if there is a surface $F \in \bar{S}_{\rho}$ and a representative $\delta \in \Delta(F)$ such that $\delta_{0}=\delta \cap F_{0}$ and $\beta_{1}, \beta_{2}$ are contained in the same connected component of $\delta$. If no two connected components of $\delta_{0}$ are coherent, we say that $\rho$ is reduced.

Definition 8.8. Let $\rho$ and $\rho^{\prime}$ be two divided ribbon graphs such that $\rho^{\prime}$ viewed as an ordinary spatial ribbon graph is obtained from $\rho$ by a handle addition. Let also $F, F^{\prime}$, and $d$ be as in Definition 4.13 We say that the transition $\rho \mapsto \rho^{\prime}$ is a propagation, and the inverse transition is a shrinking if the hole patched by $d$ is negligible, and we have $F^{\prime} \subset_{\mathrm{d}} F$.

Let us explain this operation in different terms. One can see that if $F$ is a compact surface, and $\delta$ is an abstract dividing set on $F$ consisting of pairwise disjoint arcs, then there exists a unique equivalence class of divided spatial ribbon graphs $\rho$ such that $F \in S_{\rho}$ and $\delta \in \Delta(F)$. Thus, isotopy classes of such pairs $(F, \delta)$, which we call divided surfaces, are in a natural one-to-one correspondence with equivalence classes of divided spatial ribbon graphs.

For $(F, \delta)$ a divided surface, a connected component $\gamma$ of $\partial F$ is negligible if $\gamma$ is inessential and there are exactly two endpoints of $\delta$ on $\gamma$. 
If a connected component $\beta$ of $\delta$ has one endpoint on a negligible component $\gamma$ of $\partial F$, and the other endpoint on a component $\gamma^{\prime} \neq \gamma$, the removal of a tubular neighborhood $U$ of $\beta$ such that $U \cap \delta=\beta$ from $F$ represents a shrinking. This is illustrated in Figure 8.1, where the dividing sets of the divided surfaces are shown in green. It is understood that the new boundary component, which is the 'connected

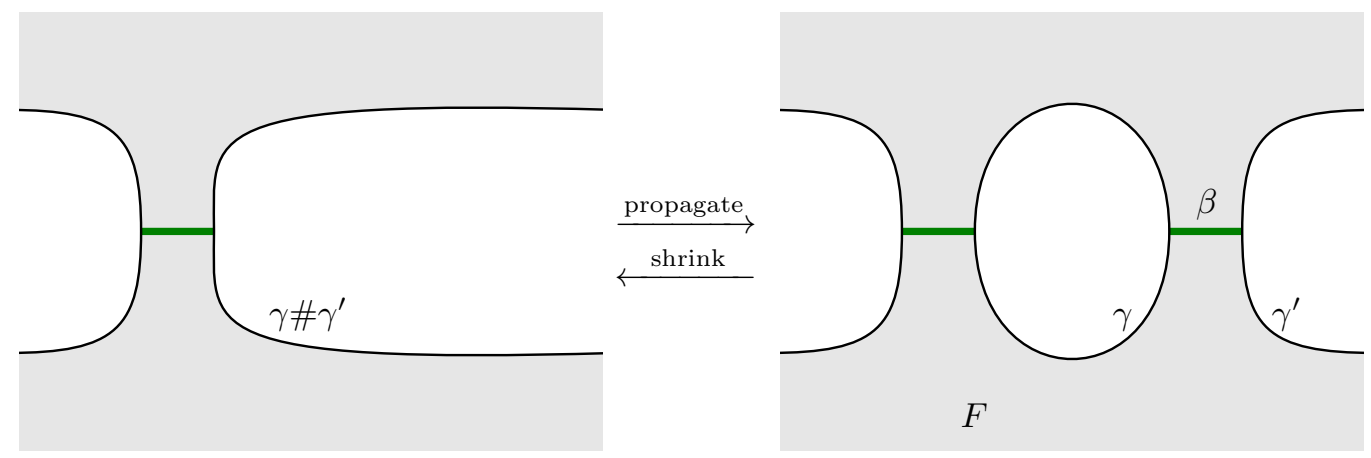

Figure 8.1. Propagation and shrinking in terms of divided surfaces

sum' $\gamma \# \gamma^{\prime}$ of $\gamma$ and $\gamma^{\prime}$ is essential if and only if so is $\gamma^{\prime}$. This implies, in particular, that $\gamma \# \gamma^{\prime}$ is negligible if and only if so is $\gamma^{\prime}$.

It is easy to see that if $\beta_{1}, \beta_{2}$ are two connected components of $\delta$ distinct from $\beta$, then they are coherent if and only if they remain coherent after the shrinking. So, connected components of $\delta$ fall into several coherence classes, and a propagation adds an element to one of these classes, whereas a shrinking removes an element from a class. The number of coherence classes remains fixed.

With this explanation at hand the following statement is obvious.

Proposition 8.1. (i) Two divided ribbon graphs $\rho$ and $\rho^{\prime}$ are stably equivalent if and only if there is an ribbon graph $\rho^{\prime \prime}$ equivalent to $\rho^{\prime}$ that is obtained from $\rho$ by a finite sequence of propagations and shrinkings.

(ii) For any divided spatial ribbon graphs $\rho$, there is a sequence of shrinkings that produces a reduced divided ribbon graph from $\rho$.

(iii) Two reduced divided spatial ribbon graphs are equivalent if and only if they are stably equivalent.

Now we plug back in the contact structure $\xi_{+}$.

Definition 8.9. A divided Legendrian ribbon graph is a divided spatial ribbon graph $\rho$ such that $\Gamma_{\rho}$ is a Legendrian graph, and there are representatives $F \in S_{\rho}$ and $\delta \in \Delta(F)$ with the following properties:

(1) $\delta$ is disjoint from $V_{\rho}$;

(2) each connected component of $\delta$ is an arc intersecting $\Gamma_{\rho}$ exactly once;

(3) each edge of $\rho$ has at most one intersection with $\delta$;

(4) $F$ is tangent to $\xi_{+}$along all edges of $\rho$ that are disjoint from $\delta$;

(5) if an edge $e$ of $\rho$ intersects $\delta$ (such edges are called divided), then $e$ is a -1-arc of the characteristic foliation of $F$, i.e. a Legendrian arc such that the contact plane $\xi_{+}(p)$ makes a negative half-turn relative to the tangent plane $T_{p} F$ when $p$ traverses the edge $e$, and $\xi_{+}(p) \neq T_{p} F$ for $p \in \operatorname{int}(e)$;

(6) the orientation of each connected component $\beta \subset \delta$ agrees with the coorientation of $\xi_{+}$at the point $\beta \cap \Gamma_{\rho}$.

It is not hard to see that, if $\Gamma$ is a Legendrian graph with the set of vertices $V$ such that any edge is a smooth simple arc, and $X$ is a subset of edges of $\Gamma$, then there is a unique divided Legendrian ribbon graph $\rho$ with $\Gamma_{\rho}=\Gamma, V_{\rho}=V$ such that the set of divided edges of $\Gamma$ is $X$.

The main source of divided Legendrian ribbon graphs is the class of extended Giroux graphs of convex surfaces, see [12, Definition 27]. Let $F$ be a Giroux's convex surface with Legendrian boundary and very nice characteristic foliation, and let $\delta$ be a dividing set of $F$. Let also $\mathscr{G}$ be the Giroux graph, and $\widetilde{\mathscr{G}}$ an extended Giroux graph of $F$. By declaring the -1 -arcs in $\widetilde{\mathscr{G}}$, which are not contained in $\mathscr{G}$, 
to be divided edges we get a divided Legendrian ribbon graph, which we denote by $\rho(F, \widetilde{\mathscr{G}})$. To get representatives $F^{\prime} \in S_{\rho(F, \widetilde{\mathscr{G}})}$ and $\delta^{\prime} \in \Delta\left(F^{\prime}\right)$ it suffices to take a small open neighborhood $U$ of $\widetilde{\mathscr{G}}$ in $F$ and put $F^{\prime}=\bar{U}, \delta^{\prime}=\delta \cap F^{\prime}$.

The point is that the divided Legendrian ribbon graph $\rho(F, \widetilde{\mathscr{G}})$ constructed in this way carries essentially all interesting information about $F$ if the latter is viewed up to isotopy in the class of Giroux's convex surfaces. This means that we can study convex surfaces by operating essentially one-dimensional objects, namely, Legendrian graphs with some additional structure.

The link to extended Giroux graphs also motivates introducing the following equivalence relation, up to which it is natural to consider divided Legendrian ribbon graphs.

Definition 8.10. Two divided Legendrian ribbon graphs $\rho_{0}$ and $\rho_{1}$ are said to be Legendrian equivalent if there is a 1-parametric family of divided Legendrian ribbon graphs $\rho_{t}, t \in[0,1]$, connecting $\rho_{0}$ and $\rho_{1}$, and an isotopy $F_{t}, t \in[0,1]$, from a surface $F_{0} \in S_{\rho_{0}}$ to a surface $F_{1} \in S_{\rho_{1}}$ such that $F_{t} \in S_{\rho_{t}}$ and this isotopy brings $\Delta\left(F_{0}\right)$ to $\Delta\left(F_{t}\right)$ for all $t \in[0,1]$.

In terms of Legendrian graphs this definition means the following. We consider Legendrian graphs some edges of which are declared divided. Two such Legendrian graphs are regarded equivalent if one can be obtained from the other by an isotopy in the class of Legendrian graphs and operations of edge contraction/blow-up which are allowed only on non-divided edges. The divided edges are forbidden to collapse and to be born as a result of an edge blow up.

There is an arbitrariness in the definition of an extended Giroux graph $\widetilde{\mathscr{G}}$ of a convex surface $F$ that allows to add more divided edges to it at our will. Such an addition will change the Legendrian equivalence class of $\rho(F, \widetilde{\mathscr{G}})$. Propagations and shrinkings take care of this.

Definition 8.11. In the case of divided Legendrian ribbon graphs, a propagation and a shrinking have the same meaning as in the case of general divided spatial ribbon graphs (see Definition 8.8). Two divided ribbon graphs $\rho$ and $\rho^{\prime}$ are said to be stably Legendrian equivalent if there is a divided Legendrian ribbon graph $\rho^{\prime \prime}$ such that $\rho^{\prime \prime}$ is Legendrian equivalent to $\rho^{\prime}$ and is obtained from $\rho$ by finitely many propagations and shrinkings.

Theorem 8.3. Let $F$ and $F^{\prime}$ be two connected Giroux's convex surfaces with Legendrian boundary and very nice characteristic foliation, and let $\mathscr{G}, \mathscr{G}^{\prime}$ be their respective extended Giroux graphs. Suppose that all connected components of $\partial F$ and $\partial F^{\prime}$ are essential. Then divided Legendrian ribbon graphs $\rho(F, \mathscr{G})$ and $\rho\left(F^{\prime}, \mathscr{G}^{\prime}\right)$ are stably Legendrian equivalent if and only if the surfaces $F$ and $F^{\prime}$ are isotopic through the class of Giroux's convex surfaces with Legendrian boundary.

We omit the proof, which will be published elsewhere.

With every mirror diagram $M$ we associate a divided Legendrian ribbon graph, which we denote by $\rho^{+}(M)$, by letting the underlying spatial ribbon graph be $\widehat{M}$, and letting $\delta_{+}$be a representative of $\Delta(\overparen{M})$, where $\delta_{+}$is the first entry of a canonic dividing configuration of $\overparen{M}$ (see Definition [5.11).

One can, of course, define $\rho^{-}(M)$ similarly using the symmetry between $\xi_{+}$and $\xi_{-}$.

Theorem 8.4. (i) Any divided Legendrian ribbon graph is stably Legendrian equivalent to $\rho^{+}(M)$ for some mirror diagram $M$.

(ii) Let $M$ and $M^{\prime}$ be mirror diagrams. The divided Legendrian ribbon graphs $\rho^{+}(M)$ and $\rho^{+}\left(M^{\prime}\right)$ are stably Legendrian equivalent if and only if $M$ and $M^{\prime}$ are related by a finite sequence of type I elementary moves.

We need a little preparation before the proof.

Definition 8.12. A boundary circuit $c$ of a(n enhanced) mirror diagram $M$ is called +-negligible (respectively, --negligible) if $c$ is inessential and $\mathrm{tb}_{+}(c)=-1$ (respectively, $\mathrm{tb}_{-}(c)=-1$ ).

Recall that the equality $\operatorname{tb}_{+}(c)=-1$ is equivalent to saying that $c$ hits $\backslash$-mirrors of $M$ exactly twice. Thus $c$ is +-negligible if and only if the respective boundary circuit $\widehat{c}$ of $\rho^{+}(M)$ is negligible. 
Definition 8.13. Two distinct $\backslash$-mirrors $\mu$ and $\mu^{\prime}$ of a(n enhanced) mirror diagram $M$ are said to be immediately coherent if there is a +-negligible boundary circuit that hits both $\mu$ and $\mu^{\prime}$.

Two mirrors $\mu$ and $\mu^{\prime}$ are said to be coherent if there are mirrors $\mu_{0}=\mu, \mu_{1}, \mu_{2}, \ldots, \mu_{k}=\mu^{\prime}$ such that $\mu_{i-1}$ and $\mu_{i}$ are immediately coherent for all $i=1, \ldots, k$.

Immediate coherence and coherence of $/$-mirrors is defined similarly, using --negligible boundary circuits.

Let $M$ be a mirror diagram, and let $\left(\delta_{+}, \delta_{-}\right)$be a canonic dividing configuration of $\overparen{M}$. It is easy to see that two \-mirrors (respectively, $/$-mirrors) of $M$ are coherent if and only if so are the respective components of $\delta_{+}$(respectively, $\delta_{-}$).

Recall that Definition 5.9 introduces the mirror diagram $M(\Pi)$ associated with a rectangular diagram $\Pi$. This definition makes perfect sense even if $\Pi$ is any finite collection of pairwise compatible rectangles, that is, a collection of rectangles that forms a subset of a rectangular diagram of a surface. Recall also that, in the proof of Lemma 6.1, we introduced the union of two mirror diagrams if they agree on the common mirrors.

Definition 8.14. Let $M$ be an enhanced mirror diagram, and let $c$ be an inessential circuit of $M$. Let also $\Pi$ be a collection of rectangles in $\mathbb{T}^{2}$ such that $\widehat{\Pi}=\cup_{r \in \Pi} \widehat{r}$ is a patching disc for $\widehat{c}$ in $\widehat{M}$. Denote by $M^{\prime}$ the enhanced mirror diagram whose underlying mirror diagram is $M \cup M(\Pi)$, and the set of essential boundary circuits is the same as that of $M$.

Then the transition from $M$ to $M^{\prime}$ is called a patching of $c$. With such an operation we associate a morphism $\eta$ defined by $\left(\overparen{M}, \widehat{M}^{\prime},\left.\mathrm{id}\right|_{\widehat{M}}\right) \in \eta$.

The mirrors explicitly mentioned in Definitions 6.1, 6.2, and 6.3 are said to participate in the respective moves.

Lemma 8.1. Let $M$ be an enhanced mirror diagram, and let $c$ be a +-negligible boundary circuit of $M$. Then any patching of $c$ admits a neat decomposition into type I extensions and elementary bypass additions. Moreover, if $c$ hits two distinct $\backslash$-mirrors of $M$, then the decomposition of any patching of $c$ into type I elementary moves can be chosen so that one of the \-mirrors, at our choice, does not participate in the moves.

Proof. Let $\Pi$ be a collection of rectangles in $\mathbb{T}^{2}$ such that $\widehat{\Pi}=\cup_{r \in \Pi} \widehat{r}$ is a patching disc for $\widehat{c}$ in $\widehat{M}$. We proceed by induction in the number $k$ of rectangles in $\Pi$. If $k=1$ there is nothing to prove, since $M=M \cup M(\Pi)$.

Suppose that $k>1$. Let $r$ be a rectangle in $\Pi$ such that $\partial r$ hits a $\backslash$-mirror of $M$. Denote the $\backslash$-vertices of $r$ by $\mu_{1}$ and $\mu_{2}$, and the $/$-vertices of $r$ by $\mu_{3}$ and $\mu_{4}$. Denote also by $M^{\prime}$ the diagram $M \cup M(\{r\})$ having the same essential boundary circuits as $M$ has.

Only one of $\mu_{1}$ and $\mu_{2}$ can be a mirror of $M$. Indeed, otherwise the diagram $M^{\prime}$ would have an inessential boundary circuit hitting only $/$-mirrors, that is, with zero Thurston-Bennequin number $\mathrm{tb}_{+}$, which would mean the existence of an overtwisted disc. For the same reason the intersection of $\partial \widehat{r}$ with $\Gamma_{\widehat{M}}$ must be connected.

If $c$ hits two distinct \-mirrors and we want one of them $\mu_{0}$, say, not to be involved in the moves from the sought-for decomposition, the rectangle $r$ should be chosen disjoint from $\mu_{0}$. This is possible as no rectangle in $\Pi$ hits both \-mirrors on $c$.

We may assume without loss of generality that $\mu_{1} \in E_{M}$ and $\mu_{2} \notin E_{M}$. If $\mu_{3}, \mu_{4} \in E_{M}$, then $M \mapsto M^{\prime}$ is a type I elementary bypass addition. Suppose that $\mu_{3} \notin E_{M}$. Let $x$ be the meridian or longitude passing through $\mu_{2}$ and $\mu_{3}$. We must have $x \notin L_{M}$, since otherwise the intersection $\partial \widehat{r} \cap \Gamma_{\widehat{M}}$ would not be connected. The addition of $\mu_{3}$ and $x$ to $M$ is, therefore, a type I extension move. Similarly, if $\mu_{4} \notin E_{M}$, then the addition of $\mu_{4}$ and the meridian or longitude passing through $\mu_{2}$ and $\mu_{4}$ is a type I extension move.

Thus, we can proceed from $M$ to $M^{\prime}$ by zero, one, or two type I extension moves followed by a type I elementary bypass addition.

Now let $\Pi^{\prime}=\Pi \backslash\{r\}$. One can see that $\widehat{\Pi}^{\prime}$ is a patching disc for $\widehat{M}^{\prime}$, and $M^{\prime} \cup M\left(\Pi^{\prime}\right)=M \cup M(\Pi)$. The induction step follows. 
All the morphisms associated with the discussed moves have a representative identical on $\overparen{M}$, which implies that the obtained decomposition is neat.

Lemma 8.2. Let $M$ be an enhanced mirror diagram, and let $c$ be a +-negligible boundary circuit that hits two distinct \-mirrors $\mu_{1}$ and $\mu_{2}$. Let also $c^{\prime}$ be the boundary circuit that hits the other side of $\mu_{2}$.

Denote by $M^{\prime}$ the enhanced mirror diagram obtained from $M$ by removing the mirror $\mu_{2}$ and declaring the new boundary circuit, which is naturally denoted $c \# c^{\prime}$, essential or inessential depending on whether $c^{\prime}$ is essential or not. Denote also by $\eta$ the morphism from $M$ to $M^{\prime}$ defined by $\left(F, F,\left.\mathrm{id}\right|_{F}\right) \in \eta$, where $F$ is an arbitrary surface carried by $\widehat{M}$. Then the transformation $M \stackrel{\eta}{\mapsto} M^{\prime}$ admits a neat decomposition into type I elementary moves.

Proof. Let $M^{\prime \prime}$ be an enhanced mirror diagram obtained from $M$ by a patching of $c$. By Lemma 8.1 there exists a sequence of type I extensions and elementary bypass additions

$$
M=M_{0} \mapsto M_{1} \mapsto \ldots \mapsto M_{k}=M^{\prime \prime}
$$

not involving the mirror $\mu_{2}$.

In each diagram $M_{i}, i=0, \ldots, k$, the mirror $\mu_{2}$ is hit by $c^{\prime}$ on one side and by an inessential boundary circuit $c_{i}$ on the other side. Let $M_{i}^{\prime}$ be obtained from $M_{i}$ by removing $\mu_{2}$ and declaring the new boundary circuit $c_{i} \# c^{\prime}$ essential or inessential depending on whether $c^{\prime}$ is essential or inessential. Then $M_{k} \mapsto M_{k}^{\prime}$ is a type I elementary bypass removal, and $M_{i}^{\prime} \mapsto M_{i-1}^{\prime}$ is either a type I elimination move or a type I elementary bypass removal depending on whether $M_{i-1} \mapsto M_{i}$ is an extension move or an elementary bypass addition.

Thus, the sought-for decomposition is

$$
M=M_{0} \mapsto M_{1} \mapsto \ldots \mapsto M_{k} \mapsto M_{k}^{\prime} \mapsto M_{k-1}^{\prime} \mapsto \ldots \mapsto M_{1}^{\prime} \mapsto M_{0}^{\prime}=M^{\prime} .
$$

The details are left to the reader.

Proof of Theorem 8.4. The part (i) follows from the Approximation Principle of [12, and holds without 'stably'. Namely, for a given divided Legendrian ribbon graph $\rho$ we can obtain a mirror diagram $M$ with $\rho^{+}(M)$ equivalent to $\rho$, as follows. Make a generic Legendrian perturbation of $\Gamma_{\rho}$ and take the torus projection of the obtained graph. Then approximate this torus projection by a rectangular diagram of a graph $G$ as described in the proof of [12, Proposition 8].

We may assume that the torus projection of each edge $e$ of $\Gamma_{\rho}$ (after a generic perturbation) is approximated in $G$ by a staircase arc consisting of more than one edge. If $e$ is a divided edge, choose a vertex of $G$ in the respective staircase arc distinct from the endpoints (the latter may be shared with other staircase arcs) and assign type ' ' to it. Do it for all divided edges. All the other vertices of $G$ are assigned type ' $/$ '. We get a mirror diagram $M$ that represents a divided Legendrian ribbon graph equivalent to $\rho$.

Now proceed with proving part (ii) of the theorem. The fact that type I elementary moves of mirror diagrams preserve the stable Legendrian equivalence class of $\rho^{+}(M)$ follows from a direct check: type I extensions and slides preserve the equivalence class, whereas a type I elementary bypass addition translates into a propagation.

The fact that these moves suffice to transform $M$ to $M^{\prime}$, provided that $\rho^{+}(M)$ and $\rho^{+}\left(M^{\prime}\right)$ are stably Legendrian equivalent, is established similarly to Theorem 8.1. Lemma 8.2 implies that propagations and shrinkings of divided Legendrian ribbon graphs of the form $\rho^{+}(M)$ also can be realized as compositions type I moves.

8.3. Type II moves from the point of view of $\xi_{+}$-divided Legendrian ribbon graphs. Here we consider what happens to the divided Legendrian ribbon graph $\rho^{+}(M)$ when a type II move is applied to $M$. More honestly, we look at what happens to the stable equivalence class of the underlying divided ribbon graph. The type II moves that we discuss include extension, elimination, elementary bypass addition, elementary bypass removal, split, and merge moves.

Let $M \mapsto M^{\prime}$ be one of the listed moves, and let $F \in \bar{S}_{\rho^{+}(M)}$ and $F^{\prime} \in \bar{S}_{\rho^{+}\left(M^{\prime}\right)}$ be obtained from $\overparen{M}$ and $\overparen{M}^{\prime}$, respectively, by patching all negligible holes. Choose some representatives $\delta \in \Delta(F)$ and $\delta^{\prime} \in$ 
$\Delta\left(F^{\prime}\right)$. We are going to discuss how the divided surfaces $(F, \delta)$ and $\left(F^{\prime}, \delta^{\prime}\right)$ are related in each case depending on the kind of the move $M \mapsto M^{\prime}$, which we consider now one by one. The moves come in three pairs of mutually inverses, so we need to consider only one move in each pair.

We assume that the surfaces $F$ and $F^{\prime}$ are chosen to have as much in common as possible. In particular, the common negligible boundary circuits of $\rho^{+}(M)$ and $\rho^{+}\left(M^{\prime}\right)$ are patched in $F$ and $F^{\prime}$ in the same way.

Case 1: $M \mapsto M^{\prime}$ is a type II extension move. Denote by $c$ the boundary circuit of $M$ on which a new mirror is added, and by $c^{\prime}$ the respective boundary circuit of $M^{\prime}$.

The hole $\widehat{c}^{\prime} \subset \partial \widehat{M^{\prime}}$ is never negligible (since either $c$ and $c^{\prime}$ are essential, or $\operatorname{tb}_{+}(c)<0$ and hence $\left.\operatorname{tb}_{+}\left(c^{\prime}\right)<-1\right)$, but it may happen that $\widehat{c}$ is negligible. In this case, $\widehat{c}$ is patched in $F$, but $\widehat{c}^{\prime}$ is not patched in $F^{\prime}$. One can see that $\left(F^{\prime}, \delta^{\prime}\right)$ is obtained from $(F, \delta)$ by removing an open disc that intersects $\delta$ in an open arc and then attaching to $\widehat{c}$ a half-disc with a new component of $\delta^{\prime}$ in it whose endpoints lie on $\widehat{c}^{\prime}$; see Figure 8.2 (a). The overall effect, viewed up to isotopy, of these two operations

(a)

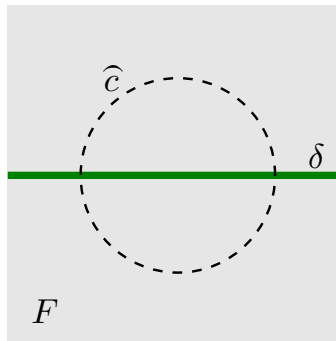

(b)

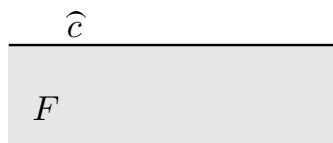

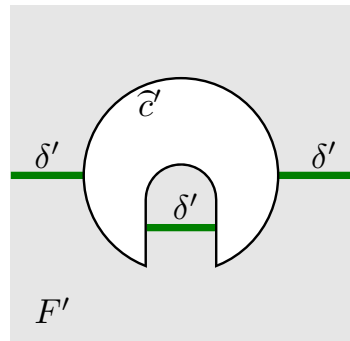

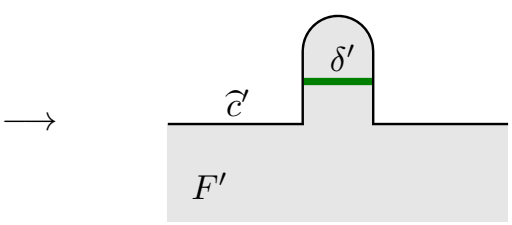

Figure 8.2. A type II extension move from the divided surface associated with $\rho^{+}(M)$ point of view

can also be viewed as a removal of an open disc intersecting $\delta$ in two open arcs.

If $\widehat{c}$ is not negligible, then $F^{\prime}$ is obtained from $F$ just by attaching a half-disc with a new component of $\delta^{\prime}$ inside; see Figure 8.2 (b).

Case 2: $M \mapsto M^{\prime}$ is a type II elementary bypass removal.

By the definition of the move, there are two boundary circuits $c_{1}, c_{2}$ of $M$ that are replaced by a single one in $M^{\prime}$, which we denote by $c_{1} \# c_{2}$. One of $\widehat{c}_{1}$ and $\widehat{c}_{2}$ must be negligible, and we assume that so is $\widehat{c}_{1}$. If $\widehat{c}_{2}$ is negligible, too, then $\left(F^{\prime}, \delta^{\prime}\right)$ is obtained from $(F, \delta)$ by removing two open discs, each intersecting $\delta$ in an open arc, and then removing a 1-handle 'in between'. The overall effect can be viewed as a removal of an open disc intersecting $\delta$ in two open arcs; see Figure 8.3 (a).

Otherwise, the effect of the move $M \mapsto M^{\prime}$ on $(F, \delta)$ is a removal of a half-disc intersecting $\delta$ in a single open arc; see Figure 8.3 (b).

Case 3: $M \mapsto M^{\prime}$ is a type II split move.

Let $c$ be the boundary circuit of $M$ containing the snip point of the move $M \mapsto M^{\prime}$. As we saw in Subsection 7.4 the surface $\widehat{M}^{\prime}$ is obtained from $\overparen{M}$ by cutting along an arc $\beta$ that has exactly one endpoint on $\partial \overparen{M}$, and intersects $\delta$ transversely in a single point, followed by a small deformation. If $c$ is not negligible, then the corresponding component of $\partial \widehat{M}$ is not patched in $F$, and the effect of the move $M \mapsto M^{\prime}$ on the surface $F$ consists in a removal of a half-disc intersecting $\delta$ in a single open arc. 
(a)
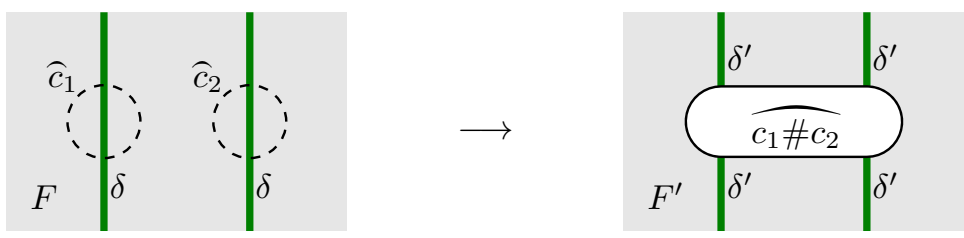

(b)
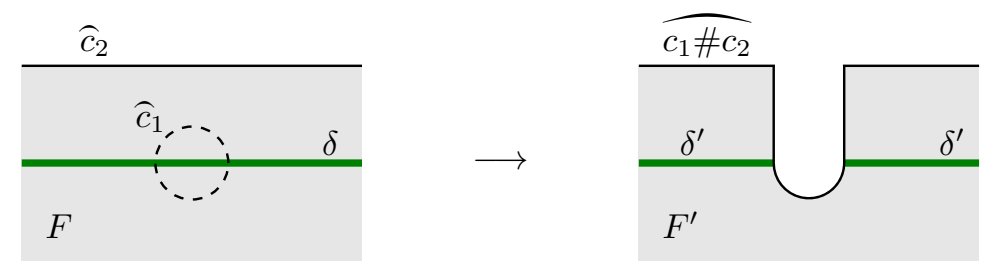

FiguRE 8.3. A type II elementary bypass removal from the divided surface associated with $\rho^{+}(M)$ point of view

If $c$ is negligible, then the corresponding connected component of $\partial \widehat{M}$ is patched, and the transition from $F$ to $F^{\prime}$ consists in removing the respective patching disc and then removing a half-disc as in the previous case. The overall effect is a removal of an open disc intersecting $\delta$ in two open $\operatorname{arcs}$.

We see that the effect of a type II split move on the divided surface $(F, \delta)$ is analogous to that of a type II elementary bypass removal. In the case of a type II extension move the effect is also a particular case of this, provided that the modified boundary circuit has negative Thurston-Bennequin number tb+ before the move.

Indeed, in the case of a negligible boundary circuit the negativity of tb $b_{+}$is automatic, and the topological effect of an extension is a removal of an open disc intersecting $\delta$ in two open arcs as noticed above. If a type II extension move modifies a non-negligible boundary circuit with $t b_{+}<0$, then the addition of a half-disc containing a new connected component of $\delta^{\prime}$ can be viewed, up to isotopy, as a removal of a half-disc intersecting $\delta$ in an open arc. This is illustrated in Figure 8.4.

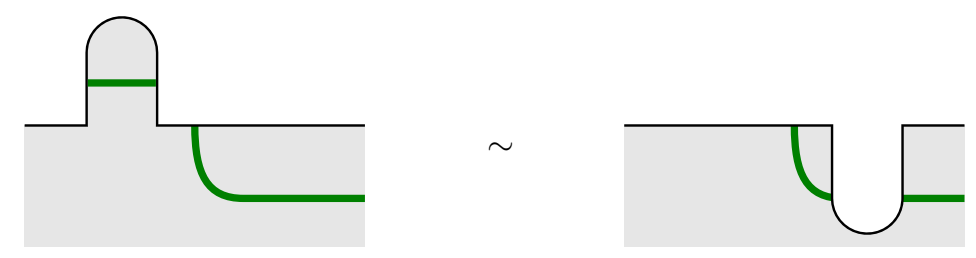

FiguRE 8.4. Attaching a half-disc with a new component of $\delta^{\prime}$ is equivalent to removing a closed half-disc intersecting $\delta$ in an open $\operatorname{arc}$

These observations give a hint for the idea behind generalized type II moves introduced in Section 9 , The generalized moves are designed to have the same effect on the isotopy class of the respective divided surfaces as split/merge and elementary bypass removal/addition moves have: either removing/gluing up a disc intersecting the dividing set in two open arcs, or removing/gluing up a half-disc intersecting the dividing set in a single open arc.

We also see that type II extension moves modifying a boundary circuit with $t_{+}=0$ fall out of this scheme. A natural generalization of such extension moves is not 'good enough' like other generalized moves, which is, roughly, the reason for including the flexibility assumptions in Theorems 9.1 and 9.2

\subsection{Subdiagrams of mirror diagrams.}


Definition 8.15. An enhanced mirror diagram $M_{1}$ is said to be a subdiagram of an enhanced mirror diagram $M_{2}$ if the following holds:

$$
L_{M_{1}} \subset L_{M_{2}}, \quad E_{M_{1}} \subset E_{M_{2}},\left.\quad T_{M_{2}}\right|_{E_{M_{1}}}=T_{M_{1}}, \quad \partial M_{1} \cap \partial M_{2} \cap H_{M_{1}}=\partial M_{1} \cap \partial M_{2} \cap H_{M_{2}} .
$$

In this case we write $M_{1} \subset M_{2}$.

In other words, this definition means that $M_{2}$ is obtained from $M_{1}$ by adding some (may be none) new occupied levels and mirrors without modifying the enhancement on the preserved boundary circuits.

Proposition 8.2. Let $M_{1}$ be a subdiagram of an enhanced mirror diagram $M_{2}$, and let $M_{1} \mapsto M_{1}^{\prime}$ be a move from the following list:

- a type I extension, elimination, split, or merge move;

- a jump or twist move.

Then there exist a mirror diagram $M_{2}^{\prime}$ and a sequence $s$ of type I elementary moves moves producing $M_{2}^{\prime}$ from $M_{2}$ such that $M_{1}^{\prime}$ is a subdiagram of $M_{2}^{\prime}$, and the composition of the moves in s transforms any essential boundary circuit $c \in \partial M_{1} \cap \partial M_{2}$ in exactly the same way as the move $M_{1} \mapsto M_{1}^{\prime}$ does. This means, in particular, that the boundary circuit of $M_{2}^{\prime}$ corresponding to such c belongs to $\partial M_{1}^{\prime}$.

We need the following preparatory lemma.

Lemma 8.3. Let $M$ be an enhanced mirror diagram, and let $\theta_{1}, \theta_{2}, \varphi_{1}, \varphi_{2} \in \mathbb{S}^{1}$ be such that $\theta_{1} \neq \theta_{2}$, $\varphi_{1} \neq \varphi_{2}$. Let also $\Omega \subset \mathbb{T}^{2}$ be either $\left(\theta_{1} ; \theta_{2}\right] \times\left(\varphi_{1} ; \varphi_{2}\right]$ or $\left[\theta_{1} ; \theta_{2}\right) \times\left[\varphi_{1} ; \varphi_{2}\right)$. Then there exists a sequence of type I elementary moves transforming $M$ to another enhanced mirror diagram $M^{\prime}$ so that $M^{\prime}$ does not have any mirrors in $\Omega$, and all boundary circuits of $M$ that don't hit any mirror in $\Omega$ are preserved by all these moves. Moreover, for any $\varepsilon>0$ chosen in advance such that $\theta_{2}+\varepsilon \in\left(\theta_{2} ; \theta_{1}\right)$ and $\varphi_{2}+\varepsilon \in\left(\varphi_{2} ; \varphi_{1}\right)$, we can ensure that

(1) all mirrors in $E_{M^{\prime}} \backslash E_{M}$ are contained in $\left(\theta_{1} ; \theta_{2}+\varepsilon\right) \times\left(\varphi_{1} ; \varphi_{2}+\varepsilon\right) \backslash \Omega$ if $\Omega=\left(\theta_{1} ; \theta_{2}\right] \times\left(\varphi_{1} ; \varphi_{2}\right]$, and in $\left(\theta_{1}-\varepsilon ; \theta_{2}\right) \times\left(\varphi_{1}-\varepsilon ; \varphi_{2}\right) \backslash \Omega$ if $\Omega=\left[\theta_{1} ; \theta_{2}\right) \times\left[\varphi_{1} ; \varphi_{2}\right)$;

(2) if $\varphi \in\left(\varphi_{1} ; \varphi_{2}\right)$ and $M$ has no mirrors on $\ell_{\varphi} \backslash \Omega$, then $M^{\prime}$ has no mirrors on $\ell_{\varphi}$

Proof. Due to symmetry it suffices to consider the case $\Omega=\left(\theta_{1} ; \theta_{2}\right] \times\left(\varphi_{1} ; \varphi_{2}\right]$. The proof is by induction in the number of mirrors of $M$ contained in $\Omega$. If there are no such mirrors then there is nothing to prove. Suppose that there are some.

Then there exists a longitude $\ell_{\varphi_{3}}$ such that the following holds:

(1) $\varphi_{3} \in\left(\varphi_{1} ; \varphi_{2}\right]$;

(2) there are some mirrors of $M$ in $\left(\theta_{1} ; \theta_{2}\right] \times\left\{\varphi_{3}\right\}$;

(3) either $\varphi_{3}=\varphi_{2}$ or there is no mirror of $M$ in $\left(\theta_{1} ; \theta_{2}\right] \times\left(\varphi_{3} ; \varphi_{2}\right]$.

Pick a $\varphi_{4} \in\left(\varphi_{2} ; \varphi_{2}+\varepsilon\right)$ such that $M$ has no mirrors in $\mathbb{S}^{1} \times\left(\varphi_{2} ; \varphi_{4}\right]$.

If all mirrors of $M$ lying on $\ell_{\varphi_{3}}$ are in $\Omega$, we apply a jump move to $M$ that shifts all these mirrors to $\ell_{\varphi_{4}}$; see Figure 8.5 (a). Denote by $M^{\prime \prime}$ the obtained enhanced mirror diagram.

If not all mirrors of $M$ lying on $\ell_{\varphi_{3}}$ are in $\Omega$, we pick a $\theta_{3} \in\left(\theta_{2} ; \theta_{2}+\varepsilon\right)$ so that the domain $\left(\theta_{2} ; \theta_{3}\right] \times \mathbb{S}^{1}$ contains no mirrors of $M$, and then apply to $M$ a sequence of the following three moves:

(1) a type I extension move that adds a new mirror $\mu$ at $\left(\theta_{3}, \varphi_{3}\right)$;

(2) a type I split move that splits $\ell_{\varphi_{3}}$ at $\mu$ so that all mirrors in $\left(\theta_{3} ; \theta_{1}\right) \times\left\{\varphi_{3}\right\}$ stay fixed, and mirrors in $\left(\theta_{1} ; \theta_{2}\right) \times\left\{\varphi_{3}\right\}$ shift upwards slightly;

(3) a jump move that shifts the successors of the mirrors in $\left(\theta_{1} ; \theta_{2}\right) \times\left\{\varphi_{3}\right\}$ to $\ell_{\varphi_{4}}$.

The result is again denoted by $M^{\prime \prime}$; see Figure 8.5 (b).

In both cases, the operations used to produce $M^{\prime \prime}$ from $M$ do not modify any boundary circuit that is disjoint from $\Omega$. The transformation $M \mapsto M^{\prime \prime}$ admits a neat decomposition into type I elementary moves, due to Lemmas 7.3 and 7.4, and $M^{\prime \prime}$ has fewer mirrors in $\Omega$ than $M$ has. The induction step follows.

Proof of Proposition 8.2. We consider different kinds of moves $M_{1} \mapsto M_{1}^{\prime}$ case by case. In each case, except the one of elimination move, the sequence $s$ consists of two parts. The first part, which we refer to 
(a)

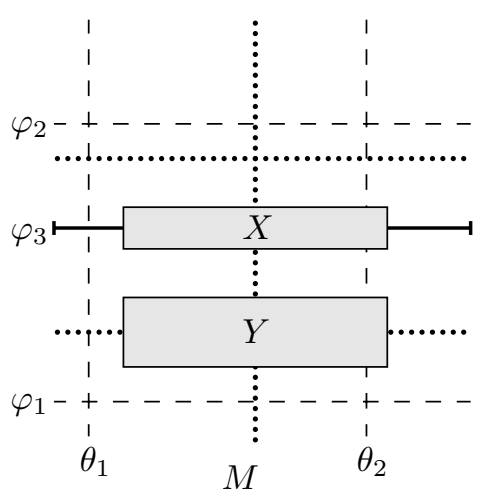

(b)

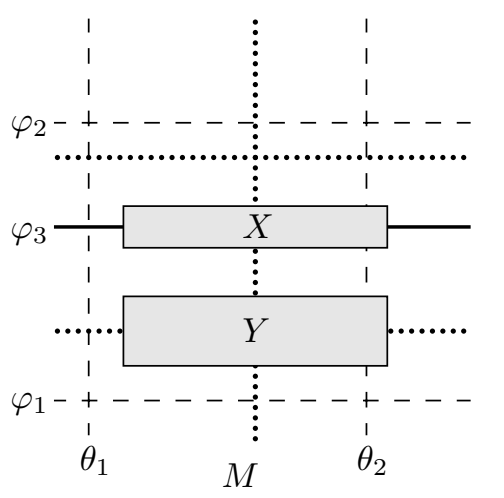

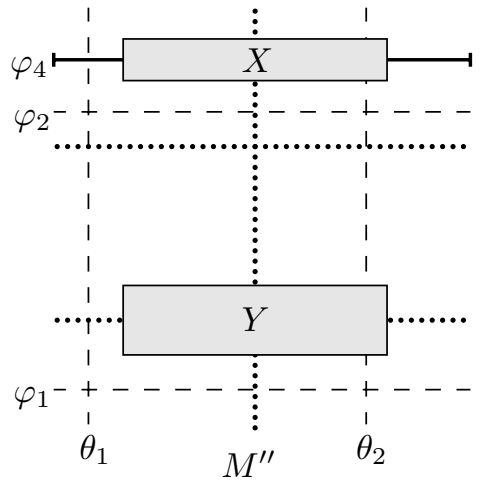

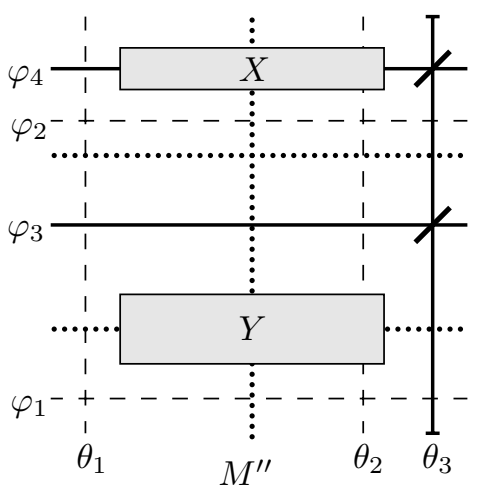

FiguRE 8.5. Removing mirrors from $\Omega=\left(\theta_{1} ; \theta_{2}\right] \times\left(\varphi_{1} ; \varphi_{2}\right]$. The meridians $m_{\theta_{1}}, m_{\theta_{2}}$ and the longitudes $\ell_{\varphi_{1}}, \ell_{\varphi_{2}}$ may or may not be occupied levels of both diagrams

as the removing of obstacles, includes only moves that do not alter any boundary circuit in $\partial M_{1} \cap \partial M_{2}$, and produces a diagram for which the preconditions of the move $M_{1} \mapsto M_{1}^{\prime}$ hold. This part is trivial if the preconditions hold already for $M_{2}$.

The removing of obstacles part is described in terms of moves that are not elementary, but admit a neat decomposition into type I elementary moves by Lemmas 7.3 and 7.4 .

The second part of the sequence $s$ is a neat decomposition into type I elementary moves of a move that 'does the same thing as $M_{1} \mapsto M_{1}^{\prime}$ does'.

Case 1: $M_{1} \mapsto M_{1}^{\prime}$ is a type I extension move.

Denote by $\mu$ the mirror added by this move and by $x$ and $y$ the two levels passing through $\mu$ so that $x$ is an occupied level of both $M_{1}$ and $M_{1}^{\prime}$, and $y$ is the occupied level of $M_{1}^{\prime}$ added by the move.

If $y$ is an occupied level of $M_{2}$ we start $s$ by a jump move that replaces $y$ by a parallel occupied level $y^{\prime}$ close to $y$ and shifts the mirrors on $y$ accordingly, decomposed neatly into a sequence of type I elementary moves. Such a decomposition exists by Lemma 7.3. Since $y$ is not an occupied level of $M_{1}$ no boundary circuit in $\partial M_{1} \cap \partial M_{2}$ can have an edge at $y$, hence all the boundary circuits in $\partial M_{1} \cap \partial M_{2}$ remain untouched. This is the removing of obstacles part.

If $y$ is not an occupied level of $M_{2}$ there is no obstacle to remove.

Now append $s$ by an extension move that adds the occupied level $y$ and the mirror $\mu$ to the diagram. The obtained diagram is taken for $M_{2}^{\prime}$.

Case 2: $M_{1} \mapsto M_{1}^{\prime}$ is a type I elimination move.

Denote by $\mu$ and $y$ the eliminated mirror and occupied level, respectively, and by $c$ the boundary circuit of $M_{1}$ modified by the move. 
If there are some other mirrors of $M_{2}$ on $y$, this means that $c \notin \partial M_{2}$. In this case, we take $\varnothing$ for $s$, and $M_{2}$ for $M_{2}^{\prime}$. If $\mu$ is the only mirror of $M_{2}$ at the level $y$, then we take for $s$ a sequence consisting of a single elimination move $M_{2} \mapsto M_{2}^{\prime}$ that removes $\mu$ and $y$ from the diagram.

Case 3: $M_{1} \mapsto M_{1}^{\prime}$ is a type I split move.

We use the notation from Definition 7.9 and substitute $M_{1}$ and $M_{1}^{\prime}$ for $M$ and $M^{\prime}$, respectively. There are two preconditions that may not hold for $M_{2}$ :

(1) the diagram $M_{2}$ may have a mirror at the snip point, which is $\left(\theta_{1}, \varphi_{0}\right)$,

(2) the diagram $M_{2}$ may have occupied levels in $\mathbb{S}^{1} \times\left[\varphi_{1} ; \varphi_{2}\right]$ other than $\ell_{\varphi_{0}}$.

An obstacle of the first kind is removed by a small alteration of the snip point. An obstacle of the second kind is removed by applying a few jump moves that shift all occupied levels contained in $\mathbb{S}^{1} \times\left[\varphi_{1} ; \varphi_{2}\right]$ upward or downward. The positions of occupied levels of $M_{1}$ need not be altered for that.

When obstacles are removed we can apply a split move $M_{2} \mapsto M_{2}^{\prime}$ so that all mirrors of the subdiagram $M_{1}$ have the same successors in $M_{2}^{\prime}$ as in $M_{1}^{\prime}$.

Case 4: $M_{1} \mapsto M_{1}^{\prime}$ is a type I merge move.

We again use the notation from Definition [7.9, but now substitute $M_{1}^{\prime}$ and $M_{1}$ for $M$ and $M^{\prime}$, respectively. If there are some mirrors of $M_{2}$ in the splitting gap of the move $M_{1}^{\prime} \mapsto M_{1}$, we apply a split move that splits the occupied level $m_{\theta_{2}}$ at $\mu^{\prime}$ and has the snip point at $\left(\theta_{2}, \varphi_{3}\right)$, where $\varphi_{3} \in\left(\varphi_{1} ; \varphi_{2}\right)$ is chosen so that $M_{2}$ has no mirrors in $\left\{\theta_{2}\right\} \times\left[\varphi_{3} ; \varphi_{2}\right)$. This split move is chosen to keep the mirrors of $M_{1}$ in $\left\{\theta_{2}\right\} \times\left[\varphi_{2} ; \varphi_{1}\right)$ fixed.

Then we apply Lemma 8.3 twice to remove all mirrors of $M_{2}$, as well as their successors, from $\left[\theta_{1} ; \theta_{2}\right) \times$ $\left[\varphi_{1} ; \varphi_{2}\right)$, and then from $\left(\theta_{2} ; \theta_{1}\right] \times\left(\varphi_{1} ; \varphi_{2}\right]$. The second application does not introduce any new mirrors in $\left[\theta_{1} ; \theta_{2}\right) \times\left[\varphi_{1} ; \varphi_{2}\right)$, but some empty horizontal occupied levels of the obtained diagram may remain in $\mathbb{S}^{1} \times\left(\varphi_{1} ; \varphi_{2}\right)$. These can be shifted outside this domain by jump moves. This creates preconditions for a merge move as described in Definition 7.9, where we now substitute the currently obtained diagram for $M^{\prime}$ and the sought-for diagram $M_{2}^{\prime}$ for $M$.

Case 5: $M_{1} \mapsto M_{1}^{\prime}$ is a jump move.

Clearly, the obstacles, if any, can be removed by jump moves that alter the positions of occupied level of $M_{2}$ that do not belong to $L_{M_{1}}$.

Case 6: $M_{1} \mapsto M_{1}^{\prime}$ is a twist move.

We use the notation from Definition 7.5. The obstacles, if any, can be removed by one or two double split moves with the splitting mirrors $\mu_{1}$ and $\mu_{2}$ (see Definition 7.8). These moves should be chosen to keep the occupied level $\ell_{\varphi_{0}}$ in the diagram, and to clear the intervals $\left(\theta_{1} ; \theta_{2}\right) \times\left\{\varphi_{0}\right\}$ and $\left(\theta_{2} ; \theta_{1}\right) \times\left\{\varphi_{0}\right\}$ from the mirrors of $M_{2}$.

\subsection{Invariance of $\mathscr{I}$.}

Lemma 8.4. Let $M \stackrel{\eta}{\mapsto} M^{\prime}$ be a type I elementary move of enhanced mirror diagrams, and let $\Pi, \Pi^{\prime}$ be rectangular diagrams of a surface properly carried by $M$ and $M^{\prime}$, respectively (see Definitions 4.10 and 5.10). Let also $\left(\delta_{+}, \delta_{-}\right)$and $\left(\delta_{+}^{\prime}, \delta_{-}^{\prime}\right)$ be canonic dividing configurations of $\widehat{\Pi}$ and $\widehat{\Pi}^{\prime}$, respectively, and $\phi$ be a homeomorphism $\widehat{\Pi} \rightarrow \widehat{\Pi}^{\prime}$ such that $\left(\widehat{\Pi}, \widehat{\Pi}^{\prime}, \phi\right) \in \eta$.

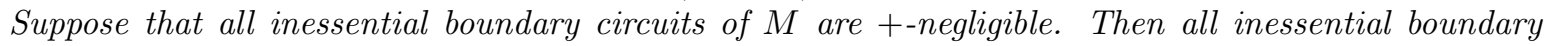
circuits of $M^{\prime}$ are also +-negligible, and the abstract dividing sets $\phi\left(\delta_{+}\right)$and $\delta_{+}^{\prime}$ are equivalent.

Proof. This statement follows easily from the discussion in Subsection 8.2

Proof of Theorem [2.1. Since $\widehat{R}$ and $\widehat{R}^{\prime}$ are Legendrian equivalent, there exists a sequence of exchange moves, type I stabilizations, and type I destabilizations producing $R^{\prime}$ from $R$ (see [38, Proposition 4.4]):

$$
R=R_{0} \mapsto R_{1} \mapsto \ldots \mapsto R_{k}=R^{\prime} .
$$

Such a sequence can be chosen so that all stabilizations in it occur before all destabilizations. This ensures that all the diagrams $R_{i}, i=0, \ldots, k$, are--compatible with $F$. Indeed, each $R_{i}$ is obtained by exchange 
moves and type I stabilizations either from $R$ or from $R^{\prime}$. Exchange moves preserve Thurston-Bennequin numbers of the connected components of the link, whereas stabilizations may only decrease them.

Thus, to prove the theorem it suffices to assume that $R \mapsto R^{\prime}$ is either a single exchange move, or a single type I stabilization, or a single type I destabilization, and to show, in all three cases, that $\mathscr{I}_{F, L,+}(R) \subset \mathscr{I}_{F, L,+}\left(R^{\prime}\right)$.

Let $\delta \in \mathscr{I}_{F, L+}(R)$, and let $(\Pi, \phi)$ be a proper +-realization of $\delta$. Let also $M=M(\Pi)$ be the enhanced mirror diagram associated with $\Pi$ (see Definition 5.9). Denote by $C$ the simple collection of essential boundary circuits of $M$ representing $R$, that is, such that $R(C)=R$ (see Subsection 5.2). Denote by $M_{0}$ the subdiagram of $M$ defined by the conditions: $E_{M_{0}}=R, L_{M_{0}}=\left\{x \in L_{M}: x\right.$ contains an edge of $\left.R\right\}$. One can see that $C$ is a simple collection of essential boundary circuits of $M_{0}$, too.

We claim that there is a sequence of moves

$$
M_{0} \mapsto M_{1} \mapsto \ldots \mapsto M_{k}
$$

including only the moves listed in the assumption of Proposition 8.2 such that, for the induced transformations $C_{0}=C \mapsto C_{1} \mapsto \ldots \mapsto C_{k}$, we have $R\left(C_{k}\right)=R^{\prime}$.

We take this claim for granted for the moment and deduce the assertion $\delta \in \mathscr{I}_{F, L,+}\left(R^{\prime}\right)$.

Let $C^{\prime}=C_{k}$. Since $M_{0}$ is a subdiagram of $M$, and $C \subset \partial M \cap \partial M_{0}$, by Proposition 8.2 there exists a sequence $M \stackrel{\eta_{1}}{\longmapsto} M_{1}^{\prime} \stackrel{\eta_{2}}{\longmapsto} M_{2}^{\prime} \stackrel{\eta_{3}}{\longmapsto} \ldots \stackrel{\eta_{k^{\prime}}}{\longmapsto} M_{k^{\prime}}^{\prime}$ of type I elementary moves that induces a sequence of transformations $C \mapsto C_{1}^{\prime} \mapsto C_{2}^{\prime} \mapsto \ldots \mapsto C_{k^{\prime}}^{\prime}$ such that $C_{k^{\prime}}^{\prime}=C^{\prime}$. Denote $M_{k^{\prime}}^{\prime}$ by $M^{\prime}$.

By Theorem 6.1 the enhanced spatial ribbon graphs $\widehat{M}$ and $\widehat{M}^{\prime}$ are stably equivalent, and, moreover, this equivalence can be realized by an isotopy inducing the morphism $\eta=\eta_{k^{\prime}} \circ \ldots \circ \eta_{2} \circ \eta_{1}$ and bringing $\widehat{\Pi}$ to a surface $F^{\prime}$ carried by $\widehat{M}^{\prime}$. Since this isotopy induces the morphism $\eta$, it also brings $\widehat{R}$ to $\widehat{R}^{\prime}$.

Thus, we have a homeomorphism $\psi: \mathbb{S}^{3} \rightarrow \mathbb{S}^{3}$ such that $\psi(\widehat{R})=\widehat{R}^{\prime}$ and $\psi(\widehat{\Pi})=F^{\prime}$. By Lemma 5.1 the surface $F^{\prime}$ can be chosen in the form $\widehat{\Pi^{\prime}}$, where $\Pi^{\prime}$ is a rectangular diagram of a surface properly carried by $M^{\prime}$. It now follows from Lemma 8.4 that $\left(\Pi^{\prime}, \psi \circ \phi\right)$ is a proper +-realization of $\delta$ at $R^{\prime}$.

It remains to prove the claim about the existence of the sequence (8.1). There are several cases and subcases to consider.

Case 1: $R \mapsto R^{\prime}$ is an exchange move. The sought-for sequence can obviously be made of jump moves (at most three are needed).

Case 2: $R \mapsto R^{\prime}$ is a type I stabilization move. Due to symmetry, we may assume that a vertex $\left(\theta_{0}, \varphi_{0}\right)$ is replaced by the vertices $\left(\theta_{0}+\varepsilon, \varphi_{0}\right),\left(\theta_{0}, \varphi_{0}+\varepsilon\right)$, and $\left(\theta_{0}+\varepsilon, \varphi_{0}+\varepsilon\right)$, with a small $\varepsilon$. First, we perform an extension move that creates a mirror at $\left(\theta_{0}+\varepsilon, \varphi_{0}\right)$, and then a split move that splits the occupied level $\ell_{\varphi_{0}}$ at $\left(\theta_{0}+\varepsilon, \varphi_{0}\right)$ and moves the mirror at $\left(\theta_{0}, \varphi_{0}\right)$ to $\left(\theta_{0}, \varphi_{0}+\varepsilon\right)$; see Figure 8.6. There are

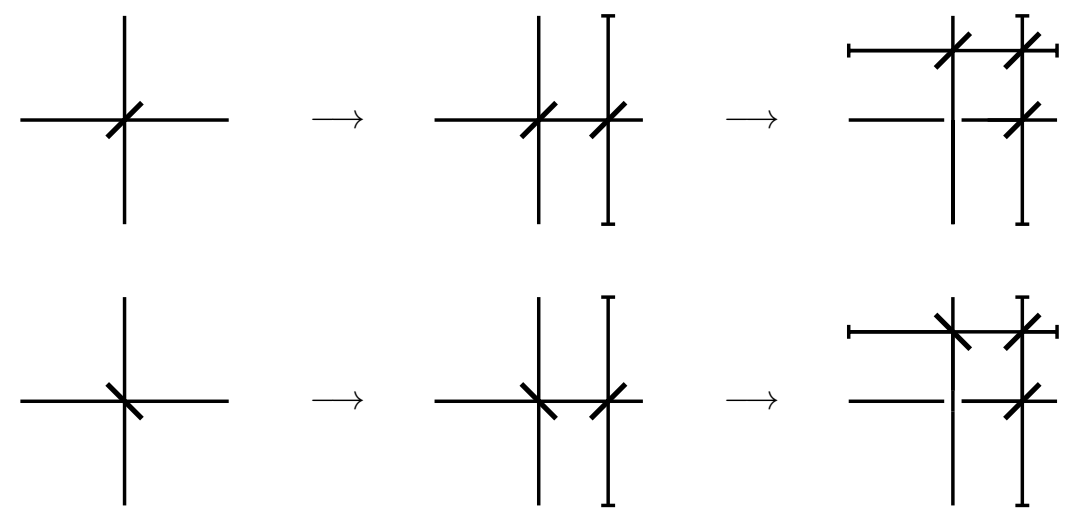

FIGURE 8.6. Doing a type I stabilization of a boundary circuit by means of type I moves of a mirror diagram 
two boundary circuits modified by these moves, and both undergo a stabilization as the result of these operations.

Case 3: $R \mapsto R^{\prime}$ is a type I destabilization move. Due to symmetry, we may assume that, for some $\theta_{0}, \varphi_{0}$, and a small $\varepsilon$, the vertices $\left(\theta_{0}+\varepsilon, \varphi_{0}\right),\left(\theta_{0}, \varphi_{0}+\varepsilon\right)$, and $\left(\theta_{0}+\varepsilon, \varphi_{0}+\varepsilon\right)$ are replaced by a vertex $\left(\theta_{0}, \varphi_{0}\right)$. Let $c \in C$ be the boundary circuit of $M$ on which the destabilization occurs. Since the destabilization makes the Thurston-Bennequin number of the knot $\widehat{c}$ larger, and after the destabilization the diagram $R^{\prime}$ is still --compatible with $F$, the relative Thurston-Bennequin number tb_ $(\widehat{c}, \widehat{\Pi})$, which is the same thing as tb_ $(c)$, is strictly negative. Therefore $c$ hits $/$-mirrors at least twice.

By applying twist moves to $M_{0}$ we can permute $/$ - and \-mirrors arbitrarily along a connected component of $R$. In particular, we can achieve that the mirrors at $\left(\theta_{0}+\varepsilon, \varphi_{0}\right)$ and $\left(\theta_{0}+\varepsilon, \varphi_{0}+\varepsilon\right)$ become $/$-mirrors. After that we can apply the moves from the decompositions in Case 2, in the reverse order.

\section{Commutation theorems}

9.1. Formulation and the strategy of the proof. In this section we formulate and prove the key technical result of this paper. We start from a warm-up version of it. Recall (see Definition 5.3) that the connectedness of a mirror diagram is defined in terms of the corresponding spatial ribbon graph.

Theorem 9.1. Let $M$ and $M^{\prime}$ be mirror diagrams such that each connected component of $M$ contains at least one \-mirror, and each connected component of $M^{\prime}$ contains at least one /-mirror. Assume that $M$ and $M^{\prime}$ are related by a sequence of elementary moves

$$
M=M_{0} \stackrel{\eta_{1}}{\longmapsto} M_{1} \stackrel{\eta_{2}}{\longmapsto} \ldots \stackrel{\eta_{n}}{\longmapsto} M_{n}=M^{\prime} .
$$

Then there exists an enhanced mirror diagram $M^{\prime \prime}$ related to $M$ by a sequence

$$
M=M_{0}^{\prime} \stackrel{\eta_{1}^{\prime}}{\longmapsto} M_{1}^{\prime} \stackrel{\eta_{2}^{\prime}}{\longmapsto} \ldots \stackrel{\eta_{k}^{\prime}}{\longmapsto} M_{k}^{\prime}=M^{\prime \prime}
$$

of type I elementary moves, and to $M^{\prime}$ by a sequence

$$
M^{\prime \prime}=M_{0}^{\prime \prime} \stackrel{\eta_{1}^{\prime \prime}}{\longmapsto} M_{1}^{\prime \prime} \stackrel{\eta_{2}^{\prime \prime}}{\longmapsto} \ldots \stackrel{\eta_{l}^{\prime \prime}}{\longmapsto} M_{l}^{\prime \prime}=M^{\prime}
$$

of type II elementary moves such that

$$
\eta_{n} \circ \ldots \circ \eta_{2} \circ \eta_{1}=\eta_{l}^{\prime \prime} \circ \ldots \circ \eta_{2}^{\prime \prime} \circ \eta_{1}^{\prime \prime} \circ \eta_{k}^{\prime} \circ \ldots \circ \eta_{2}^{\prime} \circ \eta_{1}^{\prime} .
$$

The whole of this section is devoted to the proof of this theorem and Theorem 9.2, which is more elaborate. Before formulating the latter and proceeding with the actual proof we make a few remarks about the ideas, and provide the vague outline.

If the sequence (9.1) consists of just two moves the first of which is of type II and the second one is of type I, then the respective sequences (9.2) and (9.3) are found fairly easily even without additional assumptions on the presence of $\backslash$-mirrors in $M$ and $/$-mirrors in $M^{\prime}$. It is then tempting to try the following strategy in the general case: whenever a type II move in the sequence (9.1) is followed by a type I move replace the subsequence of these two moves by a sequence in which all type I moves occur before all type II moves, and keep repeating this procedure until all type I moves occur before all type II moves.

The problem with this approach is to show that the process ever stops, as the number of moves of both types in the sequence may grow. To overcome this difficulty we introduce a larger family of moves, which we call generalized type II moves, and prove two commutation properties for them. The first commutation property is a statement similar to Theorem 9.1 in which type II elementary moves are replaced by generalized type II moves, and the assertion claims additionally that the number of generalized type II moves in the sequence (9.3) is the same as that in the sequence (9.1). To establish the first commutation property in the general case it suffices to do so when a single generalized type II move is followed by a single elementary type I move. 
The second commutation property of generalized type II moves is a statement that these moves admit a decomposition into a sequence of elementary moves such that all type I moves in it occur before all type II moves.

So, the strategy to prove Theorem 9.1 is as follows. First, we modify the initial sequence of moves so that it consists of elementary type I moves and generalized type II moves for which we establish the two commutation properties mentioned above. Then we proceed by induction in the number of generalized type II moves preceding some elementary type I moves in the sequence. To make the induction step we take the last generalized type II move that is immediately followed by a type I elementary move, use the first commutation property to 'shift' it to the end of the sequence, and then decompose into elementary moves so that all type I moves occur before all type II moves in this decomposition.

This method works perfectly for Theorem 9.1 but in order to have applications to Legendrian knots we need a relative version of Theorem 9.1 in which a collection $C$ of essential boundary circuits is taken special care. It would be ideal for the relative version of Theorem 9.1 to assume that $C$ is preserved by all the moves in (9.1) and then to assert the same about the sequences (9.2) and (9.3). However, the required relative versions of the commutation properties of generalized type II moves do not always hold, so, in order to make the general scheme outlined above work, we weaken the relative formulation by letting the sequences of moves modify $C$ in a 'delicate' way (namely, so that $C$ undergoes only exchange moves). To overcome the difficulties we are also forced to include jump moves into the list of moves occurring in the discussed sequences. According to Lemma 7.3 jump moves can be decomposed into elementary moves of either type, but such a decomposition may no longer be delicate with $C$, so, in the relative case, some jump moves have to be left as is, not decomposed into elementary moves.

In order to formulate the relative version of the commutation theorem we need a couple more definitions.

Definition 9.1. Let $M$ and $M^{\prime}$ be enhanced mirror diagrams, and let $C \subset \partial_{\mathrm{e}} M$ be a collection of essential boundary circuits of $M$. Let also

$$
M=M_{0} \stackrel{\eta_{1}}{\longmapsto} M_{1} \stackrel{\eta_{2}}{\longmapsto} \ldots \stackrel{\eta_{n}}{\longmapsto} M_{n}=M^{\prime}
$$

be a sequence of moves including elementary moves and jump moves, and

$$
C=C_{0} \mapsto C_{1} \mapsto \ldots \mapsto C_{n}
$$

be the induced by (9.4) sequence of transformations of the boundary circuits that starts from $C$. The sequence of moves (9.4) is called $C$-delicate if $C_{i-1}=C_{i}$ whenever $M_{i-1} \mapsto M_{i}$ is an elementary move. A $C$-delicate sequence of moves is said to be of type $I$ (respectively, of type $I I$ ) if all the elementary moves in it are of type I (respectively, of type II).

In other words, a $C$-delicate sequence consists of elementary moves preserving the chosen family of essential boundary circuits, and jump moves that are allowed to modify this family.

Definition 9.2. Let $M$ be an enhanced mirror diagram, and let $C$ be a collection of essential boundary circuits of $M$. The diagram $M$ is said to be +-flexible relative to $C$ (respectively, --flexible relative to $C$ ) if, for any boundary circuit $c \in \partial M$ that does not belong to $C$, there exists a sequence

$$
c_{1}, c_{2}, \ldots, c_{m}=c
$$

of boundary circuits of $M$ not belonging to $C$ such that $\operatorname{tb}_{+}\left(c_{1}\right)<0$ (respectively, $\mathrm{tb}_{-}\left(c_{1}\right)<0$ ), and for all $i=1, \ldots, m$, the boundary circuits $c_{i-1}$ and $c_{i}$ are adjacent to one another in the sense that some mirror of $M$ is hit by both of them.

$M$ is said to be flexible relative to $C$ if it is both +-flexible and --flexible relative to $C . M$ is called flexible (respectively, +-flexible or --flexible) if it is flexible relative to $\varnothing$ (respectively, +-flexible or --flexible relative to $\varnothing$ ).

The reader may be puzzled for the moment why 'flexibility' refers to this strange property. The name will be justified in Subsection 9.8 .

The following statement is trivial. 
Proposition 9.1. An enhanced mirror diagram $M$ is +-flexible (respectively, --flexible) if and only if every connected component of $M$ contains a \-mirror (respectively, a /-mirror).

Thus, the assumption of Theorem 9.1 on the presence of $\backslash$ - and $/$-mirrors can be rephrased by requesting that $M$ and $M^{\prime}$ be +-flexible and --flexible, respectively.

The following statement demonstrates that flexibility is not a very restrictive condition, and it holds in the most important case, which motivated all this machinery.

Proposition 9.2. If the essential boundary of an enhanced mirror diagram $M$ is simple, and no connected component of $\Gamma_{\widehat{M}}$ is a tree, then $M$ is flexible relative to $C$ for any $C \subset \partial_{\mathrm{e}} M$.

Proof. Let $c$ be an inessential boundary circuit of $M$, and let $\left(\mathbb{D}^{2}, \phi\right)$ be a patching disc for $\widehat{c}$. Since the connected component of $\Gamma_{\widehat{M}}$ containing $\widehat{c}$ is not a tree, the image $\phi\left(\mathbb{D}^{2}\right)$ is not a sphere. Therefore, $\phi$ can be disturbed slightly to become and embedding so that the image $\phi\left(\mathbb{D}^{2}\right)$ becomes a disc with Legendrian boundary having relative Thurston-Bennequin number equal to tb $+(c)$.

It follows from Bennequin's theorem on the non-existence of overtwisted discs that $\operatorname{tb}_{+}(c)<0$. Similarly, we have tb_ $(c)<0$ for any inessential boundary circuit of $M$.

If the essential boundary is simple, then any boundary circuit is either inessential or adjacent to an inessential one. The claim follows.

Here is the relative version of the commutation theorem.

Theorem 9.2. Let $M$ and $M^{\prime}$ be enhanced mirror diagrams related by a $C$-delicate sequence of moves

$$
M=M_{0} \stackrel{\eta_{1}}{\longmapsto} M_{1} \stackrel{\eta_{2}}{\longmapsto} \ldots \stackrel{\eta_{n}}{\longmapsto} M_{n}=M^{\prime},
$$

where $C$ is a collection of essential boundary circuits of $M$. Let $C^{\prime}$ be the respective collection of essential boundary circuits of $M^{\prime}$. Assume that $M$ is +-flexible relative to $C$, and $M^{\prime}$ is--flexible relative to $C^{\prime}$.

Then there exists an enhanced mirror diagram $M^{\prime \prime}$ related to $M$ by a type I C-delicate sequence of moves

$$
M=M_{0}^{\prime} \stackrel{\eta_{1}^{\prime}}{\longmapsto} M_{1}^{\prime} \stackrel{\eta_{2}^{\prime}}{\longmapsto} \ldots \stackrel{\eta_{k}^{\prime}}{\longrightarrow} M_{k}^{\prime}=M^{\prime \prime}
$$

and to $M^{\prime}$ by a type II $C^{\prime \prime}$-delicate sequence of moves

$$
M^{\prime \prime}=M_{0}^{\prime \prime} \stackrel{\eta_{1}^{\prime \prime}}{\longmapsto} M_{1}^{\prime \prime} \stackrel{\eta_{2}^{\prime \prime}}{\longmapsto} \ldots \stackrel{\eta_{l}^{\prime \prime}}{\longmapsto} M_{l}^{\prime \prime}=M^{\prime},
$$

where $C^{\prime \prime}$ is the collection of essential boundary circuits of $M^{\prime \prime}$ which $C$ is transformed to by (9.5), such that

$$
\eta_{n} \circ \ldots \circ \eta_{2} \circ \eta_{1}=\eta_{l}^{\prime \prime} \circ \ldots \circ \eta_{2}^{\prime \prime} \circ \eta_{1}^{\prime \prime} \circ \eta_{k}^{\prime} \circ \ldots \circ \eta_{2}^{\prime} \circ \eta_{1}^{\prime} .
$$

Before proceeding with the proof of Theorems 9.1 and 9.2 we demonstrate that the flexibility assumption is essential in each of them.

Example 9.1. Let $M$ be an enhanced mirror diagram consisting of a single occupied level without any mirrors, and let $M^{\prime}$ be the mirror diagram shown in Figure 9.1. All boundary circuits of both diagrams

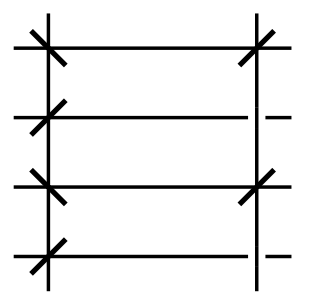

Figure 9.1. The diagram $M^{\prime}$ in Example 9.1

are inessential. These two diagrams are related by elementary moves. Indeed, there are two coherent 
$\backslash$-mirrors in $M^{\prime}$, one of which can be eliminated by means of a sequence of three type I elementary moves (see Lemma 8.2). The respective spatial graph will become a tree. So, all the mirrors now can be reduced by elimination moves.

We claim that it is impossible to get $M^{\prime}$ from $M$ by a sequence of elementary moves such that all type I moves in it precede all type II moves. This can be seen as follows. Let $M^{\prime \prime}$ be obtained from $M$ by type I elementary moves. Since $\Gamma_{\widehat{M}}$ is a single point, the spatial graph $\Gamma_{\widehat{M}^{\prime \prime}}$ is a tree. The diagram $M^{\prime \prime}$ cannot have any \-mirrors, hence no two $/$-mirrors of $M^{\prime \prime}$ are coherent.

The graph $\Gamma_{\widehat{M}^{\prime}}$ is not simply connected, and no two $/$-mirrors of $M^{\prime}$ are coherent. It follows from Proposition 8.1 that $\rho^{-}\left(M^{\prime}\right)$ and $\rho^{-}\left(M^{\prime \prime}\right)$ viewed as divided spatial ribbon graphs are not stably equivalent. By Theorem 8.4 (ii) the diagrams $M^{\prime \prime}$ and $M^{\prime}$ are not related by type II elementary moves.

Example 9.2. This example is similar in nature to the previous one, but slightly more complicated. Consider the mirror diagrams $M$ and $M^{\prime}$ shown in Figure 9.2 Again, $M$ can be transformed to $M^{\prime}$ by means of a sequence of elementary moves, but any such sequence have a type II move preceding a type I move. The details are left to the reader.

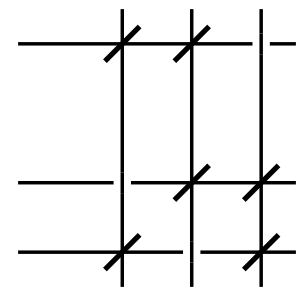

$M$

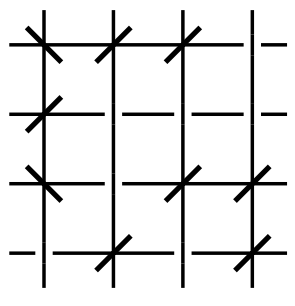

$M^{\prime}$

Figure 9.2. The diagrams in Example 9.2

We now proceed with the constructions needed to prove Theorems 9.1 and 9.2 .

9.2. Splitting routes and splitting paths. For $x$ an occupied level of a mirror diagram $M$ and $p$ a point at $x$ distinct from all mirrors of $M$, we denote by $\widehat{p}$ the point at $\partial \widehat{x}$ defined by $\varphi(\widehat{p})=\varphi(p)$ if $x$ is a meridian and $\theta(\widehat{p})=\theta(p)$ if $x$ is a longitude of $\mathbb{T}^{2}$. By using this notation we assume that the surface $\overparen{M}$ is chosen slim enough, so that $\widehat{p}$ is outside of all the strips $\widehat{\mu}, \mu \in E_{M}$. This means, in particular, that $\widehat{p}$ lies on $\partial \widehat{M}$.

A sequence $\left(\mu_{1}, \ldots, \mu_{m}\right)$ of mirrors of a mirror diagram will be called cancellable if there is a balanced parenthesis sequence of length $m$ such that whenever the $i$ th and the $j$ th parentheses in it are matched we have $\mu_{i}=\mu_{j}$. (In particular, this means that $m$ is even.) For instance, $\left(\mu, \mu^{\prime}, \mu^{\prime}, \mu^{\prime \prime}, \mu^{\prime \prime \prime}, \mu^{\prime \prime \prime}, \mu^{\prime \prime}, \mu\right)$ is cancellable for any mirrors $\mu, \mu^{\prime}, \mu^{\prime \prime}, \mu^{\prime \prime \prime}$.

Definition 9.3. By a single-headed type II splitting path in the surface $\overparen{M}$ we mean an oriented simple normal arc $\widehat{\omega} \subset \overparen{M}$ such that the following holds:

(1) $\widehat{\omega}$ starts at an interior point of $\overparen{M}$ and arrives at a point on $\partial \widehat{M}$, that is, a point of the form $\widehat{p}$, where $p \in y \in L_{M}, p \notin E_{M}$

(2) $\widehat{\omega}$ can be cut into subarcs $\widehat{\omega}^{1}, \ldots, \widehat{\omega}^{k}$ (following in $\widehat{\omega}$ in this order) so that

(a) for all $2 \leqslant i \leqslant k-1$ each endpoint of $\widehat{\omega}^{i}$ lies in the interior of a disc of the form $\widehat{x}, x \in L_{M}$;

(b) each $\widehat{\omega}^{i}, i=1, \ldots, k$, intersects a single strip $\widehat{\mu}_{i}, \mu_{i} \in E_{M}$;

(c) $\mu_{1}$ is a $\backslash$-mirror of $M$, all the other $\mu_{i}$ 's are $/$-mirrors;

(d) whenever $i, i^{\prime}, j, j^{\prime} \in\{2, \ldots, k\}$ are such that $i^{\prime}>i, j^{\prime}>j, \mu_{i}=\mu_{i^{\prime}}=\mu_{j}=\mu_{j^{\prime}}$ and the sequences $\left(\mu_{i}, \mu_{i+1}, \ldots, \mu_{i^{\prime}}\right)$ and $\left(\mu_{j}, \mu_{j+1}, \ldots, \mu_{j^{\prime}}\right)$ are cancellable, we have $i \equiv j(\bmod 2)$.

The sequence $\omega=\left(\mu_{1}, \ldots, \mu_{k}, p\right)$ arising in this way is referred to as a single-headed type II splitting route in $M$, and the path $\widehat{\omega}$ is said to be associated with $\omega$. 
If $k=1$, then $\omega$ is called an elementary type II splitting route, and $\widehat{\omega}$ an elementary type II splitting path.

A single-headed (or elementary) type I splitting path and a single-headed (or elementary) type I splitting route are defined similarly, with the roles of $/$-mirrors and $\backslash$-mirrors exchanged.

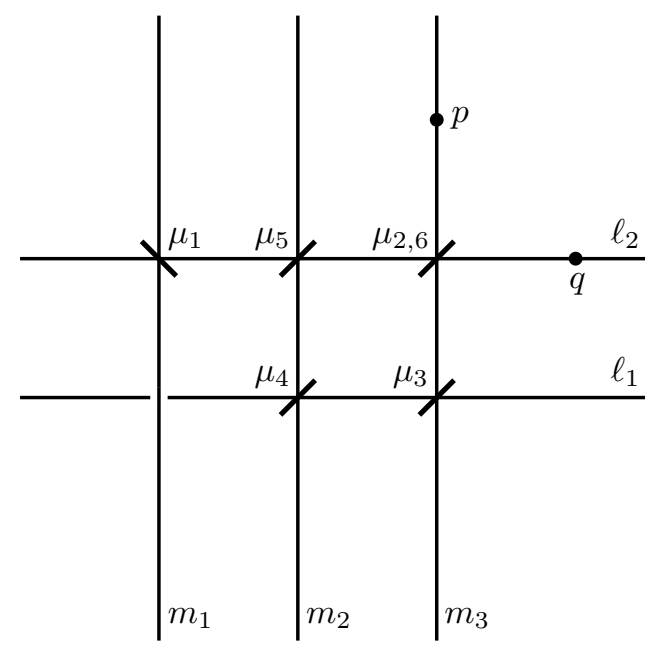

FIGURE 9.3. A single-headed splitting path associated with the splitting route $\left(\mu_{1}, \mu_{2}, \mu_{3}, \mu_{4}, \mu_{5}, \mu_{6}=\mu_{2}, p\right)$

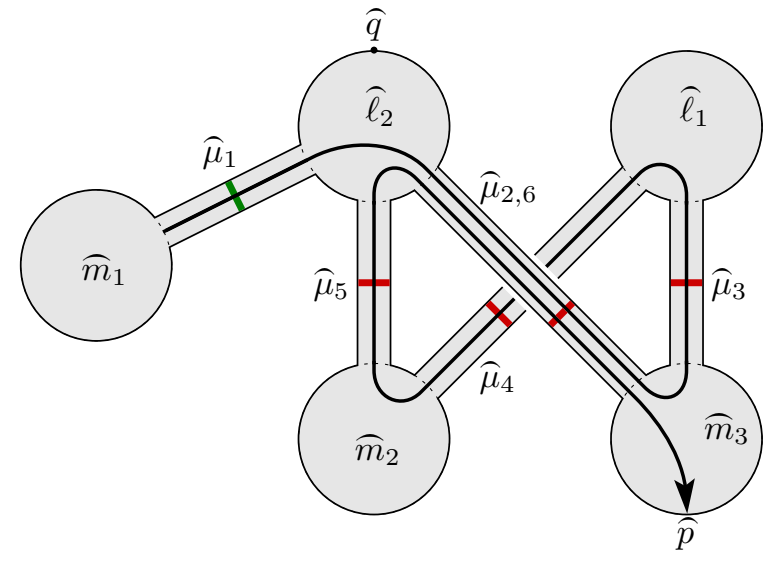

Example 9.3. Shown in Figure 9.3 is a mirror diagram $M$ and a single-headed splitting path in $\overparen{M}$ associated with the single-headed type II splitting route $\left(\mu_{1}, \mu_{2}, \mu_{3}, \mu_{4}, \mu_{5}, \mu_{6}=\mu_{2}, p\right)$. Observe that the sequence $\left(\mu_{1}, \mu_{2}, \mu_{3}, \mu_{4}, \mu_{5}, q\right)$ is not a single-headed type II splitting route as an arc crossing the 1-handles in the indicated order and ending at $\widehat{q}$ must have a self-intersection in $\widehat{\ell}_{2}$.

Note that a $/$-mirror may appear in a single-headed type II splitting route arbitrarily many times but at most two times in a row. This constraint is a part of Condition (2d) in Definition 9.3

Condition (2d), which looks somewhat artificial, is added in order for any single-headed type II splitting route to define the associated single-headed splitting path uniquely up to isotopy in the class of normal arcs. If this condition is omitted, then the path $\widehat{\omega}$ may be defined by $\omega$ too loosely, which, in turn, would complicate the exposition of some arguments below.

Condition (2d) prohibits, in particular, having $\mu_{i}=\mu_{i+1}=\mu_{i+2}$ or $\mu_{i}=\mu_{i+1}=\mu_{i+2 m-1}=\mu_{i+2 m}$ with $m>1$. The issues that are avoided by these prohibitions are illustrated in Figures 9.4 and 9.5 , where pairs of fragments of normal arcs are shown such that, for each pair, replacing one fragment of a normal arc by the other does not change the combinatorial presentation of the path by a sequence of mirrors.
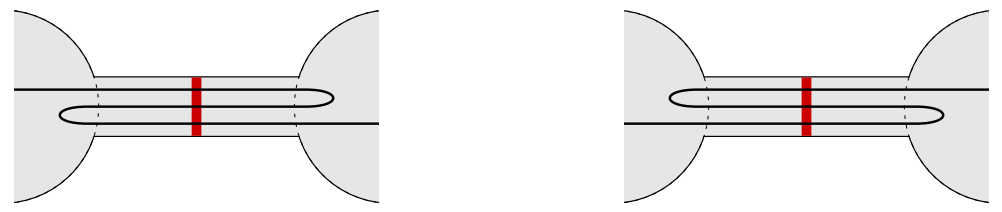

Figure 9.4. Non-uniqueness of $\widehat{\omega}$ if we allow $\mu_{i}=\mu_{i+1}=\mu_{i+2}$ 

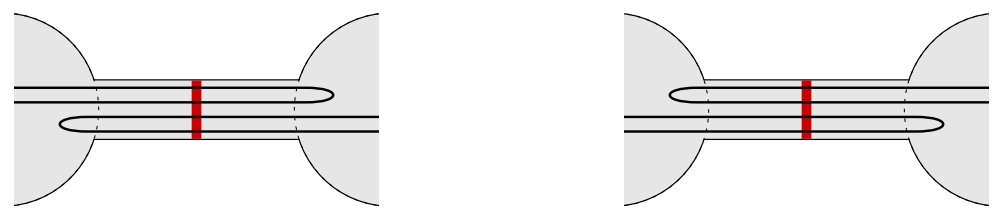

Figure 9.5. Non-uniqueness of $\widehat{\omega}$ if we allow $\mu_{i}=\mu_{i+1}=\mu_{i+2 m-1}=\mu_{i+2 m}, m>1$

The general form of Condition (2d) allows to avoid similar issues with longer pieces of $\widehat{\omega}$, for instance, with sequences of the form

$$
(\ldots, \mu, \nu, \nu, \mu, \mu, \nu \ldots), \quad(\ldots, \mu, \nu, \nu, \mu, \ldots, \nu, \mu, \mu, \nu, \ldots)
$$

and also guarantees that such pieces do not appear if a cancellable portion of a splitting route is removed.

Note that the equalities $\mu_{i}=\mu_{i+1}=\mu_{j}=\mu_{j+1}$ on their own cannot cause the problem shown in Figure 9.5 if $j-i$ is even and is larger than 2 in absolute value. Indeed, for $l=1, \ldots, k-1$, denote by $x_{l}$ the occupied level of $M$ containing $\mu_{l}$ and $\mu_{l+1}$. If $i$ and $j$ are of the same parity, then the occupied levels $x_{i}$ and $x_{j}$ are parallel. Therefore, the equality $\mu_{i}=\mu_{j}$ implies $x_{i}=x_{j}$, and hence, the 'tongues' $\widehat{\omega}^{i} \cup \widehat{\omega}^{i+1}$ and $\widehat{\omega}^{j} \cup \widehat{\omega}^{j+1}$ are directed coherently unlike the ones in Figure 9.5 .
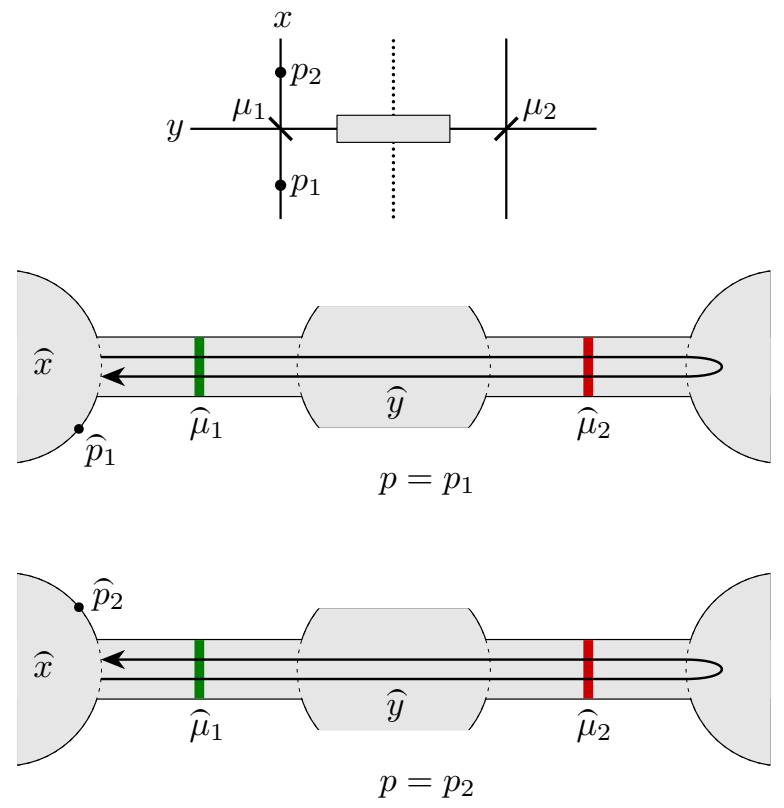

FIgURE 9.6. Dependence of $\widehat{\omega}$ on $p$ in the case of a double-headed splitting route $\omega=\left(\mu_{1}, \mu_{2}, \mu_{2}, \mu_{1}, p\right)$

Definition 9.4. By a double-headed type II splitting path in the surface $\overparen{M}$ we mean an oriented normal simple arc $\widehat{\omega} \subset \overparen{M}$ such that the following holds:

(1) both endpoints of $\widehat{\omega}$ are interior points of $\overparen{M}$

(2) $\widehat{\omega}$ can be cut into subarcs $\widehat{\omega}^{1}, \ldots, \widehat{\omega}^{k}$ (following in $\widehat{\omega}$ in this order), $k \geqslant 2$, so that

(a) for all $2 \leqslant i \leqslant k-1$, each endpoint of $\widehat{\omega}^{i}$ lies in the interior of a disc of the form $\widehat{x}, x \in L_{M}$;

(b) each $\widehat{\omega}^{i}, i=1, \ldots, k$, intersects a single strip $\widehat{\mu}_{i}, \mu_{i} \in E_{M}$;

(c) $\mu_{1}$ and $\mu_{k}$ are $\backslash$-mirrors of $M$, all the other $\mu_{i}$ 's are $/$-mirrors;

(d) whenever $i, i^{\prime}, j, j^{\prime} \in\{2, \ldots, k-1\}$ are such that $i^{\prime}>i, j^{\prime}>j, \mu_{i}=\mu_{i^{\prime}}=\mu_{j}=\mu_{j^{\prime}}$ and the sequences $\left(\mu_{i}, \mu_{i+1}, \ldots, \mu_{i^{\prime}}\right)$ and $\left(\mu_{j}, \mu_{j+1}, \ldots, \mu_{j^{\prime}}\right)$ are cancellable, we have $i \equiv j(\bmod 2)$. 
To encode $\widehat{\omega}$ we use a sequence $\omega=\left(\mu_{1}, \ldots, \mu_{k}, p\right)$ in which $p \in \mathbb{T}^{2}$ satisfies the following conditions:

(i) $p$ lies on the occupied level $x_{0}$ of $M$ characterized by $z \in \partial \widehat{x}_{0}$, where $z$ is the terminal point of $\widehat{\omega}$;

(ii) $p \notin E_{M}$;

(iii) the shorter of the two open arcs into which $z$ and $\widehat{p}$ cut the circle $\partial \widehat{x}_{0}$ is disjoint from $\widehat{\omega}$ and from the strips of the form $\widehat{\mu}, \mu \in E_{M} \backslash\left\{\mu_{k}\right\}$.

The sequence $\omega$ is then referred to as a double-headed type II splitting route in $M$, and the path $\widehat{\omega}$ is said to be associated with $\omega$.

A double-headed type II splitting route is called special if it has the form $\left(\mu_{1}, \mu_{2}, p\right)$.

By a type II splitting route or path we mean either a single-headed or a double-headed type II splitting route or path, respectively.

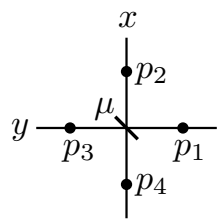

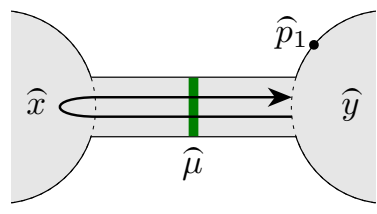

$\omega=\left(\mu, \mu, p_{1}\right)$

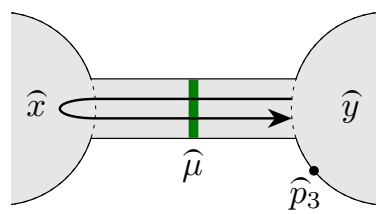

$\omega=\left(\mu, \mu, p_{3}\right)$

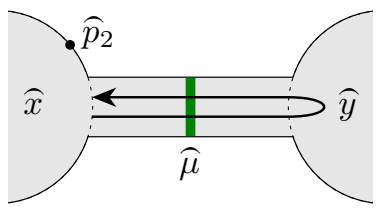

$\omega=\left(\mu, \mu, p_{2}\right)$

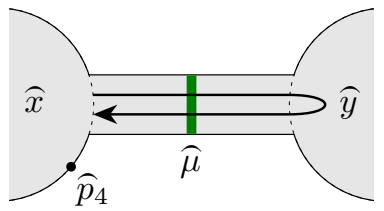

$\omega=\left(\mu, \mu, p_{4}\right)$

Figure 9.7. Dependence of $\widehat{\omega}$ on $p$ in the case of a special splitting route $\omega$ with $\mu_{1}=\mu_{2}$

Let $\omega=\left(\mu_{1}, \ldots, \mu_{k}, p\right)$ be a double-headed type II splitting route. If $\mu_{1} \neq \mu_{k}$, then the last entry in it, the point $p$, carries no additional information. Neither does it if $k \equiv 0(\bmod 2)$ and we have $\mu_{i} \neq \mu_{k+1-i}$

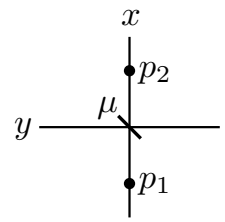

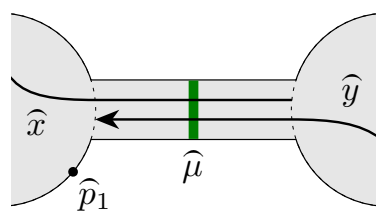

$p=p_{1}$

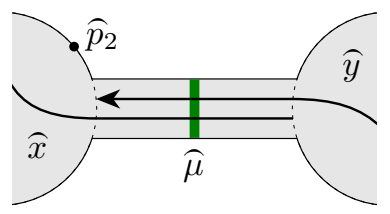

$p=p_{2}$

FiguRE 9.8. Dependence of $\widehat{\omega}$ on $p$ in the case of a double-headed splitting route $\omega=$ $\left(\mu, \mu_{2}, \mu_{3}, \ldots, \mu_{k-1}, \mu, p\right)$ 
for some $i \in\{2, \ldots, k / 2\}$. If $k \equiv 0(\bmod 2), k>2$, and $\mu_{i}=\mu_{k+1-i}$ for all $i=1, \ldots, k / 2$, then $p$ is important only for specifying the orientation of $\widehat{\omega}$; see Figure 9.6. If $\mu_{1}=\mu_{k}$ and $k$ is either odd (it should then be greater than four) or equal to two, then even the isotopy class of the unoriented arc underlying $\widehat{\omega}$ depends on $p$; see Figures 9.7 and 9.8 .

Another reason to keep $p$ in the notation for a double-headed splitting routes is the similarity with the notation for single-headed splitting routes, which sometimes allows for shorter formulations.

Proposition 9.3. If $\omega$ is a type II splitting route in a mirror diagram $M$, then the associated type II splitting path $\widehat{\omega}$ in $\widehat{M}$ is defined uniquely up to isotopy in the class of normal arcs.

We skip the easy proof.

Definition 9.5. Two type II (or type I) splitting routes $\omega$ and $\omega^{\prime}$ are said to be equivalent if the respective splitting paths $\widehat{\omega}$ and $\widehat{\omega}^{\prime}$ are isotopic in the class of normal arcs.

Clearly, it two splitting routes $\omega=\left(\mu_{1}, \ldots, \mu_{k}, p\right)$ and $\omega^{\prime}=\left(\mu_{1}^{\prime}, \ldots, \mu_{k^{\prime}}^{\prime}, p^{\prime}\right)$ are equivalent, then $k^{\prime}=k$ and $\mu_{i}=\mu_{i}^{\prime}$ for $1 \leqslant i \leqslant k$, so the only difference that can occur in equivalent splitting routes is $p \neq p^{\prime}$.

9.3. Generalized split and generalized merge moves. For proving Theorems 9.1 and 9.2 we need to generalize merge moves. However, it is more intuitive to speak in terms of the inverse operations, which are generalized split moves and which we now define.

Definition 9.6. Let $M$ be an enhanced mirror diagram, and let $\omega=\left(\mu_{1}, \mu_{2}, \ldots, \mu_{k}, p\right)$ be a type II splitting route in $M$. A generalized type II split move $M \stackrel{\eta}{\mapsto} M^{\prime}$ associated with $\omega$ is defined inductively as follows.

Case 1: $k=1$.

$M \stackrel{\eta}{\mapsto} M^{\prime}$ is a type II split move for which $\mu_{1}$ is the splitting mirror and $p$ is the snip point.

Case 2: $k>1, \omega$ is a single-headed splitting route.

Let $M \stackrel{\eta_{1}}{\longmapsto} M_{1}$ be a type I split move for which $\mu_{k}$ is the splitting mirror and $p$ the snip point, and let $M_{1} \stackrel{\eta_{2}}{\longmapsto} M^{\prime}$ be a generalized type II split move associated with a type II splitting route $\omega^{\prime}=$ $\left(\mu_{1}^{\prime}, \mu_{2}^{\prime}, \ldots, \mu_{k-1}^{\prime}, p^{\prime}\right)$ in which $\mu_{i}^{\prime}$ is a successor of $\mu_{i}, i=1, \ldots, k-1$, and $p^{\prime}$ lies in the splitting gap of the move $M \stackrel{\eta_{1}}{\longmapsto} M_{1}$. Then $M \stackrel{\eta}{\mapsto} M^{\prime}$ with $\eta=\eta_{2} \circ \eta_{1}$ is a generalized type II split move associated with $\omega$. Case 3: $k>2, \omega$ is a double-headed splitting route.

Let $M \stackrel{\eta_{1}}{\longmapsto} M_{1}$ be a double split move for which $\mu_{k}$ and $\mu_{k-1}$ are the splitting mirrors (see Definition 7.8 ). Denote by $\mu_{k}^{\prime}$ and $\mu_{k}^{\prime \prime}$ the successors of $\mu_{k}$ ordered so that the $\backslash$-splitting gap of the move is $\left(\mu_{k}^{\prime} ; \mu_{k}^{\prime \prime}\right)$ if $\left(\mu_{k} ; p\right)$ is shorter than $\left(p ; \mu_{k}\right)$, and $\left(\mu_{k}^{\prime \prime} ; \mu_{k}^{\prime}\right)$ otherwise.

Let $M_{1} \stackrel{\eta_{2}}{\longmapsto} M^{\prime}$ be a generalized type II split move associated with a single-headed type II splitting route $\omega^{\prime}=\left(\mu_{1}^{\prime}, \mu_{2}^{\prime}, \ldots, \mu_{k-2}^{\prime}, p^{\prime}\right)$ in which $\mu_{i}^{\prime}$ is a successor of $\mu_{i}, i=1, \ldots, k-2$, and $p^{\prime}$ lies in the $/$-splitting gap of the move $M \stackrel{\eta_{1}}{\longmapsto} M_{1}$. If $\mu_{1}=\mu_{k}$ we demand additionally that $\mu_{1}^{\prime}=\mu_{k}^{\prime}$.

Then $M \stackrel{\eta}{\mapsto} M^{\prime}$ with $\eta=\eta_{2} \circ \eta_{1}$ is a generalized type II split move associated with $\omega$.

Case 4: $\omega=\left(\mu_{1}, \mu_{2}, p\right)$ is a special double-headed splitting route. In this case, the generalized type II split move $M \stackrel{\eta}{\mapsto} M^{\prime}$ defined below is also called special.

Denote by $y$ the occupied level of $M$ containing $\mu_{1}$ and $\mu_{2}$, and not containing $p$. Let $M \stackrel{\eta_{1}}{\longmapsto} M_{1}$ be a type I extension move that adds a new mirror $\mu_{0}$ on $y$, and let $M_{1} \stackrel{\eta_{2}}{\longmapsto} M^{\prime}$ be a generalized type II split move associated with the splitting route $\left(\mu_{1}, \mu_{0}, \mu_{0}, \mu_{2}, p\right)$. Then $M \stackrel{\eta}{\mapsto} M^{\prime}$ with $\eta=\eta_{2} \circ \eta_{1}$ is a generalized type II split move associated with $\omega$. The mirror $\mu_{0}$ is referred to as the auxiliary mirror of the move $M \stackrel{\eta}{\mapsto} M^{\prime}$. The new occupied level added by the extension move $M \stackrel{\eta_{1}}{\longmapsto} M_{1}$ is called the auxiliary level of the move $M \stackrel{\eta}{\mapsto} M^{\prime}$.

The inverse of a generalized type II split move is called a generalized type II merge move.

One can see that generalized split moves are safe-to-bring-forward, whereas generalized merge moves are safe-to-postpone (see Definition 7.3 ). 
Proposition 9.4. For any type II splitting route $\omega$ in an enhanced mirror diagram $M$, there exists an associated generalized type II split move $M \mapsto M^{\prime}$. Unless $\omega$ is special, the combinatorial type of the resulting diagram $M^{\prime}$ is prescribed by the combinatorial class $M$ and the equivalence class of $\omega$. If $\omega$ is special, then the combinatorial type of the resulting diagram $M^{\prime}$ is prescribed by $M, \omega$, and the position of the auxiliary mirror.

Proof. We use the notation from Definition [9.6. We only need to prove the existence of the type II splitting route $\omega^{\prime}$ used for the inductive step in the Cases 2 and 3 of Definition 9.6, and show that it is unique up to replacing $p^{\prime}$ by another point in the $\left(/\right.$-) splitting gap of the move $M \stackrel{\eta_{1}}{\longmapsto} M_{1}$.

One can see that the requirements on $\omega^{\prime}$ can be reformulated in topological terms by means of the partial homeomorphisms $h_{M}^{M^{\prime}}$ introduced in Subsection 7.4 as follows: the image under $h_{M_{1}}^{M}$ of a type II splitting path $\widehat{\omega}^{\prime}$ associated with $\omega^{\prime}$ is a subarc of a type II splitting path $\widehat{\omega}$ associated with $\omega$.
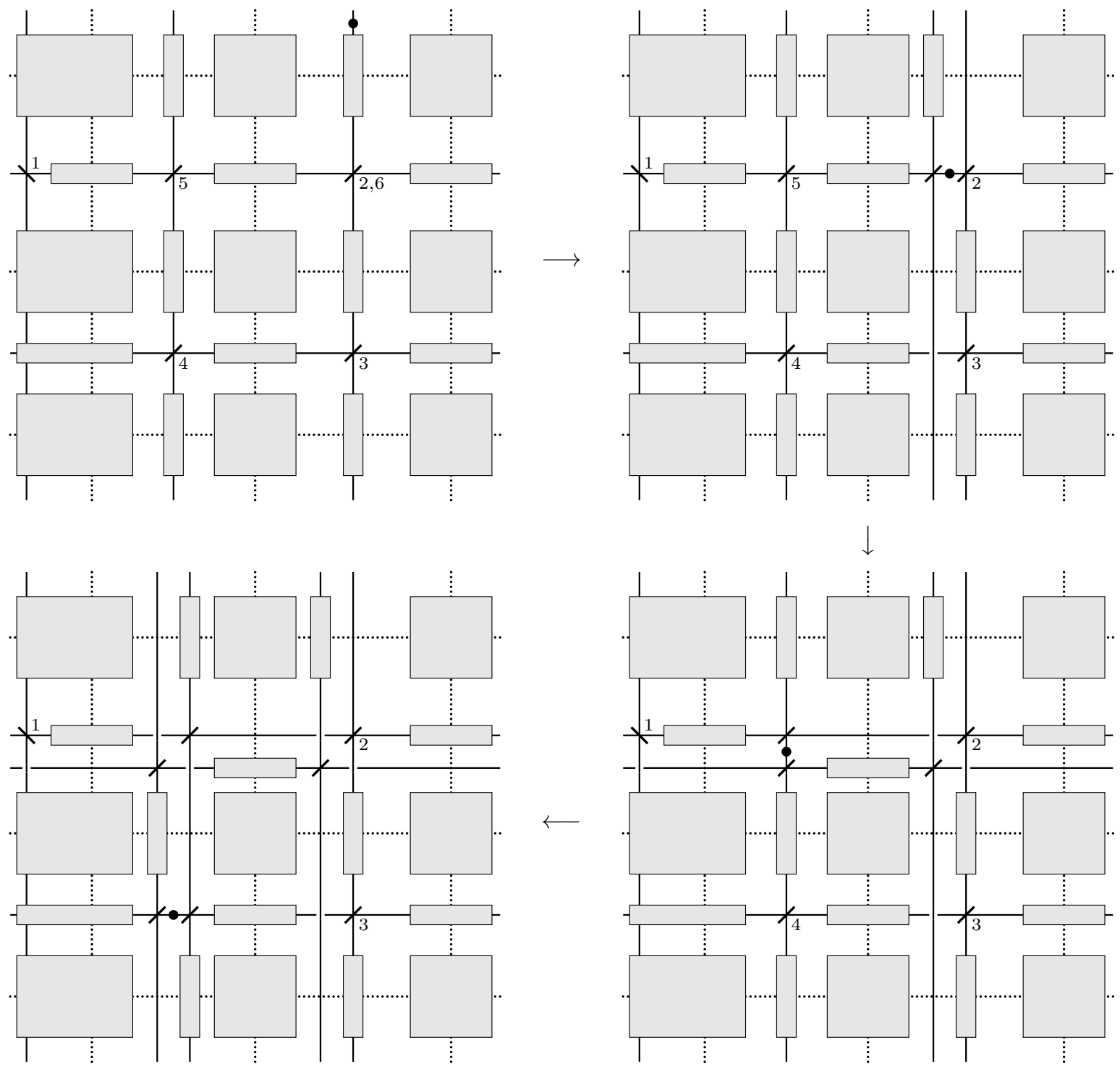

FIGURE 9.9. An example of a generalized type II splitting, part 1 

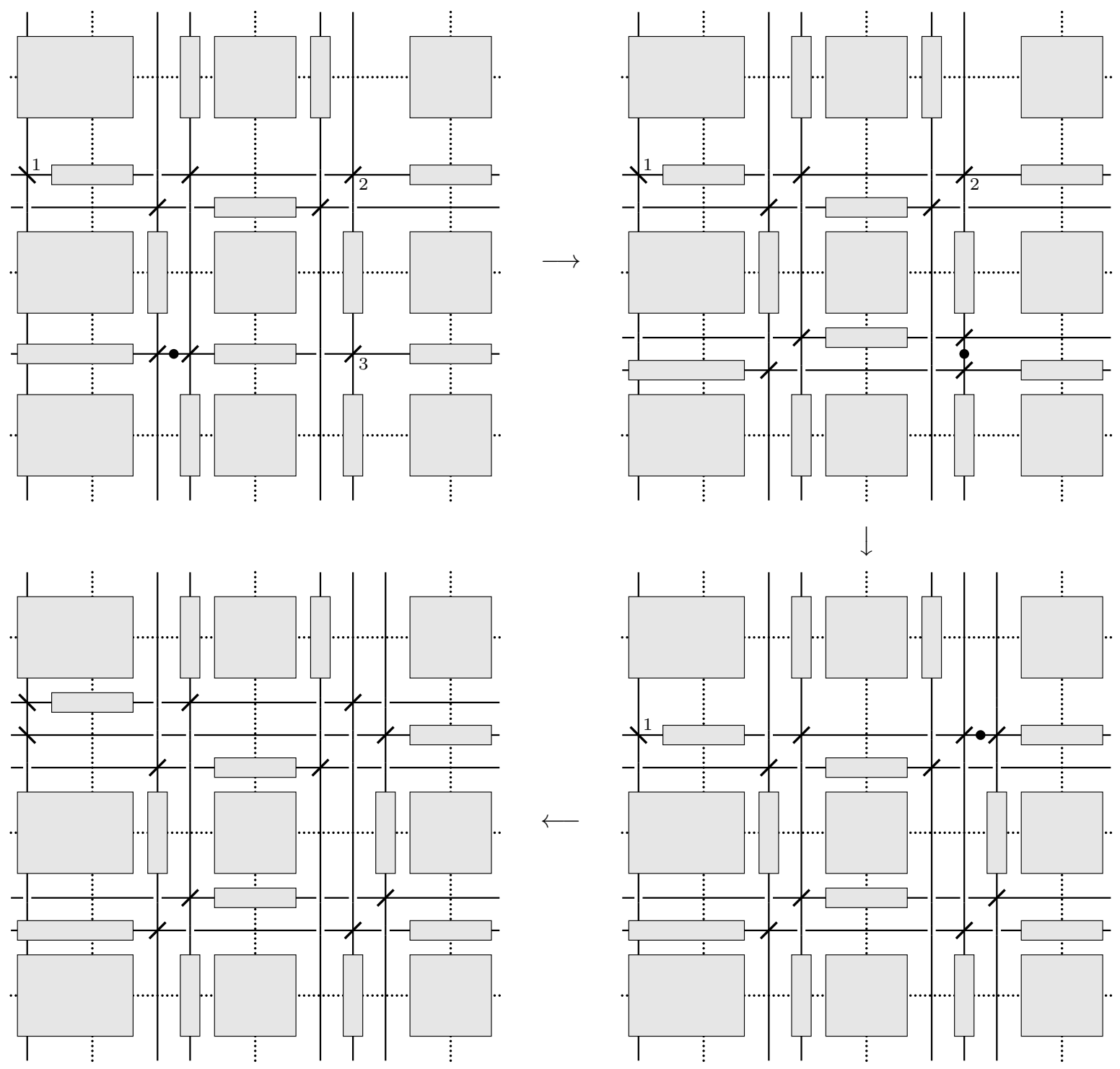

FIgURE 9.10. An example of a generalized type II splitting, part 2

This means that the sought-for $\omega^{\prime}$ can be obtained as follows. Take any type II splitting path $\widehat{\omega}$ associated with $\omega$ and decompose it into subarcs $\widehat{\omega}^{i}, i=1, \ldots, k$, as in Definition 9.3 . The partial homeomorphism $h_{M}^{M_{1}}$ can be chosen so that the intersection of the domain of $h_{M}^{M_{1}}$ with $\widehat{\omega}$ is precisely $\widehat{\omega}^{1} \cup$ $\ldots \widehat{\omega}^{k-1}$ if $\omega$ is a single-headed splitting route and $\widehat{\omega}^{1} \cup \ldots \widehat{\omega}^{k-2}$ if it is a double-headed one. We can take for $\widehat{\omega}^{\prime}$ the image $h_{M}^{M_{1}}(\widehat{\omega})$. One can see that any eligible choice of $\widehat{\omega}^{\prime}$ fits into this construction. This means that the choice of $\mu_{i}^{\prime}, i=1, \ldots, k-1$, in Definition 9.6 is unique.

Example 9.4. Consider a general mirror diagram obtained from that in Example 9.3 by adding more occupied levels and mirrors, and take the same splitting route $\omega$ as in Example 9.3 .

Figures 9.9 and 9.10 show a sequence of ordinary splittings arising from the definition of a generalized type II split move associated with $\omega$. In each picture we mark the snip point of the forthcoming split move, and number the mirrors of the splitting route with which the remaining part of the process (which 
is by definition also a generalized type II split move) is associated. Each mirror $\mu_{i}$ and its successors in the respective splitting routes are marked by the respective number $i$.

Convention 9.1. Though a generalized split move associated with a splitting route $\omega$ depends only on the equivalence class of $\omega$, by saying that $M \mapsto M^{\prime}$ is a (generalized) split move we assume that a concrete splitting route for this move has been chosen and fixed.

Recall that equivalent splitting routes may be different only in the last entry, which is the snip point in the case of single-headed splitting routes.

For every non-special generalized type II split move $M \mapsto M^{\prime}$ and its inverse, which is the generalized type II merge move $M^{\prime} \mapsto M$, we define the partial homeomorphisms $h_{M}^{M^{\prime}}, h_{M^{\prime}}^{M}$ as follows. Let $M=$ $M_{0} \stackrel{\eta_{1}}{\longmapsto} M_{1} \stackrel{\eta_{2}}{\longmapsto} \ldots \stackrel{\eta_{k}}{\longmapsto} M_{k}=M^{\prime}$ be the sequence of split moves that arises from the inductive definition of a non-special generalized type II split move. We take for $h_{M^{\prime}}^{M}$ the composition $h_{M_{1}}^{M_{0}} \circ h_{M_{2}}^{M_{1}} \circ \ldots \circ h_{M_{k}}^{M_{k-1}}$. Its domain is the whole of the surface $\widehat{M}^{\prime}$, and the image is obtained from $\overparen{M}$ by cutting along an arc of the form $\widehat{\omega}$, where $\omega$ is the type II splitting route with which the split move $M \mapsto M^{\prime}$ is associated. Clearly the triple $\left(\widehat{M}^{\prime}, \widehat{M}, h_{M^{\prime}}^{M}\right)$ represents the morphism from $\widehat{M}^{\prime}$ to $\widehat{M}$ associated with the move $M^{\prime} \stackrel{\eta^{-1}}{\longmapsto} M$, where $\eta=\eta_{k} \circ \ldots \circ \eta_{2} \circ \eta_{1}$.

Accordingly, $h_{M}^{M^{\prime}}$ is defined as the inverse of $h_{M^{\prime}}^{M}$. Its domain is obtained by cutting $\widehat{M}$ along $\widehat{\omega}$, and the image is the whole of $\widetilde{M}^{\prime}$. Clearly, there is a surface $F \in \widetilde{S}_{\widehat{M}^{\prime}}$ and an extension $\widetilde{h}$ of the partial homeomorphism $h_{M}^{M^{\prime}}$ to a homeomorphism $\overparen{M} \rightarrow F$ such that the triple $(\overparen{M}, F, \widetilde{h})$ represents the morphism $\eta$, which is associated with the move $M \mapsto M^{\prime}$.

Successors and predecessors of mirrors and occupied levels are defined for the moves $M \mapsto M^{\prime}$ and $M^{\prime} \mapsto M$ by following the general rule (see Definition (7.11). One can see that every mirror and every occupied level of $M^{\prime}$ has a predecessor in $M$ for the move $M \mapsto M^{\prime}$. The number of successors of a mirror $\mu$ in $M$ is one greater than the number of entries in $\omega$ equal to $\mu$.

Definition 9.7. Let $C$ be a collection of boundary circuits of a mirror diagram $M$, and let $\omega$ be a splitting route in $M$. Denote by $\widehat{C}$ the union of the corresponding boundary components of $\overparen{M}$. We say that $\omega$ separates $C$ if there exists an occupied level $x$ of $M$ such that at least two connected components of $\widehat{x} \backslash \widehat{\omega}$ have a non-empty intersection with $\widehat{C}$.

Proposition 9.5. Let $C$ be a collection of boundary circuits of a mirror diagram $M$, and let $\omega$ be a non-special splitting route in $M$. The following four conditions are equivalent:

(1) $\omega$ does not separate $C$;

(2) for any two successive mirrors $\mu$ and $\mu^{\prime}$ in $\omega$, and also, if $\omega$ is single-headed, for $\mu$ the last mirror and $\mu^{\prime}$ the snip point of $\omega$, either $\left(\mu, \mu^{\prime}\right)$ or $\left(\mu^{\prime}, \mu\right)$ is disjoint from $\bigcup_{c \in C} c$;

(3) every split move in the decomposition of $M \mapsto M^{\prime}$ arising from Definition 9.6 can be chosen so as to preserve all the boundary circuits in $C$;

(4) a generalized split move $M \mapsto M^{\prime}$ associated with $\omega$ can be chosen so as to preserve all the boundary circuits in $C$.

This proposition is an easy consequence of the correspondence between boundary circuits of $M$ and boundary components of $\overparen{M}$ described in Subsection [5.1, and the definition of a split move. We omit the proof.

Definition 9.8. Let $M \mapsto M^{\prime}$ be a generalized type II split move, and let

$$
M=M_{0} \mapsto M_{1} \mapsto \ldots \mapsto M_{k}=M^{\prime}
$$

be a sequence of moves including a type II split move, some number of type I split moves, and possibly a double split move and a type I extension move, that arises from the inductive definition of a generalized type II split move. We say that the decomposition (9.6) of the move $M \mapsto M^{\prime}$ is canonical if it is neat.

It follows from Proposition 9.5 that any generalized type II split move always has a canonical decomposition. 
9.4. Commutation of moves. Formally speaking, two moves of mirror diagrams $M_{1} \mapsto M_{2}$ and $M_{3} \mapsto$ $M_{4}$ are composable only if $M_{2}=M_{3}$. However, in certain situation, a natural meaning can be given to a composition of non-trivial moves $M \mapsto M_{1}$ and $M \mapsto M_{2}$ starting from the same diagram. If they can be 'composed' in either way it makes sense to ask whether the results of the two compositions are (combinatorially) the same. If they are, then it is natural to say that the moves commute. In the following definition we list specific cases in which we use such terminology. All mirror diagrams in it are assumed to be enhanced.

Definition 9.9. Let $M \stackrel{\eta}{\mapsto} M_{1}$ be a move of one of the following kinds: generalized split, double split, wrinkle creation, jump, elementary bypass removal, or elimination move, and let $M \stackrel{\zeta}{\mapsto} M_{2}$ be one of the moves for which we have defined the partial homeomorphism $h_{M}^{M_{2}}$. Let also $C$ be the set of all boundary circuits of $M$ preserved by both moves.

We say that the move $M \stackrel{\zeta}{\mapsto} M_{2}$ is friendly to the move $M \stackrel{\eta}{\mapsto} M_{1}$ if one of the cases $1-7$ listed below occurs (one extra case will be introduced in Definition 9.18). In each case we define (non-uniquely) a move $M_{2} \stackrel{\eta^{\zeta}}{\longmapsto} M_{21}$ resembling the move $M \stackrel{\eta}{\longmapsto} M_{1}$. To be called such it must preserve all boundary circuits in $C$. If $M \stackrel{\zeta}{\mapsto} M_{2}$ is a jump move, then, additionally, $M_{2} \stackrel{\eta^{\zeta}}{\longmapsto} M_{21}$ is demanded to preserve any boundary circuit of $M_{2}$ corresponding to a boundary circuit of $M$ preserved by the move $M \stackrel{\eta}{\mapsto} M_{1}$.

Case 1: $M \stackrel{\eta}{\mapsto} M_{1}$ is a generalized type II (respectively, type I) split move associated with a type II (respectively, type I) splitting route $\omega$, and there is a unique, up to equivalence, type II (respectively, type I) splitting route $\omega^{\prime}$ in $M_{2}$ such that the partial homeomorphism $h_{M}^{M_{2}}$ can be chosen to take $\widehat{\omega}$ to $\widehat{\omega}^{\prime}$.

The move $M_{2} \stackrel{\eta^{\zeta}}{\longmapsto} M_{21}$ is defined as a generalized type II (respectively, type I) split move associated with $\omega^{\prime}$.

Case 2: $M \stackrel{\eta}{\mapsto} M_{1}$ is a jump move, $M \stackrel{\zeta}{\mapsto} M_{2}$ is a move different from a jump move such that every occupied level and mirror of $M_{2}$ has exactly one predecessor in $M$.

Suppose that the jump move $M \stackrel{\eta}{\mapsto} M_{1}$ shifts a meridian $m_{\theta_{1}} \in L_{M}$. Let $m_{\theta_{1}}, m_{\theta_{2}}, \ldots, m_{\theta_{n}}$ be all occupied meridians of $M$ listed so that $\theta_{1}<\theta_{2}<\ldots<\theta_{n}<\theta_{1}+2 \pi$, and let $m_{\theta_{11}}, \ldots, m_{\theta_{1 l}}$ be all the successors of $m_{\theta_{1}}$ in $M_{2}$. By construction of our moves of mirror diagrams, there are $2 n$ real numbers $\theta_{1}^{\prime}<\theta_{1}^{\prime \prime}<\theta_{2}^{\prime}<\theta_{2}^{\prime \prime}<\ldots<\theta_{n}^{\prime}<\theta_{n}^{\prime \prime}<\theta_{1}^{\prime}+2 \pi$ such that any successor of the meridian $m_{\theta_{i}}$ is contained in $\left(\theta_{i}^{\prime} ; \theta_{i}^{\prime \prime}\right) \times \mathbb{S}^{1}$. We may also assume that $\theta_{1}^{\prime}<\theta_{11}<\theta_{12}<\ldots<\theta_{1 l}<\theta_{1}^{\prime \prime}$.

If the move $M \stackrel{\eta}{\mapsto} M_{1}$ shifts $m_{\theta_{1}}$ to a position between $m_{\theta_{j}}$ and $m_{\theta_{j+1}}$, then the move $M_{2} \stackrel{\eta^{\zeta}}{\longmapsto} M_{21}$ is a composition of jump moves that replaces the meridians $m_{\theta_{11}}, \ldots, m_{\theta_{1 l}}$ by $m_{\theta_{11}^{\prime}}, \ldots, m_{\theta_{1 l}^{\prime}}$ such that $\theta_{j}^{\prime \prime}<$ $\theta_{11}^{\prime}<\theta_{12}^{\prime}<\ldots<\theta_{1 l}^{\prime}<\theta_{j+1}^{\prime}$.

If $M \stackrel{\eta}{\mapsto} M_{1}$ shifts not a meridian but a longitude, the move $M_{2} \stackrel{\eta^{\zeta}}{\longmapsto} M_{21}$ is defined similarly with $\varphi$ 's instead of $\theta$ 's.

Case 3: $M \stackrel{\eta}{\mapsto} M_{1}$ is a jump move that replaces an occupied level $x$ by another occupied level $y$, and $M \stackrel{\zeta}{\mapsto} M_{2}$ is an extension move such that replacing of $x$ by $y$ in $M_{2}$ can still be realized by a jump move.

Then this jump move is the one that is taken for $M_{2} \stackrel{\eta^{\varsigma}}{\longmapsto} M_{21}$.

Case 4: $M \stackrel{\eta}{\mapsto} M_{1}$ is an elimination move removing a mirror $\mu$ and an occupied level $x$ such that $\mu$ and $x$ have unique successors $\mu^{\prime}$ and $x^{\prime}$, respectively, in $M_{2}$, and $x^{\prime}$ contains no mirrors of $M_{2}$ other than $\mu^{\prime}$. The move $M_{2} \stackrel{\eta^{\zeta}}{\longmapsto} M_{21}$ is then the elimination of $\mu^{\prime}$ and $x^{\prime}$.

Case 5: $M \stackrel{\eta}{\mapsto} M_{1}$ is an elementary bypass removal, $c$ is the corresponding inessential boundary circuit having the form of the boundary of a rectangle, and the move $M \stackrel{\zeta}{\mapsto} M_{2}$ transforms $c$ to a boundary circuit $c^{\prime}$ that also has the form of the boundary of a rectangle.

Let $\mu$ be the mirror removed by $M \stackrel{\eta}{\mapsto} M_{1}$. The move $M_{2} \stackrel{\eta^{\varsigma}}{\longmapsto} M_{21}$ removes a successor of $\mu$ lying on $c^{\prime}$, which is unique. 
Case 6: $M \stackrel{\eta}{\mapsto} M_{1}$ is a double split move with splitting mirrors $\mu_{1}, \mu_{2}$ lying on an occupied level $x, M \stackrel{\zeta}{\mapsto}$ $M_{2}$ is any move for which the partial homeomorphism $h_{M}^{M_{2}}$ is defined, and there is a unique triple $\left(\mu_{1}^{\prime}, \mu_{2}^{\prime}, x^{\prime}\right) \in$ $E_{M_{2}} \times E_{M_{2}} \times L_{M_{2}}$ such that $\mu_{1}^{\prime}, \mu_{2}^{\prime}$, and $x^{\prime}$ are successors of $\mu_{1}, \mu_{2}$ and $x$, respectively, and $\mu_{1}^{\prime}, \mu_{2}^{\prime} \in x^{\prime}$. The move $M_{2} \stackrel{\eta^{\zeta}}{\longmapsto} M_{21}$ is then defined as a double split move with splitting mirrors $\mu_{1}^{\prime}$ and $\mu_{2}^{\prime}$.

Case 7: $M \stackrel{\eta}{\mapsto} M_{1}$ is a wrinkle creation move with ramification mirrors $\mu_{1}, \mu_{2}$, and the conditions from the previous case hold.

The move $M_{2} \stackrel{\eta^{\zeta}}{\longmapsto} M_{21}$ is a wrinkle creation move with ramification mirrors $\mu_{1}^{\prime}$ and $\mu_{2}^{\prime}$.

One can see that in each case listed in Definition 9.9 the move $M_{2} \stackrel{\eta^{\zeta}}{\longmapsto} M_{21}$ does exist.

Definition 9.10. Let $M \stackrel{\eta}{\mapsto} M_{1}$ and $M \stackrel{\zeta}{\mapsto} M_{2}$ be two moves that are friendly to each other and neither of which is a special generalized split move, and let $C$ be the set of all boundary circuits of $M$ preserved by both moves. Let also $M_{1} \stackrel{\zeta^{\eta}}{\longmapsto} M_{12}$ and $M_{2} \stackrel{\eta^{\zeta}}{\longmapsto} M_{21}$ be moves that resemble $M \stackrel{\zeta}{\mapsto} M_{2}$ and $M \stackrel{\eta}{\mapsto} M_{1}$, respectively. We say that the moves $M \stackrel{\eta}{\mapsto} M_{1}$ and $M \stackrel{\zeta}{\mapsto} M_{2}$ commute with each other if the move

$$
M_{21} \stackrel{\zeta^{\eta} \circ \eta \circ \zeta^{-1} \circ\left(\eta^{\zeta}\right)^{-1}}{\longmapsto} M_{12}
$$

admits a $C$-neat decomposition into jump moves that preserve the combinatorial type of the diagram.

If the transformation (9.7) admits a $C$-neat decomposition into jump moves each of which either preserves the combinatorial type of the diagram or modifies it only by exchanging occupied levels that have a common predecessor in $M$, then we say that the moves $M \stackrel{\eta}{\mapsto} M_{1}$ and $M \stackrel{\zeta}{\mapsto} M_{2}$ almost commute.

Below we prove a number of statements about almost commutation. In the proofs, we don't make a special check that the involved jump moves exchange only the successors of the same occupied level of the original diagram, because this is kind of automatic. The long sequences of moves that we consider are designed so that the families of successors of different parallel occupied occupied levels never mix with each other, and the non-trivial part of establishing the almost commutation of the discussed moves is to show that the order of successors in each family can be changed by jump moves in the expected way.

Lemma 9.1. Let $M \stackrel{\eta}{\mapsto} M_{1}$ and $M \stackrel{\zeta}{\mapsto} M_{2}$ be two split moves (not generalized, but of any type) associated with splitting routes $\omega_{1}=(\mu, p)$ and $\omega_{2}=(\nu, q)$, respectively, and let $x$ and $y$ be the respective occupied levels of $M$ that are split by these moves. Assume that $p \neq q$.

Then the moves $M \stackrel{\eta}{\mapsto} M_{1}$ and $M \stackrel{\zeta}{\mapsto} M_{2}$ are friendly to each other unless one of the following situations occurs:

(1) we have $\mu=\nu$ and $x \neq y$;

(2) we have $x=y, \mu \neq \nu$, and the pairs $\{\mu, p\}$ and $\{\nu, q\}$ interleave, which means that the interval $(\mu ; p)$ contains exactly one of the points $\nu$ and $q$. This is equivalent to saying that $\widehat{\omega}_{1}$ and $\widehat{\omega}_{2}$ have an unavoidable intersection.

If the moves $M \stackrel{\eta}{\mapsto} M_{1}$ and $M \stackrel{\zeta}{\mapsto} M_{2}$ are friendly to each other, then they almost commute.

Proof. The claim of the lemma is quite obvious if $\mu \neq \nu$ and $x \neq y$. In this case, the moves $M \stackrel{\eta}{\mapsto} M_{1}$ and $M \stackrel{\zeta}{\mapsto} M_{2}$, when viewed combinatorially, 'do not interfere' which each other and clearly commute.

The moves $M \stackrel{\eta}{\mapsto} M_{1}$ and $M \stackrel{\zeta}{\mapsto} M_{2}$ are friendly to each other if and only if the following two conditions hold:

(1) the splitting paths $\widehat{\omega}_{1}, \widehat{\omega}_{2}$ associated with $\omega_{1}$ and $\omega_{2}$ can be chosen to be disjoint;

(2) if $\widehat{\omega}_{1}^{\prime}$ and $\widehat{\omega}_{2}^{\prime}$ are another disjoint splitting paths associated with $\omega_{1}$ and $\omega_{2}$, respectively, then there is a self-homeomorphism of $\overparen{M}$ isotopic to the identity, and preserving the handle decomposition structure, that takes the pair $\left(\widehat{\omega}_{1}, \widehat{\omega}_{2}\right)$ to $\left(\widehat{\omega}_{1}^{\prime}, \widehat{\omega}_{2}^{\prime}\right)$

Indeed, the first condition means that we can define the image of $\omega_{1}$ in $M_{2}$ and the image of $\omega_{2}$ in $M_{1}$ in a consistent way, and the second condition means that the choice of these images is essentially unique. 
The only possible reason for $\widehat{\omega}_{1}$ and $\widehat{\omega}_{2}$ to have an unavoidable intersection is the coincidence of $x$ and $y$ and a cyclic order of $\mu, p, \nu, q$ such that the pairs $\{\mu, p\}$ and $\{\nu, q\}$ interleave; see Figure 9.11 (a).

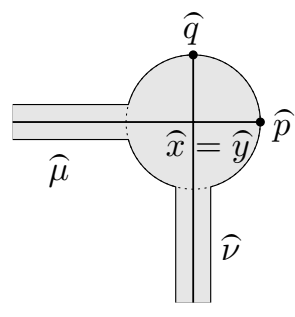

(a)

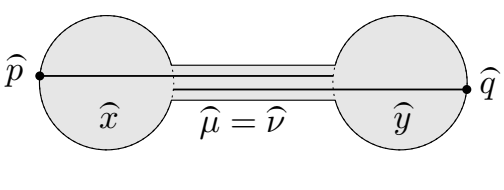

(b)

Figure 9.11. Mutually unfriendly splitting paths $\widehat{(\mu, p)}$ and $\widehat{(\nu, q)}$

Assume that $\widehat{\omega}_{1}$ and $\widehat{\omega}_{2}$ can be chosen to be disjoint. Their mutual position is ambiguous only if $\mu$ has two successors for the move $M \stackrel{\zeta}{\mapsto} M_{2}$, or $\nu$ has two successors for the move $M \stackrel{\eta}{\mapsto} M_{1}$. Both conditions are equivalent to $\mu=\nu$. However, if $x=y$, then the ambiguity does not occur since the mutual position of $\widehat{p}$ and $\widehat{q}$ on $\partial \widehat{x}$ prescribes the mutual position of $\widehat{\omega}_{1} \cap \widehat{\mu}$ and $\widehat{\omega}_{2} \cap \widehat{\mu}$ in $\widehat{\mu}$. The ambiguity does occur if $\mu=\nu$ and $x \neq y$; see Figure 9.11 (b).

It remains to show that the moves $M \stackrel{\eta}{\mapsto} M_{1}$ and $M \stackrel{\zeta}{\mapsto} M_{2}$ almost commute with one another in the case when they are mutually friendly, and we have $x=y$. Up to various symmetries there are the following five cases to consider. In each case, we denote by $\nu_{1}$ the splitting mirror of the move $M_{1} \stackrel{\zeta^{\eta}}{\longrightarrow} M_{12}$, by $x_{1}$ the successor of $x$ in $M_{1}$ containing $\nu_{1}$, by $\mu_{2}$ the splitting mirror of the move $M_{2} \stackrel{\eta^{\zeta}}{\longmapsto} M_{21}$, by $x_{2}$ the successor of $x$ in $M_{2}$ containing $\mu_{2}$, and by $q_{1} \in x_{1}, p_{2} \in x_{2}$ points such that $h_{M}^{M_{1}}(\widehat{q})=\widehat{q}_{1}, h_{M}^{M_{2}}(\widehat{p})=\widehat{p}_{2}$.

Case 1: the moves $M \stackrel{\eta}{\mapsto} M_{1}$ and $M \stackrel{\zeta}{\mapsto} M_{2}$ are of type II and type I, respectively, and we have $q \in(\mu ; \nu)$ and $p \in(\nu ; \mu)$.

One can see from Figure 9.12 that the results of the transformations $M \stackrel{\zeta^{\eta} \circ \eta}{\longmapsto} M_{12}$ and $M \stackrel{\eta^{\zeta} \circ \zeta}{\longmapsto} M_{21}$ are combinatorially the same. It follows easily that the moves commute.

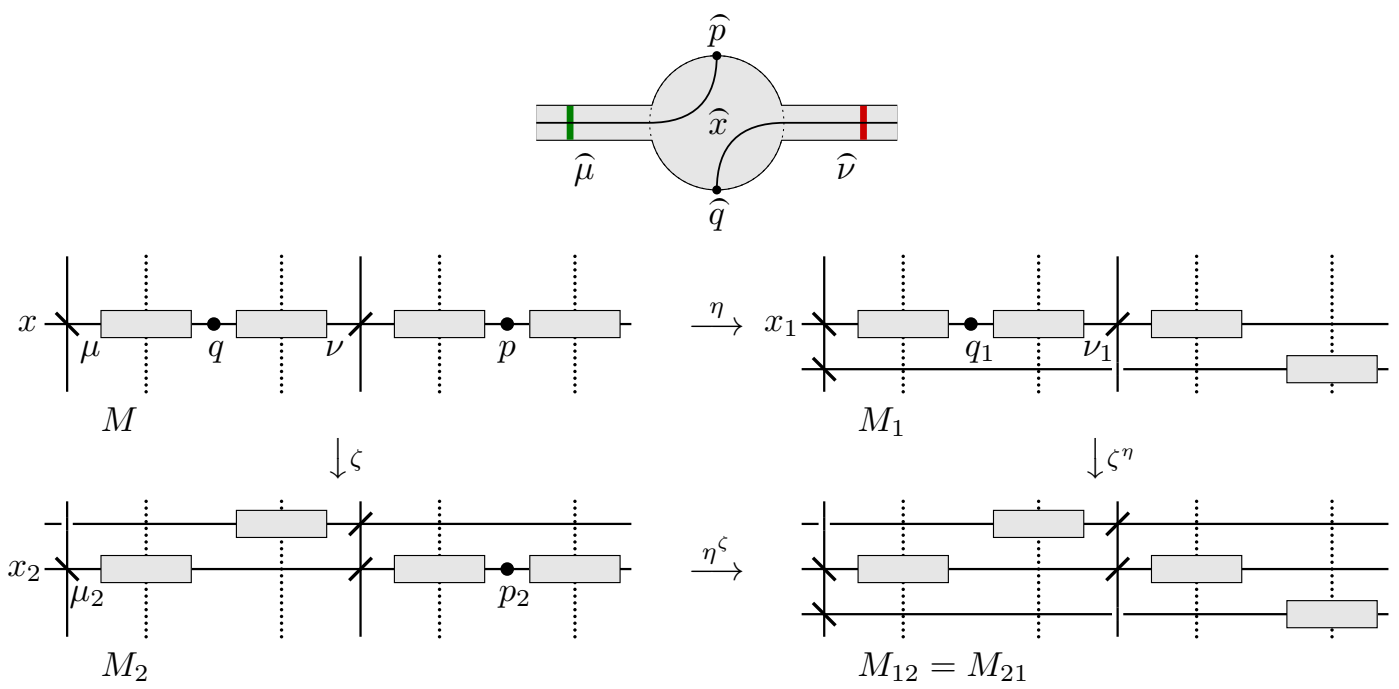

Figure 9.12. Proof of Lemma 9.1 in the case $x=y, \mu$ is of type ' $\, \nu$ is of type ' $\angle$ ', $q \in(\mu ; \nu), p \in(\nu ; \mu)$ 
Case 2: The moves $M \stackrel{\eta}{\mapsto} M_{1}$ and $M \stackrel{\zeta}{\mapsto} M_{2}$ are of type II and type I, respectively, and we have $q \in(\nu ; \mu)$ and $p \in(q ; \mu)$.

One can see from Figure 9.13 that the combinatorial types of the diagrams $M_{12}$ and $M_{21}$ are obtained from each other by exchanging two successors of $x$, and this exchange can be realized by jump moves. This implies that the moves almost commute.

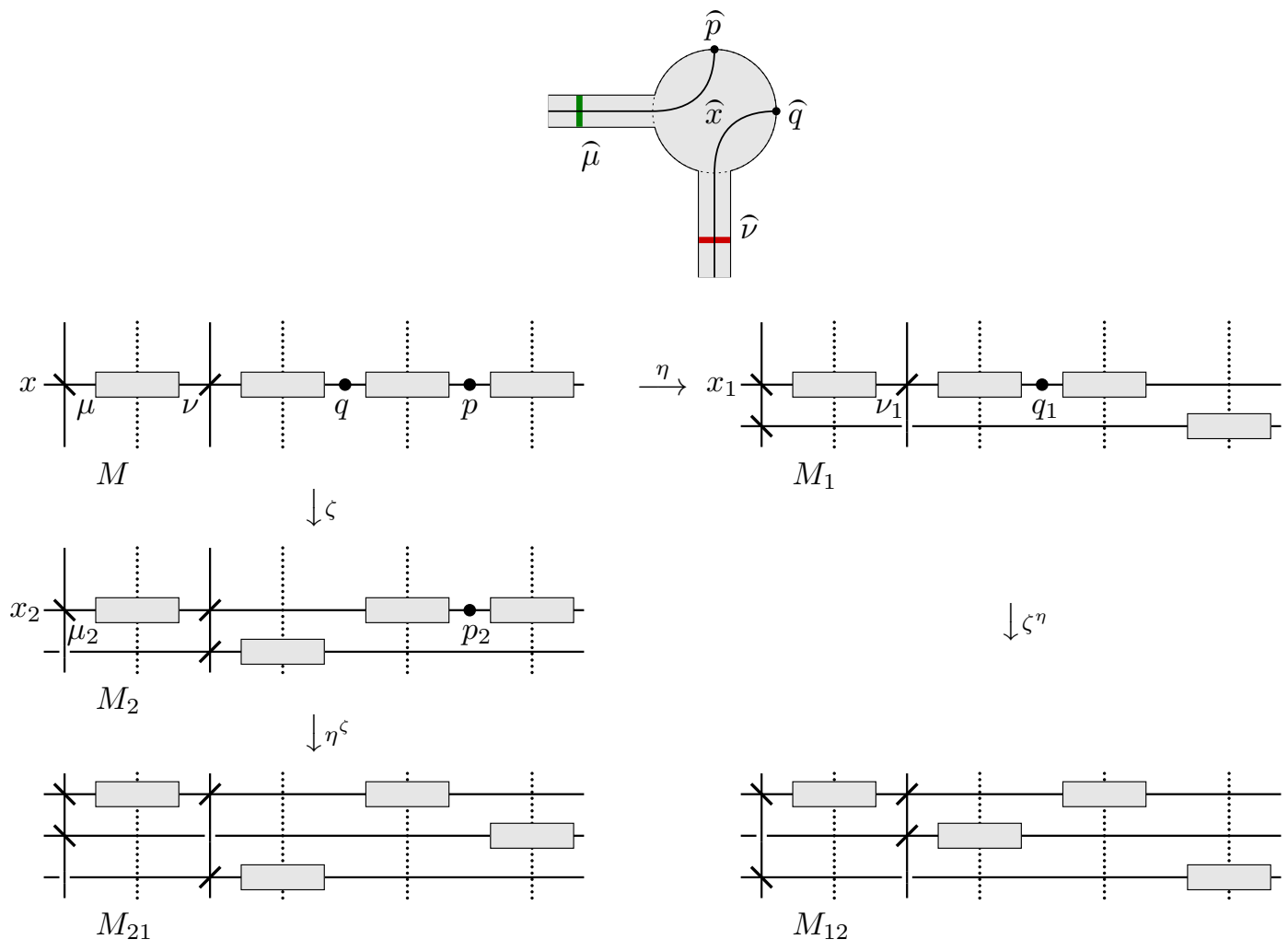

Figure 9.13. Proof of Lemma 9.1 in the case $x=y, \mu$ is of type ' $\backslash$ ', $\nu$ is of type ' $/$ ', $q \in(\nu ; \mu), p \in(q ; \mu)$

Case 3: The moves $M \stackrel{\eta}{\mapsto} M_{1}$ and $M \stackrel{\zeta}{\mapsto} M_{2}$ are both of type I, and we have $\mu=\nu$. As one can see from Figure 9.14 the diagrams $M_{12}$ and $M_{21}$ are combinatorially equivalent, which means that the moves commute.

Case 4: The moves $M \stackrel{\eta}{\mapsto} M_{1}$ and $M \stackrel{\zeta}{\mapsto} M_{2}$ are both of type I, and we have $q \in(\mu ; \nu), p \in(\nu ; \mu)$. This case is similar to Case 2. The almost commutation of the moves is illustrated in Figure 9.15.

Case 5: The moves $M \stackrel{\eta}{\mapsto} M_{1}$ and $M \stackrel{\zeta}{\mapsto} M_{2}$ are both of type I, and we have $q \in(\nu ; \mu), p \in(q ; \mu)$. This case is similar to Case 1. The commutation of the moves is illustrated in Figure 9.16.

Thus, in all five cases the moves $M \stackrel{\eta}{\mapsto} M_{1}$ and $M \stackrel{\zeta}{\mapsto} M_{2}$ almost commute.

Lemma 9.2. Let $M \stackrel{\eta}{\mapsto} M_{1}$ be a double split move with splitting $\backslash$-mirror $\mu_{1}$ and splitting /-mirror $\mu_{2}$, and let $M \stackrel{\zeta}{\mapsto} M_{2}$ be a type I split move associated with a splitting route $\omega_{2}=(\nu, q)$. Let also $x$ and $y$ be the respective occupied levels of $M$ that are split by these moves.

Then the moves $M \stackrel{\eta}{\mapsto} M_{1}$ and $M \stackrel{\zeta}{\mapsto} M_{2}$ are friendly to each other unless one of the following situations occurs:

(1) we have $\mu_{2}=\nu$ and $x \neq y$; 


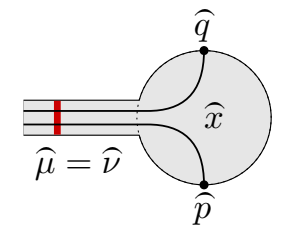

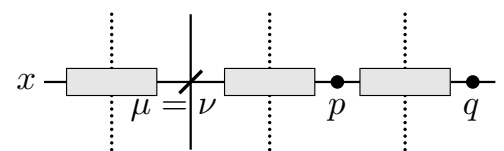

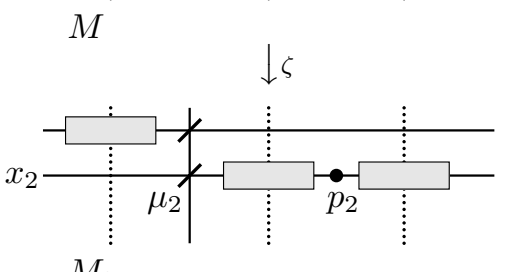

$M_{2}$

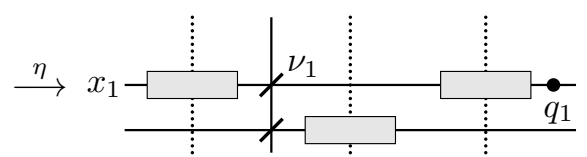

$M_{1}$

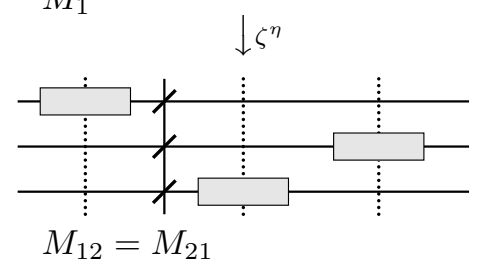

Figure 9.14. Proof of Lemma 9.1 in the case $x=y, \mu=\nu$ are of type ' $/$ '

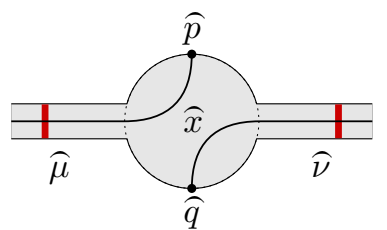

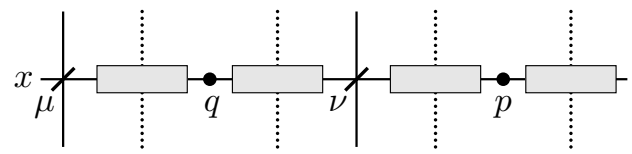

M

$\downarrow \zeta$

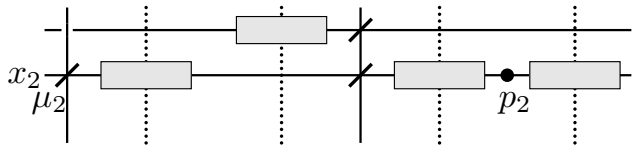

$M_{2}$

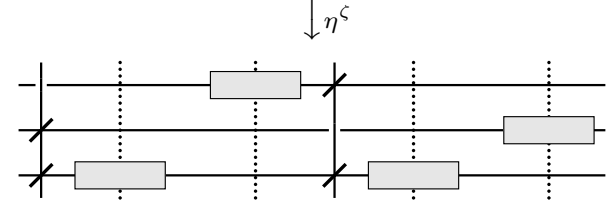

$M_{21}$

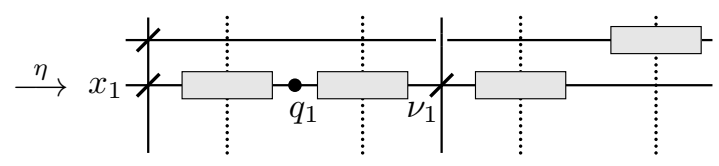

$M_{1}$

Figure 9.15. Proof of Lemma 9.1 in the case $x=y, \mu \neq \nu$ are of type ' $/$ ' $, q \in(\mu ; \nu), p \in(\nu ; \mu)$

(2) we have $x=y, \mu_{2} \neq \nu$, and the pairs $\left\{\mu_{1}, \mu_{2}\right\}$ and $\{\nu, q\}$ interleave. This is equivalent to saying that $\widehat{\omega}_{2}$ has an unavoidable intersection with a splitting path associated with some (and then any) type II splitting route starting from $\left(\mu_{1}, \mu_{2}\right)$.

If the moves $M \stackrel{\eta}{\mapsto} M_{1}$ and $M \stackrel{\zeta}{\mapsto} M_{2}$ are friendly to each other, then they almost commute.

We omit the proof, which is completely analogous to the proof of Lemma 9.1 


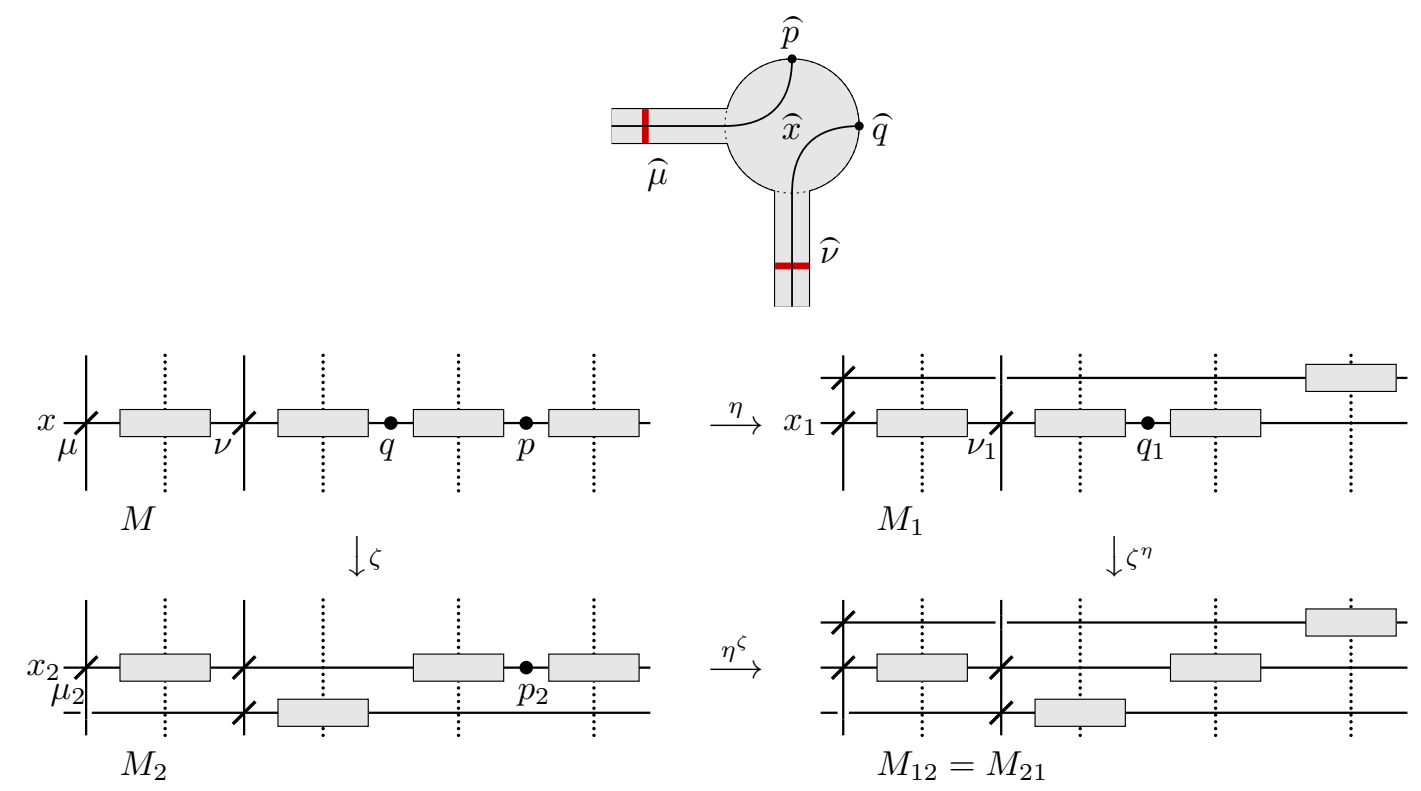

Figure 9.16. Proof of Lemma 9.1 in the case $x=y, \mu \neq \nu$ are of type ' $/, q \in(\nu ; \mu), p \in(q ; \mu)$

Lemma 9.3. Any non-special generalized type II split move commutes with any jump move.

We omit the easy proof.

The following is an analogue of Lemma 9.3 for the case of a special generalized split move.

Lemma 9.4. Let $M \stackrel{\eta}{\mapsto} M_{1}$ be a special generalized type II split move, and let $M \stackrel{\zeta}{\mapsto} M_{2}$ be a jump move. Let also $M \stackrel{\eta_{1}}{\longmapsto} M_{1}^{\prime}$ be the first move of a canonical decomposition of $M \stackrel{\eta}{\longmapsto} M_{1}$, which is a type I extension move, and let $M_{1}^{\prime} \stackrel{\eta_{2}}{\longmapsto} M_{1}$ be the 'remaining part' of the decomposition, which is a generalized type II split move such that $\eta=\eta_{2} \circ \eta_{1}$. Denote by $C$ the set of all boundary circuits preserved by both moves $M \stackrel{\eta}{\mapsto} M_{1}$ and $M \stackrel{\zeta}{\mapsto} M_{2}$.

Suppose that the move $M \stackrel{\eta_{1}}{\longmapsto} M_{1}^{\prime}$ is friendly to $M \stackrel{\zeta}{\mapsto} M_{2}$. Then the move $M_{2} \stackrel{\eta^{\zeta}}{\longmapsto} M_{21}$ can be chosen so that it preserves all boundary circuits in $C$, and the transformation $M_{1} \stackrel{\eta^{\zeta} \circ \zeta \circ \eta^{-1}}{\longrightarrow} M_{21}$ admits a C-neat decomposition into jump moves.

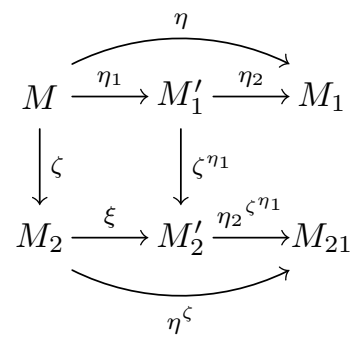

Figure 9.17. Scheme of the moves in the proof of Lemma 9.4

Proof. By the assumption of the lemma there is a well defined jump move $M_{1}^{\prime} \stackrel{\zeta^{\eta_{1}}}{\longrightarrow} M_{2}^{\prime}$. One can see that $M_{2} \stackrel{\xi}{\mapsto} M_{2}^{\prime}$, where $\xi=\zeta^{\eta_{1}} \circ \eta_{1} \circ \zeta^{-1}$, is a type I extension move (consult Figure 9.17 for the scheme). The moves $M_{1}^{\prime} \stackrel{\eta_{2}}{\longmapsto} M_{1}$ and $M_{1}^{\prime} \stackrel{\zeta^{\eta_{1}}}{\longmapsto} M_{2}^{\prime}$ commute by Lemma 9.3 , so we can define the non-special 
generalized type II split move $M_{2}^{\prime} \stackrel{\eta_{2}^{\zeta_{1}}}{\longmapsto} M_{21}$ so that the transformation $M_{1} \stackrel{\eta_{2}^{\zeta_{1}} \circ \zeta^{\eta_{1}} \circ \eta_{2}^{-1}}{\longmapsto} M_{21}$ admits a $C$-neat decomposition into jump moves. It remains to note that $M_{2} \stackrel{\eta_{2}^{\zeta_{1}} \circ{ }^{\circ}}{\longrightarrow} M_{21}$ is a special generalized type II split move that can be taken for $M_{2} \stackrel{\eta^{\zeta}}{\longmapsto} M_{21}$.

Lemma 9.5. Let $M \stackrel{\eta}{\mapsto} M_{1}$ be a generalized type II split move associated with a non-special type II splitting route $\omega$ in $M$, and let $M \stackrel{\zeta}{\mapsto} M_{2}$ be a type I elimination move such that the mirror being removed does not appear in $\omega$. Then these moves are friendly to one another and, moreover, they commute.

We omit the easy proof.

Lemma 9.6. Let $M \stackrel{\eta}{\mapsto} M_{1}$ be a generalized type II split move associated with a non-special type II splitting route $\omega$ in $M$, and let $M \stackrel{\zeta}{\mapsto} M_{2}$ be a type I split move that is friendly to $M \stackrel{\eta}{\mapsto} M_{1}$. Then the move $M \stackrel{\eta}{\mapsto} M_{1}$ is also friendly to $M \stackrel{\zeta}{\mapsto} M_{2}$, and the moves almost commute with one another.

Proof. Let $C$ be the set of all boundary circuits of $M$ preserved by both moves $M \stackrel{\eta}{\mapsto} M_{1}$ and $M \stackrel{\zeta}{\mapsto} M_{2}$, and let $\omega_{1}=(\nu, q)$ be the type I splitting route with which the move $M \stackrel{\zeta}{\mapsto} M_{2}$ is associated. Since this move is friendly to the move $M \stackrel{\eta}{\mapsto} M_{1}$ the path $\widehat{\omega}_{1}$ has no unavoidable intersections with $\widehat{\omega}$, and, moreover, the mutual position of $\widehat{\omega}$ and $\widehat{\omega}_{1}$ is essentially unique. This implies that the move $M \stackrel{\eta}{\mapsto} M_{1}$ is also friendly to $M \stackrel{\zeta}{\mapsto} M_{2}$. We will now show, by induction in the length of $\omega$, that the moves $M \stackrel{\eta}{\mapsto} M_{1}$ and $M \stackrel{\zeta}{\mapsto} M_{2}$ almost commute.

Let $\omega=\left(\mu_{1}, \mu_{2}, \ldots, \mu_{k}, p\right)$. If $k=1$, then $M \stackrel{\eta}{\mapsto} M_{1}$ is an ordinary type II split move, whose almost commutation with $M \stackrel{\zeta}{\mapsto} M_{2}$ follows from Lemma 9.1 .

Now assume that $k>1$ and the lemma is proved for generalized type II split moves associated with shorter non-special splitting routes than $\omega$. Let $M \stackrel{\eta_{1}}{\longmapsto} M^{\prime}$ be the first move of a canonical decomposition of the move $M \stackrel{\eta}{\longmapsto} M_{1}$, and let $M^{\prime} \stackrel{\eta_{2}}{\longmapsto} M_{1}$ be the 'remaining part' of this decomposition, that is, a generalized type II split move such that $M \stackrel{\eta}{\longmapsto} M_{1}$ is the composition of $M \stackrel{\eta_{1}}{\longmapsto} M^{\prime}$ and $M^{\prime} \stackrel{\eta_{2}}{\longmapsto} M_{1}$. Let also $M_{2} \stackrel{\xi}{\mapsto} M_{2}^{\prime}$ be the first move of a canonical decomposition of the move $M_{2} \stackrel{\eta^{\zeta}}{\longmapsto} M_{21}$, and $M^{\prime} \stackrel{\chi}{\longmapsto} M_{2}^{\prime}$ be the composition of the moves $M^{\prime} \stackrel{\eta_{1}^{-1}}{\longmapsto} M, M \stackrel{\zeta}{\longmapsto} M_{2}$, and $M_{2} \stackrel{\xi}{\mapsto} M_{2}^{\prime}$.

By construction, the transformation $M^{\prime} \stackrel{\chi}{\longmapsto} M_{2}^{\prime}$ is friendly to the generalized type II split move $M^{\prime} \stackrel{\eta_{2}}{\longmapsto}$ $M_{1}$, and the move $M_{2}^{\prime} \stackrel{\eta_{2}{ }^{x}}{\longmapsto} M_{21}$ is the 'remaining part' of a canonical decomposition of the move $M_{2} \stackrel{\eta^{\zeta}}{\longmapsto}$ $M_{21}$. Thus, we have the commutative diagram of moves shown in Figure 9.18 on the left, in which all the transformations preserve the boundary circuits in $C$.
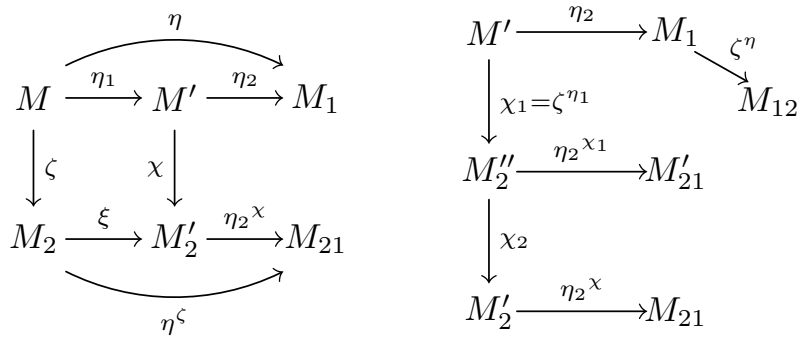

FIGURE 9.18. The scheme of the induction step in the proof of Lemma 9.6

Suppose that the moves $M \stackrel{\eta_{1}}{\longmapsto} M^{\prime}$ and $M \stackrel{\zeta}{\longmapsto} M_{2}$ almost commute. Then $\xi=\eta_{1}{ }^{\zeta}$, and the move $M^{\prime} \stackrel{\chi}{\longmapsto}$ $M_{2}^{\prime}$ admits a $C$-neat decomposition into a type I split move of the form $M^{\prime} \stackrel{\chi_{1}}{\longmapsto} M_{2}^{\prime \prime}$ with $\chi_{1}=\zeta^{\eta_{1}}$, followed by a transformation $M_{2}^{\prime \prime} \stackrel{\chi_{2}}{\longmapsto} M_{2}^{\prime}$ that can be further $C$-neatly decomposed into jump moves. 
The move $M^{\prime} \stackrel{\zeta^{\eta_{1}}}{\longmapsto} M_{2}^{\prime \prime}$ is friendly to $M^{\prime} \stackrel{\eta_{2}}{\longmapsto} M_{1}$, and the latter is a generalized type II split move associated with a non-special type II splitting route which is shorter than $\omega$.

By the induction hypothesis there is a generalized type II split move $M_{2}^{\prime \prime} \stackrel{\eta_{2}{ }^{\chi_{1}}}{\longmapsto} M_{21}^{\prime}$ and a type I split move $M_{1} \stackrel{\chi_{1}^{\eta_{2}}=\zeta^{\eta}}{\longrightarrow} M_{12}$, both preserving the boundary circuits in $C$, such that $M_{21}^{\prime}$ and $M_{12}$ are related by a sequence of jump moves also preserving the boundary circuits in $C$ and realizing the morphism $\zeta^{\eta} \circ$ $\eta_{2} \circ \chi_{1}^{-1} \circ\left(\eta_{2} \chi_{1}\right)^{-1}$.

It follows from Lemma 9.3 that the moves $M_{2}^{\prime \prime} \stackrel{\eta_{2} \chi_{1}}{\longmapsto} M_{21}^{\prime}$ and $M_{2}^{\prime \prime} \stackrel{\chi_{2}}{\longmapsto} M_{2}^{\prime}$ commute, and by construction we have $\eta_{2}{ }^{\chi}=\left(\eta_{2}{ }^{\chi_{1}}\right)^{\chi_{2}}$. Therefore, the transformation $M_{21}^{\prime} \stackrel{\eta_{2}{ }^{\chi} \circ \chi_{2} \circ\left(\eta_{2}{ }^{\chi_{1}}\right)^{-1}}{\longmapsto} M_{21}$ also admits a $C$-neat decomposition into jump moves. We see from here that the moves $M \stackrel{\eta}{\mapsto} M_{1}$ and $M \stackrel{\zeta}{\mapsto} M_{2}$ almost commute.

Now we turn to the case when the moves $M \stackrel{\eta_{1}}{\longmapsto} M^{\prime}$ and $M \stackrel{\zeta}{\longmapsto} M_{2}$ do not almost commute. Suppose that $\omega$ is a single-headed splitting route, so $M \stackrel{\eta_{1}}{\longmapsto} M^{\prime}$ is a type I split move.

The splitting paths $\widehat{\left(\mu_{k}, p\right)}$ and $\widehat{\omega}_{1}$ don't have an unavoidable intersection, since neither do $\widehat{\omega}$ and $\widehat{\omega}_{1}$. By Lemma 9.1 the only reason for $M \stackrel{\eta_{1}}{\longmapsto} M^{\prime}$ and $M \stackrel{\zeta}{\longmapsto} M_{2}$ to be unfriendly to one another is an ambiguity in the mutual position of $\widehat{\left(\mu_{k}, p\right)}$ and $\widehat{\omega}_{1}$. This is the issue illustrated in Figure 9.11(b).

However, by the assumption of the lemma the mutual position of $\widehat{\omega}$ and $\widehat{\omega}_{1}$ is not ambiguous if they are chosen disjoint. Let us fix such a choice. Then there is a unique splitting route $\omega_{1}^{\prime}=\left(\nu^{\prime}, q^{\prime}\right)$ in $M^{\prime}$ such that $h_{M}^{M^{\prime}}\left(\widehat{\omega}_{1}\right)=\widehat{\omega}_{1}^{\prime}$. We define the move $M^{\prime} \stackrel{\chi_{1}}{\longmapsto} M_{2}^{\prime \prime}$ as a type I split move associated with $\omega_{1}^{\prime}$ and preserving all boundary circuits in $C$.

As one can learn from Figure 9.19 the diagrams $M_{2}^{\prime \prime}$ and $M_{2}^{\prime}$ are combinatorially equivalent, so one of them can be obtained from the other by jump moves adjusting the positions of the occupied levels without changing the combinatorial type. In this figure we denote by $p^{\prime}, p_{2}$, and $p_{2}^{\prime}$ the snip points of the generalized type II split moves $M^{\prime} \stackrel{\eta_{2}}{\longmapsto} M_{1}, M_{2} \stackrel{\eta^{\zeta}}{\longmapsto} M_{21}$, and $M_{2}^{\prime} \stackrel{\eta_{2} \times}{\longmapsto} M_{21}$, respectively. We also denote by $x$ and $y$ the occupied levels of $M$ that are split by the moves $M \stackrel{\eta_{1}}{\longmapsto} M^{\prime}$ and $M \stackrel{\zeta}{\mapsto} M_{2}$, respectively.

Figure 9.19 shows, up to symmetry, what happens in the particular case when $\mu_{k-1} \neq \mu_{k}$. It is, however, possible that $\mu_{k-1}=\mu_{k}$. Since there is no ambiguity in the mutual position of $\widehat{\omega}$ and $\widehat{\omega}_{1}$, there must be another arc in $\widehat{y} \cap \widehat{\omega}$ separating $\widehat{q}$ from the 'tail' of $\widehat{\omega}$ in $\widehat{y}$. This means that, for some $j \notin\{k, k-1\}$, we have $\mu_{j}=\mu_{k}$ and, for some $\epsilon \in\{-1,1\}$, we have $\mu_{j+\epsilon} \in y, \mu_{j+\epsilon} \neq \mu_{k}$. One should then substitute $\mu_{j+\epsilon}$ for $\mu_{k-1}$ in Figure 9.19 .

If $\omega$ is a double-headed splitting route, then the reasoning is almost the same with the roles of $\mu_{k}$ and $\mu_{k-1}$ played by $\mu_{k-1}$ and $\mu_{k-2}$, respectively. In place of $p$ we have the mirror $\mu_{k}$, which has two successors in each of $M^{\prime}, M_{2}^{\prime}$, and $M_{2}^{\prime \prime}$, and Lemma 9.2 should be used instead of Lemma 9.1 .

Thus, the scheme described above and sketched in Figure 9.18 works perfectly in the case when the moves $M \stackrel{\eta_{1}}{\longmapsto} M^{\prime}$ and $M \stackrel{\zeta}{\mapsto} M_{2}$ do not almost commute, too, though $\chi$ and $\xi$ are not interpreted as $\zeta^{\eta_{1}}$ and $\eta_{1}$, respectively. So, the almost commutation of the moves $M \stackrel{\eta}{\mapsto} M_{1}$ and $M \stackrel{\zeta}{\mapsto} M_{2}$ also holds in this case.

Lemma 9.7. Let $M \stackrel{\eta}{\mapsto} M_{1}$ be a generalized type II split move associated with a non-special type II splitting route $\omega$ in $M$, and let $M \stackrel{\zeta}{\mapsto} M_{2}$ be a type I elementary bypass removal. Denote by $\mu_{0}$ the $\backslash$-mirror removed by $M \stackrel{\zeta}{\mapsto} M_{2}$ and by $c$ the inessential boundary circuit of $M$ hitting $\mu_{0}$ and having the form $\partial r$, where $r$ is a rectangle with interior disjoint from $E_{M}$.

Suppose that the following conditions hold:

(1) the mirror $\mu_{0}$ does not appear in $\omega$;

(2) either $\omega$ is double-headed, or $\omega$ is single-headed and the snip point of the move $M \stackrel{\eta}{\mapsto} M_{1}$ does not lie on $c$.

Then the moves $M \stackrel{\eta}{\mapsto} M_{1}$ and $M \stackrel{\zeta}{\mapsto} M_{2}$ are friendly to one another and, moreover, they commute. 


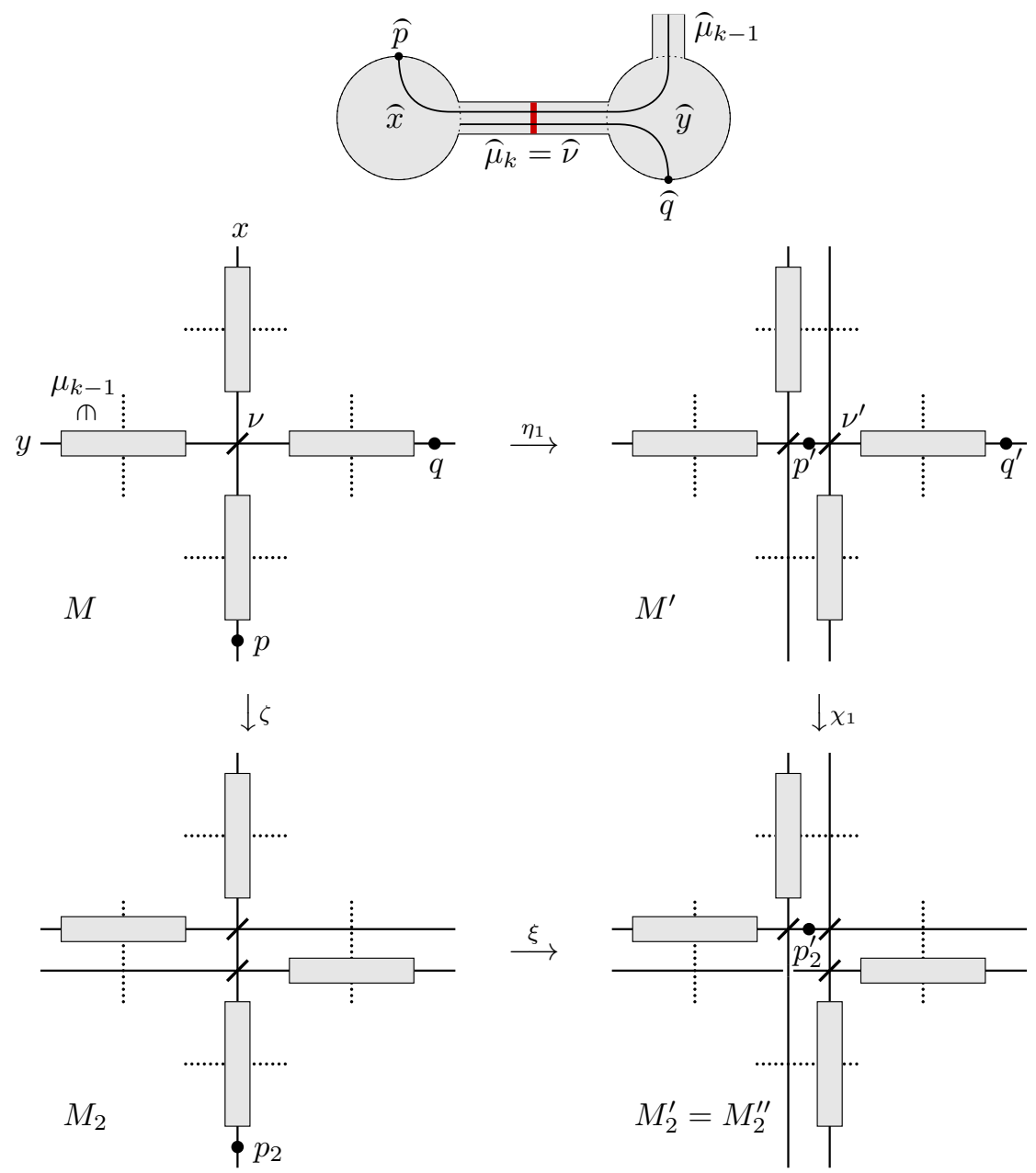

Figure 9.19. Induction step in the proof of Lemma 9.6 in the case when the moves $M \stackrel{\eta_{1}}{\longmapsto} M^{\prime}$ and $M \stackrel{\zeta}{\longmapsto} M_{2}$ do not almost commute

Proof. The move $M \stackrel{\zeta}{\mapsto} M_{2}$ is clearly friendly to $M \stackrel{\eta}{\mapsto} M_{1}$, since it only removes a single mirror that does not appear in $\omega$.

If $r$ is a rectangle such that $\partial r \in \partial M$ and $\operatorname{int}(r) \cap E_{M}=\varnothing$, then any double split move $M \mapsto M^{\prime}$ takes the boundary circuit $\partial r$ to a boundary circuit of the same kind, that is $\partial r^{\prime} \in \partial M^{\prime}, \operatorname{int}\left(r^{\prime}\right) \cap E_{M^{\prime}}=\varnothing$, where $r^{\prime}$ is a rectangle. This follows easily from the definition of a double split move.

An ordinary split move can 'destroy' $\partial r$ only if the snip point of the move lies on $\partial r$. Thus, the first move in a canonical decomposition does not destroy $c$. The subsequent moves don't do so either because they are split moves whose snip point lies in the splitting gap of the previous move, and this splitting gap cannot be a part of $c$ or of the boundary circuit into which $c$ is transformed. One can see that the removal of $\mu_{0}$ or its successor is a type I elementary bypass removal for all diagrams arising in the canonical decomposition of the move $M \stackrel{\eta}{\mapsto} M_{1}$, and this bypass removal commutes with the ordinary split moves from the canonic decomposition. The claim follows.

\subsection{Similarity of splitting routes.}

Definition 9.11. Let $\omega=\left(\mu_{1}, \mu_{2}, \ldots, \mu_{k}, p\right)$ and $\omega^{\prime}$ be type II splitting routes in a mirror diagram $M$. We say that $\omega^{\prime}$ is obtained from $\omega$ by a pulling tight operation, and that $\omega$ is obtained from $\omega^{\prime}$ by a 
loosening operation, if, for some $i \in\{2, \ldots, k-1\}$, we have $\mu_{i}=\mu_{i+1}$, and $\omega^{\prime}$ is the result of removing the $i$ th and the $(i+1)$ st entries in $\omega$.

Definition 9.12. Let $\omega=\left(\mu_{1}, \mu_{2}, \ldots, \mu_{k}, p\right)$ and $\omega^{\prime}$ be single-headed type II splitting routes in a mirror diagram $M$. We say that $\omega^{\prime}$ is obtained from $\omega$ by a tail shrinking operation, and that $\omega$ is obtained from $\omega^{\prime}$ by a tail stretching operation, if $\omega^{\prime}$ has the form $\left(\mu_{1}, \ldots, \mu_{k-1}, q\right)$, and one of the unions of intervals $\left(\mu_{k} ; p\right) \cup\left(q ; \mu_{k}\right)$ and $\left(p ; \mu_{k}\right) \cup\left(\mu_{k} ; q\right)$ does not contain mirrors of $M$.

Observe that each union of intervals mentioned in Definition 9.12 is composed of two mutually orthogonal straight line portions of the same boundary circuit of $M$.
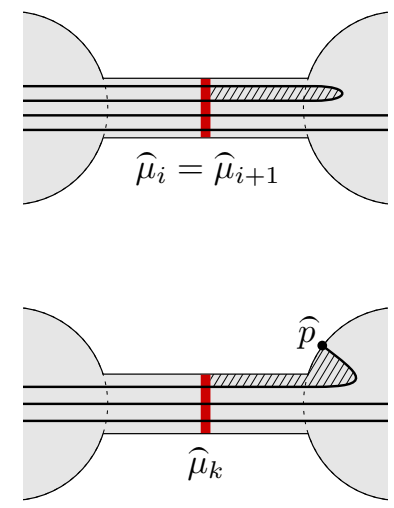
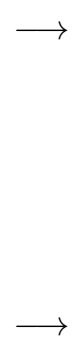

FIGURE 9.20. Pulling tight and tail shrinking operations as (half-)bigon reductions

Definitions 9.11 and 9.12 have a simple topological meaning, which we now explain.

Let $M$ be a mirror diagram, and let $\left(\delta_{+}, \delta_{-}\right)$be a canonic dividing configuration of $\overparen{M}$ (see Definition (5.11) such that $\delta_{+} \cup \delta_{-}$is contained in the union of 1-handles of $\overparen{M}$. If $\omega=\left(\mu_{1}, \ldots, \mu_{k}, p\right)$ is a type II splitting route, and the associated splitting path $\widehat{\omega}$ is chosen to have minimal possible number of intersections with $\delta_{+} \cup \delta_{-}$, then each arc $\widehat{\omega}^{i}, i=1, \ldots, k$, from Definitions 9.3 and 9.4 intersects $\delta_{+} \cup \delta_{-}$ exactly once. We say in this case that $\widehat{\omega}$ is chosen in the optimal way.

Let $\omega \mapsto \omega^{\prime}$ be a pulling tight operation, and let $\widehat{\omega}$ and $\widehat{\omega}^{\prime}$ be chosen in the optimal way. One can see that, topologically, the transition from $\widehat{\omega}$ to $\widehat{\omega}^{\prime}$ is a reduction of a bigon of $\widehat{\omega}$ and $\delta_{-}$. Similarly, if $\omega \mapsto \omega^{\prime}$ is a tail shrinking operation then the transition from $\widehat{\omega}$ to $\widehat{\omega}^{\prime}$ is a reduction of a half-bigon of $\widehat{\omega}$ and $\delta_{-}$. This is illustrated in Figure 9.20 .

Definition 9.13. A type II splitting route $\omega=\left(\mu_{1}, \ldots, \mu_{k}, p\right)$ and the associated type II splitting path are said to be reduced if $\mu_{i} \neq \mu_{i+1}$ for all $i=2, \ldots, k-1$, and one of the following two conditions holds:

(1) $\omega$ is single-headed and both intervals $\left(\mu_{k} ; p\right)$ and $\left(p ; \mu_{k}\right)$ contain at least one mirror of $M$;

(2) $\omega$ is double-headed.

Proposition 9.6. For any type II splitting route $\omega$ in a mirror diagram $M$, there exists a reduced type II splitting route in $M$ obtained from $\omega$ by a finite sequence of pulling tight and tail shrinking operations.

Proof. Pulling tight and tail shrinking operations shorten the corresponding splitting route, hence it suffices to show that, whenever a type II splitting route is not reduced, one of these operations can be applied. The latter would be obvious without Condition (2d) in Definitions 9.3 and 9.4 This condition is designed to be robust under pulling tight and shrinking operations, so it does not prohibit any of them. The claim follows.

Lemma 9.8. Let $M \mapsto M^{\prime}$ be a generalized type II split move associated with a type II splitting route $\omega$, and let $\omega^{\prime}$ be a type II splitting route obtained from $\omega$ either by a pulling tight or tail shrinking operation. Let also $C$ be the set of boundary circuits of $M$ preserved by the move $M \mapsto M^{\prime}$. Then there is a choice 
of the generalized type II split move $M \mapsto M^{\prime \prime}$ associated with $\omega^{\prime}$ such that all the boundary circuits in $C$ are also preserved by this move.

Proof. If $\omega^{\prime}$ is not special, the statement follows from Proposition 9.5 and the obvious fact that pulling tight and tail shrinking operations cannot take a splitting route that does not separate $C$, to a splitting route that does.

Now suppose that $\omega^{\prime}$ is special. This means that $\omega$ and $\omega^{\prime}$ have the form $\left(\mu_{1}, \mu_{2}, \mu_{2}, \mu_{3}, p\right)$ and $\left(\mu_{1}, \mu_{3}, p\right)$, respectively. There is a boundary circuit $c$ that hits $\mu_{2}$ and does not belong to $C$, since at least one of the boundary circuits hitting $\mu_{2}$ is modified by the move $M \mapsto M^{\prime}$. Choose the auxiliary mirror $\mu_{0}$ for $M \mapsto M^{\prime \prime}$ on $c$ in a vicinity of $\mu_{2}$. Then the extension that introduces $\mu_{0}$ will not modify any boundary circuit in $C$, and the splitting path $\left(\mu_{1}, \mu_{0}, \mu_{0}, \mu_{3}, p\right)$ will not separate $C$. The claim follows.

Definition 9.14. Let $M$ be a mirror diagram, and let $\omega$ and $\omega^{\prime}$ be type II splitting routes in $M$ having the form $\omega=\left(\mu_{1}, \mu_{2}, \ldots, \mu_{k}, p\right)$ and $\omega^{\prime}=\left(\mu_{1}^{\prime}, \mu_{1}^{\prime \prime}, \mu_{2}, \ldots, \mu_{k}, p\right)$. Assume that $M$ has an inessential boundary circuit $c$ such that the following conditions hold:

(1) $c$ has the form of the boundary of a rectangle $r$, and $\operatorname{int}(r) \cap E_{M}=\varnothing$;

(2) $\mu_{1}, \mu_{1}^{\prime}, \mu_{1}^{\prime \prime} \in c$;

(3) $p \notin c$.

Then we say that $\omega$ and $\omega^{\prime}$ are obtained from one another by a head wandering operation.

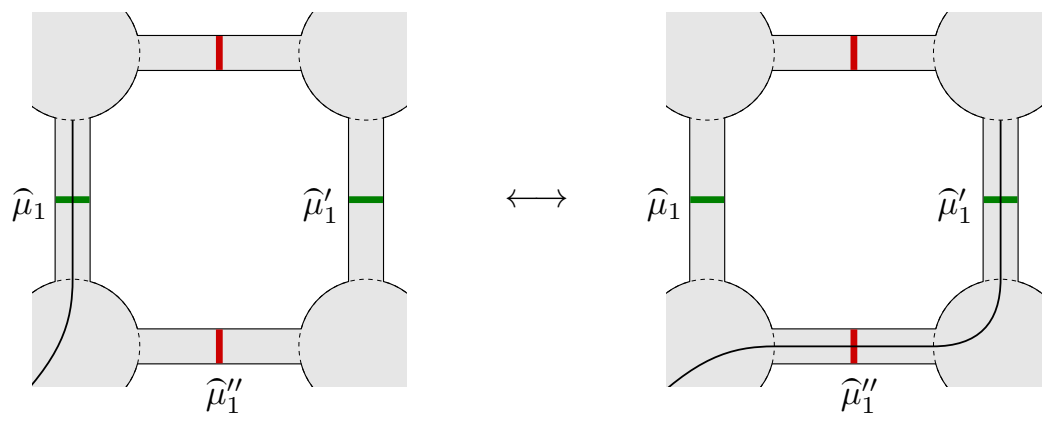

FiguRE 9.21. Head wandering

The idea of this move is illustrated in Figure 9.21

Definition 9.15. Let $M$ be a mirror diagram, and let $\omega=\left(\mu_{1}, \mu_{2}, \ldots, \mu_{k}, p\right)$ be a single-headed type II splitting route in $M$. Let also $\omega^{\prime}$ be a double-headed type II splitting route in $M$ of the form $\omega^{\prime}=$ $\left(\mu_{1}, \mu_{2}, \ldots, \mu_{k}, \mu_{k+1}, p^{\prime}\right)$. Assume that $M$ has an inessential boundary circuit $c$ such that the following conditions hold:

(1) $c$ has the form of the boundary of a rectangle $r$, and $\operatorname{int}(r) \cap E_{M}=\varnothing$;

(2) $\mu_{k+1}, p, p^{\prime} \in c$.

Then we say $\omega^{\prime}$ is obtained from $\omega$ by a tail-to-head conversion, and $\omega$ is obtained from $\omega^{\prime}$ by a head-to-tail conversion.

The tail-to-head and head-to-tail conversion moves are illustrated in Figure 9.22 .

Definition 9.16. Two splitting routes $\omega$ and $\omega^{\prime}$ are said to be similar if there is a sequence of splitting routes

$$
\omega=\omega_{0}, \omega_{1}, \ldots, \omega_{N}=\omega^{\prime}
$$

in which $\omega_{i}$ and $\omega_{i+1}$ are either equivalent or obtained from each other by one of the transformations introduced in Definitions 9.11, 9.12, 9.14, and 9.15, for all $i=0, \ldots, N-1$. 

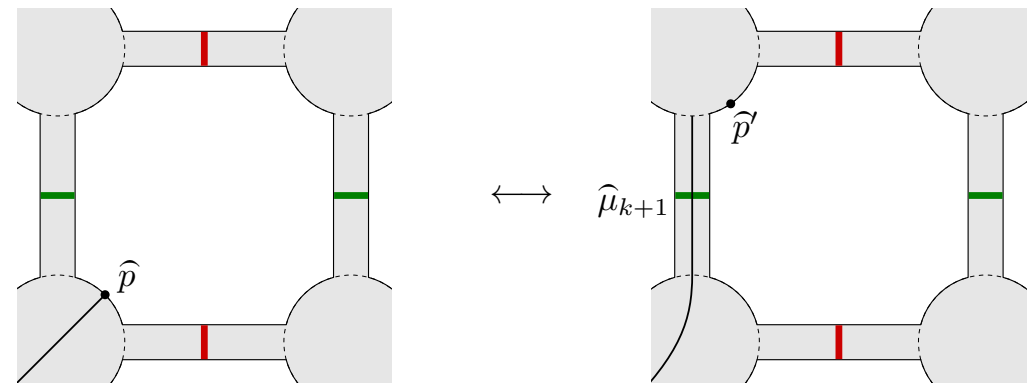

FiguRE 9.22. Tail-to-head and head-to-tail conversions

Proposition 9.7. Let $\omega$ and $\omega^{\prime}$ be similar type II splitting routes in a mirror diagram $M$, and let $M \stackrel{\eta}{\mapsto}$ $M_{1}$ and $M \stackrel{\zeta}{\mapsto} M_{2}$ be generalized type II split moves associated with $\omega$ and $\omega^{\prime}$, respectively. Let also $C$ be the set of essential boundary circuits of $M$ preserved by both moves.

Then the transformation $M_{1} \stackrel{\zeta \circ \eta^{-1}}{\longmapsto} M_{2}$ admits a $C$-neat decomposition into type I elementary moves.
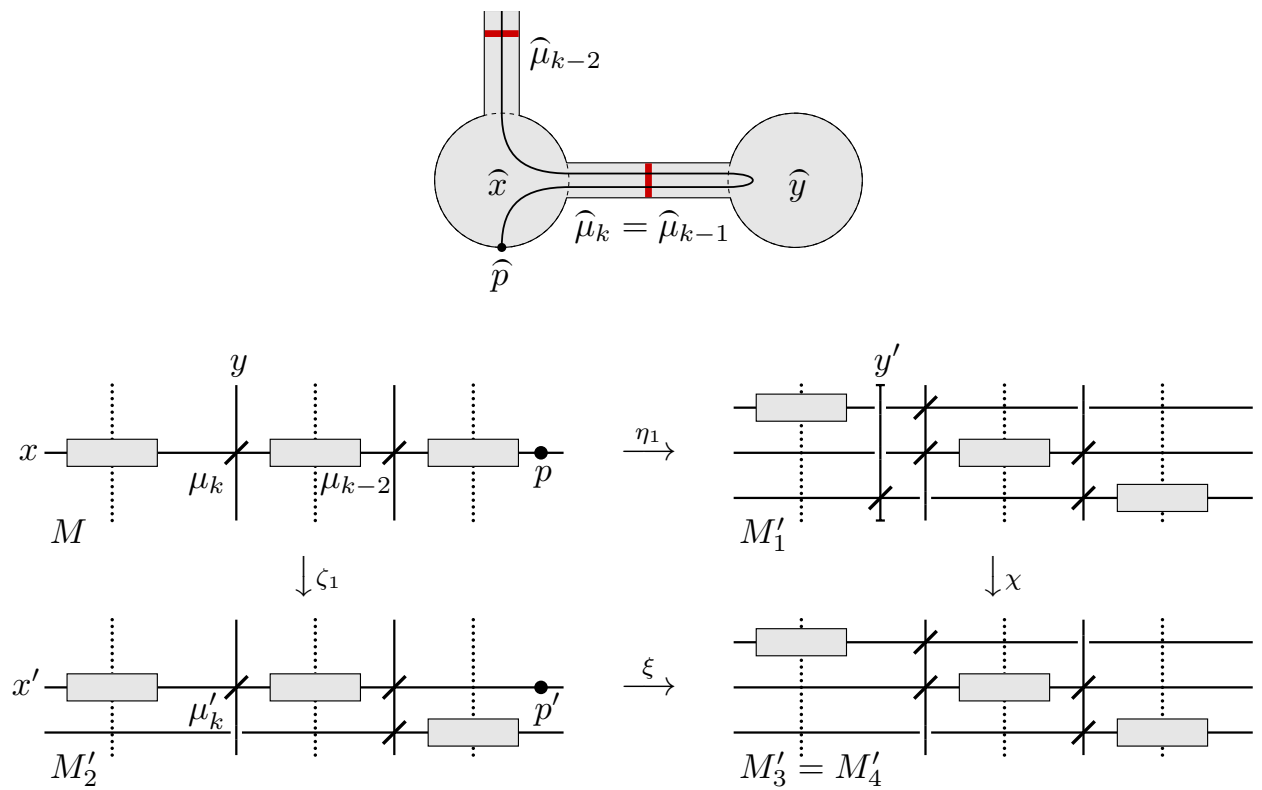

Figure 9.23. Proof of Proposition 9.7 in the pulling tight case, $\omega$ and $\omega^{\prime}$ are singleheaded, $k>3$

Proof. It suffices to prove the proposition in the case when $\omega \mapsto \omega^{\prime}$ is one of the transformations introduced in Definitions 9.11, 9.12, 9.14, and 9.15, and in the case when $\omega=\omega^{\prime}$ is a special route. Indeed, let $\omega_{0}, \ldots, \omega_{N}$ be as in Definition 9.16, and let $M \stackrel{\eta_{i}}{\longmapsto} M_{i}^{\prime}$ be a generalized type II split move associated with $\omega_{i}, i=0,1, \ldots, N$, and preserving all the boundary circuits in $C$.

Generalized type II merge moves are safe-to-postpone, which implies, by induction in $N$, that

$$
M_{0}^{\prime} \stackrel{\eta_{1} \circ \eta_{0}^{-1}}{\longmapsto} M_{1}^{\prime} \stackrel{\eta_{2} \circ \eta_{1}^{-1}}{\longmapsto} \ldots \stackrel{\eta_{N} \circ \eta_{N-1}^{-1}}{\longmapsto} M_{N}^{\prime} \stackrel{\eta_{N}^{-1}}{\longmapsto} M
$$

is a $C$-neat decomposition of $M_{0}^{\prime} \stackrel{\eta_{0}^{-1}}{\longmapsto} M$. Therefore, the first $N$ moves in this sequence form a $C$-neat decomposition of the move $M_{1}=M_{0}^{\prime} \stackrel{\zeta \circ \eta^{-1}}{\longmapsto} M_{N}^{\prime}=M_{2}$. 
Thus, in what follows we assume $N=1$.

Case 1: $\omega \mapsto \omega^{\prime}$ is a pulling tight operation.

We use the notation from Definition [9.11, that is, we assume that $\omega^{\prime}$ is obtained from $\omega$ by removing the $i$ th and the $(i+1)$ st entries, which are equal. Suppose that $\omega$ and $\omega^{\prime}$ are single-headed.

The first $k-1-i$ steps of a canonical decomposition of the move $M \stackrel{\eta}{\mapsto} M_{1}$ coincide - combinatoriallywith those of a canonical decomposition of the move $M \stackrel{\zeta}{\mapsto} M_{2}$. This reduces the general case to the case when $k=i+1$, that is, when the two entries in $\omega$ being cancelled are the last two mirrors.

Let $M \stackrel{\eta_{1}}{\longmapsto} M_{1}^{\prime}$ be the composition of the first three split moves in a canonical decomposition of the generalized type II split move $M \stackrel{\eta}{\mapsto} M_{1}$, and let $M_{1}^{\prime} \stackrel{\eta_{2}}{\longmapsto} M_{1}$ be the remaining part of the decomposition. Let also $M \stackrel{\zeta_{1}}{\longmapsto} M_{2}^{\prime}$ be the first split move in a canonical decomposition of the move $M \stackrel{\zeta}{\longmapsto} M_{2}$, and $M_{2}^{\prime} \stackrel{\zeta_{2}}{\longmapsto}$ $M_{2}$ the remaining part.
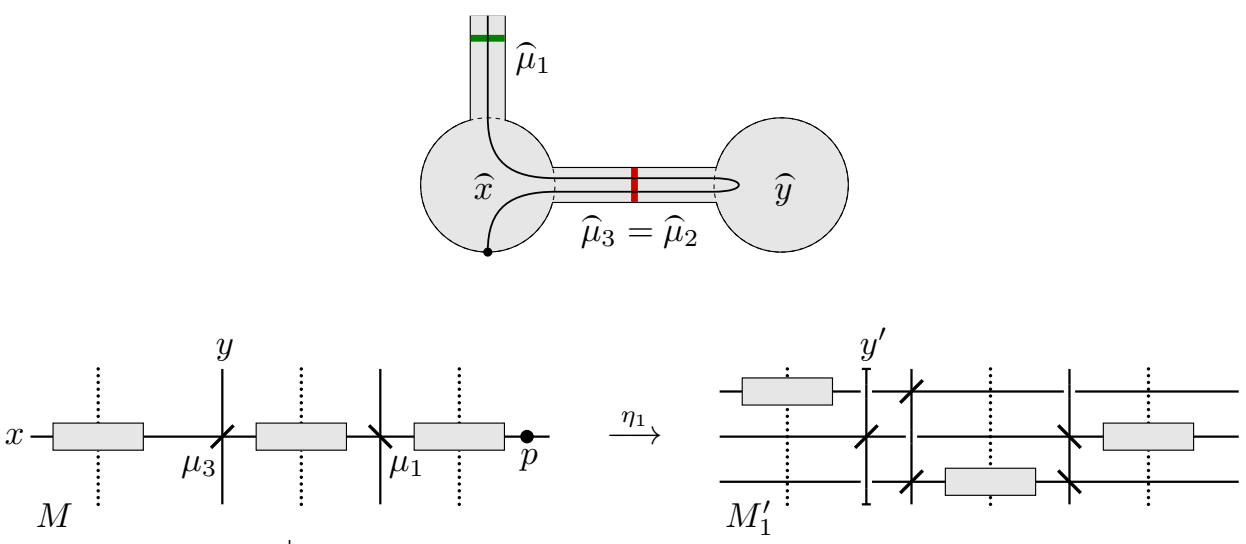

$\downarrow \varsigma_{1}$
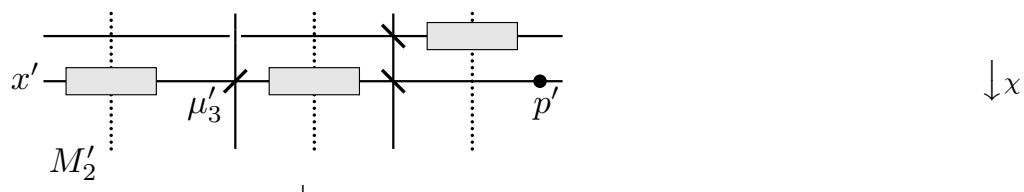

$\downarrow \xi$
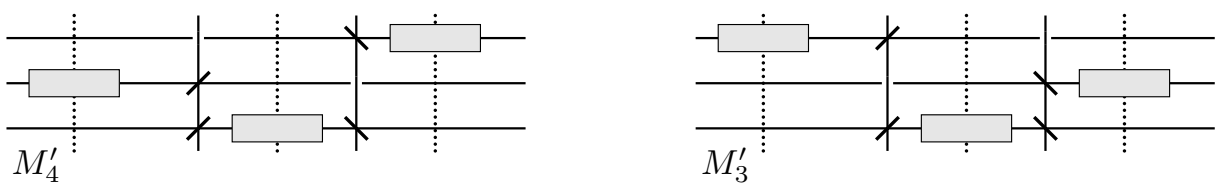

Figure 9.24. Proof of Proposition 9.7 in the pulling tight case, $\omega$ and $\omega^{\prime}$ are singleheaded, $k=3$

Denote by $x$ the occupied level of $M$ containing $\mu_{k}=\mu_{k-1}$ and $\mu_{k-2}$, and by $y$ the other occupied level passing through $\mu_{k}$. The occupied level $y$ has two successors in $M_{1}^{\prime}$ one of which $y^{\prime}$, say, carries only one mirror of $M_{1}^{\prime}$. Let $M_{1}^{\prime} \stackrel{\chi}{\mapsto} M_{3}^{\prime}$ be the elimination move that removes this mirror and the level $y^{\prime}$.

The occupied level $x$ has two successors in $M_{2}^{\prime}$ only one of which contains a successor of $\mu_{k}$. Denote these successors of $x$ and $\mu_{k}$ by $x^{\prime}$ and $\mu_{k}^{\prime}$, respectively. Denote also by $p^{\prime}$ a point on $x^{\prime}$ such that $\hat{p}^{\prime}$ is the image of an interior point of $\overparen{M}$ under $h_{M}^{M_{2}^{\prime}}$. Let $M_{2}^{\prime} \stackrel{\xi}{\mapsto} M_{4}^{\prime}$ be a type I split move associated with the splitting route $\left(\mu_{k}^{\prime}, p^{\prime}\right)$. We can choose it to preserve all boundary circuits in $C$.

We claim that the transformation $M_{3}^{\prime} \stackrel{\psi}{\longmapsto} M_{4}^{\prime}$, where $\psi=\xi \circ \zeta_{1} \circ \eta_{1}^{-1} \circ \chi^{-1}$, admits a $C$-neat decomposition into jump moves. Indeed, if $k>3$, then $\mu_{k-2}$ is of type ' $/$ ', and one can directly check that the combinatorial types of $M_{3}^{\prime}$ and $M_{4}^{\prime}$ just coincide. This is illustrated in Figure 9.23. If $k=3$, 
then $\mu_{k-2}=\mu_{1}$ is of type ' $\searrow$ ', and the combinatorial type of $M_{4}^{\prime}$ differs from that of $M_{3}^{\prime}$ only in the mutual position of two successors of $x$; see Figure 9.24 .

The moves $M_{1}^{\prime} \stackrel{\chi}{\longmapsto} M_{3}^{\prime}$ and $M_{2}^{\prime} \stackrel{\xi}{\mapsto} M_{4}^{\prime}$ are friendly to the moves $M_{1}^{\prime} \stackrel{\eta_{2}}{\longmapsto} M_{1}$ and $M_{2}^{\prime} \stackrel{\zeta_{2}}{\longmapsto} M_{2}$, respectively, so we can define the moves $M_{3}^{\prime} \stackrel{\eta_{2}{ }^{\chi}}{\longmapsto} M_{3}$ and $M_{4}^{\prime} \stackrel{\zeta_{2}{ }^{\xi}}{\longmapsto} M_{4}$, again preserving all the boundary circuits in $C$. The scheme of all the involved moves is shown in Figure 9.25.

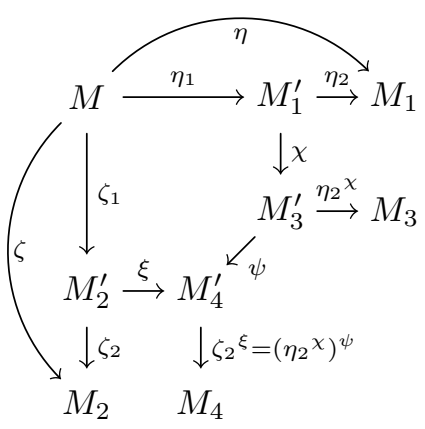

Figure 9.25. The scheme of the moves in the proof of Proposition 9.7, the pulling tight case, single-headed splitting routes

Now note that the splitting routes of the moves $M_{3}^{\prime} \stackrel{\eta_{2} \times}{\longmapsto} M_{3}$ and $M_{4}^{\prime} \stackrel{\zeta_{2}^{\xi}}{\longmapsto} M_{4}$ originate from the coincident starting portions of the splitting routes $\omega$ and $\omega^{\prime}$. One can see from this that $M_{4}^{\prime} \stackrel{\zeta_{2}{ }^{\xi}}{\longmapsto} M_{4}$ is actually the same thing as $M_{4}^{\prime} \stackrel{\left(\eta_{2}\right)^{\psi}}{\longrightarrow} M_{4}$. It follows from Lemmas 9.5, 9.3, 9.6, and 7.3 that all three transformations

$$
M_{1} \stackrel{\eta_{2}{ }^{\chi} \circ \chi \circ \eta_{2}^{-1}}{\longmapsto} M_{3}, \quad M_{3} \stackrel{\zeta_{2} \xi^{\circ} \circ \psi \circ\left(\eta_{2}\right)^{-1}}{\longmapsto} M_{4}, \quad M_{4} \stackrel{\zeta_{2} \circ \xi^{-1} \circ\left(\zeta_{2}\right)^{-1}}{\longmapsto} M_{2}
$$

admit $C$-neat decompositions into type I elementary moves.

Now suppose that $\omega$ and $\omega^{\prime}$ are double-headed. Then the first $k-2-i$ steps of canonical decompositions of the moves $M \stackrel{\eta}{\longmapsto} M_{1}$ and $M \stackrel{\zeta}{\longmapsto} M_{2}$ are the same from the combinatorial point of view, and if $k>i+2$, then the rest of the proof is literally the same as above, since, starting from the second step of a canonical decomposition, we deal with single-headed splitting routes.

Suppose $k=i+2>4$. The above scheme still works with the only change occurring in the numeration of the mirrors, which should be shifted by 1 . The discussed parts of the diagrams are shown in Figure 9.26

Finally, suppose that $k=4, \omega=\left(\mu_{1}, \mu_{2}, \mu_{2}, \mu_{3}, p\right)$, and $\omega^{\prime}=\left(\mu_{1}, \mu_{3}, p\right)$. Let $M \stackrel{\zeta_{1}}{\longmapsto} M_{2}^{\prime}$ be the first move in a canonical decomposition of $M \stackrel{\zeta}{\longmapsto} M_{2}$, and let $M_{2}^{\prime} \stackrel{\zeta_{2}}{\longmapsto} M_{2}$ be the remaining part of the decomposition. By definition, $M \stackrel{\zeta_{1}}{\longmapsto} M_{2}^{\prime}$ is an extension move, and $M_{2}^{\prime} \stackrel{\zeta_{2}}{\longmapsto} M_{2}$ is a generalized type II split move associated with the double-headed type II splitting path $\omega^{\prime \prime}=\left(\mu_{1}, \mu_{0}, \mu_{0}, \mu_{3}, p\right)$, where $\mu_{0}$ is the auxiliary mirror of the move $M \stackrel{\zeta}{\mapsto} M_{2}$.

At least one of the sequences $\left(\mu_{1}, \mu_{0}, \mu_{0}, \mu_{2}, \mu_{2}, \mu_{3}, p\right)$ and $\left(\mu_{1}, \mu_{2}, \mu_{2}, \mu_{0}, \mu_{0}, \mu_{3}, p\right)$ is a double-headed type II splitting route in $M_{2}^{\prime}$. We denote it by $\omega^{\prime \prime \prime}$. Which one of the two can be taken for $\omega^{\prime \prime \prime}$ depends on the cyclic order of $\mu_{0}, \mu_{1}, \mu_{2}, \mu_{3}$ on the occupied level of $M_{2}^{\prime}$ that contains all of them, and, in the case $\mu_{1}=\mu_{3}$, also on the position of $p$. Both splitting routes $\omega$ and $\omega^{\prime \prime}$ are obtained from $\omega^{\prime \prime \prime}$ by pulling tight operations.

Let $M_{2}^{\prime} \stackrel{\xi}{\mapsto} M_{3}$ be a generalized type II split move associated with $\omega^{\prime \prime \prime}$. Choose it to preserve all the boundary circuits in $C$.

The extension move $M \stackrel{\zeta_{1}}{\longmapsto} M_{2}^{\prime}$ is friendly to $M \stackrel{\eta}{\longmapsto} M_{1}$, and the move $M_{2}^{\prime} \stackrel{\eta^{\zeta_{1}}}{\longmapsto} M_{1}^{\prime}$ resembling $M \stackrel{\eta}{\longmapsto} M_{1}$ (chosen to preserve the boundary circuits in $C$ ) is a generalized type II split move associated with $\omega$, which is also a type II splitting route in $M_{2}^{\prime}$. Consult Figure 9.27 for the general scheme. 

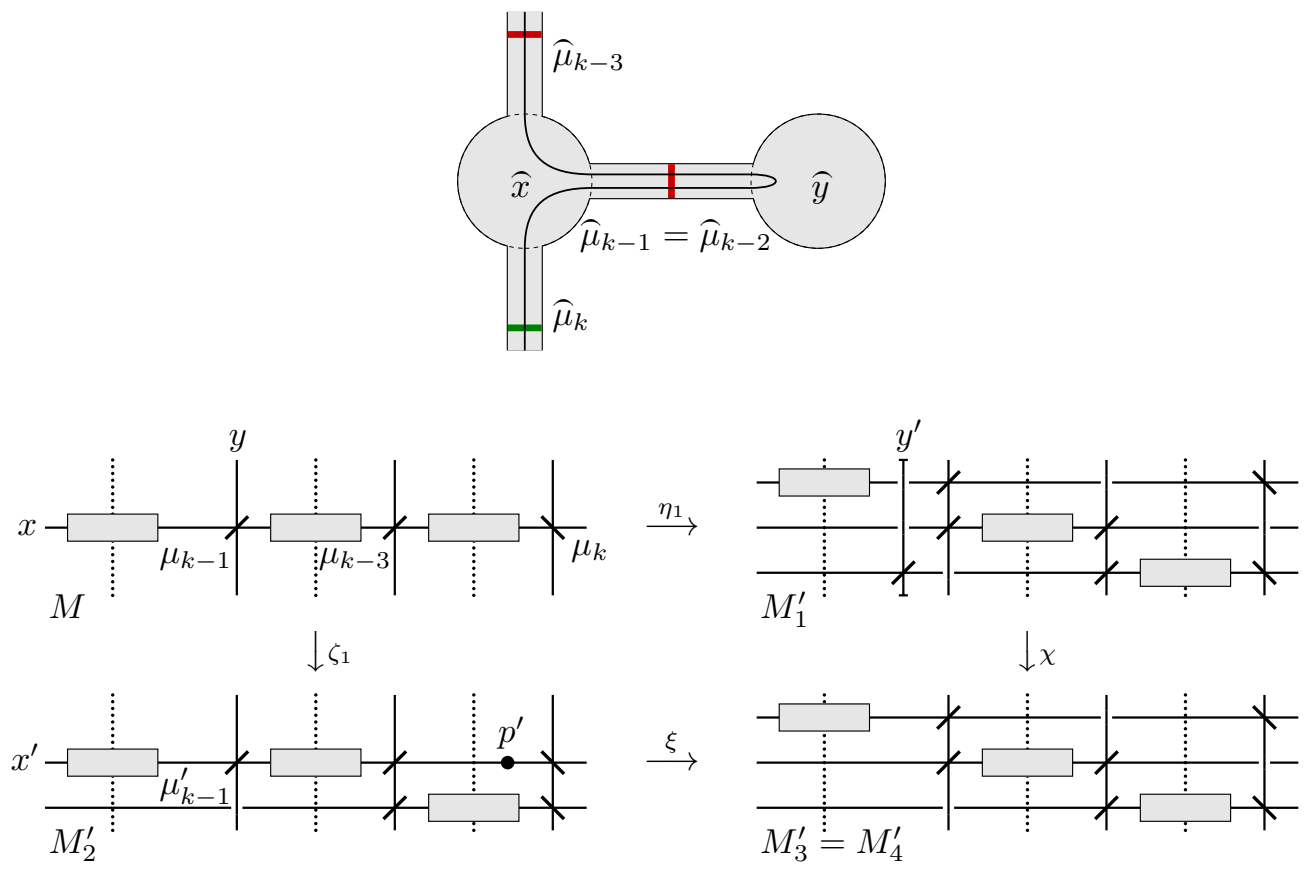

Figure 9.26. Proof of Proposition 9.7 in the pulling tight case, $\omega$ and $\omega^{\prime}$ are doubleheaded, $k>4$

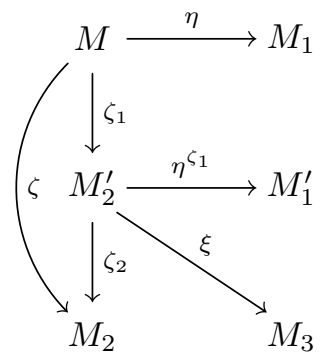

Figure 9.27. The scheme of the moves in the proof of Proposition9.7 the pulling tight case, double-headed splitting routes, $k=4$

The transformation $M_{1} \stackrel{\eta^{\zeta_{1}} \circ \zeta_{1} \circ \eta^{-1}}{\longmapsto} M_{1}^{\prime}$ is a type I extension move preserving the boundary circuits in $C$ by Lemma 9.5 (applied to the moves $M_{2}^{\prime} \stackrel{\eta^{\zeta_{1}}}{\longmapsto} M_{1}^{\prime}$ and $M_{2}^{\prime} \stackrel{\zeta_{1}^{-1}}{\longmapsto} M$ ), and the transformations $M_{1}^{\prime} \stackrel{\xi \circ\left(\eta^{\zeta_{1}}\right)^{-1}}{\longmapsto}$ $M_{3}, M_{3} \stackrel{\zeta_{2} \circ \xi^{-1}}{\longrightarrow} M_{2}$ admit a $C$-neat decompositions into type I elementary moves by the established above cases of Proposition 9.7 This completes the proof in the pulling tight case.

Case 2: $\omega \mapsto \omega^{\prime}$ is a tail shrinking operation.

We use the notation from Definition [9.12, Let $M \stackrel{\eta_{1}}{\longmapsto} M_{1}^{\prime}$ be the first split move of a canonical decomposition of $M \stackrel{\eta}{\mapsto} M_{1}$, and let $M_{1}^{\prime} \stackrel{\eta_{2}}{\longmapsto} M_{1}$ be the remaining part. From the combinatorial point of view the move $M \stackrel{\eta_{1}}{\longmapsto} M_{1}^{\prime}$ is a type I extension move, whose inverse is friendly to $M_{1}^{\prime} \stackrel{\eta_{2}}{\longmapsto} M_{1}$, so $M \stackrel{\zeta}{\longmapsto} M_{2}$ can be interpreted as $M \stackrel{\eta_{2}{ }^{-1}}{\longmapsto} M_{2}$. We leave the details, which are easy in this case, to the reader.

Case 3: $\omega=\omega^{\prime}$ is a special type II splitting route, $\omega=\left(\mu_{1}, \mu_{2}, p\right)$.

Let $\nu_{1}, x_{1}$ be the auxiliary mirror and the auxiliary level, respectively, of the move $M \stackrel{\eta}{\mapsto} M_{1}$, and 
let $\nu_{2}, x_{2}$ be the auxiliary mirror and the auxiliary level, respectively, of the move $M \stackrel{\zeta}{\mapsto} M_{2}$. Let also $M \stackrel{\eta_{1}}{\longmapsto} M_{1}^{\prime}$ and $M \stackrel{\zeta_{1}}{\longmapsto} M_{2}^{\prime}$ be the extension moves from which the canonic decompositions of the moves $M \stackrel{\eta}{\mapsto} M_{1}$ and $M \stackrel{\zeta}{\mapsto} M_{2}$, respectively, start, and let $M_{1}^{\prime} \stackrel{\eta_{2}}{\longmapsto} M_{1}$ and $M_{2}^{\prime} \stackrel{\zeta_{2}}{\longmapsto} M_{2}$ be the remaining parts of the canonical decompositions. If $\nu_{1}=\nu_{2}$, there is nothing to prove, so we assume $\nu_{1} \neq \nu_{2}$, which implies $x_{1} \neq x_{2}$.

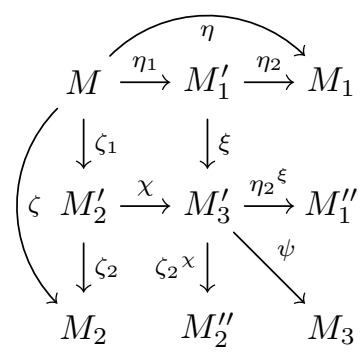

Figure 9.28. The scheme of the moves in the proof of Proposition 9.7 in the case $\omega=$ $\omega^{\prime}=\left(\mu_{1}, \mu_{2}, p\right)$

Denote by $M_{3}^{\prime}$ the mirror diagram obtained from $M$ by adding both occupied levels $x_{1}$ and $x_{2}$ and both mirrors $\nu_{1}, \nu_{2}$. We have two extension moves $M_{1}^{\prime} \stackrel{\xi}{\mapsto} M_{3}^{\prime}$ and $M_{2}^{\prime} \stackrel{\chi}{\mapsto} M_{3}^{\prime}$ such that $\xi \circ \eta_{1}=\chi \circ \zeta_{1}$, and these moves are friendly to $M_{1}^{\prime} \stackrel{\eta_{2}}{\longmapsto} M_{1}$ and $M_{2}^{\prime} \stackrel{\zeta_{2}}{\longmapsto} M_{2}$, respectively; see Figure 9.28. Thus, we have generalized type II split moves $M_{3}^{\prime} \stackrel{\eta_{2}{ }^{\xi}}{\longmapsto} M_{1}^{\prime \prime}$ and $M_{3}^{\prime} \stackrel{\zeta_{2}{ }^{x}}{\longmapsto} M_{2}^{\prime \prime}$ resembling $M_{1}^{\prime} \stackrel{\eta_{2}}{\longmapsto} M_{1}$ and $M_{2}^{\prime} \stackrel{\zeta_{2}}{\longmapsto} M_{2}$, respectively.

At least one of the sequences $\left(\mu_{1}, \nu_{1}, \nu_{1}, \nu_{2}, \nu_{2}, \mu_{2}, p\right)$ and $\left(\mu_{1}, \nu_{2}, \nu_{2}, \nu_{1}, \nu_{1}, \mu_{2}, p\right)$ is a double-headed type II splitting route in $M_{3}^{\prime}$, which we denote by $\omega^{\prime \prime}$. There exists a generalized type II split move $M_{3}^{\prime} \longmapsto$ $M_{3}$ associated with $\omega^{\prime \prime}$ and preserving the boundary circuits in $C$.

The splitting routes that the moves $M_{3}^{\prime} \stackrel{\eta_{2} \xi}{\longmapsto} M_{1}^{\prime \prime}$ and $M_{3}^{\prime} \stackrel{\zeta_{2} \times}{\longmapsto} M_{2}^{\prime \prime}$ are associated with, which are $\left(\mu_{1}, \nu_{1}, \nu_{1}, \mu_{2}, p\right)$ and $\left(\mu_{1}, \nu_{2}, \nu_{2}, \mu_{2}, p\right)$, respectively, are obtained from $\omega^{\prime \prime}$ by pulling tight operations and are not special. Thus, the existence of a $C$-neat decomposition of the transformations $M_{1}^{\prime \prime} \stackrel{\psi \circ\left(\eta_{2}{ }^{\xi}\right)^{-1}}{\longleftrightarrow}$ $M_{3}$ and $M_{3} \stackrel{\zeta_{2}{ }^{\circ} \circ \psi^{-1}}{\longrightarrow} M_{2}^{\prime \prime}$ into type I elementary moves follows from the previously considered cases of Proposition 9.7. The existence of such a decomposition for the transformations $M_{1} \stackrel{\eta_{2}{ }^{\xi} \circ \xi \circ \eta_{2}^{-1}}{\longmapsto} M_{1}^{\prime \prime}$ and $M_{2}^{\prime \prime} \stackrel{\zeta_{2} \circ \chi^{-1} \circ\left(\zeta_{2} \times\right)^{-1}}{\longmapsto} M_{2}$ follows from Lemma 9.5

Case 4: $\omega \mapsto \omega^{\prime}$ is a head wandering operation.

We use the notation from Definition 9.14. Suppose that $\omega$ and $\omega^{\prime}$ are single-headed. The first $k-1$ steps in canonical decompositions of the moves $M \stackrel{\eta}{\mapsto} M_{1}$ and $M \stackrel{\zeta}{\mapsto} M_{2}$ are combinatorially the same, and the type II splitting routes that the remaining parts of the decompositions are associated with are still related by a head wandering operation. So, it suffices to consider the case $k=1, \omega=(\mu, p), \omega^{\prime}=\left(\mu^{\prime}, \mu^{\prime \prime}, p\right)$.

Denote by $x$ the occupied level of $M$ containing $p$ and $\mu$, and by $y$ the occupied level of $M$ containing $\mu^{\prime}$ and $\mu^{\prime \prime}$. Denote also by $c_{1}$ and $c_{2}$ the inessential boundary circuits corresponding to $c$ in $M_{1}$ and $M_{2}$, respectively. Up to various symmetries the portion of $M$ in question looks as shown at the top of Figure 9.29 .

The mirror $\mu$ has a unique successor in $M_{1}$ that is hit by $c_{1}$. Let $M_{1} \stackrel{\xi}{\mapsto} M_{1}^{\prime}$ be the elementary bypass removal that deletes this successor. This move will preserve all boundary circuits except $c_{1}$, which is inessential, and another one that is already modified by $M \stackrel{\eta}{\mapsto} M_{1}$. Thus all the boundary circuits in $C$ are preserved in $M_{1}^{\prime}$.

The occupied level $y$ has a unique successor in $M_{2}$ containing a portion of $c_{2}$. It carries exactly two mirrors of $M_{2}$, which are successors of $\mu^{\prime}$ and $\mu^{\prime \prime}$. Let $M_{2} \stackrel{\chi}{\longmapsto} M_{2}^{\prime}$ be the bridge removal that deletes $y$ 

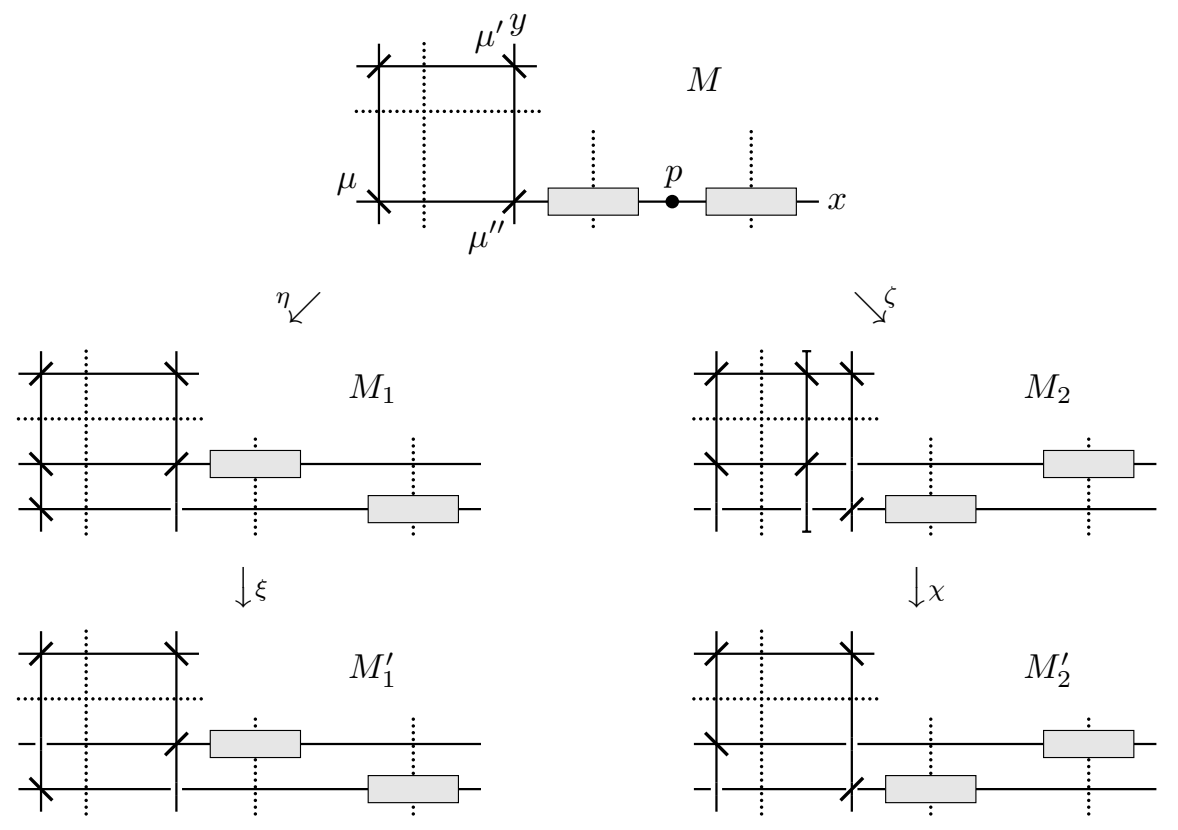

Figure 9.29. Proof of Proposition 9.7 in the head wandering case, $\omega$ and $\omega^{\prime}$ are single-headed

together with these two mirrors. This move preserves all boundary circuits except $c_{2}$ and another one that is already modified by $M \stackrel{\eta}{\mapsto} M_{1}$. Thus all the boundary circuits in $C$ are also preserved in $M_{2}^{\prime}$.

One can see that $M_{1}^{\prime}$ and $M_{2}^{\prime}$ are obtained from one another by exchanging the two occupied levels that are successors of $x$. One can also see that there is no obstruction to exchange them by means of jump moves preserving all the boundary circuits that have not yet been modified by other moves discussed above. To complete the proof in this case it remains to decompose neatly the jump moves as well as the bridge addition $M_{2}^{\prime} \stackrel{\chi^{-1}}{\longrightarrow} M_{2}$ into elementary type I moves, which is possible by Lemma 7.3 .

Now suppose that $\omega$ and $\omega^{\prime}$ are double-headed. If $k>2$, then the first moves in canonical decompositions of $M \stackrel{\eta}{\mapsto} M_{1}$ and $M \stackrel{\zeta}{\mapsto} M_{2}$ are combinatorially the same, and the type II splitting routes responsible for the remaining parts of the canonical decompositions are single-headed, so we can proceed as above.

Suppose that $k=2$ and $\mu_{1} \neq \mu_{2}$. Denote by $x$ the occupied level of $M$ passing through $\mu_{1}$ and $\mu_{2}$. Since $\mu_{1} \neq \mu_{2}$, the mirror $\mu_{2}$ lies outside $c$, which means that the union of boundary circuits in $C$ does not cover $x \backslash c$, and hence there exists a generalized type II split move $M \stackrel{\xi}{\mapsto} M_{3}$ associated with $\omega$ and preserving the boundary circuits in $C$, such that the auxiliary mirror of this move is outside $c$. We have seen in Case 3 above that $M_{1} \stackrel{\xi \circ \eta^{-1}}{\longrightarrow} M_{3}$ admits a $C$-neat decomposition into type I elementary moves. This reduces the currently considered case to the subcase when the auxiliary mirror of the move $M \stackrel{\eta}{\mapsto} M_{1}$ is outside $c$. We assume this in the sequel.

Denote by $\mu_{0}$ the auxiliary mirror. Let $M \stackrel{\eta_{1}}{\longmapsto} M_{1}^{\prime}$ be the first move of a canonical decomposition of $M \stackrel{\eta}{\longmapsto} M_{1}$, and let $M_{1}^{\prime} \stackrel{\eta_{2}}{\longmapsto} M_{1}$ be the remaining part. The type I extension move $M \stackrel{\eta_{1}}{\longmapsto} M_{1}^{\prime}$ is friendly to $M \stackrel{\zeta}{\mapsto} M_{2}$, so we can define $M_{1}^{\prime} \stackrel{\zeta^{\eta_{1}}}{\longmapsto} M_{2}^{\prime}$ in such a way that the transformation $M_{2}^{\prime} \stackrel{\zeta \circ \eta_{1}^{-1} \circ\left(\zeta^{\eta_{1}}\right)^{-1}}{\longmapsto} M_{2}$ is a type I elimination move (see Lemma 9.5). This move is associated with the type II splitting route $\omega^{\prime}=$ $\left(\mu_{1}^{\prime}, \mu_{1}^{\prime \prime}, \mu_{2}, p\right)$ in $M_{1}^{\prime}$.

Let $\omega^{\prime \prime}$ be the type II splitting route $\left(\mu_{1}^{\prime}, \mu_{1}^{\prime \prime}, \mu_{0}, \mu_{0}, \mu_{2}, p\right)$ in $M_{1}^{\prime}$, and let $M_{1}^{\prime} \stackrel{{ }^{\chi}}{\mapsto} M_{3}$ be the respective generalized type II split move; see the scheme in Figure 9.30. By construction, the generalized type II split move $M_{1}^{\prime} \stackrel{\eta_{2}}{\longmapsto} M_{1}$ is associated with the splitting route $\omega_{1}=\left(\mu_{1}, \mu_{0}, \mu_{0}, \mu_{2}, p\right)$ in $M_{1}^{\prime}$. 


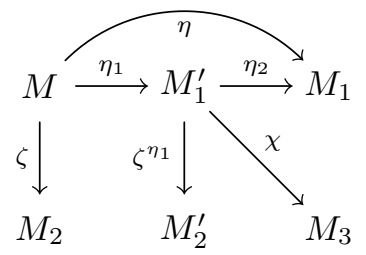

Figure 9.30. The scheme of the moves in the proof of Proposition 9.7 in the head wandering case with special $\omega$

Thus, the splitting routes $\omega_{1}$ and $\omega^{\prime \prime}$ are related by a head wandering operation, and neither of them is special. As has been shown above, in this case, the transformation $M_{1} \stackrel{\chi \circ \eta_{2}^{-1}}{\longrightarrow} M_{3}$ admits a $C$-neat decomposition into type I elementary moves.

The splitting route $\omega^{\prime}$ in $M_{1}^{\prime}$ is obtained from $\omega^{\prime \prime}$ by a pulling tight operation. So, the required decomposition for $M_{3} \stackrel{\zeta^{\eta_{1}} \circ \chi^{-1}}{\longmapsto} M_{2}^{\prime}$ exists by Case 1 considered above.

It remains to consider the subcase when $\omega$ and $\omega^{\prime}$ have the form $\omega=(\mu, \mu, p), \omega^{\prime}=\left(\mu^{\prime}, \mu^{\prime \prime}, \mu, p\right)$. Denote by $\mu_{0}$ and $y$ the auxiliary mirror and the auxiliary occupied level of the move $M \stackrel{\eta}{\mapsto} M_{1}$, by $x$ the occupied level of $M$ containing $\mu$ and $\mu_{0}$ (and $\mu^{\prime \prime}$ ), and by $z$ the occupied level of $M$ containing $\mu^{\prime}$ and $\mu^{\prime \prime}$.
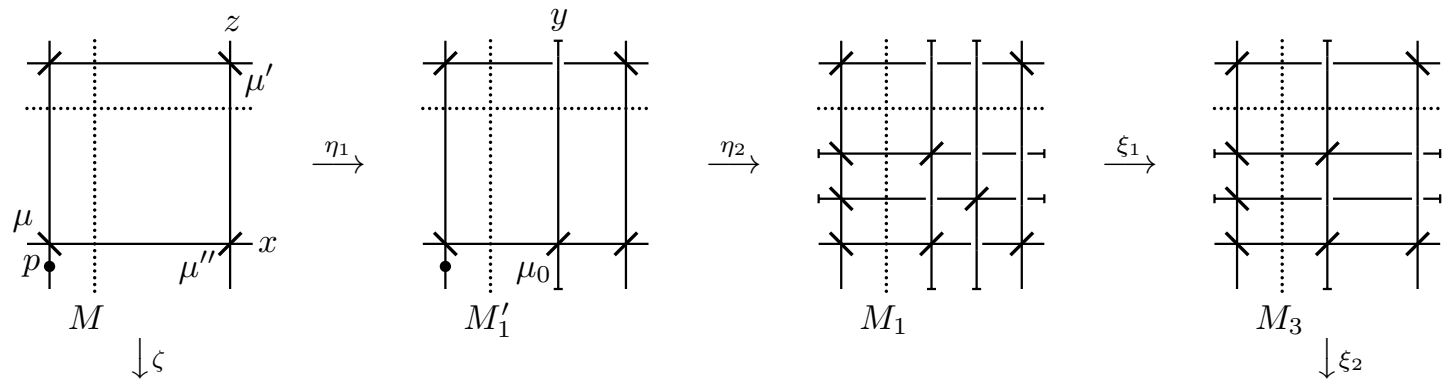

$M_{3}$
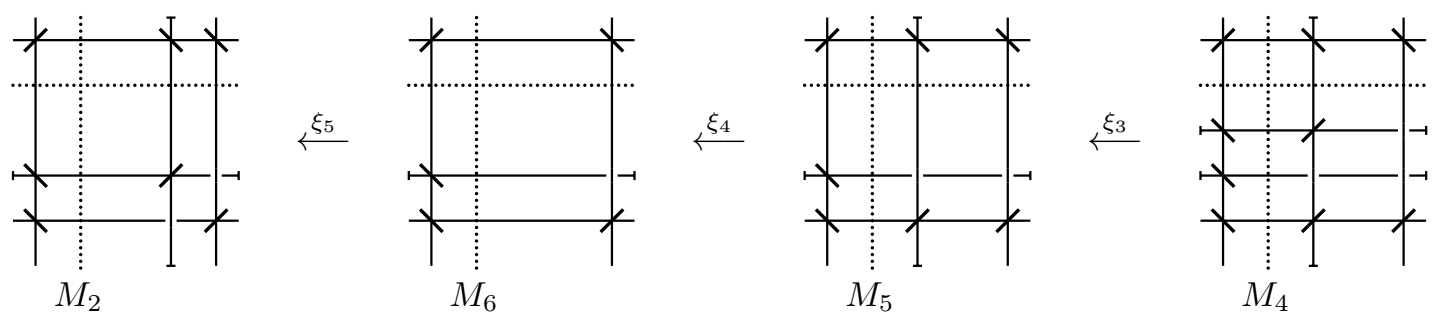

Figure 9.31. Proof of Proposition 9.7 in the head wandering case, $\omega=(\mu, \mu, p), \omega^{\prime}=\left(\mu^{\prime}, \mu^{\prime \prime}, \mu, p\right)$

If $\mu_{0}$ is outside $c$ we proceed exactly as before, when we had $\mu_{1} \neq \mu_{2}$. Suppose that $\mu_{0} \in c$. The sought-for decomposition is sketched in Figure 9.31 (the other cases are obtained by symmetries), in which:

- the move $M \stackrel{\eta_{1}}{\longmapsto} M_{1}^{\prime}$ is the first move of a canonical decomposition of $M \stackrel{\eta}{\mapsto} M_{1}$, and $M_{1}^{\prime} \stackrel{\eta_{2}}{\longmapsto} M_{1}$ is the remaining part,

- $M_{1} \stackrel{\xi_{1}}{\longmapsto} M_{3}$ is a type I elimination move that deletes a successor of $y$ and a successor of $\mu_{0}$,

- $M_{3} \stackrel{\xi_{2}}{\longmapsto} M_{4}$ is a type I elementary bypass addition,

- $M_{4} \stackrel{\xi_{3}}{\longmapsto} M_{5}$ is a bridge removal, which deletes a successor of $x$ with two mirror on it,

- $M_{5} \stackrel{\xi_{4}}{\longmapsto} M_{6}$ is a composition of a jump move that shifts the remaining successor of $y$ toward $z$ and a double merge move that merges this successor with $z$, 
- and finally, $M_{6} \stackrel{\xi_{5}}{\longmapsto} M_{2}$ is a bridge addition.

If the moves $M \stackrel{\eta}{\mapsto} M_{1}$ and $M \stackrel{\zeta}{\mapsto} M_{2}$ preserve all boundary circuits except $c$, then so do all the moves in Figure 9.31 Otherwise, some adjustment may be needed by means of jump moves. All jump moves and bridge moves should be decomposed neatly into type I moves to get the sought-for decomposition of $M_{1} \stackrel{\zeta \circ \eta^{-1}}{\longmapsto} M_{2}$.

Case 5: $\omega \mapsto \omega^{\prime}$ is a tail-to-head conversion.

We use the notation from Definition 9.15. Suppose that $k>1$, that is, $\omega^{\prime}$ is not special. Let $M \stackrel{\eta_{1}}{\longmapsto} M_{1}^{\prime}$ and $M \stackrel{\zeta_{1}}{\longmapsto} M_{2}^{\prime}$ be the first moves of canonical decompositions of $M \stackrel{\eta}{\mapsto} M_{1}$ and $M \stackrel{\zeta}{\mapsto} M_{2}$, respectively, and let $M_{1}^{\prime} \stackrel{\eta_{2}}{\longmapsto} M_{1}$ and $M_{2}^{\prime} \stackrel{\zeta_{2}}{\longmapsto} M_{2}$ be the remaining parts of the decompositions.

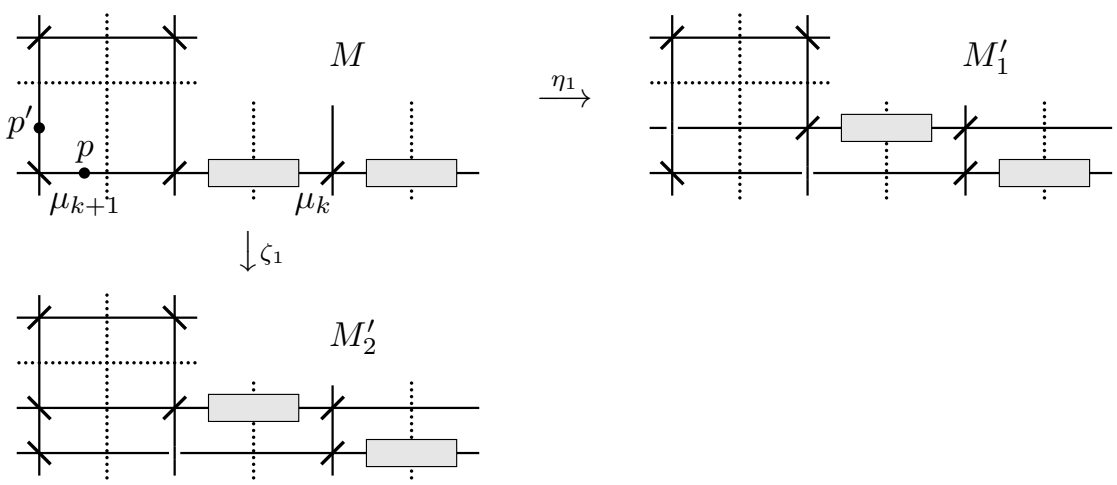

Figure 9.32. Proof of Proposition 9.7 in the tail-to-head conversion case

One can see from Figure 9.32 that $M_{1}^{\prime} \stackrel{\xi}{\mapsto} M_{2}^{\prime}$ with $\xi=\zeta_{1} \circ \eta_{1}^{-1}$ is a type I elementary bypass addition possibly composed with jump moves that do not change the combinatorial type of the diagram. It is also clear that $M_{2}^{\prime} \stackrel{\zeta_{2}}{\longmapsto} M_{2}$ can be interpreted as $M_{2}^{\prime} \stackrel{\eta_{2}{ }^{\xi}}{\longmapsto} M_{2}$; refer to Figure 9.33 for the general scheme. We

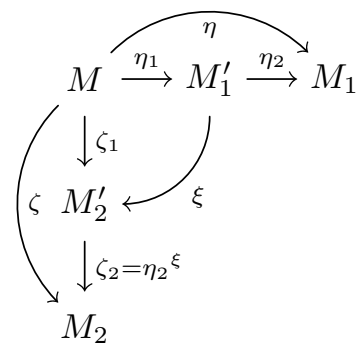

Figure 9.33. The scheme of the moves in the proof of Proposition 9.7 the tail-to-head conversion case with non-special $\omega^{\prime}$

skip the details, which are similar to many previously considered cases.

Finally, suppose that $k=1$. Let $x$ be the occupied level of $M$ containing $\mu_{1}, \mu_{2}$, and $p$. There is a unique $/$-mirror in $c \cap x$, which we denote by $\mu_{0}$. The sequences $\omega^{\prime \prime}=\left(\mu_{1}, \mu_{0}, \mu_{0}, p\right)$ and $\omega^{\prime \prime \prime}=$ $\left(\mu_{1}, \mu_{0}, \mu_{0}, \mu_{2}, p^{\prime}\right)$ are non-special type II splitting routes related by a tail-to-head conversion operation, and in each of the pairs $\omega^{\prime \prime}, \omega$ and $\omega^{\prime}, \omega^{\prime \prime \prime}$ the splitting routes are related by a pulling tight operation. The assertion of the proposition follows from the previously considered cases. 


\subsection{First commutation property of generalized type II merge moves.}

Lemma 9.9. Let $M \stackrel{\eta}{\mapsto} M_{1}$ be a generalized type II split move associated with a splitting route $\omega$, and let $M \stackrel{\zeta}{\mapsto} M_{2}$ be a type I move of one of the following kinds: elimination, extension, split, merge, elementary bypass addition, or elementary bypass removal. Denote by $C$ the set of all boundary circuits of $M$ preserved by both moves.

Then there exists a generalized type II split move $M \stackrel{\eta^{\prime}}{\longmapsto} M_{1}^{\prime}$ associated with a type II splitting route $\omega^{\prime}$ such that the following holds:

(1) the splitting route $\omega^{\prime}$ is similar to $\omega$;

(2) the move $M \stackrel{\eta^{\prime}}{\longmapsto} M_{1}^{\prime}$ preserves all boundary circuits in $C$.

(3) the move $M \stackrel{\zeta}{\longmapsto} M_{2}$ is friendly to $M \stackrel{\eta^{\prime}}{\longmapsto} M_{1}^{\prime}$;

(4) if $M_{2} \stackrel{\eta^{\prime \zeta}}{\longmapsto} M_{21}$ is a move resembling $M \stackrel{\eta^{\prime}}{\longmapsto} M_{1}^{\prime}$, then the transformation $M_{21} \stackrel{\eta^{\prime} \circ \zeta^{-1} \circ\left(\eta^{\prime \zeta}\right)^{-1}}{\longmapsto} M_{1}^{\prime}$ admits a C-neat decomposition into type I elementary moves.

Proof. Let $\omega^{\prime \prime}$ be a reduced type II splitting route obtained from $\omega$ by pulling tight and tail shrinking operations, which exists by Proposition 9.6, and let $M \stackrel{\eta^{\prime \prime}}{\longmapsto} M_{1}^{\prime \prime}$ be a generalized type II split move associated with $\omega^{\prime \prime}$. By Lemma 9.8 the move $M \stackrel{\eta^{\prime \prime}}{\longmapsto} M_{1}^{\prime \prime}$ can be chosen to preserve all the boundary circuits in $C$. We assume that such a choice has been made.

If the move $M \stackrel{\zeta}{\mapsto} M_{2}$ is friendly to $M \stackrel{\eta^{\prime \prime}}{\longmapsto} M_{1}^{\prime \prime}$ and the moves almost commute with one another, then due to Lemma 7.3 Condition (4) above is satisfied for $\omega^{\prime}=\omega^{\prime \prime}$, and we are done. Similarly, we are done if the move $M_{2} \stackrel{\eta^{\prime \prime \zeta}}{\longmapsto} M_{21}$ almost commutes with $M_{2} \stackrel{\zeta^{-1}}{\longmapsto} M$. If neither of these occurs, additional arguments are required and/or $\omega^{\prime \prime}$ has to be modified. There are again a number of cases to consider.

Case 1: $\omega^{\prime \prime}$ is not special.

Case $1 a: M \stackrel{\zeta}{\mapsto} M_{2}$ is a type I elimination move.

If $x$ is an occupied level of $M$ containing a single mirror $\mu$, and this mirror is of type ' $/$, then no reduced type II splitting route visits $\mu$. Hence, such a $\mu$ cannot appear in $\omega^{\prime \prime}$, and any type I elimination move is friendly to $M \stackrel{\eta^{\prime \prime}}{\longmapsto} M_{1}^{\prime \prime}$. The moves $M \stackrel{\eta^{\prime \prime}}{\longmapsto} M_{1}^{\prime \prime}$ and $M \stackrel{\zeta}{\longmapsto} M_{2}$ commute with one another by Lemma 9.5 . so we can take $\omega^{\prime \prime}$ for $\omega^{\prime}$.

Case $1 b: M \stackrel{\zeta}{\mapsto} M_{2}$ is a type I extension move.

An extension move can be unfriendly to the non-special generalized split move $M \stackrel{\eta^{\prime \prime}}{\longmapsto} M_{1}^{\prime \prime}$ only if $\omega^{\prime \prime}$ is single-headed, and the place where a new mirror is inserted coincides with the snip point of the move $M \stackrel{\eta^{\prime \prime}}{\longmapsto} M_{1}^{\prime \prime}$. However, a small shift of the snip point has no combinatorial effect on the respective generalized type II split move, hence we may consider all extension moves being friendly to $M \stackrel{\eta^{\prime \prime}}{\longmapsto} M_{1}^{\prime \prime}$. Then the move $M_{2} \stackrel{\eta^{\prime \prime \zeta}}{\longmapsto} M_{21}$ is friendly to the elimination move $M_{2} \stackrel{\zeta^{-1}}{\longmapsto} M$, and these moves commute with one another, again, by Lemma 9.5

Case $1 c: M \stackrel{\zeta}{\mapsto} M_{2}$ is a type I merge move.

Denote by $\nu_{1}, \nu_{2}$ the two mirrors of $M$ that have a common successor in $M_{2}$, which we denote by $\nu$. We number them so that $\left(\nu_{1} ; \nu_{2}\right)$ is the splitting gap of the inverse move $M_{2} \stackrel{\zeta^{-1}}{\longmapsto} M$. Denote also by $x$ the occupied level of $M$ passing through both $\nu_{1}$ and $\nu_{2}$, and by $y_{1}$ and $y_{2}$ the occupied levels of $M$ perpendicular to $x$ and passing through $\nu_{1}$ and $\nu_{2}$, respectively. Denote the common successor of $y_{1}$ and $y_{2}$ in $M_{2}$ by $y$.

Under an appropriate choice of $h_{M}^{M_{2}}$ the image of $\widehat{\omega}^{\prime \prime}$ in $\overparen{M}_{2}$ is a normal arc unless the splitting route $\omega^{\prime \prime}$ is single-headed and its snip point lies in $\left(\nu_{1} ; \nu_{2}\right)$. If the latter is the case, apply a tail stretching operation to $\omega^{\prime \prime}$ to obtain $\omega^{\prime}$. Otherwise, put $\omega^{\prime}=\omega^{\prime \prime}$.

Even if we have to apply a tail stretching to $\omega^{\prime \prime}$, the obtained splitting route $\omega^{\prime}$ still does not separate $C$, since $\widehat{\omega}^{\prime}$ is obtained from $\widehat{\omega}^{\prime \prime}$ by pulling the snip point along a boundary circuit of $M$ which is modified 
by the move $M \stackrel{\eta}{\mapsto} M_{1}$, and hence is not in $C$. So, we can always choose the move $M \stackrel{\eta^{\prime}}{\longmapsto} M_{1}^{\prime}$ associated with $\omega^{\prime}$ so that it preserves all boundary circuits in $C$.

Let $\left(\mu_{1}, \ldots, \mu_{k}, p\right)=\omega^{\prime}$. In the case when $\omega^{\prime}$ is double-headed we assume that $p$ is chosen so close to $\mu_{k}$ that no occupied level passes between $\mu_{k}$ and $p$. For $i=1, \ldots, k$, denote by $\mu_{i}^{\prime}$ be the successor of $\mu_{i}$ in $M_{2}$. Denote also by $p^{\prime}$ the point in $\mathbb{T}^{2}$ such that $h_{M}^{M_{2}}(\widehat{p})=\widehat{p}^{\prime}$. The sequence $\omega^{\prime \prime \prime}=\left(\mu_{1}^{\prime}, \mu_{2}^{\prime}, \ldots, \mu_{k}^{\prime}, p^{\prime}\right)$ is a type II splitting route in $M_{2}$, provided that Condition (2d) of the corresponding Definition 9.3 or 9.4 holds.

We claim that this is the case due to the fact that $\omega^{\prime}$ is either reduced or is obtained from the reduced splitting route $\omega^{\prime \prime}$ by a single tail stretching operation. Indeed, we can have $\mu_{i}^{\prime}=\mu_{i+1}^{\prime}$ only if $\left\{\mu_{i}, \mu_{i+1}\right\}=\left\{\nu_{1}, \nu_{2}\right\}$. In this case, if $3 \leqslant i \leqslant k-2$, then one of the mirrors $\mu_{i-1}$ and $\mu_{i+2}$ lies on $y_{1}$ and the other on $y_{2}$, and we have $\mu_{i-1}, \mu_{i+2} \notin\left\{\nu_{1}, \nu_{2}\right\}, \mu_{i-1} \neq \mu_{i+2}$. Therefore, we cannot have $\mu_{i-1}^{\prime}=\mu_{i+2}^{\prime}$.

One can see from this that the only cancellable portions of $\omega^{\prime \prime \prime}$ have the form $\left(\mu_{i}^{\prime}, \mu_{i+1}^{\prime}\right)=(\nu, \nu)$. One can also see that if $\left\{\mu_{i}, \mu_{i+1}\right\}=\left\{\mu_{j}, \mu_{j+1}\right\}=\left\{\nu_{1}, \nu_{2}\right\}$, then $i-j=0(\bmod 2)$, so Condition (2d) holds.

Thus, the image of $\omega^{\prime}$ in $M_{2}$ is a type II splitting route and we can define the associated generalized type II split move $M_{2} \stackrel{\eta^{\prime \zeta}}{\longmapsto} M_{21}$. We now show that the move $M_{2} \stackrel{\zeta^{-1}}{\longmapsto} M$ is friendly to $M_{2} \stackrel{\eta^{\prime \zeta}}{\longmapsto} M_{21}$.

The move $M_{2} \stackrel{\zeta^{-1}}{\longmapsto} M$ is a type I split move associated with a splitting route of the form $(\nu, q)$, where $q \in y$. By construction, the associated splitting path $\widehat{(\nu, q)}$ is disjoint from $\widehat{\omega}^{\prime \prime \prime}$. We claim that their mutual position is unambiguous.

Indeed, suppose otherwise. Then there is a type II splitting route in $M$ not equivalent to $\omega^{\prime}$ whose image in $M_{2}$ coincides with $\omega^{\prime \prime \prime}$. Such a route should be obtained from $\omega^{\prime}$ by replacing some entries equal to $\nu_{1}$ or $\nu_{2}$ with $\nu_{2}$ or $\nu_{1}$, respectively. By construction we have $\mu_{i} \neq \mu_{i+1}$ for all $i=1, \ldots, k-1$. Therefore, if $\mu_{i}=\nu_{1}, 2 \leqslant i \leqslant k$, then at least one of $\mu_{i-1}$ and $\mu_{i+1}$ is not in $\left\{\nu_{1}, \nu_{2}\right\}$ and lies on $y_{1}$ (if $\omega^{\prime}$ is single-headed we assume that $\mu_{k+1}$ refers to $p$ ). This means that replacing $\mu_{i}$ by $\nu_{2}$ will produce a sequence that is not a type II splitting route even if some other $\nu_{1}$ 's are replaced by $\nu_{2}$ 's or vice versa. Similarly, no $\nu_{2}$-entry in $\omega^{\prime}$ can be replaced by $\nu_{1}$. A contradiction.

Thus the move $M_{2} \stackrel{\zeta^{-1}}{\longmapsto} M$ is friendly to $M_{2} \stackrel{\eta^{\prime \zeta}}{\longmapsto} M_{21}$, and by Lemma 9.6 these moves almost commute.

Case $1 d: M \stackrel{\zeta}{\mapsto} M_{2}$ is a type I split move.

Let $(\nu, q)$ be the respective type I splitting route, and let $\left(\mu_{1}, \mu_{2}, \ldots, \mu_{k}, p\right)=\omega^{\prime \prime}$. Denote by $x$ the occupied level of $M$ containing $\nu$ and $q$.

The only possible reason for $M \stackrel{\zeta}{\mapsto} M_{2}$ to be unfriendly to $M \stackrel{\eta^{\prime \prime}}{\longmapsto} M_{1}^{\prime \prime}$ in this case is an unavoidable intersection of $\widehat{(\nu, q)}$ and $\widehat{\omega}^{\prime \prime}$, since the other possible reason, an ambiguity in the relative position of $\widehat{(\nu, q)}$ and $\widehat{\omega}^{\prime \prime}$ is ruled out by the fact that $\omega^{\prime \prime}$ is reduced. Clearly, all intersections of $\widehat{(\nu, q)}$ and $\widehat{\omega}^{\prime \prime}$ occur in $\widehat{x}$ and each of them is due to the fact that, for some $i \in\{2,3, \ldots, k\}$, the following condition holds:

$$
\text { the mirrors } \mu_{i}, \mu_{i+1} \text { lie on } x \text {, and the pairs }\left\{\mu_{i}, \mu_{i+1}\right\} \text { and }\{\nu, q\} \text { interleave, }
$$

where we put $\mu_{k+1}=p$ if $\omega^{\prime}$ is single-headed; see the left picture in Figure 9.34
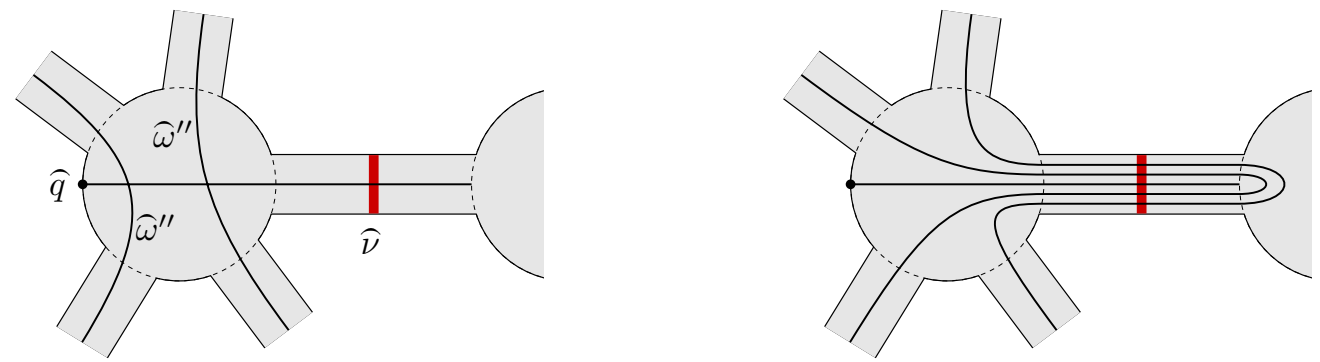

FIGURE 9.34. Loosening $\omega^{\prime \prime} \mapsto \omega^{\prime}$ that resolves intersections with $\widehat{(\nu, q)}$ 
Let $\omega^{\prime}$ be obtained from $\omega^{\prime \prime}$ by inserting the subsequence $(\nu, \nu)$ between $\mu_{i}$ and $\mu_{i+1}$ whenever condition (9.8) holds. Topologically this means that we replace $\widehat{\omega}^{\prime \prime}$ by an isotopic path $\widehat{\omega}^{\prime}$ avoiding intersections with $\widehat{(\nu, q)}$. Clearly, the new path can be chosen to be a normal arc. Since $\omega^{\prime \prime}$ is reduced, one can see that $\omega^{\prime}$ satisfies Conditions (2d) of the corresponding Definition 9.3 or 9.4 so $\omega^{\prime}$ is a type II splitting route.

By construction, the move $M \stackrel{\zeta}{\mapsto} M_{2}$ is now friendly to $M \stackrel{\eta^{\prime}}{\longmapsto} M_{1}^{\prime}$ if the latter is a generalized type II split move associated with $\omega^{\prime}$. It remains to show that $\omega^{\prime}$ does not separate $C$, and hence the move $M \stackrel{\eta^{\prime}}{\longmapsto} M_{1}^{\prime}$ can be chosen to preserve all the boundary circuits in $C$.

Let $d_{1}, \ldots, d_{l}$ be the connected components of $\widehat{x} \backslash\left(\widehat{(\nu, q)} \cup \widehat{\omega}^{\prime \prime}\right)$. For any $i, j \in\{1, \ldots, l\}, i \neq j$, the components $d_{i}$ and $d_{j}$ are separated by at least one of $\widehat{(\nu, q)}$ and $\widehat{\omega}^{\prime \prime}$. This means that the respective portions of $x$ appear at different occupied levels either in $M_{1}$, or in $M_{2}$ (or in both). Therefore, for at most one $i$ the component $d_{i}$ may have a non-empty intersection with the boundary components corresponding to boundary circuits from $C$. This means that at most one connected component of $x \backslash\left(\{\nu, q,\} \cup \omega^{\prime \prime}\right)=$ $x \backslash\left(\{\nu, q,\} \cup \omega^{\prime}\right)$ has a non-empty intersection with $\bigcup_{c \in C} c$. Therefore, $\omega^{\prime}$ does not separate $C$.

Case $1 e: M \stackrel{\zeta}{\mapsto} M_{2}$ is a type I elementary bypass removal.

Let $c$ be the inessential boundary circuit of $M$ whose presence allows this move. This means that $c$ has the form of the boundary of a rectangle $r$ such that $\operatorname{int}(r) \cap E_{M}=\varnothing$. Let $\nu_{1}, \nu_{2}, \nu_{3}, \nu_{4}$ be the mirrors at the corners of $r$ numbered so that $c=\left[\nu_{1} ; \nu_{2}\right] \cup\left[\nu_{1} ; \nu_{3}\right] \cup\left[\nu_{2} ; \nu_{4}\right] \cup\left[\nu_{3} ; \nu_{4}\right]$. This means, in particular, that $\nu_{1}$ and $\nu_{4}$ are $\backslash$-mirrors, and $\nu_{2}, \nu_{3}$ are $/$-mirrors.

We may assume without loss of generality that the move $M \stackrel{\zeta}{\mapsto} M_{2}$ removes $\nu_{1}$, since the case of removing $\nu_{4}$ is symmetric to this one. By Lemma 9.7 the moves $M \stackrel{\zeta}{\mapsto} M_{2}$ and $M \stackrel{\eta^{\prime \prime}}{\longmapsto} M_{1}^{\prime \prime}$ commute with one another unless one of the following holds:

- $\nu_{1}$ is an entry of $\omega^{\prime \prime}$;

- $\omega^{\prime \prime}$ is single-headed and the snip point of the move $M \stackrel{\eta^{\prime \prime}}{\longmapsto} M_{1}^{\prime \prime}$ lies on $c$.

So, it suffices to find a non-special type II splitting route $\omega^{\prime}$ similar to $\omega^{\prime \prime}$ such that $\nu_{1}$ is not an entry of $\omega^{\prime}$ and either $\omega^{\prime}$ is double-headed or $\omega^{\prime}$ is a single-headed splitting route ending outside $c$.

There are a number of cases in each of which we either take $\omega^{\prime \prime}$ for $\omega^{\prime}$ or apply a few operations transforming $\omega^{\prime \prime}$ to $\omega^{\prime}$, which include tail stretching, tail-to-head and head-to-tail conversions, and head wandering. All the operations modify the corresponding splitting path near the boundary component $\widehat{c} \subset$ $\partial \widehat{M}$. One can see from this and from the fact that $c \notin C$ that, in each case, the obtained type II splitting route $\omega^{\prime}$ still does not separate $C$.

Let $\left(\mu_{1}, \mu_{2}, \ldots, \mu_{k}, p\right)=\omega^{\prime \prime}$. Denote by $x$ the occupied level of $M$ passing through $\nu_{1}$ and $\nu_{2}$. The roles of $\nu_{2}$ and $\nu_{3}$ are symmetric to each other in the present context, so we omit the cases obtained from the considered ones by the exchange $\nu_{2} \leftrightarrow \nu_{3}$.

Suppose first that $\omega^{\prime \prime}$ is single-headed.

If $\mu_{1} \neq \nu_{1}, p \notin c$ take $\omega^{\prime \prime}$ for $\omega^{\prime}$.

If $\mu_{1} \neq \nu_{1}$ and $p \in\left(\nu_{1} ; \nu_{2}\right)$ take $\left(\mu_{1}, \ldots, \mu_{k}, \nu_{2}, \nu_{4}, q\right)$ for $\omega^{\prime}$, where $q \in\left(\nu_{3} ; \nu_{4}\right)$ is close to $\nu_{4}$. This splitting route is obtained from $\omega^{\prime \prime}$ by a tail stretching followed by a tail-to-head conversion:

$$
\left(\mu_{1}, \mu_{2}, \ldots, \mu_{k}, p\right) \mapsto\left(\mu_{1}, \mu_{2}, \ldots, \mu_{k}, \nu_{2}, p^{\prime}\right) \mapsto\left(\mu_{1}, \ldots, \mu_{k}, \nu_{2}, \nu_{4}, q\right),
$$

where $p^{\prime} \in\left(\nu_{2} ; \nu_{4}\right)$.

If $\mu_{1} \neq \nu_{1}$ and $p \in\left(\nu_{2} ; \nu_{4}\right)$ take $\left(\mu_{1}, \ldots, \mu_{k}, \nu_{4}, q\right)$ for $\omega^{\prime}$, where $q \in\left(\nu_{3} ; \nu_{4}\right)$ is close to $\nu_{4}$. This splitting route is obtained from $\omega^{\prime \prime}$ by a tail-to-head conversion.

If $\mu_{1}=\nu_{1}, p \notin c$, and $\mu_{2} \in x$ (here and in the next case, if $k=1$, then $\mu_{2}$ refers to $p$ ), take $\left(\nu_{4}, \nu_{2}, \mu_{2}, \ldots, \mu_{k}, p\right)$ for $\omega^{\prime}$. This splitting route is obtained from $\omega^{\prime \prime}$ by a head wandering operation.

If $\mu_{1}=\nu_{1}, p \in\left(\nu_{1} ; \nu_{2}\right)$, and $\mu_{2} \in x$, take $\left(\nu_{4}, \nu_{2}, \mu_{2}, \ldots, \mu_{k}, \nu_{2}, \nu_{4}, q\right)$ for $\omega^{\prime}$, where $q \in\left(\nu_{4} ; \nu_{3}\right)$ is close to $\nu_{4}$. This splitting route is obtained from $\omega^{\prime \prime}$ by a sequence of four operations including a tail stretching, a tail-to-head conversion, replacing the splitting route by an equivalent one, and then a head wandering 
operation:

$\omega^{\prime \prime} \mapsto\left(\mu_{1}, \ldots, \mu_{k}, \nu_{2}, p^{\prime}\right) \mapsto\left(\mu_{1}, \ldots, \mu_{k}, \nu_{2}, \nu_{4}, q^{\prime}\right) \mapsto\left(\mu_{1}, \ldots, \mu_{k}, \nu_{2}, \nu_{4}, q\right) \mapsto\left(\nu_{4}, \nu_{2}, \mu_{2}, \ldots, \mu_{k}, \nu_{2}, \nu_{4}, q\right)$,

where $q^{\prime} \in\left(\nu_{3} ; \nu_{4}\right)$ is close to $\nu_{4}$, and $p^{\prime} \in\left(\nu_{2} ; \nu_{4}\right)$.

If $\mu_{1}=\nu_{1}, p \in\left(\nu_{1} ; \nu_{3}\right)$, and $\mu_{2} \in x$, take $\left(\nu_{4}, \nu_{2}, \mu_{2}, \ldots, \mu_{k}, \nu_{3}, \nu_{4}, q\right)$ for $\omega^{\prime}$, where $q \in\left(\nu_{4} ; \nu_{2}\right)$ is close to $\nu_{4}$. The sequence of transformations producing $\omega^{\prime}$ from $\omega^{\prime \prime}$ is similar to the previous case:

$\omega^{\prime \prime} \mapsto\left(\mu_{1}, \ldots, \mu_{k}, \nu_{3}, p^{\prime}\right) \mapsto\left(\mu_{1}, \ldots, \mu_{k}, \nu_{3}, \nu_{4}, q^{\prime}\right) \mapsto\left(\mu_{1}, \ldots, \mu_{k}, \nu_{3}, \nu_{4}, q\right) \mapsto\left(\nu_{4}, \nu_{2}, \mu_{2}, \ldots, \mu_{k}, \nu_{3}, \nu_{4}, q\right)$,

where $q^{\prime} \in\left(\nu_{2} ; \nu_{4}\right)$ is close to $\nu_{4}$, and $p^{\prime} \in\left(\nu_{3} ; \nu_{4}\right)$.

If $\mu_{1}=\nu_{1}, p \in\left(\nu_{2} ; \nu_{4}\right)$, and $\mu_{2} \in x$, take $\left(\nu_{4}, \nu_{2}, \mu_{2}, \ldots, \mu_{k}, \nu_{4}, q\right)$ for $\omega^{\prime}$, where $q \in\left(\nu_{4} ; \nu_{3}\right)$ is close to $\nu_{4}$. This splitting route is obtained from $\omega^{\prime \prime}$ by a sequence of three operations including a tail-to-head conversion, replacing the splitting route by an equivalent one, and then a head wandering operation:

$$
\omega^{\prime \prime} \mapsto\left(\mu_{1}, \ldots, \mu_{k}, \nu_{4}, q^{\prime}\right) \mapsto\left(\mu_{1}, \ldots, \mu_{k}, \nu_{4}, q\right) \mapsto\left(\nu_{4}, \nu_{2}, \mu_{2}, \ldots, \mu_{k}, \nu_{4}, q\right),
$$

where $q^{\prime} \in\left(\nu_{3} ; \nu_{4}\right)$ is close to $\nu_{4}$.

If $\mu_{1}=\nu_{1}, p \in\left(\nu_{3} ; \nu_{4}\right)$, and $\mu_{2} \in x$, take $\left(\nu_{4}, \nu_{2}, \mu_{2}, \ldots, \mu_{k}, \nu_{4}, q\right)$ for $\omega^{\prime}$, where $q \in\left(\nu_{4} ; \nu_{2}\right)$ is close to $\nu_{4}$. The splitting route $\omega^{\prime}$ is produced from $\omega^{\prime \prime}$ similarly to the previous case:

$$
\omega^{\prime \prime} \mapsto\left(\mu_{1}, \ldots, \mu_{k}, \nu_{4}, q^{\prime}\right) \mapsto\left(\mu_{1}, \ldots, \mu_{k}, \nu_{4}, q\right) \mapsto\left(\nu_{4}, \nu_{2}, \mu_{2}, \ldots, \mu_{k}, \nu_{4}, q\right),
$$

where $q^{\prime} \in\left(\nu_{2} ; \nu_{4}\right)$ is close to $\nu_{4}$.

Now suppose that the splitting route $\omega^{\prime \prime}$ is double-headed.

If $\mu_{1} \neq \nu_{1}$ and $\mu_{k} \neq \nu_{1}$ take $\omega^{\prime \prime}$ for $\omega^{\prime}$.

If $\mu_{k}=\nu_{1}$ and $p \in\left(\nu_{1} ; \nu_{3}\right)$, apply a head-to-tail conversion to $\omega^{\prime \prime}$ :

$$
\left(\mu_{1}, \ldots, \mu_{k-1}, \nu_{1}, p\right) \mapsto\left(\mu_{1}, \ldots, \mu_{k-1}, q\right),
$$

where $q \in\left(\nu_{1} ; \nu_{2}\right)$. We obtain a single-headed type II splitting route similar to $\omega^{\prime \prime}$ and proceed as in one of the above listed cases.

If $\mu_{k}=\nu_{1}, \mu_{1} \neq \nu_{1}, p \in\left(\nu_{3} ; \nu_{1}\right)$, pick a point $p^{\prime} \in\left(\nu_{1} ; \nu_{3}\right)$ close to $\nu_{1}$. The type II splitting route $\left(\mu_{1}, \ldots, \mu_{k}, p^{\prime}\right)$ is then equivalent to $\omega^{\prime \prime}$ and we can proceed as in the previous case.

If $\mu_{1}=\nu_{1}, p \notin c$, and $\mu_{2} \in x$, apply a head wandering

$$
\left(\nu_{1}, \mu_{2}, \ldots, \mu_{k}, p\right) \mapsto\left(\nu_{4}, \nu_{2}, \mu_{2}, \ldots, \mu_{k}, p\right)
$$

and then proceed as in one of the above listed cases (or symmetric to them).

If $\mu_{1}=\nu_{1}, \mu_{k} \neq \nu_{1}, p \in c$, replace $\omega^{\prime \prime}$ with an equivalent splitting route with $p \notin c$, and then proceed as in the previous case.

Case $1 f: M \stackrel{\zeta}{\mapsto} M_{2}$ is a type I elementary bypass addition.

If $\omega^{\prime \prime}$ is double-headed, then the move $M_{2} \stackrel{\eta^{\prime \prime} \zeta}{\longmapsto} M_{21}$ is defined and commutes with $M_{2} \stackrel{\zeta^{-1}}{\longmapsto} M$ by Lemma 9.7, so we can take $\omega^{\prime \prime}$ for $\omega^{\prime}$.

Suppose that $\omega^{\prime \prime}=\left(\mu_{1}, \ldots, \mu_{k}, p\right)$ is single-headed. Let $c=\left[\nu_{1} ; \nu_{2}\right] \cup\left[\nu_{1} ; \nu_{3}\right] \cup\left[\nu_{2} ; \nu_{4}\right] \cup\left[\nu_{3} ; \nu_{4}\right]=\partial r$ be the inessential boundary circuit of $M_{2}$ created by this move. We may assume without loss of generality that the mirror added by the move is $\nu_{1}$ (the case when the added mirror is $\nu_{4}$ is symmetric to this one). So, $\nu_{1}$ is not present in $M$.

If $p \in\left[\nu_{1} ; \nu_{2}\right) \cup\left[\nu_{1} ; \nu_{3}\right)$ there is an equivalent type II splitting route $\left(\mu_{1}, \ldots, \mu_{k}, p^{\prime}\right)$ with $p^{\prime} \notin c$. Take such a route for $\omega^{\prime}$.

If $p \in\left(\nu_{2} ; \nu_{4}\right) \cup\left(\nu_{3} ; \nu_{4}\right)$, then a tail stretching operation on $\omega^{\prime \prime}$ yields a type II splitting route of the form $\left(\mu_{1}, \ldots, \mu_{k}, \nu_{j}, p^{\prime}\right)$ with $j \in\{2,3\}, p^{\prime} \in\left[\nu_{1} ; \nu_{2}\right) \cup\left[\nu_{1} ; \nu_{3}\right)$, which can then be changed to an equivalent one $\left(\mu_{1}, \ldots, \mu_{k}, \nu_{j}, p^{\prime \prime}\right)$ with $p^{\prime \prime} \notin c$. We take the obtained splitting route for $\omega^{\prime}$.

The move $M \stackrel{\zeta}{\longmapsto} M_{2}$ is friendly to the type II split move $M \stackrel{\eta^{\prime}}{\longmapsto} M_{1}^{\prime}$ associated with $\omega^{\prime}$, and, moreover, it follows from Lemma 9.7 that the moves $M_{2} \stackrel{\eta^{\prime \zeta}}{\longmapsto} M_{21}, M_{2} \stackrel{\zeta^{-1}}{\longmapsto} M$ commute. 
Case 2: $\omega^{\prime \prime}$ is special, $\omega^{\prime \prime}=\left(\mu_{1}, \mu_{2}, p\right)$.

We may assume without loss of generality that the interval $\left(\mu_{2} ; p\right)$ is shorter than $\left(p ; \mu_{2}\right)$, since the other case is symmetric to this one.

The special case requires special care, but in the present context, it is actually easier than the nonspecial one. There are several subcases to consider in each of which the proof is by a straightforward check. We skip some boring details and provide only the most essential ones.

By Proposition 9.7 it suffices to prove the assertion of the lemma for a concrete eligible choice of the auxiliary mirror of the move $M_{2} \stackrel{\eta^{\prime \zeta}}{\longmapsto} M_{21}$. So, in each case, we specify this choice.

Case 2a: $M \stackrel{\zeta}{\mapsto} M_{2}$ is a type I elimination move. We take $\omega^{\prime \prime}$ for $\omega^{\prime}$ and take the auxiliary mirror of the move $M \stackrel{\eta}{\longmapsto} M_{1}$ for the auxiliary mirror of the move $M_{2} \stackrel{\eta^{\prime \zeta}}{\longmapsto} M_{21}$.

Case $2 b: M \stackrel{\zeta}{\mapsto} M_{2}$ is a type I extension move.

We take $\omega^{\prime \prime}$ for $\omega^{\prime}$ unless the mirror $\mu_{*}$ added by the move $M \stackrel{\zeta}{\mapsto} M_{2}$ appears in $\left(\mu_{2} ; p\right)$, in which case we put $\omega^{\prime}=\left(\mu_{1}, \mu_{2}, p^{\prime}\right)$, where $p^{\prime} \in\left(\mu_{2} ; \mu_{*}\right)$ is any point not lying at the intersection of two occupied levels of $M$. Then the move $M_{2} \stackrel{\eta^{\prime \zeta}}{\longmapsto} M_{21}$ is also associated with $\omega^{\prime}$.

We choose the same auxiliary level for the move $M_{2} \stackrel{\eta^{\prime \zeta}}{\longmapsto} M_{21}$ as the one for $M \stackrel{\eta}{\longmapsto} M_{1}$, provided that it does not coincide with the occupied level created by the move $M \stackrel{\zeta}{\mapsto} M_{2}$. In the latter case, the position of the auxiliary level should be disturbed slightly.

Case $2 c: M \stackrel{\zeta}{\mapsto} M_{2}$ is a type I merge move.

Let $\left(\nu_{1} ; \nu_{2}\right)$ be the splitting gap of the inverse move $M_{2} \stackrel{\zeta^{-1}}{\longrightarrow} M$. We take $\omega^{\prime \prime}$ for $\omega^{\prime}$. Since $\mu_{1}, \mu_{2}$ are $\backslash$-mirrors, and $\nu_{1}, \nu_{2}$ are $/$-mirrors, the image $\left(\mu_{1}^{\prime}, \mu_{2}^{\prime}, p^{\prime}\right)$ of $\omega^{\prime}$ in $M_{2}$ is a well defined (up to equivalence) special type II splitting route.

At least one of the two (not necessarily distinct) boundary circuits of $M$ that hit $\mu_{1}$ is modified by the move $M \stackrel{\eta}{\mapsto} M_{1}$, and hence, does not belong to $C$. So, we can choose the auxiliary mirror $\mu_{0}$ of the move $M \stackrel{\eta^{\prime}}{\longmapsto} M_{1}^{\prime}$ on such a circuit in a small vicinity of $\mu_{1}$. Similarly, we choose the auxiliary mirror of the move $M_{2} \stackrel{\eta^{\prime \zeta}}{\longmapsto} M_{21}$ in a small vicinity of $\mu_{1}^{\prime}$, on the same side of $\mu_{1}^{\prime}$ on which $\mu_{0}$ is located relative to $\mu_{1}$.

Case 2d: $M \stackrel{\zeta}{\mapsto} M_{2}$ is a type I split move.

Let $(\nu, q)$ be the splitting route with which the move $M \stackrel{\zeta}{\mapsto} M_{2}$ is associated. If the splitting paths $\widehat{\omega}^{\prime \prime}$ and $\widehat{(\nu, q)}$ have no unavoidable intersection, we take $\omega^{\prime \prime}$ for $\omega^{\prime}$. The positions of the auxiliary mirrors for the moves $M \stackrel{\eta^{\prime}}{\longmapsto} M_{1}^{\prime}$ and $M_{2} \stackrel{\eta^{\prime \zeta}}{\longmapsto} M_{21}$ should be chosen in a small vicinity of the mirror $\mu_{1}$ and its successor in $M_{2}$, respectively.

Otherwise we put $\omega^{\prime}=\left(\mu_{1}, \nu, \nu, \mu_{2}, p\right)$, similarly to Case $1 \mathrm{~d}$.

Case $2 e: M \stackrel{\zeta}{\mapsto} M_{2}$ is a type I elementary bypass removal.

We use the same notation as in Case 1e, as well as a similar strategy. Unless $\nu_{1}$ or $\nu_{4}$ appear in $\omega^{\prime \prime}$ we take $\omega^{\prime \prime}$ for $\omega^{\prime}$ and use the same positions of the auxiliary mirrors for the moves $M \stackrel{\eta^{\prime}}{\longmapsto} M_{1}^{\prime}$ and $M_{2} \stackrel{\eta^{\prime \varsigma}}{\longmapsto}$ $M_{21}$. These positions should be chosen outside $r$.

If $\nu_{1}$ does not appear in $\omega^{\prime \prime}$, but $\nu_{4}$ does, we take for $\omega^{\prime}$ a splitting route of the form $\left(\mu_{1}, \nu_{2}, \nu_{2}, \mu_{2}, p^{\prime}\right)$ or $\left(\mu_{1}, \nu_{3}, \nu_{3}, \mu_{2}, p^{\prime}\right)$, whichever fits the definition. One of these must do so. The splitting route $\omega^{\prime}$ is now non-special, and the associated move $M \stackrel{\eta^{\prime}}{\longmapsto} M_{1}^{\prime}$ and the move $M \stackrel{\zeta}{\mapsto} M_{2}$ are mutually friendly.

If $\nu_{1}$ appears in $\omega^{\prime \prime}$ we resolve this by means of head wandering operations, tail-to-head conversions, and head-to-tail conversions, similarly to the procedure in Case 1e. This produces a double-headed splitting route with no entry equal $\nu_{1}$ and we proceed as before. 
Case $2 f: M \stackrel{\zeta}{\mapsto} M_{2}$ is a type I elementary bypass addition.

We use the same notation as in Case $1 f$ above.

If $\nu_{4}$ does not appear in $\omega^{\prime \prime}$ we take $\omega^{\prime \prime}$ for $\omega^{\prime}$ and use the same position of the auxiliary mirrors for the moves $M \stackrel{\eta^{\prime}}{\longmapsto} M_{1}^{\prime}$ and $M_{2} \stackrel{\eta^{\prime \zeta}}{\longmapsto} M_{21}$ chosen outside $r$.

If $\nu_{4}=\mu_{1}$ or $\mu_{2}$, we take for $\omega^{\prime}$ a splitting route of the form $\left(\mu_{1}, \nu_{2}, \nu_{2}, \mu_{2}, p\right)$ or $\left(\mu_{1}, \nu_{3}, \nu_{3}, \mu_{2}, p\right)$, whichever fits the definition. One of these must do so. Such splitting route $\omega^{\prime}$ is now non-special, and the associated move $M \stackrel{\eta^{\prime}}{\longmapsto} M_{1}^{\prime}$ and the move $M \stackrel{\zeta}{\longmapsto} M_{2}$ are mutually friendly.

Thus, in each case, we have a recipe how to produce $\omega^{\prime}$, and to choose the associated generalized split move, and it is straightforward to check that the assertion of the Lemma holds in each case.

We are ready to establish the first commutation property of generalized type II merge moves.

Proposition 9.8. Let $M_{1}$ be an enhanced mirror diagram, and let $C$ be a collection of essential boundary circuits of $M_{1}$. Let also $M_{1} \stackrel{\eta}{\mapsto} M_{2}$ be a generalized type II merge move preserving all $c \in C$, and $M_{2} \stackrel{\zeta}{\mapsto} M_{3}$ a transformation that admits a $C$-neat decomposition into type I elementary moves.

Then there exists a composition $M_{1} \stackrel{\zeta^{\prime}}{\longmapsto} M_{4}$ of type I elementary moves, and a generalized type II merge move $M_{4} \stackrel{\eta^{\prime}}{\longmapsto} M_{3}$ that give rise to a $C$-neat decomposition of the transformation $M_{1} \stackrel{\zeta \circ \eta}{\longmapsto} M_{3}$.

Proof. A type I slide move admits a neat decomposition into a sequence of three moves: a type I split move, a jump move, and a type I merge move (refer to Figure 7.9, where a similar decomposition for a type II slide move is shown). Therefore, it suffices to prove the proposition, substituting for $M_{2} \stackrel{\zeta}{\mapsto} M_{3}$ a single move from the following list: a type I elimination, extension, split, or merge move, an elementary bypass addition or removal, or a jump move.

If $M_{2} \stackrel{\zeta}{\mapsto} M_{3}$ is a jump move, then the assertion of the proposition follows from Lemmas 9.3 and 7.3 provided that the generalized split move $M_{2} \stackrel{\eta^{-1}}{\longmapsto} M_{1}$ is not special.

Suppose that $M_{2} \stackrel{\zeta}{\mapsto} M_{3}$ is a jump move and the move $M_{2} \stackrel{\eta^{-1}}{\longmapsto} M_{1}$ is special. Let $\left(\mu_{1}, \mu_{2}, p\right)$ be the respective special splitting route. By choosing the position of the auxiliary mirror close enough to $\mu_{1}$, and outside $\bigcup_{c \in C} c$, we can ensure that the obtained generalized type II split move $M_{2} \stackrel{\xi}{\mapsto} M_{1}^{\prime}$, also associated with $\left(\mu_{1}, \mu_{2}, p\right)$, has the following properties:

(1) the move $M_{2} \stackrel{\xi}{\mapsto} M_{1}^{\prime}$ preserves the boundary circuits in $C$;

(2) the first move in the canonic decomposition of $M_{2} \stackrel{\xi}{\mapsto} M_{1}^{\prime}$ is friendly to $M_{2} \stackrel{\zeta}{\mapsto} M_{3}$.

The assertion of the proposition now follows from Proposition 9.7 and Lemmas 9.4 and 7.3 .

If $M_{2} \stackrel{\zeta}{\mapsto} M_{3}$ is a type I elimination, extension, split, or merge move, or an elementary bypass addition or removal, the assertion follows from Lemma 9.9 (where we substitute $M_{2} \stackrel{\eta^{-1}}{\longmapsto} M_{1}$ and $M_{2} \stackrel{\zeta}{\mapsto} M_{3}$ for $M \stackrel{\eta}{\mapsto} M_{1}$ and $M \stackrel{\zeta}{\mapsto} M_{2}$, respectively), and Proposition 9.7 .

9.7. Second commutation property of generalized type II merge moves, non-special case. Generalized type II split moves come with a decomposition into split moves (and a single extension move in the special case), which are all of type I except the last one, which is of type II. By Lemma 7.4 this implies that any generalized type II split move admits a neat decomposition into a sequence of elementary moves such that all type I moves in it precede all type II moves. So, for generalized type II split moves the second commutation property holds trivially, but this is not what we need. In order to establish the second commutation property for the inverse moves we need a decomposition of the opposite kind, in which type II elementary moves precede type I elementary moves.

Proposition 9.9. Any non-special generalized type II split move $M \stackrel{\eta}{\mapsto} M^{\prime}$ admits a neat decomposition into a sequence of elementary moves such that all type II moves in it occur before all type I moves.

Proof. It suffices to prove the proposition in the case when the move $M \stackrel{\eta}{\mapsto} M^{\prime}$ is associated with a singleheaded type II splitting route. Indeed, suppose otherwise, namely that $M \stackrel{\eta}{\mapsto} M^{\prime}$ is associated with a 
non-special double-headed type II splitting route. The canonic decomposition of the move $M \stackrel{\eta}{\mapsto} M^{\prime}$ starts with a double split move, which can be decomposed neatly into type II elementary moves according to Lemma 7.3. and the rest of the decomposition compiles into a generalized type II split move associated with a single-headed splitting route. If the latter admits a desired decomposition, then so does the move $M \stackrel{\eta}{\mapsto} M^{\prime}$.

Thus, in the sequel we assume that the move $M \stackrel{\eta}{\mapsto} M^{\prime}$ is associated with a single-headed splitting route. Let $\omega=\left(\mu_{1}, \mu_{2}, \ldots, \mu_{k}, p\right)$ be that route. We proceed by induction in $k$ and construct a neat decomposition of the move $M \mapsto M^{\prime}$ into a sequence of moves in which the first $k-1$ moves are wrinkle creation moves, the $k$ th move is a type II split move, and all the other moves are type I elementary moves (including elementary bypass removals and eliminations) and jump moves. Such a decomposition of the move $M \mapsto M^{\prime}$ will be referred to as anticanonical.

Each wrinkle creation move and the type II split move in the anticanonical decomposition should be then neatly decomposed into type II elementary moves, and the jump moves should be neatly decomposed into type I elementary moves. This is possible according to Lemmas 7.3 and 7.4 .

If $k=1$ the move $M \stackrel{\eta}{\mapsto} M^{\prime}$ is an ordinary type II move whose anticanonical decomposition consists of the move itself.

Suppose that $k \geqslant 2$. The first move $M \stackrel{\xi}{\mapsto} M_{1}$ of the anticanonical decomposition is a wrinkle creation move for which $\mu_{1}$ and $\mu_{2}$ are the ramification mirrors. Denote by $\mu_{1}^{\prime}$ and $\mu_{2}^{\prime}$ the $/$-mirror and the $\backslash$ mirror in $M_{1}$ that have no predecessors in $M$ (they are located near $\mu_{1}$ and $\mu_{2}$, respectively).

The proposition will follow from the Claims 1-4 proven below.

Claim 1: there is a unique, up to equivalence, type II splitting route $\omega^{\prime}=\left(\mu_{2}^{\prime}, \mu_{3}^{\prime}, \ldots, \mu_{k}^{\prime}, p^{\prime}\right)$ in $M_{1}$ such that $h_{M}^{M_{1}}(\widehat{\omega} \cap F)$ is a subarc of $\widehat{\omega}^{\prime}$, where $F$ is the domain of $h_{M}^{M_{1}}$ (the partial homeomorphism $h_{M}^{M_{1}}$ is associated with the move $M \stackrel{\xi}{\mapsto} M_{1}$ as explained in Subsection (7.4).

Let $M_{1} \stackrel{\eta^{\prime}}{\longmapsto} M_{2}$ be a generalized type II split move associated with $\omega^{\prime}$. Let $\mu_{1}^{\prime \prime}$ be the successor of $\mu_{1}^{\prime}$, and $\mu_{2}^{\prime \prime}, \mu_{2}^{\prime \prime \prime}$ be the successors of $\mu_{2}^{\prime}$ for this move. These three mirrors lie on the same occupied level of $M_{2}$, which contains no other mirrors. Let $M_{3}$ be the diagram obtained from $M_{2}$ by removing the mirrors $\mu_{1}^{\prime \prime}, \mu_{2}^{\prime \prime}$, and $\mu_{2}^{\prime \prime \prime}$ together with the occupied level of $M_{2}$ passing through them. This eliminates the two inessential boundary circuits that have been created by the wrinkle move $M \stackrel{\xi}{\mapsto} M_{1}$ and modifies one more boundary circuit, whose essentialness or inessentialness is assumed to be preserved by the transformation $M_{2} \mapsto M_{3}$ (consult Figures 9.35 and 9.36 below). It means, in particular, that any surface carried by $\widehat{M}_{2}$ is also carried by $\widehat{M}_{3}$. Define a morphism $\chi: \widehat{M}_{2} \rightarrow \widehat{M}_{3}$ by $\left(F, F\right.$, id $\left.\left.\right|_{F}\right) \in \chi$, where $F$ is any such surface.

Claim 2: the transformation $M_{2} \stackrel{\chi}{\longmapsto} M_{3}$ admits a decomposition into type I elementary moves.

Claim 3: the transformation $M_{3} \stackrel{\zeta}{\mapsto} M^{\prime}$, where $\zeta=\eta \circ \xi^{-1} \circ \eta^{\prime-1} \circ \chi^{-1}$, admits a decomposition into jump moves shifting only the successors of the occupied levels of $M$ that are being split by the move $M \mapsto M^{\prime}$.

Claim 4: the moves $M \stackrel{\xi}{\mapsto} M_{1}, M_{1} \stackrel{\eta^{\prime}}{\longmapsto} M_{2}$ and the decompositions of $M_{2} \stackrel{\chi}{\longmapsto} M_{3}$ and $M_{3} \stackrel{\zeta}{\mapsto} M^{\prime}$ into type I elementary moves and jump moves, respectively, can be chosen to give rise to a neat decomposition of the move $M \stackrel{\eta}{\mapsto} M^{\prime}$.

Thus, an anticanonical decomposition of the move $M \stackrel{\eta}{\mapsto} M^{\prime}$ is defined as the concatenation of the following four sequences of moves:

(1) the wrinkle creation move $M \stackrel{\xi}{\mapsto} M_{1}$,

(2) an anticanonical decomposition of the generalized type II split move $M_{1} \stackrel{\eta^{\prime}}{\longmapsto} M_{2}$;

(3) a decomposition into type I elementary moves of the transformation $M_{2} \stackrel{\chi}{\longmapsto} M_{3}$ (this will be referred to as a clean-up);

(4) a sequence of jump moves that compiles into the transformation $M_{3} \stackrel{\zeta}{\mapsto} M^{\prime}$. 
Example 9.5. Consider again the generalized type II split move from Example 9.4 Figure 9.35 shows the transitions $M \stackrel{\xi}{\mapsto} M_{1}, M_{1} \stackrel{\eta^{\prime}}{\longmapsto} M_{2}$, and $M_{2} \stackrel{\chi}{\longmapsto} M_{3}$. By $1,2, \ldots$ we mark the mirrors $\mu_{1}, \mu_{2}, \ldots$, and by $1^{\prime}, 2^{\prime}, \ldots$ the mirrors $\mu_{1}^{\prime}, \mu_{2}^{\prime}, \ldots$, respectively. One can see that the final diagram in Figure 9.10 can be obtained from $M_{3}$ by two jump moves.
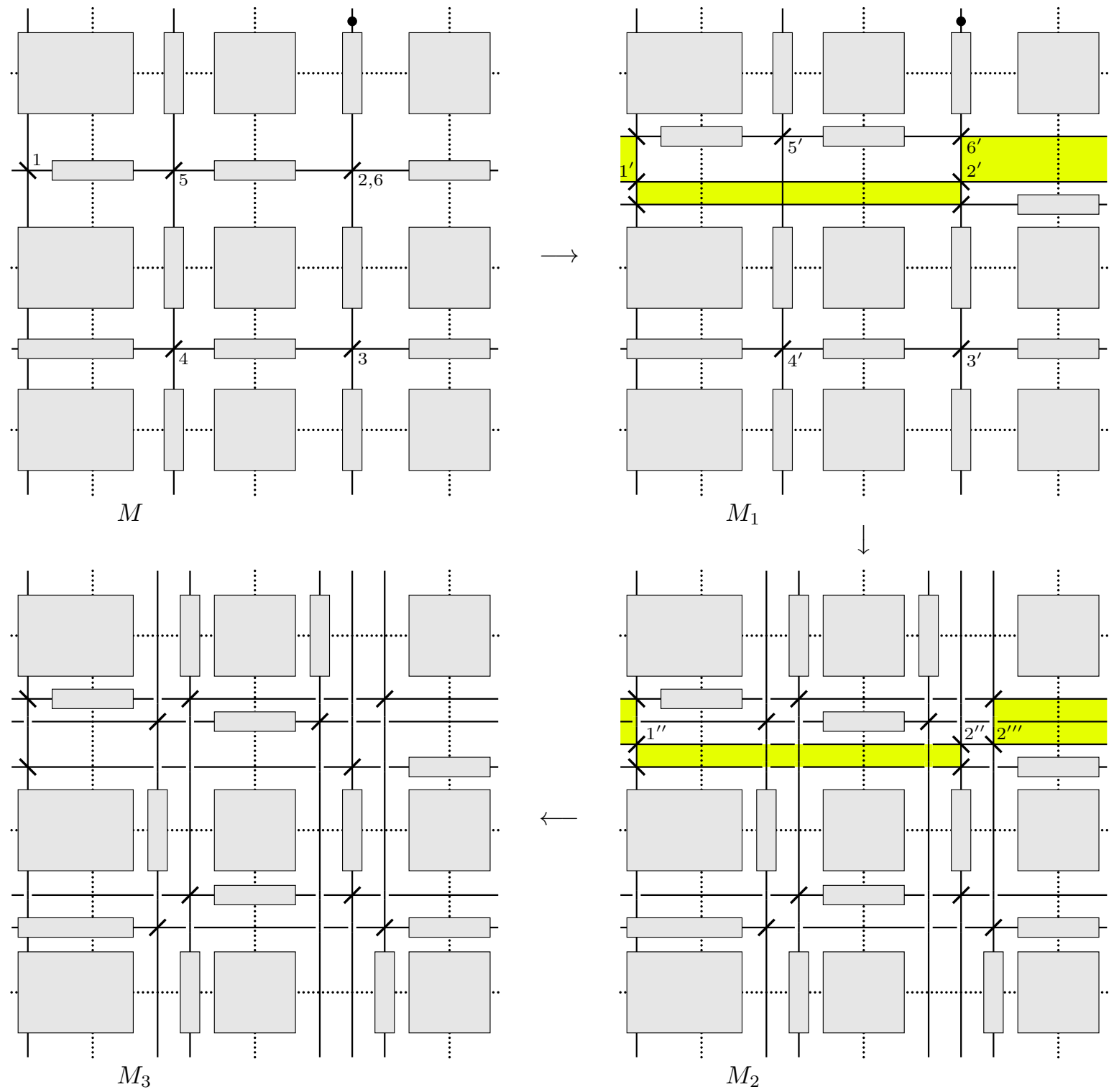

FiguRE 9.35. First three steps of the anticanonical decomposition of a generalized type II split move in Example 9.5

Now we proceed with the proof of Claims 1-4. Let $\widehat{\omega}=\widehat{\omega}^{1} \cup \ldots \cup \widehat{\omega}^{k}$ be the decomposition of the $\operatorname{arc} \widehat{\omega}$ into subarcs as in Definition 9.3. This decomposition can be chosen so that the domain $F$ of $h_{M}^{M_{1}}$ is obtained from $\widehat{M}$ by cutting along $\left(\widehat{\mu}_{1} \cup \widehat{x} \cup \widehat{\mu}_{2}\right) \cap\left(\widehat{\omega}^{1} \cup \widehat{\omega}^{2}\right)$, where $x$ is the occupied level of $M$ containing $\mu_{1}$ and $\mu_{2}$, and the intersection of $F$ with $\widehat{\omega}$ is $\widehat{\omega}^{3} \cup \ldots \cup \widehat{\omega}^{k}$. We can also ensure that the starting point of $h_{M}^{M_{1}}(\widehat{\omega} \cap F)$ appears on the boundary of the strip $\widehat{\mu}_{2}^{\prime}$ but not on $\partial \widehat{M}_{1}$. The $\operatorname{arc} h_{M}^{M_{1}}(\widehat{\omega} \cap F)$ is then 
continued uniquely (up to isotopy of $\widehat{M}_{1}$ preserving the handle decomposition structure) to a type II splitting path; see Figure 9.36. Claim 1 follows.
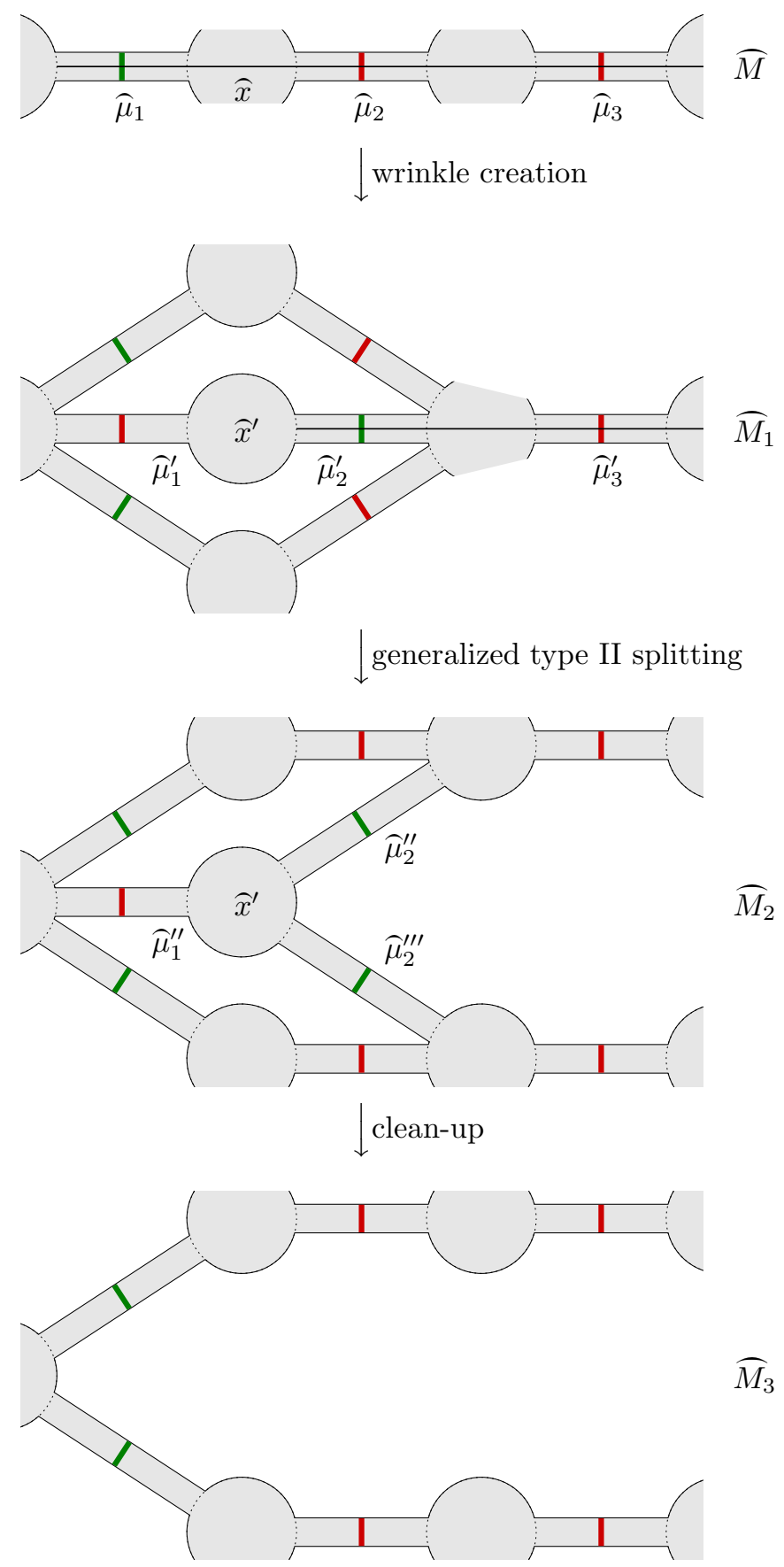

Figure 9.36. Anticanonical decomposition of a generalized type II split move from the 'internal' point of view of the surface $\overparen{M}$

The wrinkle move $M \stackrel{\xi}{\mapsto} M_{1}$ creates two new boundary circuits, the ones that hit the mirror $\mu_{1}^{\prime}$. These circuits have the form $\partial r_{1}, \partial r_{2}$, where $r_{1}$ and $r_{2}$ are some rectangles with no mirrors in the interior. (In Figure 9.35 these rectangles are highlighted.) One can see that the move $M_{1} \mapsto M_{2}$ transforms them to 
circuits that still have the form of the boundary of a rectangle with no mirrors inside. No splitting of the level $x^{\prime}$ containing $\mu_{1}^{\prime}$ and $\mu_{2}^{\prime}$ occurs in the canonical decomposition of $M_{1} \stackrel{\eta^{\prime}}{\longmapsto} M_{2}$, since the mirror $\mu_{1}^{\prime}$ cannot appear in $\omega^{\prime}$. Hence $\mu_{1}^{\prime \prime}, \mu_{2}^{\prime \prime}$, and $\mu_{2}^{\prime \prime \prime}$ remain on the level $x^{\prime}$.

One can now see that the deletions of $\mu_{2}^{\prime \prime}$ and $\mu_{2}^{\prime \prime \prime}$ are type I elementary bypass removals, and the subsequent deletion of $\mu_{1}^{\prime \prime}$ together with the occupied level $x^{\prime}$ is a type I elimination move. Thus, Claim 2 has been settled.

We define the partial homeomorphisms $h_{M_{2}}^{M_{3}}$ and $h_{M_{3}}^{M_{2}}$ for the transformation $M_{2} \stackrel{\chi}{\longmapsto} M_{3}$ in the obvious way: $h_{M_{3}}^{M_{2}}$ is the embedding $\overparen{M}_{3} \rightarrow \overparen{M}_{2}$, and $h_{M_{2}}^{M_{3}}$ is the inverse map. Clearly, these partial homeomorphisms decompose into the partial homeomorphisms corresponding to the elementary type I moves involved in the decomposition of the transformation $M_{2} \stackrel{\chi}{\longmapsto} M_{3}$ discussed above.

From the 'internal' point of view of the surface $\overparen{M}$ the composition of the transformations $M \stackrel{\xi}{\mapsto}$ $M_{1} \stackrel{\eta^{\prime}}{\longmapsto} M_{2} \stackrel{\chi}{\longmapsto} M_{3}$ and the generalized type II split move $M \stackrel{\eta}{\mapsto} M^{\prime}$ do the same thing: they cut the surface $\overparen{M}$ along the path $\widehat{\omega}$ and deform the obtained surface so that the parts of each 1 - or 0 handle are brought to positions near the original position of the handle. The latter formally means that whenever $x_{1}, x_{2}, \ldots, x_{l}$ are parallel occupied levels of $M$ and $x_{1}^{\prime}, x_{2}^{\prime}, \ldots, x_{l}^{\prime}$ are their respective successors for either of the transformations $M \stackrel{\eta}{\mapsto} M^{\prime}$ and $M \stackrel{\chi \circ \eta^{\prime} \circ \xi}{\longmapsto} M_{3}$, the cyclic order of $x_{1}^{\prime}, x_{2}^{\prime}, \ldots, x_{l}^{\prime}$ is the same as that of $x_{1}, x_{2}, \ldots, x_{l}$. So, the combinatorial difference between $M^{\prime}$ and $M_{3}$ is in that the successors of each individual occupied level of $M$ in the diagrams $M^{\prime}$ and $M_{3}$ may appear in different orders, and to prove Claim 3 it is only needed to show that the required change of the order of successors of each occupied level of $M$ can be achieved by means of jump moves.

The proof of Claim 3 is by induction in $k$. The induction base, $k=2$, is verified by a direct check illustrated in Figure 9.37, where the top row shows a canonical decomposition of the move $M \stackrel{\eta}{\mapsto} M^{\prime}$. One can see that the combinatorial types of $M_{3}$ and $M^{\prime}$ are different only in the order in which appear
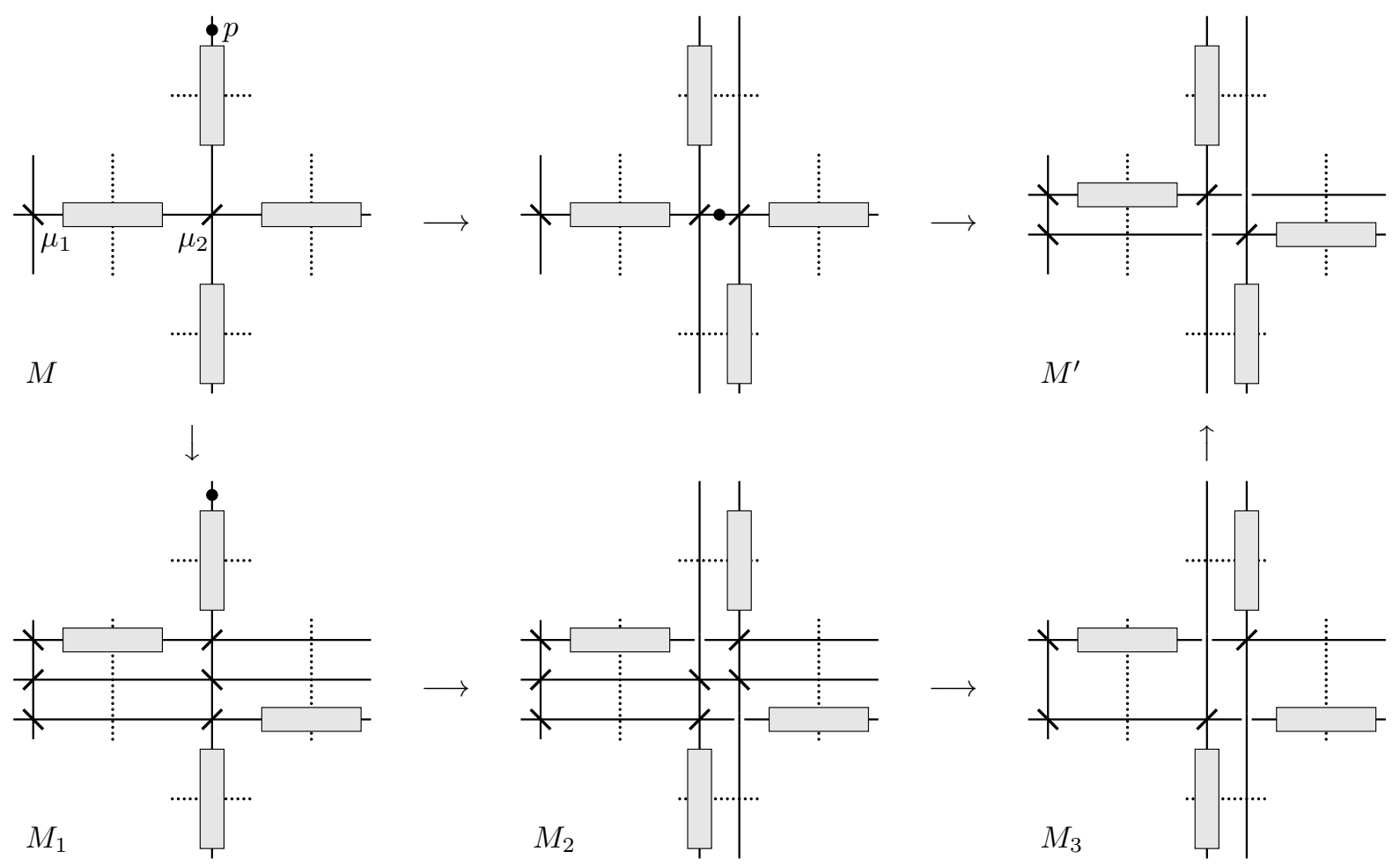

Figure 9.37. Proof of Claim 3 in the case $k=2$ 
the two successors of the occupied level of $M$ containing $\mu_{2}$ and $p$, and this difference can be eliminated by a single jump move.

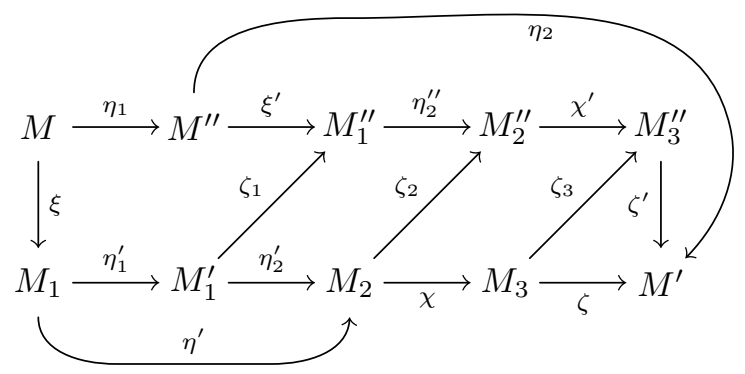

Figure 9.38. Proof of Claim 3, the scheme of the induction step

To make the induction step, let $M \stackrel{\eta_{1}}{\longmapsto} M^{\prime \prime}$ be the first type I split move in a canonical decomposition of the generalized type II split move $M \stackrel{\eta}{\longmapsto} M^{\prime}$, and let $M_{1} \stackrel{\eta_{1}^{\prime}}{\longmapsto} M_{1}^{\prime}$ be the first type I split move in a canonical decomposition of the generalized type II split move $M_{1} \stackrel{\eta^{\prime}}{\longmapsto} M_{2}$. Let also $M^{\prime \prime} \stackrel{\eta_{2}}{\longmapsto} M^{\prime}$ and $M_{1}^{\prime} \stackrel{\eta_{2}^{\prime}}{\longmapsto} M_{2}$ be the remaining parts of the decompositions.

The transformation $M^{\prime \prime} \stackrel{\eta_{2}}{\longmapsto} M^{\prime}$ is a generalized type II split move associated with a splitting route having $k-1$ mirror entries, so we can use the induction hypothesis and decompose it into the following transformations as described above (consult Figure 9.38):

(1) a wrinkle creation move $M^{\prime \prime} \stackrel{\xi^{\prime}}{\longmapsto} M_{1}^{\prime \prime}$;

(2) a generalized type II split move $M_{1}^{\prime \prime} \stackrel{\eta_{2}^{\prime \prime}}{\longmapsto} M_{2}^{\prime \prime}$ associated with a splitting route of length $k-2$;

(3) a clean-up transformation $M_{2}^{\prime \prime} \stackrel{\chi^{\prime}}{\longmapsto} M_{3}^{\prime \prime}$;

(4) a composition of jump moves $M_{3}^{\prime \prime} \stackrel{\zeta^{\prime}}{\longmapsto} M^{\prime}$.

Let $\omega^{\prime \prime}$ and $\omega^{\prime \prime \prime}$ be the type II splitting routes with which associated are the moves $M_{1}^{\prime \prime} \stackrel{\eta_{2}^{\prime \prime}}{\longmapsto} M_{2}^{\prime \prime}$ and $M_{1}^{\prime} \stackrel{\eta_{2}^{\prime}}{\longmapsto} M_{2}$, respectively. We claim that the transformation $M_{1}^{\prime} \stackrel{\zeta_{1}}{\longmapsto} M_{1}^{\prime \prime}$, where $\zeta_{1}=\xi^{\prime} \circ \eta_{1} \circ \xi^{-1} \circ \eta_{1}^{\prime-1}$, decomposes neatly into jump moves, and takes $\omega^{\prime \prime \prime}$ to $\omega^{\prime \prime}$.

Again, if we look at the transformations $M \stackrel{\eta_{1}^{\prime} \circ \xi}{\longmapsto} M_{1}^{\prime}$ and $M \stackrel{\xi^{\prime} \circ \eta_{1}}{\longmapsto} M_{1}^{\prime \prime}$ from the 'internal' point of view of the surface $\overparen{M}$, there is no distinction: both transformations result in cutting $\overparen{M}$ along the initial (corresponding to $\mu_{1}, \mu_{2}$ ) and terminal (corresponding to $\mu_{k}$ ) parts of $\widehat{\omega}$ and adding a 'bridge' across the new hole, which is the result of the cutting along the initial part of $\widehat{\omega}$. This means, in particular, that there is a homeomorphism $\psi: \overparen{M}_{1}^{\prime} \rightarrow \overparen{M}_{1}^{\prime \prime}$ preserving the handle decomposition structure and representing the morphism $\zeta_{1}$, such that $h_{M_{1}}^{M} \circ h_{M_{1}^{\prime}}^{M_{1}}=h_{M^{\prime \prime}}^{M} \circ h_{M_{1}^{\prime \prime}}^{M^{\prime \prime}} \circ \psi$, and for an appropriate choice of the partial homeomorphisms $h_{*}^{*}$, we have $\psi\left(\widehat{\omega}^{\prime \prime \prime}\right)=\widehat{\omega}^{\prime \prime}$.

Let $y_{1}$ and $y_{2}$ be the occupied levels of $M$ perpendicular to $x$ and containing $\mu_{1}$ and $\mu_{2}$, respectively. Without loss of generality we may assume that the occupied level $x$ is horizontal, that is, has the form $\ell_{\varphi_{0}}$ for some $\varphi_{0}$. The occupied levels $y_{1}, y_{2}$ have then the form $m_{\theta_{1}}$ and $m_{\theta_{2}}$, respectively, for some $\theta_{1}, \theta_{2}$. To show that the transformation $M_{1}^{\prime} \stackrel{\zeta_{1}}{\longmapsto} M_{1}^{\prime \prime}$ decomposes neatly into jump moves we need to consider the following five cases.

Case 1: $\mu_{k}$ does not lie on the occupied level $x$.

In this case it is quite obvious that the moves $M \stackrel{\eta_{1}}{\longmapsto} M^{\prime \prime}$ and $M \stackrel{\xi}{\mapsto} M_{1}$ commute.

Case 2: $\mu_{k}$ coincides with $\mu_{2}$, and $k$ is even.

The occupied level being split by the move $M \mapsto M^{\prime \prime}$ is $y_{2}$. Let $y_{2}^{\prime}=m_{\theta_{2}^{\prime}}$ and $y_{2}^{\prime \prime}=m_{\theta_{2}^{\prime \prime}}$ be the two successors of $y_{2}$ for the split move $M \mapsto M^{\prime \prime}$, and let $x^{\prime \prime}=\ell_{\varphi_{0}^{\prime \prime}}, x^{\prime \prime \prime}=\ell_{\varphi_{0}^{\prime \prime \prime}}$ be the successors of $x$ for the 


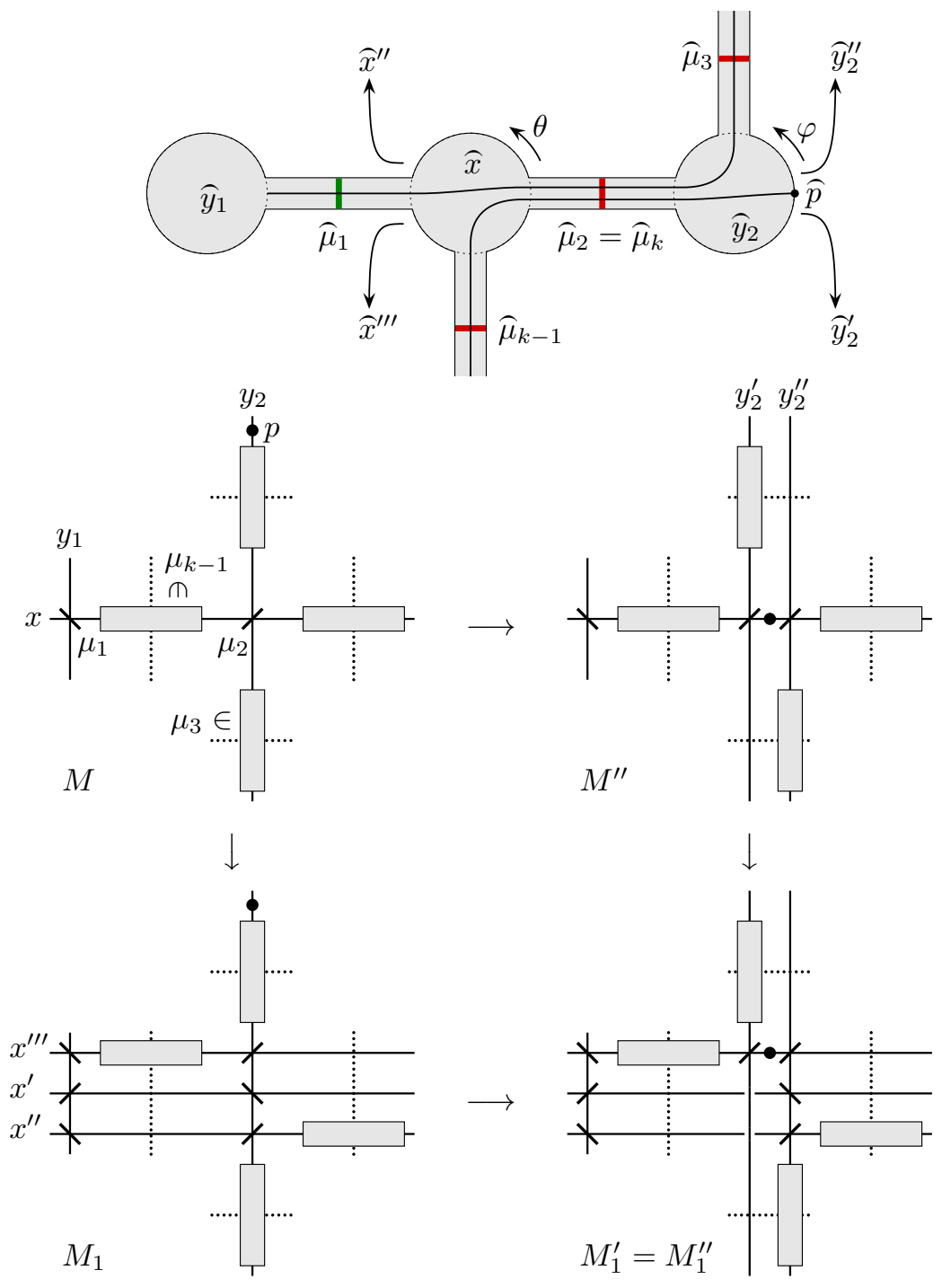

Figure 9.39. Induction step in the proof of Claim 3 , the case $\mu_{k}=\mu_{2}, k \equiv 0(\bmod 2)$, $\mu_{k-1} \in\left(\mu_{1} ; \mu_{2}\right), \mu_{3} \in\left(p ; \mu_{2}\right)$

wrinkle creation move $M \mapsto M_{1}$. Let also $x^{\prime}$ be the occupied level of $M_{1}$ with no predecessor. We order these levels so that $\theta_{2}^{\prime} \in\left(\theta_{1} ; \theta_{2}^{\prime \prime}\right)$ and $\varphi_{0}^{\prime} \in\left(\varphi_{0}^{\prime \prime} ; \varphi_{0}^{\prime \prime \prime}\right)$, where $x^{\prime}=\ell_{\varphi_{0}^{\prime}}$.

Due to the fact that $\omega=\left(\mu_{1}, \mu_{2}, \ldots, \mu_{k}, p\right)$ is a type II splitting route, we have either $\mu_{k-1} \in\left(\mu_{1} ; \mu_{2}\right)$ and $\mu_{3} \in\left(p ; \mu_{2}\right)$, or $\mu_{k-1} \in\left(\mu_{2} ; \mu_{1}\right)$ and $\mu_{3} \in\left(\mu_{2} ; p\right)$. These two subcases are obtained from one another by the symmetry $(\theta, \varphi) \mapsto(-\theta,-\varphi)$, so it suffices to consider the first one, when $\mu_{k-1} \in\left(\mu_{1} ; \mu_{2}\right)$ and $\mu_{3} \in\left(p ; \mu_{2}\right)$. In this case, the $/$-ramification mirror of the move $M^{\prime \prime} \stackrel{\xi^{\prime}}{\longmapsto} M_{1}^{\prime \prime}$ is located at $x \cap y_{2}^{\prime \prime}$, whereas the splitting mirror of the move $M_{1} \stackrel{\eta_{1}^{\prime}}{\longrightarrow} M_{1}^{\prime}$ is located at $x^{\prime \prime \prime} \cap y_{2}$. One can see from Figure 9.39 that the diagrams $M_{1}^{\prime}$ and $M_{1}^{\prime \prime}$ are combinatorially equivalent.

Case 3: $\mu_{k}$ coincides with $\mu_{2}$, and $k$ is odd. 
We may assume that $p \in\left(\mu_{2} ; \mu_{1}\right)$ as the other case is obtained by applying the transformation $(\theta, \varphi) \mapsto$ $(-\theta,-\varphi)$. One can see from Figure 9.40 that the moves $M \stackrel{\eta_{1}}{\longmapsto} M^{\prime \prime}$ and $M \stackrel{\xi}{\mapsto} M_{1}$ commute with one another.
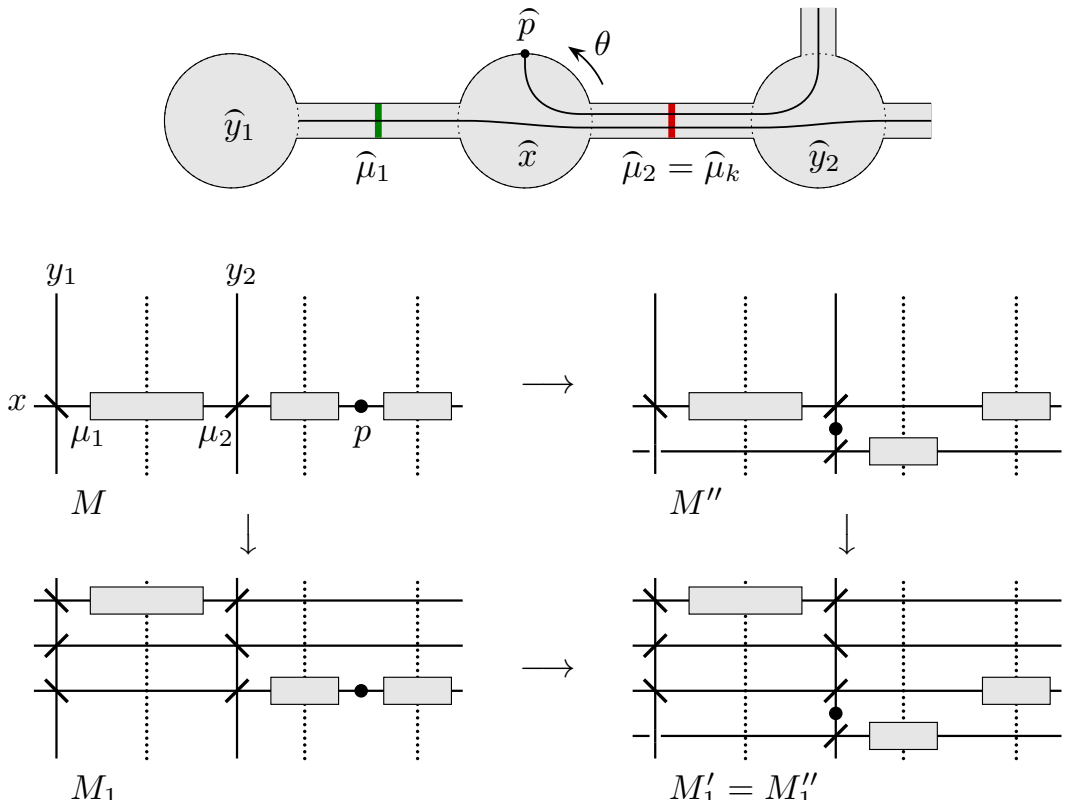

Figure 9.40. Induction step in the proof of Claim 3 , the case $\mu_{k}=\mu_{2}, k \equiv 1(\bmod 2)$
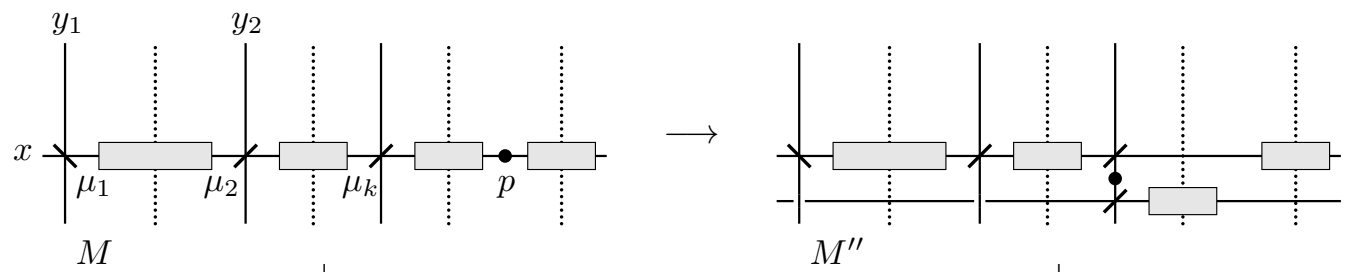

$M$

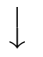

$$
M^{\prime \prime}
$$
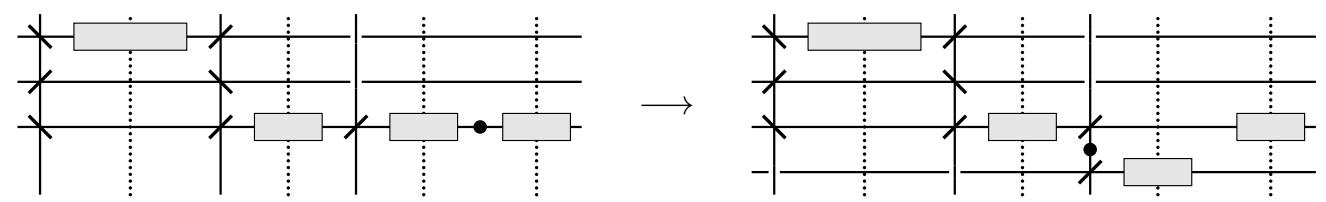

$M_{1}$

$$
M_{1}^{\prime}=M_{1}^{\prime \prime}
$$

FiguRE 9.41. Induction step in the proof of Claim 3, the case $\mu_{k} \in x, \mu_{k} \neq \mu_{2}, k \equiv 1$ $(\bmod 2), p \in\left(\mu_{k} ; \mu_{1}\right)$

\section{Case $4: \mu_{k}$ lies on $x, \mu_{k} \neq \mu_{2}, k$ is even.}

It is quite easy to see in this case that the moves $M \stackrel{\eta_{1}}{\longmapsto} M^{\prime \prime}$ and $M \stackrel{\xi}{\mapsto} M_{1}$ commute with one another. We leave this to the reader.

Case 5: $\mu_{k}$ lies on $x, \mu_{k} \neq \mu_{2}, k$ is odd.

The point $p$ lies on the occupied level $x$ in this case. We may assume that $\mu_{k} \in\left(\mu_{2} ; \mu_{1}\right)$ as the other case is obtained by applying the transformation $(\theta, \varphi) \mapsto(-\theta,-\varphi)$. We then also have $p \in\left(\mu_{2} ; \mu_{1}\right)$ as otherwise $\omega=\left(\mu_{1}, \mu_{2}, \ldots, \mu_{k}, p\right)$ would not be a type II splitting route. 
There are, however, two different subcases, $p \in\left(\mu_{k} ; \mu_{1}\right)$ and $p \in\left(\mu_{2} ; \mu_{k}\right)$. In the former subcase the moves producing $M_{1}^{\prime}$ and $M_{1}^{\prime \prime}$ are shown in Figure 9.41, One can see that the moves $M \stackrel{\eta_{1}}{\longmapsto} M^{\prime \prime}$ and $M \stackrel{\xi}{\mapsto} M_{1}$ again commute with one another.

The latter subcase is the only situation when the diagrams $M_{1}^{\prime}$ and $M_{1}^{\prime \prime}$ are not combinatorially equivalent. The moves in question are shown in Figure 9.42, One can see that the moves $M \stackrel{\eta_{1}}{\longmapsto} M^{\prime \prime}$ and $M \stackrel{\xi}{\mapsto} M_{1}$ almost commute with one another, so $M_{1}^{\prime \prime}$, viewed up to combinatorial equivalence, is obtained from $M_{1}^{\prime}$ by a single jump move. This jump move exchanges one of the successors of the

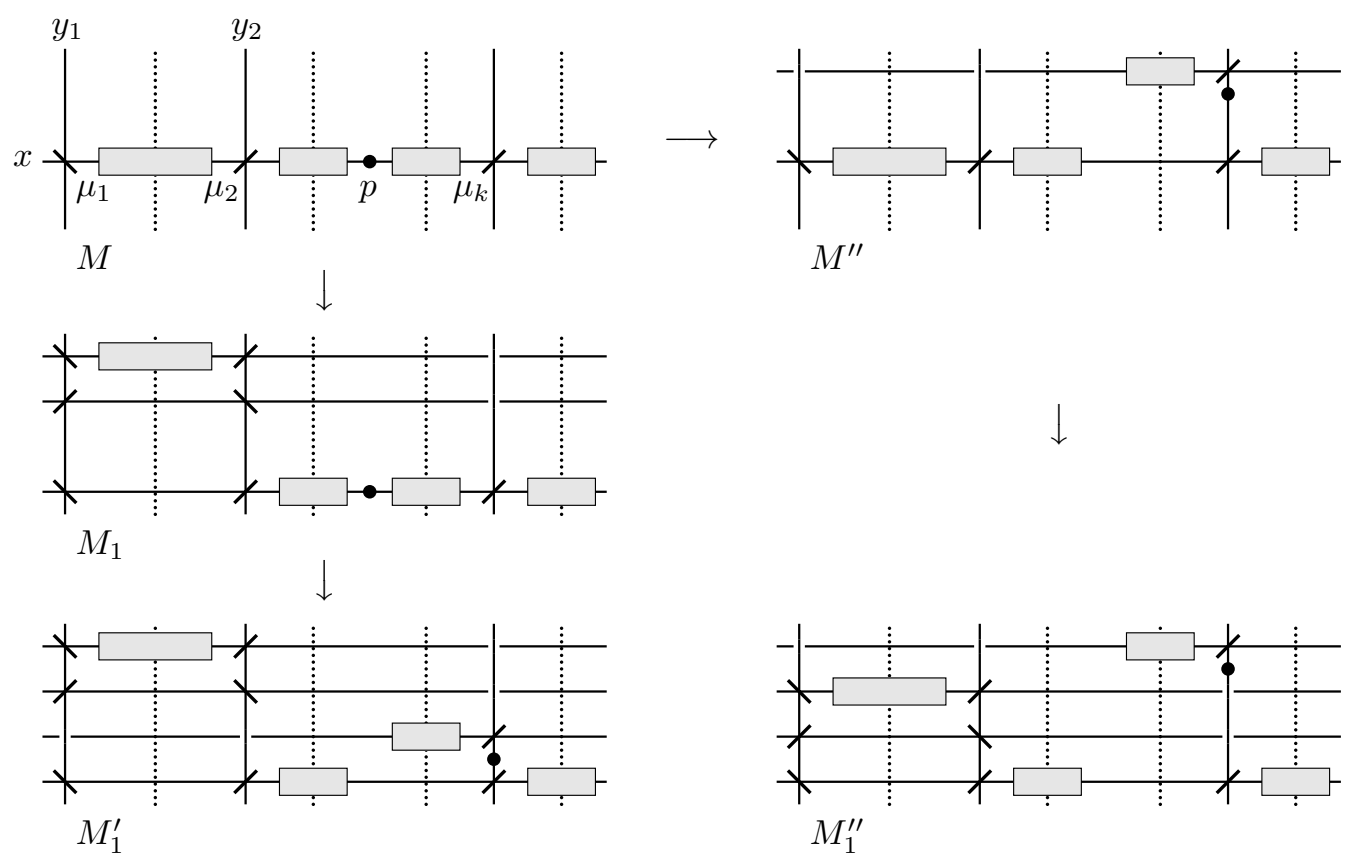

Figure 9.42. Induction step in the proof of Claim 3 , the case $\mu_{k} \in x, \mu_{k} \neq \mu_{2}, k \equiv 1$ $(\bmod 2), p \in\left(\mu_{2} ; \mu_{k}\right)$

occupied level $x$ with two successive occupied levels of $M_{1}^{\prime}$, one of which is also a successor of $x$, and the other is $x^{\prime}$. Recall that the occupied level $x^{\prime}$ is untouched by the move $M_{1} \stackrel{\eta^{\prime}}{\longmapsto} M_{2}$ and eliminated by the subsequent clean-up $M_{2} \stackrel{\chi}{\longmapsto} M_{3}$.

Thus, in all cases the diagrams $M_{1}^{\prime}$ and $M_{1}^{\prime \prime}$ are related by a sequence of jump moves that takes $\omega^{\prime \prime \prime}$ to $\omega^{\prime \prime}$ and represents the morphism $\zeta_{1}=\xi^{\prime} \circ \eta_{1} \circ \xi^{-1} \circ \eta_{1}^{\prime-1}$. It follows from Lemma 9.3 that the transformation $M_{2} \stackrel{\zeta_{2}}{\longrightarrow} M_{2}^{\prime \prime}$, where $\zeta_{2}=\eta_{2}^{\prime \prime} \circ \zeta_{1} \circ \eta_{2}^{\prime-1}$, also admits a decomposition into jump moves. Moreover, one can see that this transformation establishes a bijection between the elements of $M_{2}$ (occupied levels and mirrors) and those of $M_{2}^{\prime \prime}$ so that an element of $M_{2}$ is removed by the clean-up $M_{2} \stackrel{\chi}{\longmapsto} M_{3}$ if and only if the respective element of $M_{2}^{\prime \prime}$ is removed by the clean-up $M_{2}^{\prime \prime} \stackrel{\chi^{\prime}}{\longmapsto} M_{3}^{\prime \prime}$. Therefore, the transformation $M_{3} \stackrel{\zeta_{3}}{\longmapsto} M_{3}^{\prime \prime}$, where $\zeta_{3}=\chi^{\prime} \circ \zeta_{2} \circ \chi^{-1}$, also decomposes into jump moves. The induction step follows, which concludes the proof of Claim 3.

It remains to prove Claim 4, that is, to show that an anticanonical decomposition of the generalized type II split move $M \mapsto M^{\prime}$ can be chosen to be neat.

Let $C$ be a collection of boundary circuits of $M$ untouched by the move $M \mapsto M^{\prime}$, and let $x$ be an occupied level of $M$. Among the successors of $x$, only one can coincide with $x$. This means that at most one connected component of $\widehat{x} \backslash \widehat{\omega}$ has a non-empty intersection with the union of the boundary components of $\overparen{M}$ corresponding to the circuits in $C$. Let $d$ be such a component. 
As noted above, the principal difference of an anticanonical decomposition from a canonical one, is that we start cutting the surface $\overparen{M}$ along the path $\widehat{\omega}$ from the other end. However, the cutting path is still the same, so $d$ is not going to be cut as a result of a wrinkle creation move included in an anticanonical decomposition. This implies that we can keep the position of the 1-handles that remain attached to $d$ fixed when choosing a concrete wrinkle creation move that splits the occupied level $x$ or one of its successors.

The clean-up operations modify only the boundary circuits that has already been modified (or created) by the preceding moves of an anticanonical decompositions. Finally, the jump moves involved in an anticanonical decomposition are needed to exchange some successors of $x$ with each other and are not needed to exchange them with successors of another occupied level of $\overparen{M}$. So, there is no problem to keep one selected successor of $x$ fixed while reordering the successors by jump moves.

Thus, we see that an anticanonical decomposition of the move $M \mapsto M^{\prime}$ can be chosen to satisfy Condition (1) of Definition 7.2. One can see that the other two conditions are satisfied automatically.

This concludes the proof of Proposition 9.9.

9.8. Generalized wrinkle moves and flexibility. In order to prove the second commutation property for generalized merge moves in the special case we need some preparations.

Lemma 9.10. Let $M$ be an enhanced mirror diagram, and let $C$ be a collection of essential boundary circuits of $M$. The following two conditions are equivalent.

(1) $M$ is +-flexible (respectively, --flexible) relative to $C$ (see Definition 9.2).

(2) For any /-mirror (respectively, \-mirror) $\mu$ hit by some boundary circuit $c \notin C$, there exists a type II (respectively, type I) single-headed splitting route $\omega=\left(\mu_{1}, \ldots, \mu_{k}, p\right)$ with $\mu_{k}=\mu$ such that $\omega$ does not separate $C$.

Proof. $(1) \Rightarrow(2)$. We consider only the case of +-flexibility as the other is symmetric to it.

Let $\mu$ be a $/$-mirror on $c \in \partial M \backslash C$, and let $c_{1}, c_{2}, \ldots, c_{m}=c$ be a sequence of boundary circuits of $M$ satisfying the conditions from Definition 9.2 It follows from these conditions that there exists a sequence of mirrors $\mu_{1}, \mu_{2}, \ldots, \mu_{i_{1}}, \mu_{i_{1}+1}, \ldots, \mu_{i_{2}}, \mu_{i_{2}+1}, \ldots, \mu_{i_{m}}$ of $M$ such that:

(i) $\mu_{1}$ is a $\backslash$-mirror and all the others are $/$-mirrors;

(ii) the last mirror $\mu_{i_{m}}$ is $\mu$;

(iii) for all $j=1, \ldots, m$ and $i=i_{j-1}, i_{j-1}+1, \ldots, i_{j}-1$ the mirrors $\mu_{i}$ and $\mu_{i+1}$ lie on the same occupied level of $M$ (we put $i_{0}=1$ ), and either $\left[\mu_{i} ; \mu_{i+1}\right]$ or $\left[\mu_{i+1} ; \mu_{i}\right]$ is a subset of $c_{j}$.

The following condition on a sequence $\mu_{1}, \ldots, \mu_{k}$ is clearly weaker than (iii):

(iii') for all $i=1, \ldots, k-1$, the mirrors $\mu_{i}$ and $\mu_{i+1}$ lie on the same occupied level of $M$, and either $\left[\mu_{i} ; \mu_{i+1}\right]$ or $\left[\mu_{i+1} ; \mu_{i}\right]$ is a subset of $\bigcup_{c \in \partial M \backslash C} c$.

So, there exists a sequence satisfying (i), (ii), and (iii'). Take a shortest such sequence and choose a point $p \neq \mu$ on a boundary circuit $c \notin C$ in a small neighborhood of $\mu$ so that $\mu, \mu_{k-1}$, and $p$ don't lie on the same occupied level of $M$. Then $\left(\mu_{1}, \ldots, \mu_{k}, p\right)$ is a type II splitting route not separating $C$.

The implication $(2) \Rightarrow(1)$ is easy and left to the reader. We don't use it in the sequel.

In the previous subsection we defined an anticanonical decomposition of a generalized type II split move. An anticanonical decomposition of a generalized type I split move is defined by symmetry, exchanging the roles of $/$ - and $\backslash$-mirrors.

Definition 9.17. Let $\omega=\left(\mu_{1}, \ldots, \mu_{k}, p\right), k \geqslant 2$, be a single-headed splitting route (of either type) in an enhanced mirror diagram $M$. By a generalized wrinkle creation move associated with $\omega$ we mean the composition of the first $k-1$ moves (which are wrinkle creation moves) of an anticanonical decomposition of a generalized split move $M \mapsto M^{\prime}$ associated with $\omega$. Thus defined generalized wrinkle creation move will also be said to comply with the move $M \mapsto M^{\prime}$.

The inverse operation is referred to as a generalized wrinkle reduction move.

Figure 9.43 illustrates the idea of a generalized wrinkle creation move. Note that if $k=2$, then a generalized wrinkle creation move associated with $\left(\mu_{1}, \mu_{2}, p\right)$ is an ordinary wrinkle creation move having $\mu_{1}$ and $\mu_{2}$ as the ramification mirrors. 

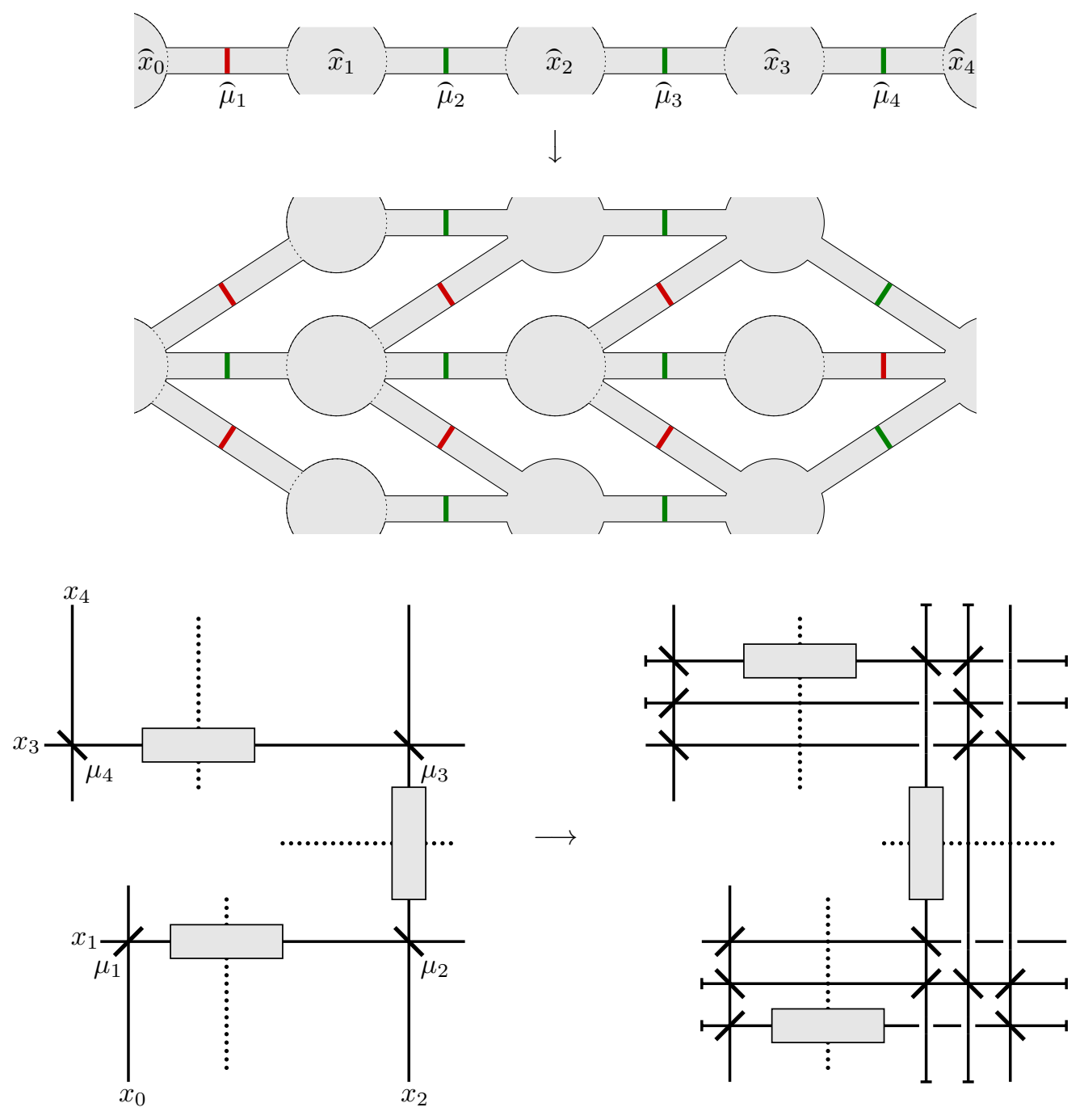

FIGURE 9.43. Generalized wrinkle creation move associated with a type I splitting route

The existence of an anticanonical decomposition of a non-special generalized split move implies that, for every single-headed splitting route $\omega=\left(\mu_{1}, \mu_{2}, \ldots, \mu_{k}, p\right)$ with $k \geqslant 2$ such that $\omega$ does not separate a chosen collection $C$ of essential boundary circuits, there exists a generalized wrinkle creation move associated with $\omega$ that preserves all boundary circuits in $C$.

So, the meaning of Lemma 9.10 is, roughly, that we can create wrinkles anywhere in the diagram without disturbing selected boundary circuits, provided that the diagram is flexible relative to these boundary circuits.

Another thing the flexibility allows us to do is to avoid the use of type II extension moves.

Lemma 9.11. Let $M$ be an enhanced mirror diagram, and let $C$ be a collection of essential boundary circuits of $M$ such that $M$ is +-flexible relative to $C$. Then any type II extension move that preserves the boundary circuits in $C$ admits a $C$-neat decomposition into type I elementary moves combined with type II split/merge moves.

Proof. We call a boundary circuit $c \in \partial M \backslash C$ flexible if any type II extension move $M \mapsto M^{\prime}$ that modifies $c$ admits a $C$-neat decomposition into type I elementary moves and type II split/merge moves. It follows from Lemmas 7.6 and 7.3 that any boundary circuit $c \in \partial M \backslash C$ with $\operatorname{tb}_{+}(c)<0$ is flexible. 
It also follows that $c$ is flexible, once some type II extension move $M \mapsto M^{\prime}$ that modifies $c$ admits a $C$-neat decomposition into type I elementary moves and type II split/merge moves.

It therefore suffices to show that whenever $c, c^{\prime} \in \partial M \backslash C$ are adjacent boundary circuits one of which is flexible, the other is flexible, too. This is demonstrated in Figure 9.44, where we assume that $c^{\prime}$ is flexible and shares a $/$-mirror with $c$.

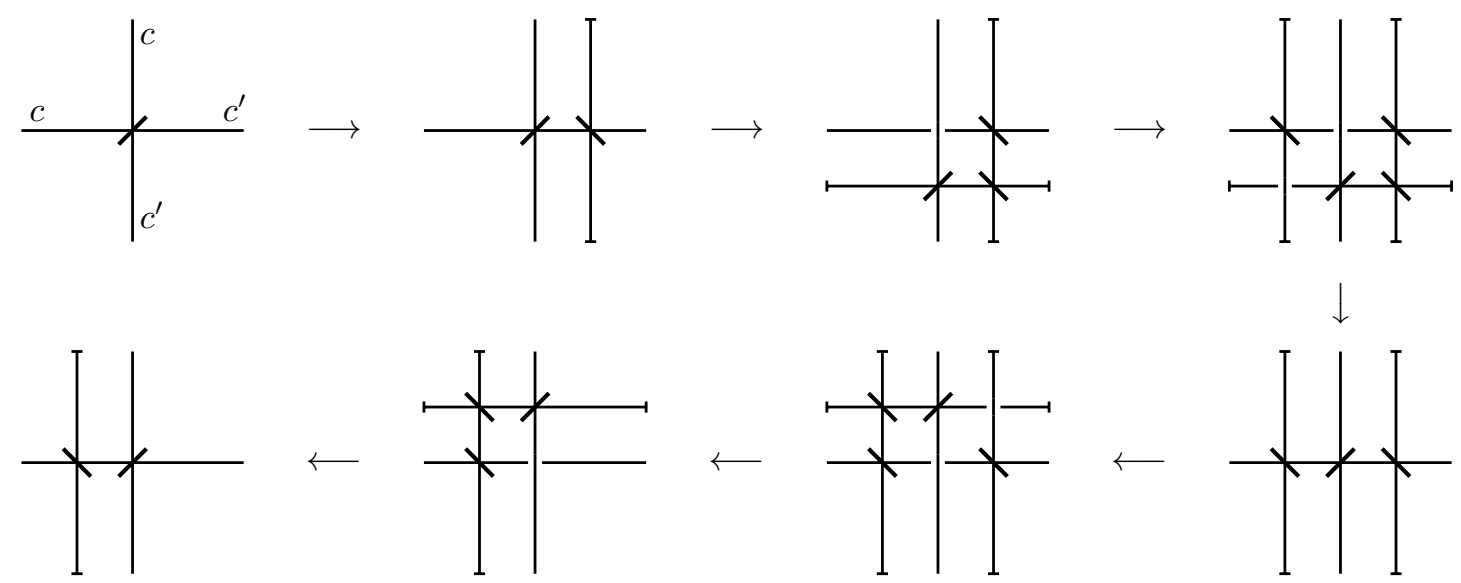

Figure 9.44. If $c^{\prime}$ is flexible, then so is $c$

We apply the following moves:

(1) a type II extension move that modifies $c^{\prime}$;

(2) a type II split move;

(3) a type II extension move that modifies the boundary circuit obtained from $c$ by the previous transformations, which now has $\mathrm{tb}_{+}<0$;

(4) a type II merge move;

(5) a type II split move;

(6) a type II elimination move that modifies the boundary circuit obtained from $c^{\prime}$ by the previous transformations, which now has $\mathrm{tb}_{+}<-1$;

(7) a type II merge move.

None of these moves modifies any boundary circuit of the original diagram except for $c$ and $c^{\prime}$.

9.9. Second commutation property of generalized type II merge moves, special case. Here we complete the proof of the second commutation property of generalized type II merge moves. Special generalized type II merge moves don't have this property in general, and a --flexibility assumption on the obtained diagram is needed.

To construct the required decomposition we will need generalized wrinkle creation moves. In order to make them friendlier to certain split moves we introduce the following

Convention 9.2. A generalized wrinkle creation move $M \mapsto M_{1}$ associated with a single-headed splitting route $\omega=\left(\mu_{1}, \ldots, \mu_{k}, p\right)$ does not depend on $p$. However, in the context where generalized wrinkle moves are used we will assume that a concrete choice for $p$ has been made.

If $M \stackrel{\eta_{1}}{\longmapsto} M_{1}$ is a generalized wrinkle creation move that complies with a generalized split move $M \stackrel{\eta}{\longmapsto}$ $M^{\prime}$, and $M_{1} \stackrel{\eta_{2}}{\longmapsto} M_{2} \stackrel{\eta_{3}}{\longmapsto} \ldots \stackrel{\eta_{m}}{\longmapsto} M_{m}=M^{\prime}$ are the remaining moves from an anticanonical decomposition of $M \stackrel{\eta}{\mapsto} M^{\prime}$, then we define $h_{M}^{M_{1}}$ as $h_{M_{2}}^{M_{1}} \circ h_{M_{3}}^{M_{2}} \circ \ldots h_{M_{m}}^{M_{m-1}} \circ h_{M}^{M^{\prime}}$. (Thus, the domain of $h_{M}^{M_{1}}$ is the same as that of $h_{M}^{M^{\prime}}$, and it does depend essentially on $p$ though the move $M \mapsto M_{1}$ does not.) 
Definition 9.18. Let $M \stackrel{\eta_{1}}{\longmapsto} M_{1}$ be a generalized wrinkle creation move complying with a generalized split move $M \stackrel{\eta}{\mapsto} M^{\prime}$, and let $M \stackrel{\zeta}{\mapsto} M_{2}$ be one of the moves for which the partial homeomorphism $h_{M}^{M_{2}}$ has been defined.

We say that the move $M \stackrel{\zeta}{\mapsto} M_{2}$ is friendly to the move $M \stackrel{\eta_{1}}{\longmapsto} M_{1}$ if it is friendly to $M \stackrel{\eta}{\mapsto} M^{\prime}$. In this case, the move $M_{2} \stackrel{\eta_{1}{ }^{\zeta}}{\longmapsto} M_{21}$ resembling $M \stackrel{\eta_{1}}{\longmapsto} M_{1}$ is defined as a generalized wrinkle creation move complying with a move $M_{2} \stackrel{\eta^{\varsigma}}{\longmapsto} M_{2}^{\prime}$ resembling $M \stackrel{\eta}{\mapsto} M^{\prime}$.

Now Definition 9.10 extends to generalized wrinkle moves accordingly.

Lemma 9.12. Let $M$ be an enhanced mirror diagram in which a /-mirror $\mu_{0}$ can be removed by a type I elimination move, and let $\omega$ be a type II splitting route in $M$ of the form $\omega=\left(\mu_{1}, \mu_{0}, \mu_{0}, \mu_{2}, p\right)$. Let also $\sigma=\left(\nu_{1}, \nu_{2}, \ldots, \nu_{k}, q\right)$ be a single-headed type I splitting route such that, $\nu_{k} \in\left\{\mu_{1}, \mu_{2}\right\}, \nu_{1}, \ldots, \nu_{k-1} \notin$ $\left\{\mu_{0}, \mu_{1}, \mu_{2}\right\}$, and the associated splitting paths $\widehat{\sigma}$ and $\widehat{\omega}$ have no unavoidable intersection. Finally, let $M \stackrel{\eta}{\mapsto}$ $M_{1}$ and $M \stackrel{\zeta}{\mapsto} M_{2}$ be a generalized type II split move associated with $\omega$, and a generalized wrinkle creation move associated with $\sigma$, respectively.

Then the moves $M \stackrel{\eta}{\mapsto} M_{1}$ and $M \stackrel{\zeta}{\mapsto} M_{2}$ almost commute.

Proof. The proof is by induction in the length of the decomposition of $M \stackrel{\zeta}{\mapsto} M_{2}$ into a sequence of ordinary wrinkle creation moves, and is similar in nature to that of Lemma 9.6 We omit most of the detail leaving it to the reader.

The induction base, $k=2$, amounts to considering the six, up to symmetries, possible mutual positions of the splitting paths $\widehat{\omega}$ and $\widehat{\sigma}$ shown in Figure 9.45,

For the induction step, one uses the fact that generalized wrinkle moves commute with jump moves, which is obvious, and shows that an ordinary wrinkle creation move with ramification mirrors $\nu_{1}, \nu_{2}$ disjoint from $\mu_{0}, \mu_{1}, \mu_{2}$ almost commutes with the move $M \stackrel{\eta}{\mapsto} M_{1}$. To do so one considers six cases, in one of which the moves do not interfere, so their commutation is obvious, and the five others are shown in Figure 9.46 .

Proposition 9.10. Let $M$ be an enhanced mirror diagram, and let $C$ be a collection of essential boundary circuits of $M$ such that $M$ is --flexible relative to $C$. Let also $M \stackrel{\eta}{\mapsto} M_{1}$ be a special generalized type II split move preserving all boundary circuits in $C$.

Then the move $M \stackrel{\eta}{\mapsto} M_{1}$ admits a $C$-neat decomposition into a sequence of elementary moves such that all type II moves in it occur before all type I moves.

Proof. Let $\omega=\left(\mu_{1}, \mu_{2}, p\right)$ be the special type II splitting route with which the move $M \stackrel{\eta}{\mapsto} M_{1}$ is associated. Let also $M \stackrel{\eta^{\prime}}{\longmapsto} M_{1}^{\prime}$ be another such move associated with $\omega$, and preserving all $c \in C$. It follows from Proposition 9.7 that the sought-for decomposition of the move $M \stackrel{\eta}{\mapsto} M_{1}$ exists if and only if it exists for $M \stackrel{\eta^{\prime}}{\longmapsto} M_{1}^{\prime}$. This means that, without loss of generality, we can prescribe the position of the auxiliary mirror, which we denote by $\mu_{0}$, of the move $M \stackrel{\eta}{\mapsto} M_{1}$ at our wish.

By Lemma 9.10 there exists a type I single-headed splitting route $\sigma=\left(\nu_{1}, \ldots, \nu_{k}, q\right)$ with $\nu_{k} \in\left\{\mu_{1}, \mu_{2}\right\}$ such that $\sigma$ does not separate $C$. Take a shortest such $\sigma$. This will ensure that $\nu_{i} \notin\left\{\mu_{1}, \mu_{2}\right\}$ for all $i=1, \ldots, k-1$. Moreover, the associated splitting path $\widehat{\sigma}$ will have no unavoidable intersection with $\widehat{\omega}$.

Now choose the position of $\mu_{0}$ using the following rules. If $\mu_{1}=\nu_{k}$ put $\mu_{0}$ close to $\mu_{2}$ and outside $\bigcup_{c \in C} c \cup\{q\}$. If $\mu_{2}=\nu_{k}$ put $\mu_{0}$ close to $\mu_{1}$ and outside $\bigcup_{c \in C} c \cup\{q\}$. Let $M \stackrel{\eta_{1}}{\longmapsto} M^{\prime}$ be the first move in a canonical decomposition of the move $M \stackrel{\eta}{\mapsto} M_{1}$, that is, the type I extension move that adds the mirror $\mu_{0}$ to the diagram. Then the remaining part of the canonical decomposition is a generalized type II split move $M^{\prime} \stackrel{\eta_{2}}{\longmapsto} M_{1}$ associated with $\omega^{\prime}=\left(\mu_{1}, \mu_{0}, \mu_{0}, \mu_{2}, p\right)$.

Define $M^{\prime} \stackrel{\zeta}{\mapsto} M_{2}^{\prime}$ to be a generalized wrinkle creation move associated with $\sigma$. We choose it to preserve all the boundary circuits in $C$. This move clearly commutes with the elimination move $M^{\prime} \stackrel{\eta_{1}^{-1}}{\longmapsto} M$, 

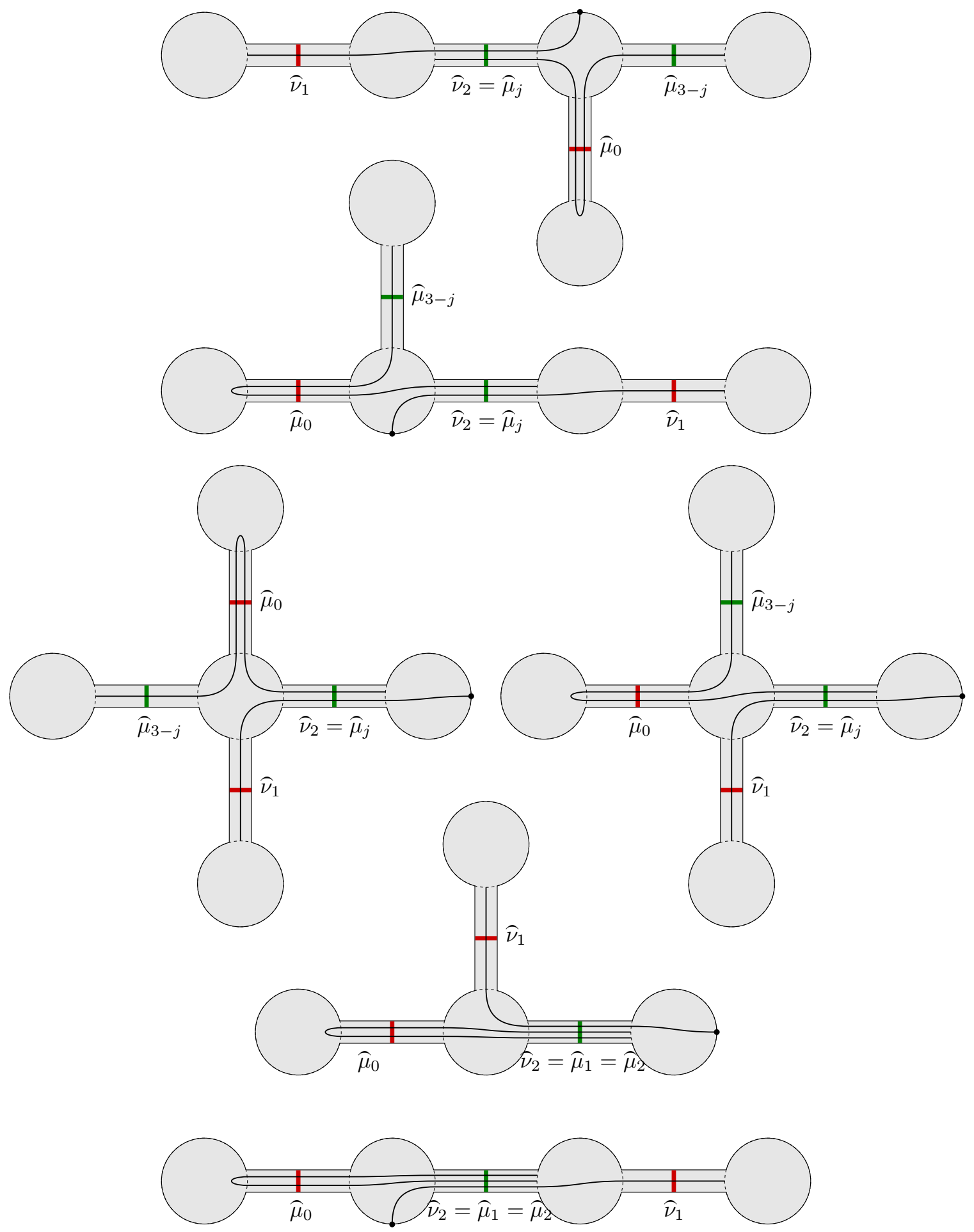

Figure 9.45. Possible mutual positions of $\widehat{\omega}$ and $\widehat{\sigma}$ in the case $k=2$ in Lemma $9.12(j=1,2)$

so we have a generalized wrinkle creation move $M \stackrel{\zeta^{\eta_{1}^{-1}}}{\longmapsto} M_{2}$ and an elimination move $M_{2}^{\prime} \stackrel{\xi}{\mapsto} M_{2}$, where $\xi=\left(\eta_{1}^{-1}\right)^{\zeta}$. 


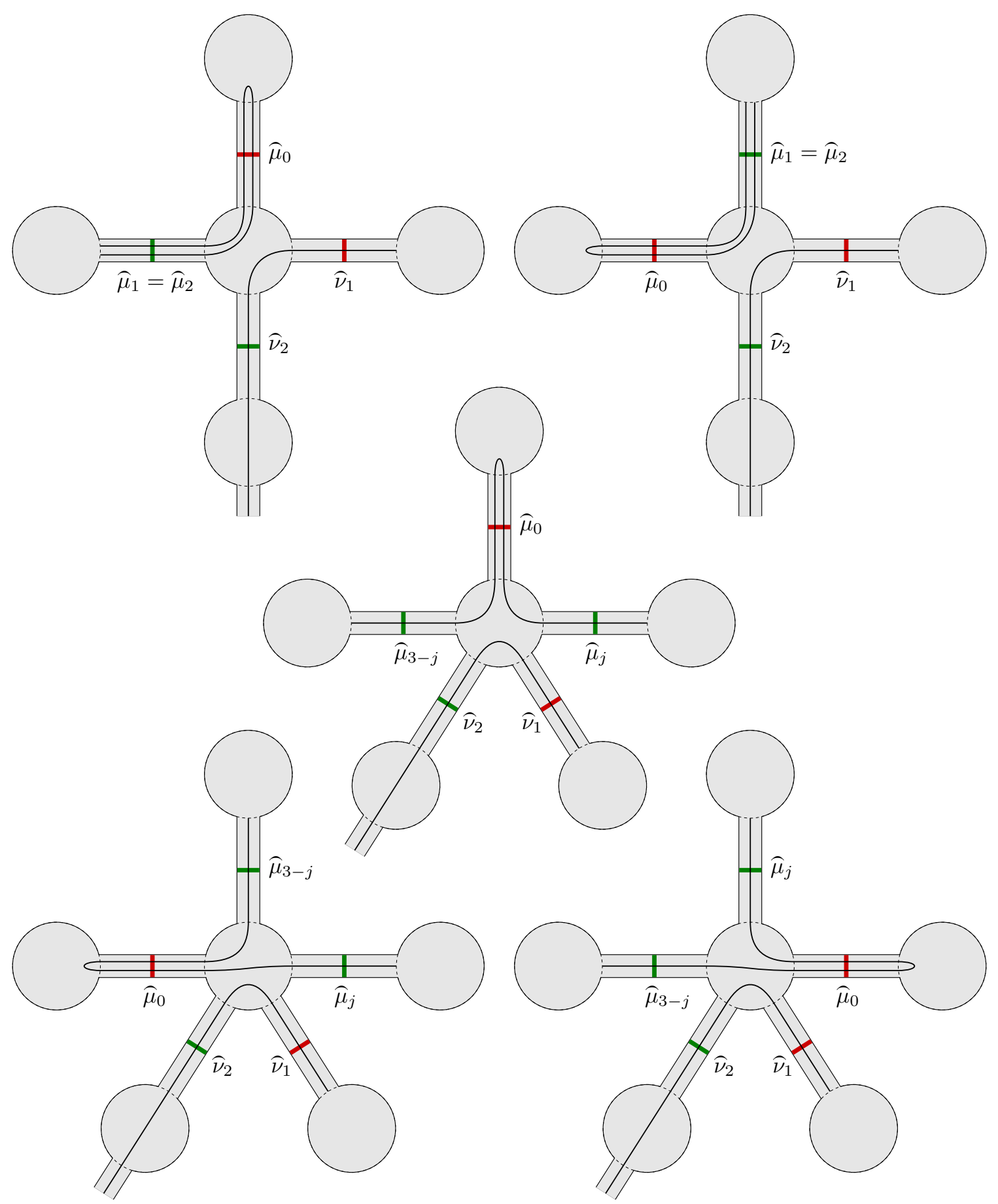

Figure 9.46. Cases to consider for the induction step in the proof of Lemma $9.12(j=1,2)$

By construction, the splitting paths $\widehat{\omega}^{\prime}$ and $\widehat{\sigma}$ in $\widehat{M}^{\prime}$ have no unavoidable intersection, so we can apply Lemma 9.12 to conclude that the moves $M^{\prime} \stackrel{\eta_{2}}{\longmapsto} M_{1}$ and $M^{\prime} \stackrel{\zeta}{\longmapsto} M_{2}^{\prime}$ almost commute. Let $M_{1} \stackrel{\zeta^{\eta_{2}}}{\longmapsto} M_{12}$ 
and $M_{2}^{\prime} \stackrel{\eta_{2}^{\zeta}}{\longmapsto} M_{21}$ be the moves resembling $M^{\prime} \stackrel{\zeta}{\longmapsto} M_{2}^{\prime}$ and $M^{\prime} \stackrel{\eta_{2}}{\longmapsto} M_{1}$, respectively (see Figure 9.47 for the general scheme of moves).

Denote by $\omega^{\prime \prime}=\left(\mu_{1}^{\prime}, \mu_{0}^{\prime}, \mu_{0}^{\prime}, \mu_{1}^{\prime}, p^{\prime}\right)$ the type II splitting route in $M_{2}^{\prime}$ with which the move $M_{2}^{\prime} \stackrel{\eta_{2}^{\zeta}}{\longmapsto} M_{21}$ is associated. This route is the image of $\omega^{\prime}$ under the generalized wrinkle creation move $M^{\prime} \zeta^{\zeta} M_{2}^{\prime}$. Denote also by $x$ the occupied level of $M_{2}^{\prime}$ containing the mirrors $\mu_{0}^{\prime}, \mu_{1}^{\prime}$, and $\mu_{2}^{\prime}$ (since $\mu_{0}^{\prime} \neq \mu_{1}^{\prime}$ this level is unique).

The move $M^{\prime} \stackrel{\zeta}{\mapsto} M_{2}^{\prime}$ creates a number of inessential boundary circuits having form of the boundary of a rectangle. Since $\nu_{k} \in\left\{\mu_{1}, \mu_{2}\right\}$, one of these circuits $c$, say, hits either $\mu_{1}^{\prime}$ or $\mu_{2}^{\prime}$. There is a unique $/$-mirror $\mu_{*}$ of $M_{2}^{\prime}$ on $x$ hit by $c$. One can see that $\omega^{\prime \prime \prime}=\left(\mu_{1}^{\prime}, \mu_{*}, \mu_{*}, \mu_{2}^{\prime}, p^{\prime}\right)$ is a type II splitting route similar to $\omega^{\prime \prime}$. One can also see that $\omega^{\prime \prime \prime}$ does not separate $C$.

Let $M_{2}^{\prime} \stackrel{\chi}{\longmapsto} M_{3}$ be a generalized type II splitting route associated with $\omega^{\prime \prime \prime}$ and preserving all the boundary circuits in $C$. Since $\mu_{0}^{\prime}$ does not appear in $\omega^{\prime \prime \prime}$, this move commutes with the elimination move $M_{2}^{\prime} \stackrel{\xi}{\mapsto} M_{2}$, so we have a type I elimination move $M_{3} \stackrel{\xi^{\chi}}{\longmapsto} M_{4}$ and a generalized type II split move $M_{2} \stackrel{\chi^{\xi}}{\longmapsto} M_{4}$ that preserve all the boundary circuits in $C$.

We are ready to produce the sought-for decomposition of the move $M \stackrel{\eta}{\mapsto} M_{1}$. It consists of the following parts:

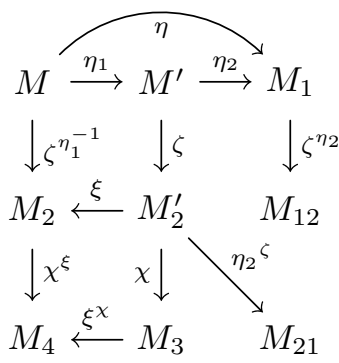

Figure 9.47. The scheme of the moves in the proof of Proposition 9.10

(1) a decomposition of the generalized wrinkle creation move $M \stackrel{\zeta^{\eta_{1}^{-1}}}{\longmapsto} M_{2}$ into type II elementary moves, (the decomposition exists by Lemma 7.3);

(2) a decomposition of the non-special type II split move $M_{2} \stackrel{\chi^{\xi}}{\longmapsto} M_{4}$ into a sequence of elementary moves such that all type II moves in it occur before type I moves (the decomposition exists by Proposition 9.9)

(3) type I extension move $M_{4} \stackrel{\left(\xi^{\chi}\right)^{-1}}{\longmapsto} M_{3}$;

(4) a decomposition into type I elementary moves of the transformation $M_{3} \stackrel{\eta_{2}^{\zeta} \circ \chi^{-1}}{\longmapsto} M_{21}$ (the decomposition exists by Proposition 9.7);

(5) a decomposition into type I elementary moves of the transformation $M_{21} \stackrel{\zeta^{\eta_{2}} \circ \eta_{2} \circ \zeta^{-1} \circ\left(\eta_{2}^{\zeta}\right)^{-1}}{\longmapsto} M_{12}$ (the decomposition exists by Lemmas 9.12 and 7.3 );

(6) and finally, a decomposition into type I elementary moves of the generalized wrinkle reduction $M_{12} \stackrel{\left(\zeta^{\eta_{2}}\right)^{-1}}{\longmapsto} M_{1}$ (the decomposition exists by Lemma 7.3);

All the listed decompositions are assumed to be $C$-neat.

9.10. Generalized type II bypass removals. First commutation property. Generalized type II split moves don't have the first commutation property, and should be replaced by something that has it. Generalized type II bypass removals, which are defined below, provide for a proper replacement. If the mirror diagrams are viewed up to type I moves, then generalized type II bypass removals do exactly the 
same job as generalized type II splittings do (see Subsection 8.3), but unlike the latter they do have the first commutation property as we will see in a moment. Another important property of generalized type II bypass removals is that these moves, unlike generalized type II split moves, are always --safe-to-postpone (see Definition 7.3).

Definition 9.19. Let $M$ and $M^{\prime}$ be enhanced mirror diagram such that the following holds:

(1) the transition $\widehat{M} \mapsto \widehat{M}^{\prime}$ is a handle removal;

(2) all mirrors in $E_{M} \backslash E_{M^{\prime}}$ are /-mirrors;

(3) there is a +-negligible boundary circuit $c \in \partial M \backslash \partial M^{\prime}$ (see Definition 8.12).

Then we say that $M^{\prime}$ is obtained from $M$ by a generalized type $I I$ bypass removal. With this operation we associate a morphism $\eta: \widehat{M} \rightarrow \widehat{M}^{\prime}$ by requesting that $\left(F, F\right.$, id $\left.\left.\right|_{F}\right) \in \eta$, where $F$ is a surface obtained from $\overparen{M}$ by a patching of $\widehat{c}$. The inverse operation $M^{\prime} \stackrel{\eta^{-1}}{\longmapsto} M$ is called a generalized type II bypass addition.

According to this definition, if $M \mapsto M^{\prime}$ is a generalized type II bypass removal, then the closure of $\widehat{M} \backslash \widehat{M}$ ' is an arc with endpoints on $\widehat{M}^{\prime}$. (The endpoints may coincide, thus making this arc a loop.) This means that there is a sequence $\beta=\left(y_{0}, \nu_{1}, y_{1}, \nu_{2}, \ldots, \nu_{k}, y_{k}\right)$ in which $y_{i} \in L_{M}, \nu_{i}=y_{i-1} \cap y_{i} \in E_{M} \backslash E_{M^{\prime}}$, $i=1, \ldots, k$, and $y_{0}, y_{k} \in L_{M^{\prime}}$. This sequence, which is defined uniquely up to reversing the order, will be referred to as the type II bypass removed by the move $M \mapsto M^{\prime}$. Note that the elements that are actually removed do not include the first and the last ones, which are the occupied levels $y_{0}$ and $y_{k}$. Note also that the occupied levels $y_{i}, i=0,1, \ldots, k$, must be pairwise distinct, with the only exception that $y_{0}$ may coincide with $y_{k}$. Each level $y_{i}$ with $1 \leqslant i \leqslant k-1$ must contain exactly two mirrors of $M$, which are $\nu_{i}$ and $\nu_{i+1}$.

Definition 9.19 requires the existence of a + -negligible boundary circuit $c$ of $M$ hitting each of $\nu_{i}$, $i=1, \ldots, k$, exactly once. Since $c$ is inessential, there must exist a patching disc for $\widehat{c}$, and, as follows from Lemma 5.1, the patching disc can be chosen in the form $\bigcup_{r \in \Pi} \widehat{r}$, where $\Pi$ is a collection of rectangles. This can be visualized by drawing the rectangles from $\Pi$ together with the mirror diagram $M$, and following the conventions made for both kinds of pictures, presenting mirror diagrams and presenting rectangular diagrams of a surface.

There is another boundary circuit of $M$ hitting each of $\nu_{i}, i=1, \ldots, k$, exactly once, which we denote by $c^{\prime}$. The boundary circuits $c$ and $c^{\prime}$ are said to be adjacent to the bypass $\beta$. They are replaced in $M^{\prime}$ by a single boundary circuit, which is referred to as $c \# c^{\prime}$.

Example 9.6. Shown in Figure 9.48 is an example of a generalized type II bypass removal $M \mapsto M^{\prime}$. The top left picture shows the diagram $M$ together with a collection of rectangles representing a patching disc for a +-negligible circuit $c$ which is destroyed by the move. The circuit $c$ is shown in orange. The dashed lines show two meridians that are not occupied levels of $M$. They correspond to two intersection points of the interior of the patching disc with $\mathbb{S}_{\tau=1}^{1}$. Denoted by $\mu^{\prime}$ and $\mu^{\prime \prime}$ are the only two \-mirrors on $c$ due to which $c$ is +-negligible.

The bottom picture shows the structure of a canonic dividing configuration $\left(\delta_{+}, \delta_{-}\right)$of the patching disc. The crucial point about this dividing configuration is that $\delta_{+}$is a single arc. This follows from the condition $\mathrm{tb}_{+}(c)=-1$.

Proposition 9.11. Let $M \stackrel{\eta}{\mapsto} M^{\prime}$ be a generalized type $I I$ bypass removal, and let $M^{\prime} \zeta^{\zeta} M^{\prime \prime}$ be a type $I$ elementary move. Let also $C$ be the set of all boundary circuits of $M$ preserved by both moves. Then there exist transformations $M \stackrel{\zeta^{\prime}}{\longmapsto} M^{\prime \prime \prime}$ and $M^{\prime \prime \prime} \stackrel{\eta^{\prime}}{\longmapsto} M^{\prime \prime}$ preserving all the boundary circuits in $C$ such that the following holds:

(1) we have $\eta^{\prime} \circ \zeta^{\prime}=\zeta \circ \eta$;

(2) the transformation $M^{\prime \prime \prime} \stackrel{\eta^{\prime}}{\longmapsto} M^{\prime \prime}$ is a generalized type II bypass removal;

(3) the transformation $M \stackrel{\zeta^{\prime}}{\longmapsto} M^{\prime \prime \prime}$ admits a neat decomposition into a sequence of type I elementary moves which yields a $C$-neat decomposition of $M \stackrel{\zeta \circ \eta}{\longmapsto} M^{\prime \prime}$ after appending the move $M^{\prime \prime \prime} \stackrel{\eta^{\prime}}{\longmapsto} M^{\prime \prime}$ at the end of the sequence. 

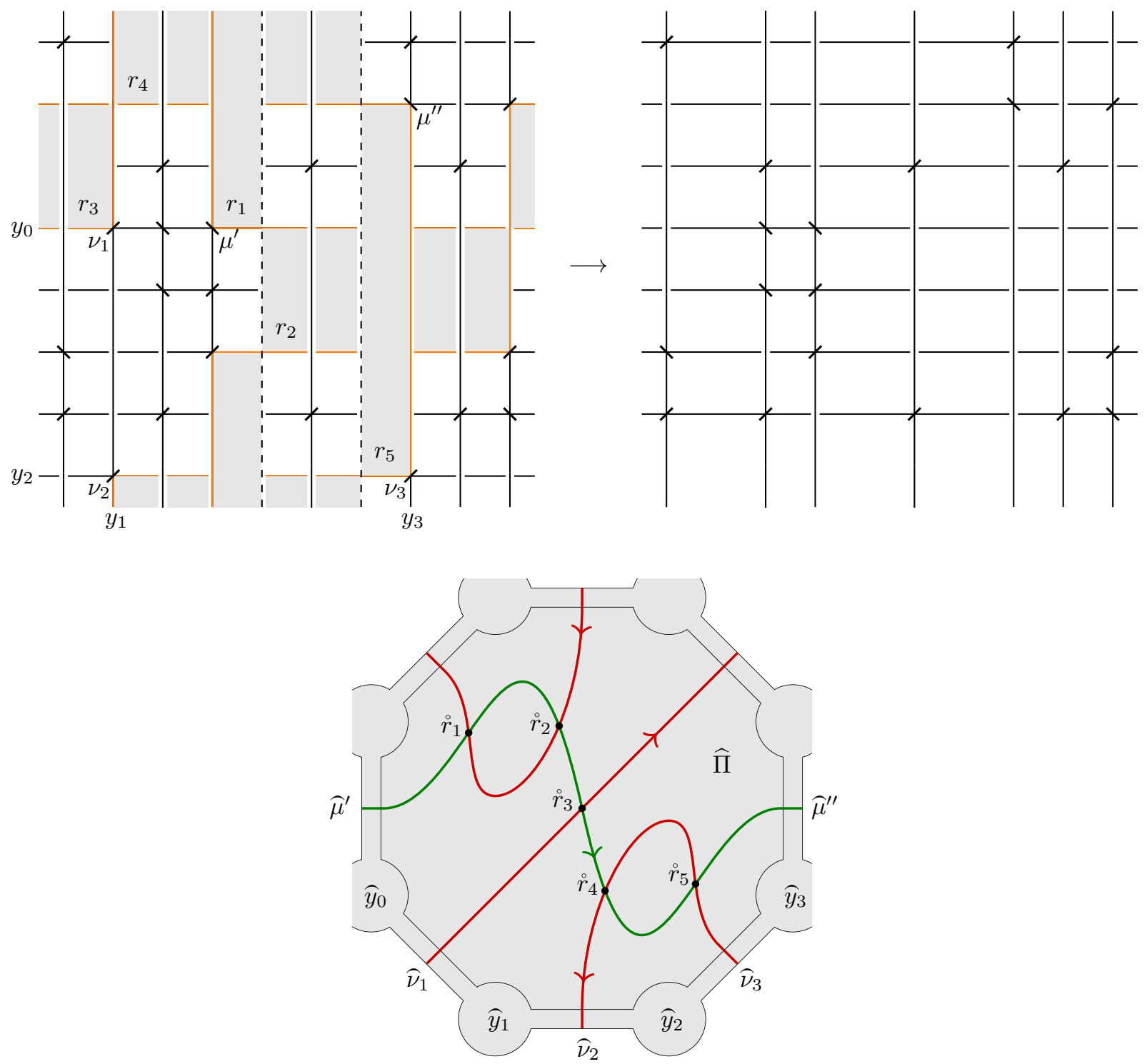

FIGURE 9.48. Generalized type II bypass removal and the structure of the corresponding patching disc $\widehat{\Pi}=\widehat{r}_{1} \cup \widehat{r}_{2} \cup \widehat{r}_{3} \cup \widehat{r}_{4} \cup \widehat{r}_{5}$

Proof. Generalized type II bypass removals are --safe-to-postpone. Therefore, we need not worry about the $C$-neatness of the decomposition of $M \stackrel{\zeta \circ \eta}{\longmapsto} M^{\prime \prime}$, once the constructed decomposition of $M \stackrel{\zeta^{\prime}}{\longmapsto} M^{\prime \prime \prime}$ is neat.

The statement is established similarly to [11, Proposition 2] and to Proposition 8.2 above. So, we will be a little sketchy, providing only the details that are specific in the present case.

As usually, we have to consider different kinds of moves $M^{\prime} \stackrel{\zeta}{\mapsto} M^{\prime \prime}$ one by one. The bypass removed by $M \stackrel{\eta}{\mapsto} M^{\prime}$ is denoted $\left(y_{0}, \nu_{1}, y_{1}, \ldots, \nu_{k}, y_{k}\right)$.

Case 1: $M^{\prime} \stackrel{\zeta}{\mapsto} M^{\prime \prime}$ is a type I extension move.

Let $\mu$ and $x$ be the mirror and the occupied level that are added by the move. The move $M \stackrel{\zeta^{\prime}}{\longmapsto} M^{\prime \prime \prime}$ 
should also add $x$ and $\mu$. If $x=y_{i}$ for some $i \in\{1, \ldots, k-1\}$, then prior to that the diagram should be modified by a jump move that disturbs the position of $y_{i}$ slightly.

Case 2: $M^{\prime} \stackrel{\zeta}{\mapsto} M^{\prime \prime}$ is a type I elimination move.

Let $\mu$ and $x$ be the mirror and the occupied level that are eliminated by the move. Let also $z$ be the other occupied level of $M$ passing through $\mu$. The move $M \stackrel{\zeta^{\prime}}{\longmapsto} M^{\prime \prime \prime}$ simply removes $\mu$ and $x$, unless $x \in\left\{y_{0}, y_{k}\right\}$.

If $x=y_{0} \neq y_{k}$ we put $M^{\prime \prime \prime}=M$ and $\zeta^{\prime}=\left.\mathrm{id}\right|_{\widehat{M}}$. In this case, the transformation $M \stackrel{\zeta \circ \eta}{\longmapsto} M^{\prime \prime}$ is already a generalized type II bypass removal, and the removed bypass is $\left(z, \mu, y_{0}, \nu_{1}, y_{1}, \ldots, \nu_{k}, y_{k}\right)$.

Similarly, if $x=y_{k} \neq y_{0}$, we can take $M$ for $M^{\prime \prime \prime}$ as $M \stackrel{\zeta \circ \eta}{\longrightarrow} M^{\prime \prime}$ removes the bypass $\left(y_{0}, \nu_{1}, y_{1}, \ldots, \nu_{k}, y_{k}, \mu, z\right)$.

Suppose that $x=y_{0}=y_{k}$. Pick a point $p$ on $x$ distinct from $\mu, \nu_{1}$, and $\nu_{2}$ so that the pair $\{\mu, p\}$ interleaves with $\left\{\nu_{1}, \nu_{k}\right\}$, and take for $M \stackrel{\zeta^{\prime}}{\longmapsto} M^{\prime \prime \prime}$ a type I split move with splitting mirror $\mu$ and snip point $p$. The occupied level $x$ will have two successors in $M^{\prime \prime \prime}$, which we denote by $x_{1}, x_{2}$, numbering so that $x_{1}$ contains the successor of $\nu_{1}$ (which is unique), and $x_{2}$ contains the successor of $\nu_{k}$ (which is also unique). We also denote the successors of $\nu_{1}$ and $\nu_{k}$ by $\nu_{1}^{\prime}$ and $\nu_{k}^{\prime}$, respectively, and the two successors of $\mu$ contained in $x_{1}$ and $x_{2}$ by $\mu_{1}$ and $\mu_{2}$, respectively.

One can see that $M^{\prime \prime \prime} \stackrel{\eta^{\prime}}{\longmapsto} M^{\prime \prime}$, where $\eta^{\prime}=\zeta \circ \eta \circ \zeta^{\prime-1}$, removes the following bypass:

$$
\left(z, \mu_{1}, x_{1}, \nu_{1}^{\prime}, y_{1}, \nu_{2}, \ldots, \nu_{k-1}, y_{k-1}, \nu_{k}^{\prime}, x_{2}, \mu_{2}, z\right) \text {. }
$$

Case 3: $M^{\prime} \stackrel{\zeta}{\mapsto} M^{\prime \prime}$ is a type I elementary bypass addition.

We use the notation from Definition 6.2 in which we substitute $M^{\prime \prime}$ for $M$. Denote also by $\mu$ the mirror being added by the move, and by $\mu_{1}, \mu_{2}, \mu_{3}$ the mirrors at $\left(\theta_{1}, \varphi_{1}\right),\left(\theta_{2}, \varphi_{1}\right)$, and $\left(\theta_{1}, \varphi_{2}\right)$, respectively.

If no mirrors of the bypass appear in $r=\left[\theta_{1} ; \theta_{2}\right] \times\left[\varphi_{1} ; \varphi_{2}\right]$, then $M \stackrel{\zeta^{\prime}}{\longmapsto} M^{\prime \prime \prime}$ simply adds a $\backslash$-mirror at $\left(\theta_{2}, \varphi_{2}\right)$, and this is also a type I elementary bypass addition. Otherwise we have to modify the bypass in order to remove the obstacles.

There are the following two possible kinds of obstacles:

(1) one of, or both, of the mirrors $\nu_{1}, \nu_{k}$ appear in $\left[\mu_{1} ; \mu_{2}\right] \cup\left[\mu_{1} ; \mu_{3}\right]$;

(2) some of the mirrors $\nu_{i}$ appear in $\Omega=\left(\theta_{1} ; \theta_{2}\right] \times\left(\varphi_{1} ; \varphi_{2}\right]$.

Obstacles of the first kind are removed by type I split moves with splitting mirror $\mu_{2}$ or $\mu_{3}$. For instance, suppose that $\nu_{1} \in\left(\mu_{1} ; \mu_{2}\right)$ and $\nu_{k} \notin\left(\nu_{1} ; \mu_{2}\right)$. Let $\theta_{0}=\theta\left(\nu_{1}\right)$.

For a small enough $\varepsilon>0$, the longitude $\ell_{\varphi_{1}+\varepsilon}$ is not an occupied level of $M$, and there are no mirrors of $M$ in $\left[\theta_{0} ; \theta_{2}\right] \times\left(\varphi_{1}, \varphi_{1}+\varepsilon\right]$. We apply a type I split move that adds a $/$-mirrors $\nu_{1}^{\prime}$ and $\mu_{2}^{\prime}$ at $\left(\theta_{0}, \varphi_{1}+\varepsilon\right)$ and $\left(\theta_{2}, \varphi_{1}+\varepsilon\right)$, respectively, to $M$, and removes $\nu_{1}$.

The new bypass is

$$
\left(m_{\theta_{2}}, \mu_{2}^{\prime}, \ell_{\varphi_{1}+\varepsilon}, \nu_{1}^{\prime}, y_{1}, \nu_{2}, \ldots, \nu_{k}, y_{k}\right)
$$

Obstacles of the second kind are removed by applying Lemma 8.3. Note, however, that we have to use here not only the formulation of the Lemma, but also the proof. One can see that the procedure used to remove mirrors from $\Omega$ transforms a bypass to another bypass.

The removing of obstacles procedure gives us the sought-for transformation $M \stackrel{\zeta^{\prime}}{\longmapsto} M^{\prime \prime \prime}$ coming with a decomposition into type I split moves and jump moves, which can be decomposed neatly into type I elementary moves. These moves do not alter any part of the diagram $M$ except for the bypass.

Case $4: M^{\prime} \stackrel{\zeta}{\mapsto} M^{\prime \prime}$ is a type I elementary bypass removal.

We again use the notation from Definition [6.2, in which we now substitute $M^{\prime}$ and $M^{\prime \prime}$ for $M$ and $M^{\prime}$, respectively, and denote by $\mu$ and $\mu^{\prime}$ the mirrors at $\left(\theta_{2}, \varphi_{2}\right)$ and $\left(\theta_{1}, \varphi_{1}\right)$, respectively. Denote also by $c$ and $c^{\prime}$ the boundary circuits of $M$ adjacent to the bypass being removed by $M \stackrel{\eta}{\mapsto} M^{\prime}$.

If the circuit $c \# c^{\prime}$ of $M^{\prime}$ is patchable, then so are both $c$ and $c^{\prime}$, and hence $\operatorname{tb}_{+}\left(c \# c^{\prime}\right)=\operatorname{tb}_{+}(c)+$ $\operatorname{tb}_{+}\left(c^{\prime}\right) \leqslant-2$. This means that we cannot have $\partial r=c \# c^{\prime}$, since $t_{+}(\partial r)=-1$. Therefore, $\partial r$ is a boundary circuit of $M^{\prime}$ preserved by the generalized type II bypass addition $M^{\prime} \stackrel{\eta^{-1}}{\longmapsto} M$. 
If a patchable hole is preserved by a generalized bypass addition, this hole remains patchable. Thus, $\partial r$ is a patchable hole of $M$, which means that the mirrors $\mu$ and $\mu^{\prime}$ are coherent (see Definition 8.13), and by Lemma 8.2 the removal of $\mu$ can be decomposed neatly into type I elementary moves. So, in this case, the transformation $M \stackrel{\zeta^{\prime}}{\longmapsto} M^{\prime \prime \prime}$ is just the removal of $\mu$ endowed with the morphism $\zeta^{\prime}$ defined by the identical map on a surface obtained from $\overparen{M}$ by a patching of $\widehat{c}$.

Case 5: $M^{\prime} \stackrel{\zeta}{\mapsto} M^{\prime \prime}$ is a type I slide move.

This case is treated similarly to Case 3 . If the bypass being removed by the move $M \stackrel{\eta}{\mapsto} M^{\prime}$ creates an obstacle for doing the desired slide move on $M$, the bypass is modified by the same means.

Definition 9.20. Let $M_{0} \stackrel{\eta_{1}}{\longmapsto} M_{1} \stackrel{\eta_{2}}{\longmapsto} \ldots \stackrel{\eta_{k}}{\longmapsto} M_{k}$ be a sequence of transformations of enhanced mirror diagrams, and let $C=C_{0}$ be a family of boundary circuits of $M_{0}$. For each $i=1, \ldots, k$, let $C_{i}$ be the image of $C_{0}$ in $M_{i}$ under the transformation $M_{0} \stackrel{\eta_{i} \circ \eta_{i-1} \circ \ldots \circ \eta_{1}}{\longrightarrow} M_{i}$.

The sequence $M_{0} \stackrel{\eta_{1}}{\longmapsto} M_{1} \stackrel{\eta_{2}}{\longmapsto} \ldots \stackrel{\eta_{k}}{\longmapsto} M_{k}$ is called an almost $C$-neat decomposition of the transformation $M_{0} \stackrel{\eta_{k} \circ \eta_{k-1} \circ \ldots, \eta_{1}}{\longrightarrow} M_{k}$ if the following two condition hold:

(1) for any $i=1, \ldots, k$, either $M_{i-1} \stackrel{\eta_{i}}{\longmapsto} M_{i}$ is a jump move or it preserves all the boundary circuits in $C_{i-1}$;

(2) for any $0 \leqslant i_{1}<i_{2} \leqslant k$, whenever an essential boundary circuit $c \in \partial M_{i_{1}} \backslash C_{i_{1}}$ and the respective boundary circuit $c^{\prime} \in \partial M_{i_{2}} \backslash C_{i_{2}}$ have negative Thurston-Bennequin number tb $\mathrm{b}_{+}$ (respectively, tb - ), so also have the corresponding boundary circuits in each $\partial M_{j} \backslash C_{j}$ with $j \in$ $\left[i_{1}, i_{2}\right]$.

An almost $C$-neat decomposition is said to be of type $I$ (respectively, of type II) if it includes only type I (respectively, type II) elementary moves and jump moves.

Note that an almost $C$-neat decomposition is also $C$-delicate, provided that $C$ includes only essential boundary circuits, and that the moves include only jump moves and elementary ones.

Proposition 9.12. Let $M \stackrel{\eta}{\mapsto} M^{\prime}$ be a generalized type II bypass removal, and let $M^{\prime} \stackrel{\zeta}{\mapsto} M^{\prime \prime}$ be a jump move. Let also $C$ be the set of all boundary circuits of $M$ preserved by the move $M \stackrel{\eta}{\mapsto} M^{\prime}$ (but not necessarily by $\left.M^{\prime} \stackrel{\zeta}{\longmapsto} M^{\prime \prime}\right)$. Then there exist transformations $M \stackrel{\zeta^{\prime}}{\longmapsto} M^{\prime \prime \prime}$ and $M^{\prime \prime \prime} \stackrel{\eta^{\prime}}{\longmapsto} M^{\prime \prime}$ such that the following holds:

(1) we have $\eta^{\prime} \circ \zeta^{\prime}=\zeta \circ \eta$;

(2) the transformation $M \stackrel{\zeta^{\prime}}{\longmapsto} M^{\prime \prime \prime}$ admits a type I almost C-neat decomposition;

(3) the transformation $M^{\prime \prime \prime} \stackrel{\eta^{\prime}}{\longmapsto} M^{\prime \prime}$ is a generalized type II bypass removal;

(4) the transformation $M \stackrel{\zeta^{\prime}}{\longmapsto} M^{\prime \prime \prime}$ takes $C$ to a collection $C^{\prime} \subset \partial_{\mathrm{e}} M^{\prime \prime \prime}$ of boundary circuits such that the move $M^{\prime \prime \prime} \stackrel{\eta^{\prime}}{\longmapsto} M^{\prime \prime}$ preserves them.

Proof. We proceed similarly to Case 3 of the proof of Proposition 9.11 The bypass removed by $M \stackrel{\eta}{\mapsto} M^{\prime}$ is denoted $\left(y_{0}, \nu_{1}, y_{1}, \ldots, \nu_{k}, y_{k}\right)$.

We use the notation from Definition [7.6 substituting $M^{\prime}$ and $M^{\prime \prime}$ for $M$ and $M^{\prime}$, respectively. So, the jump move $M^{\prime} \stackrel{\zeta}{\mapsto} M^{\prime \prime}$ takes the occupied level $\ell_{\varphi_{1}}$ with all mirrors on it to $\ell_{\varphi_{2}}$. The almost $C$-neat decomposition of the move $M \stackrel{\zeta^{\prime}}{\longmapsto} M^{\prime \prime \prime}$ should end up with a jump move that does the same thing, namely, shifts $\ell_{\varphi_{1}}$ to $\ell_{\varphi_{2}}$. The preconditions for this move may not hold for $M$, and the possible obstacles may be only of the following two kinds:

(1) one of, or both of, the mirrors $\nu_{1}$ and $\nu_{k}$ appear in $\left[\theta_{2} ; \theta_{1}\right] \times\left\{\varphi_{1}\right\}$;

(2) some mirrors $\nu_{i}$ appear in $\left(\theta_{1} ; \theta_{2}\right) \times\left(\varphi_{1} ; \varphi_{2}\right]$.

Obstacles of the first kind are removed by sliding 'the attachment points' of the bypass along $\ell_{\varphi_{1}}$ into the interval $\left(\theta_{1} ; \theta_{2}\right) \times\left\{\varphi_{1}\right\}$. For instance, suppose that $\nu_{1} \in\left[\theta_{2} ; \theta_{1}\right] \times\left\{\varphi_{1}\right\}$ and $\nu_{k} \notin\left[\theta_{2} ; \theta_{0}\right) \times\left\{\varphi_{1}\right\}$, where $\theta_{0}=\theta\left(\nu_{1}\right)$. Then for a small enough $\varepsilon$ there are no mirrors of $M$ in $\left[\theta_{2}-\varepsilon ; \theta_{0}\right) \times\left[\varphi_{1}-\varepsilon, \varphi_{1}\right)$, and $m_{\theta_{2}-\varepsilon}, \ell_{\varphi_{1}-\varepsilon}$ are not occupied levels of $M$. 
We apply to $M$ two type I extension moves that add a $/$-mirror $\nu_{1}^{\prime}$ at $\left(\theta_{2}-\varepsilon, \varphi_{1}\right)$, and a $/$-mirror $\nu_{1}^{\prime \prime \prime}$ at $\left(\theta_{0}, \varphi_{1}-\varepsilon\right)$, and then apply a type I slide move that replaces $\nu_{1}$ by a $/$-mirror $\nu_{1}^{\prime \prime}$ at $\left(\theta_{2}-\varepsilon, \varphi_{1}-\varepsilon\right)$. The new bypass is

$$
\left(\ell_{\varphi_{1}}=y_{0}, \nu_{1}^{\prime}, m_{\theta_{2}-\varepsilon}, \nu_{1}^{\prime \prime}, \ell_{\varphi_{1}-\varepsilon}, \nu_{1}^{\prime \prime \prime}, m_{\theta_{0}}=y_{1}, \nu_{2}, \ldots, \nu_{k}, y_{k}\right)
$$

The obstacles of the second kind are again removed by means of Lemma 8.3 . We leave the details to the reader.

9.11. Second commutation property of generalized type II bypass removals. The whole of this subsection is devoted to the proof of the following statement.

Proposition 9.13. Let $M$ be an enhanced mirror diagram, and let $C$ be a collection of essential boundary circuits of $M$. Let also $M \stackrel{\eta}{\mapsto} M^{\prime}$ be a generalized type II bypass removal that preserves all the boundary circuits in $C$. Assume that $M^{\prime}$ is --flexible relative to $C$.

Then the move $M \stackrel{\eta}{\mapsto} M^{\prime}$ admits an almost $C$-neat decomposition into a sequence of elementary moves and jump moves such that all type I elementary moves in it occur before all type II elementary moves.

Proof. Let $\beta=\left(y_{0}, \nu_{1}, y_{1}, \ldots, \nu_{k}, y_{k}\right)$ be the bypass removed by the move $M \stackrel{\eta}{\mapsto} M^{\prime}$. Denote by $c$ the +-negligible circuit of $M$ adjacent to $\beta$ (there can be two such boundary circuits, in which case take any of them), and by $c^{\prime}$ the other boundary circuit of $M$ adjacent to $\beta$.

As noted above there exists a patching disc for $\widehat{c}$ having the form $\bigcup_{r \in \Pi} \widehat{r}$, where $\Pi$ is a collection of rectangles. For brevity we will refer to $\Pi$ as a patching disc. Though $\Pi$ is not necessarily a rectangular diagram of a surface (topologically $\widehat{\Pi}$ can be a surface with finitely many identifications between boundary points), the associated mirror diagram $M(\Pi)$ (see Definition 5.9) still has perfect sense for $\Pi$.

The complexity of the patching disc $\Pi$ is defined as the sum $n_{1}+n_{2}+n_{3}$, where $n_{1}$ is the number of rectangles in $\Pi, n_{2}$ is the number of mirrors in $E_{M^{\prime}}$ hit by $c$, and $n_{3}$ is the number of occupied levels of $M(\Pi)$ that do not belong to $L_{M}$.

We proceed by induction in the complexity of the patching disc.

We say that the patching disc $\Pi$ can be simplified if there are transformations $M \stackrel{\zeta}{\mapsto} M_{1}$ and $M^{\prime} \stackrel{\zeta^{\prime}}{\longmapsto} M_{1}^{\prime}$ with the following properties:

(1) $M \stackrel{\zeta}{\mapsto} M_{1}$ (respectively, $M^{\prime} \stackrel{\zeta^{\prime}}{\mapsto} M_{1}^{\prime}$ ) admits a type I (respectively, type II) almost $C$-neat decomposition;

(2) the transformation $M_{1} \stackrel{\eta_{1}}{\longmapsto} M_{1}^{\prime}$, where $\eta_{1}=\zeta^{\prime} \circ \eta \circ \zeta^{-1}$ is a generalized type II bypass removal;

(3) if $a \in C$, and $M \stackrel{\zeta}{\mapsto} M_{1}$ transforms $a$ to $a_{1}$, then the move $M_{1} \stackrel{\eta_{1}}{\longmapsto} M_{1}^{\prime}$ preserves $a_{1}$;

(4) there is a patching disc $\Pi^{\prime}$ associated with $M_{1} \stackrel{\eta_{1}}{\longmapsto} M_{1}^{\prime}$ that has lower complexity than $\Pi$ has.

Clearly, if such a simplification exists and the assertion of the proposition holds for the move $M_{1} \stackrel{\eta_{1}}{\longmapsto}$ $M_{1}^{\prime}$, then it also holds for the original move $M \stackrel{\eta}{\mapsto} M^{\prime}$.

For $j=1,2, \ldots, k-1$ we denote by $I_{j}$ the one of the two subintervals $\left(\nu_{j} ; \nu_{j+1}\right),\left(\nu_{j+1} ; \nu_{j}\right)$ of $y_{j}$ that is contained in $c$. By $I_{0}$ (respectively, $I_{k}$ ) we denote the minimal subinterval of $y_{0}$ (respectively, $y_{k}$ ) contained in $c$ that either has the form $\left(\mu ; \nu_{1}\right)$ or $\left(\nu_{1} ; \mu\right)$ (respectively, $\left(\mu ; \nu_{k}\right)$ or $\left.\left(\nu_{k} ; \mu\right)\right)$ with $\mu \in E_{M}$. Denote also by $\mu^{\prime}$ (respectively, $\left.\mu^{\prime \prime}\right)$ the mirror of $M$ such that $I_{0}$ is either $\left(\mu^{\prime} ; \nu_{1}\right)$ or $\left(\nu_{1} ; \mu^{\prime}\right)$ (respectively, $I_{k}$ is either $\left(\mu^{\prime \prime} ; \nu_{k}\right)$ or $\left.\left(\nu_{k} ; \mu^{\prime \prime}\right)\right)$.

Lemma 9.13. If $\bigcup_{j=0}^{k} I_{j} \cup\left\{\mu^{\prime}, \mu^{\prime \prime}\right\}$ contains a /-mirror of $M(\Pi)$, then $\Pi$ can be simplified.

Proof. Suppose there is a /-mirror $\lambda$ of $M(\Pi)$ in $I_{j}, j \in\{1, \ldots, k-1\}$. Denote by $x$ the occupied level of $M(\Pi)$ perpendicular to $y_{j}$ and passing through $\lambda$. We claim that $x \notin L_{M}$. Indeed, if $x \in L_{M}$, then the addition of $\lambda$ to $M$ would split the boundary circuit $c$ into two patchable boundary circuits $c_{1}, c_{2}$, say, such that $\operatorname{tb}_{+}\left(c_{1}\right)+\operatorname{tb}_{+}\left(c_{2}\right)=-1$, which is impossible.

Pick a point $p$ in $y_{j} \backslash \bar{I}_{j}$. Let $M \stackrel{\zeta}{\mapsto} M_{1}$ be the composition of the type I extension move that adds the $/$-mirror $\lambda$ together with the occupied level $x$, and a type I split move associated with $(\lambda, p)$. The mirrors $\nu_{j}, \nu_{j+1}$ have unique successors in $M_{1}$, which we denote by $\nu_{j}^{\prime}$ and $\nu_{j+1}^{\prime}$, respectively. The 
occupied level $y_{j}$ will have two successors, which we denote by $y_{j}^{\prime}$ and $y_{j}^{\prime \prime}$ so as to have $\nu_{j}^{\prime} \in y_{j}^{\prime}, \nu_{j+1}^{\prime} \in y_{j}^{\prime \prime}$. The mirror $\lambda$ will also have two successors, $\lambda^{\prime} \in y_{j}^{\prime}$ and $\lambda^{\prime \prime} \in y_{j}^{\prime \prime}$.

Now let $\beta^{\prime}$ be the sequence obtained from $\beta$ by replacing the subsequence $\left(\nu_{j}, y_{j}, \nu_{j+1}\right)$ with

$$
\left(\nu_{j}^{\prime}, y_{j}^{\prime}, \lambda^{\prime}, x, \lambda^{\prime \prime}, y_{j}^{\prime \prime}, \nu_{j+1}^{\prime}\right) \text {. }
$$

We put $M_{1}^{\prime}=M^{\prime}$ and $\zeta^{\prime}=$ id. One can see that $M_{1} \stackrel{\eta \circ \zeta^{-1}}{\longmapsto} M_{1}^{\prime}$ is a generalized bypass removal, and the respective bypass is $\beta^{\prime}$.

One can also see that a patching disc $\Pi^{\prime}$ associated with $M_{1} \stackrel{\eta \circ \zeta^{-1}}{\longrightarrow} M_{1}^{\prime}$ can be obtained from $\Pi$ by a small perturbation of rectangles having one of the sides on $y_{j}$ (see the second move in Figure 3.5 for an example of what happens with $\Pi$ ).

In the passage from $\Pi$ to $\Pi^{\prime}$, the numbers $n_{1}$ and $n_{2}$ in the definition of the complexity of $\Pi$ are unchanged, whereas $n_{3}$ drops by 1 , since $x$ is now an occupied level of $M_{1}$. Hence, a simplification of the patching disc occurs.

In the case when $\lambda \in I_{0}$ or $\lambda \in I_{k}$ we proceed as above substituting $\mu^{\prime}$ for $\nu_{0}$ if $j=0$, and $\mu^{\prime \prime}$ for $\nu_{k+1}$ if $j=k$. If $j=0$ (respectively, $j=k$ ) we also choose $p$ close to $\nu_{1}$ (respectively, to $\nu_{k}$ ), and define the move $M \stackrel{\zeta}{\mapsto} M_{1}$ so as to have $y_{0}^{\prime}=y_{0}$ (respectively, $y_{k}^{\prime \prime}=y_{k}$ ). We also drop the first two (respectively, the last two) entries in (9.9).

If $\mu^{\prime}$ is a $/$-mirror, we let $M \stackrel{\zeta}{\mapsto} M_{1}$ be a type I split move associated with the splitting route $\left(\mu^{\prime}, p\right)$, where $p \in y_{0} \backslash \bar{I}_{0}$ is close to $\nu_{1}$, such that the successor of $y_{0}$ that does not contain the successor of $\nu_{1}$ coincides with $y_{0}$.

We again put $M_{1}^{\prime}=M^{\prime}$ and $\zeta^{\prime}=\mathrm{id}$, and have that a patching disc associated with $M_{1} \stackrel{\eta \circ \zeta^{-1}}{\longmapsto} M_{1}^{\prime}$ is obtained from $\Pi$ by a small perturbation of rectangles having a side on $y_{0}$.

In this case the numbers $n_{1}, n_{3}$ are preserved, and $n_{2}$ drops by 1 , since $c$ transforms to a boundary circuit that does not hit $\mu^{\prime}$.

The case when $\mu^{\prime \prime}$ is a $/$-mirror is similar.

So, we assume in the sequel that the mirrors $\mu^{\prime}$ and $\mu^{\prime \prime}$ of $M$ are of type ' $\backslash$ '. Since $\operatorname{tb}_{+}(c)=-1$, these are the only $\backslash$-mirrors hit by $c$. We also assume that there are no $/$-mirrors of $M(\Pi)$ in $\bigcup_{j=0}^{k} I_{j}$. This implies that $I_{0}$ and $I_{k}$ contain no mirror of $M(\Pi)$, and each $I_{j}$ with $j \in\{1, \ldots, k-1\}$ contains exactly one \-mirror, which we denote by $\mu_{j}$. We also put $\mu_{0}=\mu^{\prime}$ and $\mu_{k}=\mu^{\prime \prime}$.

For every $j \in\{1, \ldots, k\}$ we have a unique rectangle in $\Pi$ with a vertex at $\nu_{j}$. Denote this rectangle by $r_{j}$. It follows from the assumptions we have just made that $\mu_{j-1}$ and $\mu_{j}$ are also vertices of $r_{j}$. Denote the remaining, the fourth vertex of $r_{j}$ by $\lambda_{j}$. It is opposite to $\nu_{j}$ in $r_{j}$, and it is a $/$-mirror of $M(\Pi)$. It may or may not be a mirror of $M$, and if it is, then its type in $M$ is also ' $/$ '.

Let $\left(\delta_{+}, \delta_{-}\right)$be a canonic dividing configuration of $\widehat{\Pi}$, and let $d=\widehat{\Pi} \backslash \widehat{c}$. By construction, $d$ is an open disc. Since $\operatorname{tb}_{+}(c)=-1$, the intersection $d \cap \delta_{+}$consists of a single open arc that approaches the midpoints of $\widehat{\mu}^{\prime}$ and $\widehat{\mu}^{\prime \prime}$ at the ends. This implies that there are no rectangles in $\Pi$ except $r_{1}, \ldots, r_{k}$, as otherwise we would have another connected component of $\delta_{+}$.

Lemma 9.14. If $k>2$ and, for some $j \in\{1, \ldots, k-1\}$, we have $\lambda_{j}=\lambda_{j+1} \notin E_{M^{\prime}}$, then $\Pi$ can be simplified.

Proof. Suppose that $\lambda_{j}=\lambda_{j+1} \notin E_{M^{\prime}}, 1 \leqslant j \leqslant k-1, k>2$. Without loss of generality we may assume that the rectangles $r_{j}$ and $r_{j+1}$ have the form $\left[\theta_{1} ; \theta_{2}\right] \times\left[\varphi_{1} ; \varphi_{2}\right]$ and $\left[\theta_{2} ; \theta_{3}\right] \times\left[\varphi_{2} ; \varphi_{1}\right]$ for some $\theta_{1}, \theta_{2}, \theta_{3}, \varphi_{1}, \varphi_{2} \in \mathbb{S}^{1}$. Indeed, the other cases are obtained from this one by applying the symmetries $r / r \backslash$, and $r / \circ r \backslash$ (see Subsection 6.1 for notation).

Our constructions are also invariant under the substitution $\nu_{j} \mapsto \nu_{k+1-j}, \lambda_{j} \mapsto \lambda_{k+1-j}, \mu_{j} \mapsto \mu_{k-j}$, $y_{j} \mapsto y_{k-j}$. So, we may also safely assume that $j \leqslant k-2$. Shown in Figure 9.49 is the case when we also have $j>1$, though we don't assume this in the sequel. If $j=1$, one should remove $y_{j-2}, \nu_{j-1}$, and $r_{j-1}$ from the pictures in Figure 9.49 and add $\mu_{0}$ and $\ell_{\varphi_{1}}$ to the right picture.

By construction, there are no mirrors of $M$ inside $r_{j}$ and $r_{j+1}$ and in $\left[\nu_{j} ; \nu_{j+1}\right] \cup\left[\mu_{j-1} ; \mu_{j+1}\right]$. Pick an $\varepsilon>$ 0 such that there are no occupied levels of $M$ in the domains $\Omega_{1}=\left(\theta_{1}-\varepsilon ; \theta_{1}\right) \times \mathbb{S}^{1}$ and $\Omega_{2}=\left(\theta_{3} ; \theta_{3}+\varepsilon\right) \times \mathbb{S}^{1}$. 

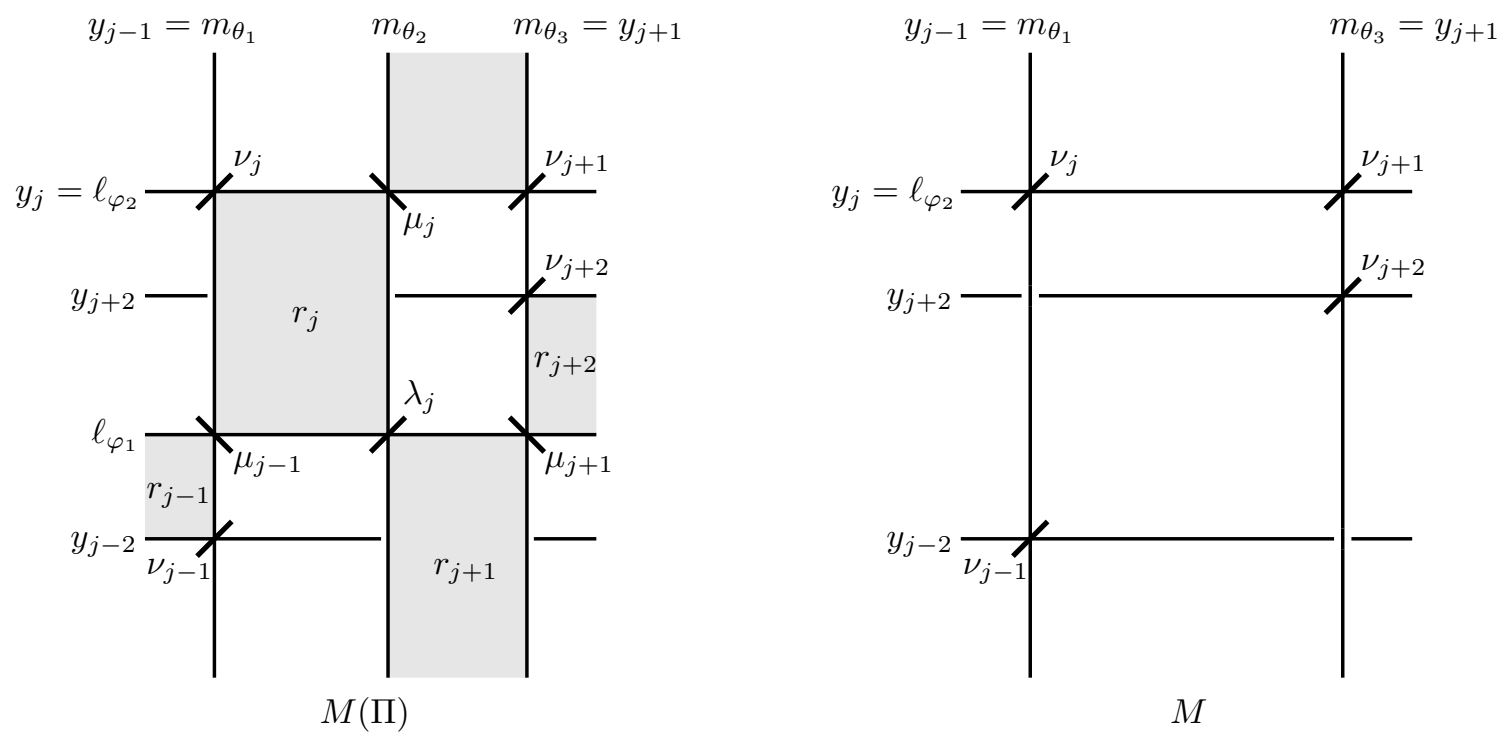

FiguRE 9.49. The mutual position of $r_{j}$ and $r_{j+1}$ in the case $\lambda_{j}=\lambda_{j+1} \notin E_{M^{\prime}}$

By means of jump moves applied to the diagrams $M$ and $M^{\prime}$ we can shift all their occupied meridians intersecting $r_{j}$ into $\Omega_{2}$, and all occupied meridians intersecting $r_{j+1}$ into $\Omega_{1}$. Let $M \stackrel{\zeta_{1}}{\longmapsto} M_{2}$ and $M^{\prime} \stackrel{\zeta^{\prime}}{\longmapsto}$ $M_{1}^{\prime}$ be the obtained transformations. The transformation $M_{2} \stackrel{\zeta^{\prime} \circ \eta \circ \zeta_{1}^{-1}}{\longrightarrow} M_{1}^{\prime}$ is still a generalized type II bypass removal, and there is an associated patching disc having the same complexity as $\Pi$ has. This patching disc is produced from $\Pi$ by two or less exchange moves.

Now observe that $m_{\theta_{2}}$ is not an occupied level of $M$, and hence not an occupied level of $M_{2}$. Therefore, $M_{2}$ has no mirrors in $\left(\theta_{1} ; \theta_{3}\right) \times \mathbb{S}^{1}$, and there is no obstruction to merging the meridians $m_{\theta_{1}}=y_{j-1}$ and $m_{\theta_{3}}=y_{j+1}$, and simultaneously applying the respective wrinkle reduction move to the patching disc. Since we assumed $j \leqslant k-2$, the meridian $y_{j+1}$ does not belong to $L_{M^{\prime}}$ (which is not the case for the meridian $y_{j-1}$ when $j=1$ ). We define $M_{2} \stackrel{\zeta_{2}}{\longleftrightarrow} M_{1}$ to be the composition of a type I merge move that merges $y_{j-1}$ and $y_{j+1}$ so that the successor of both meridians is $y_{j-1}$, and a type I elimination move that removes the common successor of $\nu_{j}$ and $\nu_{j+1}$. In other words, the transformation $M_{2} \stackrel{\zeta_{2}}{\longmapsto} M_{1}$ replaces $\nu_{j}$, $\nu_{j+1}$, and $\nu_{j+2}$ by a single $/$-mirror at $y_{j-1} \cap y_{j+2}$, and removes $y_{j}$ and $y_{j+1}$.

Now we put $\zeta=\zeta_{2} \circ \zeta_{1}$ and note that the transformation $M_{1} \stackrel{\eta^{\prime}}{\longmapsto} M_{1}^{\prime}$, where $\eta^{\prime}=\zeta^{\prime} \circ \eta \circ \zeta^{-1}$, is a generalized bypass removal with the corresponding bypass being obtained from $\beta$ by replacing the subsequence $\left(\nu_{j}, y_{j}, \nu_{j+1}, y_{j+1}, \nu_{j+2}\right)$ with a single entry $\nu_{j}^{\prime}=y_{j-1} \cap y_{j+2}$.

The new patching disc will have two rectangles less than $\Pi$ has, whereas $n_{2}$ and $n_{3}$ in the definition of the complexity are unchanged. So, a simplification of the patching disc occurs.

Lemma 9.15. If either $\lambda_{j} \neq \lambda_{j+1}$ or $\lambda_{j}=\lambda_{j+1} \in E_{M^{\prime}}$ holds for all $j \in\{1, \ldots, k-1\}$, then $\lambda_{j} \in E_{M^{\prime}}$ for all $j \in\{1, \ldots, k\}$.

Proof. If $\lambda_{j} \notin E_{M^{\prime}}$, then $\lambda_{j}$ coincides with some $\lambda_{i}, i \neq j$. This means that a connected component of $\delta_{-}$is an $\operatorname{arc} \alpha$ connecting the midpoints of $\widehat{\nu}_{i}$ and $\widehat{\nu}_{j}$. The $\operatorname{arcs} \alpha$ and $\operatorname{int}\left(\delta_{+}\right)$have two intersection points, hence there is a subdisc $d^{\prime} \subset d$ bounded by a subarc of $\alpha$ and a subarc of $\delta_{+}$. Such a disc must contain a bigon of $\delta_{+}$and $\delta_{-}$, which implies that for some $l \leqslant k-1$ we have $\lambda_{l}=\lambda_{l+1} \notin E_{M^{\prime}}$. The claim follows.

Lemma 9.16. If $\lambda_{1} \in E_{M^{\prime}}$ and $k>1$, then $\Pi$ can be simplified.

Proof. If $\lambda_{1} \in E_{M^{\prime}}$ and $k>1$, the addition of $\mu_{1}$ to $M$ is a type I elementary bypass addition. Indeed, the three vertices $\nu_{1}, \mu_{0}, \lambda_{1}$ of $r_{1}$ belong to $E_{M}$, whereas the forth, $\mu_{1}$ does not. We let $M \stackrel{\zeta}{\mapsto} M_{1}$ be this 
addition, and let $M^{\prime} \stackrel{\zeta^{\prime}}{\longmapsto} M_{1}^{\prime}$ be the addition of a bridge that adds $\mu_{1}, \nu_{1}$, and $y_{1}$ to $M^{\prime}$. It can be neatly decomposed into type II elementary moves by Lemma 7.3

One can see that $M_{1} \stackrel{\zeta^{\prime} \circ \eta \circ \zeta^{-1}}{\longrightarrow} M_{1}^{\prime}$ is a generalized bypass removal, and the corresponding bypass is $\left(y_{1}, \nu_{2}, y_{2}, \ldots, \nu_{k}, y_{k}\right)$. The collection $\left\{r_{j}\right\}_{j=2}^{k}$ can be taken for a patching disc, which is simpler than $\Pi$.

Thus, the only cases in which we have not established a possibility to simplify $\Pi$ are the following two:

(1) $k=1, \lambda_{1} \in E_{M^{\prime}}$;

(2) $k=2, \lambda_{1}=\lambda_{2} \notin E_{M^{\prime}}$.

In the first case, the move $M \stackrel{\eta}{\mapsto} M^{\prime}$ is a type II elementary bypass removal, hence the assertion of the proposition holds trivially.

To treat the second case, we revisit the proof of Lemma 9.14 where we put $j=1$. We use the same notation and make the same assumption about the positions of $r_{1}$ and $r_{2}$.

The elements $\nu_{j-1}, \nu_{j+2}, y_{j-2}, y_{j+2}, r_{j-1}, r_{j+2}$ now do not exist, whereas $\mu_{0}=\mu^{\prime}, \mu_{2}=\mu^{\prime \prime}$ are $\backslash$ mirrors of $M$ and $M^{\prime}$, and $\ell_{\varphi_{1}}$ is an occupied level of both. By using the same family of jump moves as in the proof of Lemma 9.14 we can reduce the general case to the one when no meridian in $\left(\theta_{1} ; \theta_{2}\right) \times \mathbb{S}^{1}$ is an occupied level of $M$.

Thus, it remains to consider the situation shown in Figure 9.50 on the left (the gray boxes again stand for families of mirrors of the diagram, not rectangles of $\Pi$ ).
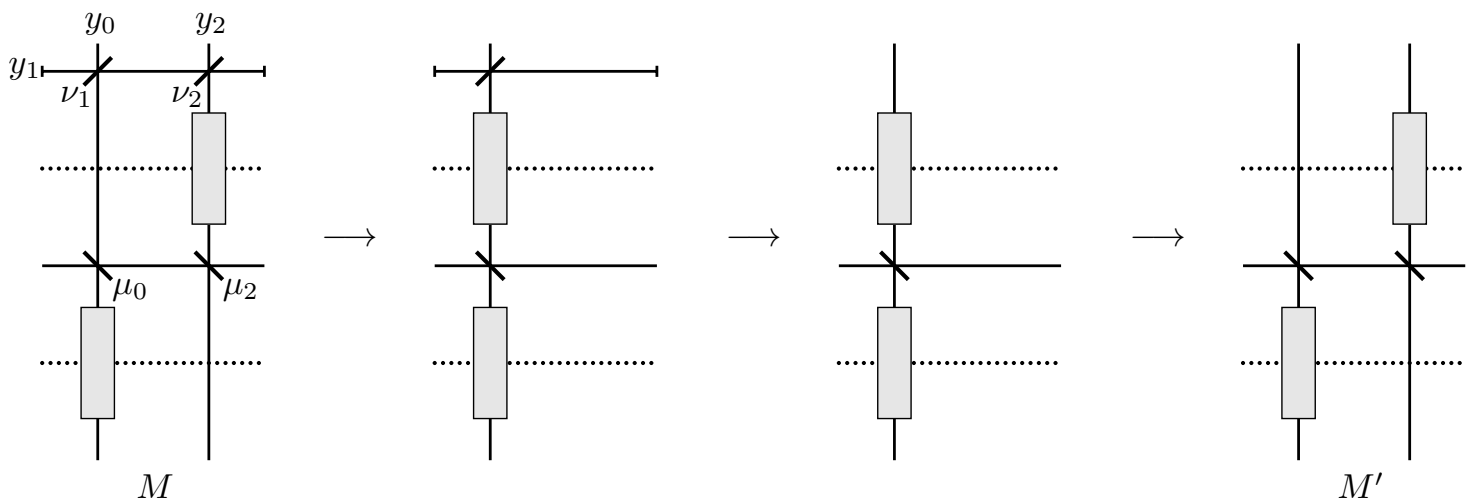

Figure 9.50. Decomposition of a generalized bypass removal into type I moves followed by type II moves in the case $k=2, \lambda_{1}=\lambda_{2} \notin E_{M^{\prime}}$

The diagram $M^{\prime}$ can be obtained from $M$ in three steps illustrated in Figure 9.50.

(1) a double merge move that merges $y_{0}$ with $y_{2}$;

(2) a type I elimination move that removes $y_{1}$ with the remaining mirror on it;

(3) a type II split move that recovers $y_{0}$ and $y_{1}$ with all mirrors on them except for $\nu_{1}$ and $\nu_{2}$.

The double merge move should then be neatly decomposed into type I elementary moves, and the type II split move should be neatly decomposed into type II elementary moves, which is possible due to Lemmas 7.3 and 7.4 .

These decompositions can be chosen to preserve either all boundary circuits visiting $y_{0}$ and not hitting $\nu_{1}$, or all boundary circuits visiting $y_{2}$ and not hitting $\nu_{2}$. If no mirror on either $y_{0}$ or $y_{2}$ is hit by a boundary circuit in $C$, we are done.

It is possible, however, that both $y_{0}$ and $y_{2}$ contain mirrors hit by boundary circuits in $C$, in which case we would get stuck without the --flexibility of $M^{\prime}$. However, --flexibility allows us to avoid the difficulty.

Suppose that there is a single-headed type I splitting route $\omega$ in $M^{\prime}$ such that $\omega$ does not separate $C$ and has the following property: no connected component of $\widehat{y}_{2} \backslash \widehat{\omega}$ has a non-empty intersection with 
both $\widehat{\nu}_{2}$ and $\bigcup_{a \in C} \widehat{a}$. By a small perturbation of the snip point we can ensure that $\omega$ is also suitable as a type I splitting route in $M$.

Let $M \stackrel{\zeta}{\mapsto} M_{1}$ be a generalized wrinkle creation move associated with $\omega$, and preserving all boundary circuits in $C$. The mirrors $\nu_{1}, \nu_{2}$, and the occupied level $y_{1}$ have unique successors in $M_{1}$, which we denote by $\nu_{1}^{\prime}, \nu_{2}^{\prime}$, and $y_{1}^{\prime}$, respectively. Let $M_{1}^{\prime}$ be obtained from $M_{1}$ by removing these three elements (the occupied level $y_{1}^{\prime}$ contains no other mirrors). One can see that $M_{1} \mapsto M_{1}^{\prime}$ is a generalized type II bypass removal, and $M^{\prime} \mapsto M_{1}^{\prime}$ is a generalized wrinkle creation move associated with $\omega$. Moreover, if $\eta_{1}: \widehat{M}_{1} \rightarrow \widehat{M}_{1}^{\prime}$ and $\zeta^{\prime}: \widehat{M}^{\prime} \rightarrow \widehat{M}_{1}^{\prime}$ are the associated morphisms, then $\eta_{1} \circ \zeta=\zeta^{\prime} \circ \eta$.

Due to the properties of $\omega$ the successor of $y_{2}$ in $M_{1}$ that contains $\nu_{2}^{\prime}$ has an empty intersection with $\bigcup_{a \in C} a$. This means that the generalized bypass removal $M_{1} \stackrel{\eta_{1}}{\longmapsto} M_{1}^{\prime}$ admits a decomposition illustrated in Figure 9.50 without disturbing any boundary circuits in $C$. Thus, it remains to show how to find $\omega$ with required properties.

Denote, as usually, by $c \# c^{\prime}$ the unique boundary circuit in $\partial M^{\prime} \backslash \partial M$. If $c \# c^{\prime}$ hits a $/$-mirror, the sought-for $\omega$ can be found by starting from such a mirror and following $c \# c^{\prime}$. More precisely, let $\kappa_{1}, \kappa_{2}, \kappa_{3}, \ldots, \kappa_{l}$ be all mirrors hit by $c \# c^{\prime}$ numbered in the order they follow on $c \# c^{\prime}$, so that $\kappa_{1}=$ $\mu_{0}$ and $\kappa_{l}=\mu_{2}$. Let $j$ be the maximal index such that $\kappa_{j}$ is a $/$-mirror. We can take for $\omega$ the sequence $\left(\kappa_{j}, \kappa_{j+1}, \ldots, \kappa_{l}, p\right)$, where $p$ is picked on the segment of $c \# c^{\prime}$ connecting $\mu_{2}$ and $\mu_{0}$.

If $c \# c^{\prime}$ does not hit any $/$-mirror, we pick any single-headed splitting route in which the last mirror appears on $c \# c^{\prime}$ (this exists by Lemma 9.10). Then we prolong this splitting route along $c \# c^{\prime}$ until the respective splitting path cuts $\widehat{y}_{2}$ as requested.

This completes the proof of Proposition 9.13 .

9.12. Proof of the commutation theorems. Theorem 9.1 is a particular case of Theorem 9.2, so it suffices to prove the latter.

A little more preparation is in order.

Definition 9.21. Let $M$ be an enhanced mirror diagram, and let $C$ be a collection of essential boundary circuits of $M$. The diagram $M$ is said to be very +-flexible (respectively, very--flexible) relative to $C$ if, for any $c \in \partial M \backslash C$ we have $\operatorname{tb}_{+}(c)<0$ (respectively, tb_ $(c)<0$ ). If $M$ is both very +-flexible and very --flexible relative to $C$, we say that it is very flexible relative to $C$.

Clearly 'very $( \pm)$-flexible' implies ' $( \pm)$-flexible'.

We call a connected component $M_{0}$ of an enhanced mirror diagram $M$ spherical if the corresponding connected component of $\widehat{M}_{0}$ is a sphere with holes, and all elements of $\partial M_{0}$ are inessential boundary circuits of $M$. A connected component $M_{0}$ of $M$ is called a disc component if the corresponding connected component of $\widehat{M}_{0}$ is a sphere with holes, and $\partial M_{0}$ contains exactly one essential boundary circuit of $M$.

Lemma 9.17. Let $M_{0} \stackrel{\eta_{1}}{\longmapsto} M_{1} \stackrel{\eta_{2}}{\longmapsto} \ldots \stackrel{\eta_{n}}{\longmapsto} M_{n}$ be an almost $C_{0}$-neat decomposition of a transformation $M_{0} \stackrel{\eta}{\mapsto} M_{n}$ of enhanced mirror diagrams into a sequence of moves of any kind introduced earlier in this paper, and let $C_{0} \mapsto C_{1} \mapsto \ldots \mapsto C_{n}$ be the induced transformations of collections of boundary circuits.

Assume that $M_{0}$ (equivalently: $M_{n}$ ) has no spherical components. Assume also that $M_{0}$ is +-flexible relative to $C_{0}$, and $M_{n}$ is very + -flexible relative to $C_{n}$. Then all diagrams $M_{1}, M_{2}, \ldots, M_{n-1}$ are +flexible relative to the respective $C_{i}$ 's, too.

Proof. Without loss of generality we may assume that all moves $M_{i-1} \stackrel{\eta_{i}}{\longmapsto} M_{i}$ are elementary or jump moves. Indeed, all the moves introduced in this paper admit a neat decomposition into elementary ones, hence, if we replace all moves in the original sequence, except jump moves, by their respective neat decompositions into elementary moves, we will still have an almost $C_{0}$-neat decomposition of $M_{0} \stackrel{\eta}{\mapsto} M_{n}$.

We proceed by induction in $n$. The induction base, $n=1$, is trivial. To make the induction step it suffices to show that $M_{1}$ is +-flexible relative to $C_{1}$.

All +-safe-to-bring-forward moves (see Definition (7.3) preserve +-flexibility by design. So, if $M_{0} \stackrel{\eta_{1}}{\longmapsto} M_{1}$ is a type I elementary move, jump move, type II extension, slide, or elementary bypass removal move, $M_{1}$ is flexible and we are done. 
Elementary bypass additions (of either type) are not always +-safe-to-bring-forward, but they preserve +-flexibility due to the fact that they replace a boundary circuit by two mutually adjacent boundary circuits one of which is +-negligible. So, the only 'dangerous' moves in the present context are type II elimination moves.

Suppose that $M_{0} \stackrel{\eta_{1}}{\longmapsto} M_{1}$ is a type II elimination move. Let $c_{0} \in \partial M_{0}$ be the modified boundary circuit of $M_{0}$, and let $c_{1}$ be the corresponding circuit of $M_{1}$.

If $c_{0}$ is inessential, then $c_{1}$ is inessential, too. Since no component of $M_{1}$ is spherical, this implies that $\mathrm{tb}_{+}\left(c_{1}\right)<0$. Indeed, otherwise one would be able produce an overtwisted disc from a patching disc for $\widehat{c}_{1}$, which is impossible according to Bennequin's result [2].

If $c_{0}$ is essential, then the inequality $\mathrm{tb}_{+}\left(c_{1}\right)<0$ follows from the assumption that the given decomposition is almost $C_{0}$-neat. Indeed, let $c_{0}$ be transformed to $c_{n}$ in $\partial M_{n}$. We must have $c_{0} \notin C_{0}, c_{n} \notin C_{n}$ as $c_{0}$ is modified by $M_{0} \stackrel{\eta_{1}}{\longmapsto} M_{1}$.

We have $\operatorname{tb}_{+}\left(c_{0}\right)=\operatorname{tb}_{+}\left(c_{1}\right)-1<0$ since $M_{0} \stackrel{\eta_{1}}{\longmapsto} M_{1}$ is a type II elimination move, and $\operatorname{tb}_{+}\left(c_{n}\right)<0$ since $M_{n}$ is assumed to be very +-flexible relative to $C_{n}$. By Definition 9.20 we must have $\mathrm{tb}_{+}\left(c_{1}\right)<0$.

Therefore, $M_{1}$ is +-flexible relative to $C_{1}$.

Now we proceed with the proof of Theorem 9.2.

Let

$$
M=M_{0} \stackrel{\eta_{1}}{\longmapsto} M_{1} \stackrel{\eta_{2}}{\longmapsto} \ldots \stackrel{\eta_{n}}{\longmapsto} M_{n}=M^{\prime}
$$

be a $C$-delicate sequence of elementary moves and jump moves. Let $C=C_{0} \mapsto C_{1} \mapsto \ldots \mapsto C_{n}=C^{\prime}$ be the induced sequence of transformations of the collection of selected boundary circuits.

Suppose, for the time being, that $M_{0}$ has no spherical components.

For every $j=0, \ldots, n$ we can find transformations $M_{j} \stackrel{\zeta_{j}^{\prime}}{\longmapsto} M_{j}^{\prime}$ and $M_{j} \stackrel{\zeta_{j}^{\prime \prime}}{\longmapsto} M_{j}^{\prime \prime}$ preserving all boundary circuits in $C_{j}$ such that the following holds (see the scheme in Figure 9.51):

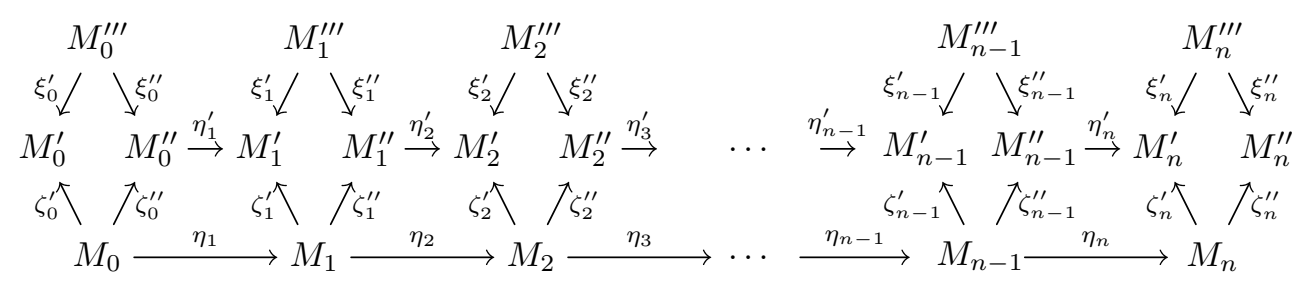

Figure 9.51. Adding flexibility to intermediate diagrams

(1) $M_{j} \stackrel{\zeta_{j}^{\prime}}{\longmapsto} M_{j}^{\prime}$ and $M_{j} \stackrel{\zeta_{j}^{\prime \prime}}{\longmapsto} M_{j}^{\prime \prime}$ are compositions of extension moves for any $j=0, \ldots, n$;

(2) $M_{0} \stackrel{\zeta_{0}^{\prime}}{\longmapsto} M_{0}^{\prime}$ is a composition of type I extension moves;

(3) $M_{n} \stackrel{\zeta_{n}^{\prime \prime}}{\longmapsto} M_{n}^{\prime \prime}$ is a composition of type II extension moves;

(4) for any $j=1, \ldots, n-1$, both diagrams $M_{j}^{\prime}$ and $M_{j}^{\prime \prime}$ are very flexible relative to $C_{j}$;

(5) the diagrams $M_{0}^{\prime}$ and $M_{0}^{\prime \prime}$ are very --flexible and very flexible, respectively, relative to $C_{0}$;

(6) the diagrams $M_{n}^{\prime}$ and $M_{n}^{\prime \prime}$ are very flexible and very +-flexible, respectively, relative to $C_{n}$;

(7) if $j<n$ the transformation $M_{j}^{\prime \prime} \stackrel{\eta_{j}^{\prime}}{\longmapsto} M_{j+1}^{\prime}$ with $\eta_{j}^{\prime}$ such that $\eta_{j}^{\prime} \circ \zeta_{j}^{\prime \prime}=\zeta_{j+1}^{\prime} \circ \eta_{j}$ is an elementary move or a jump move;

(8) for any $j=0, \ldots, n$ we have $E_{M_{j}^{\prime}} \cap E_{M_{j}^{\prime \prime}}=E_{M_{j}}$.

For each $j$, let $M_{j}^{\prime \prime \prime}$ be the union $M_{j}^{\prime} \cup M_{j}^{\prime \prime}$ (a boundary circuit of $M_{j}^{\prime \prime \prime}$ is declared essential if and only if it has a non-trivial interval in common with an essential boundary circuit of $M_{j}$ ). Both diagrams $M_{j}^{\prime}$ and $M_{j}^{\prime \prime}$ can be obtained from $M_{j}^{\prime \prime \prime}$ by a composition of elimination moves. Let $M_{j}^{\prime \prime \prime} \stackrel{\xi_{j}^{\prime}}{\longmapsto} M_{j}^{\prime}$ and $M_{j}^{\prime \prime \prime} \stackrel{\xi_{j}^{\prime \prime}}{\longmapsto} M_{j}^{\prime \prime}$ be the obtained transformations. We clearly have $\xi_{j}^{\prime \prime} \circ \xi_{j}^{\prime-1}=\zeta_{j}^{\prime \prime} \circ \zeta_{j}^{\prime-1}$. 
Thus, we have the following decomposition of the transformation $M \stackrel{\eta_{n} \circ \ldots \circ \eta_{1}}{\longmapsto} M^{\prime}$ :

$$
M_{0} \stackrel{\zeta_{0}^{\prime}}{\longmapsto} M_{0}^{\prime} \stackrel{\xi_{0}^{\prime}-1}{\longmapsto} M_{0}^{\prime \prime \prime} \stackrel{\xi_{0}^{\prime \prime}}{\longmapsto} M_{0}^{\prime \prime} \stackrel{\eta_{1}^{\prime}}{\longmapsto} M_{1}^{\prime} \stackrel{\xi_{1}^{\prime-1}}{\longmapsto} M_{1}^{\prime \prime \prime} \stackrel{\xi_{1}^{\prime \prime}}{\longmapsto} M_{1}^{\prime \prime} \stackrel{\eta_{2}^{\prime}}{\longmapsto} \ldots \stackrel{\eta_{n}^{\prime}}{\longmapsto} M_{n}^{\prime} \stackrel{\xi_{n}^{\prime}-1}{\longmapsto} M_{n}^{\prime \prime \prime} \stackrel{\xi_{n}^{\prime \prime}}{\longmapsto} M_{n}^{\prime \prime} \stackrel{\zeta_{n}^{\prime \prime}-1}{\longmapsto} M_{n},
$$

which starts from the composition $M_{0} \stackrel{\zeta_{0}^{\prime}}{\longmapsto} M_{0}^{\prime}$ of type I extension moves and ends up with the composition $M_{n}^{\prime \prime} \stackrel{\zeta_{n}^{\prime \prime-1}}{\longmapsto} M_{n}$ of type II elimination moves. So, it suffices to prove the assertion of the theorem for the transition from $M_{0}^{\prime}$ to $M_{n}^{\prime \prime}$.

All the diagrams in the truncated sequence

$$
M_{0}^{\prime} \stackrel{\xi_{0}^{\prime-1}}{\longmapsto} M_{0}^{\prime \prime \prime} \stackrel{\xi_{0}^{\prime \prime}}{\longmapsto} M_{0}^{\prime \prime} \stackrel{\eta_{1}^{\prime}}{\longmapsto} M_{1}^{\prime} \stackrel{\xi_{1}^{\prime-1}}{\longmapsto} M_{1}^{\prime \prime \prime} \stackrel{\xi_{1}^{\prime \prime}}{\longmapsto} M_{1}^{\prime \prime} \stackrel{\eta_{2}^{\prime}}{\longmapsto} \ldots \stackrel{\eta_{n}^{\prime}}{\longmapsto} M_{n}^{\prime} \stackrel{\xi_{n}^{\prime}-1}{\longmapsto} M_{n}^{\prime \prime \prime} \stackrel{\xi_{n}^{\prime \prime}}{\longmapsto} M_{n}^{\prime \prime}
$$

except for the first and the last ones are very flexible. After decomposing each transformation $M_{j}^{\prime} \stackrel{\xi_{j}^{\prime-1}}{\longmapsto}$ $M_{j}^{\prime \prime \prime}$ in (9.12) into a sequence of extension moves, and each transformation $M_{j}^{\prime \prime \prime} \stackrel{\xi_{j}^{\prime \prime}}{\longmapsto} M_{j}^{\prime \prime}$ into a sequence of elimination moves, we get an almost $C$-neat decomposition of the transformation $M_{0}^{\prime} \stackrel{{ }^{\chi}}{\longmapsto} M_{n}^{\prime \prime}$, where $\chi=$ $\xi_{n}^{\prime \prime} \circ \xi_{n}^{\prime-1} \circ \eta_{n}^{\prime} \circ \ldots \circ \eta_{2}^{\prime} \circ \xi_{1}^{\prime \prime} \circ \xi_{1}^{\prime-1} \circ \eta_{1}^{\prime} \circ \xi_{0}^{\prime \prime} \circ \xi_{0}^{\prime-1}$. We also recall that the diagram $M_{0}^{\prime}$ is + -flexible and very --flexible relative to $C_{0}$, whereas $M_{n}^{\prime \prime}$ is --flexible and very +-flexible relative to $C_{n}$.

Thus, without loss of generality, we may assume the following from the beginning:

(1) the diagram $M$ is +-flexible and very --flexible relative to $C_{0}=C$;

(2) the diagram $M^{\prime}$ is --flexible and very + -flexible relative to $C_{n}=C^{\prime}$;

(3) the decomposition (9.10) is almost $C$-neat.

In what follows we will modify this decomposition so that these conditions will always hold. Due to Lemma 9.17, in which, clearly, the roles of $M_{0}$ and $M_{n}$ can be exchanged and +-flexibility can be replaced with --flexibility, guarantees that all diagrams which will arise in our decompositions will be flexible relative to the corresponding collection of selected boundary circuits.

Now we use Lemmas 9.11, 7.5, 7.3, and 7.7 to modify the sequence (9.10) so that it includes only jump moves, type I elementary moves, type II merge moves, and type II elementary bypass removals. Type II merge moves and type II elementary bypass removals are particular cases of generalized type II merge moves and generalized type II bypass removals, which are collectively called generalized type II moves.

We are ready to follow the strategy outlined in Subsection 9.1. Namely, we deal with sequences $s$ of moves that have the following properties:

(1) $s$ transforms $M$ to $M^{\prime}$ and induces the same morphism from $\widehat{M}$ to $\widehat{M}^{\prime}$ as the original sequence does;

(2) $s$ consists of two successive parts, $s_{1}$ and $s_{2}$, such that $s_{1}$ includes only jump moves, type I elementary moves, and generalized type II moves, whereas $s_{2}$ includes only type II elementary moves;

(3) once all generalized type II moves in $s$ are neatly decomposed into elementary moves, the obtained sequence will be $C$-delicate.

The complexity of such a sequence is defined as the pair $\left(N_{1}, N_{2}\right)$ in which $N_{1}$ is the number of generalized type II moves in $s_{1}$, and $N_{2}$ is the number of elementary type I moves and jump moves in $s_{1}$ that occur after all generalized type II moves. We order such pairs lexicographically, that is, $\left(N_{1}, N_{2}\right)<\left(N_{1}^{\prime}, N_{2}^{\prime}\right)$ if either $N_{1}<N_{1}^{\prime}$, or $N_{1}=N_{1}^{\prime}$ and $N_{2}<N_{2}^{\prime}$.

Suppose that $N_{2}>0$ and $N_{1}>0$. Let $M_{j-1} \stackrel{\eta_{j}}{\longmapsto} M_{j}$ be the last generalized type II move in $s_{1}$. We apply:

- Lemmas 9.3 and 9.4 and Proposition 9.7 if $M_{j-1} \stackrel{\eta_{j}}{\longmapsto} M_{j}$ is a generalized type II merge move and $M_{j} \stackrel{\eta_{j+1}}{\longmapsto} M_{j+1}$ is a jump move,

- Proposition 9.8 if $M_{j-1} \stackrel{\eta_{j}}{\longmapsto} M_{j}$ is a generalized type II merge move and $M_{j} \stackrel{\eta_{j+1}}{\longmapsto} M_{j+1}$ is a type I elementary move, 
- Proposition 9.12 if $M_{j-1} \stackrel{\eta_{j}}{\longmapsto} M_{j}$ is a generalized type II bypass removal and $M_{j} \stackrel{\eta_{j+1}}{\longmapsto} M_{j+1}$ is a jump move,

- Proposition 9.11 if $M_{j-1} \stackrel{\eta_{j}}{\longmapsto} M_{j}$ is a generalized type II bypass removal and $M_{j} \stackrel{\eta_{j+1}}{\longmapsto} M_{j+1}$ is a type I elementary move

to reduce $N_{2}$ while keeping $N_{1}$ fixed.

Suppose that $N_{2}=0$ and $N_{1}>0$. Let again $M_{j-1} \stackrel{\eta_{j}}{\longmapsto} M_{j}$ be the last generalized type II move in $s_{1}$. We apply:

- Proposition 9.9 if $M_{j-1} \stackrel{\eta_{j}}{\longmapsto} M_{j}$ is a non-special generalized type II merge move,

- Proposition 9.10 if $M_{j-1} \stackrel{\eta_{j}}{\longmapsto} M_{j}$ is a special generalized type II merge move,

- Proposition 9.13 if $M_{j-1} \stackrel{\eta_{j}}{\longmapsto} M_{j}$ is a generalized type II bypass removal

to reduce $N_{1}$.

By following these rules we eventually get $N_{1}=0$, in which case we are done.

We are left to treat the case when $M$ has spherical components. This case does not seem to be of any importance, so we will skip some details.

Let $B_{1}, \ldots, B_{k}$ be all spherical components of $M$, and let $B_{1}^{\prime}, \ldots, B_{k}^{\prime}$ be the respective components of $M^{\prime}$. Clearly, the morphism $\eta=\eta_{n} \circ \ldots \circ \eta_{1}: \widehat{M} \rightarrow \widehat{M}^{\prime}$ can be presented by a triple $\left(F, F^{\prime}, h\right)$ such that, for any $i=1, \ldots, k$, the connected component of $F$ (respectively, of $F^{\prime}$ ) containing $\Gamma_{\widehat{B}_{i}}$ (respectively, $\left.\Gamma_{\widehat{B}_{i}^{\prime}}\right)$ is a two-disc, which we denote by $D_{i}$ (respectively, by $D_{i}^{\prime}$ ).

Let $M_{*}$ (respectively, $M_{*}^{\prime}$ ) be the enhanced mirror diagram obtained from $M$ (respectively, from $M^{\prime}$ ) by declaring all the boundary circuits corresponding to the boundaries of $D_{i}$ 's (respectively, of $D_{i}^{\prime}$ 's) essential, and let $\eta_{*}$ be the morphism $\widehat{M}_{*} \rightarrow \widehat{M}_{*}^{\prime}$ represented by $\left(F, F^{\prime}, h\right)$. The spherical connected components $B_{1}, \ldots, B_{k}$ of $M$ will turn into disc components of $M_{*}$.

One can find a $C$-delicate decomposition of the transformation $M_{*} \stackrel{\eta_{*}}{\longmapsto} M_{*}^{\prime}$ by modifying the original decomposition (9.10). This can be easily derived from Theorem 6.1 and the obvious fact that the addition of a disc component to an enhanced mirror diagram is unique up to stable equivalence (relative to the family of all boundary circuits not belonging to the added disc component).

The diagram $M_{*}$ does not have spherical components, hence we can modify the decomposition of $M_{*} \stackrel{\eta_{*}}{\longmapsto}$ $M_{*}^{\prime}$ so that all type I elementary moves occur before all type II elementary moves. Finally, we convert the artificially created disc components of $M_{*}$ back to spherical components by declaring all their boundary circuits inessential, and do so with all the respective components of the diagrams through which we transform $M_{*}$ into $M_{*}^{\prime}$, thus obtaining the sought-for decomposition of the transformation $M \stackrel{\eta}{\mapsto} M^{\prime}$.

This completes the proof of the commutation theorems.

\section{Appendix A: Finding Realizations}

Here we present a manual check that certain dividing codes have only those realizations that are mentioned in the proof of Proposition 2.3. In many cases of interest, such a manual check is infeasible, but an exhaustive search of all realizations can be implemented on a computer and takes reasonable time to run [10].

Lemma A.1. Up to combinatorial equivalence, there exists only one realization of an admissible dividing configuration having the dividing code

$$
\begin{gathered}
\{(1,2,3,4),(5,6),(7,8,9,10),(11,12),(13,14),(15,16),(17,18)\}, \\
\{(9,12,5,4,7,14,15,18,3,6,13,8,17,16,11,10,1,2)\} .
\end{gathered}
$$

Proof. Figure A.1 shows again the realization that we already know from Figure 2.5. with vertical occupied levels labeled by uppercase letters and horizontal occupied levels by lowercase letters.

Any other realization of (A.1) must have the same number of vertical and horizontal occupied levels as the one in Figure A.1, and they must admit a labeling with the same incidence between rectangles and occupied levels as in Figure A.1 rectangle 1 must be $[I ; E] \times[d ; e]$, rectangle 2 must be $[E ; I] \times[e ; f]$, 


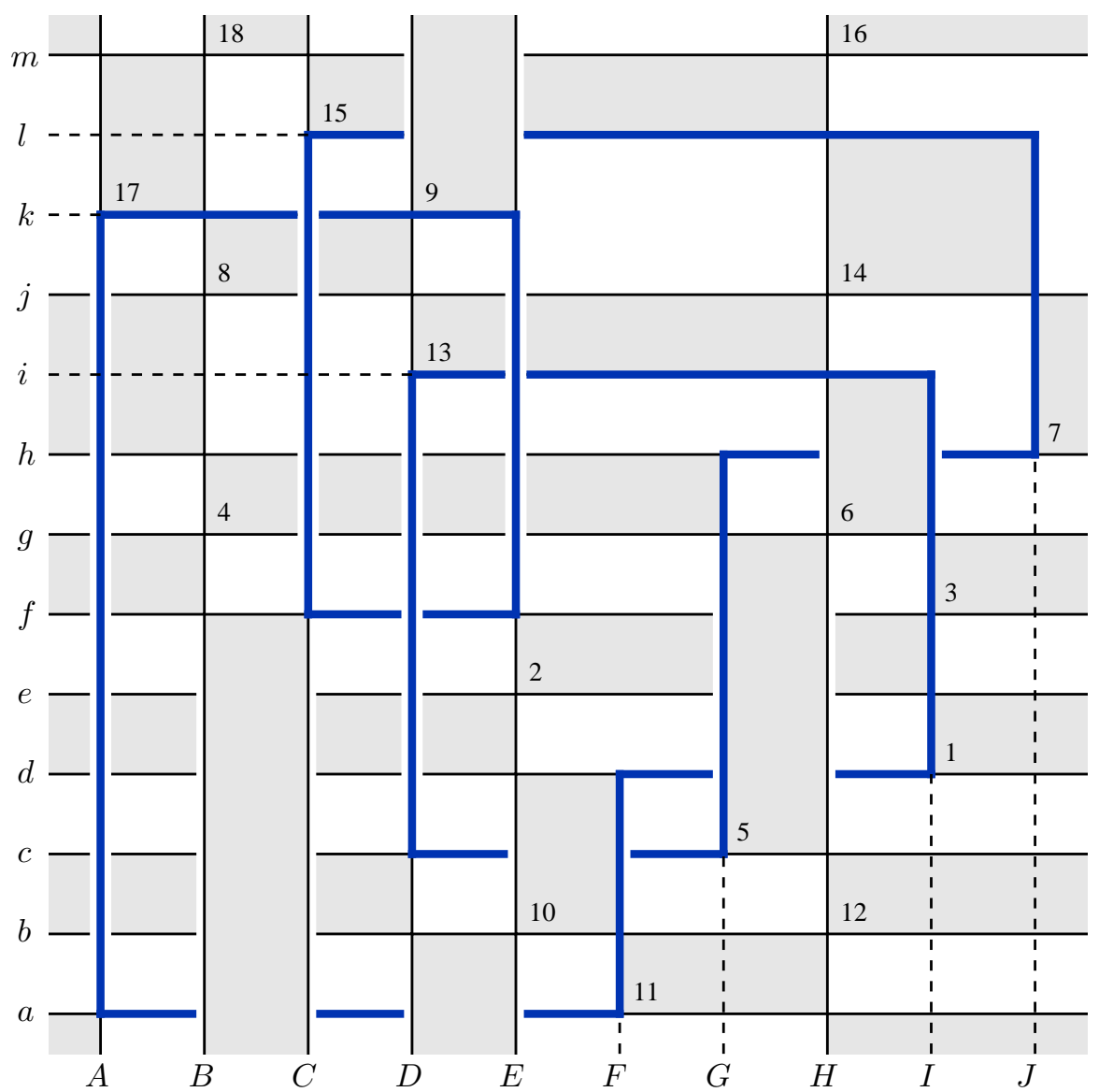

Figure A.1. A realization of A.1

etc. To find all realizations we have to search all possible ways to choose cyclic orderings of horizontal and vertical labels so that all rectangles are pairwise compatible.

To do so we introduce some notation. If $x, y, z, \ldots$ are labels of some occupied levels, we denote by $\langle x y z \ldots\rangle$ the statement that these levels follow in the indicated cyclic order.

Some information about the cyclic ordering is known from the beginning. For instance, the horizontal level $a$ in Figure A.1 contains three vertices of the diagram, $(A, a),(F, a),(H, a)$. These three vertices must follow at the level $a$ in this cyclic order for any realization of (A.1), for otherwise the rectangles 11 and 16, which have horizontal sides at the level $a$, would not be compatible. We abbreviate this implication as $a \Rightarrow\langle A F H\rangle$. A similar implication holds for each horizontal and vertical occupied level: the vertices at any level must follow in the same cyclic order for all realizations.

By $\mathrm{i}$, where $i$ is the number of a rectangle, we denote the statement that there are no vertices inside this rectangle. For instance, let this rectangle be $[X ; Y] \times[x ; y]$, and let there be a vertex at $(Z, z)$. Assume that $\langle X Z Y\rangle$ has already been established. Then $\langle x z y\rangle$ would imply that $(Z, z)$ lies inside the rectangle. Since this is forbidden, we conclude $\langle x y z\rangle$. We abbreviate this argument as $\mathrm{i} \&\langle X Z Y\rangle \Rightarrow\langle x y z\rangle$.

With this notation at hand the proof is as follows:

$$
\begin{gathered}
\text { argument } \\
b \Rightarrow\langle D E F H\rangle \\
i \Rightarrow\langle D H I\rangle \\
E \Rightarrow\langle b d e f k\rangle
\end{gathered}
$$

conclusion

$\langle D E F H I\rangle \&\langle b d e f k\rangle$ 


$$
\begin{aligned}
12 \&\langle D H I\rangle & \Rightarrow\langle b c d\rangle \\
B & \Rightarrow\langle f g h j k m\rangle \\
5 \&\langle c d g\rangle & \Rightarrow\langle F G H\rangle \\
13 \&\langle D G H\rangle & \Rightarrow\langle h i j\rangle \\
H & \Rightarrow\langle a b c j l m\rangle \\
6 \&\langle g h i\rangle & \Rightarrow\langle H I J\rangle \\
j & \Rightarrow\langle B D H J\rangle \\
9 \&\langle b k m\rangle & \Rightarrow\langle C D E\rangle \\
f & \Rightarrow\langle B C E\rangle \\
8 \&\langle B C D\rangle & \Rightarrow\langle j k l\rangle \\
m & \Rightarrow\langle A B H\rangle \\
14 \&\langle j k l\rangle & \Rightarrow\langle A H J\rangle
\end{aligned}
$$

$\langle D E F H I\rangle \&\langle b c d e f k\rangle$

$\langle D E F H I\rangle \&\langle b c d e f g h j k\rangle \&\langle f k m\rangle$

$\langle D E F G H I\rangle \&\langle b c d e f g h j k\rangle \&\langle f k m\rangle$

$\langle D E F G H I\rangle \&\langle b c d e f g h i j k\rangle \&\langle f k m\rangle$

$\langle D E F G H I\rangle \&\langle a b c d e f g h i j k m\rangle \&\langle j l m\rangle$

$\langle D E F G H I\rangle \&\langle H I J\rangle \&\langle a b c d e f g h i j k m\rangle \&\langle j l m\rangle$

$\langle B D E F G H I J\rangle \&\langle a b c d e f g h i j k m\rangle \&\langle j l m\rangle$

$\langle B D E F G H I J\rangle \&\langle C D E\rangle \&\langle a b c d e f g h i j k m\rangle \&\langle j l m\rangle$

$\langle B C D E F G H I J\rangle \&\langle a b c d e f g h i j k m\rangle \&\langle j l m\rangle$

$\langle B C D E F G H I J\rangle \&\langle a b c d e f g h i j k l m\rangle$

$\langle B C D E F G H I J\rangle \&\langle A B H\rangle \&\langle a b c d e f g h i j k l m\rangle$

$\langle A B C D E F G H I J\rangle \&\langle a b c d e f g h i j k l m\rangle$.

We see that we are left with a single option, which is the one we started with.

Lemma A.2. Up to combinatorial equivalence, there exist exactly two realizations of an admissible dividing configuration having the dividing code

$$
\begin{gathered}
\{(14,9,22,1,18,5),(2,21,10,17),(16,11,20,3),(4,19),(6,13),(12,7),(8,15)\} \\
\{(1,2,3,4,5,6,7,8,9,10,11,12,13,14,15,16,17,18,19,20,21,22)\} .
\end{gathered}
$$

Proof. We use the same notation system as in the proof of Lemma A.1. The labeling of the occupied levels is shown in Figure A.2.

The reasoning here is slightly more complicated because at some point we need to consider two cases, $\langle C M E\rangle$ and $\langle C E M\rangle$. In the former we get a contradiction. Here is the complete proof:

$$
\begin{aligned}
& \text { argument } \\
& g \Rightarrow\langle C E J\rangle \\
& c \Rightarrow\langle E H J M\rangle \\
& \text { assume }\langle C M E\rangle \\
& h \Rightarrow\langle B C E\rangle \\
& K \Rightarrow\langle e j k\rangle \\
& \text { conclusion } \\
& \langle C E J\rangle \\
& \langle C E H J\rangle \&\langle E J M\rangle \\
& \langle C M E H J\rangle \\
& \langle C M E H J\rangle \&\langle B C M E\rangle \\
& \langle C M E H J\rangle \&\langle B C M E\rangle \&\langle e j k\rangle \\
& 20 \&\langle B M E\rangle \Rightarrow\langle a k j\rangle \\
& \langle C M E H J\rangle \&\langle B C M E\rangle \&\langle a k e j\rangle \\
& E \Rightarrow\langle c e k\rangle \\
& \langle C M E H J\rangle \&\langle B C M E\rangle \&\langle a k c e j\rangle \\
& j \Rightarrow\langle C F M\rangle \\
& 21 \&\langle a k c\rangle \Rightarrow\langle F J M\rangle \\
& \text { hence }\langle C E M\rangle \\
& J \Rightarrow\langle a c f g\rangle \\
& \langle C F M E H J\rangle \&\langle B C M E\rangle \&\langle a k c e j\rangle \\
& \text { a contradiction } \\
& \langle C E H J M\rangle \\
& \langle C E H J M\rangle \&\langle a c f g\rangle \\
& 10 \&\langle C E M\rangle \Rightarrow\langle c e f\rangle \\
& \langle C E H J M\rangle \&\langle a c e f g\rangle \\
& 22 \&\langle c e f\rangle \Rightarrow\langle G H J\rangle \\
& \langle C E H J M\rangle \&\langle G H J\rangle \&\langle a c e f g\rangle \\
& g \Rightarrow\langle E G I J\rangle \\
& j \Rightarrow\langle B C M\rangle \\
& \langle C E G H J M\rangle \&\langle G I J\rangle \&\langle a c e f g\rangle \\
& \langle B C E G H J M\rangle \&\langle G I J\rangle \&\langle a c e f g\rangle \\
& 3 \&\langle B E I\rangle \Rightarrow\langle a b c\rangle \\
& \langle B C E G H J M\rangle \&\langle G I J\rangle \&\langle a b c e f g\rangle \\
& e \Rightarrow\langle G K M\rangle \quad\langle B C E G H J M\rangle \&\langle G I J\rangle \&\langle G K M\rangle \&\langle a b c e f g\rangle
\end{aligned}
$$




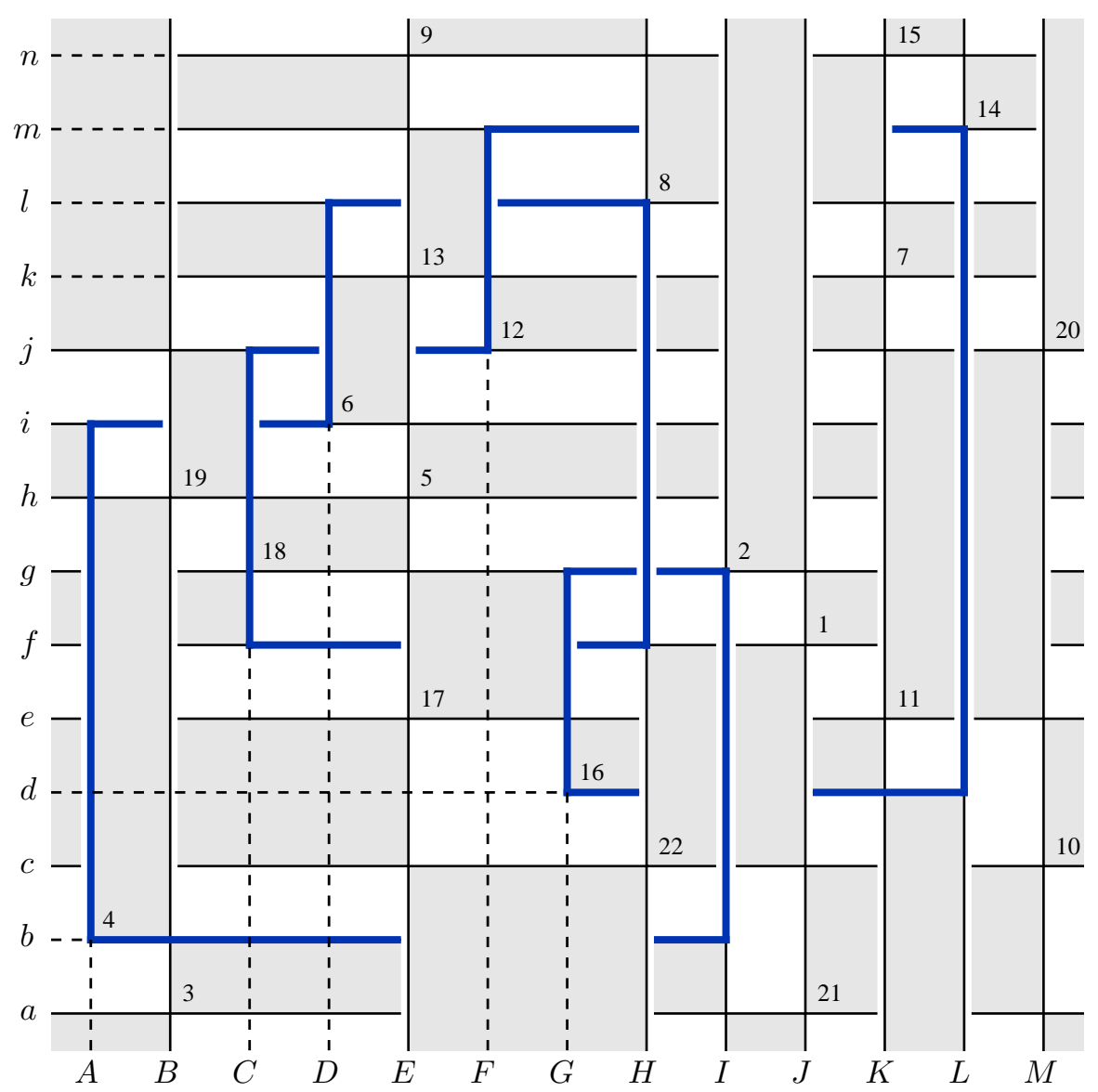

Figure A.2. A realization of A.2

$$
\begin{aligned}
& n \Rightarrow\langle E H K\rangle \quad\langle B C E G H J M\rangle \&\langle G I J\rangle \&\langle H K M\rangle \&\langle a b c e f g\rangle \\
& 22 \&\langle c e f\rangle \Rightarrow\langle H J K\rangle \quad\langle B C E G H J K M\rangle \&\langle G I J\rangle \&\langle a b c e f g\rangle \\
& E \Rightarrow\langle c g h k n\rangle \quad\langle B C E G H J K M\rangle \&\langle G I J\rangle \&\langle\text { abcefg }\rangle \text { \& }\langle\text { cgn }\rangle \\
& 21 \&\langle J K M\rangle \Rightarrow\langle a c n\rangle \quad\langle B C E G H J K M\rangle \&\langle G I J\rangle \&\langle a b c e f g h k n\rangle \\
& 9 \&\langle a c n\rangle \Rightarrow\langle E H I\rangle \quad\langle B C E G H I J K M\rangle \&\langle a b c e f g h k n\rangle \\
& 16 \&\langle G H K\rangle \Rightarrow\langle c d e\rangle \quad\langle B C E G H I J K M\rangle \&\langle\text { abcdefghkn }\rangle \\
& 15 \&\langle c d n\rangle \Rightarrow\langle K L M\rangle \quad\langle B C E G H I J K L M\rangle \&\langle a b c d e f g h k n\rangle \\
& K \Rightarrow\langle e j k l n\rangle \quad\langle B C E G H I J K L M\rangle \&\langle a b c d e f g h k l n\rangle \&\langle e j k\rangle \\
& 4 \&\langle b c h\rangle \Rightarrow\langle A B M\rangle \quad\langle A B C E G H I J K L M\rangle \&\langle a b c d e f g h k l n\rangle \&\langle e j k\rangle \\
& i \Rightarrow\langle A D E\rangle \quad\langle A B C E G H I J K L M\rangle \&\langle A D E\rangle \&\langle a b c d e f g h k l n\rangle \&\langle e j k\rangle \\
& 14 \&\langle D E L\rangle \Rightarrow\langle l m n\rangle \quad\langle A B C E G H I J K L M\rangle \&\langle A D E\rangle \&\langle\text { abcdefghklmn }\rangle \&\langle\text { ejk }\rangle \\
& 5 \&\langle A E H\rangle \Rightarrow\langle\text { hil }\rangle \quad\langle A B C E G H I J K L M\rangle \&\langle A D E\rangle \&\langle\text { abcdefghklmn }\rangle \&\langle\text { hil }\rangle \&\langle e j k\rangle \\
& D \Rightarrow\langle i k l\rangle \quad\langle A B C E G H I J K L M\rangle \&\langle A D E\rangle \&\langle\text { abcdefghiklmn }\rangle \text { \& }\langle\text { ejk }\rangle \\
& 20 \&\langle A B M\rangle \Rightarrow\langle a i j\rangle \quad\langle A B C E G H I J K L M\rangle \&\langle A D E\rangle \&\langle\text { abcdefghijklmn }\rangle
\end{aligned}
$$




$$
\begin{aligned}
& 6 \&\langle i j k\rangle \Rightarrow\langle C D E\rangle \quad\langle A B C D E G H I J K L M\rangle \&\langle\text { abcdefghijklmn }\rangle \\
& 13 \&\langle k l m\rangle \Rightarrow\langle E F H\rangle \quad\langle A B C D E G H I J K L M\rangle \&\langle E F H\rangle \&\langle a b c d e f g h i j k l m n\rangle \text {. }
\end{aligned}
$$

We are left with two options shown in Figure 2.8.

\section{Appendix B: Flypes}

This section is included in the paper for completeness of the exposition. It is moved to the very end of the paper because flypes are not involved in establishing our main result.

Definition B.1. Let $\Pi$ be a rectangular diagram of a surface, and let $\theta_{1}, \theta_{2}, \theta_{3}, \varphi_{1}, \varphi_{2}, \varphi_{3} \in \mathbb{S}^{1}$ be such that

(1) $\theta_{2} \in\left(\theta_{1} ; \theta_{3}\right), \varphi_{2} \in\left(\varphi_{1} ; \varphi_{3}\right)$;

(2) points $v_{1}, v_{2}, v_{3}, v_{4}, v_{5} \in \mathbb{T}^{2}$ having coordinates $\left(\theta_{1}, \varphi_{3}\right),\left(\theta_{2}, \varphi_{3}\right),\left(\theta_{3}, \varphi_{3}\right),\left(\theta_{3}, \varphi_{2}\right),\left(\theta_{3}, \varphi_{1}\right)$, respectively, are vertices of $\Pi$, and, moreover, $v_{1}, v_{3}, v_{5}$ are /-vertices, and $v_{2}, v_{4}$ are \-vertices;

(3) $v_{2}, v_{3}$, and $v_{4}$ do not belong to $\partial \Pi$;

(4) there are no more vertices of $\Pi$ in $\left[\theta_{1} ; \theta_{3}\right] \times\left[\varphi_{1} ; \varphi_{3}\right]$.

These assumptions imply that $\Pi$ contains, among others, four rectangles of the following form:

$$
r_{1}=\left[\theta_{1} ; \theta_{2}\right] \times\left[\varphi^{\prime} ; \varphi_{3}\right], \quad r_{2}=\left[\theta_{2} ; \theta_{3}\right] \times\left[\varphi_{3} ; \varphi^{\prime \prime}\right], \quad r_{3}=\left[\theta_{3} ; \theta^{\prime \prime}\right] \times\left[\varphi_{2} ; \varphi_{3}\right], \quad r_{4}=\left[\theta^{\prime} ; \theta_{3}\right] \times\left[\varphi_{1} ; \varphi_{2}\right]
$$

with some $\theta^{\prime}, \theta^{\prime \prime} \in\left(\theta_{3} ; \theta_{1}\right), \varphi^{\prime}, \varphi^{\prime \prime} \in\left(\varphi_{3} ; \varphi_{1}\right)$.

Let $\Pi^{\prime}$ be obtained from $\Pi$ by replacing these four rectangles with the following ones:

$$
r_{1}^{\prime}=\left[\theta_{1} ; \theta_{2}\right] \times\left[\varphi^{\prime} ; \varphi_{1}\right], \quad r_{2}^{\prime}=\left[\theta_{2} ; \theta_{3}\right] \times\left[\varphi_{1} ; \varphi^{\prime \prime}\right], \quad r_{3}^{\prime}=\left[\theta_{1} ; \theta^{\prime \prime}\right] \times\left[\varphi_{2} ; \varphi_{3}\right], \quad r_{4}^{\prime}=\left[\theta^{\prime} ; \theta_{1}\right] \times\left[\varphi_{1} ; \varphi_{2}\right] ;
$$

see Figure B.1.

Then we say that the passage from $\Pi$ to $\Pi^{\prime}$, or the other way, is a type I flype. Note that the other rectangles of $\Pi$ and $\Pi^{\prime}$, not shown in Figure B.1, are allowed to pass through $\left[\theta_{1} ; \theta_{3}\right] \times\left[\varphi_{1} ; \varphi_{3}\right]$, the region where the modification occurs.

A type II flype is defined by reversing the $\theta$-direction in the definition of a type I flype and exchanging the types of vertices: $\backslash \leftrightarrow /$.

Lemma B.1. Let $\Pi \mapsto \Pi^{\prime}$ be a flype, and let $\left(\delta_{+}, \delta_{-}\right),\left(\delta_{+}^{\prime}, \delta_{-}^{\prime}\right)$ be canonic dividing configurations of $\widehat{\Pi}$ and $\widehat{\Pi}^{\prime}$, respectively. Then there is an isotopy fixed on $\widehat{\Pi} \cap \widehat{\Pi}^{\prime}$ that brings $\left(\widehat{\Pi}, \delta_{+}\right)$to $\left(\widehat{\Pi}^{\prime}, \delta_{+}^{\prime}\right)$ if the flype is of type I, and $\left(\widehat{\Pi}, \delta_{-}\right)$to $\left(\widehat{\Pi}^{\prime}, \delta_{-}^{\prime}\right)$ if the flype is of type II. Moreover, the isotopy can be chosen to keep the surface in the class of Giroux's convex surfaces with respect to $\xi_{+}$if the flype is of type I, and with respect to $\xi_{-}$if the flype is of type II.

Proof. We use the notation from Definition B.1 and assume the flype is of type I (the type II case is similar). The surface $\widehat{\Pi}^{\prime}$ is obtained from $\widehat{\Pi}$ by replacing the union of tiles $d=\widehat{r}_{1} \cup \widehat{r}_{2} \cup \widehat{r}_{3} \cup \widehat{r}_{4}$ with $d^{\prime}=\widehat{r}_{1}^{\prime} \cup \widehat{r}_{2}^{\prime} \cup \widehat{r}_{3}^{\prime} \cup \widehat{r}_{4}^{\prime}$. One can see that both $d$ and $d^{\prime}$ are two-discs tangent to one another along their common boundary $\partial d=\partial d^{\prime}$. One can also see that they enclose a three-ball $B$ whose interior is disjoint from the common part of the surfaces $\widehat{\Pi}$ and $\widehat{\Pi}^{\prime}$. This implies the existence of an isotopy from $\widehat{\Pi}$ to $\widehat{\Pi}^{\prime}$ relative to $\widehat{\Pi} \cap \widehat{\Pi}^{\prime}$. The fact that such an isotopy brings $\delta_{+}$to an abstract dividing set isotopic to $\delta_{+}^{\prime}$ can be learned from Figure B.1.

The second assertion of the lemma follows from the fact that the boundary $\partial d$ is Legendrian, and a(ny) dividing set of $\widehat{\Pi}$ (with respect to $\xi_{+}$) intersects $d$ in two arcs. The technique of [27] allows to show that an isotopy of such a disc preserving its boundary and the tangent plane at every point of the boundary can be $C^{0}$-approximated by an isotopy within the class of Giroux's convex surfaces.

For more detail see [12, Lemma 6], where a very little modification of the proof is needed to obtain a proof of the second part of Lemma B.1. Namely, in the final part of the proof we conclude that all singularities in the interior of the disc disappear because there is just one dividing arc. This implies that 1 -arc also disappears. 

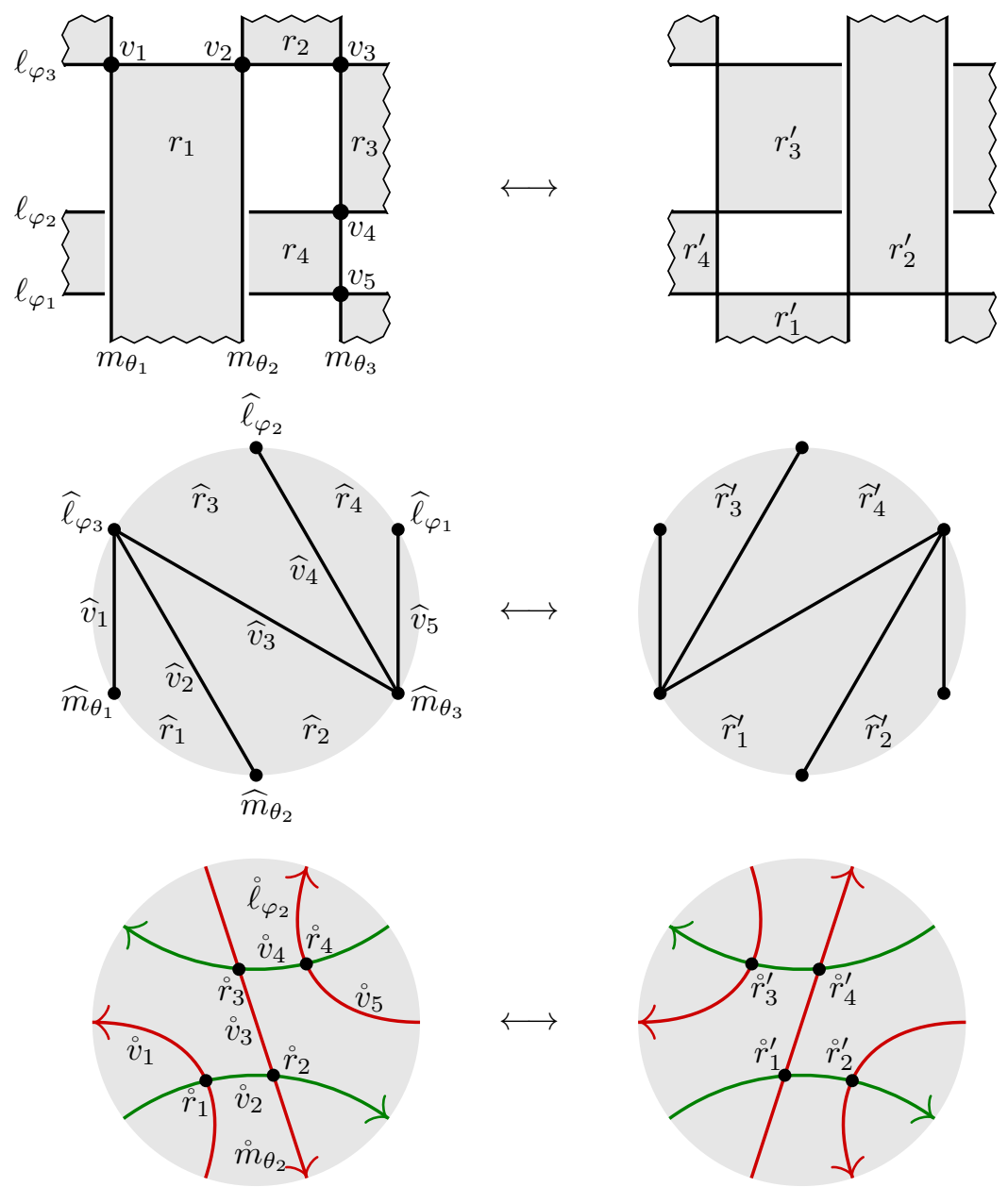

Figure B.1. A type I flype

In the present context we have two dividing arcs instead of just one, which means that, after reducing all nodes in the interior of the disc, only one saddle singularity is left, which is still not enough for the 1 -arc to survive. So the concluding argument of the proof is still valid.

Unlike (de)stabilizations, flypes of rectangular diagrams of surfaces do not necessarily change the equivalence class of the corresponding surface viewed as a Giroux's convex surface with respect to one of the contact structures $\xi_{+}$or $\xi_{-}$. From the contact topology point of view flypes of rectangular diagrams of surfaces are bypass attachments introduced by K. Honda in [28]. The concept of an ineffective flype defined below is a combinatorial counterpart of a trivial bypass attachment from [29].

Definition B.2. We use the notation from Definition B.1. We also denote by $\left(\delta_{+}, \delta_{-}\right)$a canonic dividing configuration of $\widehat{\Pi}$. The type I flype $\Pi \mapsto \Pi^{\prime}$ is called ineffective if there is an embedded disc $b \subset \widehat{\Pi}$ with boundary consisting of two arcs $\alpha$ and $\beta$ such that (consult Figures B.1 and B.2):

(1) $\alpha \subset \delta_{-} \backslash\left(\stackrel{\circ}{v}_{1} \cup \stackrel{\circ}{v}_{5}\right)$;

(2) either $\beta=\stackrel{\circ}{4}_{4}$ and $b \supset{\stackrel{\circ}{\varphi_{2}}}_{2}$, or $\beta=\stackrel{\circ}{2}_{2}$ and $b \supset{\stackrel{\circ}{m_{2}}}_{\theta_{2}}$ (note that $b$ can have more intersections

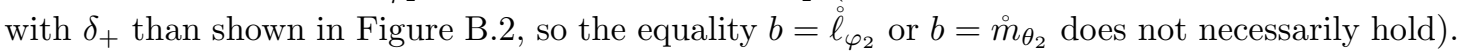

Otherwise the flype is called effective.

Effective and ineffective flypes of type II are defined similarly with the roles of $\delta_{-}$and $\delta_{+}$exchanged. 

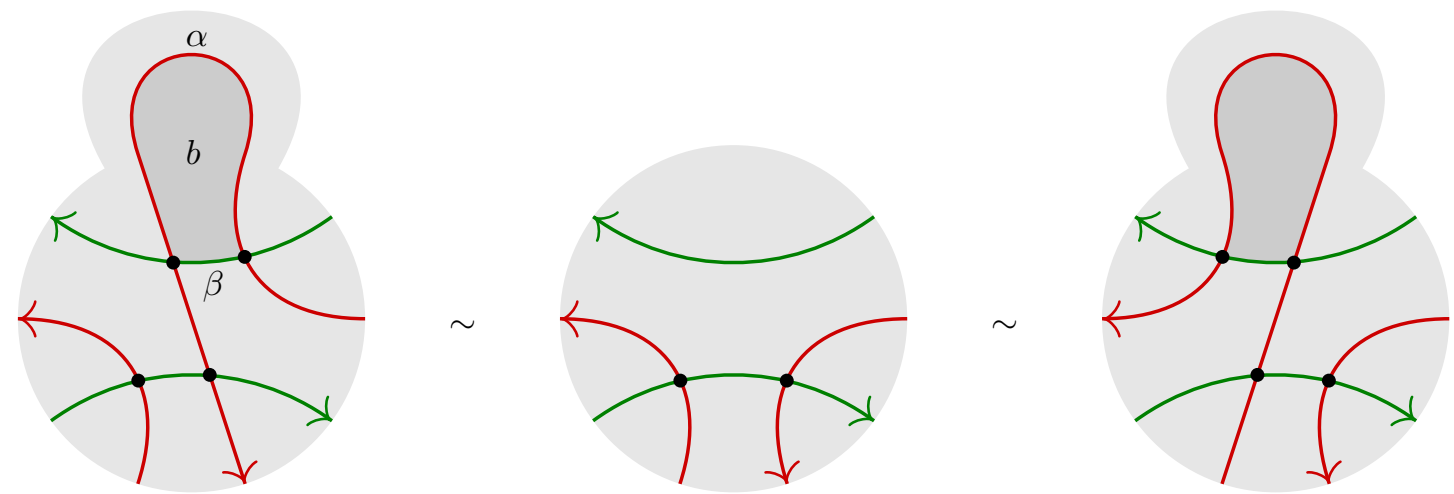

FiguRE B.2. Ineffective flype has no effect on the weak equivalence class of a canonic dividing configuration

One can see that the inverse flype of an ineffective one is also ineffective.

Proposition B.1. If $\Pi \mapsto \Pi^{\prime}$ is an ineffective flype, then $\Pi^{\prime}$ can be obtained from $\Pi$ be a finite sequence of exchange, wrinkle creation, and wrinkle reduction moves.

An ineffective flype has no effect on the equivalence class of $\widehat{\Pi}$ viewed as a Giroux's convex surface with respect to either of the contact structures $\xi_{+}$and $\xi_{-}$.

Proof. We show how to modify $\Pi$ and $\Pi^{\prime}$ by exchange and wrinkle reduction and creation moves so that they eventually coincide. We use the notation from Definitions B.1 and B.2. We may assume that $\beta=\dot{v}_{4}$ and $b \supset \dot{\ell}_{\varphi_{2}}$, as the other case is symmetric to this one.

The interior of the disc $b$ may have nontrivial intersection with $\delta_{+}$. In this case, there are bigons of $\delta_{+}$ and $\delta_{-}$in $b$ smaller than $b$. Denote by $D$ the dividing configuration obtained from $\left(\delta_{+}, \delta_{-}\right)$by reducing all bigons in $b$ including $b$ itself.

Suppose for the moment that the dividing configuration $D$ is admissible.

The proof is by induction in $k$, where $k$ is the number of intersections of the interior of $\alpha$ with $\delta_{+}$. If $k=0$, then $b$ is a bigon with corners at $\dot{r}_{3}$ and $\dot{r}_{4}$. The rectangles $r_{3}$ and $r_{4}$ share two vertices at the level $\ell_{\varphi_{2}}$. The same holds for the rectangles $r_{3}^{\prime}$ and $r_{4}^{\prime}$, and there is a respective bigon $b^{\prime}$ of a canonic dividing configuration $\left(\delta_{+}^{\prime}, \delta_{-}^{\prime}\right)$ of $\widehat{\Pi}^{\prime}$.

Reducing the bigons $b$ and $b^{\prime}$ in $\widehat{\Pi}$ and $\widehat{\Pi}^{\prime}$, respectively, as described in the proof of Lemma 3.8 produces identical diagrams from $\Pi$ and $\Pi^{\prime}$. So, the induction base $k=0$ is settled.

If $k>0$, then there must be a bigon $b_{0} \subset b$ of $\left(\delta_{+}, \delta_{-}\right)$with corners $\dot{r}^{\prime}, \dot{r}^{\prime \prime}$ distinct from $\dot{r}_{i}, i=1,2,3,4$. The rectangles $r^{\prime}$ and $r^{\prime \prime}$ are not affected by the flype, so we have $r^{\prime}, r^{\prime \prime} \in \Pi^{\prime}$, and there is a respective bigon $b_{0}^{\prime}$ of $\left(\delta_{+}^{\prime}, \delta_{-}^{\prime}\right)$.

The reduction of the bigons $b_{0}$ and $b_{0}^{\prime}$ made by following the recipe from the proof of Lemma 3.8 produces diagrams that are still related by a flype. Indeed, let $S$ be the minimal strip of the form $\left[\theta^{\prime} ; \theta^{\prime \prime}\right] \times$ $\mathbb{S}^{1}$ or $\mathbb{S}^{1} \times\left[\varphi^{\prime} ; \varphi^{\prime \prime}\right]$ containing $r^{\prime}$ and $r^{\prime \prime}$. If $S$ is disjoint from the site of the flype, which is $R=$ $\left[\theta_{1} ; \theta_{3}\right] \times\left[\varphi_{1} ; \varphi_{3}\right]$, then the bigon reductions do not interfere with the flype.

An interference occurs when either $v_{1}$ or $v_{5}$ is a vertex of $r^{\prime}$ or $r^{\prime \prime}$, or some of the vertices $v_{i}, i=$ $1,2,3,4,5$, are contained in one of the blocks that are exchanged in the course of the bigon reduction (they are denoted by $X$ and $Y$ in Figure 3.8), see Figure B.3. One can see that this interference is not essential. The moves applied to reduce the bigon, change only the shape of the region where the flype occurs but preserve all the flype preconditions.

The flype we arrive at after reducing the bigons $b_{0}$ and $b_{0}^{\prime}$ is still inessential, and $k$ is reduced. Hence the induction step follows.

Now we reduce the general case to the one considered above, where $D$ was admissible. Denote by $v_{6}$ and $v_{7}$ the vertices opposite to $v_{4}$ in the rectangles $r_{3}$ and $r_{4}$, respectively. Let $D_{1}$ be a dividing 

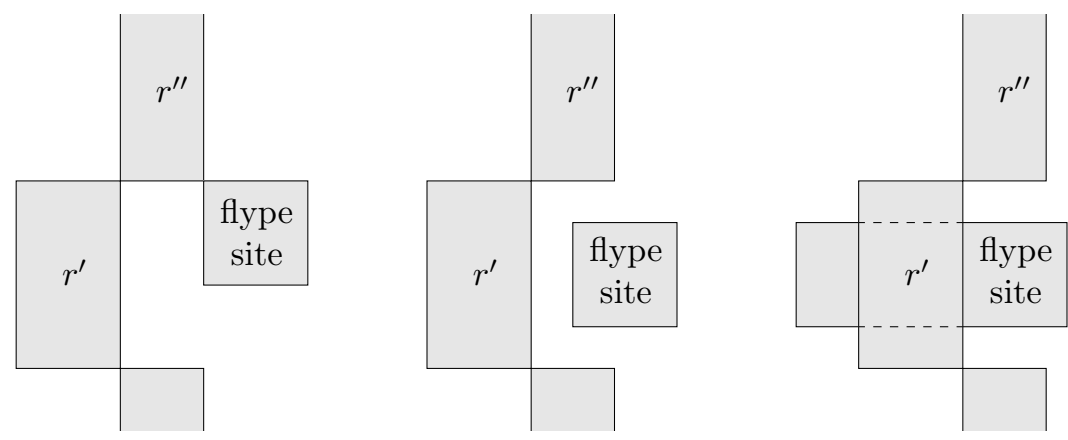

Figure B.3. Possible mutual positions of the flype site and the "large wrinkle"

configuration obtained from $\left(\delta_{+}, \delta_{-}\right)$by replacing a subarc of $\stackrel{\circ}{v}_{5}$ by an arc that intersects $\stackrel{\circ}{7}_{7}$, then goes 'parallel' to $\alpha$ until it intersects $\dot{v}_{6}$, then turns around and goes back remaining close to $\alpha$, intersects $\dot{v}_{7}$ again, and finally arrives at an endpoint of $\dot{v}_{5}$; see Figure B.4.
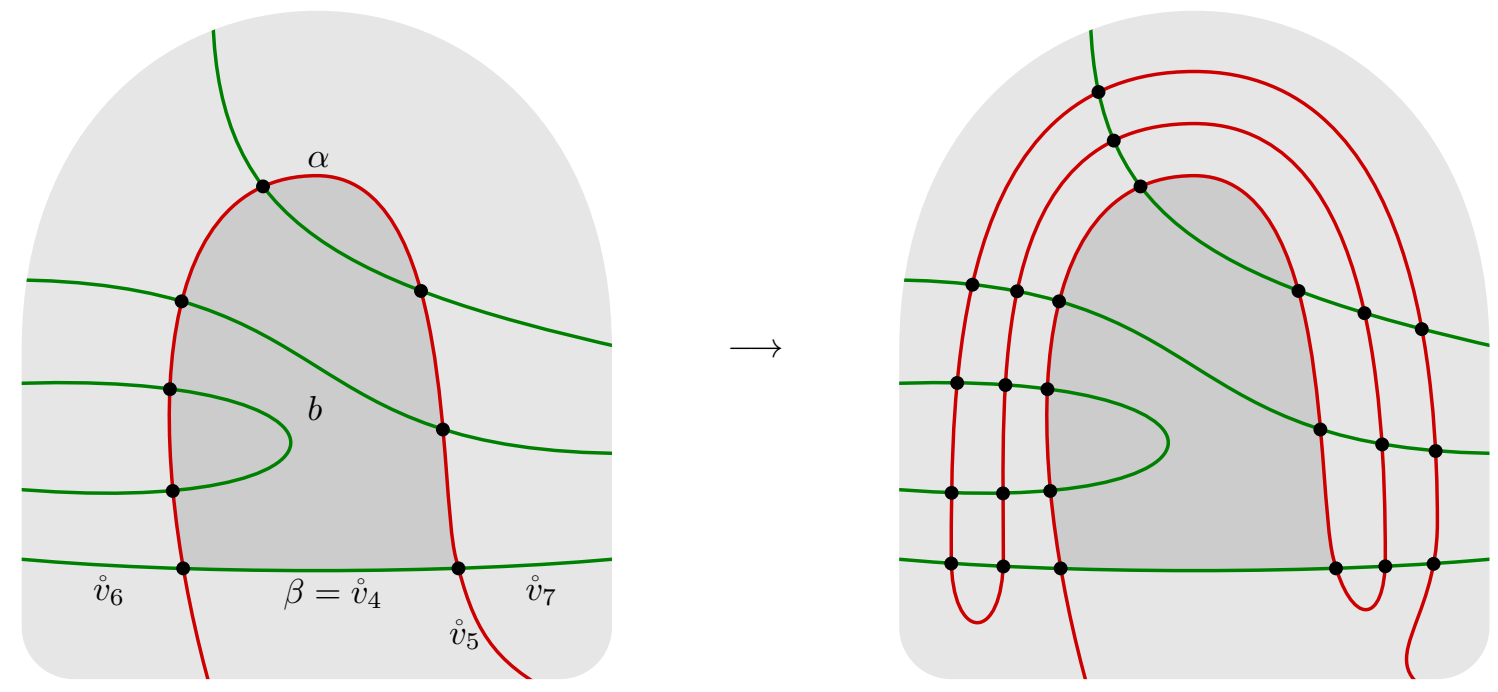

FIGURE B.4. Making the dividing configuration which is obtained by reducing all bigons in $b$ admissible

The dividing configuration $D_{1}$ is obtained from $\left(\delta_{+}, \delta_{-}\right)$by bigon creations, which, by Lemma 3.2, can be realized by wrinkle creations in $\Pi$. These wrinkle creations do not interfere with the flype we consider, which means that the same wrinkle creations can be made in $\Pi^{\prime}$ so that the new $\Pi$ and $\Pi^{\prime}$ will be still related by an ineffective flype. Reducing all bigons of $D_{1}$ contained in $b$, including $b$ itself, now produces an admissible configuration, which completes the proof.

\section{ACKNOWLEDGEMENTS}

We are indebted to our anonymous referees for their careful reading of our paper and valuable suggestions for improving the exposition.

\section{REFERENCES}

[1] S. Baader, M. Ishikawa. Legendrian graphs and quasipositive diagrams. Ann. Fac. Sci. Toulouse Math. (6) 18 (2009), no. 2, 285-305; arXiv:math/0609592.

[2] D. Bennequin. Entrelacements et equations de Pfaff. (French) Asterisque 107-108 (1983), 87-161. 
[3] J. Birman, W. Menasco. Studying links via closed braids. IV. Composite links and split links. Invent. Math. 102 (1990), no. $1,115-139$.

[4] J. Birman, W. Menasco. Studying links via closed braids. V. The unlink. Trans. Amer. Math. Soc. 329 (1992), no. 2, 585-606.

[5] Y. Chekanov. Differential algebra of Legendrian links. Invent. Math. 150 (2002), no. 3, 441-483; arXiv:math/9709233.

[6] W. Chongchitmate, L. Ng. An atlas of Legendrian knots. Exp. Math. 22 (2013), no. 1, 26-37; arXiv:1010.3997.

[7] I.Dynnikov. Arc-presentations of links: Monotonic simplification, Fund.Math. 190 (2006), 29-76; arXiv:math/0208153.

[8] P. Cromwell. Embedding knots and links in an open book. I. Basic properties. Topology Appl. 64 (1995), no. 1, 37-58.

[9] I. Dynnikov. Recognition algorithms in knot theory. (Russian) Uspekhi Mat. Nauk 58 (2003), no. 6(354), 45-92; translation in Russian Math. Surveys 58 (2003), no. 6, 1093-1139.

[10] I. Dynnikov. A Python code for searching all realizations of a dividing configuration. https://arxiv.org/src/1712.06366v2/anc

[11] I. Dynnikov, M. Prasolov. Bypasses for rectangular diagrams. A proof of the Jones conjecture and related questions (Russian), Trudy MMO $\mathbf{7 4}$ (2013), no. 1, 115-173; translation in Trans. Moscow Math. Soc. 74 (2013), no. 2, 97-144; arXiv:1206.0898.

[12] I. Dynnikov, M. Prasolov. Rectangular diagrams of surfaces: representability. (Russian) Matem. Sb. 208 (2017), no. 6, 55-108; translation in Sb. Math. 208 (2017), no. 6, 781-841; arXiv:1606.03497.

[13] I. Dynnikov, M. Prasolov. Classification of Legendrian knots of topological type 76 with maximal Thurston-Bennequin number. J. Knot Theory Ramifications, 28 (2019), no. 14, 1950089; arXiv:1901.03600.

[14] I. Dynnikov, M. Prasolov. Rectangular diagrams of surfaces: the basic moves. Proc. Int. Geom. Cent., 13 (2020), no. 4 , 50-88; arXiv: 2011.04995.

[15] I. Dynnikov, V. Shastin. On equivalence of Legendrian knots. (Russian) Uspekhi Mat. Nauk 73 (2018), no. 6, 195-196; translation in Russian Math. Surveys, 73 (2018), no. 6, 1125-1127.

[16] I. Dynnikov, V. Shastin. Distinguishing Legendrian knots with trivial orientation-preserving symmetry group. Preprint, arXiv:1810.06460.

[17] Ya. Eliashberg. Invariants in contact topology, in: Proceedings of the International Congress of Mathematicians, Vol. II (Berlin, 1998). Doc. Math. 1998, Extra Vol. II, 327-338.

[18] Y. Eliashberg, M. Fraser. Classification of topologically trivial legendrian knots. CRM Proc. Lecture Notes 15 (1998), no. $15,17-51$

[19] Y. Eliashberg, M. Fraser. Topologically trivial Legendrian knots. J. Symplectic Geom. 7 (2009), no. 2, 77-127; arXiv:0801.2553.

[20] J. Etnyre, K. Honda. Knots and Contact Geometry I: Torus Knots and the Figure Eight Knot. J. Symplectic Geom. 1 (2001), no. 1, 63-120.

[21] J. Etnyre, D. LaFountain, B. Tosun. Legendrian and transverse cables of positive torus knots. Geom. Topol. 16 (2012), 1639-1689.

[22] J. Etnyre, L. Ng, V. Vértesi. Legendrian and transverse twist knots. JEMS 15 (2013), no. 3, 969-995; arXiv:1002.2400.

[23] J. Etnyre, V. Vértesi. Legendrian satellites. Int. Math. Res. Not. IMRN 2018, no. 23, 7241-7304; arXiv: 1608.05695.

[24] D. Fuchs. Chekanov-Eliashberg invariant of Legendrian knots: existence of augmentations. J. Geom. Phys. 47 (2003), no. $1,43-65$.

[25] E. Giroux. Convexité en topologie de contact, Comment. Math. Helv. 66 (1991), 637-677.

[26] E. Giroux. Structures de contact sur les variétés fibrées en cercles au-dessus d'une surface. Comment. Math. Helv. 76 (2001), no. 2, 218-262; arXiv:math/9911235.

[27] E. Giroux. Structures de contact en dimension trois et bifurcations des feuilletages de surfaces, Invent. Math. 141 (2000), no. 3, 615-689; arXiv:math/9908178.

[28] K. Honda. On the classification of tight contact structures. I. Geom. Topol. 4 (2000), 309-368; arXiv:math/9910127.

[29] K. Honda. Gluing tight contact structures. Duke Math. J. 115 (2002), no. 3, 435-478; arXiv:math/0102029.

[30] K. Honda, W. Kazez, G. Matić. Tight contact structures and taut foliations. Geom. Topol. 4 (2000), 219-242; arXiv:math/0010138.

[31] K. Kodama, M. Sakuma. Symmetry groups of prime knots up to 10 crossings. Knots 90 (Osaka, 1990), 323-340, de Gruyter, Berlin, 1992.

[32] P. Massot. Topological methods in 3-dimensional contact geometry, Contact and symplectic topology, 27-83, Bolyai Soc. Math. Stud., 26, János Bolyai Math. Soc., Budapest, 2014; arXiv:1303.1063.

[33] S. V. Matveev. Algorithmic topology and classification of 3-manifolds. Second edition. Algorithms and Computation in Mathematics, 9. Springer, Berlin, 2007. xiv+492 pp.

[34] W. Menasco. On iterated torus knots and transversal knots. Geom. Topol. 5 (2001), 651-682.

[35] K. Murasugi. On a certain subgroup of the group of an alternating link. Amer J. Math., 85 (1963), 544-550.

[36] L. Ng. Combinatorial Knot Contact Homology and Transverse Knots. Adv. Math. 227 (2011), no. 6, 2189-2219; arXiv:1010.0451.

[37] L. Ng. Computable Legendrian Invariants. Topology 42 (2003), no. 1, 55-82; arXiv:math/0011265.

[38] P. Ozsváth, Z. Szabó, D. Thurston. Legendrian Knots, Transverse Knots and Combinatorial Floer Homology. Geom. Topol. 12 (2008), no. 2, 941-980; arXiv:math/0611841. 
[39] M. Prasolov. Rectangular diagrams of Legendrian graphs. J. Knot Theory Ramifications 23 (2014), no. 13, 1450074, 28 pp; arXiv:1412.2267.

[40] P. Pushkar', Yu. Chekanov. Combinatorics of fronts of Legendrian links and the Arnol'd 4-conjectures. Uspekhi Mat. Nauk 60 (2005), no. 1, 99-154; translation in Russian Math. Surveys 60 (2005), no. 1, 95-149.

[41] M. Sakuma. The geometries of spherical Montesinos links. Kobe J. Math. 7 (1990), no. 2, 167-190.

[42] H. Seifert. Über das Geschlecht von Knoten. (German) Math. Ann. 110 (1935), no. 1, 571-592.

[43] J. Stallings. On fibering certain 3-manifolds. Topology of 3-manifolds and related topics (Proc. The Univ. of Georgia Institute, 1961), pp. 95-100, Prentice-Hall, Englewood Cliffs, N.J.

Steklov Mathematical Institute of Russian Academy of Sciences, 8 Gubkina Str., Moscow 119991, Russia

Email address: dynnikov@mech.math.msu.su

Email address: 0x00002a@gmail.com 